\title{
NOVEL, INTEGRATED REACTOR / POWER \\ CONVERSION SYSTEM \\ (LMR-AMTEC)
}

\author{
U. S. Department of Energy \\ Nuclear Energy Research Initiative \\ Project Number: 99-0198 \\ Research Grant Number: DE-FG03-99SF21987
}

FINAL SCIENTIFIC/TECHNICAL REPORT

REPORT NUMBER: STD-ES-03-04

March 31, 2003

Lead Organization: Westinghouse Electric Company, LLC

PI: Pablo Rubiolo

1344 Beulah Road

Pittsburgh, PA 15235-5083

Telephone: $412-256-1657$

Fax: $412-256-2444$

Email: rubiolpr@westinghouse.com

Collaborating Organizations:

Institute for Space and Nuclear Power Studies, UNM

Institute for Engineering Research and Applications, UNM 
[ This page intentionally blank ] 


\section{ABSTRACT}

The main features of this project were the development of a long life (up to 10 years) Liquid Metal Reactor (LMR) and a static conversion subsystem comprising an Alkali Metal Thermalto-Electric (AMTEC) topping cycle and a ThermoElectric (TE) Bottom cycle. Various coupling options of the LMR with the energy conversion subsystem were explored and, base in the performances found in this analysis, an Indirect Coupling (IC) between the LMR and the AMTEC/TE converters with Alkali Metal Boilers (AMB) was chosen as the reference design. The performance model of the fully integrated sodium-and potassium-AMTEC/TE converters shows that a combined conversion efficiency in excess of $30 \%$ could be achieved by the plant. 
[ This page intentionally blank ] 


\section{EXECUTIVE SUMMARY}

The overall objectives of this project were to assess the feasibility, develop a conceptual plant layout and engineering solutions, and determine a range of potential applications for a Novel Integrated Reactor/Energy Conversion System. The goal was to design a proliferation resistant, reliable and economical power supply for use by developing countries and in remote locations. The main features of this project were the development of a long life (up to 10 years) Liquid Metal Reactor (LMR) and a static conversion subsystem comprising an Alkali Metal Thermal to-Electric (AMTEC) topping cycle and a ThermoElectric (TE) Bottom cycle. Furthermore, various coupling options of the LMR with the energy conversion subsystem were explored.

The project was performed by the Westinghouse Electric Company LLC (WEC), which was responsible for the long-life sodium reactor development, the University of New Mexico's Institute for Space and Nuclear Power Studies (UNM-ISNPS), which was responsible for developing the AMTEC/TE energy conversion system and designing the electric converter modules, and the Institute for Engineering Research and Applications (IERA) at the New Mexico Institute of Mining and Technology, which was responsible for supporting Westinghouse's activities related to the transport safety and waste disposal.

The work performed by Westinghouse Electric Company LLC on the reactor design and coupling with the conversion modules included three major areas:

\section{1- Selection of the reference LMR-AMTEC design concept}

Different design options were evaluated using a plant model. An Indirect Coupling (IC) plant with Alkali Metal Boilers (AMB) (see Figure 1) was chosen as the reference design as it exhibited the best performance. The main features of the design are:

- IC between the LMR and the AMTEC units: two independent loops are employed.

- Sodium and potassium are used as primary and secondary coolants, respectively.

- The net plant efficiency is $28.2 \%$ when the core outlet temperature is $1070 \mathrm{~K}$.

- The LMR core is composed of 78 fuel elements and 78 reflector elements. The fuel is $(\mathrm{U}, \mathrm{Pu}) \mathrm{N}$ and the cladding is made of the refractory alloy $\mathrm{Nb}-1 \mathrm{Zr}$.

- The AMBs generate the potassium vapor, which is fed into the AMTEC units. The AMBs can operate in once-through or in recirculation mode (with a vapor separator).

- Pool configuration: the AMB and the primary pumps are placed inside the reactor vessel. Thus, no shielding either for the secondary pipes or the AMTEC/TE converter units is needed.

\section{2- Operating parameters of the LMR-AMTEC}

The following plant characteristics and components were determined and studied: working temperatures; flow rates and pressures; core design (fuel and cladding); alkali metal boiler design and operation; primary pumps characteristics; flow-induced 
vibrations in fuel elements and AMB tubes; corrosion allowance; reactor vessel design; and in-vessel layout. A preliminary economical analysis of the plant was also performed.

\section{3- Safety features of the LMR-AMTEC}

The work performed related to the safety aspects of the LMR-AMTEC system included:

- Reactivity control systems : an actively controlled absorber rod bundle assembly was adopted as the reactivity control system. Boron carbide was selected as the Control Rod (CR) absorber material. Studies of the reactivity behavior upon sodium removal showed an acceptable core reactivity response.

- Heat removal systems: The secondary loop, composed of the alkali metal boilers and the AMTEC/TE converter units, was chosen as a normal Decay Heat Removal System (DHRS) of non-safety grade during bt and cold shutdowns. In addition, a safety grade Passive Heat Removal System (PHRS) was proposed as an emergency DHRS. The PHRS of the LMR-AMTEC is activated by the change in the sodium level after trip of the primary pumps.

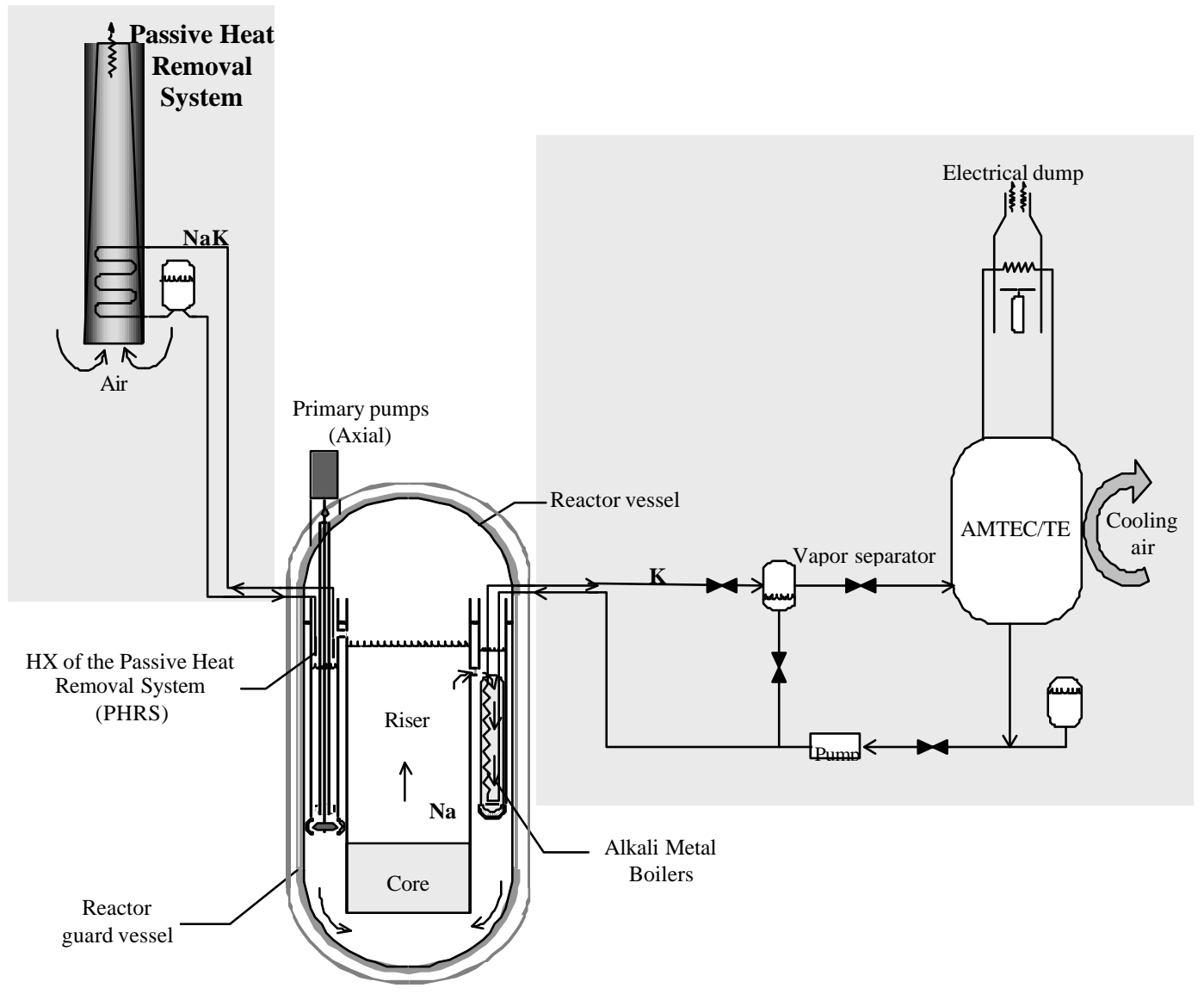

Figure 1. LMR-AMTEC plant design. 
The work performed by the Institute for Space and Nuclear Power Studies (University of New Mexico) included the design of the AMTEC/TE energy conversion and of the electrical converter modules for different coupling options with the LMR. The converters, which comprise an AMTEC top cycle and a PbTe TE bottom cycle on the condenser side of the AMTECs for both sodium and potassium working fluids, were designed and optimized for maximum overall conversion efficiency. The AMTEC topping cycle of the power conversion unit delivers high-power (> $40 \mathrm{kWe}$ ) and highvoltage ( $400 \mathrm{~V} \mathrm{DC})$. The operating temperatures of the beta"-alumina solid electrolyte (BASE) are $1006 \mathrm{~K}$ and $1127 \mathrm{~K}$, for the potassium and sodium working fluids, respectively. The heat rejected by the condenser of the AMTEC flows to the TE bottom cycle, through a conductive coupling arrangement. The electricity generated by the TE bottom cycle, which is cooled by natural convection of ambient air, contributes between $7 \%$ and $14 \%$ of the total electric power generated by the AMTEC/TE converter units.

The use of different thermoelectric materials for the bottom cycle was investigated, including both single- and multi-segment thermoelectric couples, and single-segment TE couples made of TAGS-85 and $2 \mathrm{~N}-\mathrm{PbTe}$ for the $\mathrm{P}$ and $\mathrm{N}$ legs, respectively, were selected as the best design option. Finally a performance model of the fully integrated sodium-and potassium- AMTEC/TE converters was developed, and used to optimize the converters design for maximum conversion efficiency and to investigate and determine the operation regime in which they were load-following. Performance analyses of the AMTEC/TE converters showed that a combined conversion efficiency in excess of $30 \%$ could be achieved.

The interfacing arrangement of the LMR with the converters subsystem, developed and investigated by the UNM-ISNPS used an intermediate liquid/liquid heat exchanger (IHX) between the LMR and the AMTEC/TE converter units. The overall thermal and electrical performances of the plant were evaluated using a thermal-hydraulic model of the primary and secondary loops of the LMR-AMTEC. In these designs, the secondary sodium or potassium liquid exiting the IHX is partially flash evaporated as it is introduced through an orifice in the high-pressure cavity of each AMTEC/TE converter unit. These studies showed that a $\mathrm{Na} / \mathrm{Na}$ plant (sodium in the primary loop and sodium in the secondary loop) operating at a core exit temperature of $1208 \mathrm{~K}$ could deliver a net power output of $25 \mathrm{MWe}$ at an overall conversion efficiency of $27.7 \%$, while for a $\mathrm{Na} / \mathrm{K}$ plant, which operates at a core exit temperature of $1087 \mathrm{~K}$, the net electrical power output is $25.4 \mathrm{MWe}$ at an overall plant efficiency of $28.6 \%$. In addition, these analyses showed that the K-AMTEC/PbTe converter units deliver higher efficiency (34.9\%) than the NaAMTEC/PbTe converter units (33.6\%). Even though this particular interfacing arrangement between the nuclear reactor and the AMTEC/TE converter units was not selected for the LMR-AMTEC power plant, the following important conclusions could be drawn from these analyses. The higher vapor pressure of potassium compared to sodium allows the nuclear reactor of the LMR-AMTEC plant with K-AMTEC/TE converter units to operate at a core exit temperature that is $\sim 120 \mathrm{~K}$ lower than that in the LMR-AMTEC plant with Na-AMTEC/TE converters, significantly reducing the fuel swelling and the materials compatibility issues, and increasing the reactor and plant operation lifetime. However, a K-AMTEC/PbTe converter delivers an electrical power output of $54.3 \mathrm{kWe}$, which is lower than that delivered by the Na-AMTEC/PbTe converter $(69.5 \mathrm{kWe})$, thus requiring the use of $30 \%$ more converter units in the $\mathrm{Na} / \mathrm{K}$ plant. Additional work 
performed by the UNM-ISNPS included the study of different high-energy uses and the nuclear power plant options for those applications.

Finally, the work performed by the Institute for Engineering Research and Applications (IERA) included topics related to the transport safety, corrosion control and waste disposal of the LMR-AMTEC. Based on the selected design of the LMR-AMTEC components and the coolant types, the wastes were classified and characterized according to Code of Federal Regulations. 


\section{TABLE OF CONTENTS}

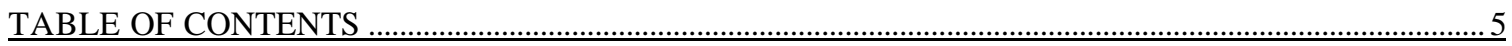

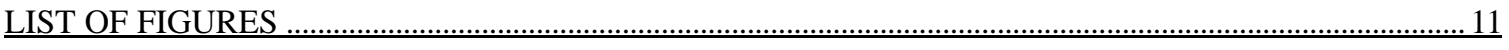

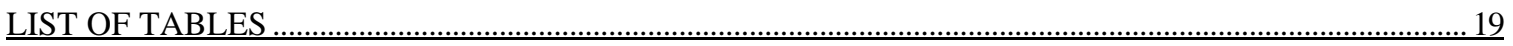

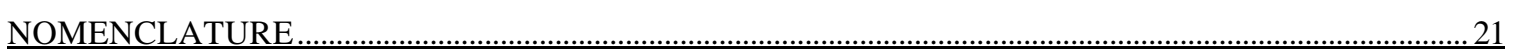

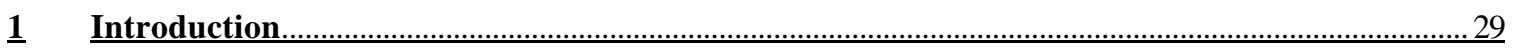

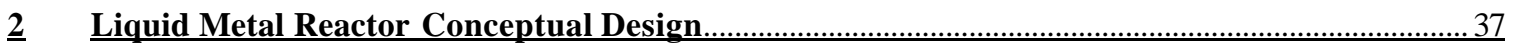

$2.1 \quad$ Coupling Between the LMR and the AMTEC/TE Units ............................................................. 37

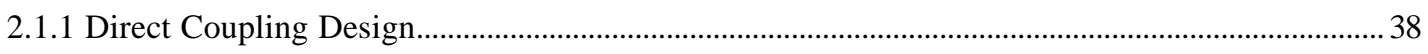

2.1.2 Indirect Coupling Designs ............................................................................................................. 39

2.1.2.1 Indirect Coupling Design with HX......................................................................................... 41

2.1.2.2 Indirect Coupling Design with Alkaline Metal Boilers ............................................................ 46

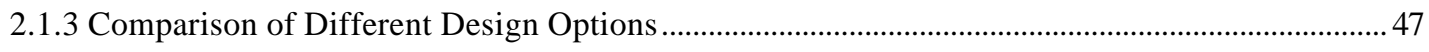

2.1.3.1 Performance of the Design with Respect to Net Efficiency and Vessel Weight..................... 47

2.1.3.2 Corrosion Behavior .......................................................................................................................... 49

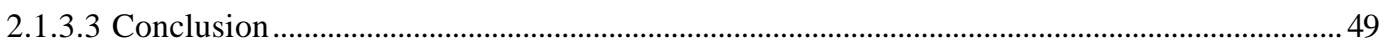

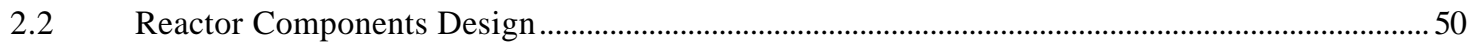

2.2.1 Layout of the Main Components of the LMR …………............................................................. 50

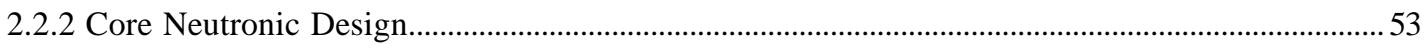

2.2.2.1 Fuel Element Design and Neutronics ...................................................................................... 53

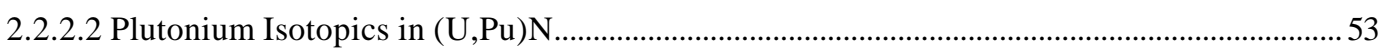

2.2.2.3 Use of Enriched Nitrogen.................................................................................................. 54

2.2.2.4 Neutron Spectrum and Fast Neutron Fluence Considerations..................................................55

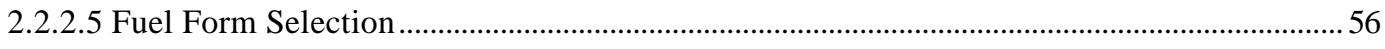

2.2.2.6 Cladding Options....................................................................................................................... 56

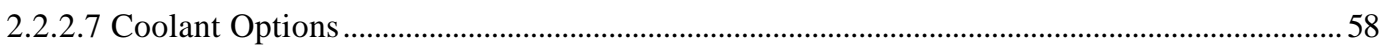

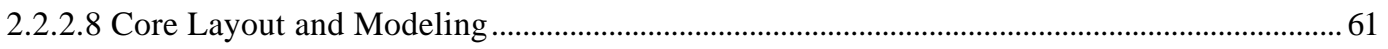

2.2.2.9 Basic Fuel and Core Parameters ………………………………………………………...... 62

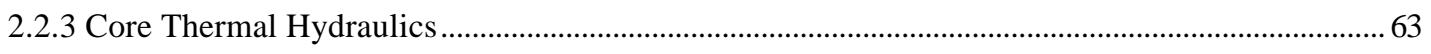

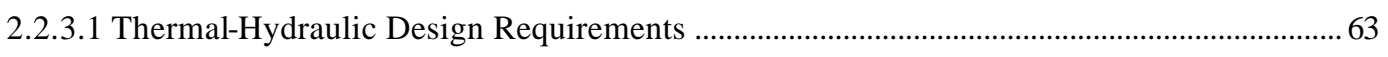

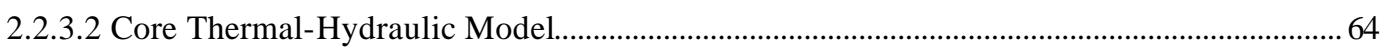

2.2.3.3 Hot-Channel analysis: The Semi-statistical Method ................................................................... 66

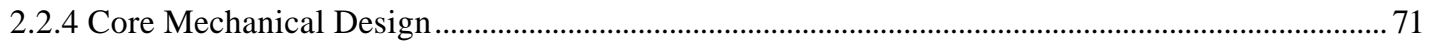

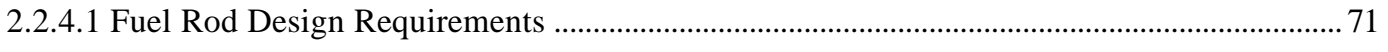

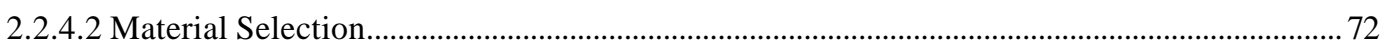

2.2.4.3 Clad Circumferential Creep …………………………………………………………….... 73 


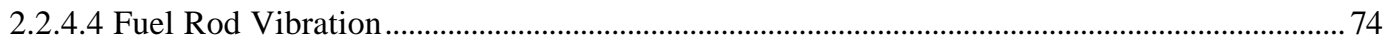

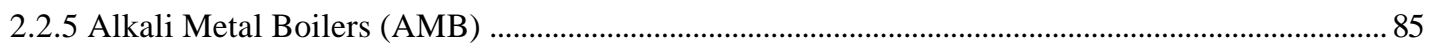

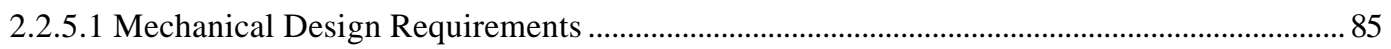

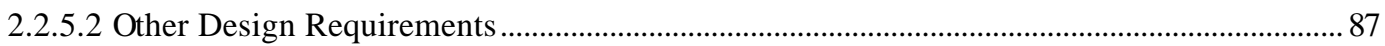

2.2.5.3 Recirculating or Once-through Operation ............................................................................. 91

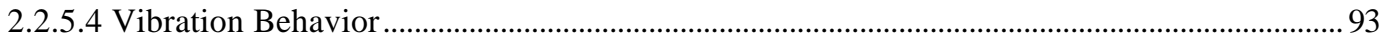

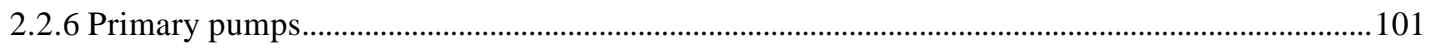

2.2.6.1 Selection of the Impeller Type: Propeller Pumps .................................................................. 101

2.2.6.2 Sizing of the Impeller and the Pump Casing ......................................................................... 103

2.2.7 Overview of Structural Material Selection .................................................................................... 104

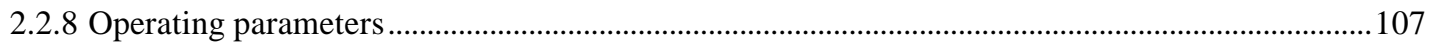

2.2.8.1 Plant Model Used for Evaluating the Design Performance ................................................... 107

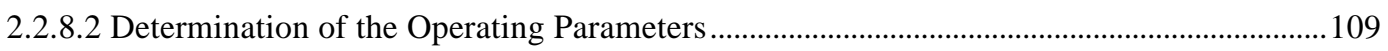

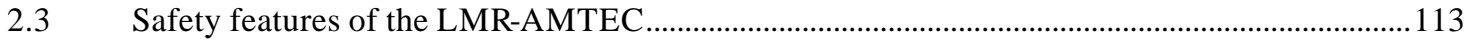

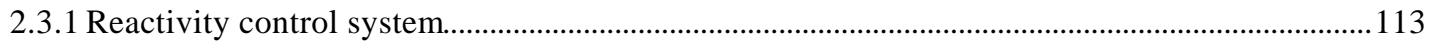

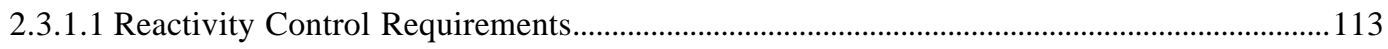

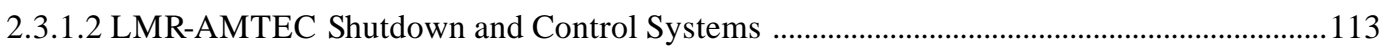

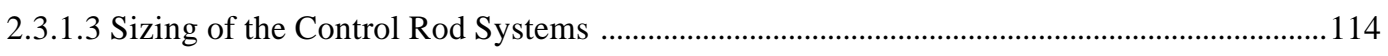

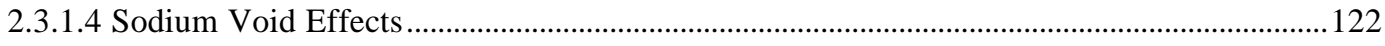

2.3.1.5 Local Boiling in the Core - Limiting Case ......................................................................... 122

2.3.1.6 Core Voiding/Boiling - A More Realistic Case ..................................................................... 123

2.3.2 Decay Heat Removal System of the LMR-AMTEC ...................................................................127

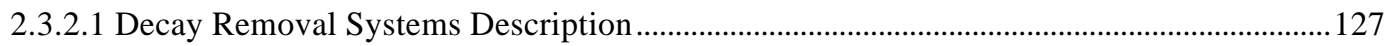

2.3.2.2 Passive Heat Removal System (PHRS) of the LMR-AMTEC............................................. 129

2.3.2.3 Transient Response of the PHRS .......................................................................................... 131

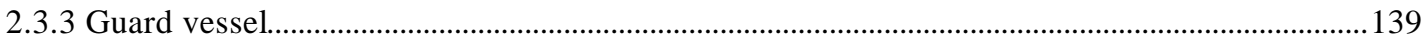

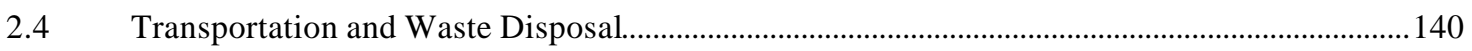

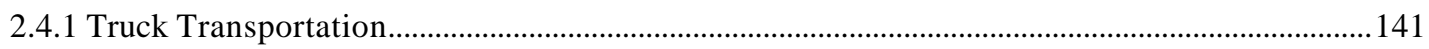

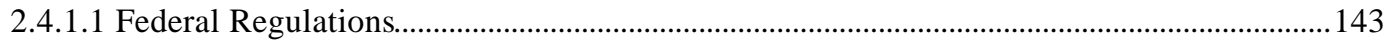

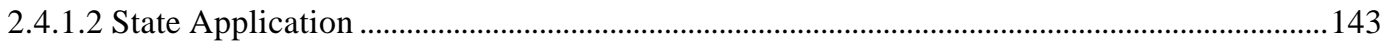

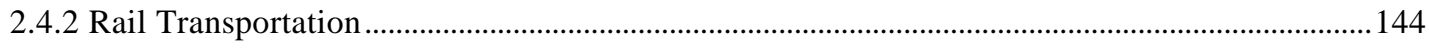

2.4.3 Transportation of the LMR-AMTEC Components ...................................................................144

2.4.3.1 Bridge Formula B (BFB) and The Alternative TTI Formula .............................................. 146

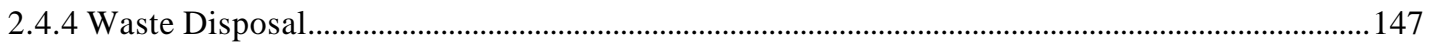

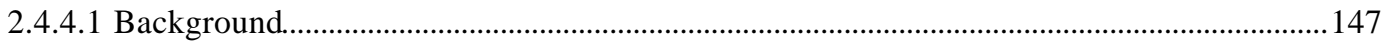

2.4.4.2 Description of the Sodium and Potassium Circuits ............................................................... 148

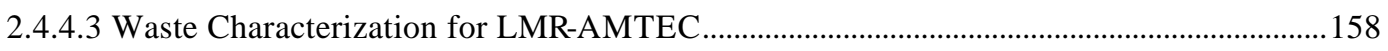




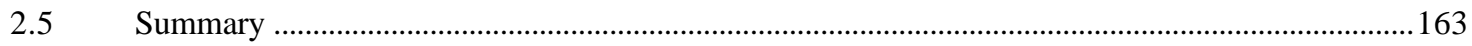

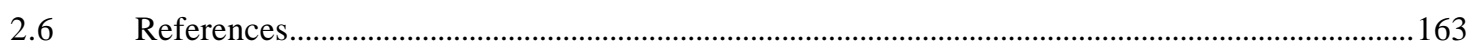

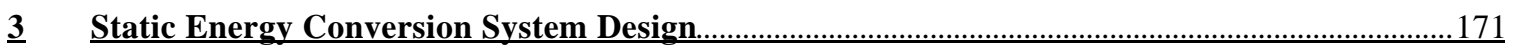

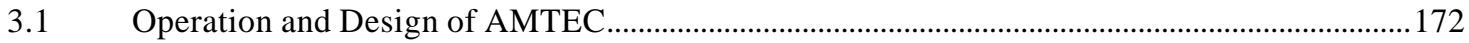

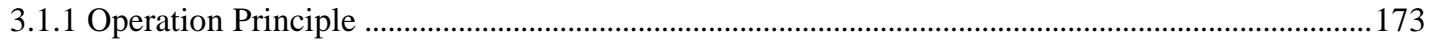

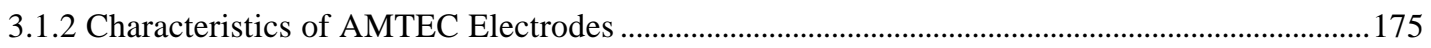

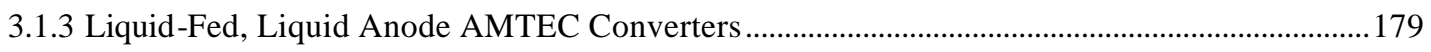

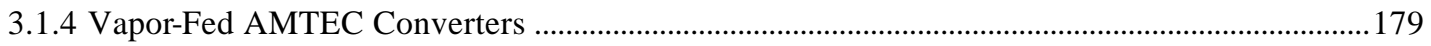

3.1.5 Vapor-Fed, Liquid Anode AMTEC Converters ............................................................................. 182

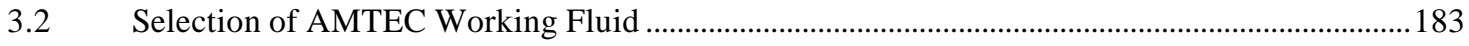

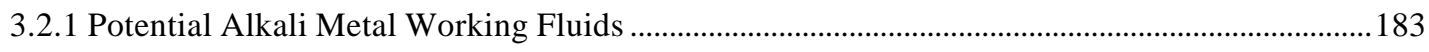

3.2.2 Induced Radioactivity in Sodium and Potassium Coolants ............................................................ 188

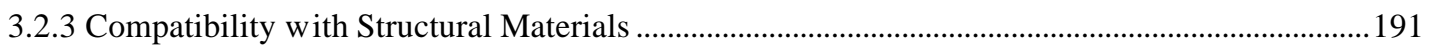

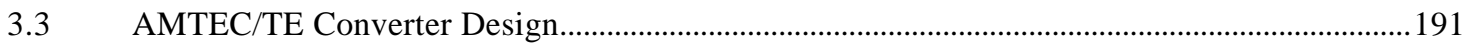

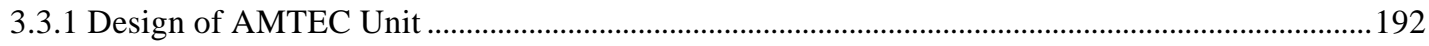

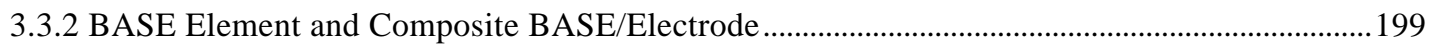

3.3.3 Breakdown Voltage in Alkali Metal Vapor in Low-Pressure Cavity of AMTEC ........................200

3.3.3.1 Experimental Setup and Measurements................................................................................2.

3.3.3.2 Experiments Conduct and Procedures …………………………………………………....2. 204

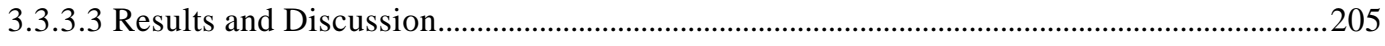

3.3.3.4 Application to Sodium and Potassium Vapors in AMTECs .................................................2.210

3.3.4 Design of Thermoelectric Bottom Cycle ...................................................................................2

3.3.5 Selection of ThermoElectric Materials for Bottom Cycle ……………………………………......215

3.3.6 Cooling of AMTEC/TE Converter Unit by Natural Convection of Air .........................................218

3.3.7 Heat Transfer Coefficient for Natural Convection of Air .............................................................220

3.3.8 Optimum Gap Size between Vertical Cooling Fins ......................................................................222

3.3.9 Estimates of Radiation Heat Exchange between Radiator and Environment ................................223

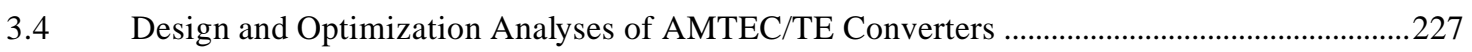

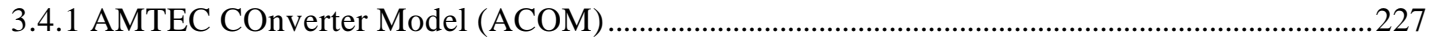

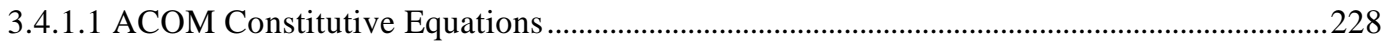

3.4.1.2 Radiation and Heat Conduction Model ..................................................................................231

3.4.1.3 Overall Energy Balance and Performance of the AMTEC/TE Converter..............................2232

3.4.2 Performance of the Thermoelectric Bottom Cycle ...........................................................................2.

3.4.3 Optimization of AMTEC/TE Converter Design for Maximum Efficiency ..................................236

3.4.3.1 Effect of the Spacing between the BASE Elements ................................................................237

3.4.3.2 Effect of the Aspect Ratio of the Dome -Shaped BASE Elements ..........................................240

3.4.3.3 Effect of the Height of the TE Modules/AMTEC Condenser Walls .......................................2242 
3.4.3.4 Effect of AMTEC Condenser Temperature ………………………………………………....24

3.4.3.5 Pressure Drop through the Orifices of AMTEC Radiation Heat Shield ..................................2245

3.4.3.6 Effect of the Number of Orifices in the AMTEC Heat Shield ...............................................250

3.4.3.7 Effect of the Diameter of the Orifices in the AMTEC Heat Shield .......................................2.250

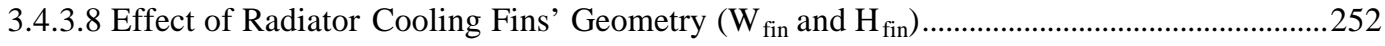

$3.5 \quad$ Reference Sodium and Potassium-AMTEC/TE Converters ..........................................................253

3.5.1 Design Parameters of Reference Na - and K-AMTEC/TE Converters............................................253

3.5.2 Thermo -Electric Modules of the Bottom Cycle ............................................................................25

3.5.3 Heat Transfer Rates and Temperatures ………………………………………………………...2.

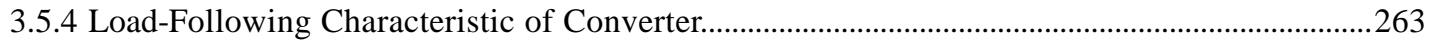

3.5.5 Selection of Nominal Operation Point.........................................................................................2. 264

3.5.6 Comparison of the Performance of the Na- and K-AMTEC/TE Converters at Identical BASE

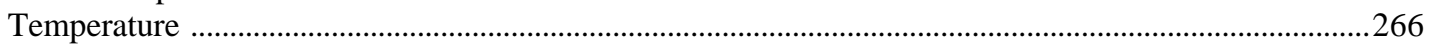

3.6 Integration of AMTEC/TE Converters into Power Modules ............................................................22

3.7 Materials Selection and Research for AMTEC Converters .......................................................22

3.7.1 Materials Selection for the Cathode Electrode and Lifetime Degradation Predictions ..............273

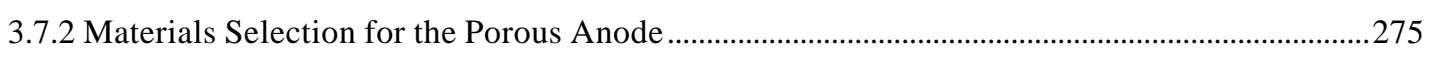

3.7.3 Recent Advances in the Fabrication of Na-BASE and K-BASE..................................................227

3.7.4 Materials Compatibility and Lifetime Issues in AMTEC Components ..........................................2.

3.7.5 Contaminant-Driven Degradation of AMTEC Components ...........................................................2.

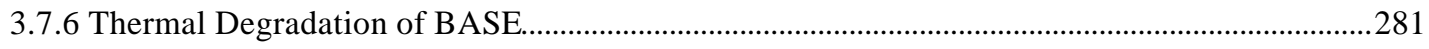

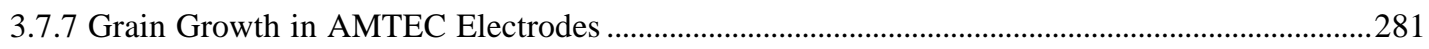

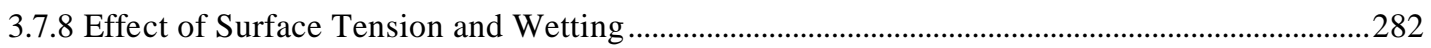

3.7.9 Degradation Mechanisms in AMTEC Cells .................................................................................2.

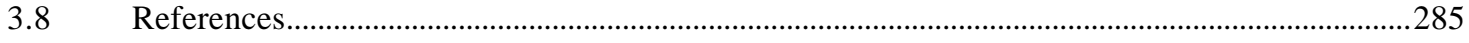

$4 \quad$ Performance Evaluation of the LMR-AMTEC Concept ……………………………………………...303

4.1 Preliminary Economic Evaluation of the LMR-AMTEC (WEC)..................................................303

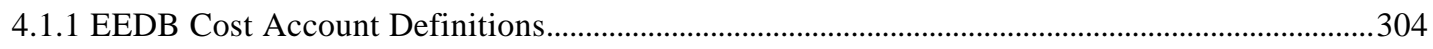

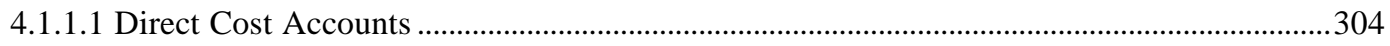

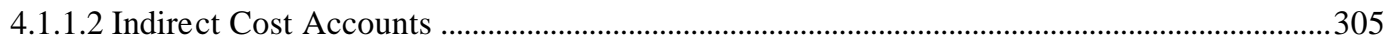

4.1.2 Factory Fabrication and Modular Construction..............................................................................307

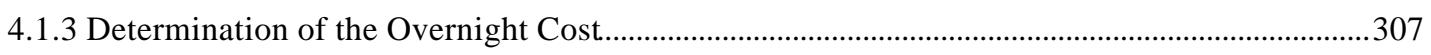

4.1.4 Optimization of the Reactor Design Parameters ..........................................................................309

4.1.4.1 Comparison Between Once-through and Recirculation AMB Operation ..............................309

4.1.4.2 Effect of the Tube Diameter of the Alkali Metal Boilers .........................................................310

4.1.5 Summary of the Preliminary Economic Evaluation ........................................................................ 311

4.2 Study on the High-Energy Utilization of LMR-AMTEC for Developing Countries and Remote

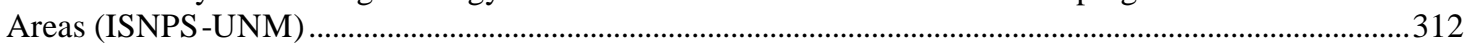


4.2.1 Production of Electricity and Co-Generation Thermal Power ……………………………….......312

4.2.2 High Figure-Of-Merit Segmented Thermoelectric Modules for Seawater Desalination.............315

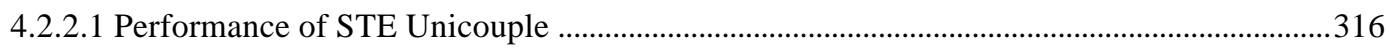

4.2.2.2 STEM Design...............................................................................................................

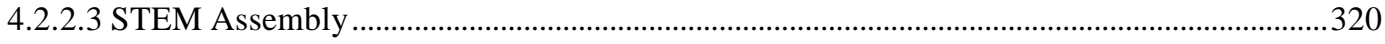

4.2.3 Potential Performance of High-Energy-Utilization LMR-AMTEC Plant.......................................322

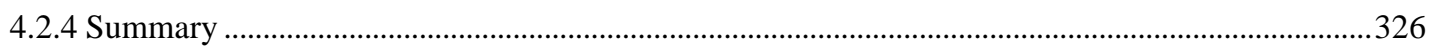

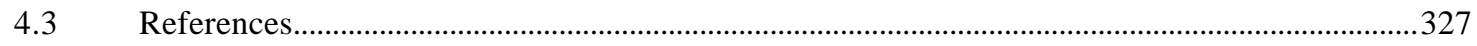

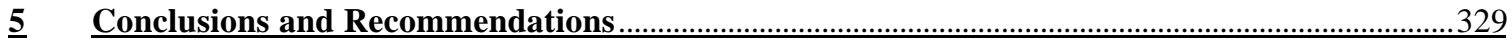

Appendix I: ISNPS's Power Plant Thermal-Hydraulic Model for IC with HXs ............................................335 
[ This page intentionally blank ] 


\section{LIST OF FIGURES}

Figure 1-1. Project schedule.

Figure 2-1. Diagram of the LMR-AMTEC direct coupling with expanders design (for simplicity only one loop is shown).

Figure 2-2. Schematic of the Na-cooled LMR-AMTEC indirect coupling concept with liquid-liquid HXs and open K-AMTEC/TE converters (only one HX loop is shown).

Figure 2-3. Schematic of the Na-cooled LMR-AMTEC indirect coupling concept with liquid-liquid HXs and closed AMTEC/TE converters (only one HX loop is shown).

Figure 2-4. Schematic of an indirect interfacing arrangement between LMR and AMTEC/TE converters in an open-unit design configuration.

Figure 2-5. Schematic of an indirect interfacing arrangement between LMR and AMTEC/TE converters in a closed-unit design configuration.

Figure 2-6. Diagram of the LMR-AMTEC indirect coupling with boilers design (for simplicity only one boiler loop is shown). Typical values of the flow rates and the pressures are shown for the $\mathrm{Na}+\mathrm{K}$ option.

Figure 2-7. Optimization procedure 47

Figure 2-8. Efficiency versus vessel weight. 48

Figure 2-9. In-vessel layout of the LMR-AMTEC reactor. The picture shows the pumps and the boilers positions and the sodium levels during normal operation of the reactor.

Figure 2-10. Power Conversion System, Passive Heat Removal Systems (PHRS), Water Cooling Guard Vessel System (WCGVS) and Primary Cold Trap

Figure 2-11. Fuel reactivity as a function of burnup for UN fuel $\left(19.95 \%{ }^{235} \mathrm{U}\right)$ and (U,Pu)N $(19.95 \% \mathrm{Pu}-\mathrm{B}$ and depleted $\mathrm{U})$.

Figure 2-12. Comparison of fuel reactivity as a function of burnup for natural and enriched nitrogen: $(\mathrm{U}, \mathrm{Pu})^{\mathrm{nat}} \mathrm{N}$ and $(\mathrm{U}, \mathrm{Pu})^{15} \mathrm{~N}$.

Figure 2-13. Comparison of $\mathrm{Nb93}$ and Fe56 total cross section.................................................................57

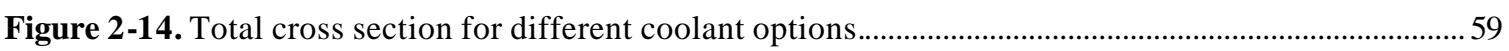

Figure 2-15. Neutron spectra for different coolant options.

Figure 2-16. Core configuration with 78 fuel elements and 13 control assemblies (outermost two rings are shield assemblies).

Figure 2-17. Cylindrical representation of core configuration with 78 fuel elements and 13 control assemblies

Figure 2-18. Core configuration with 72 fuel elements and 19 control assemblies (outermost two rings are shield assemblies).

Figure 2-19. Core configuration with 72 fuel elements and 13 control assemblies (outermost two rings are shield assemblies).

Figure 2-20. Radial fuel pin temperature distribution for the sodium-cooled core.

Figure 2-21. Nominal axial core temperatures distribution in the sodium-cooled core without uncertainties.

66

Figure 2-22. Semi-statistical method 67 
Figure 2-23. Axial core temperatures distribution in the sodium-cooled core with uncertainties $(3 \sigma)$

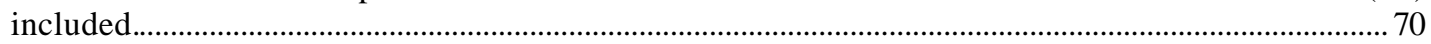

Figure 2-24. $\mathrm{Nb}-1 \mathrm{Zr}$ stress to cause $1 \%$ creep as a function of temperature ................................................. 74

Figure 2-25. Critical flow speed versus the fuel natural frequency and for different structural damping values.

Figure 2-26. Non-linear fuel rod vibration model used to study the LMR-AMTEC fuel rod........................ 77

Figure 2-27. Unconstrained vibration modes of the LMR-AMTEC fuel rod. ................................................78

Figure 2-28. Excitation force on the Yplane used to model the turbulence forces acting on the clad surface.

Figure 2-29. Rod frequency responses for zero gap case with low and high excitation forces. The first five constrained modes of vibration are identified.

Figure 2-30. Rod frequency response for non-zero gap case and with low and high excitation forces. The first four unconstrained modes of vibration are identified................................................................. 81

Figure 2-31. Motion of the center of the fuel rod at the axial position corresponding to the wire wrap. The

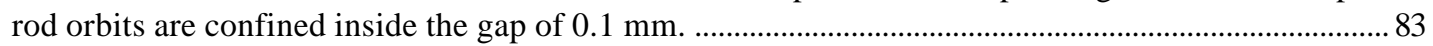

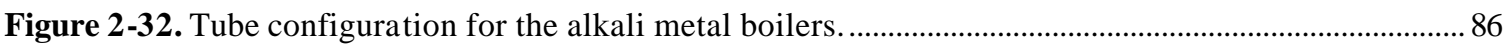

Figure 2-33. Effect of the outlet core temperature on the primary to secondary temperature drop and the

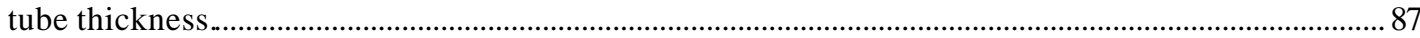

Figure 2-34. Boiling ring designed to maintain stable vapor pockets

Figure 2-35. Effect of the outlet core temperature on the two phases pressure losses and the secondary saturation temperature.

Figure 2-36. Temperature profiles for the secondary and the primary side of the once-through alkali metal boilers.

Figure 2-37. Critical flow speed versus the fuel natural frequency and for different structural damping values.

Figure 2-38. Karman vortex shedding frequency for tubes under cross flow.

Figure 2-39. Non-linear tube vibration model used in the study. The supports are modeled as a gap and a spring having a very high stiffness.

Figure 2-40. First 10 unconstrained vibration modes of the clamped tube......

Figure 2-41. Rod frequency response for zero gap case and under low and high excitation forces. The first three constrained mo des of vibration can be identified.

Figure 2-42. Rod frequency response for non zero gap case and under low and high excitation forces. The first fifth unconstrained modes of vibration can be identified.

Figure 2-43. Work rate versus the excitation force for zero gap and gap equal $0.1 \mathrm{~mm}$ cases.

Figure 2-44. Centrifugal pump characteristics for radial, propeller and mixed flow types. The working conditions of the primary pumps of the LMR-AMTEC correspond to a propeller type.

Figure 2-45. Flow diagram of the LMR-AMTEC plant model.

Figure 2-46. Gross and net efficiencies and anode temperature with respect to the outlet core temperature (for a design using potassium AMTEC).

Figure 2-47. Effect of the margin to coolant boiling temperature on the net efficiency, the normalized tube and vessel thickness'.

Figure 2-48. Effect of the margin to coolant boiling temperature on the primary pressure and the vessel weight. 
Figure 2-49. Boron carbide density as a function of ${ }^{10} \mathrm{~B}$ enrichment.

Figure 2-50. Core configuration with 13 control assemblies - actual geometry and simplified 3-D model.

Figure 2-51. Change in total worth of all control assemblies due to variability in ${ }^{10} \mathrm{~B}$ enrichment in $\mathrm{B}_{4} \mathrm{C}$.

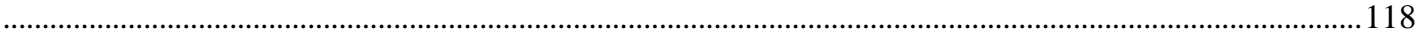

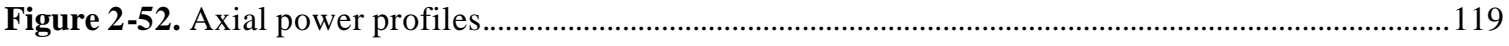

Figure 2-53. Radial normalized power profiles for different positions of control banks..............................120

Figure 2-54. Core configuration with 19 control assemblies - actual geometry and simplified 3-D model.

Figure 2-55. Reactivity vs. void fraction for central (whole core) voiding. 123

Figure 2-56. Core model of the LMR-AMTEC.

Figure 2-57. Change in core reactivity due to voiding of the core upper half and above (two sets of MCNP runs shown).

Figure 2-58. Change in core reactivity due to voiding of the core upper half and above (smoothed results).

Figure 2-59. Power Conversion System, Passive Heat Removal Systems (PHRS), Water Cooling Guard Vessel System (WCGVS) and primary Cold Trap System.

Figure 2-60. Sodium levels during normal reactor operation and during emergency decay heat removal.

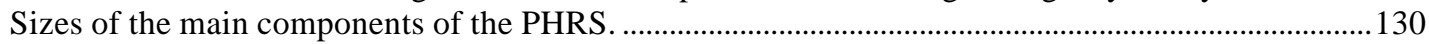

Figure 2-61. Model used for evaluating the PHRS response during postulated transient...... .132

Figure 2-62. Core power a) 0-10 s, b) up to $14000 \mathrm{~s}$. After the reactor shutdown, decay heat power are used to estimate the core power.

Figure 2-63. Temperature of the fuel, cladding, and the coolant at the core outlet. The fuel and cladding temperatures corresponds to the hot channel $(0 \mathrm{~s}<\mathrm{t}<50 \mathrm{~s})$.

Figure 2-64. Coolant mass flow rate and temperature of fuel, cladding and coolant at the core outlet. The fuel and cladding temperatures corresponds to the hot channel $(0 \mathrm{~s}<\mathrm{t}<50 \mathrm{~s})$.

Figure 2-65. Coolant mass flow in the PHRS and average temperature of the air dumps $(0 \mathrm{~s}<\mathrm{t}<50 \mathrm{~s}) \ldots 135$

Figure 2-66. Coolant mass flow in the PHRS and average temperature of the air dumps $(0 \mathrm{~s}<\mathrm{t}<14000 \mathrm{~s})$.

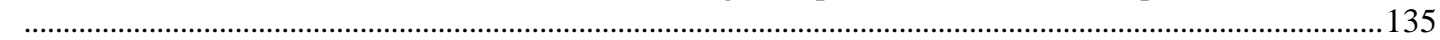

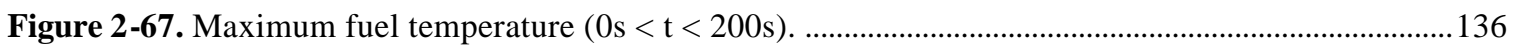

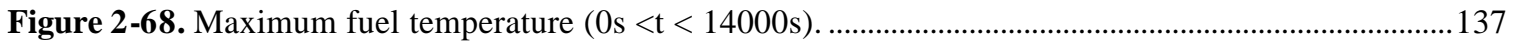

Figure 2-69. Average outlet core sodium temperature $(0 \mathrm{~s}<\mathrm{t}<200 \mathrm{~s})$........................................................ 137

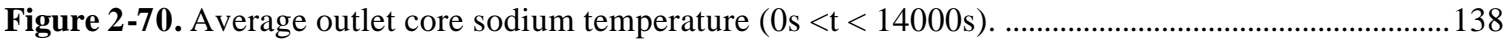

Figure 2-71. Water Cooling Guard Vessel System employed by the French PHENIX reactor....................139

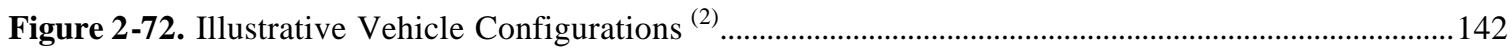

Figure 2-73: States Allowing Various Longer Combination Vehicles ${ }^{(2)}$........................................................142

Figure 2-74. LMR-AMTEC Waste Management Flowcharts - Waste Generation .....................................150

Figure 2-75. Solubility of Oxygen and Metallic Impurities in Liquid Sodium and Potassium. ...................154

Figure 2-76. LMR-AMTEC Waste Management Flowchart - Waste Characterization ................................158

Figure 2-77. LMR-AMTEC Waste Management Flowchart - Waste Treatment .........................................158

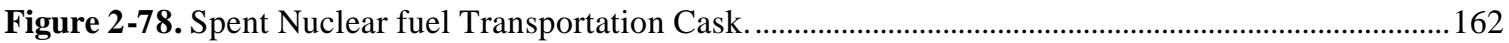


Figure 3-1. Lattice structure of sodium beta"-alumina solid electrolyte (Cole 1983).

Figure 3-2. Schematic of a vapor anode AMTEC converter.

Figure 3-3. Transport processes in the cathode electrode. (1) Transfer of Na ions to the triple phase boundary (BASE-electrode-sodium vapor); (2) Surface diffusion of Na atoms on electrode grains; (3) Desorption of $\mathrm{Na}$ atoms from surface of electrode grains; and (4) Sodiumvapor diffusion through the pores of the electrode.

Figure 3-4. Measured values of charge-exchange current coefficient of $\mathrm{Rh}_{\mathrm{x}} \mathrm{W}$ porous electrodes at the Jet

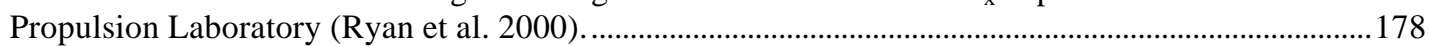

Figure 3-5. Cross-section views of a vapor anode, multi-tube AMTEC (not to scale). ..............................180

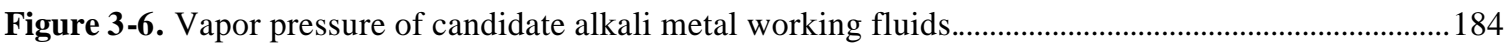

Figure 3-7. Latent heat of candidate alkali metal fluids................................................................................ 185

Figure 3-8. Ionic resistivity of sodium and potassium Beta"-alumina. ........................................................ 185

Figure 39. The difference in saturation temperature of sodium and potassium at same anode apor

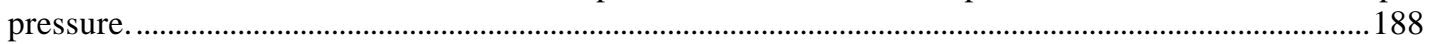

Figure 3-10. Solubility of oxygen and metallic impurities in liquid sodium and potassium. ......................190

Figure 3-11.. A plane view of the reference AMTEC/TE converter unit design developed in this work

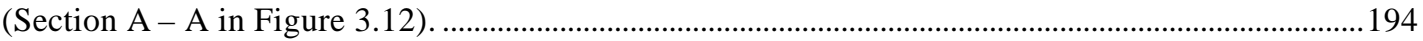

Figure 3-12. A vertical cross-section of the reference AMTEC/TE converter unit design developed in this

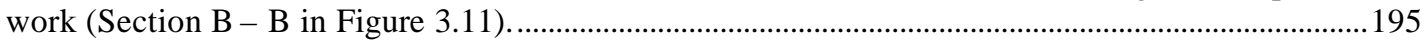

Figure 3-13. Cross-section view of two BASE elements connected in series (Section D - D in Figure 3.11).

Figure 3-14. Close-up of a composite BASE/electrode. .............................................................................200

Figure 3-15. Isometric view of narrow dome -shaped, BASE element............................................................201

Figure 3-16. Vertical cross section of a narrow dome-shaped, composite BASE element..........................201

Figure 3-17. A cross-sectional view of the test section..........................................................................203

Figure 3-18. A schematic diagram of the control and measurement circuit..............................................205

Figure 3-19. Examples of the histories of the current and voltage measurements.....................................206

Figure 3-20. Effect of cesium pressure on I-V curves and breakdown voltage..........................................207

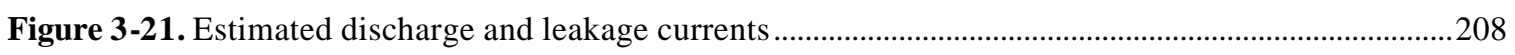

Figure 3-22. Measured breakdown voltage and discharge current as function of cesium pressure. ...........209

Figure 3-23. Effective work functions of tantalum in sodium, potassium and cesium vapors....................2211

Figure 3-24. Ionization rate constants for sodium, potassium and cesium. …..........................................211

Figure 3-25. Cross-section of bottom cycle and heat pipe radiator (Section C-C in Figure 3.11)............214

Figure 3-26. Figure-Of-Merit (FOM) of some thermoelectric materials of interest.....................................215

Figure 3-27. Design of thermoelectrics for use in the bottom cycle of the AMTEC/TE converter unit. ....217

Figure 3-28. Maximum efficiency of thermoelectrics for use in the bottom cycle of the AMTEC/TE

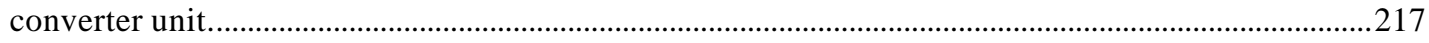

Figure 3-29. Cross-section views and dimensions of heat pipe radiator housing .......................................219

Figure 3-30. Heat transfer by natural convection of air between 2 isothermal, vertical parallel plates.......221 
Figure 3-31. Heat transfer coefficient by natural convection of air and radiator surface area of 400-V reference sodium AMTEC/TE converter unit, as functions of separation distance between cooling fins $\left(Q_{\mathrm{rad}}=109 \mathrm{~kW}, T_{\mathrm{air}}=300 \mathrm{~K}\right)$.

Figure 3-32. Radiator surface temperature of the reference sodium AMTEC/TE converter unit, as function of separation distance between cooling fins $\left(Q_{\mathrm{rad}}=109 \mathrm{~kW}, T_{\mathrm{air}}=300 \mathrm{~K}\right)$.

Figure 3-33. Air Rayleigh number at the radiator of the reference sodium AMTEC/TE converter unit, as function of separation distance between cooling fins $\left(Q_{\mathrm{rad}}=109 \mathrm{~kW}, T_{\text {air }}=300 \mathrm{~K}\right) \ldots \ldots \ldots \ldots \ldots \ldots \ldots \ldots . .224$

Figure 3-34. Hemispherical emissivity of lightly-oxid ized stainless steel. 225

Figure 3-35. Estimate of net radiative power absorbed by heat pipe radiator housing...... 226

Figure 3-36. Optimum conversion efficiency of PbTe thermoelectric couples .234

Figure 3-37. Predicted heat transfer rates and temperatures in the reference sodium AMTEC/TE converter $\left(N_{\text {rows }}=2, V_{\text {AMTEC }} \sim 400 \mathrm{~V}\right)$ at nominal operation and very near the peak efficiency $\left(T_{B}=1127 \mathrm{~K}, P_{a}\right.$ $=80 \mathrm{kPa})$

Figure 3-38. Predicted heat transfer and temperatures in the reference potassium AMTEC/TE converter $\left(N_{\text {rows }}=3, V_{\text {AMTEC }} \sim 400 \mathrm{~V}\right)$ at nominal operation and near the peak efficiency $\left(T_{B}=1006 \mathrm{~K}, P_{a}=80\right.$ $\mathrm{kPa})$..... .236

Figure 3-39. Effect of the spacing between BASE elements, $b$ on the pressure at the BASE/cathode interface and on the power output of the AMTEC top cycle, at the peak efficiency. 238

Figure 3-40. Effect of the spacing between BASE elements, $b$ on the length of the converter and on the thermal heat losses, at the peak efficiency. 239

Figure 3-41. Effect of the spacing between BASE elements, $b$ on the power output of the TE bottom cycle and on the converter peak efficiency.

Figure 3-42. Effect of the aspect ratio of the BASE elements on the width of the converter BASE support plate and on the internal radiation losses, at the peak efficiency.

Figure 3-43. Effect of the aspect ratio of the BASE elements on the pressure at the BASE/cathode interface and on the power output of the AMTEC top cycle, at the peak efficiency. .241

Figure 3-44. Effect of the aspect ratio of the BASE elements on the power output of the TE bottom cycle and on the converter peak efficiency.

Figure 3-45. Effect of the height of thermoelectric modules on the pressure at the BASE/cathode interface and on the power output of the AMTEC top cycle, at the peak efficiency.....

Figure 3-46. Effect of the height of thermoelectric modules on the internal radiation heat losses and on the power output of the TE bottom cycle, at the peak efficiency.

Figure 3-47. Effect of the height of thermoelectric modules on the temperature drop and average heat flux through the thermoelectric couples, at the peak efficiency

Figure 3-48. Effect of the height of thermoelectric modules on the power output of the converter and on the peak efficiency.

Figure 3-49. Effect of the condenser temperature of the converter on the conduction and radiation heat losses to the condenser walls, at the peak efficiency. 246

Figure 3-50. Effect of the condenser temperature of the converter on the temperature drop $\Delta T_{\mathrm{TE}}$ and on the efficiency of the thermoelectric bottom cycle 246

Figure 3-51. Effect of the condenser temperature of the converter on the pressure at the BASE/cathode interface and on the voltage output of the AMTEC top cycle, at the peak efficiency.

Figure 3-52. Effect of the condenser temperature of the converter on the electrical power output and peak conversion 
Figure 3-53. Effect of the condenser temperature of the converter on the vapor Mach number in the heat shield orifices.

Figure 3-54. Effect of the number of orifices in the heat shield on the flow area and vapor Mach number in the orifices.

Figure 3-55. Effect of the orifices diameter in the heat shield on the pressure at the BASE/cathode interface and on the power output of the AMTEC top cycle, at the peak efficiency. The total orifices flow area is kept constant.

Figure 3-56. Effect of the orifices diameter in the heat shield on the peak efficiency and corresponding power output of the converter. The total orifices flow area is kept constant

Figure 3-57. Cross-sectional views of optimized sodium-AMTEC/TE converter. .....................................254

Figure 3-58. Cross -sectional views of optimized potassium-AMTEC/TE converter. .................................255

Figure 3-59. Cross-sectional views of BASE elements, TE bottom cycle and cooling fins........................256

Figure 3-60. P-TAGS-85/2N-PbTe thermoelectric module (TEM) in reference K-AMTEC/TE unit (560 unicouples connected in series, and $P e_{\mathrm{STEM}}=43.13 \mathrm{We}$ at $\left.14.87 \mathrm{~V} \mathrm{DC}\right)$.

Figure 3-61. Unicouples for the bottom cycle of the optimized AMTEC/TE converters (nominal operation at $\left.P_{a}=80 \mathrm{kPa}\right)$. 261

Figure 3-62. Predicted heat transfer rates and temperatures in the reference sodium AMTEC/TE converter $\left(N_{\text {rows }}=2, V_{\text {AMTEC }} \sim 400 \mathrm{~V}\right)$ at nominal operation, very near the peak efficiency $\left(T_{B}=1127 \mathrm{~K}, P_{a}=80\right.$ $\mathrm{kPa})$..

Figure 363. Predicted heat transfer rates and temperatures in the reference potassium AMTEC/TE converter $\left(N_{\text {rows }}=3, V_{\text {AMTEC }} \sim 400 \mathrm{~V}\right)$ at nominal operation $\left(T_{B}=1006 \mathrm{~K}, P_{a}=80 \mathrm{kPa}\right) \ldots \ldots \ldots \ldots \ldots . .263$

Figure 3-64. Performance results of the optimized potassium-AMTEC/TE converter when operating at $T_{B}$ $=1000 \mathrm{~K}$.

Figure 3-65. Performance comparison of the optimized sodium and potassium-AMTEC/TE converters

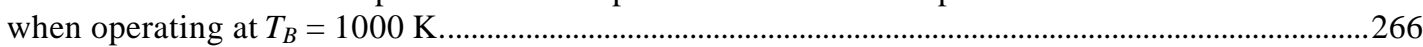

Figure 3-66. Performance of reference K-AMTEC/TE converter at nominal operation. .............................268

Figure 3-67. Performance of top cycle of reference K-AMTEC/TE converter at nominal operation. ........268

Figure 3-68. Performance of bottom cycle of reference K-AMTEC/TE converter at nominal operation. .269

Figure 3-69. Temperatures in reference K-AMTEC/TE converter at nominal operation.............................269

Figure 3-70. Configuration of the electrical power system for the LMR-AMTEC power plant................2270

Figure 3-71 . A schematic of a scalable, AMTEC/TE electric power module.

Figure 3-72. Integration of AMTEC/TE converter units in the electrical power system for a $25 \mathrm{MWe}$ power plant.

Figure 3-73. Measured values of charge-exchange current coefficient of $\mathrm{Rh}_{\mathrm{x}} \mathrm{W}$ porous electrodes at the Jet Propulsion Laboratory (Ryan et al. 2000).

Figure 3-74. Thermal expansion coefficient of Na-BASE and molybdenum refractory alloys.

Figure 3-75. Ionic resistivity of sodium- and potassium- $\beta$ "-alumina solid electrolytes (Cole et al. 1979, Steinbrück et al. 1993, Williams et al. 1992 and 1995).

Figure 3-76. Vapor pressure of structural metallic elements. 280

Figure 3-77. Effect of electrode grain growth on charge-exchange coefficient, $B$. 282

Figure 4-1. Optimization procedure used in determining the LMR-AMTEC operating parameters .309 
Figure 4-2. Net efficiency versus the reactor vessel mass for AMBs operating in recirculation or in oncethrough mode.

Figure 4-3. Cost increment of the LMR-AMTEC design with AMBs operating once-through versus the reactor vessel. The LMR-AMTEC with boilers operating in recirculation is used as reference..........310

Figure 4-4. Relative overnight cost versus the external diameter of the Alkali Metal Boilers. The reference LMR-AMTEC design is used as reference $(2.5 \mathrm{~cm})$

Figure 4-5. Characteristics of segmented TEs used in seawater desalination units....................................314

Figure 4-6. Peak conversion efficiency of STE and SiGe unicouples

Figure 4-7. Cross-sectional views of STEM/desalination preheating unit, comprised of 2 STEM panels of 18 STEMs each $\left(P e=13 \mathrm{kWe}\right.$ at $\left.360 \mathrm{~V} \mathrm{DC}, Q_{\text {water }}=100 \mathrm{~kW}\right)$. 318

Figure 4-8. Segmented ThermoElectric Module (STEM) (1280 unicouples connected in series, and $P e_{\text {STEM }}$ $=360 \mathrm{We}$ at $180 \mathrm{~V} \mathrm{DC})$. 319

Figure 4-9. Performance of optimized STEs used in conjunction with seawater desalination. 321

Figure 4-10. LMR plant with potassium AMTEC/TE converters. .323

Figure 4-11. LMR plant with potassium AMTEC/TE converters and STEM/seawater desalination...........324

Figure 4-12. LMR plant with K-AMTEC/TE converters and high-temperature process heat/steam. .324 
[ This page intentionally blank ] 


\section{LIST OF TABLES}

Table 2-1. Some characteristics of indirect and direct interfacing arrangements of the nuclear reactor and the electric energy conversion subsystem.

Table 2-2. Reference Plutonium (Pu-B) Isotopics for (U,Pu)N Fuel................................................................. 54

Table 2-3. Estimated fast fluence (relative units) for $14 \mathrm{a} / \mathrm{o}$ FPU fuel................................................................. 56

Table 2-4 K-effective for different cladding materials (Pu-A 14 a/o FPU).....................................................5

Table 2-5. Basic properties of alkali metals considered for coolant................................................................... 58

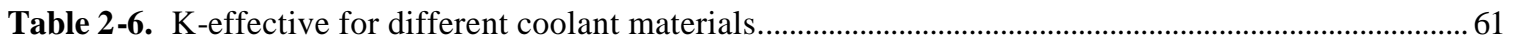

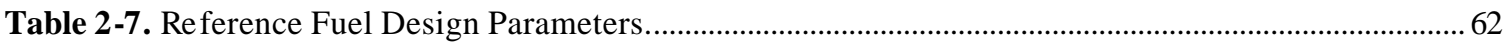

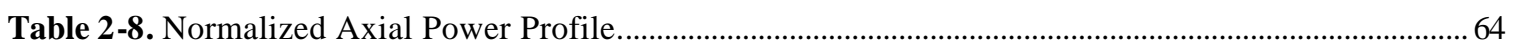

Table 2-9. Direct uncertainty subfactors, $s_{i j, d i}$. for cladding temperature evaluation $(3 \sigma)$. ............................ 68

Table 2-10. Statistical uncertainty subfactors, sij,sta for cladding temperature evaluation $(3 \sigma)$.................... 68

Table 2-11. Properties of Uranium and Plutonium fuels. Note the dependence of the thermal conductivity

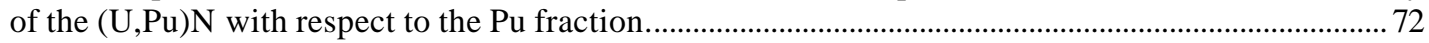

Table 2-12. Parameters used for evaluating the circumferential clad creep...................................................... 74

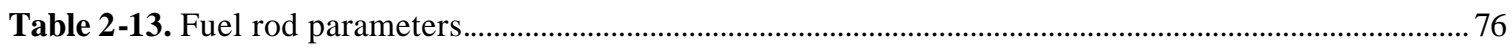

Table 2-14. Normal work rates and middle span displacement obtaining for the zero and non-zero gap cases

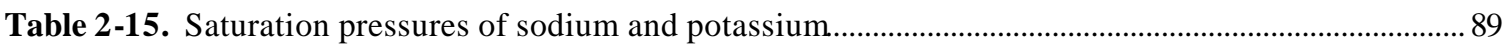

Table 2-16. Secondary parameters for the AMB operating in recirculation and once-through mode (the

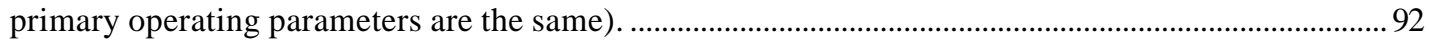

Table 2-17. AMB tube parameters used in evaluating the vibration response................................................. 95

Table 2-18. Normal work rates and middle span displacement obtaining for the zero and non-zero gap

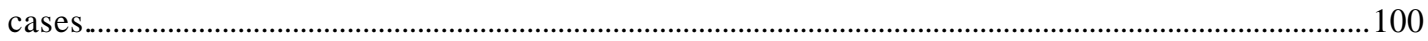

Table 2-19. Propeller pump performance data [Volney48] ........................................................................... 103

Table 2-20. Characteristics of the candidate materials for the LMR-AMTEC components. The temperature indicated on the "alloy name" column corresponds to the upper limit fixed by the mechanical properties. 105

Table 2-21. Candidate materials for the main components of the primary circuit. ........................................106

Table 2-22. Candidate materials for the main components of the secondary circuit. .....................................106

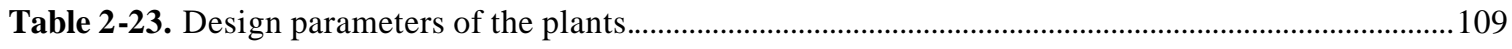

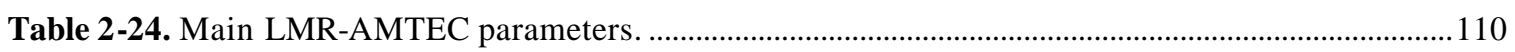

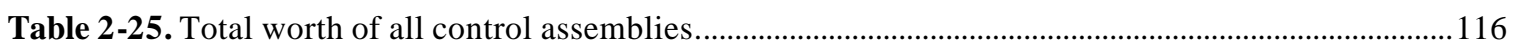

Table 2-26. Change in total worth of all control assemblies due to variability in $\mathrm{B}_{4} \mathrm{C}$ density.....................116

Table 2-27. Change in total worth of all control assemblies due to variability in $\mathrm{B}_{4} \mathrm{C}$ volume fraction.....117

Table 2-28. Change in total worth of all control assemblies due to variability in ${ }^{10} \mathrm{~B}$ enrichment in $\mathrm{B}_{4} \mathrm{C} . .117$

Table 2-29. Reactivity worth of individual control rod banks.......................................................................118

Table 2-30. Change in core reactivity due to central core voiding..................................................................122 
Table 2-31. MCNP runs performed to examine voiding in the upper core half.............................................125

Table 2-32. Change in core reactivity due to voiding of the core upper half and above.................................125

Table 2-33. Principal parameters of the helix HX's of the PHRS.................................................................129

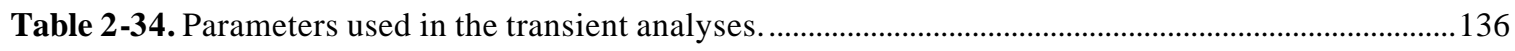

Table 2-35. Typical vehicle types and their weight and dimension specifications ${ }^{(2)}$....................................141

Table 2-36. Maximum Activity Levels Measured in Sodium of LMFBRs ${ }^{(1)}$ …............................................. 151

Table 2-37. Maximum Activity Levels Measured in The Cover Gas of LMFBRS in GBq/m3 .................157

Table 2-38. Different Techniques for Removal of Radioactivity in The Cover Gas (25) ..............................157

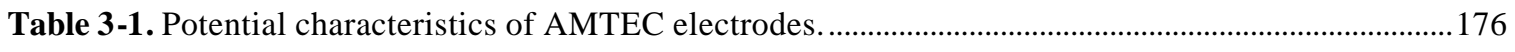

Table 3-2. Sodium ( ${ }_{11} \mathrm{Na}$ ) isotopes (El-Wakil 1978, Walker et al. 1988). ......................................................189

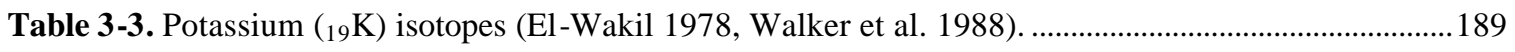

Table 3-4. Dimensions and nominal performance parameters of optimized AMTEC/TE converters

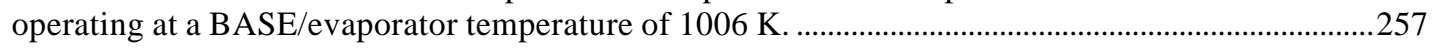

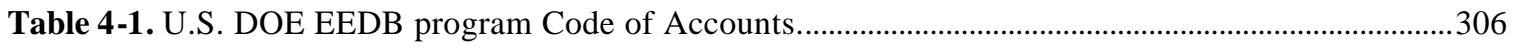

Table 4-2. Accounts used in the preliminary cost estimation of the LMR-AMTEC. …………………….....308

Table 4-3. Dimensions and performance parameters of STEM/desalination preheating unit at nominal

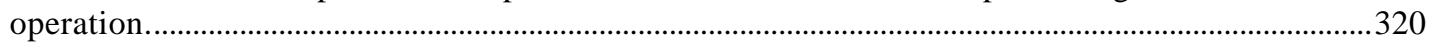

Table 44. Performance comparison of high-energy-utilization plants $\left.\eta_{\text {th }}=95 \%\right)$ with sodium- and

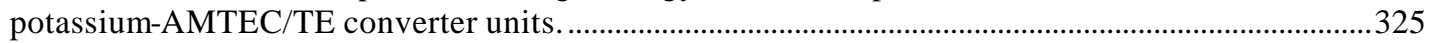




\section{NOMENCLATURE}

\section{English}

A cross-section flow area or surface area $\left(\mathrm{m}^{2}\right)$

$A_{\mathrm{E}} \quad$ Electrode area per BASE element $\left(\mathrm{m}^{2}\right), A_{\mathrm{E}}=2 L_{B} \times H_{B}$

$A_{N} \quad$ Cross-sectional area of TE $\mathrm{n}$-leg $\left(\mathrm{m}^{2}\right)$

$A_{\text {orif }} \quad$ Total flow area of orifices in internal radiation heat shield of AMTEC $\left(\mathrm{m}^{2}\right)$

$A_{P} \quad$ Cross-sectional area of TE $\mathrm{p}$-leg $\left(\mathrm{m}^{2}\right)$

$B \quad$ Temperature-independent, charge-exchange coefficient $\left(\mathrm{A} . \mathrm{K}^{1 / 2} / \mathrm{Pa} \cdot \mathrm{m}^{2}\right)$

BASE Beta"-alumina solid electrolyte

$b \quad$ Separation distance between BASE elements (m)

$C_{p} \quad$ Specific heat of liquid at constant pressure (J/ kg.K)

CVD Chemical vapor deposition

$d \quad$ Inter-electrode gap distance $(\mathrm{m})$

D Flow diffusion coefficient $\left(\mathrm{m}^{2} / \mathrm{s}\right)$

D Diameter (m)

$D_{\text {core }} \quad$ Core vessel diameter $(\mathrm{m})$

$D_{E} \quad$ Inner diameter of economizer (coil) pipe in cold trap (m)

$D^{e q} \quad$ Equivalent hydraulic diameter of flow channel (m)

$D_{f} \quad$ Outer diameter of fuel rod (m)

$D_{I H X} \quad$ Diameter of outer shell of intermediate heat exchanger (m)

$D_{P}^{i n} \quad$ Outer diameter of inlet/centerline pipe of primary coolant in IHX (m)

$D_{S}^{\text {in }} \quad$ Inner diameter of inlet pipe of secondary coolant in IHX (m)

$d_{w} \quad$ Wire diameter of screen mesh in cold trap (m)

EM Electro-magnetic

F $\quad$ Faraday's constant $(F=96,485 . \mathrm{C} / \mathrm{mol})$

$\vec{F} \quad$ Force exerted by the working fluid on solid walls

$F_{i \rightarrow j} \quad$ Geometric view factor between surfaces $A_{i}$ and $A_{j}$

$f \quad$ Darcy friction coefficient for smooth channels, dimensionless

$F_{\text {orif }} \quad$ Ratio of orifices' area in heat shield to area of two side walls of shield

$g \quad$ Earth gravity acceleration, $g=9.81 \mathrm{~m} / \mathrm{s}^{2}$ 


\begin{tabular}{|c|c|}
\hline G & Geometric pressure loss factor, dimensionless \\
\hline Gr & Air Grashof number \\
\hline$G_{\text {total }}$ & Total pressure loss factor in low-pressure cavity of AMTEC, dimensionless \\
\hline$h$ & Enthalpy of working fluid $(\mathrm{J} / \mathrm{kg})$ \\
\hline$H_{B}$ & Height of electrode in BASE element (m) \\
\hline$h^{C V}$ & Convective heat transfer coefficient $\left(\mathrm{W} / \mathrm{m}^{2} . \mathrm{K}\right)$ \\
\hline$H_{E}$ & Height of economizer in cold trap $(\mathrm{m})$ \\
\hline$H_{F}$ & Height of filtration zone of cold trap $(\mathrm{m})$ \\
\hline$H_{f}$ & Active height of fuel in nuclear reactor $(\mathrm{m})$ \\
\hline$H_{\text {fin }}$ & Length of radiator cooling fins $(\mathrm{m})$ \\
\hline$h_{f g}$ & Latent heat of vaporization of working fluid $(\mathrm{J} / \mathrm{kg})$ \\
\hline$H_{\text {gas }}$ & Height of fission gas plenum $(\mathrm{m})$ \\
\hline$H_{\text {pool }}$ & Height of flash evaporator of AMTEC unit (m) \\
\hline$h^{\text {rad }}$ & Radiative heat transfer coefficient $\left(\mathrm{W} / \mathrm{m}^{2} . \mathrm{K}\right)$ \\
\hline$H_{T E}$ & Height of AMTEC condenser walls/thermoelectric modules (m) \\
\hline$H_{t r}$ & Height of transition section between BASE support plate and TE modules (m) \\
\hline$H_{w}$ & Wire spacer lead (axial pitch) (m) \\
\hline$I$ & Electrical current (A) \\
\hline$I_{A M T E C}$ & Electrical current output of AMTEC unit (A) \\
\hline IHX & Intermediate heat exchanger \\
\hline$J$ & Average current density of AMTEC electrode (A / $\left.\mathrm{m}^{2}\right)$ \\
\hline$J^{e x}$ & Charge-exchange current density of AMTEC electrode $\left(\mathrm{A} / \mathrm{m}^{2}\right)$ \\
\hline$k$ & Boltzmann constant, $k=1.3804 \times 10^{-23} \mathrm{~J} / \mathrm{K}$ \\
\hline$k$ & Thermal conductivity ( $\mathrm{W} / \mathrm{m} . \mathrm{K}$ ) \\
\hline$K$ & Pressure form loss coefficient, dimensionless \\
\hline$L$ & Length of BASE support plate in AMTEC unit (m) \\
\hline$L_{B}$ & Width of BASE element (m) \\
\hline$L_{E}$ & Total length of economizer (coil) pipe in cold trap (m) \\
\hline$L_{H}$ & Length of diverging header box (m) \\
\hline$L_{m e s h}$ & Mesh size of wire-screen in cold trap (m) \\
\hline$L_{\text {tube }}$ & Length of straight tubes in intermediate heat exchanger $(\mathrm{m})$ \\
\hline LMFBR & Liquid metal fast breeder reactor \\
\hline$M$ & Molecular weight of working fluid ( $\mathrm{kg} /$ mole) \\
\hline
\end{tabular}




\begin{tabular}{|c|c|}
\hline$m$ & Number of fuel rods in hexagonal fuel assembly, $m=1+3 n(n+1)$ \\
\hline$\dot{m}$ & Mass flow rate of working fluid $(\mathrm{kg} / \mathrm{s})$ \\
\hline$\dot{m}^{\prime \prime}$ & Mass flux of working fluid $\left(\mathrm{kg} / \mathrm{s} . \mathrm{m}^{2}\right)$ \\
\hline$\dot{m}_{I}$ & Mass flow rate of working fluid diffusing through BASE elements $(\mathrm{kg} / \mathrm{s})$ \\
\hline $\mathrm{Ma}^{\text {orif }}$ & Vapor Mach number in orifices of internal heat shield of AMTEC unit \\
\hline$M_{C R T}$ & Chiu-Rohsenow-Todreas (CRT) geometric correction factor, Eq. (D-19) \\
\hline$N_{B}$ & Number of BASE elements connected in series in AMTEC converter \\
\hline$\vec{N}$ & Outward normal vector (unity) \\
\hline$n$ & Number of pitches of fuel rods from center in fuel assembly, $n=7$ \\
\hline$n_{\text {ass }}$ & Number of hexagonal fuel assemblies in nuclear reactor, $n_{\text {ass }}=78$ \\
\hline NIR/PCS & Novel Integrated Reactor/Power Conversion System \\
\hline$N_{\text {fins }}$ & Number of cooling fins in AMTEC heat pipe radiator \\
\hline$n_{\mathrm{H}}$ & Number of outlet pipes in diverging header \\
\hline$N_{\text {layers }}$ & Number of wire-screen layers in cold trap \\
\hline$N_{m e s h}$ & Mesh number of wire-screen in cold trap (per inch) \\
\hline$N_{\mathrm{MOD}}$ & Number of power modules connected in parallel in the power plant \\
\hline$N_{\text {orif }}$ & Number of orifices in internal radiation heat shield of AMTEC unit \\
\hline$N_{\text {rows }}$ & Number of rows of BASE elements in AMTEC unit \\
\hline$n_{\text {tubes }}$ & Number of straight tubes in intermediate heat exchanger \\
\hline $\mathrm{Nu}$ & Nusselt number \\
\hline$N_{\mathrm{UNIT}}$ & Number of AMTEC/TE units connected in parallel in a power module \\
\hline$P$ & Pressure $(\mathrm{Pa})$ \\
\hline$P$ & Triangular pitch of fuel rod in hexagonal assembly (m) \\
\hline $\mathrm{PbTe}$ & Lead telluride TE material \\
\hline$P e$ & Electrical power output (We) \\
\hline $\mathrm{Pe}$ & Peclet number of working fluid, $\operatorname{Pe}=\operatorname{Pr} \times \operatorname{Re}$ \\
\hline$P e^{\prime}$ & Average specific power of AMTEC electrode $\left(\mathrm{We} / \mathrm{cm}^{2}\right)$ \\
\hline$P_{I H X}$ & Triangular pitch of tubes in intermediate heat exchanger (m) \\
\hline $\operatorname{Pr}$ & Prandtl number, $\operatorname{Pr}=\mu C_{p} / k$ \\
\hline$P^{\text {wet }}$ & Wetted perimeter of flow channel (m) \\
\hline $\begin{array}{l}Q \\
Q_{\text {water }}\end{array}$ & $\begin{array}{l}\text { Thermal heat flow }(\mathrm{W}) \\
\text { Thermal power for desalination }(\mathrm{W})\end{array}$ \\
\hline
\end{tabular}




\begin{tabular}{|c|c|}
\hline $\begin{array}{l}q \\
q^{\prime \prime}\end{array}$ & $\begin{array}{l}\text { Radiative heat flux }\left(\mathrm{W} / \mathrm{m}^{2}\right) \\
\text { Thermal heat flux }\left(\mathrm{W} / \mathrm{m}^{2}\right)\end{array}$ \\
\hline$q_{k}^{\text {out }}$ & Outgoing radiant energy flux from surface $A_{k}\left(\mathrm{~W} / \mathrm{m}^{2}\right)$ \\
\hline$Q_{\text {core }}$ & Core thermal power $(\mathrm{W})$ \\
\hline$Q_{\text {input }}$ & Thermal heat input to AMTEC/TE converter unit (W) \\
\hline$q_{\text {Sun }}$ & Insolation heat flux, $q_{\text {Sun }}=1400 \mathrm{~W} / \mathrm{m}^{2}$ \\
\hline $\begin{array}{l}Q_{T D} \\
R\end{array}$ & $\begin{array}{l}\text { Thermodynamic heat of AMTEC top cycle (W), Equation (3.27) } \\
\text { Radius (m) }\end{array}$ \\
\hline$R_{B}$ & Ionic resistance of each BASE element $(\Omega)$ \\
\hline$R_{\text {coll }}^{\prime}$ & Specific resistance of cathode current collector $\left(\Omega . \mathrm{cm}^{2}\right)$ \\
\hline$R_{\text {cont }}^{\prime}$ & Contact resistance between BASE/electrode/current collector $\left(\Omega . \mathrm{cm}^{2}\right)$ \\
\hline$R_{c o n t}^{\prime}$ & Contact resistance per TE leg in thermoelectric couple $\left(\Omega . \mathrm{cm}^{2}\right)$ \\
\hline$R_{\text {int }}^{\prime}$ & Specific internal resistance of AMTEC converter per BASE element $\left(\Omega . \mathrm{cm}^{2}\right)$ \\
\hline $\mathrm{Ra}$ & Air Rayleigh number \\
\hline $\operatorname{Re}$ & Flow Reynolds number, $\operatorname{Re}=\rho_{L} \bar{V} D^{e q} / \mu_{L}$ \\
\hline$R_{g}$ & Perfect gas constant, $R_{g}=8.314 \mathrm{~J} / \mathrm{mol} . \mathrm{K}$ \\
\hline$R_{\mathrm{L}}$ & External load resistance per AMTEC unit $(\Omega)$ \\
\hline$R_{\text {leads }}$ & Electrical resistance of connecting leads between BASE elements $(\Omega)$ \\
\hline$R_{\mathrm{p}}$ & Average pore radius of AMTEC evaporator wick (m) \\
\hline SS & Stainless steel \\
\hline$s$ & Diameter of spacer wire between fuel rods (m), $s=P-D_{f}$ \\
\hline$T$ & Temperature $(\mathrm{K})$ \\
\hline$t$ & Thickness (m) \\
\hline TE & Thermoelectric \\
\hline$T_{b}$ & Coolant bulk temperature $(\mathrm{K})$ \\
\hline$T_{c}$ & Temperature of cold shoe of thermoelectrics $(\mathrm{K})$ \\
\hline$T_{h}$ & Temperature of hot shoe of thermoelectrics $(\mathrm{K})$ \\
\hline$T_{F}^{e x}$ & Coolant temperature at exit of filtration zone in cold trap, $T_{F}^{e x}=350 \mathrm{~K}$ \\
\hline$T_{\text {jet }}^{L}$ & Final temperature of liquid in jet entering the AMTEC flash evaporator (K) \\
\hline$T_{s h}$ & Temperature of internal radiation shield $(\mathrm{K})$ \\
\hline$U$ & Overall heat transfer coefficient $\left(\mathrm{W} / \mathrm{m}^{2} . \mathrm{K}\right)$ \\
\hline
\end{tabular}




\begin{tabular}{|c|c|}
\hline$V$ & Electrical potential $(\mathrm{V})$ \\
\hline $\bar{V}$ & Average flow velocity in channel $(\mathrm{m} / \mathrm{s})$ \\
\hline$W$ & Width of BASE support plate in AMTEC unit (m) \\
\hline$w$ & Massic work of expansion $(\mathrm{J} / \mathrm{kg})$ \\
\hline$W_{C A N}$ & Separation distance between internal heat shield and condenser wall (m) \\
\hline$W_{f i n}$ & Spacing of radiator cooling fins $(\mathrm{m}), W_{f i n}=\delta_{f i n}+\delta$ \\
\hline$w_{m e s h}$ & Width of openings in wire-screen mesh in cold trap $(\mathrm{m}), w_{m e s h}=L_{m e s h}-d_{w}$ \\
\hline $\mathrm{WRh}$ & Tungsten-rhodium AMTEC electrode \\
\hline$W_{\text {fluid }}^{\prime}$ & Mechanical work done on working fluid by pump (W) \\
\hline$y$ & Fraction of inlet mass flow rate diffusing through BASE, $y=\dot{m}_{I} / \dot{m}_{A M T E C}^{i n}$ \\
\hline$z$ & Altitude (m) \\
\hline 7 & Figure-of-merit of thermoelectric material, $Z=\alpha /(\rho k)\left(\mathrm{K}^{-1}\right)$ \\
\hline
\end{tabular}

Greek

$\begin{array}{ll}\alpha & \text { Seebeck coefficient (V / K) } \\ \alpha_{I H X} & \text { Exponential temperature coefficient of IHX, dimensionless } \\ \bar{\alpha}_{2} & \text { Velocity profile correction factor, } \bar{\alpha}_{2}=1.020 \text { for turbulent flow } \\ \bar{\alpha}_{3} & \text { Velocity profile correction factor, } \bar{\alpha}_{3}=1.056 \text { for turbulent flow } \\ \beta & \text { Area ratio for compression/expansion } \\ \beta_{\text {air }} & \text { Thermal expansion coefficient of air at constant pressure }\left(\mathrm{K}^{-1}\right) \\ \gamma & \text { Specific heat ratio of vapor }(\gamma=5 / 3 \text { for monoatomic gas) } \\ \gamma_{e v} & \text { Vaporization fraction in liquid jet entering the AMTEC flash evaporator } \\ \delta & \text { Air gap between cooling fins of heat pipe radiator (m) } \\ \delta_{\text {fin }} & \text { Thickness of cooling fins of heat pipe radiator (m) } \\ \Delta P & \text { Pressure drop (Pa) } \\ \Delta P^{a c c} & \text { Acceleration pressure loss (Pa) } \\ \Delta P^{\text {head }} & \text { Pressure head generated by pump (Pa) } \\ \Delta T & \text { Temperature drop (K) } \\ \Delta T_{\text {core }} & \text { Coolant temperature rise in reactor core (K) } \\ \varepsilon & \text { Surface radiative emissivity, dimensionless }\end{array}$




$\begin{array}{ll}\varepsilon_{E} & \text { Volume porosity of AMTEC electrode } \\ \eta & \text { Conversion efficiency, dimensionless } \\ \eta_{e} & \text { Electrical efficiency, dimensionless } \\ \eta_{f f a s h} & \text { Efficiency of flash evaporation in liquid jet, dimensionless } \\ \eta_{t h} & \text { Thermal efficiency, dimensionless } \\ \mu & \text { Dynamic viscosity }(\mathrm{kg} / \mathrm{m} . \mathrm{s}) \\ \nu & \text { Kinematic viscosity }\left(\mathrm{m}^{2} / \mathrm{s}\right), v=\mu / \rho \\ \xi_{k} & \text { Emissive flux of surface } A_{k}, \xi_{k}=\varepsilon_{k} \sigma T_{k}^{4}\left(\mathrm{~W} / \mathrm{m}^{2}\right) \\ \xi_{c} & \text { Charge-exchange polarization loss of cathode electrode }(\mathrm{V}) \\ \rho & \left.\text { Density (kg / } \mathrm{m}^{3}\right) \\ \rho & \text { Electrical resistivity }(\Omega . \mathrm{m}) \\ \sigma & \text { Stefan-Boltzmann's constant, } \sigma=5.67 \times 10^{-8} \mathrm{~W} / \mathrm{m}^{2} . \mathrm{K}^{4} \\ \sigma_{\mathrm{B}} & \text { Ionic resistivity of BASE }(\Omega . \mathrm{m}) \\ \sigma_{L} & \text { Surface tension of liquid }(\mathrm{N} / \mathrm{m}) \\ \tau_{r x} & \text { Viscous shear stress on inner wall of circular pipe (N / m) } \\ \phi & \text { Energy, work function }(\mathrm{eV}) \\ \Phi^{v i s} & \text { Viscous dissipation (mechanical energy lost by flow) }(\mathrm{W})\end{array}$

\section{Subscript / Superscript}

a Porous anode (high-pressure cavity of AMTEC unit)

air Ambient air

AMTEC AMTEC top cycle

ass Coolant channel in fuel asssembly

B Beta"-alumina solid electrolyte (BASE)

BARE Bare material

bend smooth circular bend in coolant pipe

Bun(dle) Tubes' bundle of intermediate heat exchanger (shell side)

c BASE/cathode electrode interface (low-pressure cavity of AMTEC unit)

cc Closed circuit

cd Condenser 


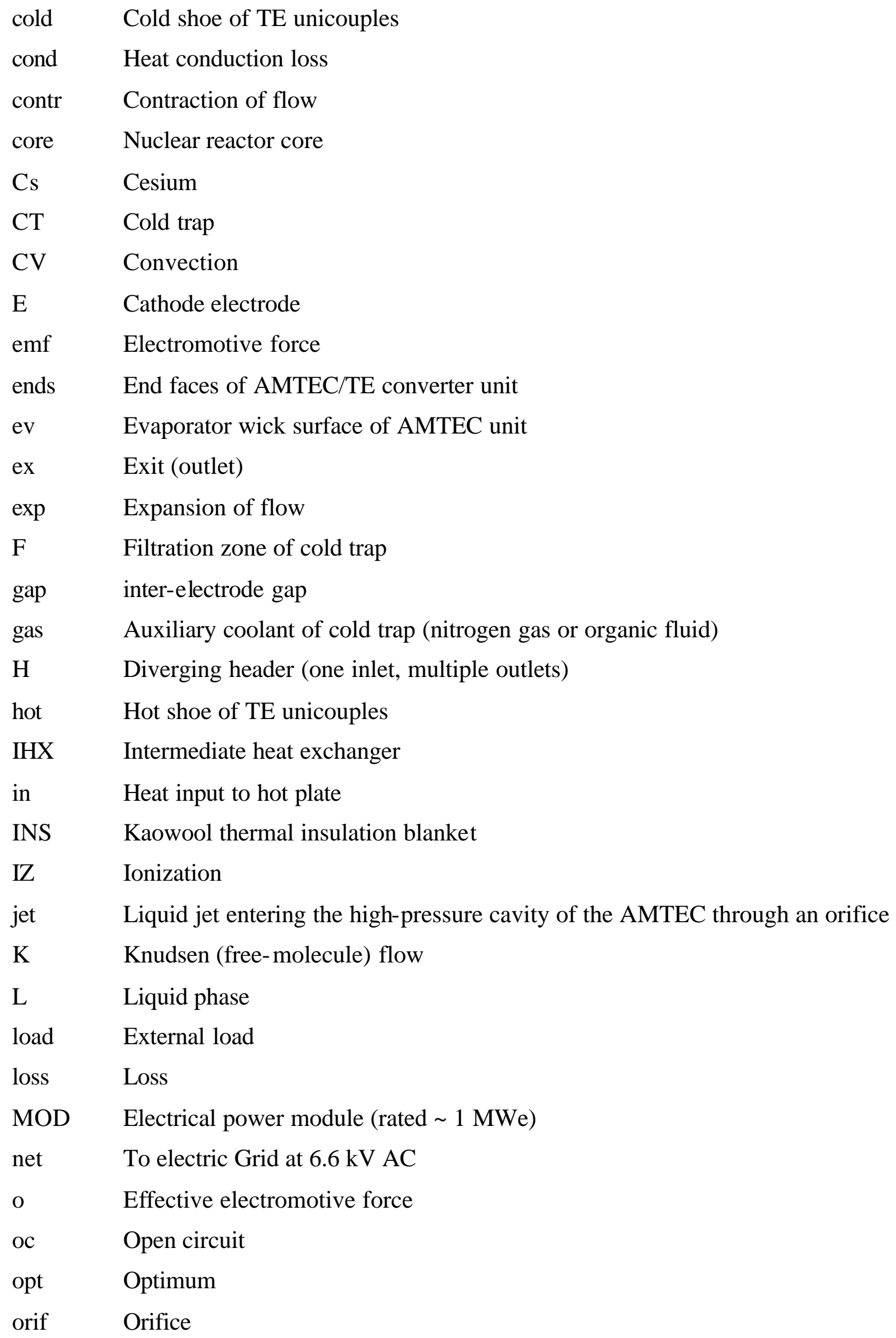




$\begin{array}{ll}\text { out } & \text { Outgoing radiant energy } \\ \text { P } & \text { Primary } \\ \text { plen } & \text { Plenum } \\ \text { pool } & \text { Liquid pool in flash evaporator } \\ \text { pump } & \text { Linear induction electro- magnetic pump } \\ \text { R } & \text { Cesium reservoir } \\ \text { rad } & \text { Heat pipe radiator of AMTEC/TE converter unit } \\ \text { rad } & \text { Radiation heat loss } \\ \text { rej } & \text { Heat rejection to the AMTEC condenser walls } \\ \text { roof } & \text { Rooftop wall of AMTEC unit containment } \\ \text { S } & \text { Secondary } \\ \text { s } & \text { Shunt resistance } \\ \text { sat } & \text { Liquid-vapor saturation line } \\ \text { sh, SH } & \text { Internal radiation heat shield in AMTEC unit } \\ \text { shell } & \text { Shell side of economizer in cold trap } \\ \text { sides } & \text { Vertical side walls of internal radiation heat shield in AMTEC unit } \\ \text { STE } & \text { Segmented thermoelectric couple } \\ \text { STEM } & \text { Segmented thermoelectric module } \\ \text { sub } & \text { Liquid subcooling } \\ \text { sup } & \text { Liquid superheat } \\ \text { surf } & \text { Outer surface of Kaowool insulation blanket } \\ \text { TE } & \text { Thermoelectric bottom cycle } \\ \text { top } & \text { Top section of internal radiation heat shield in AMTEC unit } \\ \text { tr } & \text { Flow transverse to IHX tubes' bundle (secondary coolant) } \\ \text { tube(s) } & \text { Straight tubes in intermediate heat exchanger (IHX) } \\ \text { UNIT } & \text { AMTEC/TE converter unit (combined top and bottom cycles) } \\ \text { V } & \text { Vapor phase } \\ \text { valve } & \text { Electro-magnetic valve } \\ \text { vis } & \text { Viscous flow } \\ \text { W } & \text { Metallic wall } \\ \text { WF } & \text { Workfunction } \\ \text { o } & \text { Inner wall of circular tube } \\ \text { 1 } & \text { Outer wall of circular tube } \\ & \end{array}$




\title{
1 Introduction
}

This Final Scientific/Technical Report presents the work performed under the LMR-AMTEC project. The overall objectives of this project were to assess the feasibility, develop engineering solutions, and determine a range of potential applications for a Novel Integrated Reactor/Energy Conversion System. The ultimate goal was to design a proliferation resistant, reliable and economical power supply for use by developing countries and in remote locations. The main features of this project were the development of a long life (up to 10 years) Liquid Metal Reactor (LMR) and a static conversion subsystem comprising an Alkali Metal Thermal to-Electric (AMTEC) topping cycle and a ThermoElectric (TE) Bottom cycle. Furthermore, various coupling options of the LMR with the energy conversion subsystem were explored.

The project was performed jointly by the Westinghouse Electric Company LLC (WEC), which was responsible for the long-life sodium reactor development, the University of New Mexico's Institute for Space and Nuclear Power Studies (UNMISNPS), which was responsible for developing the AMTEC/TE energy conversion subsystem and designing the electric converter modules, and the Institute for Engineering Research and Applications (IERA) at the New Mexico Institute of Mining and Technology, which was responsible for supporting Westinghouse's activities related to the transport safety and waste disposal. The following persons contributed to this work:

\section{Westinghouse Electric Company LLC}

P. R. Rubiolo (PI)

B. Petrovic

D. V. Paramonov

M. Carelli

B. Liu

H. D. Garkisch

V. N. Kucukboyaci

Institute for Space and Nuclear Power Studies (ISNPS)

Mohamed S. El-Genk

Jean-Michel Tournier

Yoichi Momozaki

Jeffrey King

Institute for Engineering Research and Applications (IERA)

\author{
Ahmed Hasan \\ Tarek F. Mohammaden \\ Yasser T. Mohamed \\ Glen L. Schmidt \\ John P. Marquis
}


The project schedule is shown in Figure 1-1. Following is a discussion of the tasks completed throughout the project, summary of the results and the problems encountered during the research.

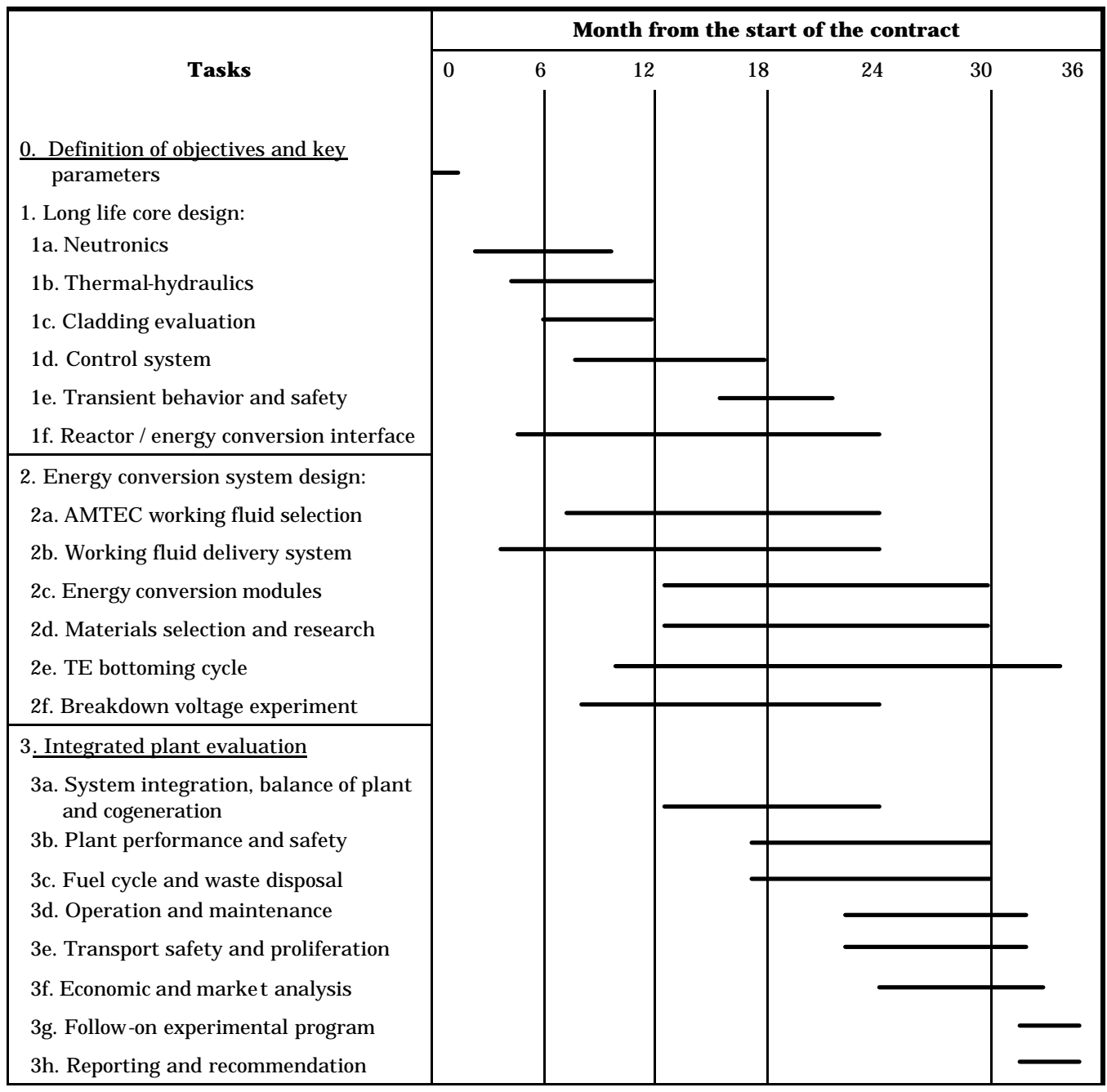

Figure 1-1. Project schedule.

\section{Task 0- Definition of Objectives and Key Parameters}

This task defined the operational objectives and desired characteristics of the LMR/AMTEC system, including the power level, lifetime, operating conditions, efficiency, proliferation resistance implementation, fabricability, transportation, waste disposal strategy, and cost objectives. 


\section{Task 1- Long life Reactor Design}

\section{Subtask 1a- Neutronics}

A neutronics design of a long-life core capable of a straight burn of 10 years or more was accomplished. The work included the characterization of the fuel, including enrichment level, core configuration, power distribution profile, reactivity feedbacks, and control requirements.

\section{Subtask 1b- Thermal Hydraulics}

The objective of this task was to perform the thermathydraulics design of the long-life core. A hexagonal fuel assembly design with wire-wrap was adopted. Initially, a canned fuel assembly was preferred. However, an open fuel assembly was finally chosen because of its ability to minimize the consequences of a coolant blockage in a core channel. One critical issue of the reactor core thermathydraulics design was the maximum cladding temperature. Hence, the temperature profiles of the fuel pellet and the cladding were analyzed. In predicting the temperature profile of the fuel rod, the semi-statistical method and a set of sub-factors developed by Westinghouse for the Clinch River Breeder Reactor Project were used.

\section{Subtask 1c- Cladding Evaluation}

This task investigated the candidate materials for the fuel rod cladding. Due to the high operating temperatures, the $\mathrm{Nb}-1 \mathrm{Zr}$ alloy was found to be the only alloy adequate for this application. However, an important drawback of this material is its strong neutron absorption, which deteriorates the neutron economy. Other important issues addressed in this task included the determination of the size of the plenum, the cladding behavior under high temperature, radiation conditions, and the flow-induced vibrations. The size of the plenum was determined to restrict the fission products pressure in the same manner as the Westinghouse LMR Ultra Long Life Core (ULLC). The evaluation of the damage due to radiation included the determination of the fast fluence and the displacement per atom (DPA). The cladding thickness was assessed according to the $\mathrm{Nb}-1 \mathrm{Zr}$ creep curves. Flow-induced vibration behavior of the fuel rods was also studied. Fretting-wear induced by vibration appears to be a concern in the design. Further studies will be required to assess this phenomenon. To accomplish this task, development of correlations applicable to the sodium coolant at high temperatures will be required.

\section{Subtask 1d- Control System}

As a result of this task, an actively-controlled absorber rod bundle assembly was adopted as a reactivity control system. Boron carbide was selected as the Control Rod (CR) absorber material. Determining the size of the CR system was challenging due to the large reactivity swing during the life of the core. If two independent shutdown systems are required, a 19 CR configuration needs to be used instead of the initial 13 CR configuration.

\section{Subtask 1e- Transient Behavior and Safety}

Safety of any LMR basically resides in its capability to provide reliable reactivity control (and shutdown) and heat removal (both during operation transients and after shutdown). In this task, the shutdown systems of the reactor were selected and the sodium void effect of the LMR-AMTEC was investigated. For the sodium void effect, two cases were considered: a hypothetical central core voiding and a more realistic case of upper core voiding. The results demonstrated acceptable core reactivity response under the two sodium voiding scenarios. In the second part of this task, a Decay Heat Removal System (DHRS) was designed. The secondary loop, composed of the alkali metal boilers and the AMTEC/TE converter units, was chosen as a normal DHRS of non-safety grade during hot and cold shutdowns. In addition, a safety grade Passive Heat Removal System (PHRS) was proposed as an emergency DHRS. The PHRS of the LMR-AMTEC is activated by changes in the sodium levels after trip of the primary pumps. To study the performance of the PHRS, a Loss of Flow Accident was chosen as limiting accident due to the high operating temperatures of the reactor. The system showed enough capacity to passively remove the decay heat by natural circulation.

\section{Subtask 1f- Reactor/Energy Conversion Interface}

The objective of this task was to investigate the feasibility of a direct coupling between the LMR and the AMTEC units (i.e. using the same coolant), and to explore other indirect coupling options. A numeric plant model was used to study the following three configurations: 1- Direct Coupling (same working fluid), 2- 
Indirect Coupling using liquid/liquid Intermediate Heat Exchangers (IHX), and 3- Indirect Coupling using Alkali Metal boilers. As a result of this study, the direct coupling configuration was abandoned because of its safety and economic drawbacks. Instead, the Indirect Coupling (IC) plant with Alkali Metal Boilers (AMB) was chosen for its superior performance with respect to the net plant efficiency, the reactor vessel weight and corrosion behavior. In addition, potassium was chosen as the secondary coolant since potassium-AMTEC converter units deliver higher efficiency than sodium-AMTEC converter units when operating at a fixed core exit temperature. The indirect coupling with IHXs and closed-unit AMTEC/TE converters was kept as an alternative design.

\section{Task 2- Energy Conversion System Design}

\section{Subtask 2a- AMTEC Working Fluid Selection}

The objective of this task was to select the working fluid. Conventional AMTEC devices use sodium as the working fluid, primarily due to the availability of the appropriate solid electrolyte. However, lithium and potassium have also been considered as AMTEC working fluids. In this Task, the thermophysical and radiological properties of alkali metal (sodium, potassium and lithium) working fluids for use in the LMRAMTEC were revie wed. Lithium was eliminated from consideration in the AMTEC based on its extremely low vapor pressure requiring operating at high temperatures $>1300 \mathrm{~K}$. Sodium has been used extensively in liquid metal reactors and AMTEC. Potassium has higher vapor pressure and lower latent heat of vaporization and specific heat than sodium, which result in a lower heat input, and higher AMTEC conversion efficiency than in sodium AMTEC when operated at the same hot side temperature. However, the condenser temperature of the potassium AMTECs could be $\sim 90 \mathrm{~K}$ lower than sodium AMTECs, resulting in a lower conversion efficiency of the TE bottom cycle. In addition, there is limited experience with potassium coolant and K-BASE solid electrolyte which has higher ionic resistivity than Na-BASE. Based on this review, potassium was chosen as the working fluid for its better performance from the efficiency point of view.

\section{Subtask 2b- Working Fluid Delivery System}

In this task various methods of delivering the working fluid from the eactor core to the AMTEC/TE converter units were assessed. This task was performed concurrently with subtask 1f, "Reactor / Energy Conversion Interface" above. Two possible design configurations for the AMTEC/TE converter units, regarding the generation of the alkali metal vapor for the AMTEC top cycle, were developed: (a) an openunit design configuration, in which hot alkali-metal (liquid or vapor) is introduced into the high-pressure cavity of the AMTEC/TE converter units; and (b) a closed-unit design configuration, in which the thermal power from the liquid metal coolant is transmitted through the metallic wall of a converter heat exchanger to an evaporator wick at the bottom of the AMTEC unit. In this configuration, the AMTEC unit carries its own charge of working fluid, and the portion that diffuses through the BASE elements is circulated back from the condenser to the evaporator wick through a porous wick. In the open-unit design configuration, the hot vapor generated by the Alkali Metal Boilers condenses inside the porous anodes of the BASE elements, and excess working fluid forms a liquid pool at the bottom of the unit's high-pressure cavity. Alternatively, alkali metal from a secondary liquid loop can be introduced into the high-pressure cavity of the AMTEC unit through an orifice, and partially flashes into vapor. A disadvantage of this design is the relatively high mass flow rate required in the secondary loop in order to obtain an adequate efficiency in the flash evaporators of the AMTEC. In the case of an open-unit design, the fraction of the mass flow rate introduced into the AMTEC which did not diffuse through the BASE membranes, and the condensate are both recirculated back to the IHXs or AMBs using electromagnetic or mechanical pumps.

\section{Subtask 2c- Energy Conversion Modules}

In this task, the energy conversion subsystem, consisting of an AMTEC top cycle and a TE bottom cycle conductively coupled to the AMTEC condenser, was designed. The top cycle uses a vapor-fed, liquid anode AMTEC design, in which the hot alkali metal vapor in the high-pressure cavity is condensed into a porous structure covering the anode side of dome-shaped, composite BASE elements. This design essentially eliminates the electrical losses on the anode side, and allows connecting a large number of BASE elements in series, to deliver a voltage output of $\sim 400 \mathrm{~V}$ DC. Furthermore, the efficient heat transfer 
to the BASE anodes by condensation of the working fluid results in a nearly uniform temperature of the BASE. This in effect increases the converters' efficiency for a given coolant core exit temperature, reduces the induced thermal stresses in the BASE membrane, and increases converter life. Furthermore, the AMTEC/TE converter was designed specifically for cooling by natural convection of air. Numerical analysis models of the AMTEC/TE converter unit were developed and used to optimize the design for maximum overall thermodynamic efficiency and compare the performances of the Na- and K-AMTEC/TE converter units. Estimates of the conversion efficiency of the static AMTEC/TE converter for the LMRAMTEC power plant showed that values in excess of 30\% could be achieved, which are based on conservative assumptions regarding the technology of the AMTECs and on using off-the-shelf TAGS-85 and $2 \mathrm{~N}-\mathrm{PbTe}$ lead telluride TE modules.

\section{Subtask 2d- Materials Selection and Research}

In this task, the properties of various materials to be used in the working fluid delivery system and AMTEC cells were evaluated. Performance criteria were based on high temperature tensile strength, high temperature and radiation creep strength, initial grain size and grain size stability, thermal conductivity, thermal expansion, compatibility with selected working fluid, fabricability, weldability, contamination resistance during manufacturing, commercial availability and cost. Based on these considerations, appropriate materials for the AMTEC module were also selected. As more advances are made in the development of thin composite BASE membranes, high performance AMTEC electrodes, and the fabrication of reliable metal-graded ceramic brazes, higher conversion efficiencies in excess of $35 \%$ for the combined AMTEC/TE converters with a long operation lifetime of 510 years, with little degradation, would be possible. Some of these technology advances have been reported very recently, and are being investigated, by industry, universities, and government research laboratories. Recent work done at the Jet Propulsion Laboratory in Pasadena, California, has shown that unlike the TiN electrodes used in the AMTEC cells developed during the space program, the new tungsten/rhodium (WRh1.5) electrodes have demonstrated excellent performance properties and long-term stability. Furthermore, efforts to develop mixed-conducting (ionic and electronic) metal/oxide electrodes such as blends of Mo/Nax-TiO2 and TiN/Nax-TiO2 are underway at both the Jet Propulsion Laboratory and Advanced Modular Power systems (AMPS), Inc.. These electrodes can be applied as a paint or slurry, are robust and inexpensive, and have demonstrated to date a performance equivalent to the best currently available refractory electrode (WRh1.5).

\section{Subtask 2e- TE Bottoming Cycle Assessment}

Taking advantage of a relatively high AMTEC heat rejection temperature, a TE bottom cycle conductively coupled to the AMTEC condenser was developed and designed to further increase the converter's efficiency. The electric power output from the AMTEC units is fed to the grid or the end user separately from that generated by the TE modules. The TE couples are electrically connected in series and in parallel to bring the voltage output to a usable value, while ensuring proper redundancy in the design. To ensure a uniform cold side temperature of the TE couples and for efficient operation and low converter weight, a metallic heat pipe spreader and cooling fins are used. They are charged with water working fluid, to reject the waste heat from the TE bottom cycle efficiently. The fins are cooled by natural convection of air for space or district heating. Three different bottom cycle converter configurations were studied: a P-TAGS85/2N-PbTe unicouple, a P-BiTe/N-BiTe unicouple, and a segmented TE unicouple (STE) that uses PTAGS-85/P-BiTe in the P-leg, and 2N-PbTe/N-BiTe in the N-leg. A one-dimensional optimization model of segmented TE unicouple was developed, and benchmarked successfully against experimental data from the Jet Propulsion Laboratory. The model determines the optimum lengths of the various segments and the ratio of the legs' cross-sectional areas for maximum efficiency and for maximum electrical power operations. Results showed that the use of the STE in the bottom cycle of the sodium-AMTEC/TE converter would only increase the unit's overall efficiency by $\sim 0.6$ percentage point, and this small gain does not justify the added complexity of the segmented design and associated issues of bonding the different TE materials. Thus, the P-TAGS-85/2N-PbTe unicouple was selected for both the sodium and the potassium converters. The single-segment TE unicouple can deliver peak efficiencies of $4.6 \%$ and $6.4 \%$ at temperature drops of $150 \mathrm{~K}$ and $230 \mathrm{~K}$ across the legs, respectively. 


\section{Subtask 2f- Breakdown Voltage Experiment}

In this task, laboratory tests were performed at UNM-ISNPS to investigate the breakdown in low-pressure cesium vapor and relate the results to sodium and potassium vapor in the AMTEC converters. A DC electrical breakdown in the low-pressure cavity of the AMTEC would increase the leakage current to the wall, hence degrading the performance of the converter, in addition to potentially damaging the cathode electrodes and the converter wall. The experiments conducted at electrodes temperatures of $1100 \mathrm{~K}$ and $625 \mathrm{~K}$ have shown clearly that when the cooler electrode was negatively biased the breakdown voltage was beyond the limit of the power supply used (> $396 \mathrm{~V}$ ). In addition to the potentially very high breakdown voltage $(>400 \mathrm{~V})$, the corresponding discharge current was quite small. Conversely, when the cooler electrode was positively biased, the breakdown voltage was in the single figure and followed a Pachen-type dependence on the cesium pressure. For the typical vapor pressures in the low-pressure cavity of an AMTEC of 20-60 Pa, the measured breakdown voltage in the cesium vapor was almost the same as the first ionization potential of cesium $(3.89 \mathrm{~V})$. The corresponding discharge currents at these values of the breakdown voltage were relatively high. Since cesium has the lowest first ionization potential of all alkali metals $(3.89 \mathrm{~V}$, versus $5.14 \mathrm{~V}$ for sodium and $4.34 \mathrm{~V}$ for potassium), and since the effective work function of tantalum in cesium vapor is the lowest, followed by that in potassium, then that in sodium vapor, it may be argued that for the same electrodes temperatures and polarity and the same vapor pressure, the breakdown voltages in potassium and sodium vapors in the low-pressure cavity of an AMTEC would be at least the same, but most likely higher than those reported in this work for cesium vapor. Based on the results of these breakdown experiments, The present AMTEC/TE converter units were designed to deliver more than $50 \mathrm{kWe}$ each at a voltage output of $\sim 400 \mathrm{~V}$ and an overall conversion efficiency $>30 \%$.

\section{Task 3- Integrated Plant Evaluation}

\section{Subtask 3a- System Integration, Balance of Plant and Cogeneration}

For this task a numerical model of the LMR-AMTEC plant was developed by WEC. For a chosen core and energy conversion subsystem configuration, a preliminary design of a plant-balance including decay heat removal system, secondary coolant circuit, support structures, power conditioning equipment, auxiliary systems, confinement, instrumentation, and radiation protection was performed. In this task, the possibility of utilizing the high AMTEC heat rejection temperature for providing a cogeneration capability was studied.

\section{Subtask 3b- Plant Performance and Safety}

In this task, the LMR-AMTEC model was used to determine the optimum operating conditions and the corresponding net plant efficiency. A design optimization procedure was developed. The optimized parameters included working temperatures, flow rates and pressures, Alkali Metal Boiler (AMB) design and operation, primary-pump characteristics and reactor vessel design. The work performed in this task was strongly coupled with Subtask 1f since for each set of operating parameters, the transient response needed to satisfy the safety margins. In addition, since the performance of the PHRS depended on the sodium relative levels inside the vessel, the in-vessel layout was optimized to obtain the best system performance. Due to a large number of uncertainties on the reliability of the main plant components (such as the AMTEC/TE converter units or the electric power conversion system), the system's response to a given component failure was not investigated.

\section{Subtask 3c: Fuel Cycle and Waste Disposal}

A once-through fuel cycle was chosen for the LMR-AMTEC. The fuel cycle cost analysis was performed iteratively with Subtask $3 \mathrm{e}$ in order to quantify the effect of fuel costs on the overall plant capital cost and the cost of electricity. Based on the selected design of the LMR-AMTEC components and the coolant types, the wastes were classified and characterized according to the Code of Federal Regulations. The applicable U.S.A. regulations governing waste transportation, treatment, storage and final disposition were also identified. The separate handling of the different types of waste appears to be the most adequate.

\section{Subtask 3d: Operation and Maintenance}

Operation of the main components was investigated. Particularly, the performance of the alkali metal boilers in once-though and in recirculation mode was investigated. 


\section{Subtask 3e: Transport Safety and Proliferation}

The truck and/or rail transportation limitations for the large components of the LMR-AMTEC were studied in this activity. The analysis was performed from weight and size points of view, according to the laws and regulations set by the Federal Administration and/or by the individual States. The proliferation issues were addressed through the adoption of a no-refueling strategy and the selection of an appropriate balance between fuel enrichment and core life, and furthermore, by adopting a pool configuration, which allows reducing the number of vessel penetrations and a complete factory assembly.

\section{Subtask 3f: Economic and Market Analysis}

A first economic analysis of the plant was performed and coupled with the LMR-AMTEC plant model. This economic evaluation was used to assist in selecting the optimal working parameters of the reactor and to assess the cost impact of using the AMTEC energy conversion. A relative cost evaluation was chosen as the methodology due to the difficulties encountered in determining the capital cost of the main components such as reactor vessel, fuel fabrication and the AMTEC/TE converter units. For this last component, only a very rough estimation was possible due to large uncertainties in cost, both in near and long terms. In this task, different high-energy utilization uses and the nuclear power plant options for those applications were also addressed.

\section{Subtask 3g: Follow-on Experimental Program}

The objective of this subtask was to design a subscale proof-of-principle demonstration of the proposed concept and verification of an AMTEC unit in prototypical configuration with an appropriate working fluid supply system. Due to the changes in the scope of the project this subtask was abandoned and the effort was redirected to support subtasks $3 \mathrm{a}$ and $3 \mathrm{~b}$.

\section{Subtask 3h: Reporting and Recommendation}

In this final subtask, the results of the long life core and the AMTEC/TE energy conversion system development and integrated plant evaluation were summarized and a final report was prepared.

The Final Scientific/Technical Report is organized into three chapters based on the major research areas:

\section{- $\quad$ Chapter 2: Liquid Metal Reactor Conceptual Design \\ - $\quad$ Chapter 3: Static Energy Conversion System Design \\ - $\quad$ Chapter 4: Performance Evaluation of the LMR-AMTEC Concept \\ - $\quad$ Conclusions and Recommendations}

Chapter 2 presents the work performed by Westinghouse Electric Company LLC on the conceptual design of the main reactor components and the determination of the operating parameters. These parameters include working temperatures, flow rates, pressures, alkali metal boiler design, primary pumps, reactor vessel, in-vessel layout and the power conversion system. This chapter also presents the work performed on the safety features of the LMR-AMTEC, including the reactivity control systems and the design of the heat removal systems. Chapter 3 presents the work performed by UNMISNPS on the design of the AMTEC/TE energy conversion subsystem and the electric conversion modules. The developed converters are comprised of an AMTEC top cycle and a PbTe TE bottom cycle on the condenser side of the AMTEC. The converters are cooled by natural convection of ambient air and are optimized for maximum overall conversion efficiency. Chapter 4 presents a preliminary economic analysis of the plant 
performed by WEC. Also in this chapter, a study of the different high-energy utilization uses and the nuclear power plant options for those applications, developed by UNMISNPS, are presented. 


\title{
2 Liquid Metal Reactor Conceptual Design
}

\author{
by Westinghouse Electric Company
}

The work performed on the "Long Life Core Design" and the "Integrated plant evaluation" (except the Economic and Market Analysis which is reported in Chapter 4) is reported in this Chapter. Section 2.1 discusses the Reactor/Energy Conversion Interface options. The design analyses and results of the long-life core (neutronics, thermat hydraulics and mechanics) and of the alkali metal boilers (AMBs) are reported in Section 2.2, and the safety features of the LMR-AMTEC are discussed in Section 2.3. The transportation and Waste disposal aspect of the design are discussed in Section 2.4. Finally the results of this Chapter are summarized in Section 2.5.

\subsection{Coupling Between the LMR and the AMTEC/TE Units}

Different design solutions can be proposed to address the technical problems arising from the coupling between the LMR and the AMTEC/TE units. Three different options for interfacing the nuclear reactor with the AMTEC/TE converter units were investigated (see also the Year Two Technical Progress Report): (a) a Direct Coupling (DC) arrangement between a liquid core and the energy conversion subsystem; (b) an Indirect Coupling (IC) arrangement between a liquid core and the energy conversion subsystem, through liquid-liquid intermediate heat exchangers (IHXs); and (c) an Indirect Coupling (IC) arrangement between a liquid core and the energy conversion subsystem, through alkali metal boilers (AMBs).

In addition to the core and converters interfacing, there are two possible design configurations for the AMTEC/TE converter units: (a) an open-unit design configuration in which the hot alkali-metal coming from the IHXs or the AMBs is directly introduced into the high-pressure cavity of the converter; and (b) a closed-unit design configuration in which the thermal power from the alkali metal is transmitted through the metallic wall of a heat exchanger to an evaporator wick at the bottom of the converter. In this configuration, the converter carries its own charge of working fluid, and the fluid in the converter that diffuses through the BASE elements is circulated back from the condenser to the evaporator wick of the AMTEC unit through a number of return artery wicks. These different possible arrangements are investigated and discussed in Section 2.1. 


\subsubsection{Direct Coupling Design}

From an engineering point of view, there are two possible options for coupling the nuclear reactor and the electrical energy conversion subsystem, namely: direct coupling (DC) and in-direct coupling (IC) interfacing arrangements. Figure 2-1 shows a schematic of the direct coupling design. In this concept, the liquid metal heated inside the core is directly transported to the AMTEC/TE converter units. The alkali metal liquid is introduced through an orifice into the high-pressure cavity of the AMTEC units, where it partially flashes into vapor as it expands. The direct coupling design is a loop-type reactor. The AMTEC/TE converter units and their condensers are placed inside of the containment building.

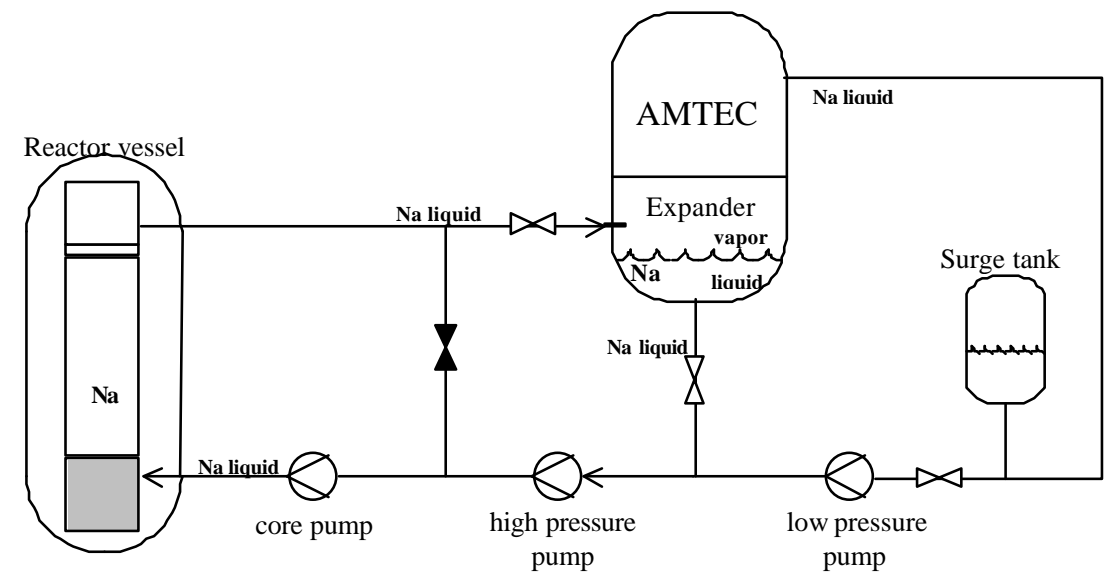

Figure 2-1. Diagram of the LMR-AMTEC direct coupling with expanders design (for simplicity only one loop is shown).

The main advantage of the DC design is the plant simplification arising from the fact that no Heat Exchangers $(\mathrm{HX})$ are required. However, the impact of this simplification is overridden by the following drawbacks:

- $\quad$ LOCA larger breaks of the primary coolant pipes are not excluded by design. Challenging when using guard vessel.

- $\quad$ Small coolant inventory increases the severity of reactor transients.

- Shielding: since the coolant is radio-activated, shielding is required around the pipes and the AMTEC units, making the operation and maintenance of the reactor difficult

- $\quad$ Size of the containment is increased due to the AMTEC unit and the expanders residing together inside the vessel.

- Corrosion will occur due to i) the transport of corrosion products between the reactor core and the AMTEC/Condenser and ii) the significant variation of the coolant temperature in the loop. 


\subsubsection{Indirect Coupling Designs}

In the indirect coupling scheme, a secondary, liquid metal coolant loop is added which is thermal-hydraulically coupled to the reactor's primary coolant loop through liquid-liquid Intermediate Heat Exchangers (IHX) or Alkali Metal Boilers (AMBs). The main advantage of the IC design is its high flexibility regarding the plant design (such as the coolant and structural material selection). Using a secondary coolant loop will keep the activation products in the reactor core within the primary coolant loop. Thus, the converters and the entire secondary coolant-loop can be kept outside of the reactor's primary containment and therefore the converters can be safely and easily serviced, without requiring a reactor shutdown and a waiting period for the induced radioactivity to drop to an acceptable level. In addition, during a scheduled maintenance or a removal of a failed converter module, the module can easily be hydro-dynamically and electrically isolated from the plant and replaced, without affecting the operation of the reactor or the rest of the power plant. This design flexibility improves the reliability of the power plant. The location of the converters outside of the primary containment also facilitates the use of natural convection cooling of the condenser side and provides easy access to highvoltage transmission lines.

Other advantages of the indirect interfacing arrangement of the nuclear reactor and the electric conversion subsystem include using separate flow management and filtering of the primary and secondary loops. This scheme also offers flexibility of selecting different working fluids and/or structural materials for the reactor and the converters, as required by the design and independent operation and testing of each. In addition, using primary and secondary loops prevents the transport of any contaminant between the reactor core and the electrical converters. Finally, the use of an indirect interfacing arrangement facilitates testing of a fully-integrated secondary side (converter) at typical operating conditions, and minimizes the impact of the failure of one or more power units on the reactor operation.

By contrast, a direct interfacing arrangement between the reactor core and the electrical energy converters does not need a liquid-liquid heat exchanger, and that is its only advantage over an indirect interfacing arrangement. In a direct interfacing arrangement, a bypass line and mixing chamber are required to allow control of the reactor inlet coolant temperature independently of the flow rate and thermal power needs of the electrical converters. Because of the relatively long half-lives of $24 \mathrm{Na}$ (15 hrs) and $42 \mathrm{~K}(12.4 \mathrm{hrs})$, the alkali-metal coolant in the converters will be radioactively hot, and all components of the power plant would require extensive radiation shielding. In the event that maintenance or servicing of the power units is required, the reactor must be shut down, and the plant must remain idle for several days to allow radioactivity levels to drop below acceptable limits. As detailed in Table 2-1, the direct interfacing arrangement of the nuclear reactor core and the electric energy conversion subsystem does not have the flexibility in design, integration, testing and maintenance offered by the indirect interfacing arrangement. However simpler it may appear in design, a direct interfacing arrangement must demonstrate a very high level of reliability and radiological safety to ensure that it can operate safely over the power plant lifetime (10 to 15 years). 
Table 2-1. Some characteristics of indirect and direct interfacing arrangements of the nuclear reactor and the electric energy conversion subsystem.

\begin{tabular}{|c|c|c|}
\hline Design characteristics & Indirect coupling & Direct coupling \\
\hline $\begin{array}{l}\text { a-Layout } \\
\text { - Requires intermediate heat } \\
\text { exchanger } \\
\text { - Requires core bypass line to } \\
\text { control the reactor inlet } \\
\text { temperature } \\
\text { - Requires shielding of the entire } \\
\text { primary coolant loop and the } \\
\text { energy conversion modules } \\
\text { - Allows natural convection heat } \\
\text { removal from the AMTEC/TE } \\
\text { electric energy converter }\end{array}$ & $\begin{array}{l}\qquad \text { Yes } \\
\qquad \text { No } \\
\text { No } \\
\text { Easily, since the entire secondary loop } \\
\text { and the energy conversion modules are } \\
\text { placed outside the primary containment } \\
\text { building in open air. }\end{array}$ & $\begin{array}{l}\qquad \text { Yes } \\
\qquad \text { Yes } \\
\text { Not easily, since the electric energy } \\
\text { converters will be kept inside the } \\
\text { reactor's containment. It may require } \\
\text { passive methods for heat rejection } \\
\text { from the reactor containment building } \\
\text { or the use of a heat rejection heat } \\
\text { exchanger. }\end{array}$ \\
\hline b-Design/integration flexibility & Extremely high & Somewhat limited \\
\hline c-Structural materials' selection & Most flexible & More limited \\
\hline $\begin{array}{l}\text { d-Primary coolant flow and } \\
\text { thermal management }\end{array}$ & $\begin{array}{l}\text { - Contains most of the induced } \\
\text { radioactivity within the primary loop } \\
\text { - Provides flexibility of selecting } \\
\text { AMTEC working fluid, which could be } \\
\text { different from the reactor coolant } \\
\text { - Converters are outside reactor } \\
\text { containment }\end{array}$ & $\begin{array}{l}\text { - Use core bypass line to control inlet } \\
\text { temperature to reactor core } \\
\text { - Radiological contamination of } \\
\text { converters and piping system } \\
\text { - Reactor coolant is same as AMTEC } \\
\text { working fluid } \\
\text { - Converters are inside reactor } \\
\text { containment }\end{array}$ \\
\hline $\begin{array}{l}\text { e-Control of contaminants, } \\
\text { radioactivity, and material } \\
\text { corrosion products in primary } \\
\text { reactor coolant }\end{array}$ & $\begin{array}{l}\text { - No transport between the reactor core } \\
\text { and the electric conversion modules. } \\
\text { - Independent filtering of the primary } \\
\text { loop could be removed using cold traps } \\
\text { that are kept inside the Rx containment }\end{array}$ & $\begin{array}{l}\text { - Limit transport by design } \\
\text { - Challenging when using different } \\
\text { structural materials in the nuclear } \\
\text { reactor core and the energy conversion } \\
\text { modules. }\end{array}$ \\
\hline f-Secondary side maintenance. & Easier than for the Direct Coupling. & $\begin{array}{l}\text { Cumbersome and would require } \\
\text { shutdown of the plant, and a waiting } \\
\text { period for the induced radioactivity in } \\
\text { the primary coolant to drop below safe } \\
\text { level }\end{array}$ \\
\hline $\begin{array}{l}\text { g-Maintenance and replacement } \\
\text { of energy conversion modules }\end{array}$ & $\begin{array}{l}\text { - In generally, no reactor shutdown } \\
\text { required }\end{array}$ & $\begin{array}{l}\text { Requires reactor shutdown, many days, } \\
\text { impacting plant reliability, lost } \\
\text { revenue, and higher cost. }\end{array}$ \\
\hline h-Impact of converters failure & $\begin{array}{l}\text { No impact on the operation of the } \\
\text { nuclear reactor or other converter } \\
\text { modules }\end{array}$ & $\begin{array}{l}\text { Larger impact on the plant operation, } \\
\text { requiring a costly reactor shutdown for } \\
\text { an extended period of time }\end{array}$ \\
\hline $\begin{array}{l}\text { i-Converters operation and } \\
\text { assembly }\end{array}$ & $\begin{array}{l}\text { Not impaired by induced radioactivity } \\
\text { in the nuclear reactor coolant }\end{array}$ & $\begin{array}{l}\text { May be impaired by radioactivity, } \\
\text { since the entire structure will become } \\
\text { contaminated with radioactivity. In } \\
\text { addition, the required radiological } \\
\text { shielding will complicate assembly, } \\
\text { maintenance and thermal management } \\
\text { of the converter modules. }\end{array}$ \\
\hline
\end{tabular}


In addition to selecting an Indirect Coupling (IC) arrangement for the LMRAMTEC plant, a pool configuration is adopted for the LMR; thus the IHXs or AMBs are placed in the annulus between the riser and the reactor vessel wall. The primary coolant flows through the shell side while the secondary coolant flows through the tube side of the IHXs. The choice of the pool configuration allows further reducing piping and shielding and has obvious safety advantages such as excluding the possibility of large LOCAs by design. Furthermore, a large primary coolant inventory reduces the severity of transients. The next subsections present two different Indirect Coupling configurations depending on the heat coupling device: (a)- IC with Heat Exchangers (HXs) and (b)- IC with Alkali Metal Boilers (AMBs).

\subsubsection{Indirect Coupling Design with $H X$}

As mentioned earlier, in the Indirect Coupling (IC) design with HX, the primary and the secondary loops have independent coolants and the HXs are placed in the annulus between the riser and the reactor vessel wall. The primary coolant flows through the shell side while the secondary coolant flows through the tube side of the HXs. Besides the advantages of the IC design, the use of HX has some drawbacks:

- The secondary mass flow rate tends to be of the same order as that on the primary side, in order to reduce the temperature drop between the primary and the secondary.

- The high secondary mass flow rate will increase the pressure losses and the required pumping power. In addition, cold traps are required for the secondary circuit to control the coolant purity.

- Corrosion is an important concern in IHXs due to the large temperature changes experienced by the coolant in this component.

Regarding the generation of the alkali metal vapor for the AMTEC top cycle, there are two possible design configurations fr the AMTEC/TE converter units: (a) an open-unit design configuration, in which the hot alkali-metal liquid coming from the reactor core or the IHXs is introduced into the high-pressure cavity of the AMTEC/TE converter units, through an orifice, and partially flashes into vapor (see Figure 2-2); and (b) a closed-unit design configuration, in which the thermal power from the alkali metal coolant is transmitted through the metallic wall of a converter heat exchanger to an evaporator wick at the bottom of the AMTEC unit (see Figure 2-3). In this configuration, the AMTEC unit carries its own charge of working fluid, and the portion that diffuses through the BASE elements is circulated back from the condenser to the evaporator wick through a porous wick. Both options are described in more details in the following SubSections. 


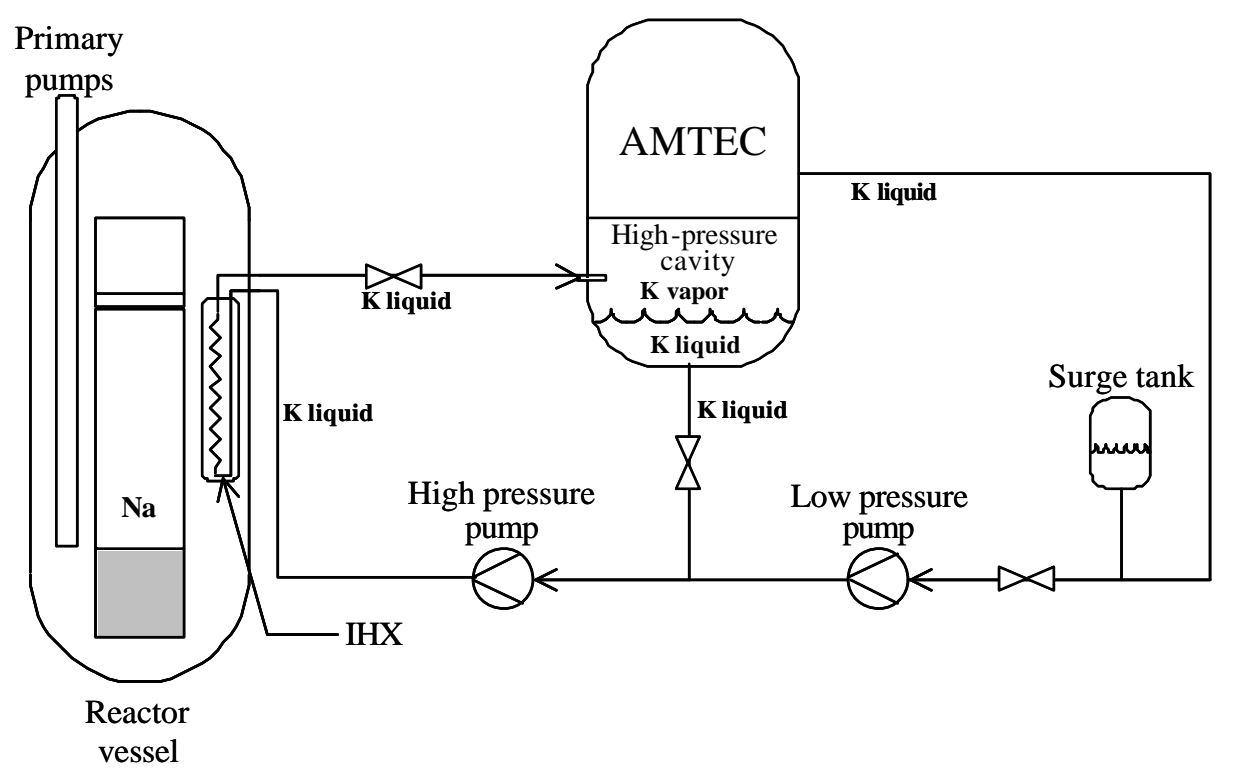

Figure 2-2. Schematic of the Na-cooled LMR-AMTEC indirect coupling concept with liquid-liquid HXs and open K-AMTEC/TE converters (only one HX loop is shown).

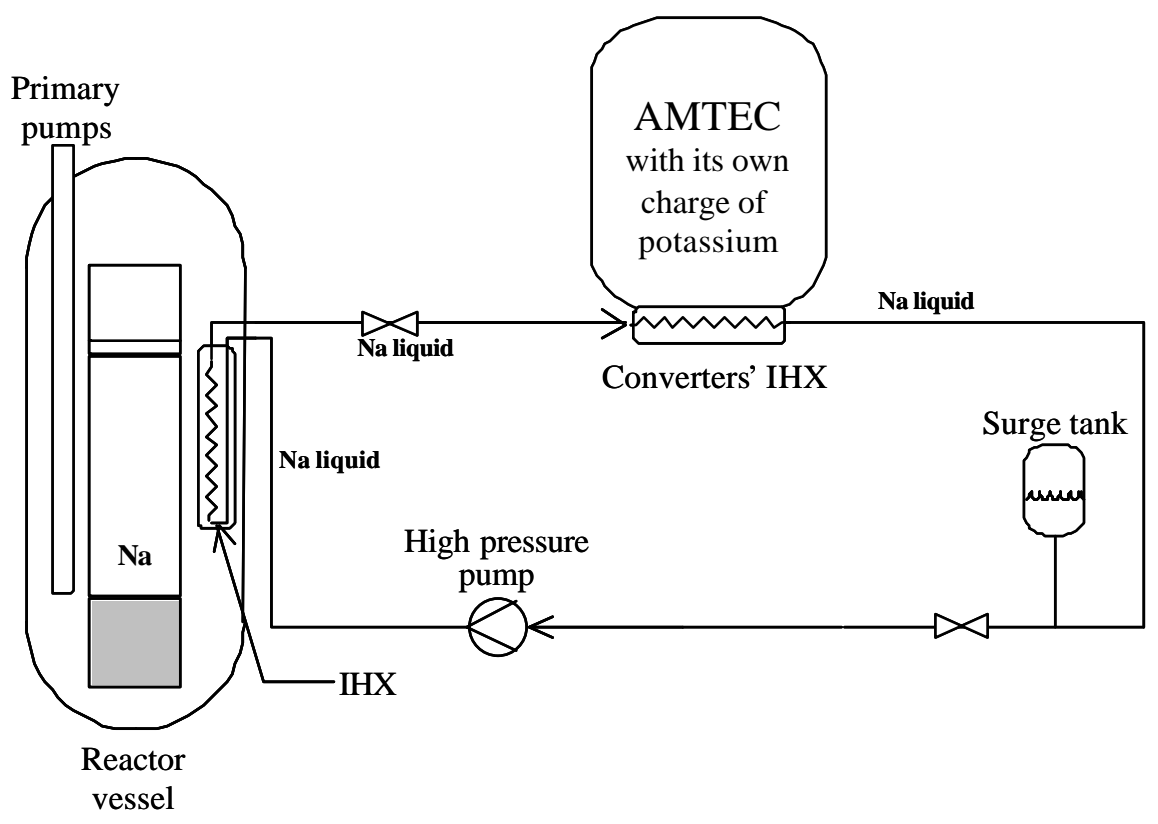

Figure 2-3. Schematic of the Na-cooled LMR-AMTEC indirect coupling concept with liquid-liquid HXs and closed AMTEC/TE converters (only one HX loop is shown). 


\subsection{HX With Open-Unit Converters (contributed by ISNPS)}

Before describing the proposed converter design configurations, it is necessary to give a brief description of the AMTEC/TE converter units developed in this work. All details on the AMTEC/TE converter design can be found in the next Chapter 3. The topping cycle of the power conversion subsystem is comprised of high-power (>50 kWe), high-voltage ( $400 \mathrm{~V}$ DC), Alkali Metal Thermal-to-Electric Converter (AMTEC) units, which nominally operate at a hot (or beta"-alumina solid electrolyte, BASE) temperature of $\sim 1000 \mathrm{~K}$ and $\sim 1120 \mathrm{~K}$, for potassium and sodium working fluids, respectively. For these working fluids, the exit coolant temperature from the nuclear reactor core would be about $50-100 \mathrm{~K}$ higher than the BASE temperature in the AMTEC converter units. These units reject excess heat through a remote condenser (along the two vertical sidewalls of the unit) that is thermally shielded from the BASE elements to minimize parasitic heat losses by radiation (Figure 2.4). The condenser temperatures are typically $\sim 530 \mathrm{~K}$ and $\sim 630 \mathrm{~K}$ for potassium and sodium working fluids, respectively.

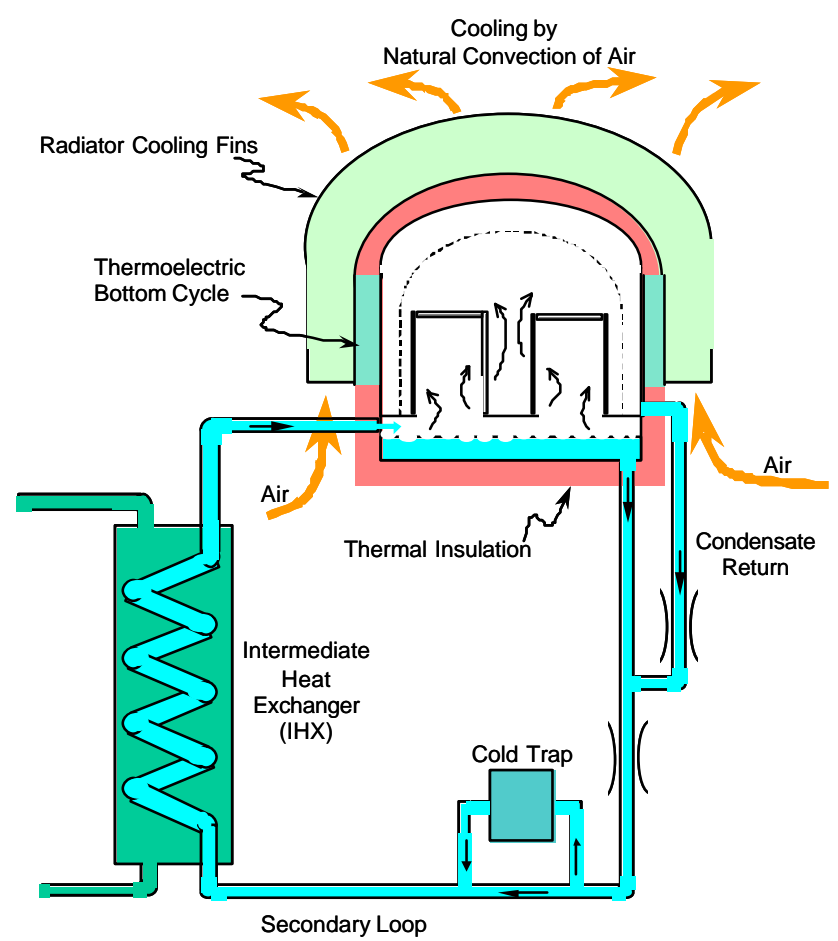

Figure 2-4. Schematic of an indirect interfacing arrangement between LMR and AMTEC/TE converters in an open-unit design configuration.

The heat imparted to the condenser then flows through the sidewalls of the AMTEC unit to a multitude of thermoelectric (TE) modules that are well insulated on the sides and cooled at the far end by natural convection of ambient air (Figure 2-4). To ensure a uniform cold side temperature of the TE modules, they are cooled using a water heat pipe having a common flat interface. The outer surface of the heat pipe enclosure in 
made into isothermal, longitudinal fins to enhance heat rejection by natural convection and minimize the temperature differential with the ambient air. This arrangement increases the temperature differential across the TE modules, increasing their efficiency for converting heat into electricity.

In the open-unit design configuration, illustrated in Figure 2-2 and Figure 2-4, the alkali metal working fluid from the hot leg of the IHX (for an indirect interfacing of the core and the converters) is continuously introduced into the high-pressure cavity of the converter through an orifice, which reduces the local pressure of the coolant. As the hot, reduced-pressure coolant exits the orifice, a fraction of it flashes into vapor at a pressure of $40-90 \mathrm{kPa}$. The resulting vapor condenses in the porous substrate supporting the Beta'-Alumina Solid Electrolyte (BASE), bringing thermal energy to the BASE elements in the form of latent heat of vaporization, as well as alkali metal ions which diffuse through the thin $(\sim 200 \mu \mathrm{m})$ BASE membrane, generating an electrical potential. The vapor desorbing from the cathode electrode/BASE interface at 30-100 Pa diffuses to the condenser of the AMTEC units where it converts into liquid. The fraction of the mass flow rate introduced through the orifice which did not diffuse through the BASE membranes, and the condensate are both recirculated back to the IHX using electromagnetic or mechanical pumps. In this open-unit design configuration, the mass flow rates of alkali metal entering and exiting the high-pressure cavity of the converter must be properly controlled by the pumps and valves, based on the electrical current produced by the AMTEC top cycle, to avoid net accumulation or depletion of working fluid in the flash evaporator. A disadvantage of this design is the high mass flow rate required in the secondary loop in order to obtain an adequate efficiency in the flash evaporators of the AMTEC top cycle.

\subsection{HX With Closed-Unit Converters (contributed by ISNPS)}

The second option is an Indirect Coupling interfacing arrangement with AMTEC/TE converter units in a closed-unit design configuration (see Figure 2-3 and Figure 2-5). In this design, the secondary coolant transfers the heat to the AMTEC/TE units' bottom wall through an intermediate heat exchanger. Each AMTEC/TE converter unit comprises its own charge of alkali metal working fluid, and a porous evaporator wick is placed at the bottom of the high-pressure cavity. The role of the evaporator wick is to distribute the alkali-metal liquid across the area where thermal energy is added, and to provide the necessary capillary pressure head to recirculate passively the condensate from the low-pressure cavity to the high-pressure cavity of the converter. The alkali metal in the condenser wick of the converter is recirculated back to the evaporator wick in the high-pressure cavity through a number of liquid-return arteries lining the two condenser sidewalls of the converter. The alkali metal working fluid in the evaporator wick is evaporated by the thermal energy from the alkali metal in the secondary coolant loop, which is transmitted through the metallic wall of a small heat exchanger at the bottom of the converter unit. 


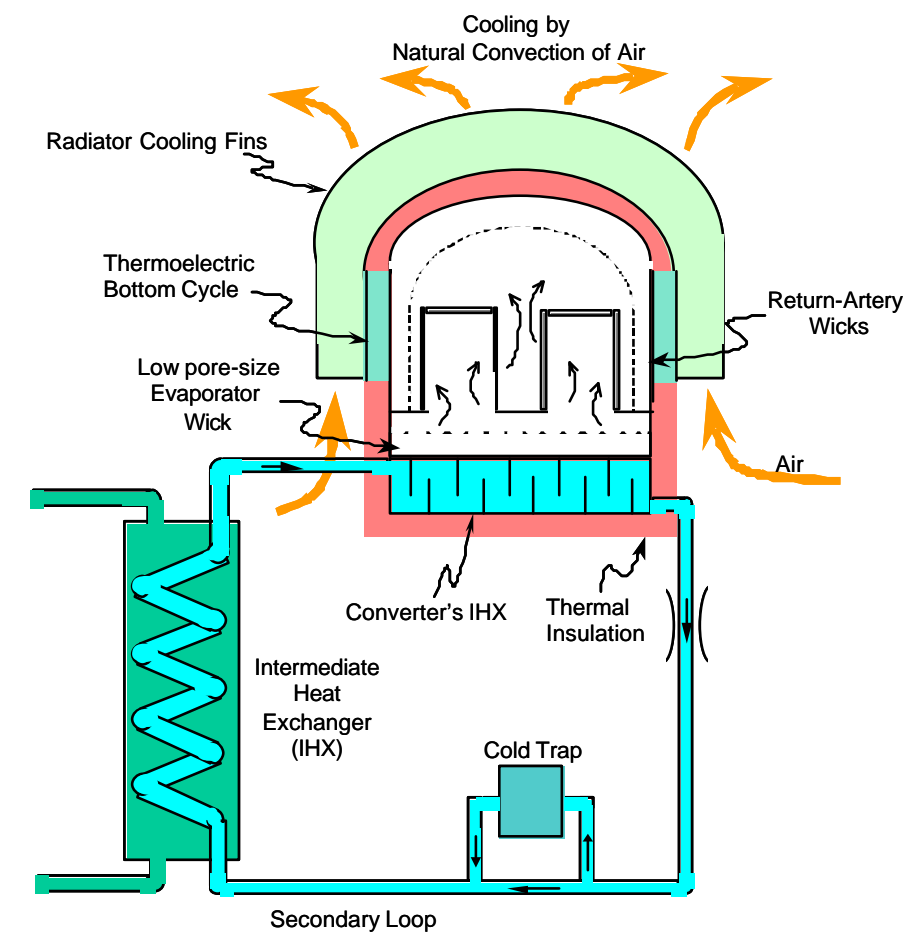

Figure 2-5. Schematic of an indirect interfacing arrangement between LMR and AMTEC/TE converters in a closed-unit design configuration.

Some disadvantages of this plant option are the temperature drop through the additional converters' IHX, and the additional components required by the AMTEC converters. Advantages include easy maintenance of the energy conversion subsystem without requiring shutdown of the nuclear reactor. A defective AMTEC/TE converter unit can easily be replaced without disturbing the coolant in neither the secondary nor the primary loops of the power plant.

The performance of the LMR-AMTEC plant with IHXs and closed-unit AMTEC/TE converters is almost identical to that using open- unit converters, except for the additional temperature drop through the converter's heat exchanger. In both designs, the thermal power of the reactor is transported to the AMTEC/TE converters by the sensible heat of the coolant in the secondary loop of the IHX. For a given BASE elements temperature in the converters, reducing the core exit temperature (which increases the lifetime of the plant) requires reducing the secondary coolant temperature drop through the IHX. In the IC with HX designs, this can only be achieved by increasing the secondary mass flow rate, which has the detrimental effect of increasing the pressure losses and the pumping power requirements, reducing the electrical efficiency of the plant. Another way of reducing the temperature difference between the core exit and the converters' BASE elements is by substituting alkali metal boilers (AMBs) in place of the liquid-liquid IHXs in the plant, whose operation will be similar to that described in Figure 2-4, except that the vapor fed to the AMTEC units is generated externally to the units. 


\subsubsection{Indirect Coupling Design with Alkaline Metal Boilers}

Figure 2-6 shows a simplified sketch of the IC plant with boilers. The Alkali Metal Boilers are placed in the annulus between the riser and the inner reactor vessel wall. The primary coolant flows through the shell side while the secondary coolant flows through the tube side of the boilers.

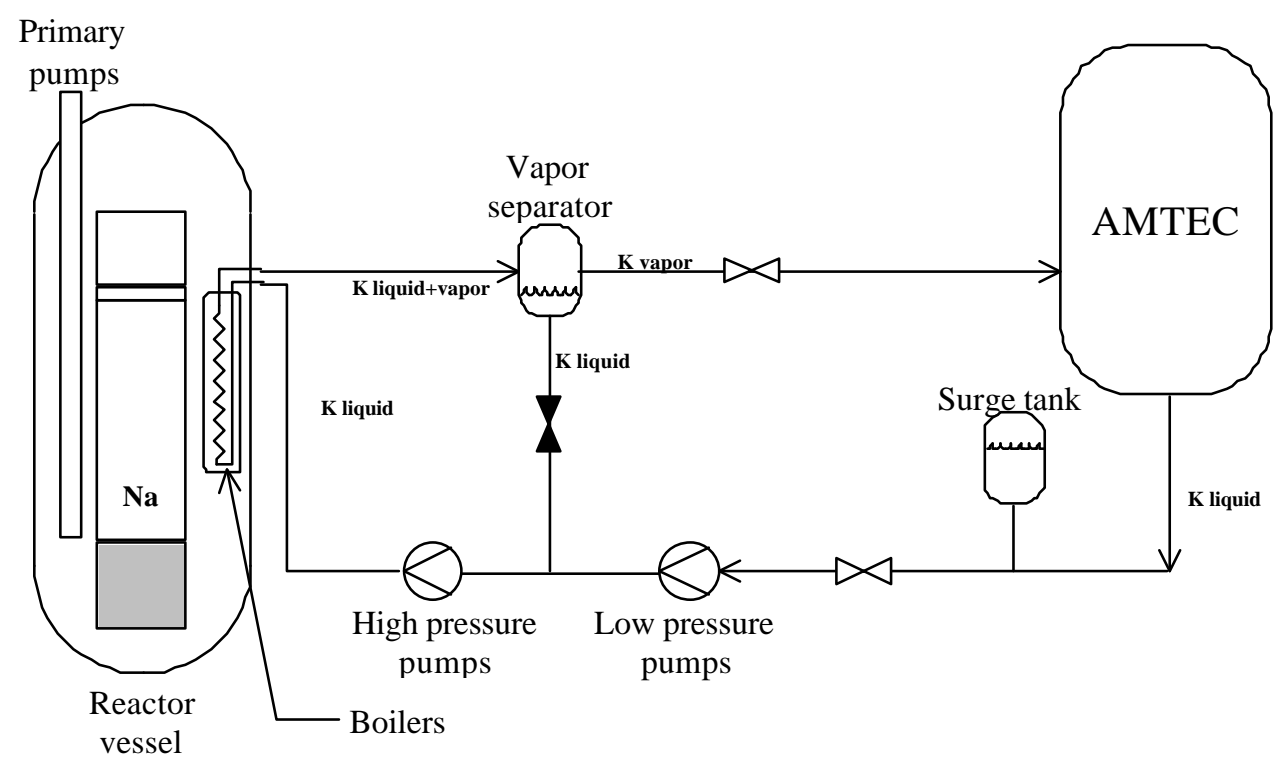

Figure 2-6. Diagram of the LMR-AMTEC indirect coupling with boilers design (for simplicity only one boiler loop is shown). Typical values of the flow rates and the pressures are shown for the Na+Koption.

In addition to the advantages of Indirect Coupling, the use of Alkali Metal Boilers (AMB) has the following incentives:

- Since the change-of-phase enthalpy is higher than the specific capacity of the liquid metal, the use of AMBs reduces the secondary coolant mass flow rate and the pumping power.

- The AMBs improve the corrosion performance of the secondary side due to low coolant flow rates. In addition, the presence of a vapor phase reduces the possibility of corrosion product transport in the secondary loop.

- No expanders or porous wick evaporator is needed.

In contrary to these advantages, the main design difficulties of the AMB are the presence of unstable boiling and the excessive temperature drop in the two-phase region. However, both problems can be solved providing adequate nucleating sites (like the boiling ring described in [Fraas82]) and increasing the cross-section area of the secondary side of the boilers. Another disadvantage is the requirement of a valving network to be able to isolate the AMTEC units for repair or maintenance if necessary. 


\subsubsection{Comparison of Different Design Options}

This section summarizes the results obtained for the comparison of different design options with respect to net efficiency, vessel weight and corrosion behavior.

\subsubsection{Performance of the Design with Respect to Net Efficiency and Vessel Weight}

The performance of each design was evaluated using a plant model developed by WEC to estimated mass flow rates, temperatures and pressure losses, thicknesses, sizes and weights of the reactor components, including core, riser, down-comer, lower-plenum, HX, AMB, expanders, pumps, secondary pipes, and the AMTEC unit. The designs were compared by determining the optimal working parameters which allow to obtain the maximal net plant efficiency for a given vessel weight. The ultimate goal of this study was to compare the flexibility of each design option with respect to the vessel size and to provide guidelines for an economic optimization procedure. At this point, it is mentioned that UNM-ISNPS developed a tool for evaluating the thermal performance of the Indirect Coupling Design with HXs. The conclusion of that study was that the maximal plant net efficiency is obtained using Potassium as secondary coolant. This is in agreement with the results found by WEC for that particular design option.

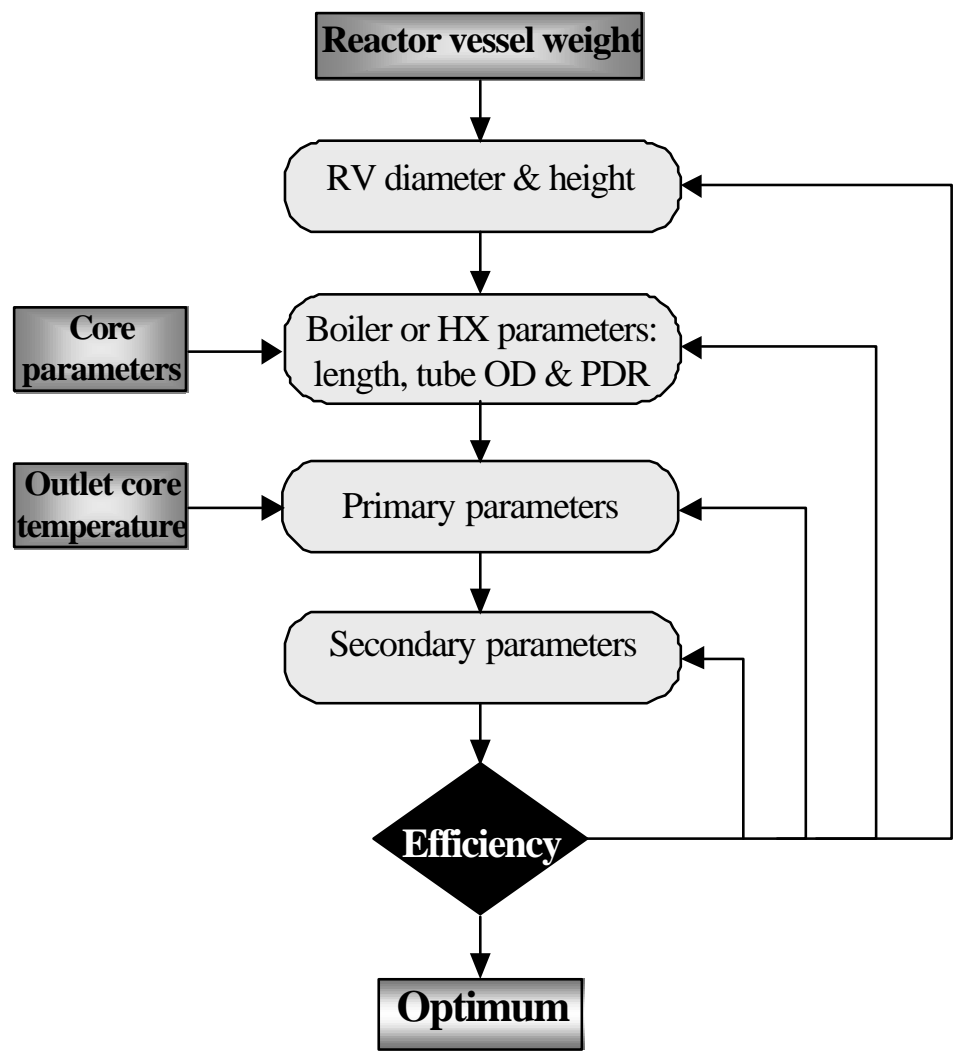

Figure 2-7. Optimization procedure 
The procedure to optimize the design parameters is shown in Figure 2-7. In this procedure, for a given RPV mass, a vessel diameter and height are chosen and the HX/AMB length is calculated. The net efficiency of the plant is then determined for different values of PDR, tube Outer Diameter (OD) and inlet/saturation temperature. Once the maximal plant efficiency is found, the optimization is repeated for new values of vessel diameter and height. In the case of the direct coupling design, the vessel weight remained fixed during the study as it depends only on the core size.

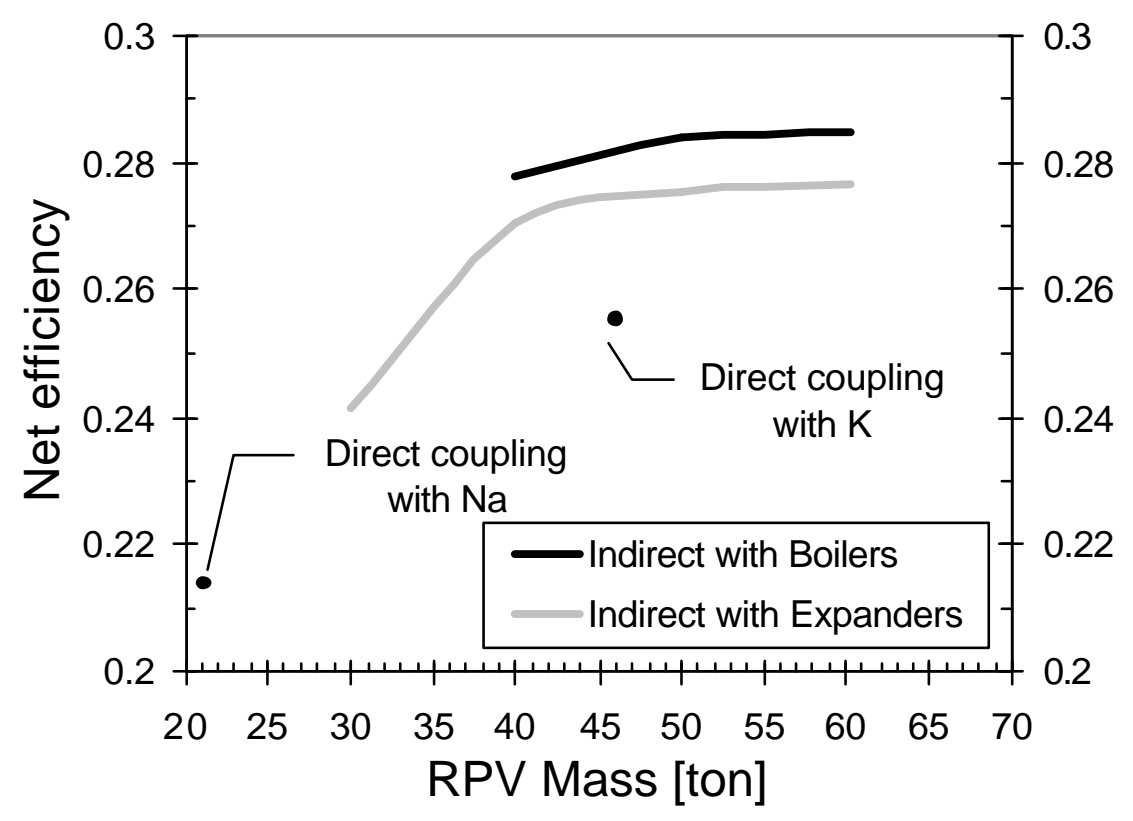

Figure 2-8. Efficiency versus vessel weight.

Figure 2-8 shows the results obtained for a case where the outlet core temperature was equal to $1050 \mathrm{~K}$ and the margin to saturation equal to $316 \mathrm{~K}$. The AMTEC efficiency was calculated using the AMTEC model developed by WEC [Paramonov01] which is more conservative than the one developed by ISNPS. Some key results of this study are:

- The curves of net efficiency versus vessel weight show an inflection point. Any further increase of the vessel weight above this point does not significantly increase the net plant efficiency. For the particular case shown in Figure 2-8, this result means that the optimal economic vessel weight is $\sim 45$ ton. In the final design this value was larger because the margin to saturation was increased to $400 \mathrm{~K}$.

- The results confirms the assumption that due to the relatively small size of the HX or the AMB, the penalty of the pool configuration on the vessel is moderate as compared to other coolants such as water.

- The use of Potassium in the DC plant increases the vessel weight by a factor two due to its high saturation pressure. This drawback and its poor neutronic performance make Potassium an undesirable coolant. Hence, the maximal efficiency for the DC plant is achieved using Sodium. 
- For the same plant efficiency, the vessel of the IC plants using AMB is 20 tons lighter than that of the IC plants using HXs.

- In the IC plant with HX (either open or close unit) requires a secondary mass flow rate of the same order of magnitude as that of the primary.

- The secondary coolant flow rate of the IC plant with AMBs is two orders of magnitude lower than that of the IC plant with heat exchangers. The secondary pumping power is also lower.

\subsubsection{Corrosion Behavior}

At this point, it is important to note the corrosion behavior of each design, since a significant difference in the corrosion patterns of boiling and non-boiling systems exist. By its characteristics, the circuit formed by the direct coupling or the indirect coupling with HX designs is similar to a forced convection system, i.e., a non-boiling system. A continuous, interconnected liquid flow throughout the heat transfer circuit exists and the gain or loss of solute at any point in the system affects all other points to some extent. Addition or removal of heat from the system, since it affects the liquid metal temperature, has a direct influence on the equilibrium solute concentration and corrosion rate value. As the coolant temperature is rapidly increased in the HX, the degree of under-saturation and the corrosion rate will be maximized at its outlet. Reduction of the coolant temperature change, with the aim of improving the corrosion behavior of the loop, could be achieved by increasing the secondary mass flow rate. However this action is limited by the adverse effect of coolant velocity on the corrosion process and the pumping power.

In contrast, the corrosion pattern of the AMB circuit is quite different. First, the solute does not flow continuously around the circuit, since the vapor-filled regions of the boilers present a high-resistance path to the solute mass transfer. Therefore, concentration changes occurring in the boiler are not directly transmitted downstream from the boiler. Secondly, heat added to the alkali metal at its boiling point produces no discernible change in the liquid temperature and, therefore does not alter either the equilibrium concentration of the solute or the solution rate in this region. The major temperature drop in the boiling system will occur in the liquid phase region of the AMB. However, this can be mitigated if the AMB is operated in a recirculating mode. Finally, because the changeof-state reaction has high latent heats, the mass flow rate is considerably small compared to a forced convection circuit, which improves the corrosion behavior of the design.

\subsubsection{Conclusion}

The indirect coupling design with $\mathrm{AMB}$ was selected as the reference design for LMR-AMTEC because of its superior performance with respect to net plant efficiency, vessel weight and corrosion behavior. In addition, Potassium was chosen as the secondary loop coolant due to its higher efficiency compared to Sodium. The indirect coupling with HX and a close-unit converter cycle is considered as an alternative design. Hence, design of the AMTEC/TE unit is performed for both open- and close-converter designs. 


\subsection{Reactor Components Design}

\subsubsection{Layout of the Main Components of the LMR}

As mentioned in the precedent section, the Indirect Coupling (IC) plant with Alkali Metal Boilers (AMB) was chosen as reference design for the LMR-AMTEC because it exhibited the best performance with respect to net plant efficiency, vessel weight and corrosion behavior. The main features of the design are:

- IC between the LMR and the AMTEC units: two independent loops are employed.

- Sodium and potassium are used as primary and secondary coolants, respectively.

- The net plant efficiency is about $34 \%$ when the core outlet temperature is $1050 \mathrm{~K}$.

- The LMR core is composed of 78 fuel elements (or 72 if 19 CR are used) and 78 reflector elements. The fuel is (U,Pu)N and the cladding is made of the refractory alloy $\mathrm{Nb}-1 \mathrm{Zr}$.

- The AMBs generate the potassium vapor, which is fed into the AMTEC units. The AMBs can operate in once-through or in recirculation mode (with a vapor separator). However, the recirculation mode was preferred due to its better corrosion behavior.

- The reactor is arranged in a pool configuration: the AMB and the primary pumps are placed inside the reactor vessel. Thus, no shielding either for the secondary pipes or the AMTEC/TE converter units is needed.

- The reactor has Passive Heat Removal System which uses the variations of the sodium levels as mechanism to initiate the heat removal. The proposed PHRS houses the HX in the cavity formed by the pump shaft and the external shaft shell. As soon as the pumps are stopped the residual heat can be extracted by the PHRS.

A detailed layout of the layout of the components inside the reactor vessel is shown in Figure 2-9. The alkali metal boilers (AMB) are arranged in the annulus between the riser and the reactor vessel. Their external shell can have other shape than circular to better fit the space in the annulus. Two types of AMB's tubes were considered: "C" tubes and concentric tubes. The reactor uses four primary pumps of propeller type. The pumps are mounted on the vessel head, pumping the sodium coolant exiting the two closest AMB. The sodium flow path is shown in the Figure 2-9. The helical HXs of the Passive Heat Removal System (PHRS) are placed in the annulus between the pump shaft and the pump external shell. The PHRS uses the variation of the sodium level inside the vessel as mechanism to initiate the heat removal. Orifice 1 in the riser is used for the normal operation coolant path while Orifice 2 is used during the operation of the PHRS. The main systems of the LMR-AMTEC including the PHRS, the Power Conversion System, the Water Cooling Guard Vessel System (WCGVS) and the Primary Cold Trap are presented in Figure 2-10. The next subsections present a brief description of the design of the main component of the reactors, including the core neutronic, thermal hydraulic and mechanic design, the Alkali Metal Boilers design, the primary pumps characteristics, the selection of the structural materials for the main reactor component and the determination of the operating parameters in order to optimize the reactor performance. A description of the safety features of the design including the void coefficient and the PHRS are given in section 2.3. 


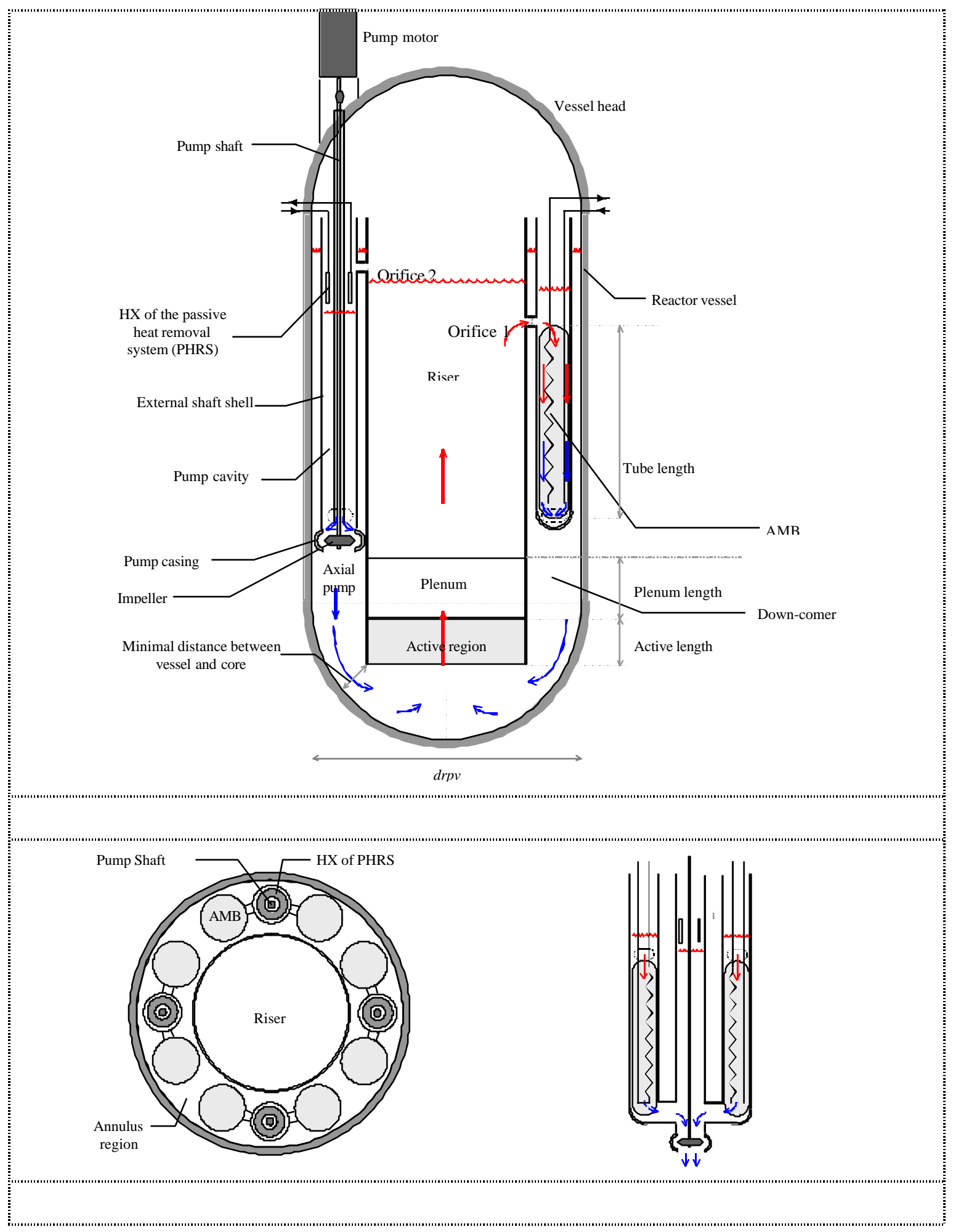

Figure 2-9. In-vessel layout of the LMR-AMTEC reactor. The picture shows the pumps and the boilers positions and the sodium levels during normal operation of the reactor. 


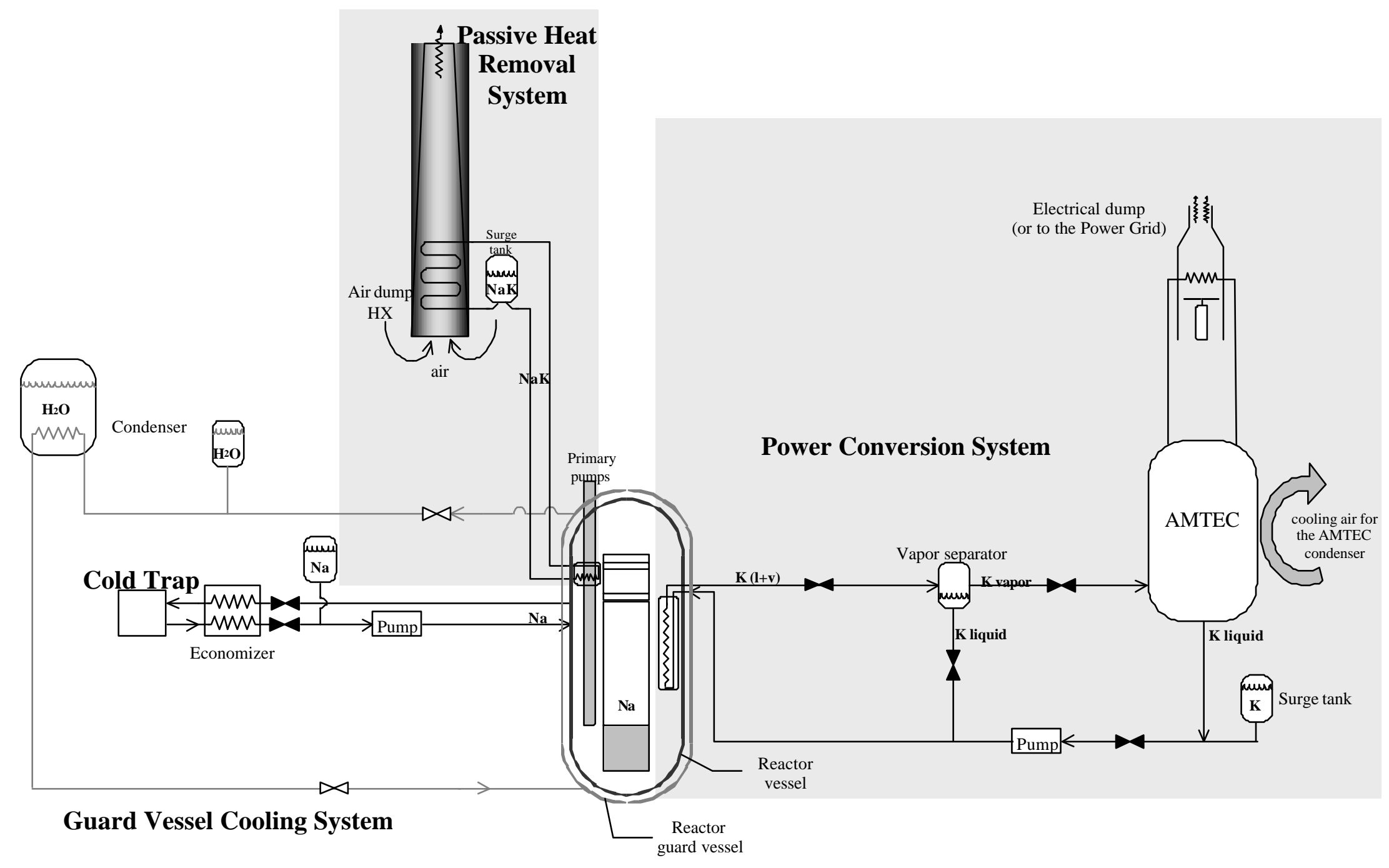

Figure 2-10. Power Conversion System, Passive Heat Removal Systems (PHRS), Water Cooling Guard Vessel System (WCGVS) and Primary Cold Trap 


\subsubsection{Core Neutronic Design}

\subsubsection{Fuel Element Design and Neutronics}

Preliminary core design and selection of basic fuel parameters were accomplished during the first project year [Paramonov01] [Petrovic01]. Some of the main design requirements and selections accrued based on these studies are:

- Nitride fuel with $\mathrm{Nb}-1 \mathrm{Zr}$ cladding is selected because of the high operating temperature requirements and the available SP-100 experience database [Dutt].

- Long core life: 10 years is currently viewed as a minimum. This feature enhances proliferation resistance.

- Non-proliferation requirements: Fissile content is kept below $20 \%$ (relatively to total heavy metal) and only reactor-grade $\mathrm{Pu}$ is considered.

- Once through fuel cycle, no reprocessing. This requirement is based on U.S. policy, and it makes deployment of radial/axial blankets technically less attractive, hence, a homogeneous core design is considered.

- Simplified reloading and single-batch (no fuel shuffling) operation.

- Fuel rod design relied on the ULLC (Ultra Long Life Core) study performed by Westinghouse in the early 80's [Calamai81].

- Linear power is selected to provide the required core lifetime of 10-15 years.

- Smear factor is selected to accommodate discharge burnup of up to about 70,000 MWd/tHM.

- Hexagonal fuel assembly design with wire-wrap spacer is employed, consistent with fast reactor design experience.

- Relatively flat core is employed $(\mathrm{H} / \mathrm{D}=0.43)$ to improve void reactivity coefficient.

- Fast neutron $(\mathrm{E}>0.1 \mathrm{MeV})$ fluence should be compatible with material properties. As the initial requirement, fast fluence should not exceed $9 \times 10^{23} \mathrm{n} / \mathrm{cm} 2$, and in this case adequate materials have to be selected to withstand such fluence.

- Cladding material should be compatible with the cladding peak temperature.

Several reactor physics studies were performed in the project to define the fuel design, as well as to address reactivity control, following general fast reactor design requirements [Bailly99][Wirtz78][Tang78]. These design studies included:

\subsubsection{Plutonium Isotopics in $(\mathrm{U}, \mathrm{Pu}) \mathrm{N}$}

Evaluation of several $\mathrm{Pu}$ isotopic compositions resulted in selecting Pur B (Table 2-2) as the preferred option if $(\mathrm{U}, \mathrm{Pu}) \mathrm{N}$ fuel is used. This isotopic composition represents $\mathrm{Pu}$ from highly-burned PWR fuel and after 20 years of cooling time. In addition to its good proliferation resistance, it provides a reduced reactivity swing over the core lifetime. 
Reference depletion curve for UN and (U,Pu)N fuel are shown in Figure 2-11. Corresponding discharge burnups are $\sim 57 \mathrm{GWd} / \mathrm{tHM}$ and $\sim 70 \mathrm{GWd} / \mathrm{thM}$.

Table 2-2. Reference Plutonium (Pu-B) Isotopics for (U,Pu)N Fuel.

\begin{tabular}{|c|c|}
\hline Components & (w/o) \\
\hline${ }^{238} \mathrm{Pu}$ & $2.39 \%$ \\
${ }^{239} \mathrm{Pu}$ & $57.76 \%$ \\
${ }^{240} \mathrm{Pu}$ & $25.89 \%$ \\
${ }^{241} \mathrm{Pu}$ & $5.73 \%$ \\
${ }^{242} \mathrm{Pu}$ & $7.65 \%$ \\
${ }^{241} \mathrm{Am}$ & $0.58 \%$ \\
\hline
\end{tabular}

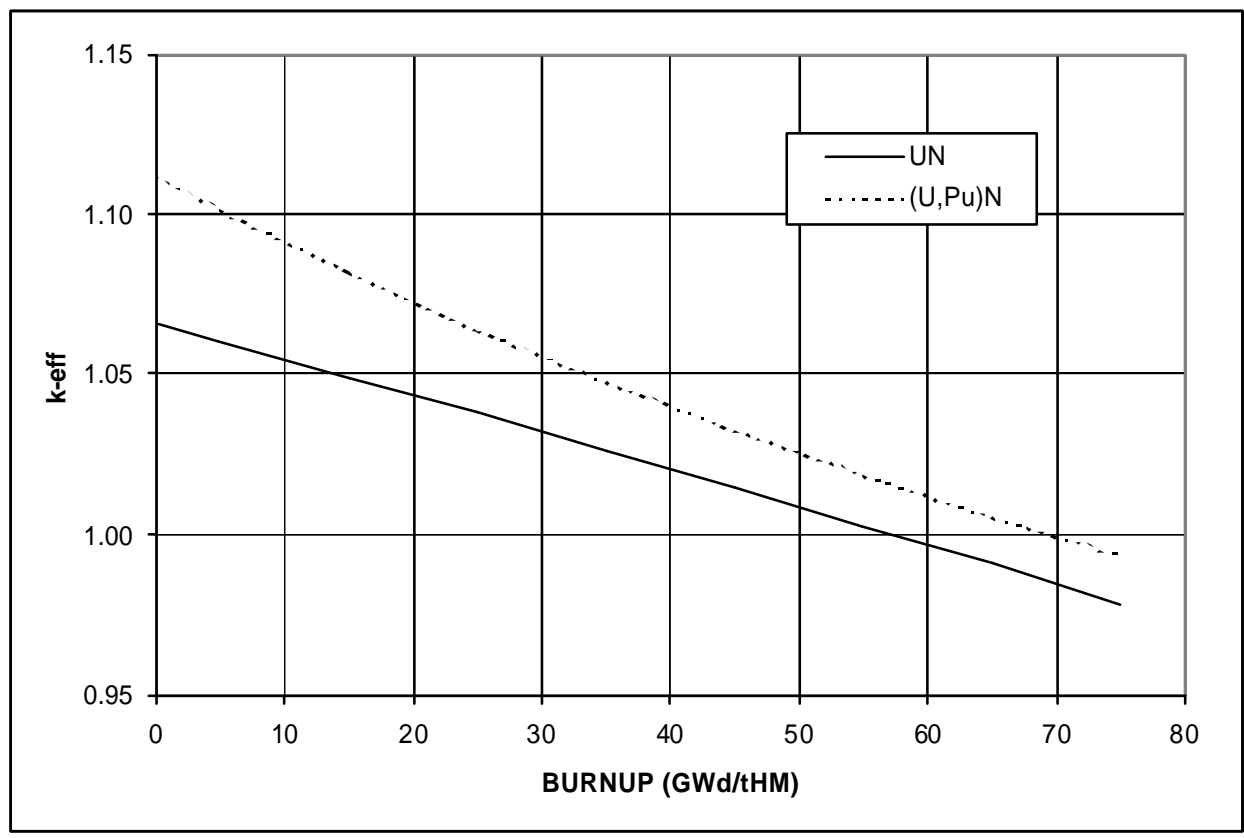

Figure 2-11. Fuel reactivity as a function of burnup for $\mathrm{UN}$ fuel $\left(19.95 \%{ }^{235} \mathrm{U}\right)$ and $(\mathrm{U}, \mathrm{Pu}) \mathrm{N}(19.95 \% \mathrm{Pu}-\mathrm{B}$ and depleted $\mathrm{U})$.

\subsubsection{Use of Enriched Nitrogen}

Natural nitrogen is composed mainly $(99.6 \%)$ of ${ }^{14} \mathrm{~N}$. This isotope has several undesirable properties [Wallenius01]:

- High capture cross section, leading to reduced reactivity and reduced burnup.

- Production of radioactive ${ }^{14} \mathrm{C}$, through ${ }^{14} \mathrm{~N}(\mathrm{n}, \mathrm{p}){ }^{14} \mathrm{C}$ reaction. The ${ }^{14} \mathrm{C}$ isotope is responsible for a significant portion of the fuel radio-toxicity during reprocessing, and of concern for irradiated fuel processing. 
- Production of helium and hydrogen through ${ }^{14} \mathrm{~N}(\mathrm{n}, \mathrm{a})$ and ${ }^{14} \mathrm{~N}(\mathrm{n}, \mathrm{p})$ reactions. These processes contribute to fuel swelling.

If nitrogen highly enriched in ${ }^{15} \mathrm{~N}$ is used instead of natural nitrogen, these negative effects are eliminated or reduced. To evaluate effect on fuel reactivity, fuel depletion analysis was performed by SAS2H code [SCALE], both for $(\mathrm{U}, \mathrm{Pu})^{\text {nat }} \mathrm{N}$ and $(\mathrm{U}, \mathrm{Pu})^{15} \mathrm{~N}$. Obtained $\mathrm{k}$-inf results were normalized via MCNP [MCNP] 3-D simulations to estimate corresponding keff for the finite reactor. Figure 2-12 presents k-eff as a function of burnup.

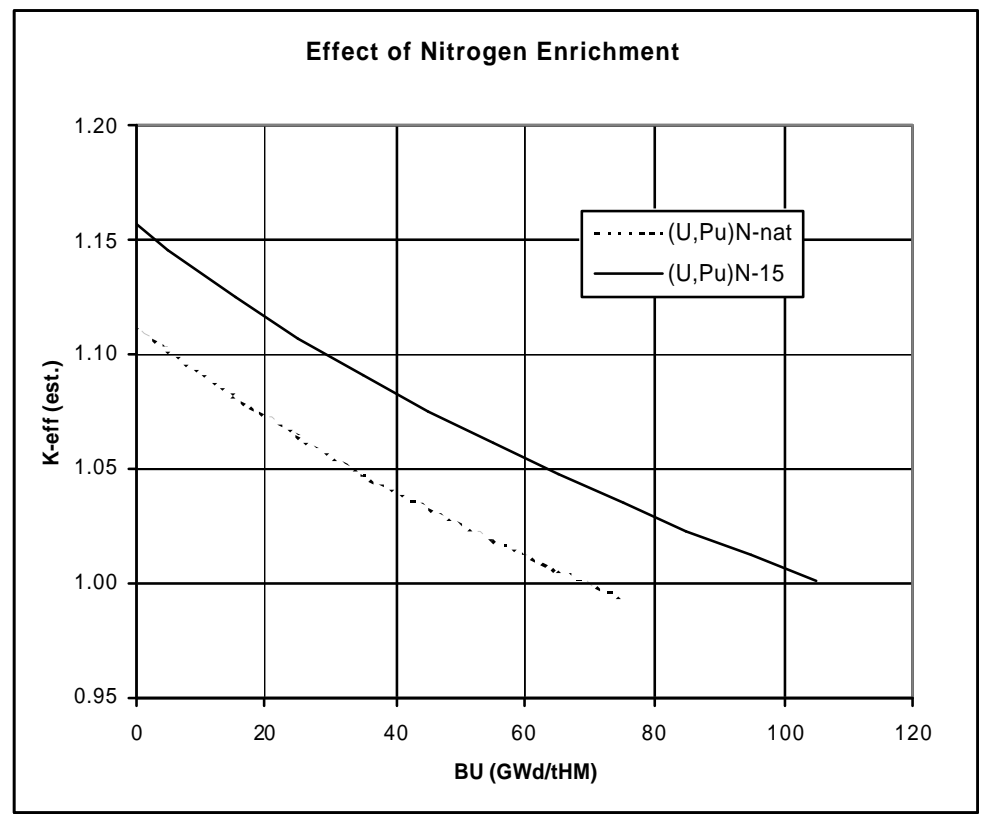

Figure 2-12. Comparison of fuel reactivity as a function of burnup for natural and enriched nitrogen:

$$
(\mathrm{U}, \mathrm{Pu})^{\text {nat }} \mathrm{N} \text { and }(\mathrm{U}, \mathrm{Pu})^{15} \mathrm{~N} \text {. }
$$

A significant gain in reactivity is observed if enriched ${ }^{15} \mathrm{~N}$ is used. Consequently, cycle length is increased by $\sim 50 \%$, from $\sim 70 \mathrm{GWd} / \mathrm{tHM}$ to $\sim 105 \mathrm{GWd} / \mathrm{tHM}$, resulting in a large fuel saving. Alternatively, fissile content may be significantly reduced with ${ }^{15} \mathrm{~N}$ while maintaining the $70 \mathrm{GWd} / \mathrm{tHM}$ cycle length. While there is a clear economic benefit associated with this reactivity gain, the cost of enriching nitrogen is also significant, and large scale production of ${ }^{15} \mathrm{~N}$ needs to be addressed. However, the general economic balance favors the use of ${ }^{15} \mathrm{~N}$.

\subsubsection{Neutron Spectrum and Fast Neutron Fluence Considerations}

Due to the fast spectrum and long core life, one of primary concerns is irradiation damage to materials (cladding in particular), and it is therefore necessary to evaluate neutron spectrum and the lifetime fast neutron fluence and DPA (displacement per atom). In a typical fast reactor design employing stainless steel as cladding, the objective is usually to limit the lifetime fast neutron fluence $\mathbf{F}_{0.1}(\mathrm{E}>0.1 \mathrm{MeV})$ to $\left\langle 4 \times 10^{23} \mathrm{n} / \mathrm{cm}^{2}\right.$. However, 
by using a different cladding material $(\mathrm{Nb}-1 \mathrm{Zr})$, a fast fluence of up to $9 \times 10^{23} \mathrm{n} / \mathrm{cm}^{2}$ is assumed to become acceptable.

A number of MC simulations was performed to obtain an estimate of the expected range of values of the fast fluence $\mathbf{F}_{0.1}$ in the LMR-AMTEC core. These simulations were performed under the following assumptions. Fuel similar to that of the 2X-ULLC core was analyzed. Simulations were performed only for BOC, and the BOC fast flux was used as the effective flux for the whole core life-time. Fast flux was tallied at $>1 \mathrm{MeV}$ and $>0.1 \mathrm{MeV}$ level. These simulations showed that depending on the linear power, $\mathrm{p} / \mathrm{d}$, smear density, and peaking factor, peak $\mathbf{F}_{0.1}$ may vary between $\sim 3 \times 10^{23} \mathrm{n} / \mathrm{cm}^{2}$ and $\sim 9 \times 10^{23} \mathrm{n} / \mathrm{cm}^{2}$ in extreme-case combinations. The peak $\mathbf{F}_{0.1}$ value exceeds the $4 \times 10^{23}$ $\mathrm{n} / \mathrm{cm}^{2}$ limit usually assumed for stainless steel cladding, but should be acceptable for $\mathrm{Nb}$ $1 \mathrm{Zr}$ cladding.

\subsubsection{Fuel Form Selection}

Monte Carlo simulations of unit fuel cell were performed to evaluate the impact of fuel selection. The following fuel form alternatives were examined:

- Oxide fuel, MOX

- Carbide fuel, $(\mathrm{U}, \mathrm{Pu}) \mathrm{C}$

- Nitride fuel, (U,Pu)N

Note that due to the AMTEC high temperature requirement, metal fuel was eliminated from further considerations. Since the fast neutron fluence may be the most limiting factor, we compared the projected fast fluence. Using MOX fuel results in the highest fast fluence, about $15 \%$ higher than for the carbide or nitride fuel. The nitride fuel also leads to the lowest fast fluence for the same reactor power density, as shown in the Table 2-3.

Table 2-3. Estimated fast fluence (relative units) for $14 \mathrm{a} / \mathrm{o}$ FPU fuel.

\begin{tabular}{|c|c|}
\hline Fuel Form & Fast Flue nce \\
\hline$(\mathrm{U}, \mathrm{Pu}) \mathrm{O}_{2}$ & 1.10 \\
\hline$(\mathrm{U}, \mathrm{Pu}) \mathrm{C}$ & 1.03 \\
\hline$(\mathrm{U}, \mathrm{Pu}) \mathrm{N}$ & 1.00 \\
\hline
\end{tabular}

Neutronically, nitride fuel has further advantages due to the highest heavy metal density among the three forms considered ( $\sim 5 \%$ more than carbide, $\sim 30 \%$ more than oxide), compatibility with the selected cladding material $\mathrm{Nb} 1-\mathrm{Zr}$, and the availability of experimental database of the SP-100 program.

\subsubsection{Cladding Options}

Selection of the cladding material is dictated by the material requirements (high temperature and radiation environment, compatibility with fuel), and it is described in 
section 2.2.4. However, it will also have a significant impact on neutronics, since some of the considered materials (primarily $\mathrm{Nb}$ ) are strong neutron absorbers. Hence, the neutronic impact of different cladding materials was examined.

Calculations were performed for the following cladding materials:

- Zr-based cladding alloy. This material is not an option under LMR-AMTEC design conditions, but provides a hypothetical limit for the smallest reactivity penalty.

- Stainless-steel-like cladding, typical in LMRs, but not satisfying LMR-AMTEC conditions.

- $\quad \mathrm{Nb}-1 \mathrm{Zr}$ cladding, current design selection.

First, we analyze the results of ORIGEN depletion calculations. As expected, there is a significant penalty when switching from stainless to $\mathrm{Nb}-1 \mathrm{Zr}$ cladding. BOL reactivity is reduced by ?? $=\sim 0.115$. It may be observed that this difference remains approximately constant with depletion. The reason for this large neutronic penalty is high niobium cross section in the fast energy range, as shown in Figure 2-13 (This figure compares total cross section for $\mathrm{Nb} 95$ and Fe 56. Natural $\mathrm{Nb}$ is composed essentially of $\mathrm{Nb} 95$, whereas iron contains $>90 \% \mathrm{Fe} 56)$.

Preliminary ORIGEN calculations confirm a notable penalty in reactivity. Furthermore, this penalty remains roughly constant, hence, based on BOL effect one can reasonably well predict lifetime behavior.

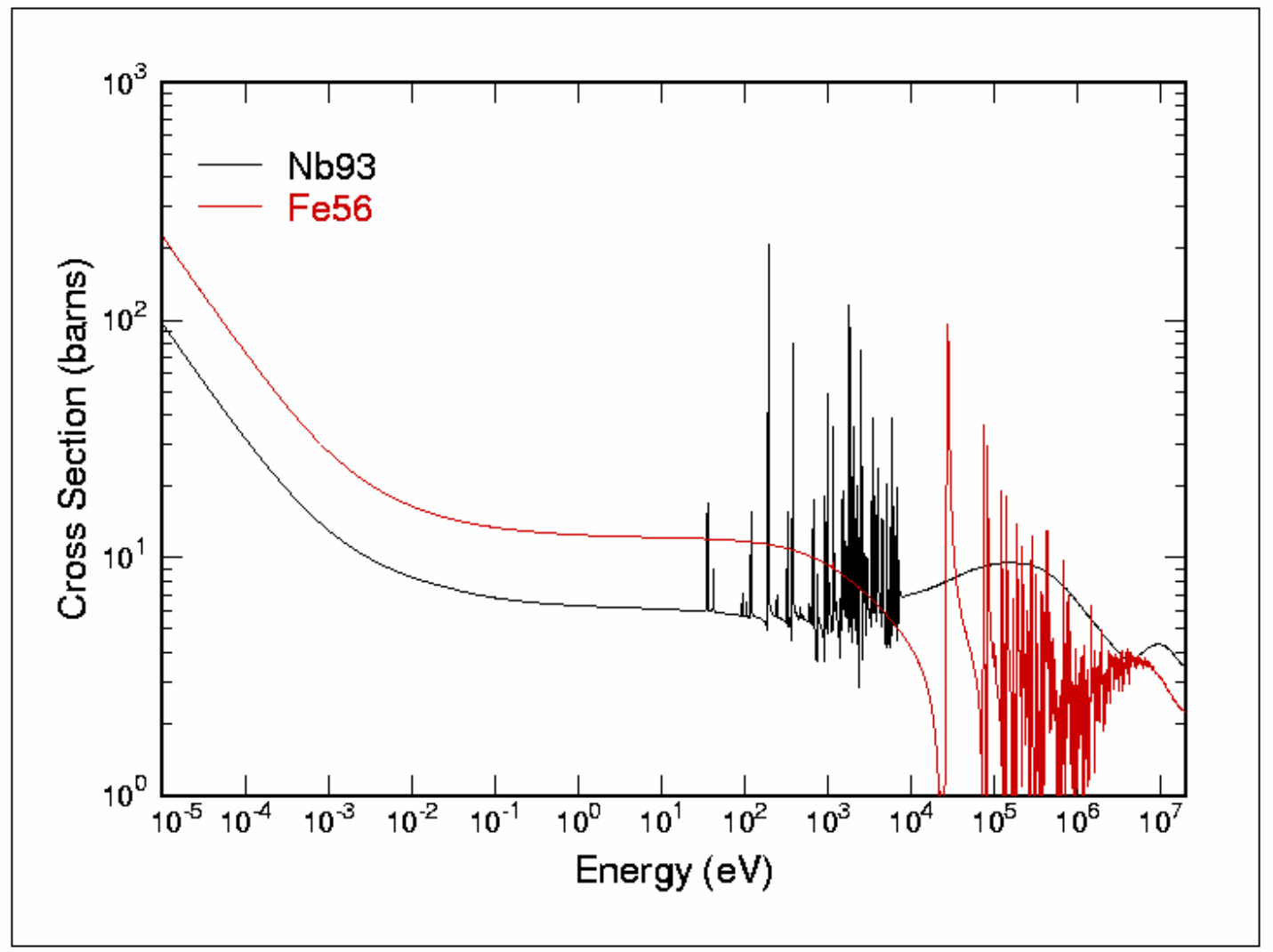

Figure 2-13. Comparison of $\mathrm{Nb} 93$ and Fe56 total cross section. 
To more accurately assess the BOL reactivity, the calculations were repeated with SCALE/SAS2H and MCNP. The two codes more accurately account for the change in neutron spectrum introduced by $\mathrm{Nb}-1 \mathrm{Zr}$ cladding.

SCALE/SAS2H estimates the $\mathrm{Nb}-1 \mathrm{Zr}$ reactivity penalty relative to stainless steel to be $? ?=\sim 0.043$, whereas stainless steel relative to $\mathrm{Zr}$ leads to a penalty of $? ?=\sim 0.016$. Results of full-core 3-D Monte Carlo simulations using MCNP for the BOL k-effective are given in Table 2-4. Again, estimated penalty due to $\mathrm{Nb}-1 \mathrm{Zr}$ is $? ?=\sim 0.045$ (or ?k= 0.050 ). This corresponds to about a third of the total reactivity swing over the core lifetime, which is quite significant. However, as compared to other cladding selections, $\mathrm{Nb}-1 \mathrm{Zr}$ is the only one that satisfies the high-temperature requirements of LMR-AMTEC.

Table 2-4 K-effective for different cladding materials (Pu-A 14 a/o FPU).

\begin{tabular}{|l|l|}
\hline Cladding & ?k vs. SS-316 \\
\hline $\mathrm{Zr}$ & $0.0100+/-0.0015$ \\
\hline $\mathrm{Nb}-1 \mathrm{Zr}$ & $-0.0502+/-0.0015$ \\
\hline
\end{tabular}

\subsubsection{Coolant Options}

The LMR-AMTEC system exploits the direct energy conversion technology using AMTEC cells. The material selection for the reactor coolant was limited to alkali metals given the design option of using the same working fluid for the reactor and the AMTEC units. Sodium is well established in fast reactors, as well as in the AMTEC technology (experience with solid electrolyte compatible with sodium). Additionally, potassium and lithium were employed and/or considered as coolant in various reactor designs. Basic properties of these three alkali metals are given in Table 2-5.

Table 2-5. Basic properties of alkali metals considered for coolant.

\begin{tabular}{|c|c|c|c|}
\hline & $\begin{array}{c}\text { Sodium (Na, } \\
\mathrm{Z}=11)\end{array}$ & Potassium (K, Z=19) & Lithium (Li, Z=3) \\
\hline $\begin{array}{c}\text { Naturally Occurring } \\
\text { Isotopic Composition }\end{array}$ & $100 \mathrm{a} / \mathrm{o} \mathrm{Na} 23$ & $\begin{array}{c}93.2581 \mathrm{a} / \mathrm{o} \text { K39 } \\
0.0117 \mathrm{a} / \mathrm{o} \mathrm{K} 40 \\
6.7302 \mathrm{a} / \mathrm{o} \text { K41 }\end{array}$ & $\begin{array}{r}7.5 \mathrm{a} / \mathrm{o} \mathrm{Li6} \\
92.5 \mathrm{a} / \mathrm{o} \mathrm{Li7}\end{array}$ \\
\hline Density $\left(\mathrm{g} / \mathrm{cm}^{3}\right)$ at $1000 \mathrm{~K}$ & 0.7778 & 0.6736 & 0.4639 \\
\hline $\begin{array}{c}\text { Density assumed in } \\
\text { MCNP simulations }\end{array}$ & 0.78 & 0.67 & 0.46 \\
\hline $\begin{array}{c}\text { Melting Point at nominal } \\
\text { pressure (K) }\end{array}$ & 370.9 & 336.5 & 553.7 \\
\hline $\begin{array}{c}\text { Boiling Point at nominal } \\
\text { pressure (K) }\end{array}$ & 1156 & 1032 & 1616 \\
\hline
\end{tabular}

Neutronic properties are depicted in Figure 2-14. The total cross section for $\mathrm{Na}$ and $\mathrm{K}$, as well as for lithium isotopes ${ }^{6} \mathrm{Li}$ and ${ }^{7} \mathrm{Li}$ is given as function of neutron energy, over the whole energy range, while enlarged view is given for energy above $100 \mathrm{eV}$. 
Several observations can be made. ${ }^{6} \mathrm{Li}$ has large cross section for lower energies, several orders of magnitude larger than other isotopes. This will lead to a large reactivity penalty if using natural $\mathrm{Li}$ as a coolant, but using only the isotope ${ }^{7} \mathrm{Li}$ may be an option. In the fast energy range, differences among the four isotopes are not as dramatic.
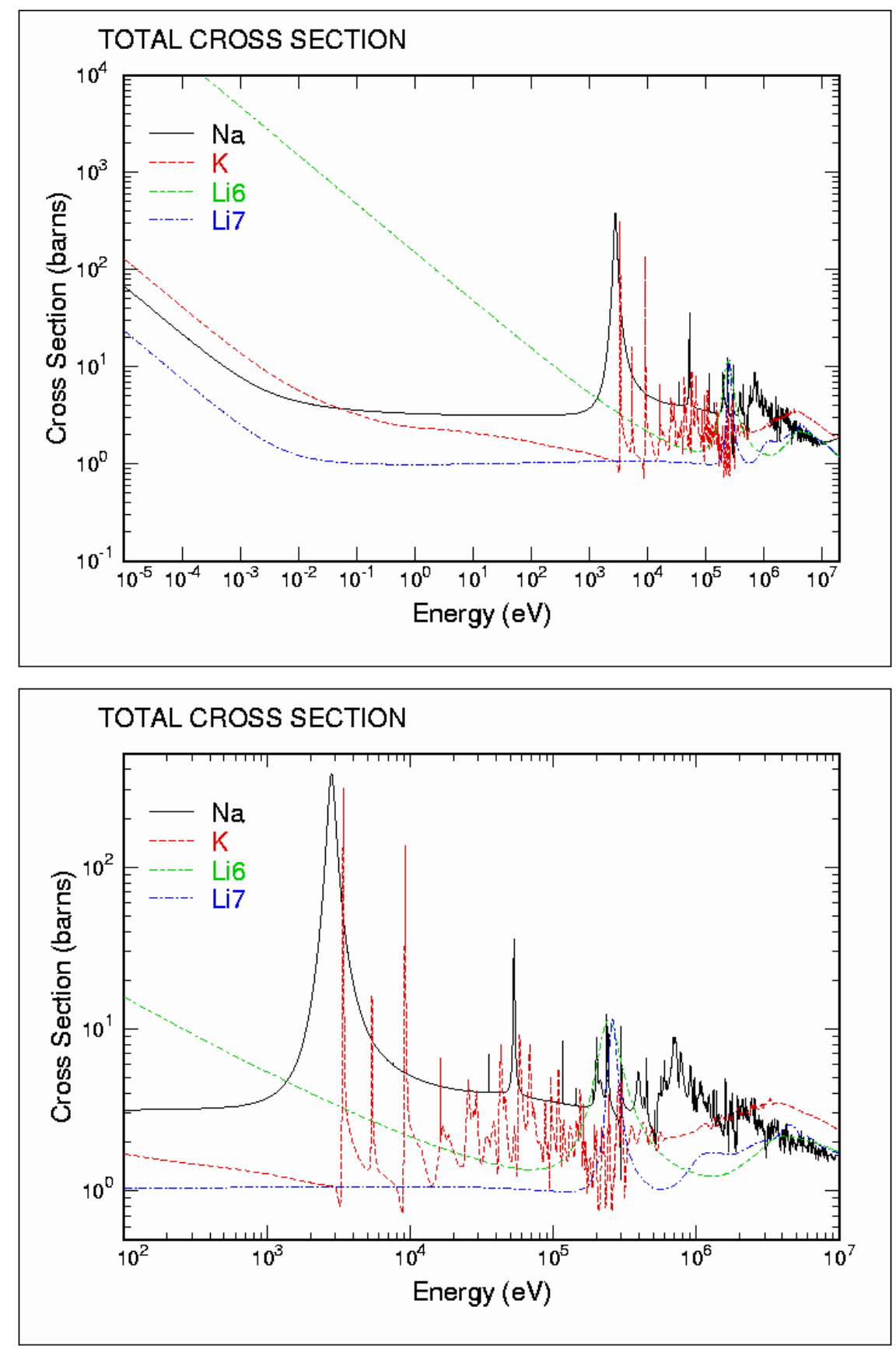

Figure 2-14. Total cross section for different coolant options.

Monte Carlo simulations were performed to evaluate core reactivity and neutron spectrum. Neutron spectra shown in Figure 2-15 demonstrate features that one would 
expect based on cross sections. Note that results for lower energies have high uncertainties, and should be taken only as indication of order-of-magnitude value.

Neutron spectrum was averaged over the whole core volume. Lower energy range (for $\mathrm{Na}, \mathrm{K}$ and ${ }^{7} \mathrm{Li}$ ) is due primarily to reflection of neutrons from the surrounding structure, and would be reduced for a larger reactor. ${ }^{\mathrm{Nat}} \mathrm{Li}$, due to its very high thermal cross section, eliminates this portion of the spectrum. Furthermore, flux depression is clearly visible corresponding to the $\mathrm{Na}$ main resonance $(\sim 2.8 \mathrm{keV})$ for sodium coolant, as well as depression due to ${ }^{239} \mathrm{Pu}(\sim 0.3 \mathrm{eV})$.

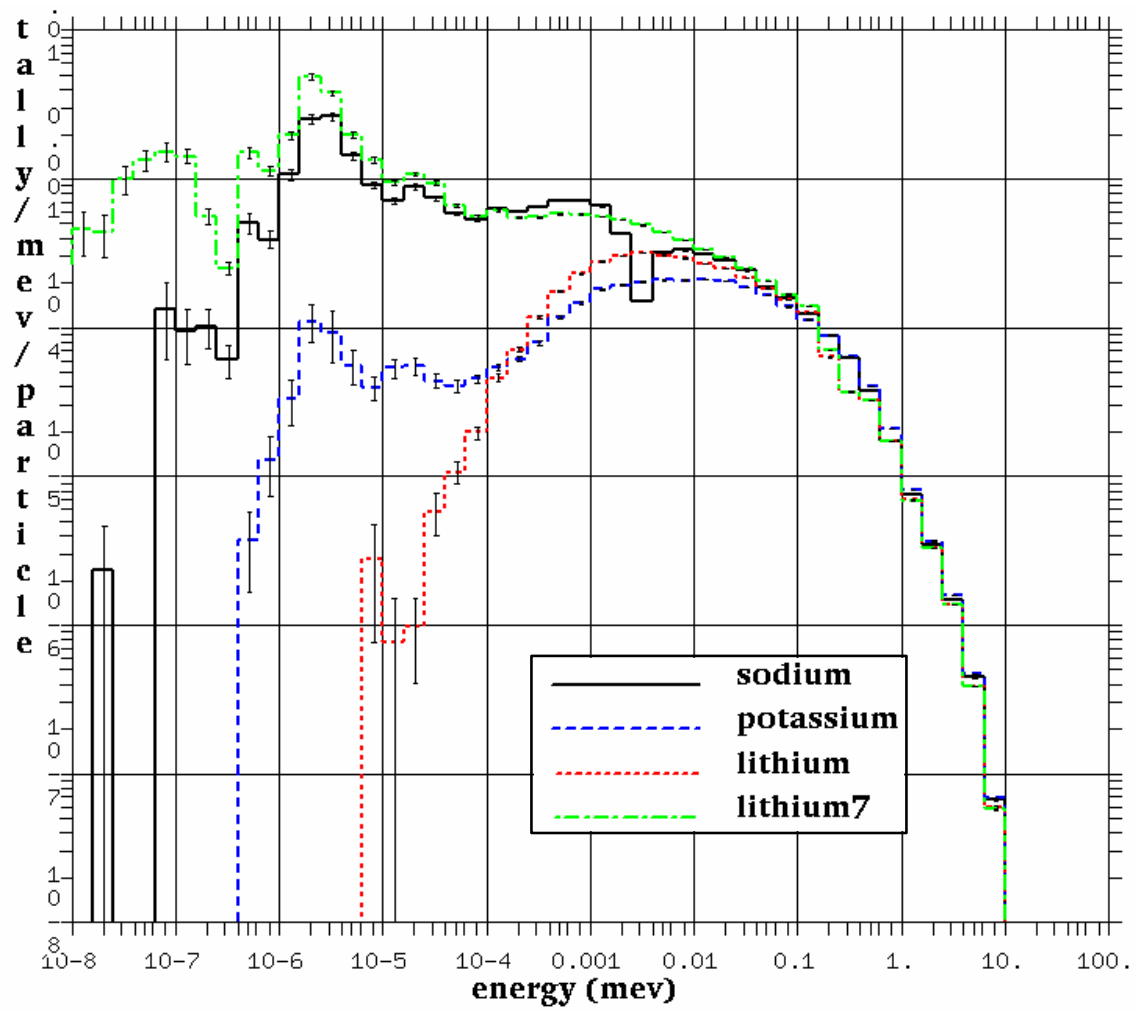

Figure 2-15. Neutron spectra for different coolant options.

Table 2-6 shows BOL K-effective for different coolant materials. Potassium leads to $\sim 5 \%$ reactivity penalty, which is rather significant, but may become acceptable $\mathrm{f}$ potassium turns out to be preferred for the energy conversion portion of LMR-AMTEC system. Lithium on the other hand leads to $\sim 15 \%$ penalty and it is difficult to imagine that such a large penalty would be compensated by some other advantage. In fact, it may require increasing the fissile content to above $20 \%$, which would be another significant drawback. Note that if only ${ }^{7} \mathrm{Li}$ is used, the penalty is reduced to $\sim 5 \%$, but then the cost is increased as well.

Depletion calculations using SCALE/SAS2H indicate that a similar reactivity swing would occur with burnup, in other words, similar BOL reactivity is needed in each case. In summary, considering neutronic properties (primarily reactivity penalty), sodium is the preferred choice, whereas potassium may be considered as an alternative provided that it offers some energy-conversion advantages. 
Table 2-6. K-effective for different coolant materials.

\begin{tabular}{|c|c|}
\hline Coolant & ?k vs. k(Na) \\
\hline $\mathrm{K}$ & $-0.0551+/-0.0014$ \\
\hline${ }^{\mathrm{Nat}} \mathrm{Li}$ & $-0.1620+/-0.0014$ \\
\hline${ }^{7} \mathrm{Li}$ & $-0.0606+/-0.0014$ \\
\hline
\end{tabular}

\subsubsection{Core Layout and Modeling}

Core physics analyses performed in the second project year assumed that the core includes 78 fuel elements. This corresponds to a hexagonal 6-ring layout with 91 total positions (Figure 2-16). The central ring/position is a control assembly, furthermore, third and fifth rings include 6 control assemblies each. To reduce fast neutron fluence on the vessel, neutron reflector/shield assemblies are employed. Monte Carlo simulations were performed mainly using a simplified cylindrical representation of this layout (Figure 2-17); note that control assemblies were individually modeled/represented.

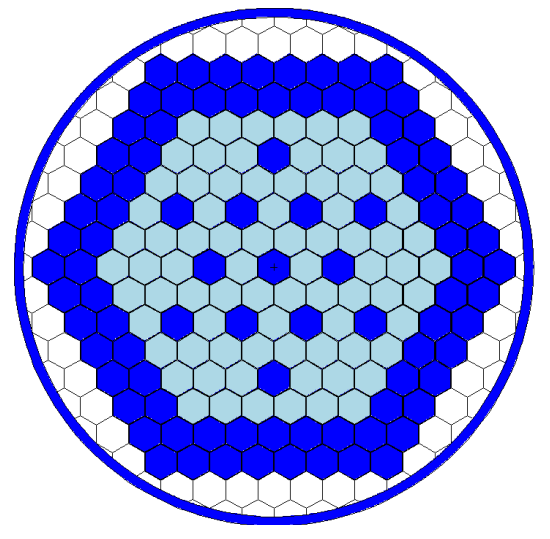

Figure 2-16. Core configuration with 78 fuel elements and 13 control assemblies (outermost two rings are shield assemblies).

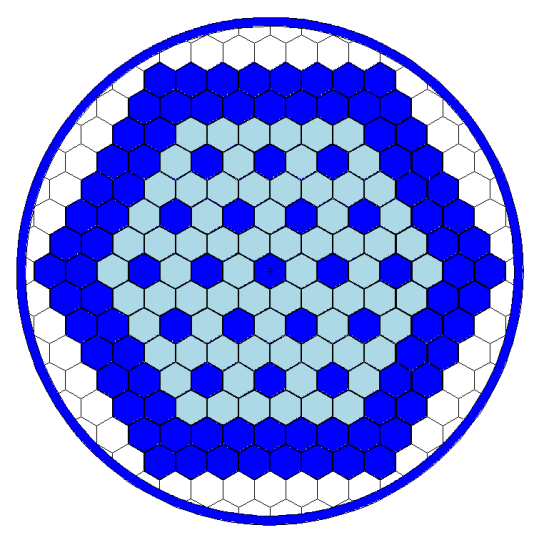

Figure 2-18. Core configuration with 72 fuel elements and 19 control assemblies (outermost two rings are shield assemblies).

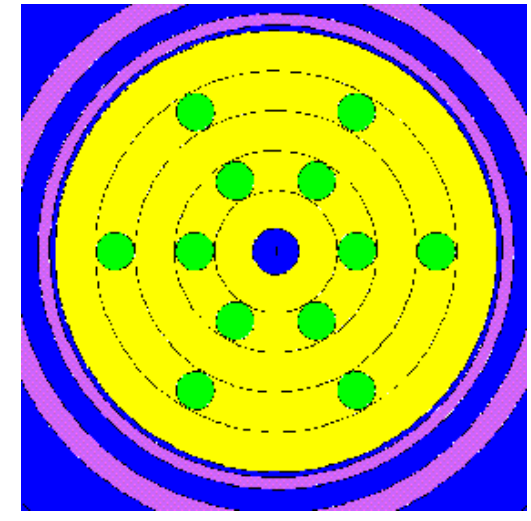

Figure 2-17. Cylindrical representation of core configuration with 78 fuel elements and 13 control assemblies.

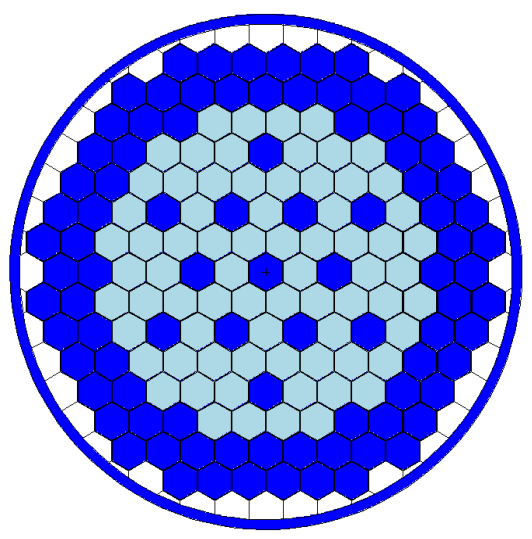

Figure 2-19. Core configuration with 72 fuel elements and 13 control assemblies (outermost two rings are shield assemblies). 
There are two issues that may lead to a core layout modification in the final design. First, if more control assemblies (19 rather than 13) are needed to provide adequate reactivity control and redundancy, the core configuration will be modified as shown in Figure 2-18. Note that the number of fuel elements would be reduced by 6 in this case. Second, the core shape would more closely match circular shape and the vessel size could be reduced if the 6 corner fuel elements were removed, as shown in Figure 2-19. This again reduces the number of fuel elements to 72. As a result, average linear power would increase by $\sim 8 \%$. The difference is relatively small, allowing the design activities to proceed. A final selection will require more detailed neutronic studies.

\subsubsection{Basic Fuel and Core Parameters}

Table 2-7 summarizes the basic design parameters determined based on neutronic calculations.

Table 2-7. Reference Fuel Design Parameters.

\begin{tabular}{|c|c|c|}
\hline Design parameter & Selected option & Comment \\
\hline Reactor power & $100 \mathrm{MWt}$ & \\
\hline Coolant & Sodium & \\
\hline No. of Fuel assemblies & 78 & $\begin{array}{l}\text { If } 19 \mathrm{CR} \text { assemblies are needed, it will be } \\
\text { reduced to } 72\end{array}$ \\
\hline Fuel assembly & Hexagonal, open & \\
\hline Fuel rods & 169 per fuel assembly & \\
\hline Spacer & Wire wrap & \\
\hline Active fuel length & $75 \mathrm{~cm}$ & \\
\hline Fission gas plenum length & $100 \mathrm{~cm}$ & \\
\hline Fuel form & Nitride fuel, UN or $(\mathrm{U}, \mathrm{Pu}) \mathrm{N}$ & Carbide may also be an option \\
\hline Pu isotopics & Reactor grade $\mathrm{Pu}$ & Highly-burnt PWR fuel \\
\hline Enrichment & $19.95 \%$ U235 & For UN fuel \\
\hline Fissile content & $19.95 \%$ total $\mathrm{Pu}$ & For $(\mathrm{U}, \mathrm{Pu}) \mathrm{N}$ fuel \\
\hline Cladding & $\mathrm{Nb}-1 \mathrm{Zr}$ & \\
\hline Fuel pellet diameter & $0.370 ”(0.9398 \mathrm{~cm})$ & \\
\hline Fuel smear factor & 0.90 & To be re-evaluated \\
\hline Fuel rod diameter & $0.417 ”(1.0592 \mathrm{~cm})$ & \\
\hline Lattice & Hexagonal & \\
\hline Lattice pitch & $0.511 ”(1.2979 \mathrm{~cm})$ & Reduced from $0.561 "$ \\
\hline P/d factor & 1.2254 & \\
\hline Linear power & $101.15 \mathrm{~W} / \mathrm{cm}$ & $\begin{array}{c}\text { If } 19 \mathrm{CR} \text { assemblies are needed, it will be } \\
\text { increased to } 109.6 \mathrm{~W} / \mathrm{cm}\end{array}$ \\
\hline Discharge burnup & $\sim 57$ and $\sim 70 \mathrm{GWd} / \mathrm{tHM}$ & For UN and $(\mathrm{U}, \mathrm{Pu}) \mathrm{N}$, respectively \\
\hline Cycle length & $\sim 13$ and $\sim 16$ EFPY & For UN and (U,Pu)N, respectively \\
\hline No. of. Control Assemblies & 13 & May be increased to 19 \\
\hline Control Assembly & Hexagonal & \\
\hline Absorber & $\mathrm{B}_{4} \mathrm{C}$ & Enriched boron \\
\hline
\end{tabular}




\subsubsection{Core Thermal Hydraulics}

\subsubsection{Thermal-Hydraulic Design Requirements}

Some of the traditional design requirements for LMR are:

1. The maximum fuel and control assembly element cladding temperatures and internal pressure during steady-state and transient conditions should be consistent with lifetime (burnup) objectives and structural design limits.

2. A substantial subcooling margin should exist between the saturation temperature and the exit coolant temperature in the hottest assembly. For an extremely unlikely event, the requirement for maintaining the coolable geometry is met by restricting the coolant temperature below its saturation temperature at the current pressure. This criterion precludes cladding from melting.

3. No significant fuel or absorber melting is allowed in the fuel or control assembly elements. In the CRBR, the criterion of non-melting at $115 \%$ overpower conditions, including design uncertainties at $3 \sigma$ was established.

4. Compatible with the balance of the primary heat transport system, the total pressure drop of the core assemblies and the reactor internals must be within the primary pumps head capability at design flow.

5. Coolant velocities must be less than the limits dictated by flow-induced vibration, cavitation, and corrosion-erosion considerations.

6. The core assembly mixed mean outlet coolant temperature and the difference in the mixed mean coolant temperature at the exit of adjacent assemblies must be within allowable limits to ensure structural integrity of the upper internals.

7. Maximum temperature levels and temperature gradients for all reactor components must be within acceptable limits to ensure structural integrity under all operating conditions.

8. Thermal hydraulic design of control assemblies must satisfy scram insertion requirements during the entire reactor lifetime.

9. The core and its surrounding interface equipment must be designed to minimize potential reactivity insertions due to such effects as sudden core compaction.

At this stage of the conceptual design of the LMR-AMTEC the first 5 criteria were studied. This section deals with the temperature margins accounting for the uncertainties. Further detailed studies are needed to verify if the design meets other criteria or if modifications in the reactor design are required. 


\subsubsection{Core Thermal-Hydraulic Model}

In order to calculate the axial temperature distributions in the hot channel and in the average channel a thermathydraulic model of the core was developed. This model was divided into a number of segments. Energy, momentum and mass conservation laws were applied in each segment. Steady state condition, angular symmetry and no fluid mixing between the channels were assumed. The last assumption yields conservative results. The thermat-physical properties were calculated based on the local temperature.

The heat transfer between the fuel and the sodium coolant is established with forced convection. The fuel thermal model assumes uniform heat generation inside the pellets and calculates the temperature profiles using the thermal properties of the uranium nitride and the cladding material. A heat exchange coefficient for the gap was selected based on the past experience with the EBR-II test fuel elements (Tang et al., 1978). The heat transfer between the clad surface and the fluid was estimated using the correlation for LMR rod bundle heat transfer recommended by Kazimi and Carelli (1976).

Table 2-8. Normalized Axial Power Profile.

\begin{tabular}{|c|c|c|}
\hline $\begin{array}{c}\text { Axial position of the } \\
\text { section center }(\mathrm{m})\end{array}$ & Radial Average & Hot Channel \\
\hline 0.0250 & 0.92 & 1.48 \\
\hline 0.0750 & 0.88 & 1.46 \\
\hline 0.1250 & 0.94 & 1.57 \\
\hline 0.1750 & 0.99 & 1.66 \\
\hline 0.2250 & 1.04 & 1.77 \\
\hline 0.2750 & 1.08 & 1.85 \\
\hline 0.3250 & 1.11 & 1.90 \\
\hline 0.3750 & 1.11 & 1.90 \\
\hline 0.4250 & 1.10 & 1.90 \\
\hline 0.4750 & 1.08 & 1.86 \\
\hline 0.5250 & 1.03 & 1.77 \\
\hline 0.5750 & 0.99 & 1.67 \\
\hline 0.6250 & 0.93 & 1.57 \\
\hline 0.6750 & 0.88 & 1.48 \\
\hline 0.7250 & 0.91 & 1.46 \\
\hline Average & 1.00 & 1.69 \\
\hline
\end{tabular}


Coolant velocity was calculated assuming a uniform inlet profile and accounting for the density variation and the pressure drop. The pressure drop comprises four parts: inlet contraction, outlet expansion, bare rod friction, and wire-wrap spacer resistance. The latter two pressure drops were determined using the Novendstern correlation (Tang et al. 1978).

The power profiles (Table 2-8) for the hot and the average channels were obtained from 3-D neutronic calculations (Monte Carlo simulations) and were used as input for the thermat hydraulic calculations.

The core thermal-hydraulic model was evaluated using reactor parameters under normal operating conditions. The pellet temperature profile at the inlet, center and outlet of the hot channel is presented in Figure 2-20. The maximum value is far below the melting point of (U,Pu)N: 2800 K (see Table 2-11).

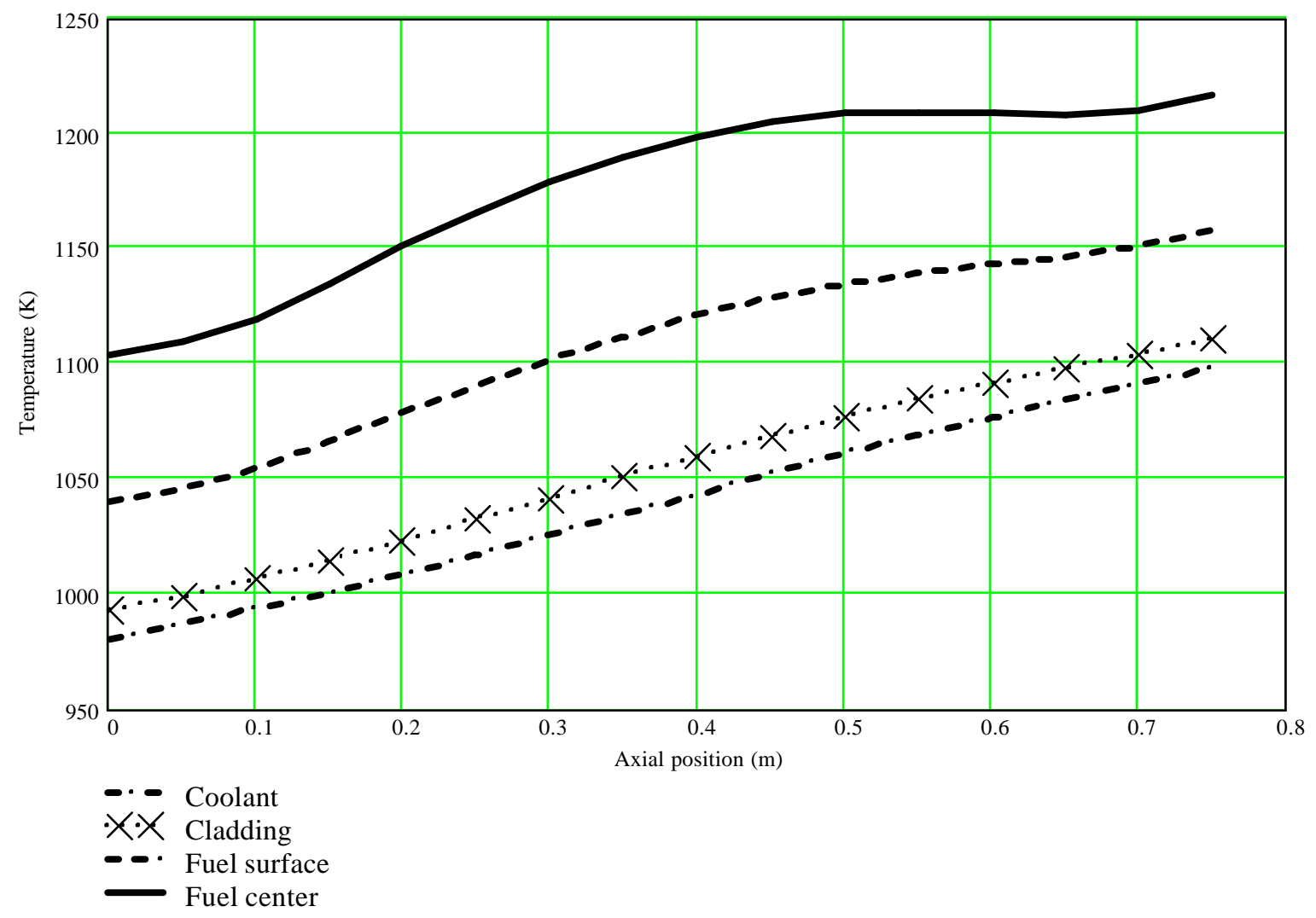

Figure 2-20. Radial fuel pin temperature distribution for the sodium-cooled core.

The axial temperature profiles for the hot channel are presented in Figure 2-21. As can be seen, a large subcooling margin exists at the cladding surface temperature. Note that the boiling point of sodium at design condition is $\sim 1400 \mathrm{~K}$. This margin prevents the cladding from melting. 


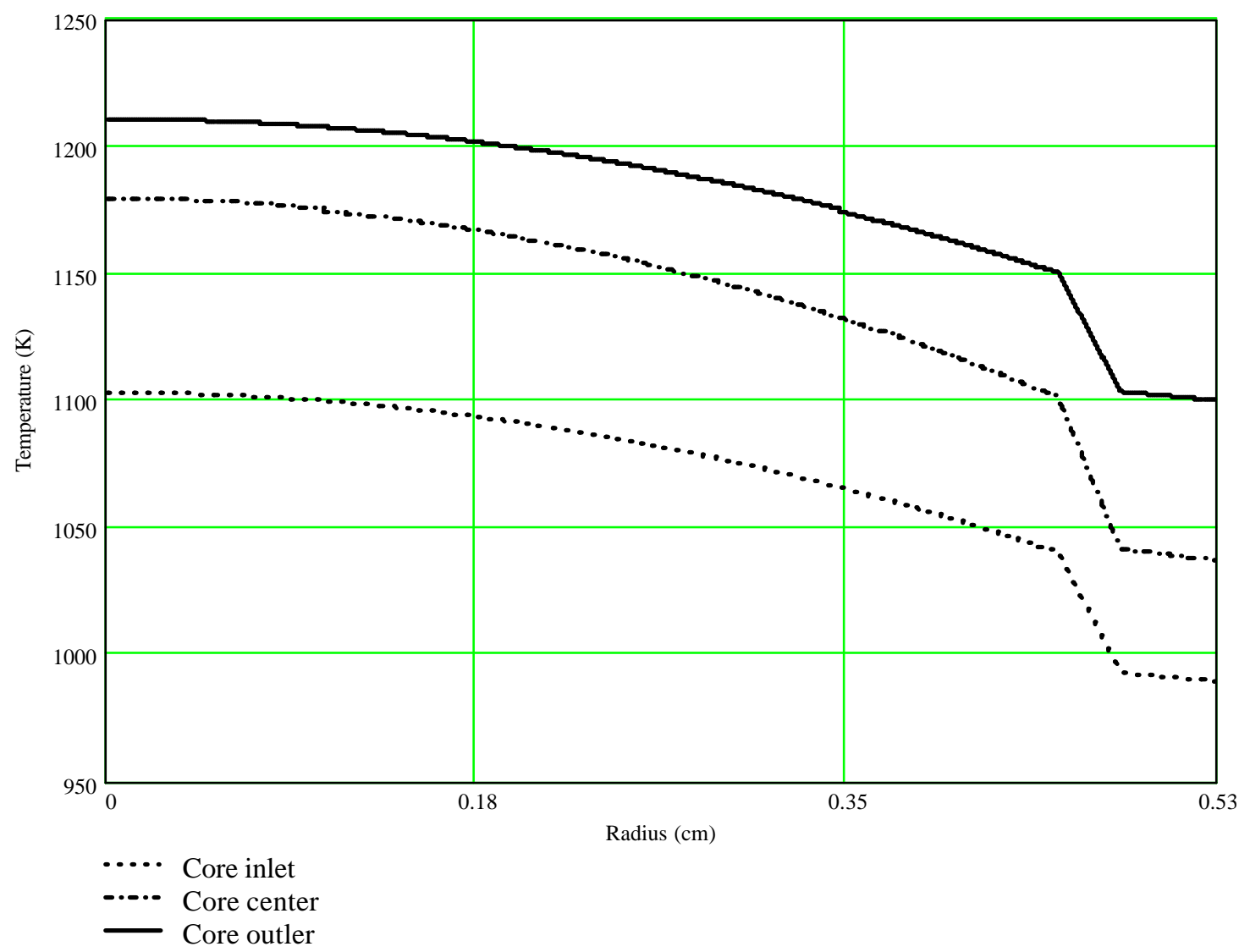

Figure 2-21. Nominal axial core temperatures distribution in the sodium-cooled core without uncertainties.

\subsubsection{Hot-Channel analysis: The Semi-statistical Method}

In the LMR-AMTEC reactor core thermal-hydraulic design, the cladding temperature is the most limiting factor. In predicting the temperature profile of the fuel rod, the impact of theoretical and experimental analysis uncertainties, instrumentation and control inaccuracies, manufacturing tolerances, material properties and correlation uncertainties, and changes in reactor conditions with lifetime must be considered. Therefore in performing the analysis, the semi-statistical method and a set of sub-factors developed by Westinghouse during the Clinch River Breeder Reactor Project was used. This method was adopted as a reasonable compromise between cumulative and fully statistical methods and is commonly used in liquid metal reactor designs. The method postulates that actually not all parameters are statistical in nature. Therefore, the sum of uncertainties is divided into two principal groups: statistical and non-statistical subfactors. By calculating the total hot-spot factor for a certain temperature rise, systematic uncertainties are treated cumulatively and statistical uncertainties are treated statistically. 
The use of the semi-statistical method requires the separation of the variables, which affect the hot spot temperatures into statistical origin and non-statistical categories. A mon-statistical (or direct) uncertainty is defined as a variation in a design variable, which is known or conservatively assumed to occur, but is not included in the nominal analysis. Computationally, the non-statistical factors represent multipliers applied to the nominal magnitudes of the variables to provide the worst value which might occur. A variable that has a random frequency distribution of occurrence is treated statistically. For example, experimental data (such as materials properties) are treated statistically since there is a random error in their evaluation. Calculation of fuel rod temperature for the hot channel is shown in Figure 2-22. The nominal temperature difference, $\Delta T_{\text {nom }}$, is multiplied by the product of the direct hot channel factors to give the temperature rise including non-statistical factors, $\Delta T_{d i r}$. Using this value, the nominal temperature is increased to $T_{d i r}$ or $T_{0 \sigma}$. $T_{d i r}$ represents the mean value about which the statistical uncertainties are applied. The nominal channel can be any channel in the assembly, with the nominal channel coolant $\Delta T$ calculated using subchannel analysis codes. In practice, the channel with the highest power density is singled out for application of hot channel factors, thus calculation of hot channel temperature and uncertainties.

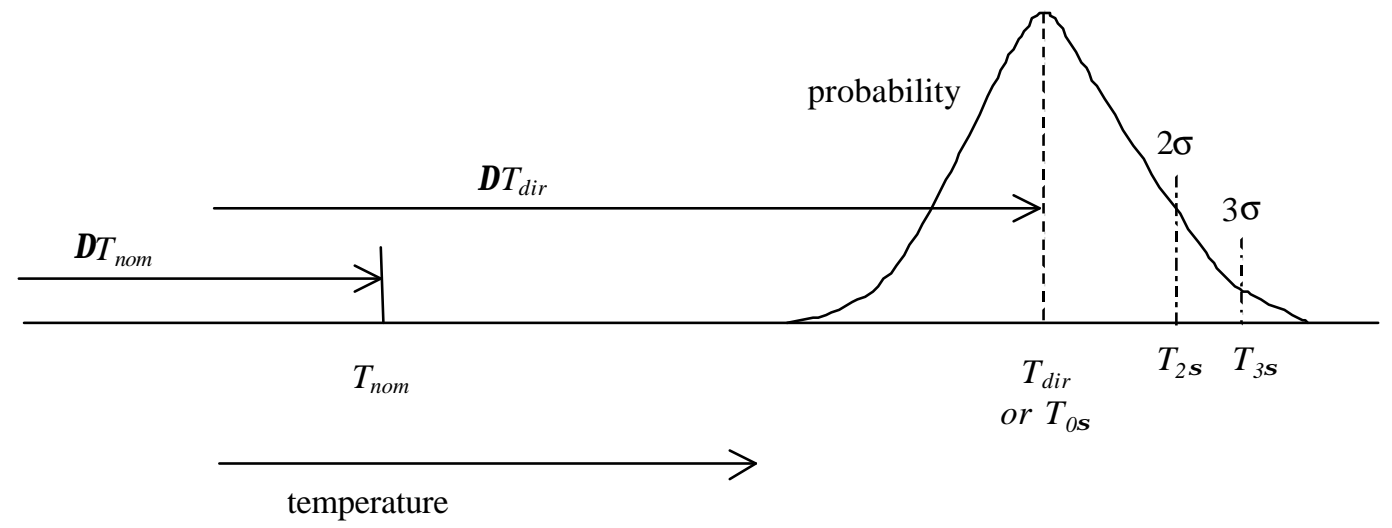

Figure 2-22. Semi-statistical method.

Direct and statistical subfactors developed during the Clinch River Breeder Reactor Project (Carelli et al., 1985) and employed for the cladding temperature calculation are reported in Table 2-9 and Table 2-10. The direct factors of each component in the last row of Table 2-9 are the products of each column of subfactors, i.e.

$$
f_{j, d i r}=\prod_{i=1}^{I I} s_{i j, d i r}
$$


Table 2-9. Direct uncertainty subfactors, $s_{i j, d i r}$. for cladding temperature evaluation ( $\left.3 \sigma\right)$.

\begin{tabular}{|l|l|l|l|}
\hline Uncertainty Fact $(i) \quad$ C Component $(j)$ & Coolant & Film & Cladding \\
\hline Reactor Power Lever & 1.03 & 1.03 & 1.03 \\
\hline Physics Modeling: 2D/3D & 1.01 & 1.1 & 1.1 \\
\hline Physics Modeling: Axial Shape (Bias) & 1.0 & 1.03 & 1.03 \\
\hline Inlet Flow Maldistribution & 1.02 & 1.0 & 1.0 \\
\hline Flow Distribution Calculational Uncertainty & 1.03 & 1.0 & 1.0 \\
\hline Pellet-Cladding Eccentricity & 1.0 & 1.11 & 1.08 \\
\hline Cladding Thermal Conductivity & 1.0 & 1.0 & 1.035 \\
\hline Combined Direct Factor $f_{j, \text { dir }}$ & 1.093 & 1.295 & 1.304 \\
\hline
\end{tabular}

Table 2-10. Statistical uncertainty subfactors, sij,sta for cladding temperature evaluation $(3 \sigma)$.

\begin{tabular}{|l|l|l|l|}
\hline Uncertainty Fact $(i) \quad$ Component $(j)$ & Coolant & Film & Cladding \\
\hline Physics Experimental & 1.055 & 1.055 & 1.055 \\
\hline Physics Modeling: Pin Interpolation & 1.01 & 1.01 & 1.01 \\
\hline Physics Modeling: Axial Shape & 1.0 & 1.01 & 1.01 \\
\hline Fissile Content & 1.052 & 1.052 & 1.052 \\
\hline Inlet Flow Maldistribution & 1.039 & 1.0 & 1.0 \\
\hline Flow Distribution Calculational Uncertainty & 1.055 & 1.0 & 1.0 \\
\hline Wire Wrap Orientation & 1.01 & 1.0 & 1.0 \\
\hline Subchannel Flow Area & 1.019 & 1.0 & 1.0 \\
\hline Film Heat Transfer Coefficient & 1.0 & 1.12 & 1.0 \\
\hline Pellet-Cladding Eccentricity & 1.0 & 1.13 & 1.11 \\
\hline Cladding Thickness & 1.0 & 1.0 & 1.08 \\
\hline Cladding Thermal Conductivity & 1.0 & 1.0 & 1.075 \\
\hline Coolant Properties & 1.017 & 1.0 & 1.0 \\
\hline
\end{tabular}


Previous practice in accounting for uncertainties affecting the cladding temperature was to set up four types of factors: coolant (multiplicative of coolant temperature rise $\Delta T_{\text {cool }}$ ), film (multiplicative of film temperature rise $\Delta T_{\text {film }}$ ), cladding (multiplicative of cladding temperature rise $\Delta T_{\text {clad }}$ ) and heat flux (multiplicative of power generation). Uncertainties affecting power generation were part of the heat flux factor, while geometry or properties uncertainties were part of the other factors. In this design, following the method of Carelli et al. (1985), no separate heat flux uncertainties are considered and power generation uncertainties are included in the coolant, film and cladding hot channel factors. If all factors were direct the two methods would yield exactly the same temperatures; however, since many factors are statistical, the second method now employed allows an expanded statistical combination and thus yields slightly less conservative temperatures.

The nominal cladding inner surface temperature was calculated as:

$$
T_{\text {gap }, \text { nom }}=T_{\text {in }}+\Delta T_{\text {cool }, \text { nom }}+\Delta T_{\text {film,nom }}+\Delta T_{\text {cald }, \text { nom }}
$$

and the mean inner cladding surface temperature with direct uncertainties included, $T_{\text {gap,dir }}$ :

$$
T_{\text {gap }, \text { dir }}=T_{i n}+\Delta T_{\text {cool,dir }}+\Delta T_{\text {film,dir }}+\Delta T_{\text {cald,dir }}
$$

where

$$
\begin{aligned}
& \Delta T_{\text {cool,dir }}=f_{\text {cool,dir }} \Delta T_{\text {cool,nom }} \\
& \Delta T_{\text {film,dir }}=f_{\text {film,dir }} \Delta T_{\text {film,nom }} \\
& \Delta T_{\text {clad,dir }}=f_{\text {clad,dir }} \Delta T_{\text {clad,nom }}
\end{aligned}
$$

The temperature uncertainty contributed by each factor was estimated as:

$$
\Delta T_{i, s t a}=\sum_{j=1}^{3}\left(s_{i j, s t a}-1\right) \cdot \Delta T_{j, d i r}
$$

Then, the temperature variance corresponding to $3 \sigma$ can be determined by statistical method:

$$
3 \sigma=\sqrt{\sum_{i=1}^{I I}\left(\Delta T_{i, s t a}\right)^{2}}
$$


Therefore, the maximum cladding temperature with $99.9 \%$ confidence level is:

$$
T_{\text {gap, } \max }=T_{i n}+\Delta T_{\text {cool,dir }}+\Delta T_{\text {film,dir }}+\Delta T_{\text {cald, dir }}+3 \sigma
$$

The core thermal-hydraulic model and semi-statistical method were evaluated with the reactor parameters under normal operating conditions to assess the thermal margin. The maximal cladding temperature with $99.9 \%$ confidence level is under $1150 \mathrm{~K}$ as seen in Figure 2-23.

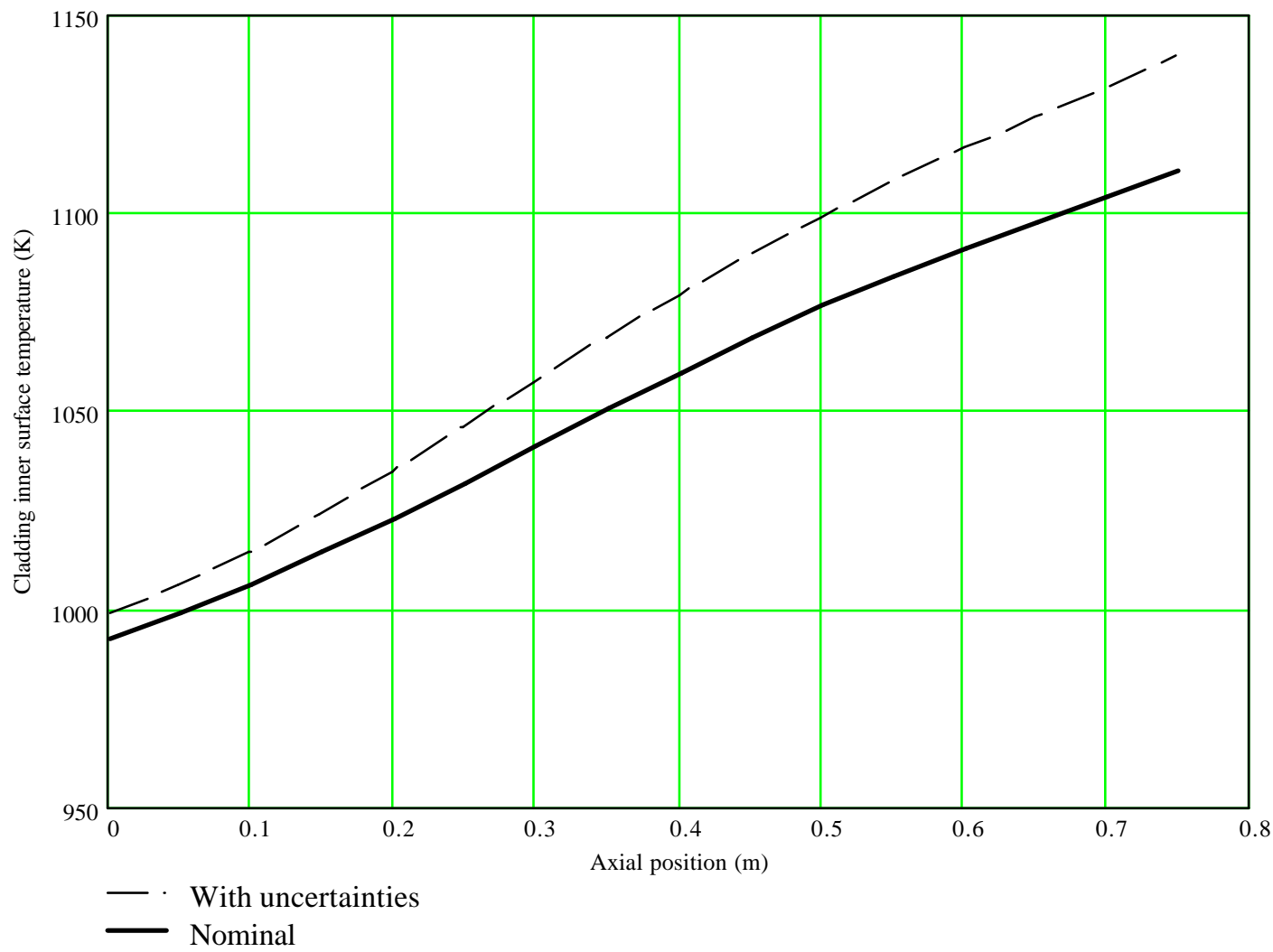

Figure 2-23. Axial core temperatures distribution in the sodium-cooled core with uncertainties $(3 \sigma)$ included. 


\subsubsection{Core Mechanical Design}

\subsubsection{Fuel Rod Design Requirements}

The design requirements for the LMR-AMTEC fuel rod are summarized as follows:

- Cladding steady state operating temperature: $900 \mathrm{~K}$ to $1200 \mathrm{~K}$,

- Cladding material: Compatible with the fuel and the coolant,

- Linear rod average power: Between 100 to $200 \mathrm{~W} / \mathrm{cm}$,

- Fuel pellet diameter: Between 0.90 to $1.05 \mathrm{~cm}$,

- Fuel smear density: Lower than $90 \%$,

- The desired fuel burnup: Up to $70 \mathrm{GWd} / \mathrm{tHM}$,

- The minimal design lifetime: 10 years,

- The design fluence $(\mathrm{E}>0.1 \mathrm{MeV})$ on the cladding: $9 \times 10^{23} \mathrm{n} / \mathrm{cm}^{2}$,

- Rod internal pressure: less than $\sim 3.4 \mathrm{MPa}$ (500 psi) provided by fission gas plenum at the rod bottom and top,

- The cladding stress: Not to exceed $28 \mathrm{MPa}$ to $34 \mathrm{MPa}$ (4000 to $5000 \mathrm{psi}$ ),

- Fission gas release: $100 \%$ during the lifetime (>10 years) at high temperature,

- High burnup fuel swelling: Limited effect on the cladding.

These design requirements will help in preventing the following fuel rod failure modes:

- Fracture or burst induced by rod internal over-pressure, assisted by reduced ductility of cladding due to irradiation,

- Cladding failure induced by mechanical interaction of fuel and cladding during steady state and transient operation,

- Cladding failure induced by excessive cladding fretting wear due to fuel vibration,

- Excessive cladding creep, including creep-ratcheting, exceeding the functional constraints of the fuel rod or the heat transfer bond between cladding and fuel pellet,

- Degradation of the cladding strength due to corrosion,

- Mass transfer from the hot leg of the system (the cladding) to the cold leg of the system (Alkali Metal Boilers).

According to the fuel rod design requirements, the following cladding and fuel rod design criteria were specified:

- Rod internal pressure shall not induce cladding creep, which affects the rod performance and cooling functions,

- The cladding stress must be less than the proof and rupture strength with an acceptable design margin,

- Circumferential creep and plastic strains during steady state and each transient power change should be less than 0.5 to $2 \%$,

- The cladding fatigue limit shall not be exceeded. This limit is generally not exceeded in reactors operated at a base load. 
- Cladding corrosion and mass transport within the coolant system shall not deteriorate the cladding and Alkali Metal Boilers functions.

In addition to these design criteria, the cladding material should possess the following characteristics:

- Acceptable degradation of effective strength and ductility due to corrosion,

- Sufficient ductility to sustain the strain generated by fuel expansion during short transients,

- Sufficient strength in irradiated and non-irradiated conditions to restrain the fuel swelling and contain the fission gas pressure,

- Sufficient ductility at high burnup to prevent failure due to PCMI during normal/offnormal transients,

- A material composition which exhibits acceptable or no volume swelling at the design fluence,

- A uniform surface corrosion rate.

\subsubsection{Material Selection}

Based on the design criteria, $(\mathrm{U}, \mathrm{Pu}) \mathrm{N}$ and $\mathrm{Nb}-1 \mathrm{Zr}$ were selected as the fuel and cladding materials, respectively. Nitride fuel was selected because of its good thermal (see Table 2-11) and neutronic properties (section 2.2.2), higher theoretical heavy metal density (allows to increase $\mathrm{Pu}$ load), and compatibility with the cladding material.

Table 2-11. Properties of Uranium and Plutonium fuels. Note the dependence of the thermal conductivity of the $(\mathrm{U}, \mathrm{Pu}) \mathrm{N}$ with respect to the $\mathrm{Pu}$ fraction.

\begin{tabular}{|c|c|c|c|c|c|c|c|c|}
\hline & \multicolumn{2}{|c|}{ Nitride } & \multicolumn{2}{|c|}{ Oxide } & \multicolumn{2}{|c|}{ Carbide } & \multicolumn{2}{|c|}{ Metal } \\
\hline & $U N$ & PuN & $\mathrm{UO}_{2}$ & $\mathrm{PuO}_{2}$ & $U C$ & $P u C$ & $U$ & $P u$ \\
\hline Melting point $[\mathrm{K}]$ & 2873 & 2773 & 3003 & 2573 & 2673 & 1923 & 1405 & 913 \\
\hline Theory density $\left[\mathrm{g} / \mathrm{cm}^{3}\right]$ & 14.32 & 14.22 & 10.96 & 11.46 & 13.63 & 13.62 & 19.05 & 19.86 \\
\hline Heavy atom density $\left[\mathrm{g} / \mathrm{cm}^{3}\right]$ & 13.51 & 13.43 & 9.66 & 10.11 & 12.97 & 12.96 & 19.05 & 19.86 \\
\hline \multirow[t]{2}{*}{ Heat conductivity $[\mathrm{W} / \mathrm{cm} . \mathrm{K}]$} & $\approx 0.21$ & $\approx 0.13$ & \multicolumn{2}{|c|}{$\approx 0.047$} & \multicolumn{2}{|c|}{$\approx 0.16$} & \multirow{2}{*}{\multicolumn{2}{|c|}{$\approx 0.3$}} \\
\hline & $\approx 0.29$ & $\approx 0.21$ & \multicolumn{2}{|c|}{$\approx 0.025$} & \multicolumn{2}{|c|}{$\approx 0.17$} & & \\
\hline Specific heat $[\mathrm{cal} / \mathrm{g} . \mathrm{K}]$ at $1773 \mathrm{~K}$ (metal $773 \mathrm{~K})$ & 0.067 & 0.061 & 0.081 & 0.084 & 0.065 & 0.058 & 0.040 & 0.044 \\
\hline
\end{tabular}

$\mathrm{Nb}-1 \mathrm{Zr}$ was selected as a cladding material because of it superior mechanical and material properties. Compared to other high temperature alloys (e.g., nickel alloys), Nb$1 \mathrm{Zr}$ has better high temperature creep strength and ductility if it is not oxidized. Also if the oxygen levels in sodium are kept lower, corrosion effects are found to be negligible, even for high temperatures up to $1400 \mathrm{~K}$. Furthermore, the $\mathrm{Nb}-1 \mathrm{Zr}$ material properties are well known and available literature data enable the conceptual design of a fuel rod with sufficient confidence. Exploratory irradiation tests at much higher temperatures than required for LMR/AMTEC have been performed, and the fuel rod performance was found to be acceptable. 
The disadvantages of using $\mathrm{Nb}-1 \mathrm{Zr}$ include:

- Niobium is neutron absorber with a large capture cross-section that penalizes the reactor neutron economy, and requires higher fuel enrichment.

- Material property degradation in the form of embrittlement occurs even in low oxygen and hydrogen environments during operation and fabrication. Consequently, material and fabrication costs are higher than for Nickel alloys or SS.

The degradation of the $\mathrm{Nb}-1 \mathrm{Zr}$ properties due to oxygen and hydrogen traces in the coolant must be considered when designing remedial systems such as cold traps. To proceed to a preliminary design and license submittal, additional information is needed on the fuel rod design, including:

- $\mathrm{Nb}-1 \mathrm{Zr}$ ductility reduction at high fluence exposure,

- Irradiation creep effects,

- Potential for volume swelling of $\mathrm{Nb}-1 \mathrm{Zr}$,

- Environmental effect of oxygen, hydrogen and other elements on $\mathrm{Nb}-1 \mathrm{Zr}$ design properties.

Acquiring this information requires a radiation test-bed with high temperature capability (e.g. FFTF case) or access to another overseas reactor.

As a final note, as mentioned in section 2.2.2, the design limit for the fluence $(\mathrm{E}>0.1 \mathrm{MeV})$ on the cladding is met according to the neutronic studies.

\subsubsection{Clad Circumferential Creep}

The circumferential creep of the clad is a more limiting factor than the ultimate tensile strength due to high core temperatures. The clad thickness must assure that the circumferential creep during steady state is kept below $2 \%$. A correlation for the $\mathrm{Nb}-1 \mathrm{Zr}$ stress to \% creep with respect to temperature can be used to estimate the maximum allowed circumferential stress. For example, Figure 3.5 shows the stress to induce $1 \%$ of creep as a function of temperature for different time periods. Using this correlation and the data in Table 2-12 the maximum allowable stress was estimated to be 5890 psi.

The rod circumferential stress $\sigma_{c}$ can be estimated from:

$$
\sigma_{c} \cong \frac{d_{c l a d} \cdot P_{f p}}{2 \cdot t_{\text {clad }}}
$$

where $d_{\text {clad }}$ is the clad diameter, $t_{\text {clad }}$ is the clad thickness, and $P_{f p}$ the internal rod pressure. The internal rod pressure is maintained below 500 psi using a $1 \mathrm{~m}$ long plenum to accommodate the fission products. Using the data in Table 2-12, $\sigma_{c}$ is calculated to be lower than 5800 psi. Therefore the value is lower than the maximum stress allowed by the $2 \%$ circumferential creep limit. 


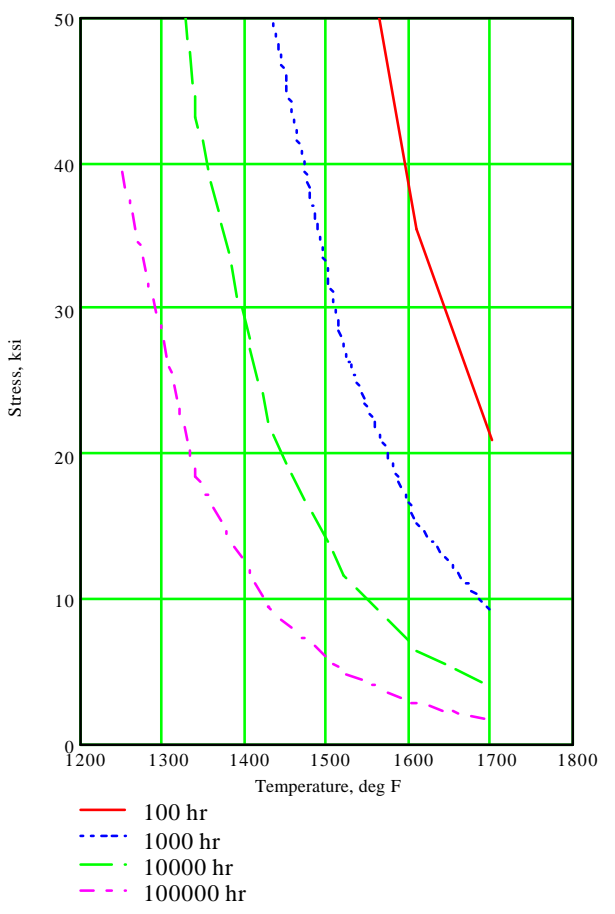

Figure 2-24. $\mathrm{Nb}-1 \mathrm{Zr}$ stress to cause $1 \%$ creep as a function of temperature.

Table 2-12. Parameters used for evaluating the circumferential clad creep.

\begin{tabular}{|l|c|}
\hline Rod internal pressure & $<500 \mathrm{psi}$ \\
\hline Clad diameter & $1.059 \mathrm{~cm}$ \\
\hline Clad thickness & $0.4572 \mathrm{~mm}$ \\
\hline Maximal clad temperature with uncertainties & $\sim 1110 \mathrm{~K}$ \\
\hline Maximal circumferential creep & $<2 \%$ \\
\hline Lifetime & $\sim 10 \mathrm{yr}$ \\
\hline
\end{tabular}

\subsubsection{Fuel Rod Vibration}

Once the fuel assemblies are loaded in the core, they will be exposed to very severe thermal, mechanical and radiation loads during the operation of the reactor. Dynamic forces generated by fluid flow will result in fuel rod vibration, which may cause fuel rod failure. In the case of LMR-AMTEC, the vibration concerns may impose a more restrictive coolant velocity limit than the one imposed by cladding corrosion. In addition to the coolant velocity limit, the designer has to measure the wire-wrap period length in order to avoid the resonance of fluid forces with the natural modes of fuel rod vibration. Both aspects, critical velocity and natural mode of vibration, will be analyzed in the following sections, in relation to the three flow-induced vibration excitation mechanisms. Finally, a brief discussion on thermoelastic instabilities will also be given. 


\section{Fluidelastic Instabilities}

Fluidelastic instabilities arise from coupling between fluid-induced dynamic forces and the motion of the structures. Instability occurs when the flow speed is sufficiently high so that the energy absorbed from the fluid forces exceeds the energy dissipated by damping. Fluidelastic instability is usually not an issue for components in axial flow (e.g., fuel rods). However, due to the flow redistribution, cross flow will exist in some regions of the core (the LMR-AMTEC fuel elements are not canned). A conservative estimation of the critical velocity can be performed using a correlation developed for cross flow.

For a single-phase cross flow, the fluidelastic instability is formulated in terms of a dimensionless flow speed, $U_{p} / f \cdot D$ and a dimensionless mass-damping parameter $2 \cdot \pi \cdot \varsigma \cdot m / \rho \cdot D^{2}$. For the case of a tube bundle subjected to uniform flow over its entire length, it takes the following form:

$$
U_{p c} / f \cdot D=K \cdot\left(2 \pi \varsigma \cdot m / \rho \cdot D^{2}\right)^{n}
$$

where $f$ is the tube frequency in the fluid, $m$ is the mass per unit length including the hydrodynamic mass, $\zeta$ is the total damping ratio (structural and fluid damping), $\rho$ is the fluid density, $D$ is the tube diameter, $U_{p c}$ is the threshold, or the critical velocity for the fluid instability and $n$ is an exponent which is often $\sim 0.5$ (note that in that case $U_{p c}$ does not depend with respect to $D$ ). The instability constant $K$ is obtained from the available experimental data. As a simple practical design guideline, an instability constant $K=0.5$ is recommended for all tube bundle configurations in single-phase cross flow.

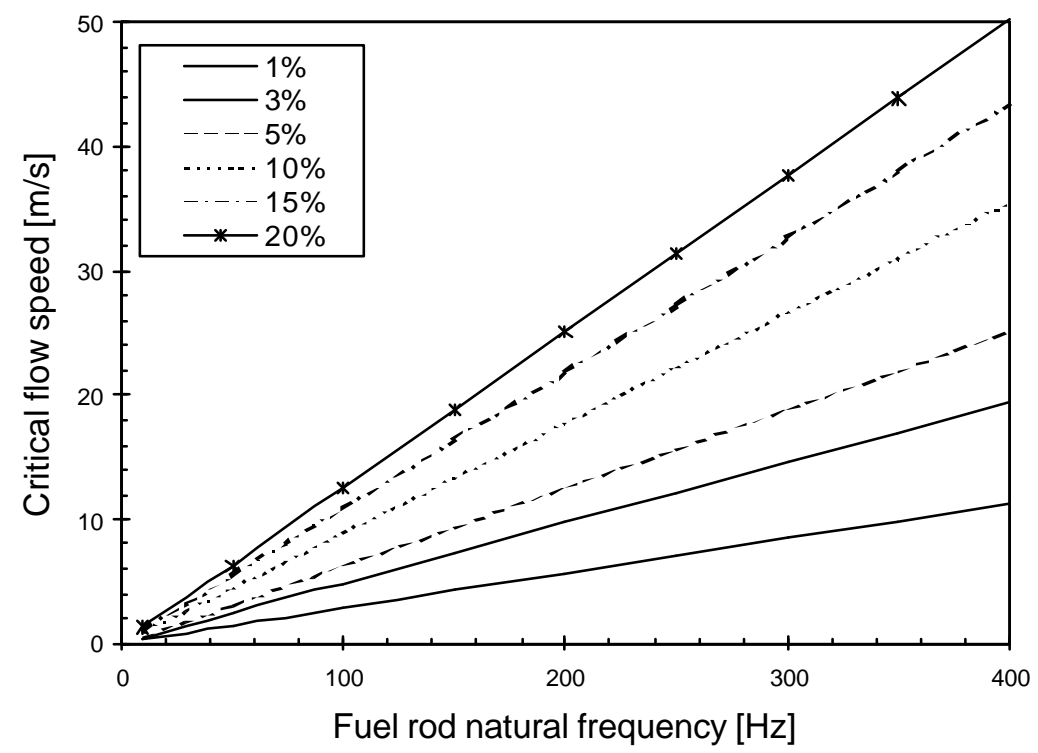

Figure 2-25. Critical flow speed versus the fuel natural frequency and for different structural damping values. 
Using this correlation and the fuel rod parameters (listed in Table 2-13) the critical flow speed was estimated and the results presented in Figure 2-25. As shown in the next section, the natural frequency of the fuel rod increases to $\sim 105 \mathrm{~Hz}$ due to the wire wrap. Using typical damping values for the fuel $\operatorname{rod}(\sim 3 \%)$, the critical speed is calculated to be $\sim 3.4 \mathrm{~m} / \mathrm{s}$. The axial flow is less than $2 \mathrm{~m} / \mathrm{s}$ and the maximum cross flow does not exceed $0.2-0.3 \mathrm{~m} / \mathrm{s}$ ( $20 \%$ is assumed as a conservative estimation), which is less than the critical speed. For these reasons, fluid elastic instabilities are not expected to cause a problem in our fuel element, except in the unlike event where the wire-wrap is completely missing.

Table 2-13. Fuel rod parameters.

\begin{tabular}{|l|c|}
\hline Fuel rod length & $0.75 \mathrm{~cm}$ \\
\hline Clad external diameter & $1.059 \mathrm{~cm}$ \\
\hline Clad thickness & $0.4572 \mathrm{~mm}$ \\
\hline Pellet diameter & $0.96756 \mathrm{~cm}$ \\
\hline Wire period length & $25 \mathrm{~cm}$ \\
\hline Structural damping & $<5 \%$ \\
\hline Clad average temperature & $1050 \mathrm{~K}$ \\
\hline Fuel rod linear density & $1.0726992 \mathrm{~kg} / \mathrm{m}$ \\
\hline Young modulus & $1.0221510^{11} \mathrm{~Pa}$ \\
\hline Inertia moment & $1.8717110^{-10} \mathrm{~m}^{4}$ \\
\hline
\end{tabular}

\section{Periodic Wake Shedding}

Periodic wake shedding, which often occurs immediately downstream of the structures subject to cross flow, generates periodic fluid forces. If the shedding frequency coincides with the natural frequency of the structure, resonances may occur. This may be an issue if the vibration response is large enough to control the wake shedding mechanism. In this case, the periodic forces become spatially correlated to the mode shape causing large vibration amplitudes. Since there are no grid-spacers in the LMR-AMTEC fuel rods, we do not expect that this phenomenon will be as significant.

\section{Turbulence}

Turbulence is the principal vibration excitation mechanism in axial flow and eventually of fuel rod failure. It can be generated locally by the fluid as it flows around the component of interest. This is called near-field excitation. Alternatively, far-field excitation can be generated by upstream components such as inlet nozzles, elbows, grids, etc. Turbulent-induced excitation generates random pressure fluctuations around the surface of components forcing them to vibrate. While fluidelastic instability and periodic wake shedding may cause failure in a very short time, turbulence excitation may induce enough vibration response to cause ong-term fretting-wear damage, especially if this vibration is coupled with other factors like temperatures, corrosion and radiation. This phenomenon will be of special concern for the LMR-AMTEC because of the high operating temperatures and the long lifetime ( 10yr) of the fuel cycle. 
Hexagonal fuel assembly design with wire-wrap spacer has been selected for the LMR-AMTEC, consistent with fast reactor design experience. In this fuel design frettingwear damage can occur at the contact region between the cladding and the wire wrap. In addition, during the residence in the core, the mechanical properties of the fuel rod will change and a gap between the rod and the wire wrap may appear. This phenomenon will not only affect the vibration behavior of the fuel but also strongly change the rate of the fretting damage. In the next section, we present the results of a study dealing with fuel rod vibration under turbulence force.

\section{Non linear vibration model}

The vibration behavior of the fuel rod was studied using a three-dimensional non-linear model, which accounts for the clad-support interaction and the external excitation force arising from the fluid. A sketch of such a model is presented in Figure 2-26. Here, the wire wrap is approximated as a set of supports spaced according to the wire period length. The existence of a gap is also accounted in the model.

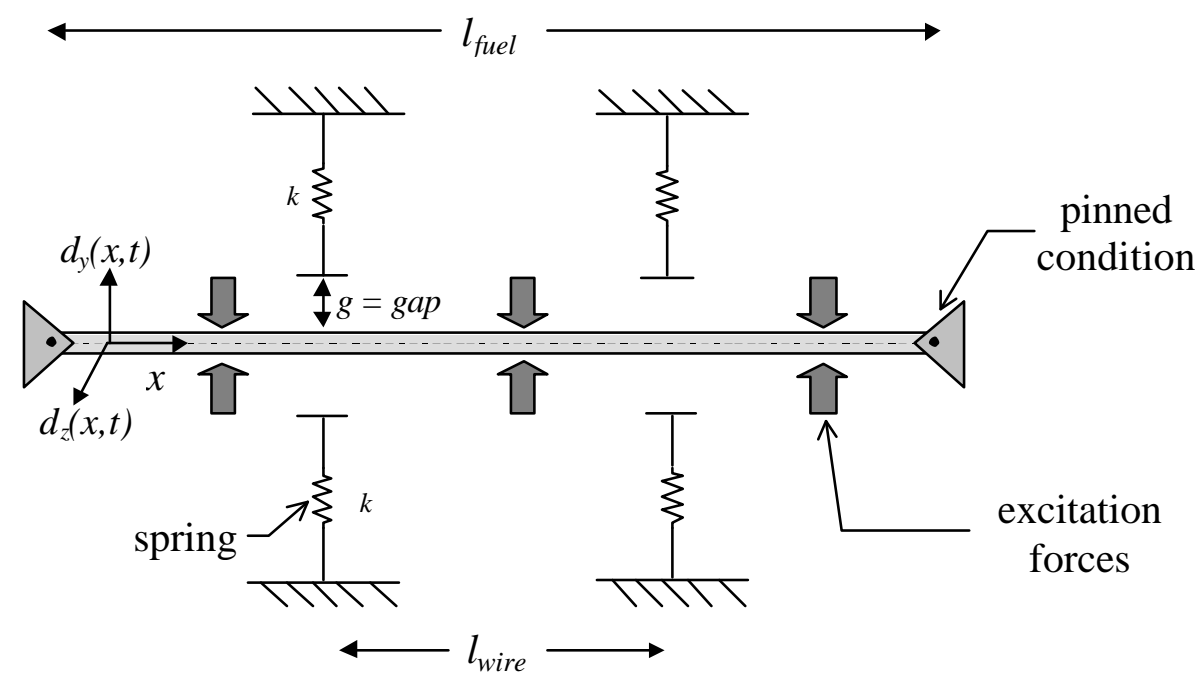

Figure 2-26. Non-linear fuel rod vibration model used to study the LMR-AMTEC fuel rod.

The equation of motion for the fuel rod can be written as:

$$
\frac{\partial^{2}}{\partial x^{2}}\left[E \cdot I \frac{\partial^{2} \vec{d}(x, t)}{\partial x^{2}}\right]+c \cdot \frac{\partial \vec{d}(x, t)}{\partial t}+\frac{\partial^{2} \vec{d}(x, t)}{\partial t^{2}} \cdot \rho(x)=\vec{f}_{e}(x, t)+\vec{f}_{s}(x, t)
$$

where $\vec{d}(x, t)$ is the displacement vector, $c$ is the viscous structural damping, $\rho(x)$ is the mass per unit length, $E \cdot I$ is the bending stiffness, and $\vec{f}_{e}(x, t)$ and $\vec{f}_{s}(x, t)$ are the excitation and support forces, respectively. The non-linearity arising from the supports (wire wraps) are defined as a function of the rod motion and incorporated in the differential equation as generalized pseudo-forces. This approach allows using the modal analysis and preserves the physical understanding of rod frequencies and modes. The modal equations were written with the help of the Laplace transformation and the rod 
displacement was expanded using the unconstrained modes of vibration of the fuel rod (see Figure 2-27) as:

$$
\vec{D}(x, s)=L[\vec{d}(x, t)]=\sum_{r=1}^{\infty} \vec{\eta}_{m}(s) \cdot \psi_{m}(x)
$$

where $L$ is the Laplace transformation, $\psi_{m}(x)$ are the unconstrained modes and $\vec{\eta}_{m}(s)$ are complex functions that contain the time dependent part of the solution. The $\psi_{m}(x)$ are calculated from the differential eigenvalue problem:

$$
\frac{d^{4} \psi_{m}(x)}{d x^{4}}-\beta_{m} \cdot \psi_{m}(x)=0
$$

where $\beta_{m}=w_{m}{ }^{2} \rho / E I$ and $w_{m}$ is the mode frequency. The boundary conditions correspond to both pinned ends seen in Figure 2-26. Using these equations and applying the Laplace transform on the equation of rod motion, a set of Ordinary Differential Equations (ODE) was obtained and integrated using an ODE solver. The model determines the rod natural frequencies (both with and without the wire wraps), the rod motion and the support impact forces.

Figure 2-27 presents the first 10 unconstrained modes of vibration of the fuel rod (see the rod parameters in Table 2-13), where "unconstrained" means without the wire wrap. As seen in the figure, the frequency of the first mode of vibration is $\sim 11.79 \mathrm{~Hz}$, well below the cutoff frequency of the turbulence force $(\sim 100 \mathrm{~Hz})$. As shown in the next sections, the wire wrap allows increasing the first vibration mode frequency above 100 $\mathrm{Hz}$ and then avoiding the resonance with the turbulence force and the concerns of fluidelastic instabilities.
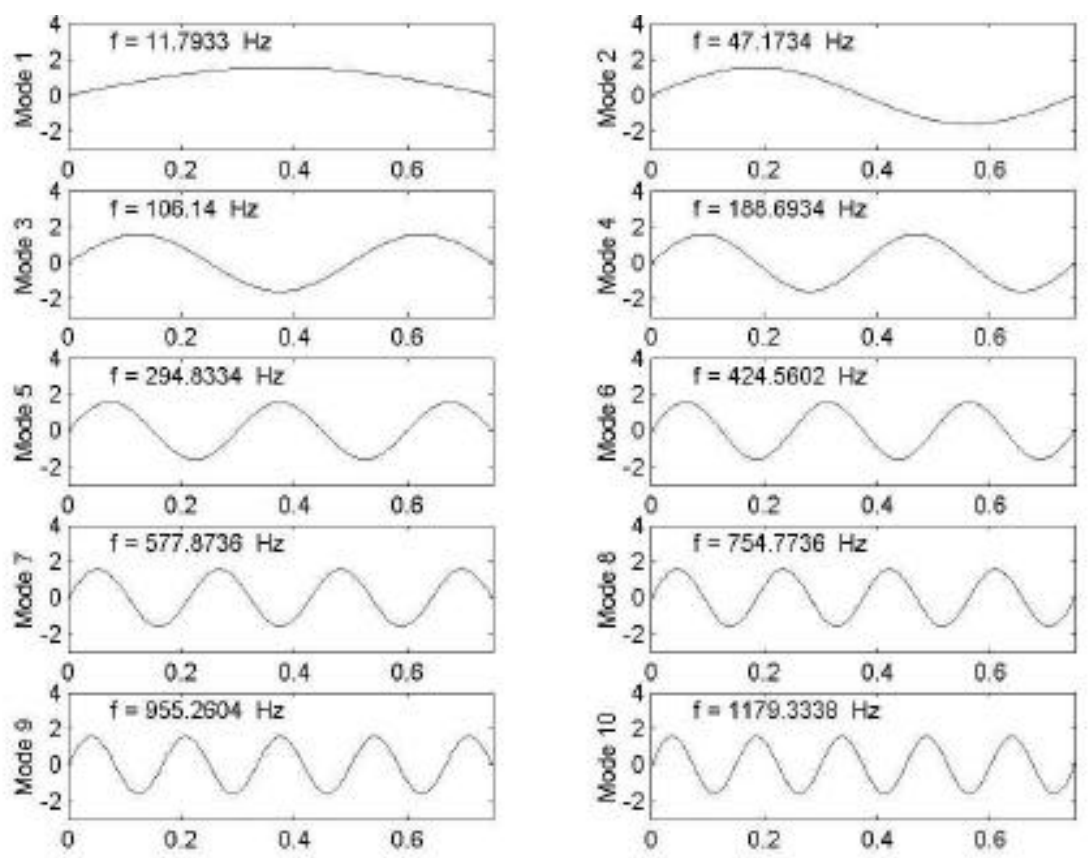

Figure 2-27. Unconstrained vibration modes of the LMR-AMTEC fuel rod. 


\section{Excitation force}

In general, the fluid force on the rod is expressed in terms of a power spectral density (PSD) of the turbulence-induced excitation forces and depends on the fluid speed. The fluid properties such as surface tension, liquid-vapor density ratio, and viscosity may also change the fluid force levels. The excitation force, generated by the fluid can be modeled with good accuracy as a random Gaussian force having a constant PSD between $0.0 \mathrm{~Hz}$ and an upper cutoff frequency of $100 \mathrm{~Hz}$. The force is then constructed by applying the inverse Fast Fourier Transformation on the force spectral components. Figure 2-28 shows an example of such an excitation force when $f_{e}=0.014143 \mathrm{~N}_{\mathrm{ms}}$ and the force frequency components are constant between 0 and $100 \mathrm{~Hz}$. Typical values of PSD forces measured for axial flows similar to that of the LMR-AMTEC were used in the simulations. An accurate correlation, however, between the fluid velocity and the PSD is required and can only be obtained from experimental data for the specific fuel design.
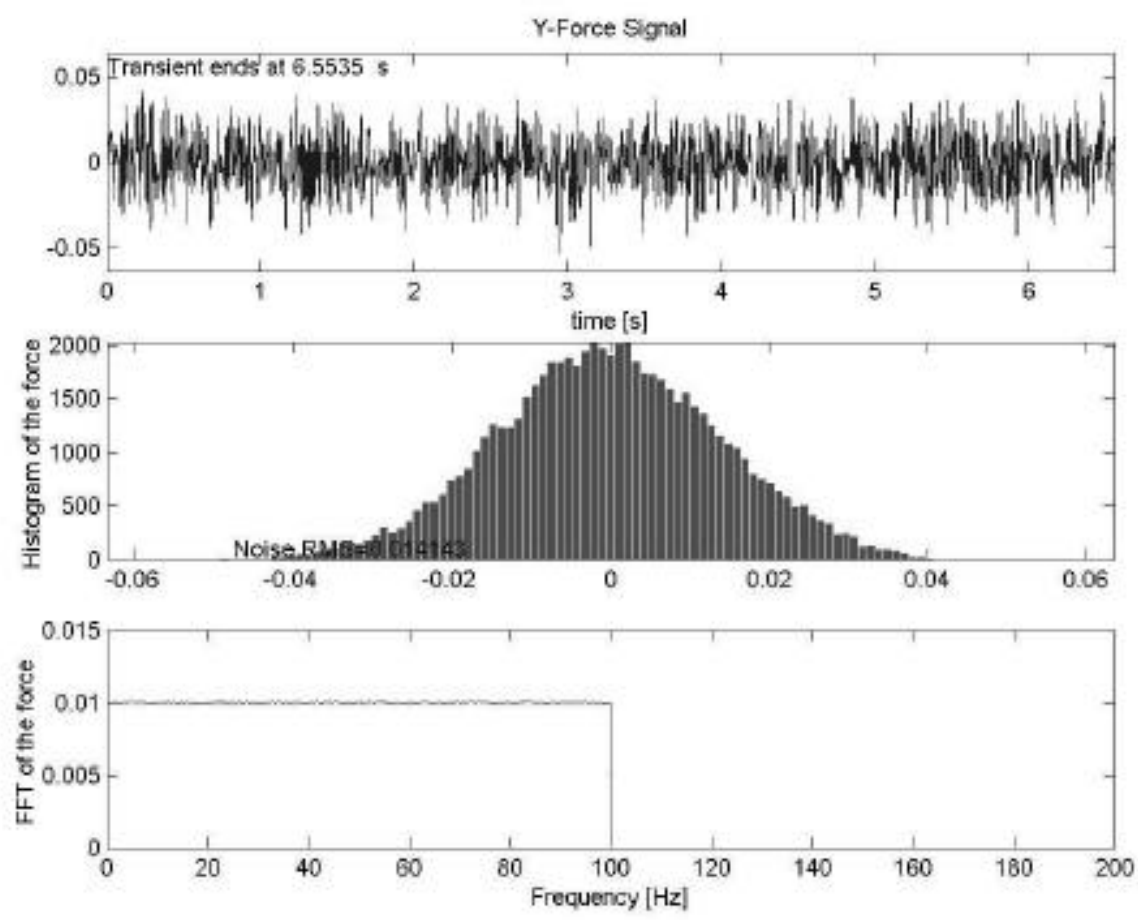

Figure 2-28. Excitation force on the Y-plane used to model the turbulence forces acting on the clad surface.

Frequency response of the fuel rod

In this analysis, the first step was to determine the rod response in the frequency domain applying the Fast Fourier Transformation on the rod displacement. Two cases were analyzed:

- No gap between the clad surface and the wire wrap.

- A 0.1-mm gap between the clad surface and the wire wrap. 
The value used for the gap is typical for a PWR fuel element. The simulations were performed for two different excitation forces, namely:

- Low excitation: $\quad 0.01414 \mathrm{~N}$ (rms)

- High excitation: $0.04472 \mathrm{~N}$ (rms)
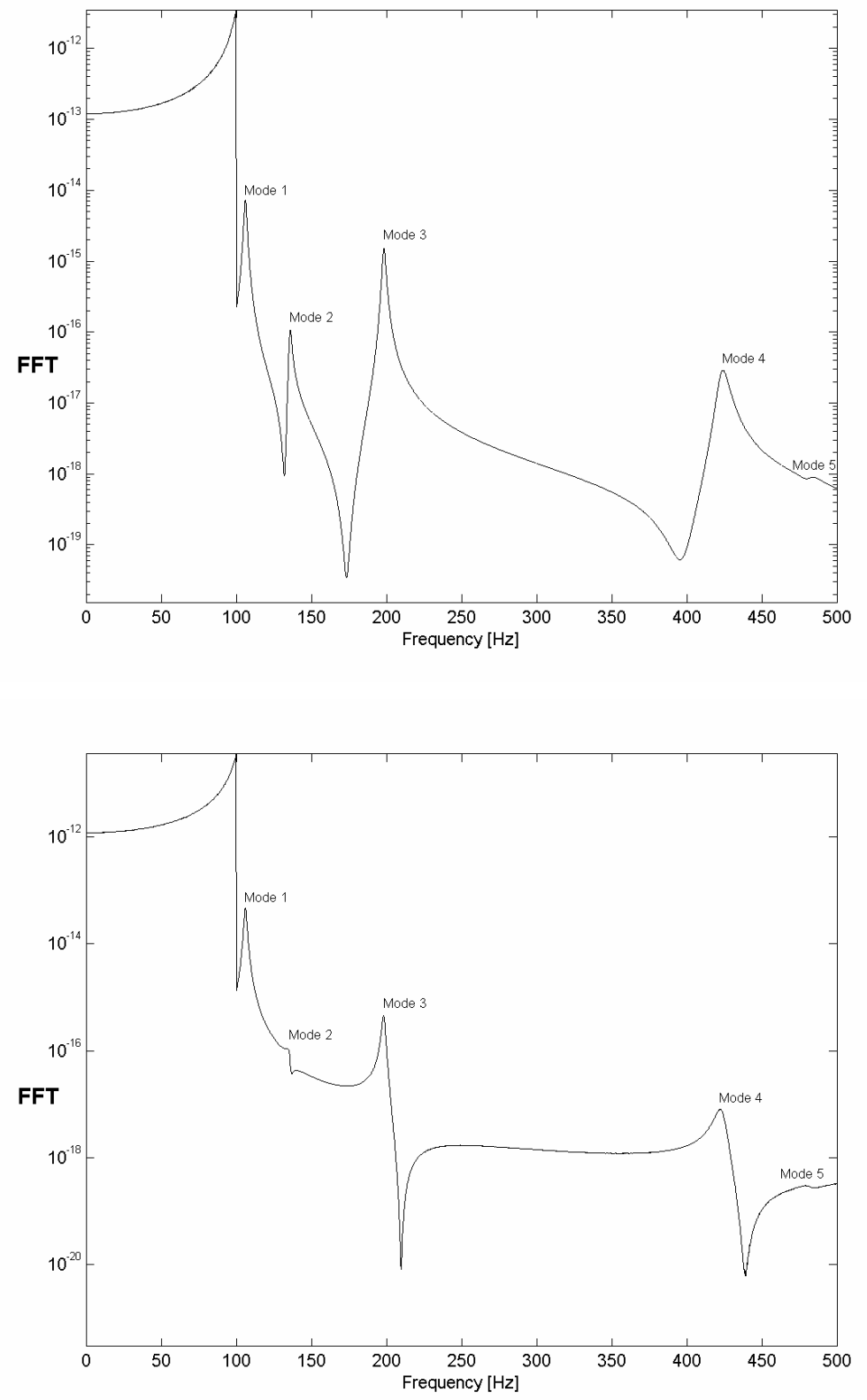

Figure 2-29. Rod frequency responses for zero gap case with low and high excitation forces. The first five constrained modes of vibration are identified.

Figure 2-29 presents the Fast Fourier Transformation (FFT) of the displacement of the fuel rod corresponding to the simulations assuming no gap with low and high force 
excitations. In these cases, the peaks correspond to the first 5 constrained modes of the fuel rod vibration. The first mode is seen to be at $\sim 105 \mathrm{~Hz}$. Hence, setting a period length of $25 \mathrm{~cm}$ for wire wrap is enough to increase the natural frequencies of the fuel rod above the upper cutoff frequency of the turbulent force. As mentioned before, with such a natural frequency, fluid elastic instabilities are not expected to be a concern in the design. Even for the high excitation force, the peak intensity does not change significantly. Note that the plateau below $100 \mathrm{~Hz}$ is caused by the PSD shape of the excitation force and the peak at $100 \mathrm{~Hz}$ is just the tail of the first mode.
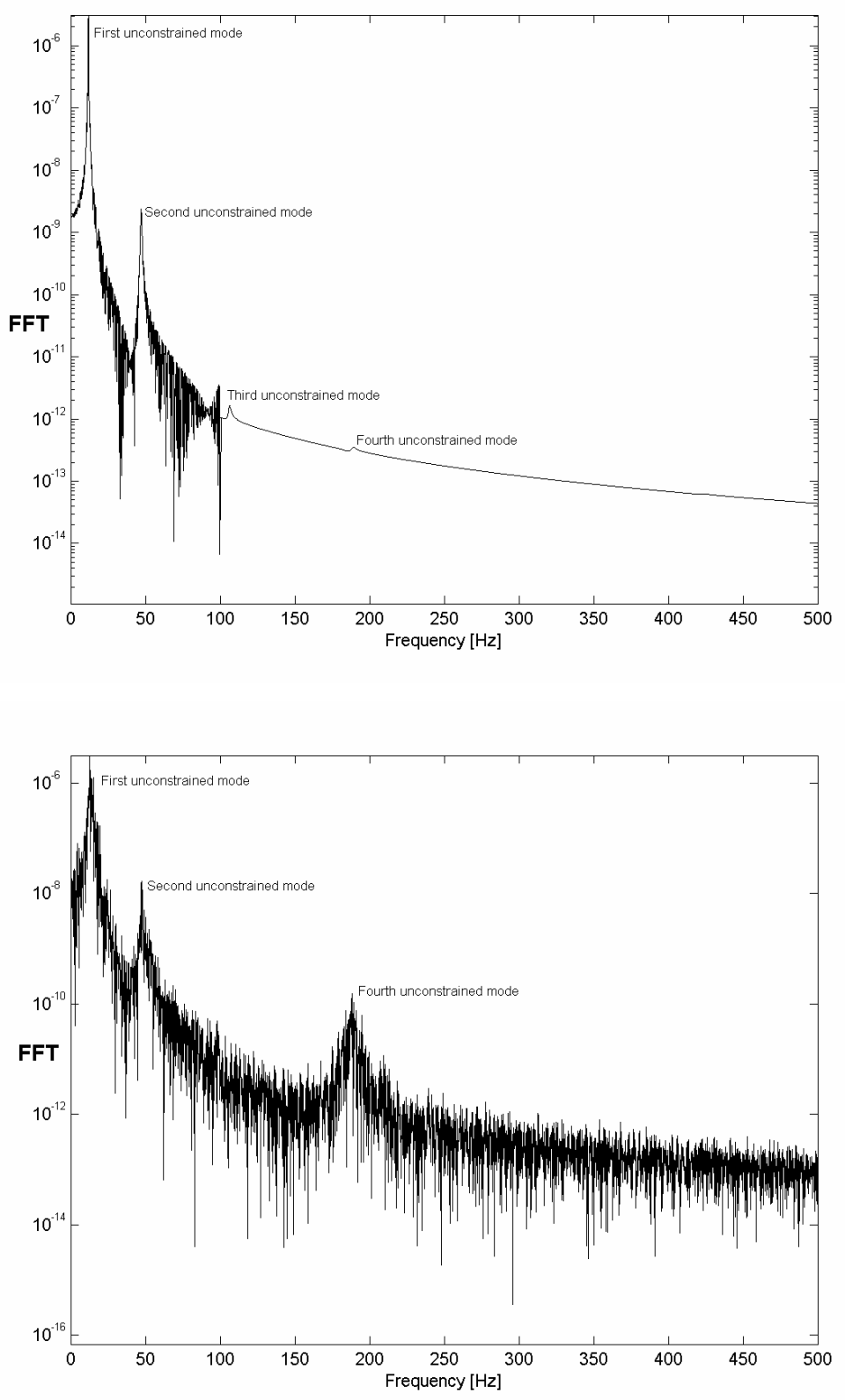

Figure 2-30. Rod frequency response for non-zero gap case and with low and high excitation forces. The first four unconstrained modes of vibration are identified. 
The results corresponding to the non-zero gap are presented in Figure 2-30. Due to the $0.1 \mathrm{~mm}$-gap, the fuel rod is now able to vibrate at the unconstrained modes. The first four modes shown in Figure 2-30 can be identified. It is important to note that for the same excitation force, the amplitude of the peaks is larger compared to the zero-gap case, particularly, for modes below $100 \mathrm{~Hz}$. In this case, those peaks can absorb larger amount of energy from the fluid, thereby increasing the available energy for fuel rod wear. Also note that when the excitation force is increased, the peaks become wider. In the next section the possible wear damage will be evaluated using the work rate concept.

\section{Work rate}

In order to evaluate the fretting wear damage, we consider the normal work-rate $\dot{W}_{n}$ defined as [Frick et al]:

$$
\dot{W}_{n}=\frac{1}{T} \cdot \int_{0}^{T} f_{s}^{n o r m a l} \cdot \frac{d S}{d t} \cdot d t=\frac{1}{T} \cdot \int_{0}^{T} f_{s}^{n o r m a l} \cdot v_{t} \cdot d t
$$

Here, $T$ is the transient duration, $d S$ is the sliding distance, $f_{s}^{\text {normal }}$ is the support contact force, and $v_{t}$ is the tangential speed.

From $\dot{W}_{n}$, the fretting-wear damage volume rate of the cladding, $\dot{V}$, can be calculated as:

$$
\dot{V}=K_{W} \cdot \dot{W}
$$

where $K_{W}$ is a wear coefficient obtained experimentally. Since wear coefficients are not readily available, and determination of them is beyond the scope of this work, the normal work rate is used to estimate the relative change in the wear damage with respect to the excitation force. It is, however, important to note that for a realistic analysis, one has to determine a PSD correlation and the wear coefficient, which is suitable for the LMRAMTEC fuel element and conditions existent in a sodium-cooled reactor.

The results obtained from simulations are presented in Table 2-14. We can draw the following conclusions:

1. The work rate of the non-zero gap case is $\sim 400$ times larger than that of the zerogap case. This result clearly shows the importance of the gap in the vibration model.

2. The normal work rate that is calculated for the LMR-AMTEC fuel rod is relatively larger than for a PWR fuel rod using the same excitation force and gap conditions. This is due to the differences on the values of linear density and rod stiffness. Furthermore, the wear coefficient is expected to be larger due to the extreme conditions existing in the LMR-AMTEC core; hence, fretting-wear might be an important concern in the fuel design. 
3. For low excitation force a zero work rate is calculated for the non-zero gap case. This means that when vibrating, the rod is not touching the neighbor rods. The opposite occurs when the excitation force is increased as seen in Figure 2-31, where the motion of the center of the rod during the simulation is plotted. The orbits of the fuel rod clearly show the presence of the supports (wire wraps) and the gap.

Table 2-14. Normal work rates and middle span displacement obtaining for the zero and non-zero gap cases.

\begin{tabular}{|c|c|c|c|c|}
\hline & \multicolumn{2}{|c|}{ Zero Gap } & \multicolumn{2}{c|}{ Gap equal 0.1 mm } \\
\hline $\begin{array}{c}\text { Excitation } \\
\text { force } \\
{[\mathrm{N}(\mathrm{rms})]}\end{array}$ & $\begin{array}{c}\text { Middle span } \\
\text { displacement } \\
{[\mu \mathrm{m}(\mathrm{rms})]}\end{array}$ & $\begin{array}{c}\text { Normal work rate } \\
\text { per support } \\
{[\mathrm{mW}]}\end{array}$ & $\begin{array}{c}\text { Middle span } \\
\text { displacement } \\
{[\mu \mathrm{m}(\mathrm{rms})]}\end{array}$ & $\begin{array}{c}\text { Normal work } \\
\text { rate per support } \\
{[\mathrm{mW}]}\end{array}$ \\
\hline 0.01414 & 0.1189 & $1.0810^{-6}$ & 22.21 & 0.0 \\
\hline 0.04472 & 0.3761 & $1.0510^{-5}$ & 38.24 & 0.00409 \\
\hline 0.14142 & 1.197 & $1.0810^{-4}$ & 43.81 & 0.0421 \\
\hline
\end{tabular}

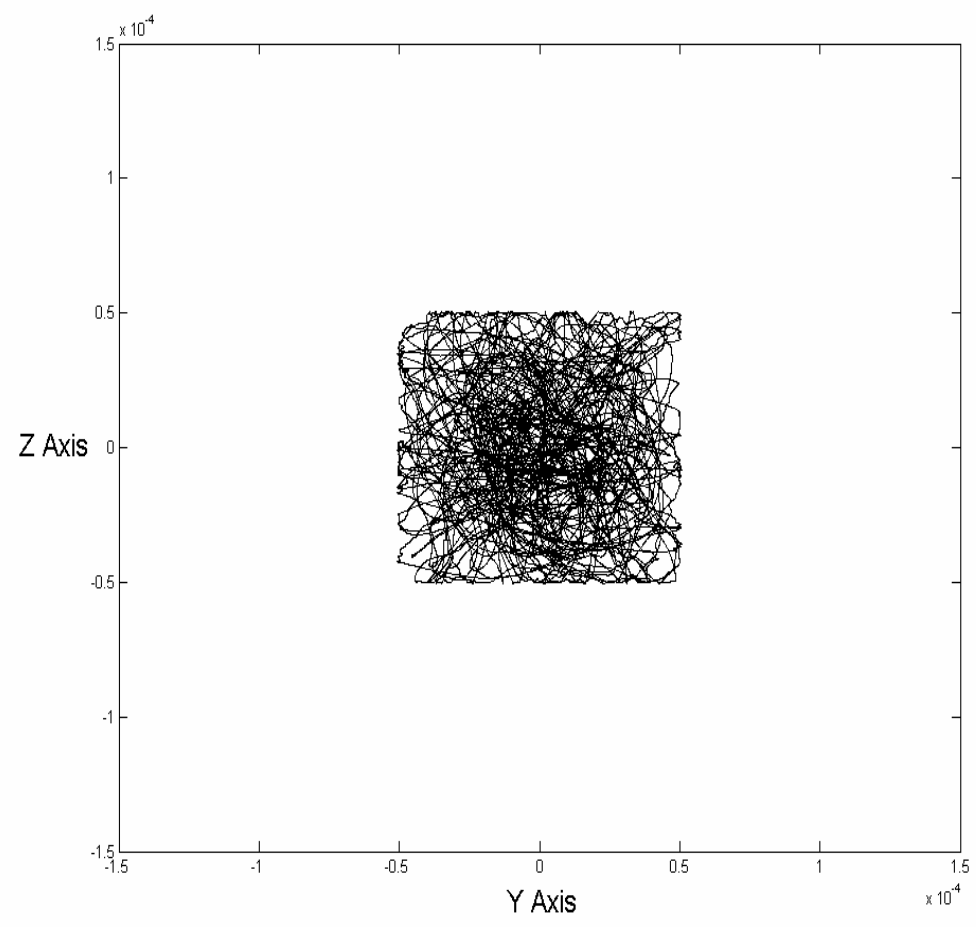

Figure 2-31. Motion of the center of the fuel rod at the axial position corresponding to the wire wrap. The rod orbits are confined inside the gap of $0.1 \mathrm{~mm}$. 


\section{Thermo-elastic instabilities}

This phenomenon is characterized by low-frequency oscillation of the fuel pins within the support clearance (grid or wire wrap) initiated by lateral movements of the pin. Therefore it is not caused by fluid induced vibration as the precedent three mechanisms. Due to the clearances between pins and the support and the free axial span, the pins have the freedom to move and to bow. This leads to a coolant flow redistribution around the circumference of the pins and subsequently to a new coolant temperature gradient field developing along the pins. These temperature gradients induce new pin bowing and new deflections, which produce a new temperature field again. Depending on the conditions concerning geometry, power and flow, this process can lead to thermo-elastic instabilities, permanent oscillations, and subsequent wear on the fuel cladding. Wear on fuel pins and spacer has been observed in the course of post irradiation examinations of the fuel pins of the KNK II/2 sodium cooled fast reactor (Germany). The clad thickness was reduced at some locations up to $50 \%$. The axial distribution of wear intensity decreased from the center of the pin bundle towards the peripheral pins. The axial distribution of wear intensity, common for all the pins, started in the heated fuel zone, increased steadily to its maximum at the upper part of the heated zone and decreased towards the top of the pin. The amount of wear was incompatible with wear caused by flow induced vibration. Experimental and theoretical studies showed that the damage was caused by thermo-elastic vibrations. This phenomenon may be of concern for the LMRAMTEC fuel rods. However, it may be controlled by proper design of the fuel rod and reducing the axial temperature gradients.

\section{Summary of the flow-induced vibration excitation mechanisms}

Although, the present studies showed a good vibration behavior of the fuel rod, more detailed studies are required. An accurate determination of the maximum coolant velocity and confirmation of fuel design reliability will require further studies, which should include evaluation of the wear coefficient for the LMR-AMTEC operating conditions, obtaining a PSD correlation for the fuel design, and evaluating the possibility of thermal vibration. Furthermore, during the cycle, the flexure rigidity of fuel rod will be affected by thermal expansion of the fuel pellets, fuel pellet cracking, densification due to intense neutron radiation, creep of the enveloping fuel sheath, and swelling due to fission gas production. All these factors will lead to considerable differences in vibration response over time. The studies should then addresses the dynamic behavior of nuclear fuel by means of a statistical treatment. 


\subsubsection{Alkali Metal Boilers (AMB)}

\subsubsection{Mechanical Design Requirements}

The alkali metal boilers are one of the key components of the LMR-AMTEC reactor. Based on past experience, the design requirements on liquid metal can be summarized as: [Fraas89]:

- The tubes should be grouped into bundles, each having a header drum and outlet pipe at either end designed to fit within the pressure ve ssel. This facilitates assembly and inspection and minimizes the number of vessel penetrations.

- Normal differential thermal expansion between the tubes and the shell should be accommodated elastically.

- Ligaments between tubes in the tube sheet should be thick enough for good welding.

- When bent tubes are employed to open up the tube spacing in the header sheet, the length of the moment arm between the bend and tube-end should be limited to avoid excessive bending stresses from the fluid drag forces on the tubes.

- The tube sheets should be curved in order to hold pressure stresses of reasonable values.

- Adequate flow-passage area into and out of the tube matrix should be provided for the shell-side fluid.

- Adequate flow-passage area should be provided inside the heater drums.

- The shell walls should be curved to hold pressure stresses to reasonable values.

- The shell walls should not be too thick if the thermal stresses are to be kept reasonably low during temperature transients.

- Thermal sleeves should be provided where the tube-side fluid pipes penetrate the heat exchanger shell.

- Flow bypass between the tube matrix and the shell should be less than $10 \%$, and preferably less than $5 \%$.

- Adequate provision for spacing the tubes should be made to assure good velocity and temperature distributions.

- Steep temperature gradients inside the shell should be avoided, for example, at the parting plane between the cold and the hot legs in a U-type heat exchanger.

- Under steady state and transient conditions, differential thermal expansions between individual tubes, between tubes and shell, and between any connected parts of the boiler must be accommodated within allowable stresses or loads.

\section{Selection of the tube configuration}

Of the above list, requirements for the thermal expansion, adequate ligaments between the tubes in the tube sheet, and the loads imposed by fluid drag forces are of a more 
challenging nature. The following tube configurations have been proposed in the past to address these requirements:

- Hockey stick

- U tube

- Circular arc or helix

- Z tube

- Units with flexible members

- Concentric tube

Among these configurations, two types appear to be more adequate for the alkali metal boilers: the $\mathrm{C}$ shape tube and the concentric tube. The $\mathrm{C}$ tube is a derivation of the $\mathrm{Z}$ tube configuration (Figure 2-32). The shape is designed to absorb the differential expansion by the short lateral sections of the tube at the header positions. One disadvantage of this design is that at least one of the tube sheets can not be placed outside the sodium coolant and therefore is subjected to significant thermal stress. In addition the headers will reduce effective annulus volume between the riser and the vessel.

The concentric tube, sketched in Figure 2-32, is generally used for mercury boilers. The fluid enters through the central tube and exits through the annulus between the central tube and the outer thimble. This type of configuration reduces the thermal and drag stresses to a minimum. Another advantage of this configuration is that the tube sheet can be placed outside the sodium in the cover gas region of the reactor vessel. The disadvantages, however, are the large spacing between tubes in both the header sheet and the tube matrix and the lower power density compared to the $\mathrm{C}$ shape tube.

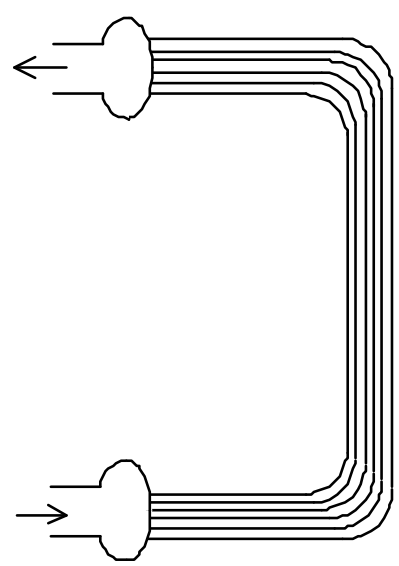

C tube

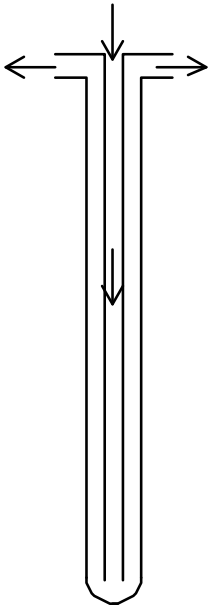

Concentric tube

Figure 2-32. Tube configuration for the alkali metal boilers.

Both configurations are retained at this stage of the conceptual design and further studies are required to select the appropriate one. The evaluation of the plant performance was mainly performed using the $\mathrm{C}$ tube configuration. 


\section{Sizing of the tube thickness}

In both tube configurations, the primary coolant flows through the shell side while the secondary flows through the tube side. Since the primary pressure is higher than that of the secondary, the tubes are under external pressure. The value of the tube thickness depends the reactor pressure and the corrosion allowance. Due to high working temperatures, the allowable stress for a given percent of creep was used as a criterion in determining the tube thickness. The wall thickness necessary to accommodate the differential pressure was determined setting the allowable stress for the niobium alloy to 60 percent of the stress to cause 1 percent creep in 100000 hours. The final wall thickness was obtained adding a corrosion allowance equal to $0.5 \mathrm{~mm}$ to this value. Since the allowable stress of the tubes strongly depends on the working temperature, the tube thickness depends on the core outlet temperature as seen in Figure 2-33. The effect of the wall thickness on the thermal performance of the AMB is only significant for wall thickness over $3 \mathrm{~mm}$ (or above $1100 \mathrm{~K}$ ). It is noted that the resulting wall thickness is thicker than that of tubes under internal pressure (i.e. when the primary coolant flows through the tube side). However, the present configuration has better mechanical performance during thermal transients and also reduces the probability of crack growing through the tube wall. Finally, regarding the two tube configurations previously mentioned, it is noted that the concentric tube will have a larger thickness, as the result of its larger outer tube diameter (thimble) than for the one of the $\mathrm{C}$ shape tube.

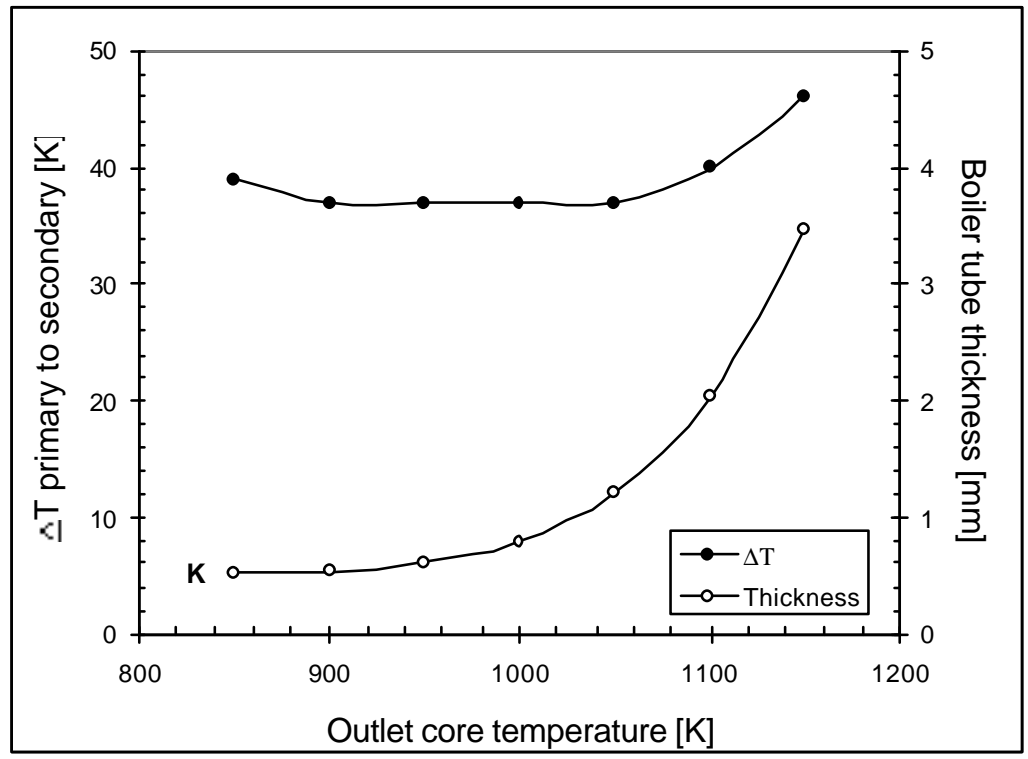

Figure 2-33. Effect of the outlet core temperature on the primary to secondary temperature drop and the tube thickness.

\subsubsection{Other Design Requirements}

Besides the mechanical requirements for the design of the AMB, the design must satisfy other requirements related to: 
- Stabilization of the boiling process,

- Pressures losses in the two phase region,

- Corrosion behavior.

\section{Stabilization of the boiling process}

Design and operating experience has shown in the past [Fraas82] that the only serious problem with alkali-metal-boilers is the difficulty to produce the nucleation of the vapor bubbles. The reason is that alkali metals wet the tube surface very tenaciously making it difficult to initiate the bubbles required for nucleation boiling. For this reason, the liquid metal can be superheated by hundreds of degrees before the bubbles become to form. When this occurs, the energy of the liquid metal is released to the vapor very rapidly producing an explosive boiling.

Different solutions have been proposed to solve this problem. MacPherson obtained stable boiling of alkali metals in pools by using a mechanism composed of two different devices [MacPherson67]. First an independently controlled device called "hot finger" to initiate the boiling and, second, a parasitic device, which is actuated by the boiler heat flux to stabilize boiling after it has been initiated. The "hot finger" was designed to provide a small, heated volume of liquid metal that can be taken to temperature levels sufficiently high to cause generation of bulk vapor in its low volume. After a vapor pocket is established, a stable liquid-vapor interface is available at which vaporization can take place. Since boiling has been initiated, the "hot finger" can be deenergized and the parasitic device (for example small crevices in the wall) maintains stable vapor pockets permitting stable operation. Other experiments were also performed in order to study the use of ultrasonic energy for stimulating nucleating sites in a saturated alkali metal [Yarosh67]. These studies showed that the localized vapor voids, generated by the high frequency minute vibrations, might serve as effective nucleating sites for vapor generation. However, some questions remained regarding the efficiency of this method and also the cavitation damage produced by the ultrasonic energy on the boiler components.

An important drawback of the techniques described above is the use of an active component to stabilize the boiling process. This active component adds a high degree of complexity both to the construction and the operation of the AMB. Fraas has proposed a simpler mechanism to replace the "hot finger" for initiating the boiling process [Fraas82]. This mechanism consists of using an inert gas dissolved in the alkali metal to provide small bubbles where vaporization can take place. A stable operation is achieved by introducing nucleating sites in the form of crevices to provide local hot spots and ensuring that a fraction of the inert gas present in the alkali metal is trapped by the crevice. A sketch of such device for the tubes of the AMBs is shown in Figure 2-34. If the concentric tube shape is adopted, the boiling ring should be placed in the annulus between the inner tube and the thimble. 


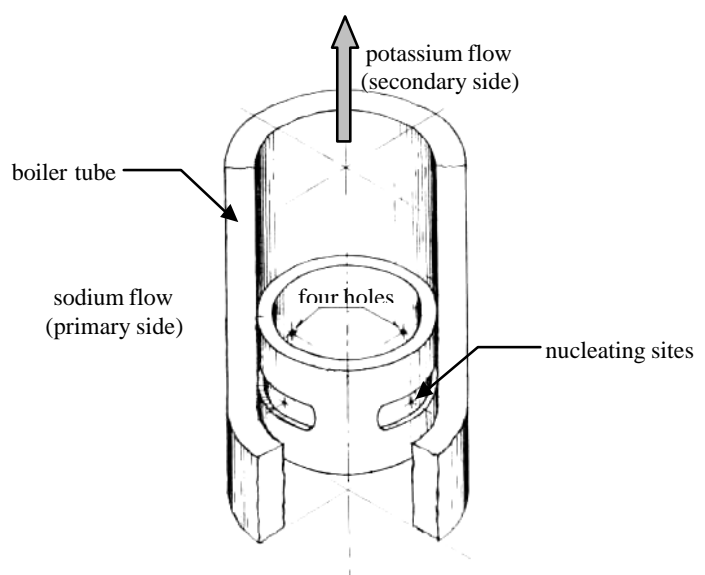

Figure 2-34. Boiling ring designed to maintain stable vapor pockets.

\section{Pressures losses in the two phase region}

As seen in Table 2-15, the saturation pressure of alkali metals is very low. A significant drop of the secondary saturation temperature can occur in the AMB, when there is significant two-phase pressure drop in the tubes. Consequently in designing the AMB, the pressure drop in the two-phase region must be kept as low as possible to avoid a significant reduction of the liquid-vapor mixture pressure and temperature.

Table 2-15. Saturation pressures of sodium and potassium

\begin{tabular}{|c|c|c|c|c|}
\hline \multirow{2}{*}{ Temperature } & \multicolumn{2}{|c|}{ Sodium } & \multicolumn{2}{c|}{ Potassium } \\
\cline { 2 - 5 } & {$[\mathrm{Pa}]$} & {$[\mathrm{atm}]$} & {$[\mathrm{Pa}]$} & {$[\mathrm{atm}]$} \\
\hline $800 \mathrm{~K}$ & 889.831 & 0.08782 & $6.14310^{3}$ & 0.061 \\
\hline $850 \mathrm{~K}$ & $2.21610^{3}$ & 0.022 & $1.28810^{4}$ & 0.127 \\
\hline $900 \mathrm{~K}$ & $4.97710^{3}$ & 0.049 & $2.48310^{4}$ & 0.245 \\
\hline $950 \mathrm{~K}$ & $1.02510^{4}$ & 0.101 & $4.45610^{4}$ & 0.44 \\
\hline $1000 \mathrm{~K}$ & $1.96210^{4}$ & 0.194 & $7.5310^{4}$ & 0.743 \\
\hline $1050 \mathrm{~K}$ & $3.52510^{4}$ & 0.348 & $1.20810^{5}$ & 1.193 \\
\hline $1100 \mathrm{~K}$ & $5.99910^{4}$ & 0.592 & $1.85510^{5}$ & 1.83 \\
\hline
\end{tabular}

The temperature drop depends on the ratio of the two-phase pressure drop to the saturation pressure, $\Delta P_{2 \phi} / P_{\text {sat }}$. The two-phase potassium friction factor depends mainly on the quality, the total flow rate and the absolute pressure, and is lower than that of water systems [Lowell60]. The saturation pressure and temperature of the AMB depends on the core working temperatures. Since the secondary flow rate ( $\propto$ friction losses) depends mainly on the core thermal power, the $\Delta P_{20} / P_{\text {sat }}$ increases as the core outlet temperature decreases. Figure 2-35 presents, for example, the ratio $\Delta P_{20} / P_{s a t}(\propto$ to the temperature drop) for an AMB with 1514 tubes of $3 \mathrm{~cm}$ OD and $2.5 \mathrm{~m}$ of length. As observed, the ratio becomes too large when the outlet core temperature is below $1050 \mathrm{~K}$. 


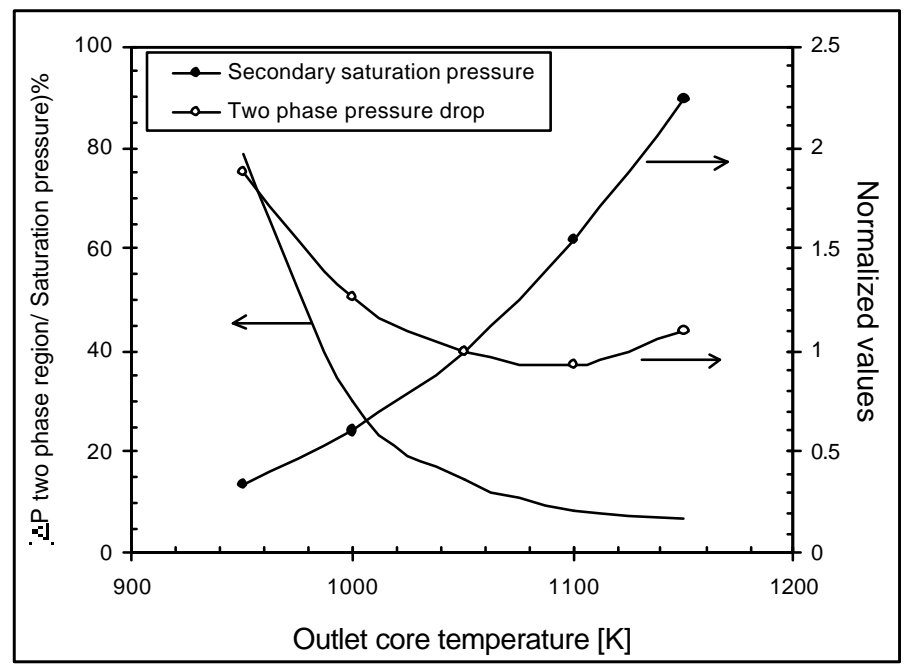

Figure 2-35. Effect of the outlet core temperature on the two phases pressure losses and the secondary saturation temperature.

The results of Figure 2-35 show that the AMB design will strongly depend on the outlet core temperature in order to avoid significant temperature losses in the two-phase region. In the reference LMR-AMTEC design, the two-phase losses of pressure were kept under $10 \%$. Thus the temperature drop in the two phases region is below $5 \mathrm{~K}$.

\section{Corrosion behavior}

Corrosion and mass transfer effects of liquid sodium or potassium must be accounted for designing the components of the boilers because the reliability of any the alkali metal system is strongly related to the corrosion behavior. In addition, the corrosion behavior of a given component depends also on the performance of other components of the loop due to the mass transport phenomena. The corrosion process of liquid alkali metals is mainly of two types:

- Simple solution leading to general surface removal, grain boundary attack, or preferential leaching of allowing constituents

- Chemical attack involving impurities in the liquid metal and in the container wall.

The cold traps, or example, are designed to control the oxygen level and other impurities in the coolant (like nitrogen and carbon) and thus reducing the second type of corrosion (In addition, a high oxygen level is quite harmful to the $\mathrm{Nb}-1 \mathrm{Zr}$ alloy). A consequence of either of these corrosion processes is that the elements of the container metal may undergo continual transport from one part of a dynamic system to another. Two factors contributing to this mass transfer are the solubility differences resulting from temperature gradients in the system and the chemical activity differences resulting from dissimilar metals in the system. Since equilibrium solubility in liquid metals nearly always change with temperature, temperature-gradient mass transfer is an inevitable effect in any hightemperature, liquid-metal system. Dissimilar-metal mass transfer, on the other hand, can be minimized through the selection of system materials that are not prone to interalloying. 
The corrosion behavior in the primary and the secondary side of the AMB are quite different as the first corresponds to a non-boiling system and the second to a boiling system:

- Primary side: A continuous, interconnected liquid flow throughout the heat transfer circuit exists. Consequently the gain or loss of solute at any point in the system is sensed to some degree by all other points in the circuit. The addition or removal of heat from the system, since it affects the liquid metal temperature, has a direct influence on the equilibrium solute concentration and corrosion rate values.

- Secondary side: First, solute does not flow continuously around the circuit, since unlike the liquid metal, the vapor-filled regions of the boiler present a high-resistance path to solute mass transfer. Hence, concentration changes in the boiler are not directly transmitted downstream from the boiler. Secondly, heat added to an alkali metal at its boiling point produces no discernible change in the liquid temperature and, therefore does not alter either the equilibrium concentration of the solute or the solution rate in this region. In fact, heating the boiler liquid fractionates it into pure vapor and impure liquid, concentrating the impurities and causing super-saturation rather than under-saturation. The major temperature drop in the boiling system occurs in the liquid phase region (in the vapor phase region there is a little attendant solute deposition). However, the temperature drop in the liquid region can be reduced by operating the AMB in a recirculating mode. Thirdly, because the change of state reactions have high latent heats, the mass flow rate required to transport energy at a given rate tends to be considerably smaller in a boiling system than in a forced convection circuit. Thus the liquid velocities are lower in boiling systems than in forced convection systems.

In the present design, $\mathrm{Nb}-1 \mathrm{Zr}$ was adopted as structural material for the AMB. Due to their compact size, it is expected that reliability gain will compensate the slight increase of the capital cost. In addition, this will reduce the corrosion concerns of the primary system as only $\mathrm{Nb}-1 \mathrm{Zr}$ will be used. In calculating the thickness of the tubes, a corrosion thickness allowance has to be added to the one determined from mechanical and thermal loading. In the present design, a conservative corrosion thickness allowance of $0.5 \mathrm{~mm}$ has been adopted. Further studies would probably refine and reduce this thickness. Finally, it is noted that the dissolution in the condenser and condensate-lines will be an important corrosion issue that can affect the performance of the boilers. Because of the distillation effects of the evaporation process (either by boiling or flash evaporation), the condensing vapor remains essentially free of solute constituents. Therefore the dissolution can continue undiminished in the condenser region for as long as the system operates. The rate of dissolution will depend on the rate of vapor condensing and the temperature [Distefano70].

\subsubsection{Recirculating or Once-through Operation}

The corrosion behavior of the secondary system will depend on the operation mode of the AMBs. In the recirculating mode, the vapor fraction is extracted at the vapor separator and the liquid fraction is mixed with the condensate return, and sent back to the inlet of the AMB. The vapor separator also traps any solute present as dust in the vapor 
leaving the boiler. It is interesting to note the experiments performed at ORNL showing that boiling potassium in a stainless-steel system with a peak temperature of about 1125 $\mathrm{K}$ caused essentially no difficulty with corrosion of mass transfer. It was also found that in a recirculating potassium boiler, the liquid in the boiler rapidly becomes saturated with iron, chromium and nickel. In addition, since only vapor moves out of the boiler, the dissolved elements were retained in the re-circulating boiler circuit. Further, the solution rates of iron, chromium and nickel in the cold zone were found to be low so no appreciable mass deposition in the boiler of corrosion products coming from the condenser was detected. Based on this experience, [DeVan76] recommended the operation of the potassium AMB delivering a low quality (10-20\%) vapor-liquid mixture to a vapor separator.

In a once-through boiler, the coolant is completely evaporated and therefore vapor separator and recirculating lines are not needed. However, the coolant enters highly subcooled and a significant temperature variation exists in the liquid region. Therefore a significant undersaturation and relative high corrosion rates (with respect to the recirculating mode) will exist until a significant vapor generation has occurred. In addition, in once-through boilers, evaporation to dryness would release an important part of the dissolved solids as dust suspended in the vapor, requiring a dust trap. However, the corrosion problem of the once-through boilers can be minimized by the use of the $\mathrm{Nb}-1 \mathrm{Zr}$ alloy.

A comparison of the temperature profiles in the AMB for operating regimes is shown in Figure 2-36 and the main parameters are listed in the Table 2-16. As can be see, the AMTEC efficiency is about the same in both cases. However, the secondary inlet temperature in the recirculation mode is $250 \mathrm{~K}$ higher than the one of once-through. Then the coolant enters less subcooled and has a smaller temperature variation in the liquid region. The corrosion performance of the recirculation $\mathrm{AMB}$ is thus expected to be superior to the once-through AMB. On the other hand, the minimum quality of the vapor in the recirculation mode is limited by the increment of the two phase pressure drop. For this reason it was set to about 30 to $40 \%$.

Table 2-16. Secondary parameters for the AMB operating in recirculation and once-through mode (the primary operating parameters are the same).

\begin{tabular}{|l|c|c|}
\hline & \multicolumn{2}{|c|}{ AMB operation mode } \\
\hline Secondary parameter & Recirculation & Once-Through \\
\hline Outlet temperature & $983.8 \mathrm{~K}$ & $992.6 \mathrm{~K}$ \\
\hline Inlet temperature & $780.4 \mathrm{~K}$ & $525.0 \mathrm{~K}$ \\
\hline Saturation temperature & $983.8 \mathrm{~K}$ & $981.5 \mathrm{~K}$ \\
\hline Mass flow rate & $95.98 \mathrm{~kg} / \mathrm{s}$ & $42.29 \mathrm{~kg} / \mathrm{s}$ \\
\hline Vapor quality & $45.2 \%$ & $100 \%$ \\
\hline Liquid length & $0.202 \mathrm{~m}$ & $0.096 \mathrm{~m}$ \\
\hline Two phase length & $1.298 \mathrm{~m}$ & $1.103 \mathrm{~m}$ \\
\hline Vapor length & $0.000 \mathrm{~m}$ & $0.302 \mathrm{~m}$ \\
\hline AMTEC efficiency & $34.7 \%$ & $34.6 \%$ \\
\hline
\end{tabular}


a) Once-through mode

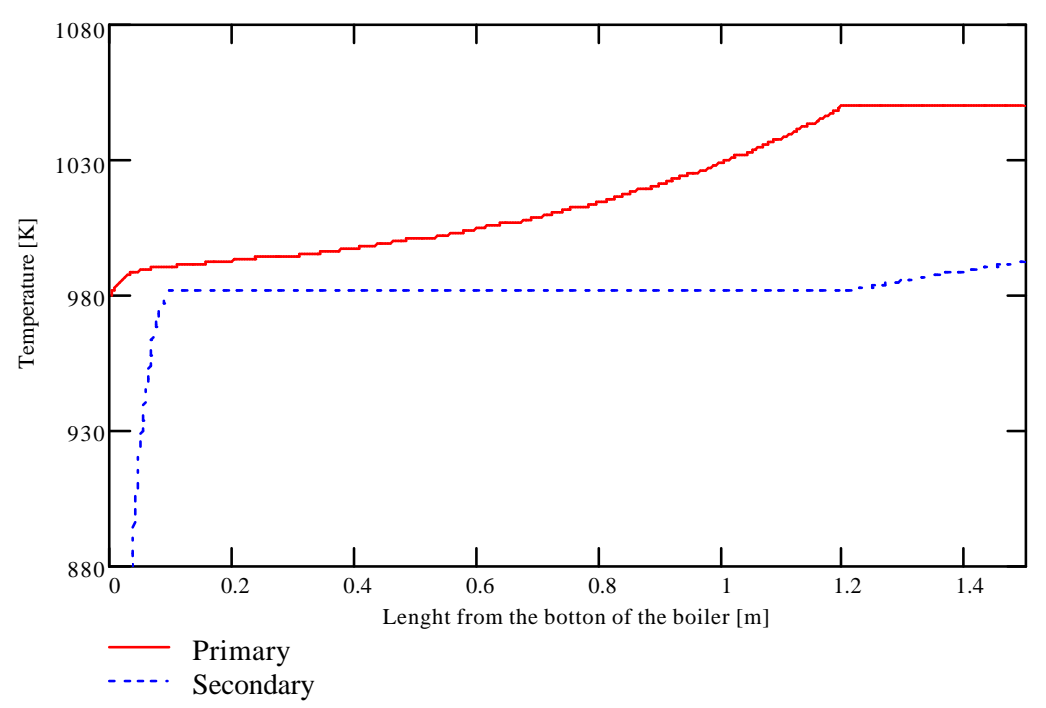

b) Recirculating mode

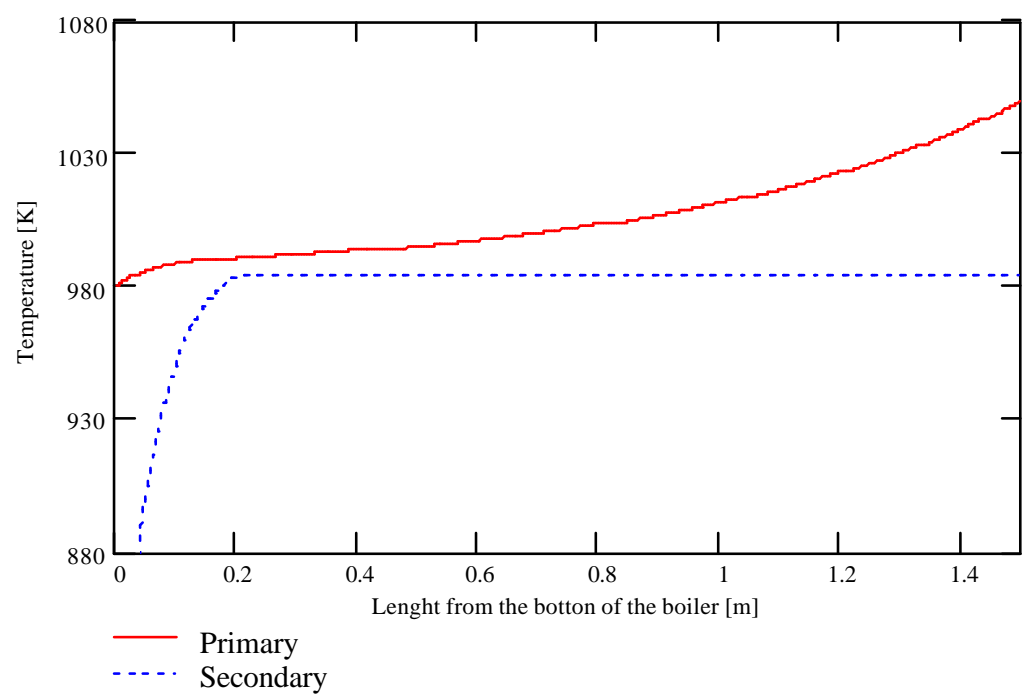

Figure 2-36. Temperature profiles for the secondary and the primary side of the once-through alkali metal boilers.

\subsubsection{Vibration Behavior}

The boilers must to be designed to withstand damage or malfunction either by internally generated vibrations, such as flow-induced vibration, or by fluid-borne or structural vibration, such as those produced by the pumps (placed on the top of the AMB). Spacing 
plates (or grid) for the tube bundle at prescribed distances that do not interfere with axial movement of the tubes is needed to increase the natural frequencies of the tubes avoiding resonance with the fluid forces. In addition, as for the fuel rods, the vibration concerns will impose a coolant velocity limit due to the fretting wear damage. Both critical speed and natural modes of vibration are analyzed in the following sections based on the relevant flow-induced vibration excitation mechanisms:

\section{1) Fluidelastic instabilities}

In the primary side of the boilers, cross flow will exist at the inlet and the outlet of the boiler, for both tube configurations. Fluidelastic instability may be a problem in those regions. An estimation of the critical speed was performed using the correlation presented in section 2.2.4. The instability constant, $K$, was assumed to be 0.5 as recommended for all tube bundle configurations in single-phase cross flow. Note that when the exponent $n$ is assumed to be 0.5 , the critical speed does not depend on the tube diameter.

Figure 2-37 presents the critical flow speed $U_{p c}$ calculated using this correlation and the tube parameters listed in Table 2-17. As shown later in this section, the first natural frequency of the tubes is above $150 \mathrm{~Hz}$. Furthermore, the damping value of the tube is greater than $1 \%$, hence the critical speed is expected to be higher than $3 \mathrm{~m} / \mathrm{s}$. In all cases, the cross-flow speed will be between 1 to $2 \mathrm{~m} / \mathrm{s}$. This value is at least $50 \%$ smaller than the critical speed. In addition, in estimating the vibration response of the tubes, conservative parameters were used as different designs were studied: For example the tube length was assumed to be $1.5 \mathrm{~m}$ to obtain the lower bound of the first natural frequency. Because of these reasons, we do not expect the fluid elastic instabilities to cause a problem in the boiler.

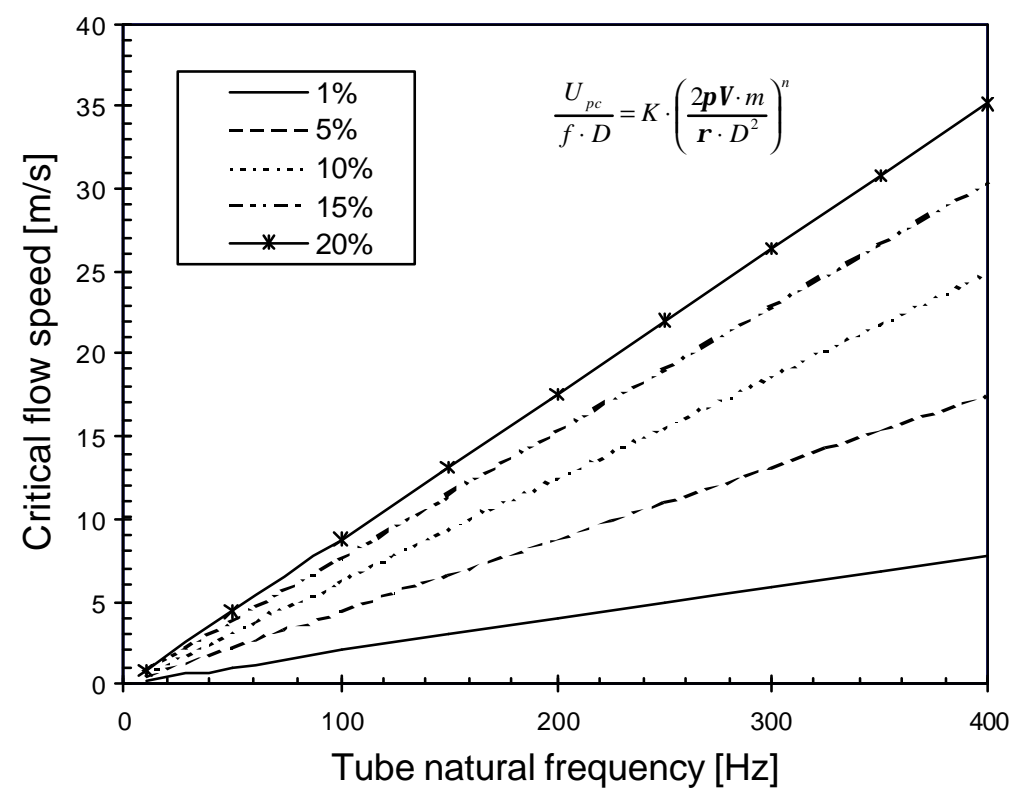

Figure 2-37. Critical flow speed versus the fuel natural frequency and for different structural damping values. 
Table 2-17. AMB tube parameters used in evaluating the vibration response.

\begin{tabular}{|l|c|}
\hline Tube length & $150 \mathrm{~cm}$ \\
\hline Tube external diameter & $2.0 \mathrm{~cm}$ \\
\hline Tube thickness & $1.0 \mathrm{~mm}$ \\
\hline Spacing plates period & $50 \mathrm{~cm}$ \\
\hline Structural damping & $>1 \%$ \\
\hline Tube average temperature & $\sim 1000 \mathrm{~K}$ \\
\hline Tube linear density & $0.745 \mathrm{~kg} / \mathrm{m}$ \\
\hline Potassium density & $685 \mathrm{~kg} / \mathrm{m}^{3}$ \\
\hline Young modulus & $1.0410^{11} \mathrm{~Pa}$ \\
\hline Inertia moment & $31.4410^{-10} \mathrm{~m}^{4}$ \\
\hline Primary coolant speed & $1-2 \mathrm{~m} / \mathrm{s}$ \\
\hline
\end{tabular}

\section{2) Periodic wake shedding}

Periodic wake shedding may occur immediately downstream of the tube bundle region subjected to cross flow. The shedding frequency is estimated according to:

$$
f=\frac{S \cdot V}{D}
$$

where $S$ is the Stroubal, $V$ the shell side speed (primary side) and $D$ the tube diameter. The Stroubal number is assumed to be 4.0 as recommended by [FOUST78] for in-line tube banks. As seen in Figure 2-38, the shedding frequency is lower than $100 \mathrm{~Hz}$ (even using $1 \mathrm{~cm}$ tube OD), hence no resonance between the shedding vortex and the natural mode of vibration of the structure is expected to occur (first mode $>150 \mathrm{~Hz}$ ).

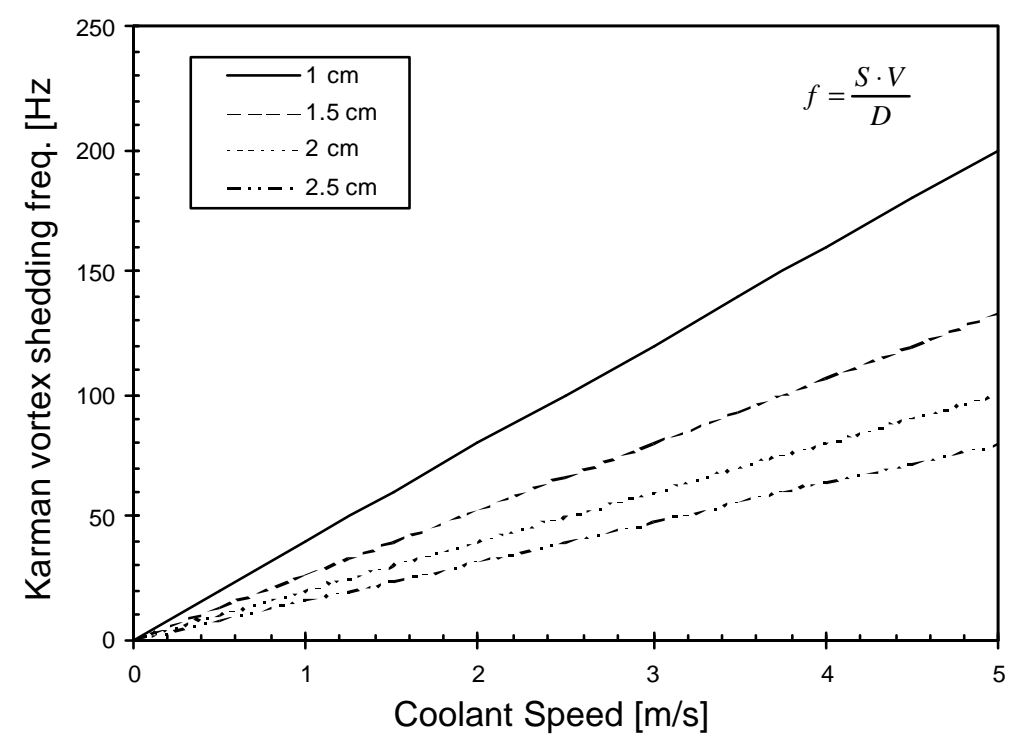

Figure 2-38. Karman vortex shedding frequency for tubes under cross flow. 


\section{3) Turbulence}

As for the fuel rod, turbulence is expected to be the principal vibration excitation mechanism for the tube and will be of special concern because of the high operating temperatures and the long component lifetimes $(\sim 10 \mathrm{yr})$. From dynamics point of view, the boiler is essentially a multitude of multispan tubes supported by clearance-supports with relatively complex geometry. This is a highly non-linear system because of the clearance between the tube and tube-supports. At a given support location, the tube may typically be: not touching the support, lightly in contact, or fully in contact with a significant pre-load depending on the tube straightness, support alignment and hydraulic drag forces. Furthermore, the tube contact loading at the support may change with time since hydrodynamic forces may vary due to thermal power changes, and tube straightness and alignment may be affected by thermal expansion. Corrosion product deposition over time can also affect the tube loading conditions at the support. Thus, the system is not stationary. The approach used here is to model the tube behavior using conservative parameters. However, a statistical approach will be needed in a more advanced design stage. The tube model is sketched in Figure 2-39. The spacer plates are separated $50 \mathrm{~cm}$ and the tubes are assumed straight (which is conservative for the $\mathrm{C}$ tube configuration). The boundary conditions at tube sheets assumed both ends clamped. These boundary conditions will not be representative for the concentric tube configuration, where one end clamped and one end free configuration is more adequate.

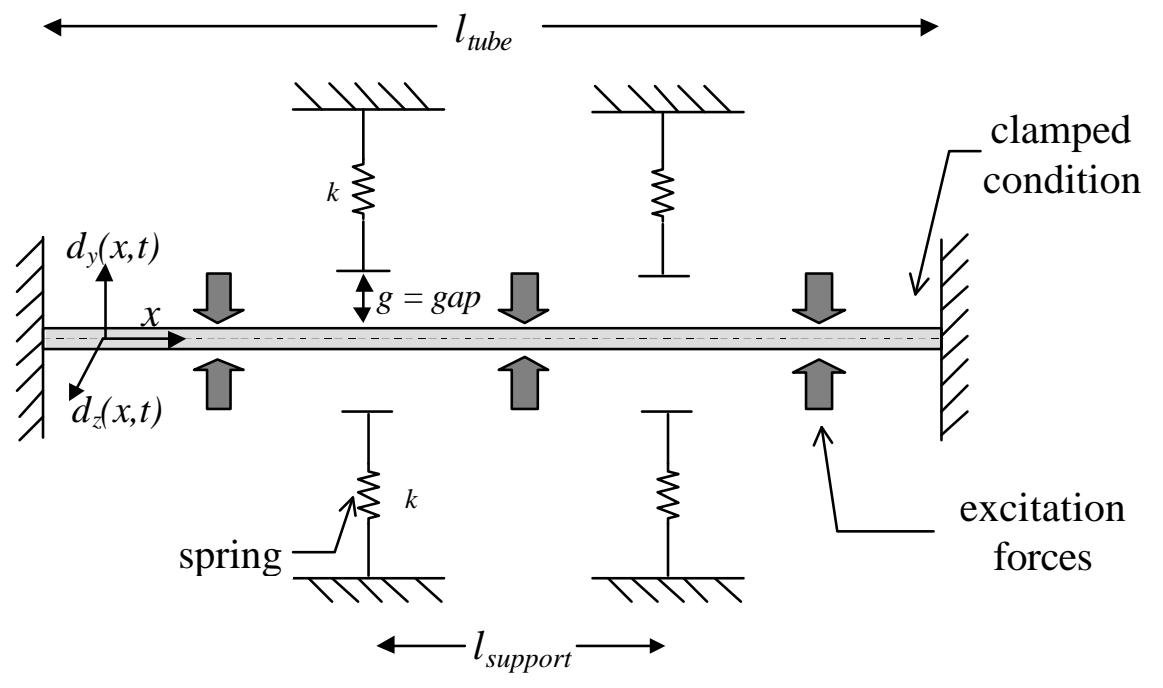

Figure 2-39. Non-linear tube vibration model used in the study. The supports are modeled as a gap and a spring having a very high stiffness.

The excitation force, generated by the fluid was modeled as a random Gaussian force with a constant power spectral density (PSD) between $0 \mathrm{~Hz}$ and an upper cutoff frequency of $100 \mathrm{~Hz}$. Note that the excitation force arising from the pump can be included in this range of frequency because the shaft speed is at $~ 300$ RPM. Therefore, even if the propeller uses a high number of blades, the excitation frequency is likely to be 
lower than $50 \mathrm{~Hz}$. Finally, to simulate the tube behavior, the tube motion was expanded using the unconstrained modes of beam vibration (Figure 2-40) with both ends clamped boundary condition.
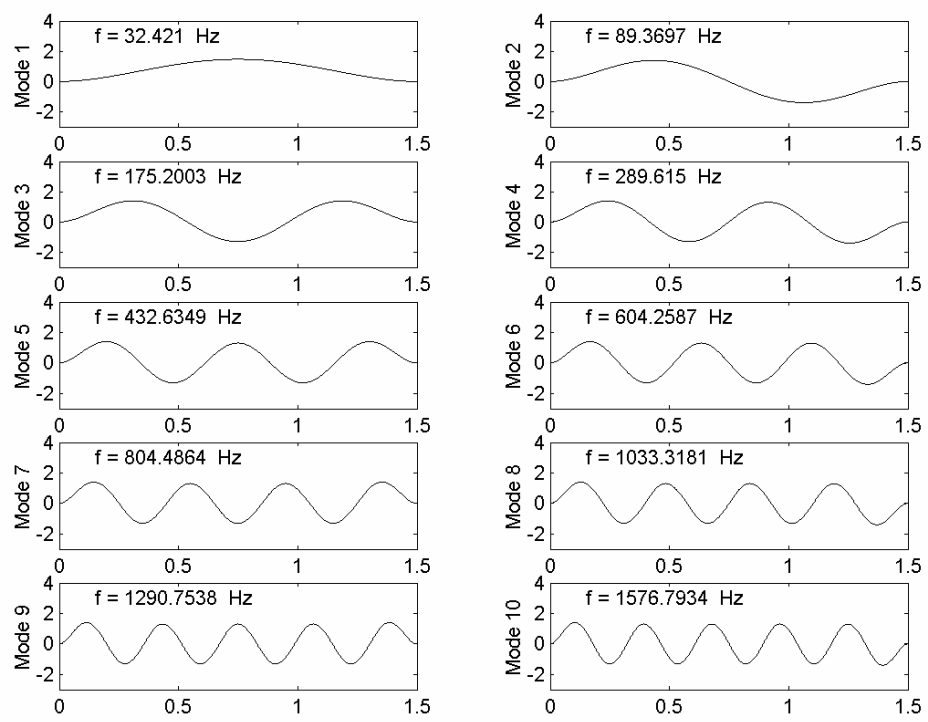

Figure 2-40. First 10 unconstrained vibration modes of the clamped tube.

\section{Frequency response of the tubes}

As a first step, the tube response in the frequency domain was analyzed by applying the Fast Fourier Transformation on the displacement of the rod. Two cases were analyzed:

- Zero gap between the tube and the support plate.

- $0.1 \mathrm{~mm}$-gap between the tube and the support plates.

The simulations were performed for four different excitation forces namely:

- low excitation: $\quad 0.01414 \mathrm{~N}(\mathrm{rms})$

- moderate excitation: $0.04473 \mathrm{~N}(\mathrm{rms})$

- high excitation: $\quad 0.14140 \mathrm{~N}(\mathrm{rms})$

- very high excitation: $0.31625 \mathrm{~N}(\mathrm{rms}$

Figure 2-41 presents Fast Fourier Transformation (FFT) of the displacement of the tube during the simulations corresponding to the case without gap and low and high excitation forces. The peaks correspond to the first 3 constrained modes of vibration. The frequency of the first mode is $\sim 165 \mathrm{~Hz}$ and it is observed that the two spacing plates sufficiently raises the natural frequencies of the tube above the upper cutoff frequency of the turbulent force (upper cutoff is at $100 \mathrm{~Hz}$ ). Increasing the excitation force changes the amplitude but not the position of the peaks. 

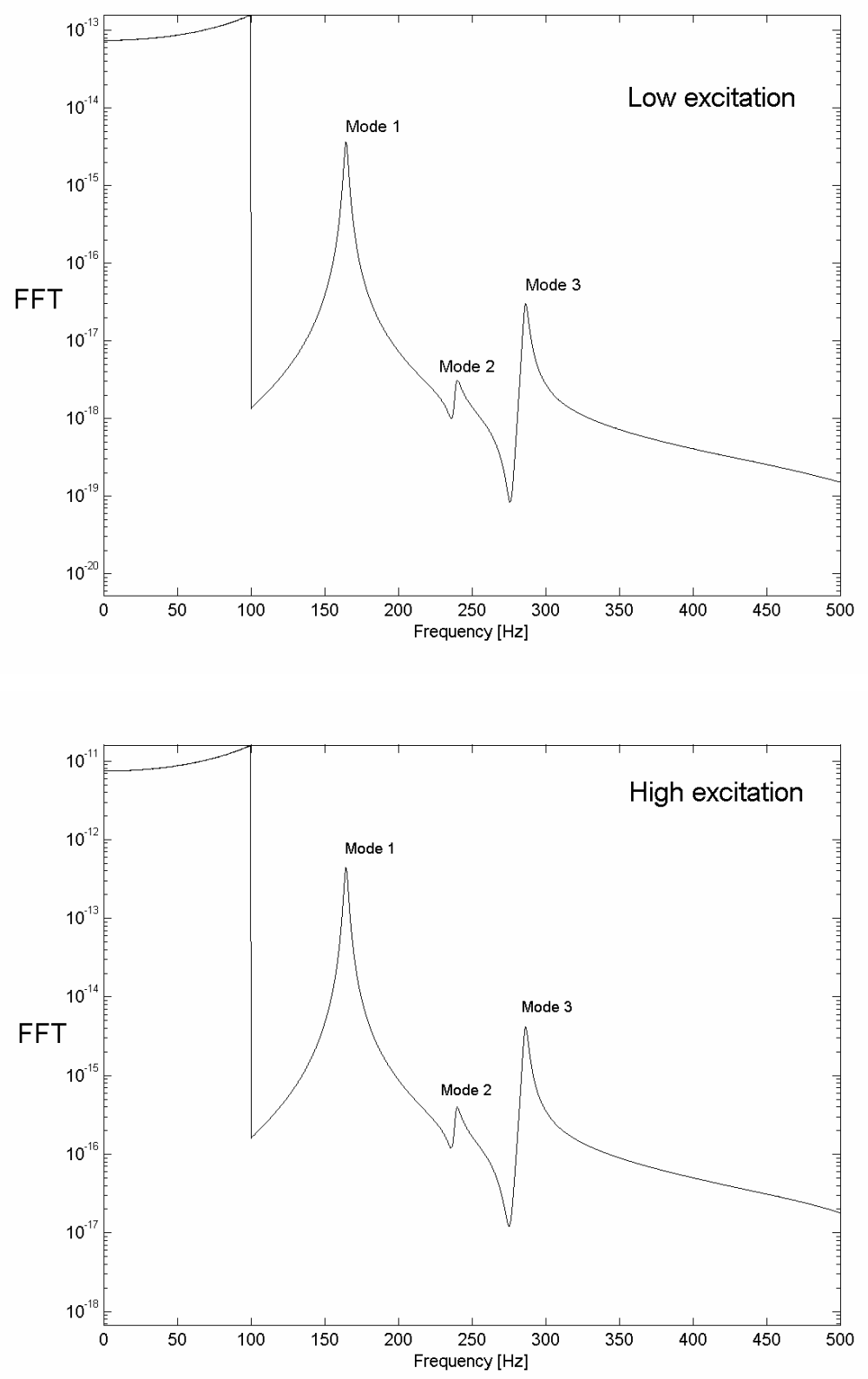

Figure 2-41. Rod frequency response for zero gap case and under low and high excitation forces. The first three constrained modes of vibration can be identified.

When a 0.1-mm gap between the tube and the support plates is allowed, the tube is able to vibrate at the unconstrained vibration modes. The first five modes are observed in the FFT of the tube motion (Figure 2-42). Note that for the same excitation force, the amplitude of the peaks is larger than for the zero gap case, because the vibration modes under $100 \mathrm{~Hz}$ can absorb the energy from the fluid. On the other hand, the increase of the force augments the thickness of the peaks. 

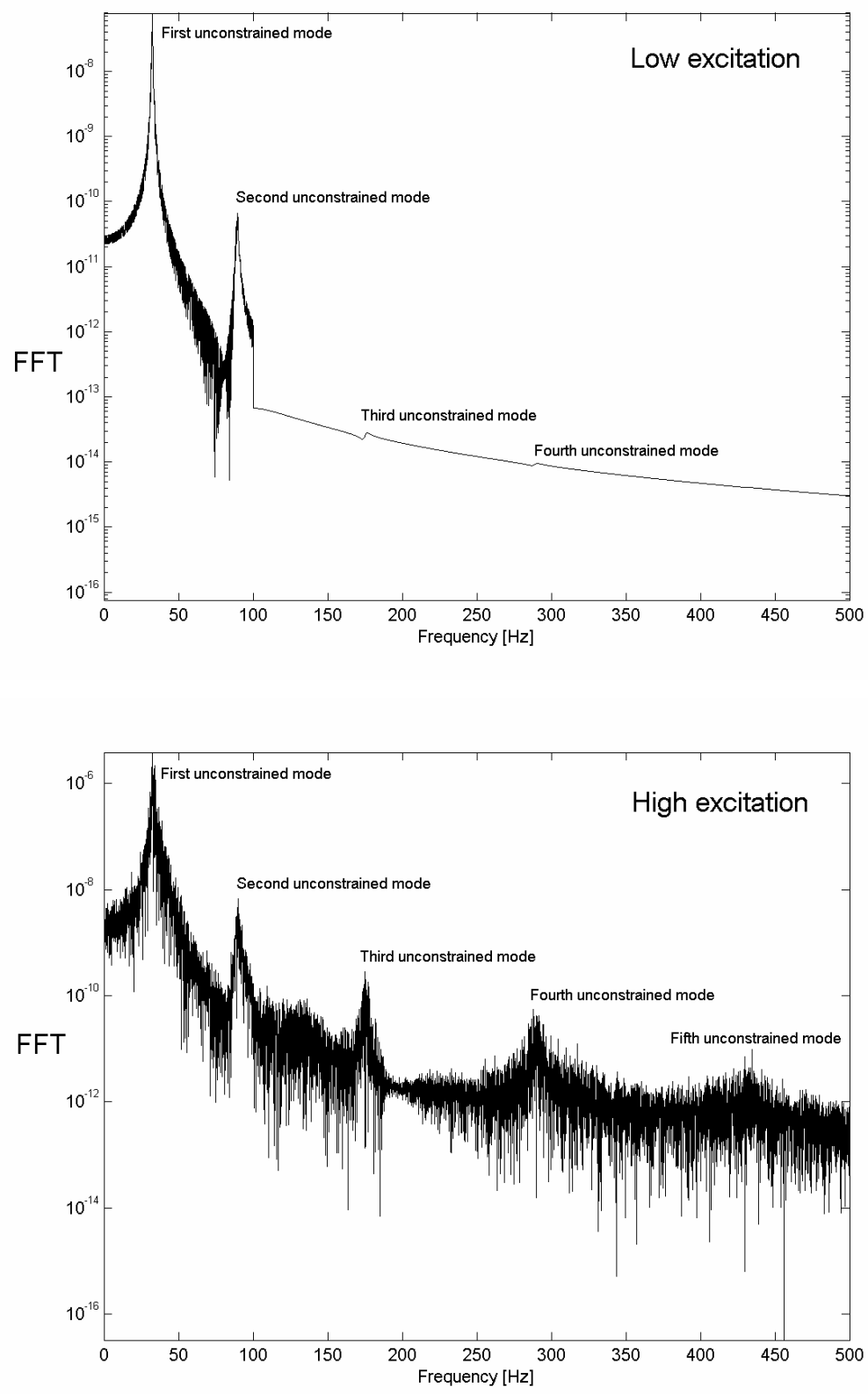

Figure 2-42. Rod frequency response for non zero gap case and under low and high excitation forces. The first fifth unconstrained modes of vibration can be identified.

\section{Work rate}

As for the fuel rod, the normal work rate was used to estimate the relative variation of the wear damage with respect to the excitation force and the gap. The results obtained from the simulations are reported in Table 2-18 and plotted in Figure 2-43. The following conclusions can be outlined: 
- The work rate of the non-zero gap case is 100 times larger than that one of the zero gap case. Therefore gap presence needs to be include in any evaluation of the tube performance.

- The normal work rate is lower than the one obtained for the fuel rods. This is due to the lower linear density of the tubes. However, it can not be concluded that the fretting-wear damage will be a least concern because the fluid forces acting on the boiler's tubes are expected to be larger than those of the fuel rods.

- As for the fuel rods, an evaluation of the wear coefficient and the PSD correlation are required for more detailed studies on the mechanics of failure.

Table 2-18. Normal work rates and middle span displacement obtaining for the zero and non-zero gap cases.

\begin{tabular}{|c|c|c|c|c|}
\hline & \multicolumn{2}{|c|}{ Zero Gap } & \multicolumn{2}{c|}{ Gap equal $0.1 \mathrm{~mm}$} \\
\hline $\begin{array}{c}\text { Excitation } \\
\text { force } \\
{[\mathrm{N}(\mathrm{rms})]}\end{array}$ & $\begin{array}{c}\text { Middle span } \\
\text { displacement } \\
{[\mu \mathrm{m}(\mathrm{rms})]}\end{array}$ & $\begin{array}{c}\text { Normal work rate } \\
\text { per support } \\
{[\mathrm{mW}]}\end{array}$ & $\begin{array}{c}\text { Middle span } \\
\text { displacement } \\
{[\mu \mathrm{m}(\mathrm{rms})]}\end{array}$ & $\begin{array}{c}\text { Normal work } \\
\text { rate per support } \\
{[\mathrm{mW}]}\end{array}$ \\
\hline 0.01414 & 0.062 & $1.2210^{-6}$ & 4.27 & 0.0 \\
\hline 0.04473 & 0.196 & $1.2210^{-5}$ & 15.3 & 0.0 \\
\hline 0.14140 & 0.620 & $1.2410^{-4}$ & 39.4 & 0.0112 \\
\hline 0.31625 & 1.390 & $6.1410^{-4}$ & 44.7 & 0.1118 \\
\hline
\end{tabular}

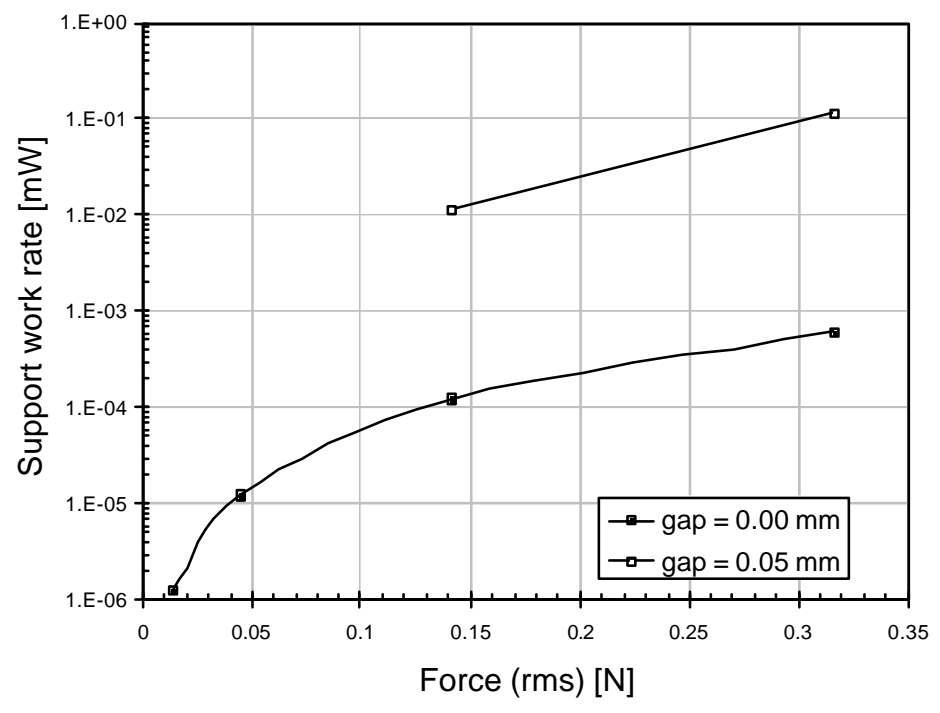

Figure 2-43. Work rate versus the exc itation force for zero gap and gap equal $0.1 \mathrm{~mm}$ cases.

As seen in Table 2-18, in the $0.1 \mathrm{~mm}$-gap case, the tubes start colliding with the supports when the middle span displacement is larger than $30 \mu \mathrm{m}$. 


\subsubsection{Primary pumps}

Centrifugal type pumps are selected for the LMR-AMTEC primary loop due to their high efficiency and significant experience in their use for molten sodium and $\mathrm{Na}+\mathrm{K}$ transport. We have rejected the use of electromagnetic pumps due to complexity of their implementation in pool reactors and difficulties in cooling them when placed inside the reactor vessel. In addition, the reliability and efficiency of the electromagnetic pumps are adversely affected by the loss of pump cooling and interruption of the liquid metal flow in the pump duct due to void formation or irradiation damage [Yevick66].

In the past, pump concepts have been strongly influenced by problems associated with liquid-metal containment and shaft sealing. According to the existing LMR experience, the primary pumps of the LMR-AMTEC are mounted vertically on the vessel head to reduce lower penetrations of the reactor vessel and to avoid the requirement for direct sealing of liquid metal by using a cover gas above the sodium. This configuration in a pool reactor has also the advantage of improving the accessibility (from a radiological point of view) if maintenance operations are required for the pump's motors.

\subsubsection{Selection of the Impeller Type: Propeller Pumps}

The centrifugal pumps are characterized by two parameters: the total head and the specific speed. The total head developed by a centrifugal pump at a given impeller rotational speed and capacity is independent of the liquid density and is normally expressed in $f t$. A centrifugal pump can only operate at one particular capacity along its total head vs. capacity characteristic curve. The specific speed is a term used to classify impellers on the basis of their performance and proportions regardless of their actual size or speed at which they operate. The specific speed of a centrifugal pump is defined as the rpm at which a theoretical homologous pump of smaller size would operate to deliver 1 GPM against a total head of $1 \mathrm{ft}$ [Holland66]. According to the specific speed the impellers can be classified in the following types:

- Radial-type impeller (or centrifugal flow): The range of the specific speed is generally between 500 and 3000 .

- Mixed-flow-type impeller (or Mixed-flow): The head created in this impeller is due partly to the centrifugal force and partly to the push of the vanes. The discharge is partly radial and partly axial. The specific speed range is usually between 4500 and 8000 .

- Propeller-type impellers (or axial flow): It has the highest specific speed (above 8000 rpm) and is used for low heads (3 to $40 \mathrm{ft}$ ), low RPM (200 to 1800), and large capacities. 
An overview of the LMR designs shows that the primary pumps are usually of the centrifugal radial type since higher head is required. However, in the LMR-AMTEC the primary pressure losses are lower (less than $20 \mathrm{ft}$ ) than in a classic LMR reactor (more than $100 \mathrm{ft}$ ), while the mass flow rates are moderate. Thus, the use of a propeller pump instead of a centrifugal radial pump is more suitable as can be seen in Figure 2-44.

The propeller pump (also called axial flow pump) creates most of its head by the propelling or lifting action of the runner vanes on the liquid. It has a single inlet runner with the flow entering axially and discharging nearly axially. This type of pump is particularly apt to handling relatively large volumes of liquid against comparatively low heads at rotational speeds much higher than those for ordinary centrifugal pumps handle. Specific speeds of ordinary double-suction centrifugal pumps do not go above 4000, but pumps of propeller type usually have a specific speed above 8000 . The inherent high speed is a definite advantage when the pump is driven by a direct-connected electric motor because the higher speed motor will be smaller for the same power, and therefore, its cost will usually be less.

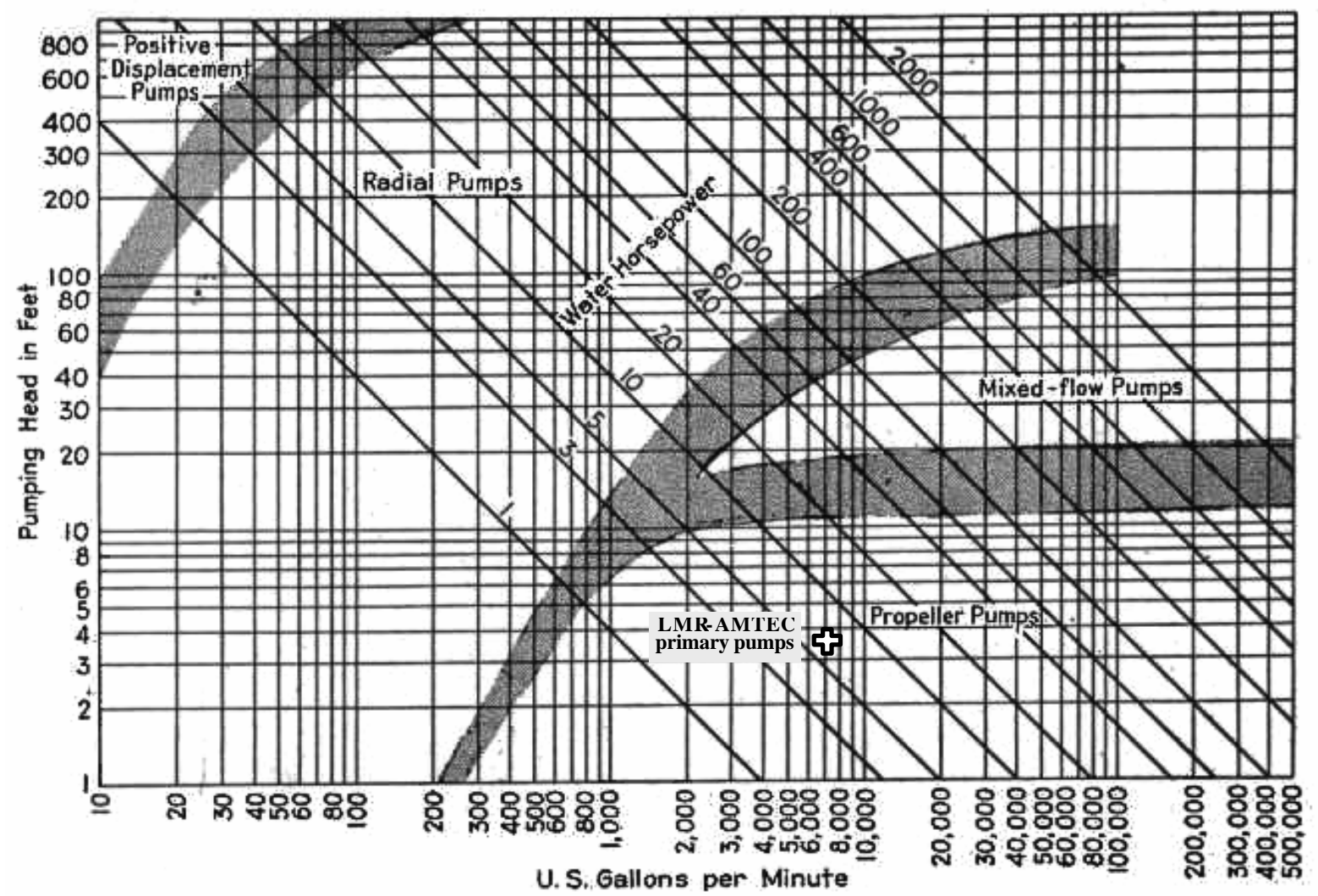

Figure 2-44. Centrifugal pump characteristics for radial, propeller and mixed flow types. The working conditions of the primary pumps of the LMR-AMTEC correspond to a propeller type. 


\subsubsection{Sizing of the Impeller and the Pump Casing}

For any type of centrifugal pump, the impeller diameter can be determined from the pump head and the specific speed [Church44]. Using the impeller size, the pumpcasing diameter can be estimated as twice the impeller diameter for pumps delivering over 1000 RPM [Foust78]. The LMR-AMTEC's pump head requirement and the capacity are estimated to be between 3 to $20 \mathrm{ft}$ and between 2000 GPM to 20000 GMP, respectively. Note that, since the pumps are placed in the annulus, the size of the pumpcasing has to be smaller than the size of the AMB in order to avoid any impact on the vessel diameter.

The requirements above (in particular the size) can only be met by employing a pump with a nominal speed above 7000, i.e. a propeller pump. Particularly for the present design using four pumps, each one pumping the sodium from two AMBs, the nominal speed is estimated equal to 7080 RPM, the capacity about 7000 GPM, and the nominal head as $3.9 \mathrm{ft}$. As seen in Table 2-19, a pump with such characteristics is in the range of commercially available propeller pumps.

Table 2-19. Propeller pump performance data [Volney48].

\begin{tabular}{|c|c|c|c|c|c|}
\hline $\begin{array}{c}\text { Pump } \\
\text { size }\end{array}$ & $\begin{array}{c}\text { Dynamic } \\
\text { head }\end{array}$ & Capacity & \multicolumn{2}{|c|}{ Electric motor } & $\begin{array}{c}\text { Specific } \\
\text { speed }\end{array}$ \\
\hline [in] & [feet] & [U.S.G.P.M.] & [HP] & [R.P.M] & \\
\hline 8 & 2 to 16 & 1000 to 2000 & 3 to 7.5 & 1760 & $10000-30000$ \\
\hline 10 & 2 to 11 & 1000 to 3000 & 3 to 7.5 & 1160 & $10000-20000$ \\
\hline 10 & 6 to 25 & 2000 to 4000 & 7.5 to 20 & 1760 & $10000-20000$ \\
\hline $\mathbf{1 4}$ & $\mathbf{4}$ to $\mathbf{1 2}$ & $\mathbf{3 0 0 0}$ to $\mathbf{6 0 0 0}$ & $\mathbf{7 . 5}$ to 15 & $\mathbf{8 7 5}$ & $\mathbf{1 0 0 0 0 - 2 0 0 0 0}$ \\
\hline $\mathbf{1 4}$ & $\mathbf{8}$ to 20 & $\mathbf{3 0 0 0}$ to 8000 & $\mathbf{1 5}$ to 40 & $\mathbf{1 1 6 0}$ & $\mathbf{1 0 0 0 0 - 2 0 0 0 0}$ \\
\hline 20 & 8 to 22 & 8000 to 16000 & 40 to 75 & 875 & $10000-15000$ \\
\hline 24 & 5 to 15 & 10000 to 20000 & 25 to 60 & 580 & $10000-20000$ \\
\hline 24 & 8 to 22 & 12000 to 24000 & 50 to 100 & 705 & $10000-15000$ \\
\hline
\end{tabular}




\subsubsection{Overview of Structural Material Selection}

The selection of suitable structural materials for the reactor components was made based on the following factors:

- Suitable mechanical properties at elevated temperatures.

- Compatibility with alkali liquid metal.

- Activation of the components.

- Cost of fabrication (material, welding, etc.).

Materials finding application under these criteria fall into three classes: austenitic stainless steels, nickel or cobalt-based super-alloys, and refractory materials [Devans67] [Distefano70] [Fraas89] [Peckner59]. A summary of the principal characteristics of these materials is presented in Table 2-20.

Based on the information presented on Table 2-20, the possible candidate material for the principal components of the primary and secondary circuits are presented in the Table 2-21 and Table 2-22, respectively.

As mentioned previously, $\mathrm{Nb}-1 \mathrm{Zr}$ was selected as the cladding material because of its superior corrosion and mechanical performances at high temperatures [Distefano70]. For the impeller of the primary pumps, both a Nickel alloy and Nb-1Zr can be used, however, the latter is more suitable because of its corrosion behavior and the erosion concerns that can arise in the unlikely event of pump cavitation. Regarding the reactor vessel, economic impact on the plant should considered when selecting the material. A refractory alloy, such as $\mathrm{Nb}-1 \mathrm{Zr}$, will be the most suitable from mechanical and corrosion points of view. However, since property degradation of $\mathrm{Nb}-1 \mathrm{Zr}$ occurs in even low oxygen and hydrogen environments, material and fabrication costs for such a large component will render it too expensive. For this reason, other less expensive alloys such as SS and Nickel-based have to be used. In particular, some Nickel alloys have enough strength at high temperatures and their corrosion behavior can be improved by using a lining made of a refractory alloy. Finally, the activation of the corrosion products precludes the use of any Cobalt based alloys in the primary circuit components. 
Table 2-20. Characteristics of the candidate materials for the LMR-AMTEC components. The temperature indicated on the "alloy name" column corresponds to the upper limit fixed by the mechanical properties.

\begin{tabular}{|c|c|c|}
\hline Alloy & Characteristics & Alloy name \\
\hline Austenitic stainless-steel & 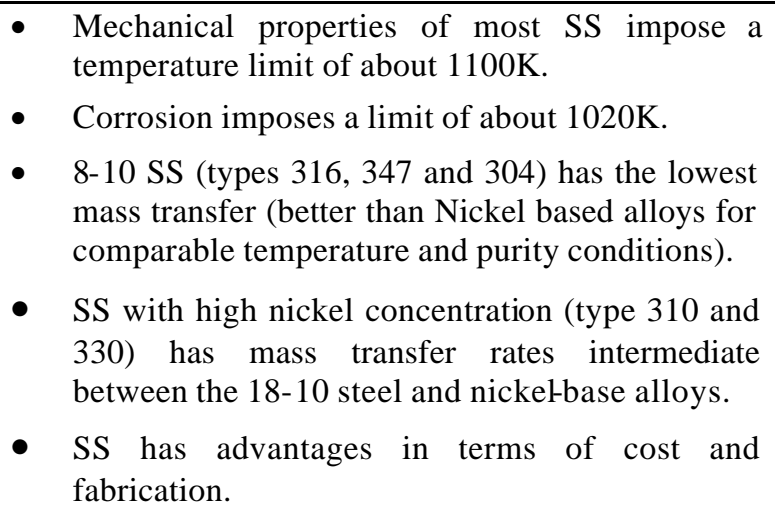 & $\begin{array}{ll}- & \text { SS316 (up to } \\
1100 K) \\
\text { - } \\
\text { SS310S and } \\
\text { SS314 (up to } \\
\text { 1350K) }\end{array}$ \\
\hline Nickel alloys & $\begin{array}{l}\text { - The majority of these alloys have mechanical } \\
\text { properties that allow temperatures up to } 1250 \mathrm{~K} \text {. } \\
\text { Studies show that nickel alloys are more } \\
\text { susceptible to temperature-gradient mass transfer } \\
\text { than iron-based alloys. However, they are less } \\
\text { affected by oxide contamination in Sodium than } \\
\text { the iron-based alloys. } \\
\text { The cost is approximately two or three times } \\
\text { higher than SS. }\end{array}$ & 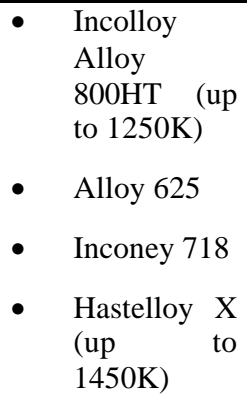 \\
\hline Cobalt alloys & $\begin{array}{l}\text { - Some Cobalt alloys can be used up to } 1450 \mathrm{~K} \text {. } \\
\text { - They resist dissolvent attack of alkali metals to at } \\
\text { least the same degree as iron and better than } \\
\text { nickel. } \\
\text { Cobalt presence precludes their use for primary } \\
\text { side components because of activation of the } \\
\text { corrosion products. } \\
\text { The cost is approximately one order of } \\
\text { magnitude more expensive than SS. }\end{array}$ & $\begin{array}{ll}\cdot & \text { Haynes } \\
& \text { Alloy } 25 \text { (up } \\
\text { to } 1250 \mathrm{~K})\end{array}$ \\
\hline$\underline{\text { Refractory metals }}$ & $\begin{array}{l}\text { - Their strength is retained in the temperature } \\
\text { region above } 1400 \mathrm{~K} \text { where most of the } \\
\text { commercial alloys cease to be useful. } \\
\text { - Alloys of niobium or tantalum containing oxide } \\
\text { formers, such as zirconium or hafnium, show } \\
\text { negligible corrosion effects up to } 1500 \mathrm{~K} \text {. } \\
\text { The presence of oxygen in alkali metals is quite } \\
\text { deleterious to most of the refractory materials. } \\
\text { The cost is approximately two orders of } \\
\text { magnitude more expensive than that of SS. }\end{array}$ & - $\mathrm{Nb}-1 \mathrm{Zr}$ \\
\hline
\end{tabular}


The secondary circuit components require more attention than those of the primary circuit because of the large temperature changes in the loop, which increase the mass transport phenomena and the corrosion issues. However, less expensive Cobalt based alloys can be utilized for the components including pipes, valves, condensers, and pumps provide that the boilers are placed in such a way to avoid excessive irradiation of the secondary coolant. On the other hand, past experience on AMBs for liquid metal and molten salts [Fraas60] [Yarosh60] [MacPherson60] indicates that more suitable candidates for these components are $\mathrm{Nb}-1 \mathrm{Zr}$ and Nickel based alloys. A distinction has to be made, though, between the designs operating an AMB in once-through mode or in recirculation mode. For example, 300 series SS are found to be adequate for service in recirculating-boiling systems up to $\sim 1100 \mathrm{~K}$, while Nickel-based alloys extend the limit up to $1170 \mathrm{~K}$ [Devans76]. As the alkali metal boilers are very compact, the increase of the capital cost due to the use of a refractory alloy may be compensated by the increase in system reliability.

Table 2-21. Candidate materials for the main components of the primary circuit.

\begin{tabular}{|l|c|c|c|}
\hline & \multicolumn{3}{|c|}{ Primary } \\
\hline Material & Cladding & Impeller of pump & Vessel \\
\hline SS (SS310 or SS316) & & & $\mathrm{x}$ (with lining) \\
\hline Nickel Alloys & & $\mathrm{x}$ & $\mathrm{x}$ \\
\hline Cobalt Alloys & & $\mathrm{X}$ & $\mathrm{x}$ \\
\hline Refractory (Nb-1Zr) & $\mathrm{X}$ & & \\
\hline
\end{tabular}

Table 2-22. Candidate materials for the main components of the secondary circuit.

\begin{tabular}{|l|c|c|c|c|}
\hline & \multicolumn{4}{|c|}{ Secondary } \\
\hline Material & $\begin{array}{c}\text { AMB } \\
\text { (once-through) }\end{array}$ & $\begin{array}{c}\text { AMB } \\
\text { (with re-circulation) }\end{array}$ & $\begin{array}{c}\text { Pipes, pumps } \\
\text { and valves }\end{array}$ & Condenser \\
\hline SS (SS310 or SS316) & & $\mathrm{X}$ & $\mathrm{x}$ & $\mathrm{x}$ \\
\hline Nickel Alloys & $\mathrm{x}$ & $\mathrm{x}$ & $\mathrm{x}$ & $\mathrm{x}$ \\
\hline Cobalt Alloys & & $\mathrm{x}$ & $\mathrm{x}$ & $\mathrm{x}$ \\
\hline Refractory (Nb-1Zr) & $\mathrm{X}$ & $\mathrm{X}$ & $\mathrm{x}$ & $\mathrm{x}$ \\
\hline
\end{tabular}




\subsubsection{Operating parameters}

\subsubsection{Plant Model Used for Evaluating the Design Performance}

An LMR-AMTEC plant model was developed to evaluate the performance of the design. The main characteristics of the model are:

- Use of lumped equations for the mass, momentum and energy balances of the reactor components, including core, riser, down-comer, HXs, boilers, expanders, condenser, pumps, AMTEC, etc.

- Determination of mass flow rates, temperatures and pressure losses of the principal reactor components. The pump power and AMTEC efficiency are calculated to determine the reactor gross and net efficiencies.

- Mechanical sizing of the principal components of the reactor: core, reactor vessel, boilers, HX, etc.

- Evaluation of different LMR-AMTEC designs (direct or indirect coupling options), boiler designs, materials and working fluids.

A sketch of the modules included in the model is shown in Figure 2-45. The main characteristics of the modules are described in the next paragraphs.

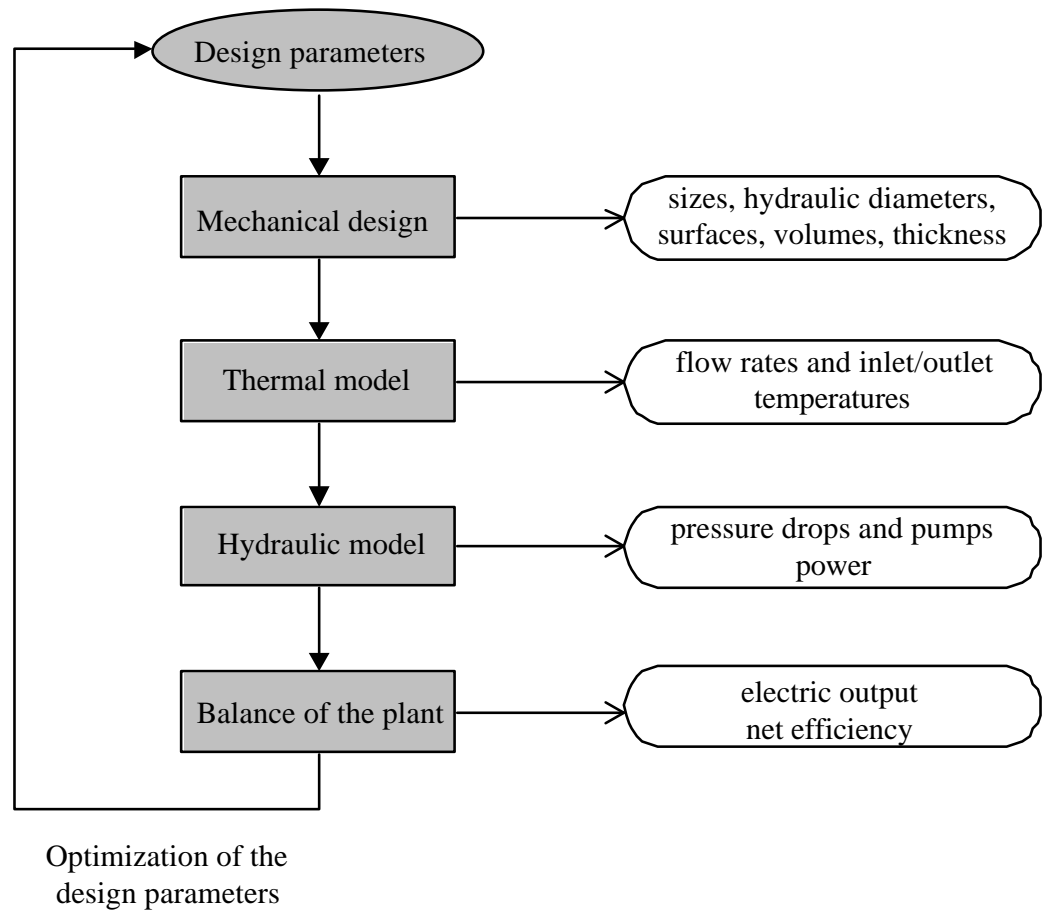

Figure 2-45. Flow diagram of the LMR-AMTEC plant model. 


\subsection{Mechanical Design}

The mechanical design module determines the size, volumes, mass, exchange surfaces, hydraulic diameters, and thickness of the main reactor components: LMR core, boilers, HXs, riser, primary pumps, reactor vessel and down comer.

In designing the boilers and the HX's, the primary coolant was assumed to flow through the shell side while the secondary coolant flows inside the tube side. The boiler external shell is circular. The tubes are placed according to a square pitch and they are straight having a bending at the ends in order to accommodate the thermal expansion $(\mathrm{C}$ tube configuration). The concentric tube configuration was also considered. The $\mathrm{Nb}-1 \mathrm{Zr}$ alloy is used as structural material for tubes and headers.

In determining the wall thickness for $\mathrm{Nb}-1 \mathrm{Zr}$ tubes under external pressure, Fraas [Fraas60, p264] estimated the allowable stress for the $\mathrm{Nb}-1 \mathrm{Zr}$ as 60 percent of the stress for 1 percent creep in 10000 hours. This value is consistent with the relation between the ASME code stresses for the iron-chrome-nickel alloys and their stresses for 1 percent creep in 10000 hours. The mechanical design module uses a more conservative value: the allowable stress for the $\mathrm{Nb}-1 \mathrm{Zr}$ is taken as 60 percent of the stress for 1 percent creep in $100000 \mathrm{~h}$ (the expected operation time of the plant). In addition, the wall thickness is calculated including a corrosion allowance equal to $0.5 \mathrm{~mm}$.

Another important component evaluated by the mechanical design module is the reactor vessel. The Incolloy Alloy $800 \mathrm{HT}$ was used as structural material for the vessel because of its good mechanical properties. The reactor vessel is assumed to have an internal lining based on a refractory alloy like $\mathrm{Nb}-1 \mathrm{Zr}$ to improve the corrosion resistance. An estimation of the thickness of the reactor vessel was achieved using the allowable stress intensity of the Incolloy Alloy 800HT. The thickness, diameter, height and weight are required for cost estimation as well as transportation issues.

Finally, based on the primary pressure losses (obtained from the hydraulic module) and the available space existing in the annulus between the riser and the reactor vessel, the impeller size and the nominal speed of the primary pumps are determined by this module.

\subsection{Thermal Model of the Plant: HXs, Boilers, Expanders}

The thermal module of the reactor uses a lumped model for obtaining the energy balance of the main reactor components during the steady state operation. These components include reactor core, riser, down-comer, HXs, boilers, expanders, condenser, pumps, AMTEC, etc. Liquid metal correlations are used to predict the transfer coefficient in single and two-phase flow [Lowell60]. The HX's are modeled using a log mean temperature model. The AMBs are modeled considering the liquid, two phases and vapor regions.

\subsection{Hydraulic Model: Primary and Secondary Pressures Losses and Pumps}

The hydraulic module determines the pressure losses in the primary and secondary circuit components. The pressure losses are determined from the momentum conservation of a control volume associated with the considered component. The balance of momentum takes into account the friction, the acceleration and the gravitational terms. The friction 
losses are calculated using correlations for contraction or expansion and single-phase flow in tubes and ducts. For the AMB, correlations for the pressure drops and the void fractions for two-phase flows of liquid metal were used. The pumping power is also estimated according to the pressure losses of the different components of the plant.

\subsection{Balance of the Plant: AMTEC/TE Units and the Net Efficiency of the Plant}

This module calculates the net electric output and the net efficiency of the plant by subtracting the pump power from the electric output of the AMTEC/TE unit. The losses of the power conversion system (lower than 5\% of the electric power) and the cold traps are not currently taken into account. Moreover, no heat losses have been considered in the secondary pipes. However they are believed to be small due to the low conductivity of the potassium vapor. The AMTEC/TE electric output is determined using a correlation for the AMTEC and the TE efficiency. This correlation determines the optimum condenser temperature and the efficiency of the AMTEC/TE unit for a given anode temperature. Two models were used to evaluate the AMTEC correlation: a conservative model developed by WEC and a more accurate model developed by ISNPS. The difference between both models is less than $7 \%$.

\subsubsection{Determination of the Operating Parameters}

The design parameters required as input for the plant model are presented in the Table 2-23. The determination of these values was achieved following an optimization procedure similar to that described in section 2.1.3 but including the economic evaluation of the reactor (see section 4.1). The goal of the optimization was to maximize the plant performance (i.e. the costs and the net efficiency) and to obtain the operating parameters of the reactor. The final values of the operating parameters of the reference design are presented in the Table 2-24.

Table 2-23. Design parameters of the plants.

\begin{tabular}{|l|l|}
\hline CORE & VESSEL \\
\hline Core coolant & Pressure vessel inner diameter \\
\hline Inlet core temperature & SECONDARY SYSTEM \\
\hline Outlet core temperature & Secondary coolant \\
\hline Margin to coolant boiling temperature & Number of boilers \\
\hline Thermal core power & Number of HX \\
\hline Active fuel length & Internal tube diameter \\
\hline Plenum length & Tube pitch to diameter ratio \\
\hline Fuel rod diameter & Length of tube \\
\hline Fuel rod pitch to diameter ratio & Secondary inlet temperature \\
\hline Power density & Secondary saturation temperature \\
\hline Height to diameter core ratio & OTHERS \\
\hline Number of reflector rings & Plant life duration \\
\hline
\end{tabular}


Table 2-24. Main LMR-AMTEC parameters.

\begin{tabular}{|c|c|c|c|}
\hline PLANT BALANCE & & PRIMARY PUMPS & \\
\hline thermal power & $100 \mathrm{MW}$ & type & impeller \\
\hline electric power & $34.74 \mathrm{MW}$ & total pump power & $13.21 \mathrm{KW}$ \\
\hline gross efficiency & $34.76 \%$ & head & $3.89 \mathrm{ft}$ \\
\hline net efficiency & $34.74 \%$ & specific speed (min.) & 7080 RPM \\
\hline CORE & & impeller diameter (max.) & $36 \mathrm{~cm}$ \\
\hline active length & $0.75 \mathrm{~m}$ & casing diameter (max.) & $71 \mathrm{~cm}$ \\
\hline plenum length & $1.0 \mathrm{~m}$ & SECONDARY CIRCUIT & \\
\hline total height & $1.75 \mathrm{~m}$ & pressure & $0.631 \mathrm{~atm}$ \\
\hline equivalent active core diameter & $1.73 \mathrm{~m}$ & mass flow rate & $95.98 \mathrm{~kg} / \mathrm{s}$ \\
\hline height to diameter ratio (active) & 0.43 & coolant & potassium \\
\hline maximal burnup & $80000 \mathrm{MWd} /$ ton & boiler inlet temperature & $780.4 \mathrm{~K}$ \\
\hline power density & $57.0 \mathrm{MW} / \mathrm{M}^{3}$ & boiler outlet temperature & $983.8 \mathrm{~K}$ \\
\hline linear power density & $10115 \mathrm{~W} / \mathrm{m}$ & vapor overheating & $0 \mathrm{~K}$ \\
\hline Number of fuel elements & $78(72)$ & vapor quality & $45.2 \%$ \\
\hline control assemblies number & $13(19)$ & secondary pump power & $11.35 \mathrm{KW}$ \\
\hline number of core rings & 5 & ALKALI METAL BOILERS & \\
\hline \multirow[t]{2}{*}{ number of reflector rings } & 2 & Operation mode & recirculation \\
\hline & & number of boilers & 8 \\
\hline FUEL ELEMENT & & tube number per boiler & 404 \\
\hline rod number & 169 & tube length & $1.5 \mathrm{~m}$ \\
\hline pitch (triangular) & $1.2979 \mathrm{~cm}$ & external tube diameter & $2.5 \mathrm{~cm}$ \\
\hline pitch to diameter ratio & 1.2254 & tube thickness & $1.45 \mathrm{~mm}$ \\
\hline outer clad diameter & $1.059 \mathrm{~cm}$ & AMTEC UNIT & \\
\hline clad thickness & $0.4572 \mathrm{~mm}$ & mass flow rate & $43.38 \mathrm{~kg} / \mathrm{s}$ \\
\hline pellet diameter & $0.9398 \mathrm{~cm}$ & anode temperature & $983.8 \mathrm{~K}$ \\
\hline pellet material & $\mathrm{UN}$ or $(\mathrm{U}, \mathrm{Pu}) \mathrm{N}$ & anode pressure & $0.631 \mathrm{~atm}$ \\
\hline cladding material & $\mathrm{Nb}-1 \mathrm{Zr}$ & condenser temperature & $525.8 \mathrm{~K}$ \\
\hline PRIMARY CIRCUIT & & condenser pressure & $7.95 \mathrm{~Pa}$ \\
\hline pressure & 8.273 at $\mathrm{m}$ & REACTOR VESSEL & \\
\hline Margin to coolant boiling temp. & $400 \mathrm{~K}$ & external diameter & $4.41 \mathrm{~m}$ \\
\hline mass flow rate & $1135 \mathrm{~kg} / \mathrm{s}$ & external height & $9.78 \mathrm{~m}$ \\
\hline coolant & sodium & head wall thickness & $7.8 \mathrm{~cm}$ \\
\hline core inlet temperature & $980 \mathrm{~K}$ & body wall thickness & $15.5 \mathrm{~cm}$ \\
\hline core outlet temperature & $1050 \mathrm{~K}$ & total weight & 125 ton \\
\hline
\end{tabular}

During the optimization procedure, the allowed values of certain design parameters were restricted in order to satisfy the design requirements described in the previous sections. For example, the cladding integrity imposes an outlet core temperature limit of about $1100 \mathrm{~K}$. This limit can only be exceeded by reducing the fuel cycle length 
(currently 10 years) or by increasing the clad thickness. Either of these actions poses drawbacks. Reducing the fuel cycle length will require more frequent refueling and thus, weakening the non-proliferation features of the reactor. Adding more $\mathrm{Nb}-1 \mathrm{Zr}$ to strengthen the clad will require the use of a higher enrichment in order to maintain the target burnup ( $\mathrm{Nb}-1 \mathrm{Zr}$ is a strong neutron absorber), thus affecting the economic performance and the proliferation resistance characteristics of the reactor. Note that even though there is not a restriction on low temperatures, low efficiency of the AMTEC units will also impose a lower temperature limit as seen in Figure 2-46.

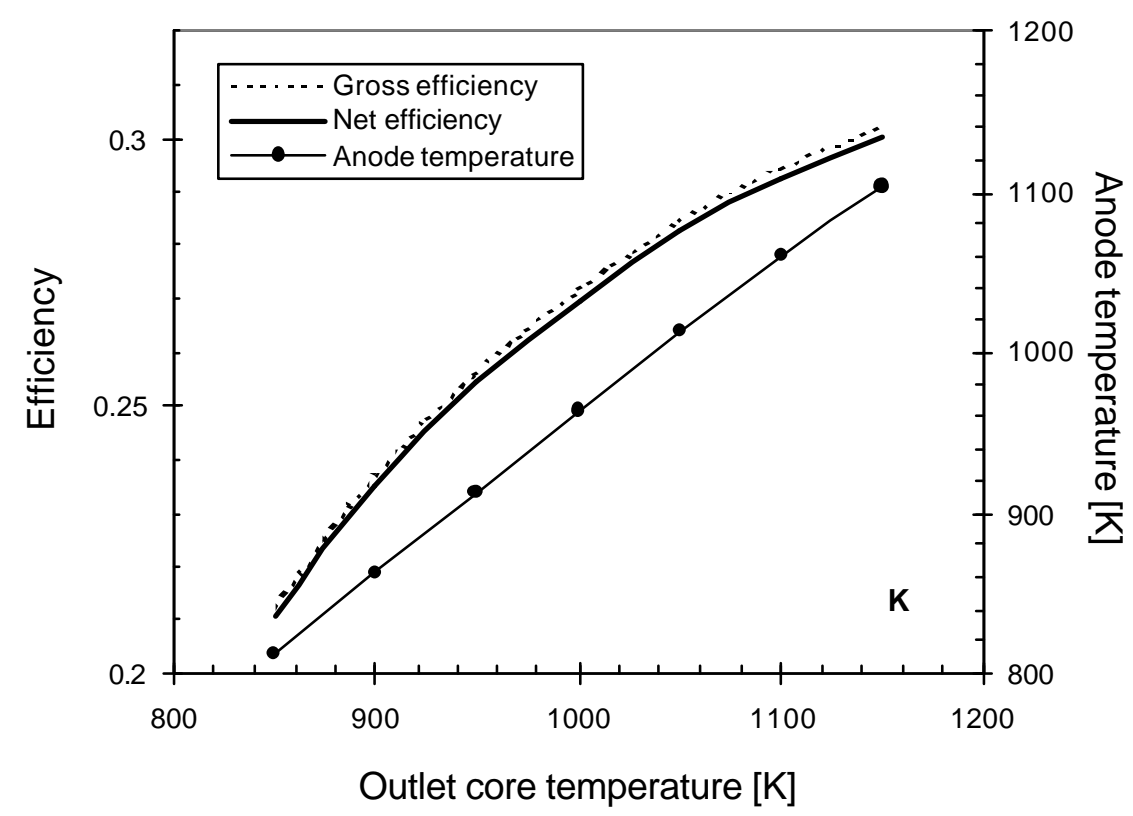

Figure 2-46. Gross and net efficiencies and anode temperature with respect to the outlet core temperature (for a design using potassium AMTEC).

Another example of a restricted parameter is the primary circuit pressure. In LMR designs, the primary pressure is set to have enough Margin to the Coolant Boiling Temperature (MCBT) and to keep an adequate cavitation margin for the primary pumps. The first limit avoids sodium boiling during postulated transients, like ATWS. The second one is especially important in alkali metal systems, as rapid deterioration of the impeller can occur when the pump is operated under cavitation conditions, even for short periods of time. Considering the plant efficiency, increasing the MCBT will have a negative impact as it increases the AMB tube thickness and their thermal resistance. This effect is moderated by the high heat transfer coefficients of liquid metals as seen in Figure 2-47. Considering the capital cost, MCBT will have an adverse effect on the reactor economy. As seen in Figure 2-48, the MCBT strongly affects the vessel weight. Since in integral reactors the capital cost of the reactor vessel is quite important, the primary pressure should be set as low as possible. Therefore, the MCBT has to be set accordingly, as low as compatible with the safety concerns. A first estimation of the minimal value of this parameter was obtained from the analysis of the loss of flow accident. The results have shown (see section 2.3) that a MCBT of at least $350 \mathrm{~K}$ is 
required to avoid sodium boiling during the transient (accounting for the uncertainties). Cavitation margin is likely not the limiting criteria for the LMR-AMTEC. An accurate determination of the primary pressure and the MCBT will require further transient studies of the LMR-AMTEC.

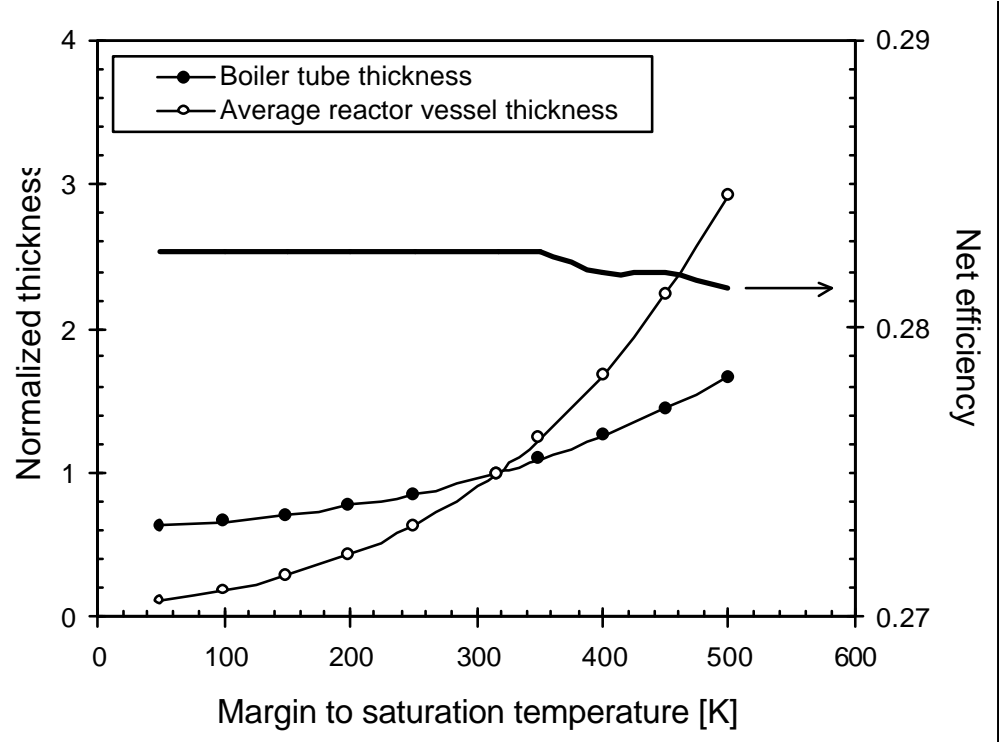

Figure 2-47. Effect of the margin to coolant boiling temperature on the net efficiency, the normalized tube and vessel thickness'.

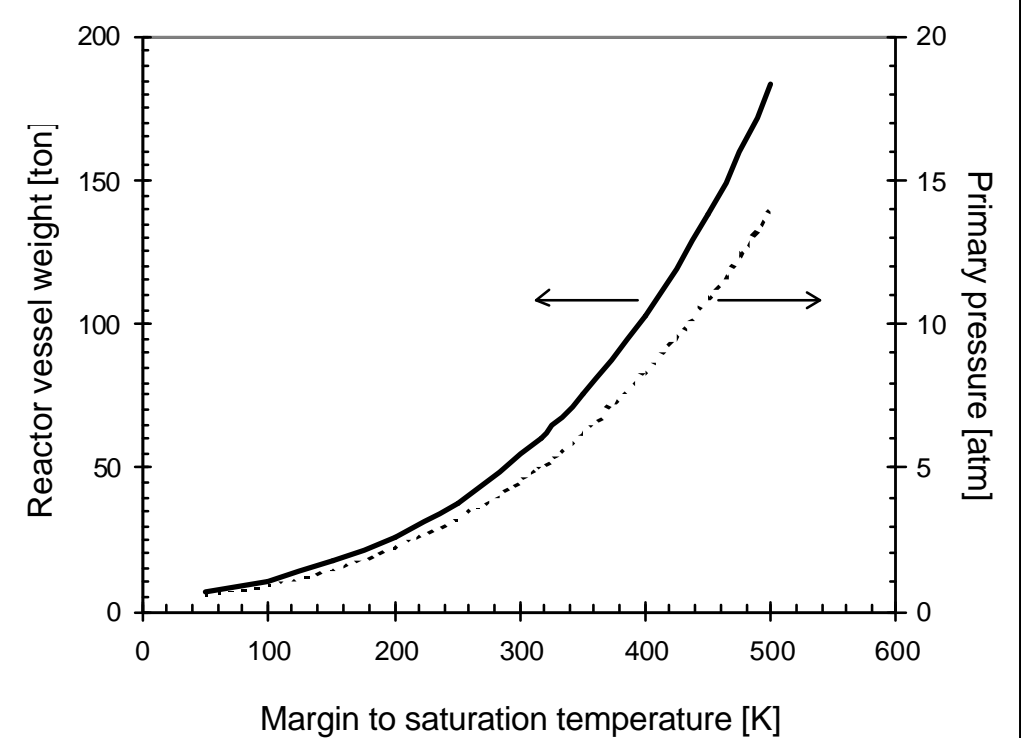

Figure 2-48. Effect of the margin to coolant boiling temperature on the primary pressure and the vessel weight. 


\subsection{Safety features of the LMR-AMTEC}

The safety of any LMR basically resides in its capability to provide reliable reactivity control (and shutdown) and heat removal (both during operation transients and after shutdown). The first part of this section describes the reactivity control systems, number of control rods and the sodium void effect of the LMR-AMTEC. In the second part the passive heat removal system and the transient results for LOFA are presented.

\subsubsection{Reactivity control system}

\subsubsection{Reactivity Control Requirements}

The LMR designs have traditionally two independent and diverse reactivity control systems. One has the primary function of controlling the reactor power and compensating for spatial and temporal changes in reactivity, the other to shutdown the reactor. Either one has the capability to scram the reactor by itself, i.e. under the assumption that one of the systems is inoperable. The safety rod assembly system, which is fully withdrawn at normal operating conditions, is therefore used with the control rod assembly system. In addition to these systems, the neutronic design of the core has to be such to ensure a negative power reactivity coefficient. Since the overall power reactivity coefficient is negative, the reactivity feedback is capable of bringing the reactor to hot shutdown even in the absence of control systems. A negative power reactivity coefficient, however, is not sufficient to make the reactor go subcritical and to achieve the ultimate, cold shutdown. Therefore, the reactor design has to incorporate a last resort system to provide cold shutdown as other designs such as ALMR or IFBR do.

\subsubsection{LMR-AMTEC Shutdown and Control Systems}

An actively controlled absorber rod bundle assembly has been adopted as the reactivity control system. An absorber assembly type has also been adopted as a secondary or shutdown system. In order to provide cold shutdown in an unlikely event of control system failure, a soluble neutron absorber, such as Indium-49 has been adopted as a last resort shutdown system. Furthermore, it is also required for the LMR-AMTEC to have a negative power reactivity coefficient. A negative void reactivity coefficient is desirable but values close to zero are also acceptable.

As an alternative control system, the use of articulated control rods and a passive release mechanism, such as the Self Actuated Shutdown System (SASS) based on the "Curie Point Principle" could be considered. Due to the long fuel cycle requirement of the LMR-AMTEC, however, the passive release mechanism must have a high reliability to avoid spurious activation during the normal operation. Further investigation is required on the feasibility of these systems. 
An important consideration on the neutronic design of the LMR-AMTEC is the reactor behavior during an Anticipated Transient without Scram (ATWS). The traditional initiator of the ATWS is the uncontrolled withdrawal of one or more control rods, namely the UTOP. At BOL, the control rods are fully inserted, and as the reactivity decreases with burnup, the rods are moved out. The worst case for a UTOP is BOL when the control rods are fully inserted, hence have the maximum worth. In order to limit the magnitude of the UTOP, insertion of control rods in the core (or control rod worth) must be limited. As mentioned in section 2.2.2, the neutronic design of the LMR-AMTEC has an objective of the reducing the reactivity swing throughout the core life. An additional advantage of reducing the insertion of control rods in the core is to limit the power peaking factors.

As a final note, in LMR-AMTEC the LOFA are an important concern because of the high working temperatures of the reactor. The use of Gas Expansion Modules (GEMS) should be evaluated as they eliminate the occurrence of a LOFA without scram. The pins of the GEMS consist of a hollow pressure tube that is capped at the top and filled with helium. The GEM's are designed to lower the power level in the core if the main coolant pumps malfunction or stop. In addition, due to the small size of the core, the required number of GEMS is not expected to be large.

The control and shutdown systems of LMR-AMTEC can be summarized as:

- Reactivity control system: absorbent $\operatorname{rod}\left(\mathrm{B}_{4} \mathrm{C}\right)$.

- Shutdown system

a) Absorbent rod $\left(\mathrm{B}_{4} \mathrm{C}\right)$ : Articulated control rods with a passive release mecha nism.

b) GEMS: depending on the results obtained from simulation of ATWS transients, the use of GEMS should be evaluated to eliminate the occurrence of a LOFA without Scram.

c) Ultimate shutdown system using a soluble neutron absorber such as Indium49.

\subsubsection{Sizing of the Control Rod Systems}

Since the LMR-AMTEC reactor core is designed to provide rather a long core life $(\sim 15$ years), requirements imposed on the reactivity control system are more stringent than in a typical fast reactor. The main reactivity control system is based on the control assembly design usually employed in fast reactors, i.e., absorber rods in a hexagonal lattice, the same size as a fuel element. Among the potential absorber materials, the largest neutron absorption cross section (averaged over the neutron spectrum) is attained by using boron (in boron carbide, $\mathrm{B}_{4} \mathrm{C}$ ), followed by europium (as $\mathrm{Eu}_{2} \mathrm{O}_{3}$ ), rhenium, tantalum and hafnium [Golden, 1969]. Using enriched boron in $\mathrm{B}_{4} \mathrm{C}$ further increases absorption. Therefore, $\mathrm{B}_{4} \mathrm{C}$ is usually selected as absorbing material in fast reactors. Ta and $\mathrm{Hf}$ have 
some attractive characteristics; e.g., they may be preferred for control assemblies that remain inserted in the core over a significant fraction of core life. In the LMR-AMTEC studies, $\mathrm{B}_{4} \mathrm{C}$ is selected as the reference option.

The theoretical density of $\mathrm{B}_{4} \mathrm{C}$ may be expressed as a function of " $\varepsilon$ ", boron enrichment (i.e., ${ }^{10} \mathrm{~B}$ isotopic fraction in B) as [Bailly, 1999]:

$$
\rho_{t h}(\varepsilon)\left[\mathrm{g} / \mathrm{cm}^{3}\right]=2.5561-0.1818 \cdot \varepsilon
$$

This relationship is graphically depicted by the solid line in Figure 2-49. However, the actual density is less than the theoretical one. For example, absorbers in SUPERPHENIX utilize $\mathrm{B}_{4} \mathrm{C}$ that has been hot-pressed at $1900 \mathrm{C}$ in graphite matrices to obtain a density corresponding to $96 \%$ of theoretical density. This particular density is also shown in Figure 2-49 (represented by the dashed curve). ${ }^{10} \mathrm{~B}$ enrichment of $90 \%$ is selected for the initial SUPERPHENIX core. Reduction of boron enrichment to $48 \%$ in some core regions or in later cores is also considered. Note that $\mathrm{B}_{4} \mathrm{C}$ melting point is $2375 \mathrm{C}$.

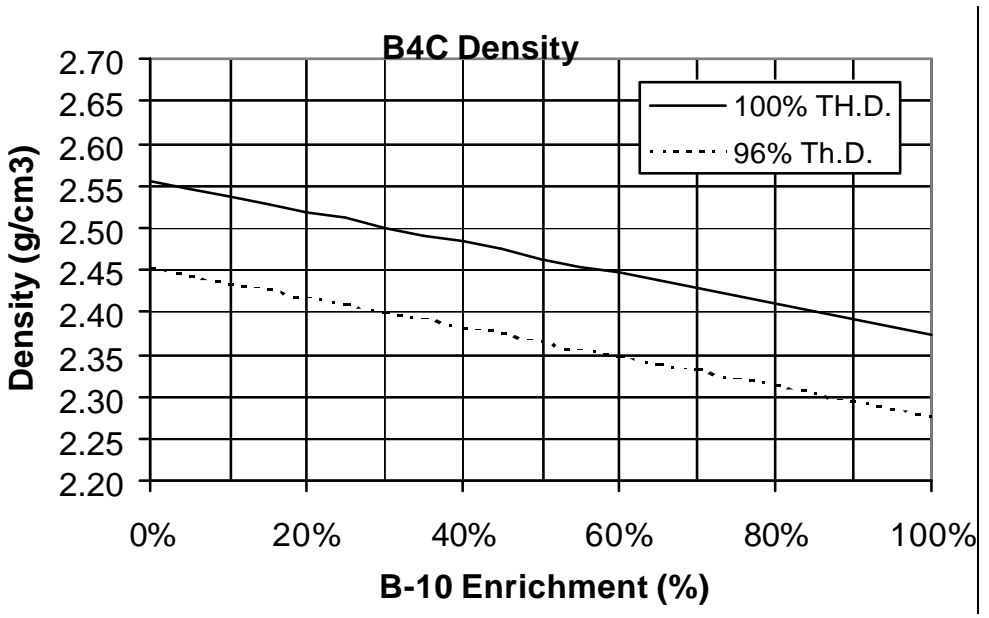

Figure 2-49. Boron carbide density as a function of ${ }^{10} \mathrm{~B}$ enrichment.

Reference absorber design in LMR-AMTEC utilizes $90 \%$ enriched $\mathrm{B}_{4} \mathrm{C}$. A density of $2.5 \mathrm{~g} / \mathrm{cm}^{3}$ is considered in most analyses, and furthermore, the control assemblies are modeled as consisting of $50 \mathrm{vol} \% \mathrm{~B}$ C and $50 \mathrm{vol} \% \mathrm{Na}$. In an actual detailed design, density will be slightly lower and the volume fraction of $\mathrm{B}_{4} \mathrm{C}$ may be somewhat different. Moreover, some fraction of the control assembly volume will be used by the absorber rod cladding. Impact of these factors is evaluated through several sensitivity studies.

The baseline analysis considers the 6-ring core configuration consisting of 78 fuel elements and 13 positions reserved for control assemblies, shown in Figure 2-50 (left). Monte Carlo simulations utilize somewhat simplified cylindrical 3-D core model, also depicted in Figure 2-50 (right). The first reference case represents all control assemblies withdrawn, with the corresponding core positions assumed to be filled with sodium. The second reference case represents all control assemblies inserted. Effective multiplication 
factors are given in Table 2-25. Their difference, expressed in terms of ?k $=\mathrm{k}_{2}-\mathrm{k}_{1}$, represents the total worth of all control assemblies, and amounts to $18.75 \%$ ?k.
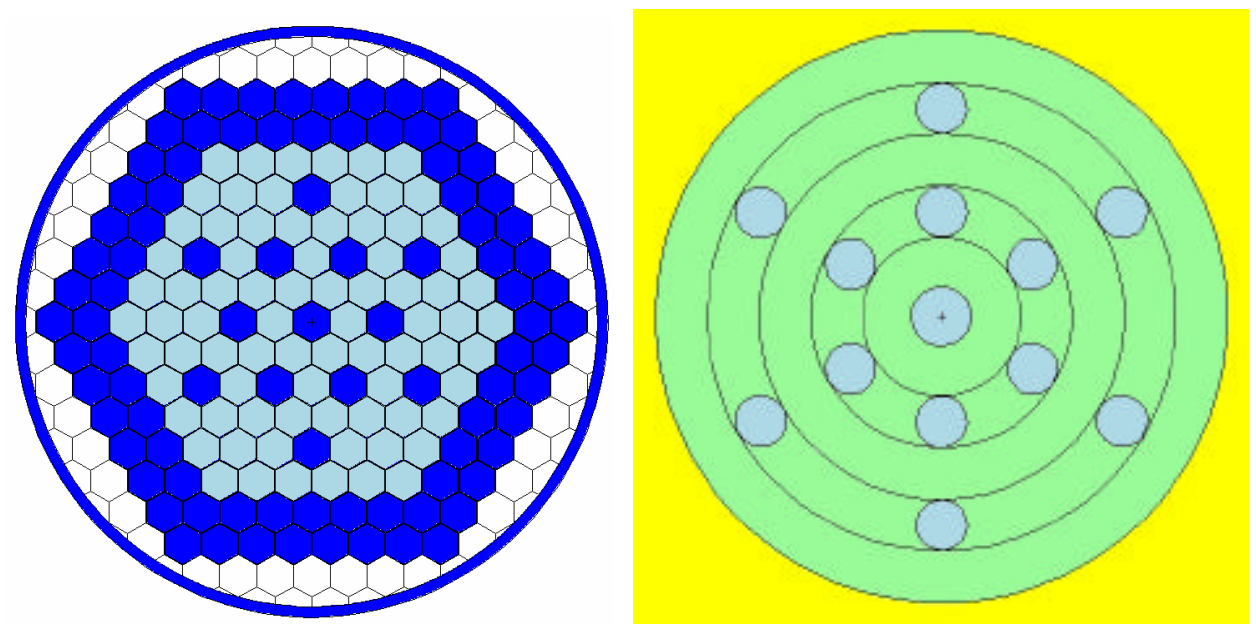

Figure 2-50. Core configuration with 13 control assemblies - actual geometry and simplified 3-D model.

Table 2-25. Total worth of all control assemblies.

\begin{tabular}{|c|c|c|}
\hline $\begin{array}{c}\text { Control } \\
\text { Assemblies }\end{array}$ & $\begin{array}{c}\text { BOL } \\
\mathrm{k}_{\text {eff }} \pm \mathrm{s}\end{array}$ & $\begin{array}{c}\text { ?k } \pm \mathrm{s} \\
\text { (relative to out case) }\end{array}$ \\
\hline Out & $1.09903 \pm 0.00052$ & \\
\hline In & $0.91154 \pm 0.00054$ & $-0.1875 \pm 0.0007$ \\
\hline
\end{tabular}

To evaluate the impact of variability in input parameters, several sensitivity analyses were performed. By examining Figure 2-49, density of $2.5 \mathrm{~g} / \mathrm{cm}^{3}$ represents the upper bound (e.g., it corresponds to $30 \%$ enriched boron in boron carbide at $100 \%$ theoretical density). Boron carbide with higher boron enrichment and $96 \%$ theoretical density with lower physical density of $2.25 \mathrm{~g} / \mathrm{cm}^{3}$ (10\% reduction) is selected as a reasonable lower bound. Total worth of control assemblies in both cases is given in Table 2-26. Reduction of the worth due to reduction in boron carbide density is relatively small, i.e., from $18.75 \%$ to $18.28 \%$ ?k.

Table 2-26. Change in total worth of all control assemblies due to variability in $\mathrm{B}_{4} \mathrm{C}$ density.

\begin{tabular}{|c|c|c|c|}
\hline $\begin{array}{c}\text { Control } \\
\text { Assemblies }\end{array}$ & $\begin{array}{c}?\left(\mathrm{~B}_{4} \mathrm{C}\right) \\
\mathrm{g} / \mathrm{cm}^{3}\end{array}$ & $\begin{array}{c}\mathrm{BOL} \\
\mathrm{k}_{\text {eff }} \pm \mathrm{s}\end{array}$ & $\begin{array}{c}? \mathrm{k} \pm \mathrm{s} \\
\text { (relative to out case) }\end{array}$ \\
\hline Out & 2.50 & $1.09903 \pm 0.00052$ & \\
\hline $\mathrm{In}$ & 2.25 & $0.91154 \pm 0.00054$ & $-0.1875 \pm 0.0007$ \\
\hline $\mathrm{In}$ & $\begin{array}{c}\text { Effect of } \mathrm{B}_{4} \mathrm{C} \\
\text { density reduction }\end{array}$ & $\begin{array}{c}\sim 3 \% \text { smaller } \\
\text { total worth }\end{array}$ \\
\hline
\end{tabular}


As stated, the basic case considers $50 \mathrm{vol} \% \mathrm{~B} 4 \mathrm{C}$ and $50 \mathrm{vol} \% \mathrm{Na}$. If necessary, a tighter lattice and a higher volume fraction of absorber rods may be achieved. Impact of increasing the absorber $\left(\mathrm{B}_{4} \mathrm{C}\right)$ volume fraction is examined in Table 2-27. Total worth is increased from $18.75 \%$ to $20.50 \%$ ?k, i.e., by $~ 9 \%$. Hence, some increase (but not very large) in worth may be achieved by tightening the absorber rod lattice.

Table 2-27. Change in total worth of all control assemblies due to variability in $\mathrm{B}_{4} \mathrm{C}$ volume fraction.

\begin{tabular}{|c|c|c|c|}
\hline $\begin{array}{c}\text { Control } \\
\text { Assemblies }\end{array}$ & $\begin{array}{c}\mathrm{B}_{4} \mathrm{C} \\
\text { vol\% }\end{array}$ & $\begin{array}{c}\mathrm{BOL} \\
\mathrm{k}_{\text {eff }} \pm \mathrm{s}\end{array}$ & $\begin{array}{c}\text { ?k } \pm \mathrm{s} \\
\text { (relative to out case) }\end{array}$ \\
\hline Out & & $1.09903 \pm 0.00052$ & \\
\hline In & $50 \%$ & $0.91154 \pm 0.00054$ & $-0.1875 \pm 0.0007$ \\
\hline In & $75 \%$ & $0.89394 \pm 0.00055$ & $-0.2050 \pm 0.0007$ \\
\hline & & $\begin{array}{c}\text { Effect of } \mathrm{B}_{4} \mathrm{C} \\
\text { vol\% increase }\end{array}$ & $\begin{array}{c}\sim 9 \% \text { larger } \\
\text { total worth }\end{array}$ \\
\hline
\end{tabular}

Another design variable of interest is boron enrichment. Table 2-28 presents results for three different ${ }^{10} \mathrm{~B}$ enrichments: $20 \%$ (natural), 50\%, and $90 \%$. Results are also graphically depicted in Figure 2-51. Increasing ${ }^{10} \mathrm{~B}$ enrichment from $20 \%$ (natural) to $90 \%$, increases reactivity worth by $\sim 50 \%$. Note that $50 \%$ enrichment provides reactivity worth almost comparable to $90 \%$, and it may offer an overall most economical option.

Table 2-28. Change in total worth of all control assemblies due to variability in ${ }^{10} \mathrm{~B}$ enrichment in $\mathrm{B}_{4} \mathrm{C}$

\begin{tabular}{|c|c|c|c|}
\hline $\begin{array}{c}\text { Control } \\
\text { Assemblies }\end{array}$ & ${ }^{10} \mathrm{~B}$ enrichment & $\begin{array}{c}\mathrm{BOL} \\
\mathrm{k}_{\mathrm{eff}} \pm \mathrm{s}\end{array}$ & $\begin{array}{c}\text { ? } \pm \mathrm{s} \\
\text { (relative to out case) }\end{array}$ \\
\hline Out & & $1.09903 \pm 0.00052$ & \\
\hline In & $20 \%$ & $0.97412 \pm 0.00057$ & $-0.1249 \pm 0.0007$ \\
\hline In & $50 \%$ & $0.93602 \pm 0.00051$ & $-0.1630 \pm 0.0007$ \\
\hline In & $90 \%$ & $0.91154 \pm 0.00054$ & $-0.1875 \pm 0.0007$ \\
\hline & & Effect of ${ }^{10} \mathrm{~B}$ enrichment & $\begin{array}{c}\text { Up to } ~ 50 \% \text { larger total } \\
\text { worth for } 90 \% \text { enriched }\end{array}$ \\
\hline
\end{tabular}




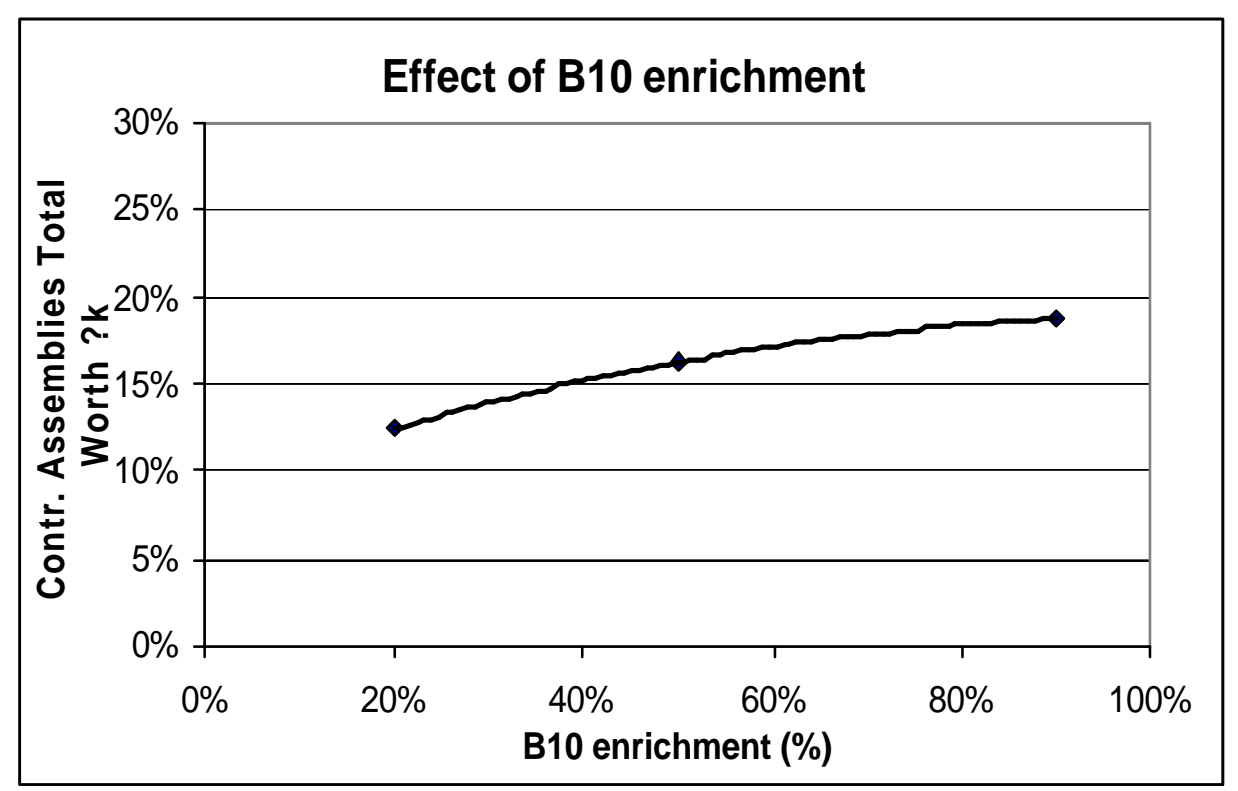

Figure 2-51. Change in total worth of all control assemblies due to variability in ${ }^{10} \mathrm{~B}$ enrichment in $\mathrm{B}_{4} \mathrm{C}$.

Worth of individual control assembly banks was also established. For that purpose, each bank (group) was inserted separately. Table 2-29 presents results, in terms of the bank worth and "per control assembly" worth.

Table 2-29. Reactivity worth of individual control rod banks.

\begin{tabular}{|c|c|c|c|c|}
\hline $\begin{array}{c}\text { Control } \\
\text { Assemblies }\end{array}$ & $\begin{array}{c}\text { No. of } \\
\text { CRs }\end{array}$ & BOL $\mathrm{k}_{\text {eff }}$ & Bank worth ?k & $\begin{array}{c}\text { Bank worth } \\
\text { ?k per CR }\end{array}$ \\
\hline Out & & 1.09903 & & \\
\hline Bank 1 In & 1 & 1.07242 & $2.66 \%$ & $2.7 \%$ \\
\hline Bank 2 In & 6 & 0.99513 & $10.39 \%$ & $1.7 \%$ \\
\hline Bank 3 In & 6 & 1.03502 & $6.40 \%$ & $1.1 \%$ \\
\hline All Banks In & 13 & 0.91154 & $18.75 \%$ & $1.4 \%$ \\
\hline
\end{tabular}

Variability in the control assembly worth seems acceptable (1.1-2.7\% per assembly, with an average value of $1.4 \%$ ). Since full insertion was considered in all cases, axial core-wide power shape profiles in all cases are very similar, as demonstrated in Figure 2-52. 


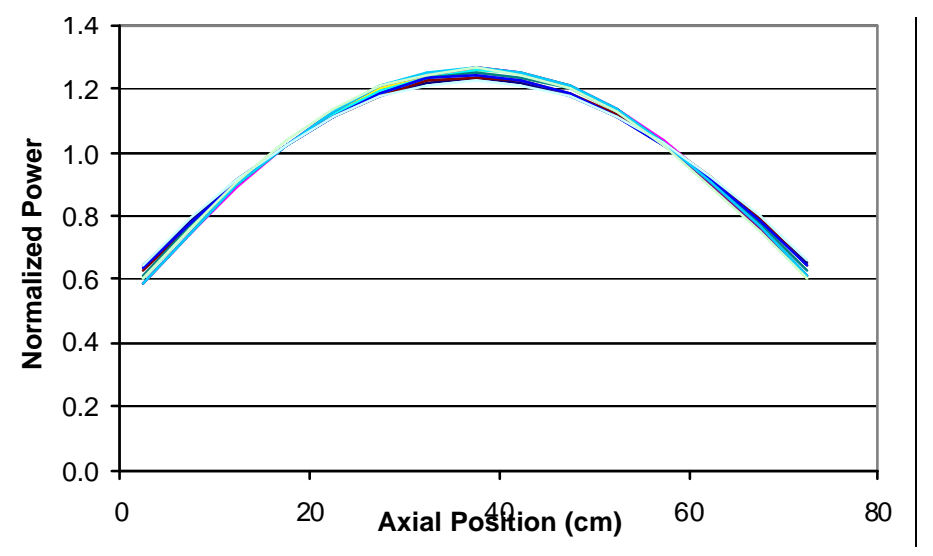

Figure 2-52. Axial power profiles.

Radial power distribution is impacted more significantly than axial distribution by insertion of control assemblies, as shown in Figure 2-53.a-e, which depict normalized radial power shapes for the five cases given in Table 2-29 (all banks out, Bank 1 in, Bank 2 in, Bank 3 in, all banks in). The radial peaking factor, however, seems to be acceptable in all cases, ranging fom 1.2 to 1.8. Note that a single control assembly occupies the central core location (whether inserted or withdrawn), hence, power density in the core ring 1 is always zero.

The required reactivity control capability may be estimated as follows. The reactivity swing due to depletion amounts to $\sim 11.5 \%$ ?k to achieve 15 years core lifetime with $(\mathrm{U}, \mathrm{Pu}) \mathrm{N}$ fuel of reference isotopic composition, as examined in section 2.2.2. This swing accounts for the main portion of the total reactivity control requirement. Therefore, a very crude estimate for the remaining reactivity components is sufficient, and it should cover geometry (expansion), temperature (Doppler), shutdown capability under all conditions, plus some margin. Considering a typical fast reactor design and characteristics (see e.g., [Wirtz71] and [Golden69]) one can estimate that the combined reactivity worth of these components may amount to some $3-6 \%$ ?k. Thus, the total estimated control requirement is in the $14-18 \%$ range.

If a more detailed analysis shows that the control requirement is closer to the lower side $(\sim 14 \%)$, the examined configuration with $13 \mathrm{CRs}$ should be sufficient. However, if the actual requirement is closer to the upper estimate $(\sim 18 \%)$, then the configuration with $13 \mathrm{CRs}$ may not be sufficient. And even if they are just sufficient, it may be desirable to provide some operational flexibility by introducing additional CRs. A configuration with 19 CRs (with six CRs added to the fifth ring) is shown in Figure 2-54, together with the corresponding simplified model. 


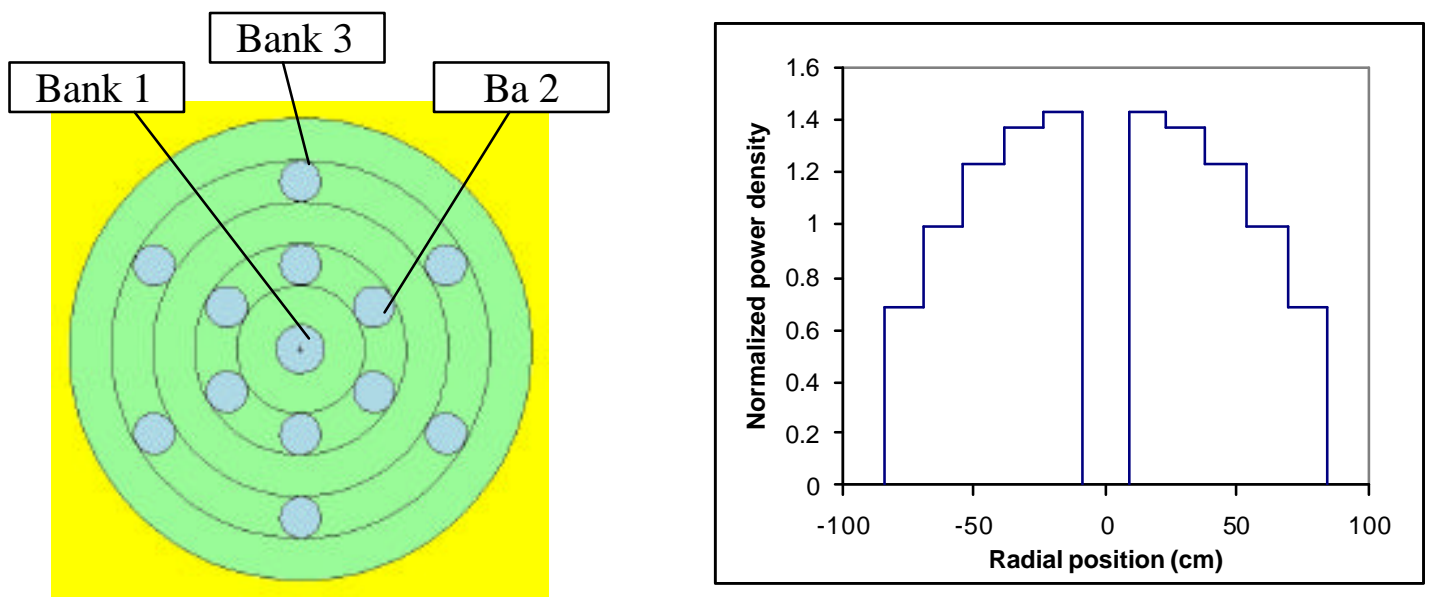

(a) All control banks out

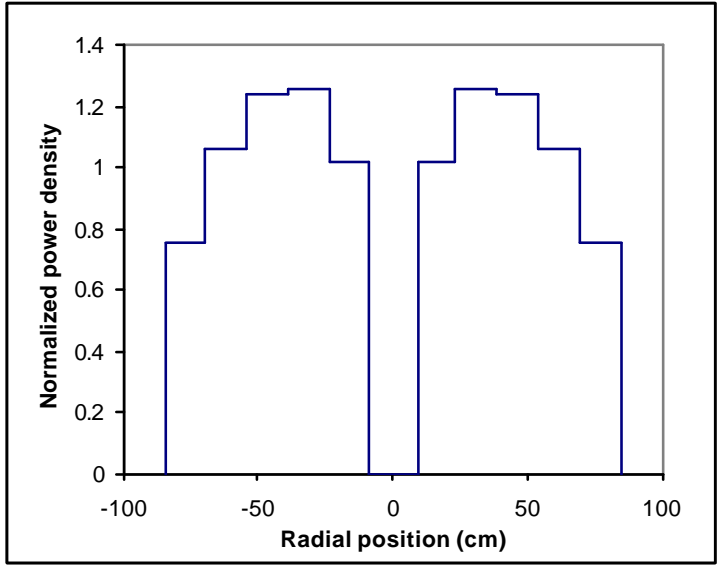

(b) Bank 1 in

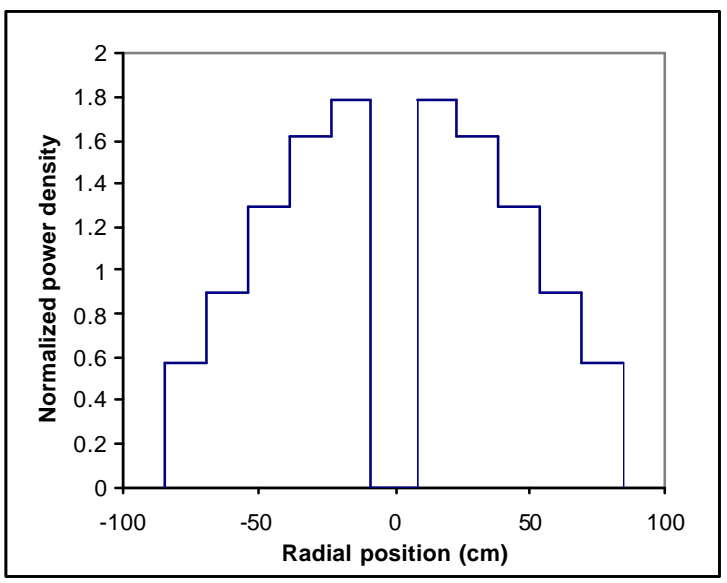

(d) Bank 3 in

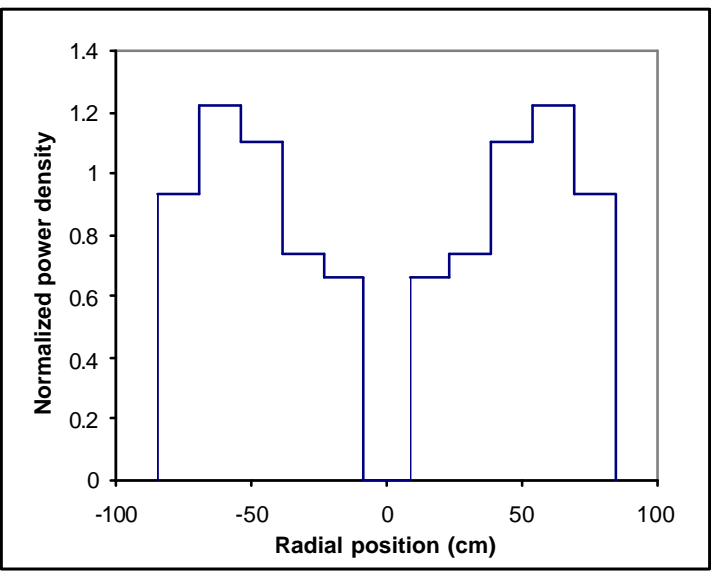

(c) Bank 2 in

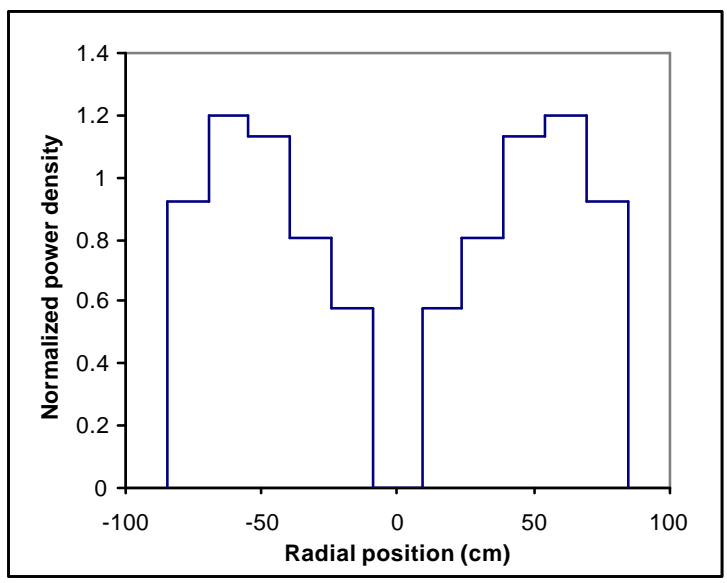

(e) All banks in

Figure 2-53. Radial normalized power profiles for different positions of control banks. 

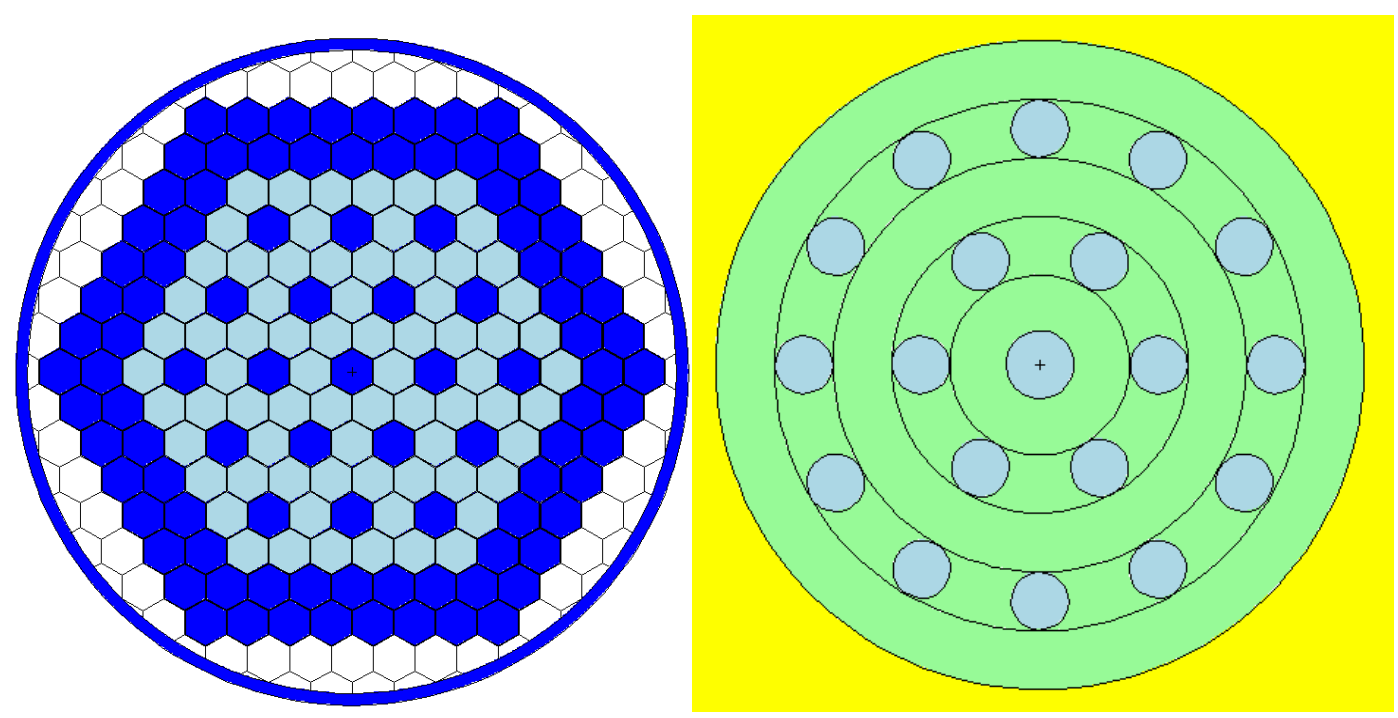

Figure 2-54. Core configuration with 19 control assemblies - actual geometry and simplified 3-D model.

A simplistic approach to estimating the total CR worth of the $19 \mathrm{CR}$ core, is to consider the ratio between the number of CRs and fuel assemblies (note that the number of fuel assemblies is reduced from 78 to 72 ). This would provide the following reactivity worth estimate:

$$
\Delta k(19 \mathrm{CR})=\Delta k(13 \mathrm{CR}) \cdot\left[\frac{19 / 72}{13 / 78}\right]=18.75 \% \cdot 1.583=29.7 \%
$$

Actual calculations result in a slightly lower value, $28.14 \%$. This still provides a margin sufficient to accommodate currently existing design uncertainties, and potentially enable reducing the ${ }^{10} \mathrm{~B}$ enrichment level in CRs to $\sim 50 \%$.

In summary, Boron Carbide is selected as the $\mathrm{CR}$ absorber material. Reference design considers $90 \%$ boron enrichment and 50 vol\% of $\mathrm{B}_{4} \mathrm{C}$ in control assemblies. Several sensitivity studies were performed to evaluate the impact of design modifications (e.g., absorber volume fraction, boron enrichment). The core design uses $13 \mathrm{CRs}$ organized in 3 banks of $1 \mathrm{CR}, 6 \mathrm{CRs}$ and $6 \mathrm{CRs}$, respectively, with option to increase the total number of CRs to 19 . More detailed studies will be required to investigate $13 \mathrm{Crs}$ configuration providing acceptable margin both during normal operational and accident conditions. Power distribution was also examined for different bank insertions and the peaking factors were found to be acceptable. Overall, the considered CR design seems to provide an adequate reactivity control capability. 


\subsubsection{Sodium Void Effects}

One important safety aspect of a LMFBR is its reactivity behavior upon sodium removal, i.e., sodium void effects. In general, these effects may be evaluated by considering sodium reactivity coefficients as well as the sodium void worth. In the subsequent analysis two cases were studied:

1. A hypothetical worst-case scenario of local boiling within the core only (while assuming that neutron leakage does not increase).

2. A more realistic scenario of voiding of the upper core half and the sodium above the core.

\subsubsection{Local Boiling in the Core - Limiting Case}

As a limiting worst-case scenario, we assume that the whole core is voided (e.g., due to blockage and boiling), yet, at the same time we assume that all neutron reflectors (both radial and axial) remain intact. Five variations of the basic core model were developed with five different sodium densities to represent voiding from $0 \%$ to $100 \%$. Table $2-30$ lists the corresponding Monte Carlo simulations. The variation of the reactivity with respect to the void fraction is also shown in Figure 2-55.

Table 2-30. Change in core reactivity due to central core voiding.

\begin{tabular}{|c|c|c|c|}
\hline Sodium Void & $\begin{array}{c}\text { Sodium density } \\
\left(\mathrm{g} / \mathrm{cm}^{3}\right)\end{array}$ & $\begin{array}{c}\text { BOL } \\
\mathrm{k}_{\text {eff }} \pm \mathrm{s}\end{array}$ & $\begin{array}{c}\text { ?k } \pm \mathrm{s} \\
\text { (relative to A42) }\end{array}$ \\
\hline $0 \%$ (reference) & 0.78 & $1.11210 \pm 0.00099$ & \\
\hline$\sim 10 \%$ & 0.70 & $1.11505 \pm 0.00096$ & $-0.0029 \pm 0.0014$ \\
\hline $50 \%$ & 0.39 & $1.12098 \pm 0.00077$ & $-0.0089 \pm 0.0013$ \\
\hline$\sim 90 \%$ & 0.08 & $1.12701 \pm 0.00065$ & $-0.00149 \pm 0.0013$ \\
\hline $99.9 \%$ & 0.00079 & $1.12746 \pm 0.00079$ & $-0.00154 \pm 0.0013$ \\
\hline
\end{tabular}




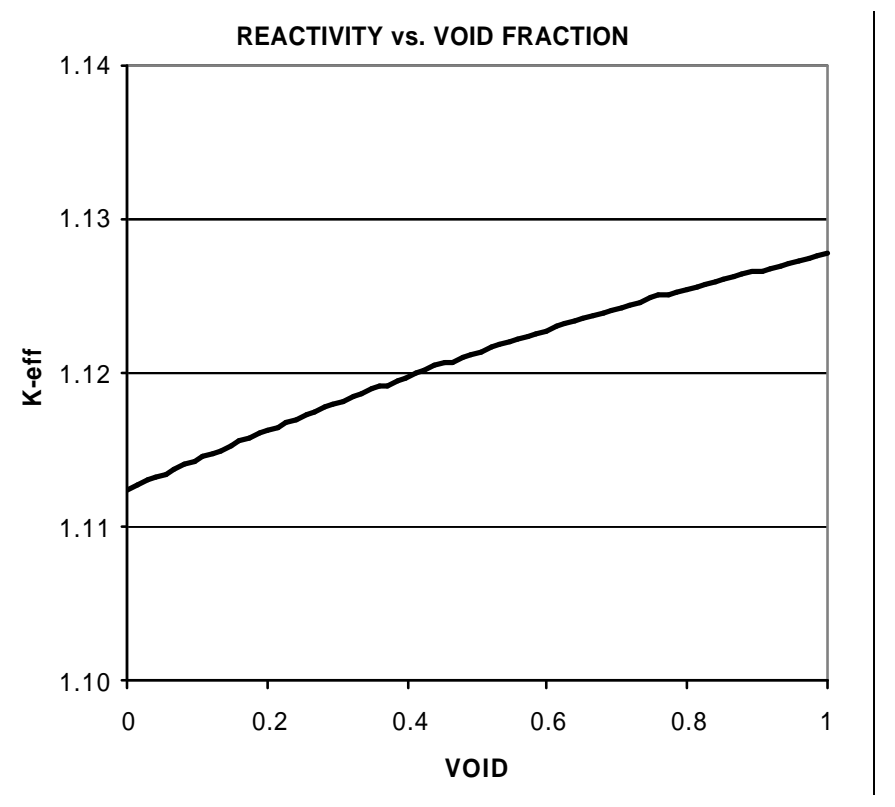

Figure 2-55. Reactivity vs. void fraction for central (whole core) voiding.

The sodium void worth (from $0 \%$ to $100 \%$ core-only void) amounts to ?k $\cong 1.5 \%$. However, sodium in the whole core cannot boil-off instantaneously and there is sufficient time to insert control rods to compensate for this reactivity increase. Hence, without any further detailed analysis, we may address the sodium void worth by requiring an extra $2 \%$ of control rod worth to be available. This amount was considered in previous section when determining the total CR worth requirements and moreover, no credit for Doppler was taken in this analysis.

\subsubsection{Core Voiding/Boiling - A More Realistic Case}

This sub-section analyzes sodium voiding under a more realistic scenario. It was assumed that the core upper half and sodium above the core is voided; while the sodium in the lower half, below the core, and in the radial reflector remains as-is. Even this is somewhat conservative, since eventually sodium will be removed from the radial reflector as well, further increasing neutron leakage and reducing the reactivity. To represent this scenario, it was necessary to develop a more realistic and more detailed MCNP geometrical model. In particular, this new model represents the core (fuel rings with CRs), radial reflector, radial zones (core barrel, sodium flow path), bottom structure of fuel elements, fission gas plenum, lower core support plate, top plate, and sodium above and below the core. Sodium density is axially varying. Three different densities were employed (below the core, core, above the core). Side-view of the model is shown in Figure 2-56. 


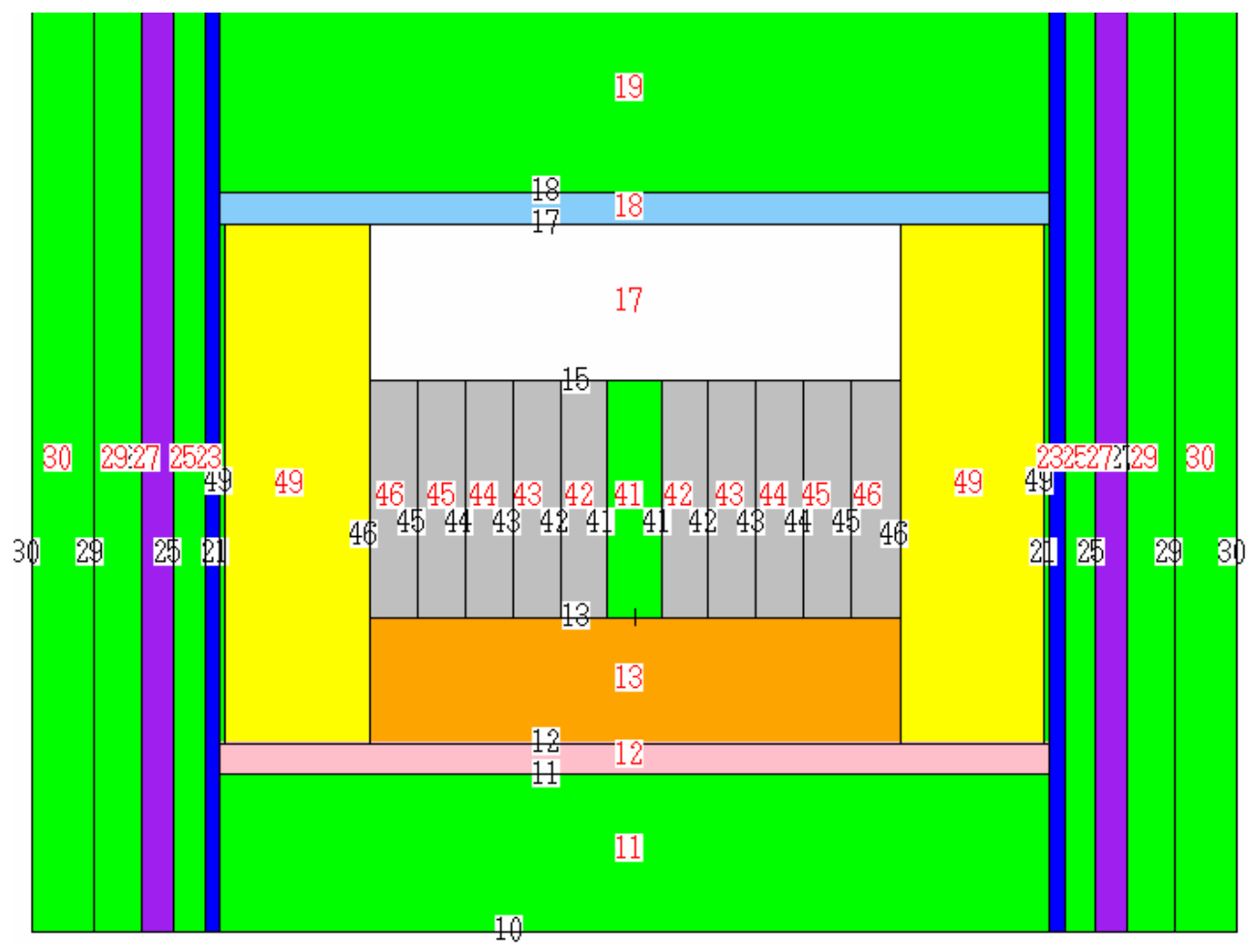

Figure 2-56. Core model of the LMR-AMTEC.

Eleven Monte Carlo models were developed to represent the sodium density change in the upper core half and above the core, corresponding to the voiding from $0 \%$ to $100 \%$. The corresponding MCNP runs are identified in Table 2-31.

These runs produced core reactivity vs. void fraction dependence as shown in Table 2-32. The reactivity change over the whole range is relatively small, and comparable in magnitude to statistical noise. To reduce these noise effects, the same eleven simulations were repeated with a different random number seed. Two sets of results (clearly demonstrating statistical noise) are shown in Figure 2-57. The reactivity values were then averaged over the two runs and additionally over two void points, each. These reduced data are shown in Figure 2-58, together with a fitted trend line; this curve reveals the core physics behavior. The maximum reactivity change (difference between the minimum and maximum reactivity in the whole range) is not more than $\sim 0.3 \%$, i.e., less than the delayed neutron fraction. Hence, it may be effectively controlled; moreover, it will also be counteracted by Doppler, which was not accounted in this analysis. It may be observed that the void reactivity coefficient is initially positive, however, when the void fraction reaches $\sim 60 \%$, it becomes negative. Again, no Doppler has been accounted for in this analysis. 
Table 2-31. MCNP runs performed to examine voiding in the upper core half.

\begin{tabular}{|c|c|c|c|c|}
\hline & \multicolumn{4}{|c|}{ Sodium density $\left(\mathrm{g} / \mathrm{cm}^{3}\right)$} \\
\hline Sodium Void & Below core & Core lower & Core upper & Plenum \\
\hline $\mathbf{0 \%}$ & 0.830 & 0.780 & 0.780 & 0.730 \\
\hline $\mathbf{1 0 \%}$ & 0.830 & 0.780 & 0.702 & 0.657 \\
\hline $\mathbf{2 0 \%}$ & 0.830 & 0.780 & 0.624 & 0.584 \\
\hline $\mathbf{3 0 \%}$ & 0.830 & 0.780 & 0.546 & 0.511 \\
\hline $\mathbf{4 0 \%}$ & 0.830 & 0.780 & 0.468 & 0.438 \\
\hline $\mathbf{5 0 \%}$ & 0.830 & 0.780 & 0.390 & 0.365 \\
\hline $\mathbf{6 0 \%}$ & 0.830 & 0.780 & 0.312 & 0.292 \\
\hline $\mathbf{7 0 \%}$ & 0.830 & 0.780 & 0.234 & 0.219 \\
\hline $\mathbf{8 0 \%}$ & 0.830 & 0.780 & 0.156 & 0.146 \\
\hline $\mathbf{9 0 \%}$ & 0.830 & 0.780 & 0.078 & 0.073 \\
\hline $\mathbf{1 0 0 \%}$ & 0.830 & 0.780 & 0 & 0 \\
\hline
\end{tabular}

Table 2-32. Change in core reactivity due to voiding of the core upper half and above.

\begin{tabular}{|c|c|c|}
\hline Sodium Void & BOL $\mathrm{k}_{\text {eff }} \pm \mathrm{s}$ & $\begin{array}{c}\text { ?k } \pm \mathrm{s} \\
\text { (relative to 0\%) }\end{array}$ \\
\hline $\mathbf{0 \%}$ & $1.09924 \pm 0.00069$ & \\
\hline $\mathbf{1 0 \%}$ & $1.09921 \pm 0.00053$ & $-0.0000 \pm 0.0009$ \\
\hline $\mathbf{2 0 \%}$ & $1.10023 \pm 0.00053$ & $0.0010 \pm 0.0009$ \\
\hline $\mathbf{3 0 \%}$ & $1.10060 \pm 0.00060$ & $0.0014 \pm 0.0009$ \\
\hline $\mathbf{4 0 \%}$ & $1.10155 \pm 0.00055$ & $0.0023 \pm 0.0009$ \\
\hline $\mathbf{5 0 \%}$ & $1.10145 \pm 0.00063$ & $0.0022 \pm 0.0009$ \\
\hline $\mathbf{6 0 \%}$ & $1.10160 \pm 0.00055$ & $0.0024 \pm 0.0009$ \\
\hline $\mathbf{7 0 \%}$ & $1.10072 \pm 0.00060$ & $0.0015 \pm 0.0009$ \\
\hline $\mathbf{8 0 \%}$ & $1.10101 \pm 0.00059$ & $0.0018 \pm 0.0009$ \\
\hline $\mathbf{9 0 \%}$ & $1.10040 \pm 0.00062$ & $0.0012 \pm 0.0009$ \\
\hline $\mathbf{1 0 0 \%}$ & $1.10121 \pm 0.00051$ & $0.0020 \pm 0.0009$ \\
\hline
\end{tabular}




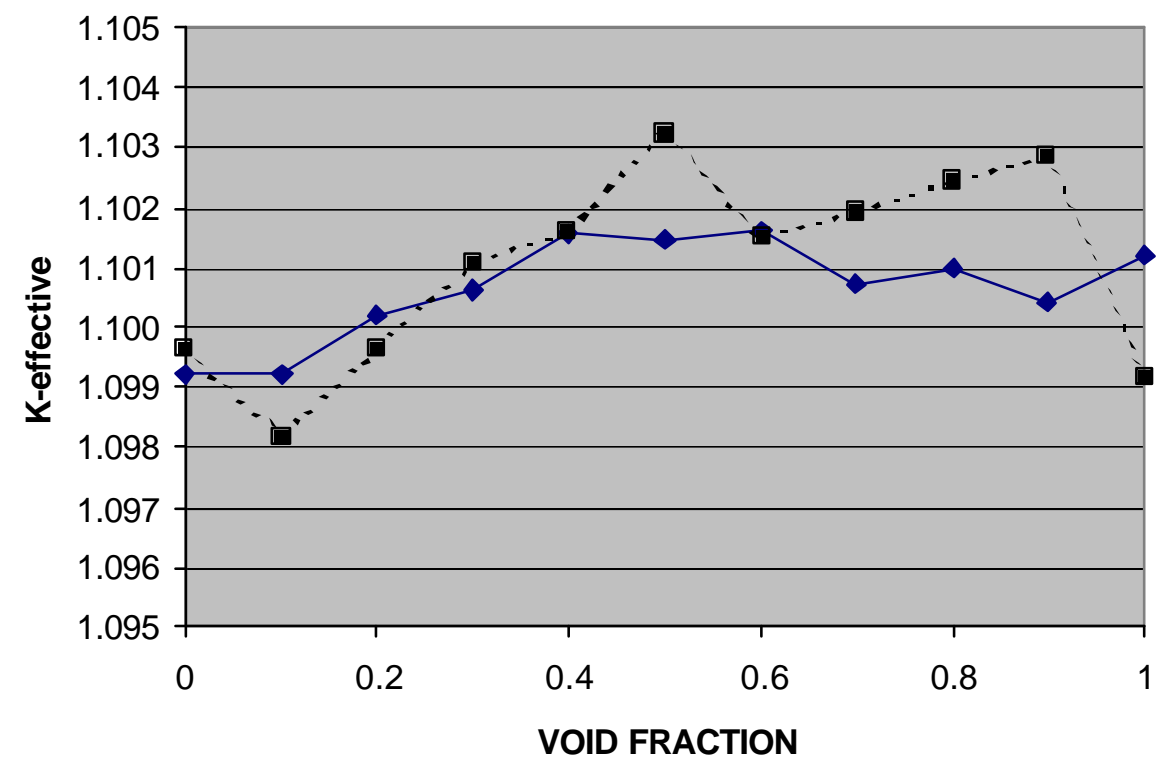

Figure 2-57. Change in core reactivity due to voiding of the core upper half and above (two sets of MCNP runs shown).

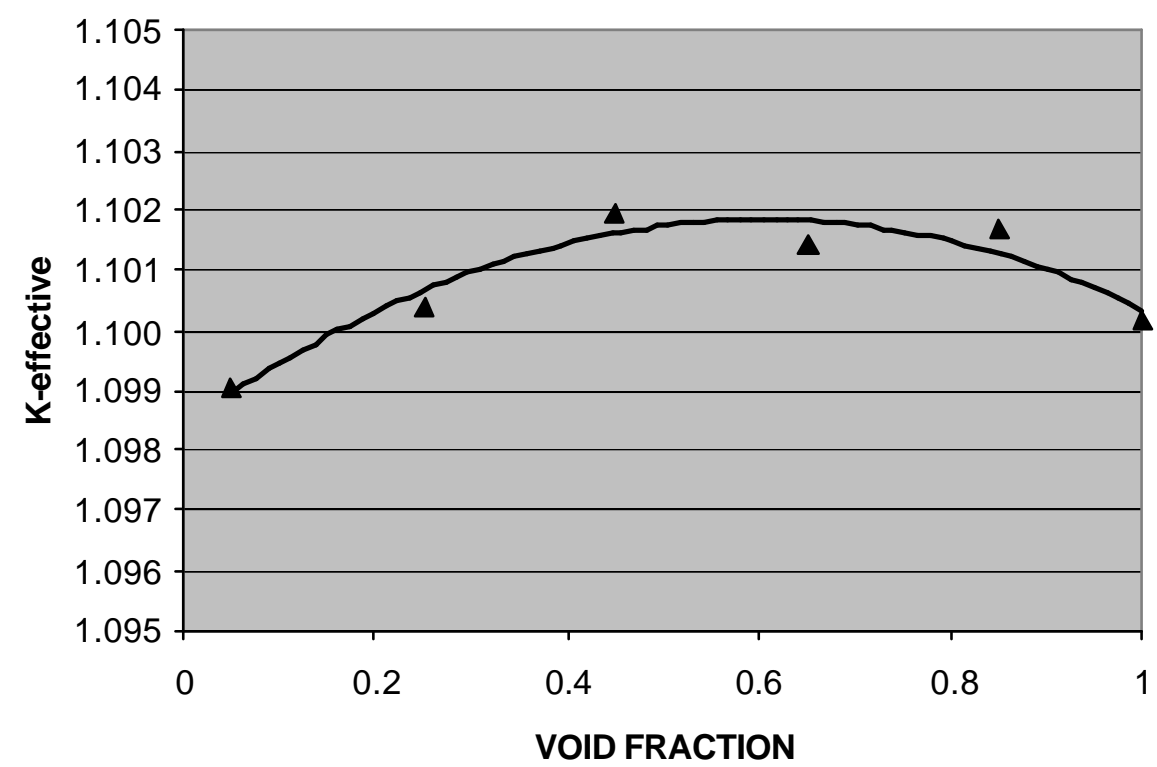

Figure 2-58. Change in core reactivity due to voiding of the core upper half and above (smoothed results).

In summary, the two considered cases (hypothetical central core voiding and a more realistic case of upper core voiding) demonstrated acceptable core reactivity response under sodium voiding scenarios. Additionally, Doppler effect (which was not included in this analysis) will make reactivity coefficients more negative and further improve the core response. 


\subsubsection{Decay Heat Removal System of the LMR-AMTEC}

\subsubsection{Decay Removal Systems Description}

Due to the elevated working temperatures of the LMR-AMTEC and the material integrity limits, the decay heat removal systems must be designed to avoid an excessive reactor temperature increase. For the present design, a Power conversion system (PCS) concept is chosen as a non-safety grade Decay Heat Removal System (DHRS) during hot shutdown. As an emergency DHRS, a safety-grade Passive Heat Removal System (PHRS) based on a Direct Reactor Auxiliary Cooling System (DRACS) concept is selected. In summary, the DHRS for the LMR-AMTEC are:

- Power Conversion System (PCS): operates in forced convection as a normal nonsafety DHRS during hot and cold shutdown.

- Passive Heat Removal System (PHRS): operates passive in natural convection as an emergency safety degree DHRS during hot and cold shutdowns.

Eventually, a Primary Auxiliary Cooling System (PACS) could be considered as an alternative non-safety DHRS when the PCS are not available for cold shutdown. This concept provides cooling from the reactor vessel using interconnections with other auxiliary systems with heat rejection capability like the cold trap. In addition, one of the three loops of the PHRS could be designed to work also in forced circulation (using EM pumps). The connection of the PHRS and the PCS of the LMR-AMTEC is shown in Figure 2-59.

Figure 2-59 also presents other LMR-AMTEC auxiliary systems not directly related to the decay removal systems: The cold traps and the Water Cooling Guard Vessel System (WCGVS). The WCGVS of the LMR-AMTEC (see section 3.3) is designed to assure the mechanical integrity of the guard vessel in the unlikely event that the reactor vessel was to leak.

Next section describes the PHRS system proposed for the LMR-AMTEC and analyzes its performance during an assumed transient. 


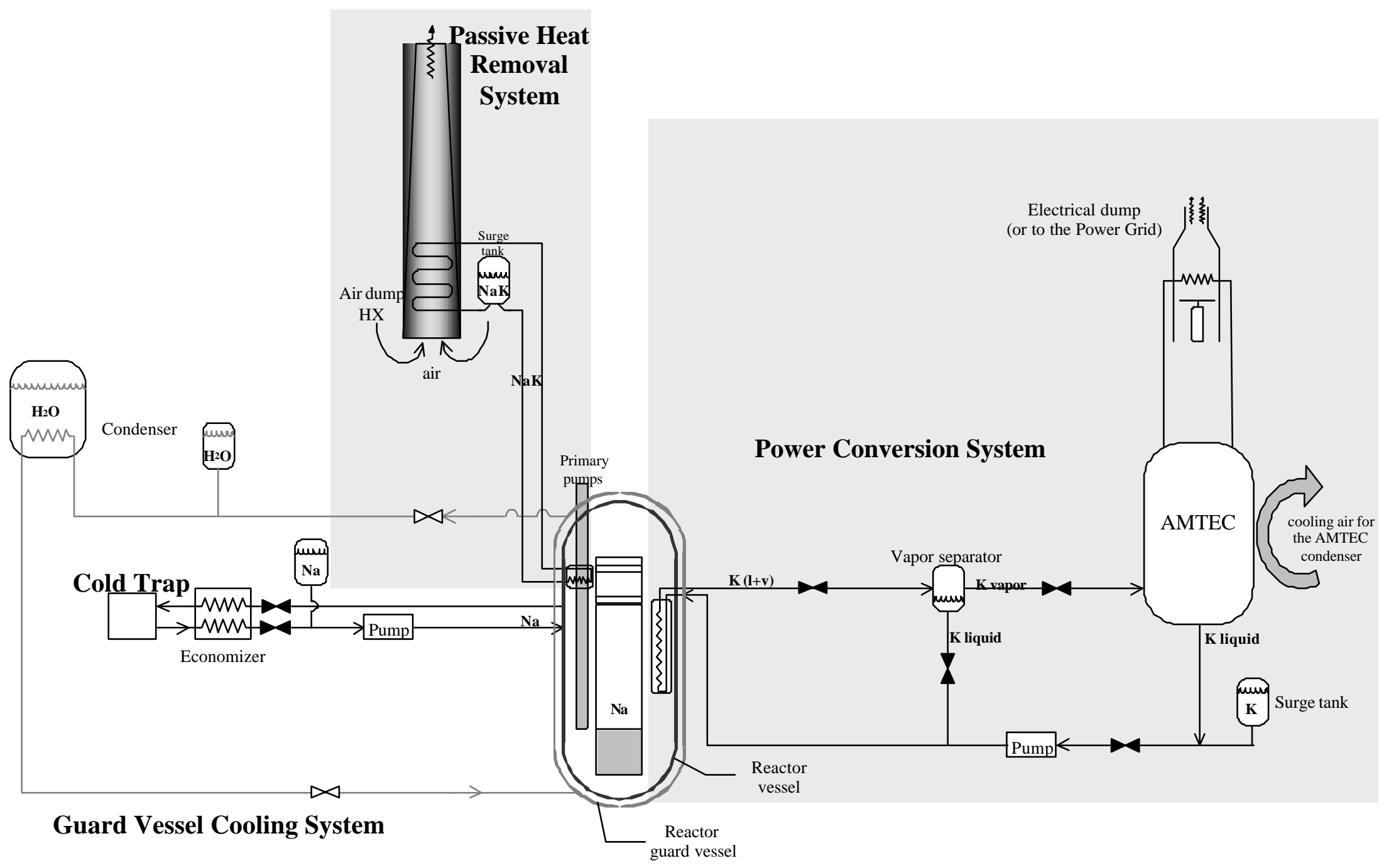

Figure 2-59. Power Conversion System, Passive Heat Removal Systems (PHRS), Water Cooling Guard Vessel System (WCGVS) and primary Cold Trap System. 


\subsubsection{Passive Heat Removal System (PHRS) of the LMR-AMTEC}

A DRACS concept has been adopted as a passive heat removal system. However, the traditional designs of DRACS [Braquilanges85] require a coolant temperature increase, which is not suitable for the LMR-AMTEC. This temperature increase is needed to ensure that the HX will be submerged due to the thermal expansion of the sodium coolant. As an example, in the present design, increasing the sodium level by about 40 $\mathrm{cm}$ will require an increase of about $300 \mathrm{~K}$. This might surpass the material temperature limits. For this reason another mechanism to start the heat removal is proposed here.

The proposed PHRS houses the HX in the cavity formed by the pump shaft and the external shaft shell (see Figure 2-60). This concept uses the variations of the sodium levels as mechanism to initiate the heat removal. As seen in Figure 2-60, during normal operation the sodium level inside the shaft cavity is lower than in the riser or in the annulus (between the riser and the reactor vessel). These differences on the coolant level are originated by the pressure losses in the AMBs and in the core, and in a less degree, by the average coolant temperature differences.

As soon as the pumps are stopped, the primary coolant flow and friction losses are reduced and the sodium levels tend to equalize. Particularly, the sodium level will increase inside the pump cavity submerging the EDRS HX. On the other hand, since during normal operation the sodium level in the annulus region is higher than that of the riser, the level will also increase in the last one. This will allow the coolant to reach orifice 2, thus establishing a natural circulation path through the EDRS HX, the pump cavity, the down-comer, the core and the riser. As the AMBs are not a concern in this natural convection path, theirs friction losses are avoided.

The level variation in the pump cavity allows to place a helix shape tubes HX of about $40 \mathrm{~cm}$. The helical HX characteristics are shown in Table 2-33. The heat transferred to the HXs of the PHRS is then transported by a mixture of Na-K to an air dump heat exchanger. The air dump HXs are supposed to be cooled by natural circulation air. A mixture of $25 \% \mathrm{Na}$ and $75 \% \mathrm{~K}$ is chosen as coolant for the PHRS because of its chemical compatibility with $\mathrm{Na}$ and its low solidification temperature $(262 \mathrm{~K})$.

Table 2-33. Principal parameters of the helix HX's of the PHRS.

\begin{tabular}{|l|r|l|r|}
\hline External tube diameter & $1.0 \mathrm{~cm}$ & Height of HX & $40 \mathrm{~cm}$ \\
\hline Internal tube diameter & $0.9 \mathrm{~cm}$ & Number of tube (per HX) & 35 \\
\hline Pitch to diameter ratio & 2.2 & Number of helix & 14 \\
\hline Average tube length & $2.35 \mathrm{~m}$ & Number of HX & 4 \\
\hline
\end{tabular}




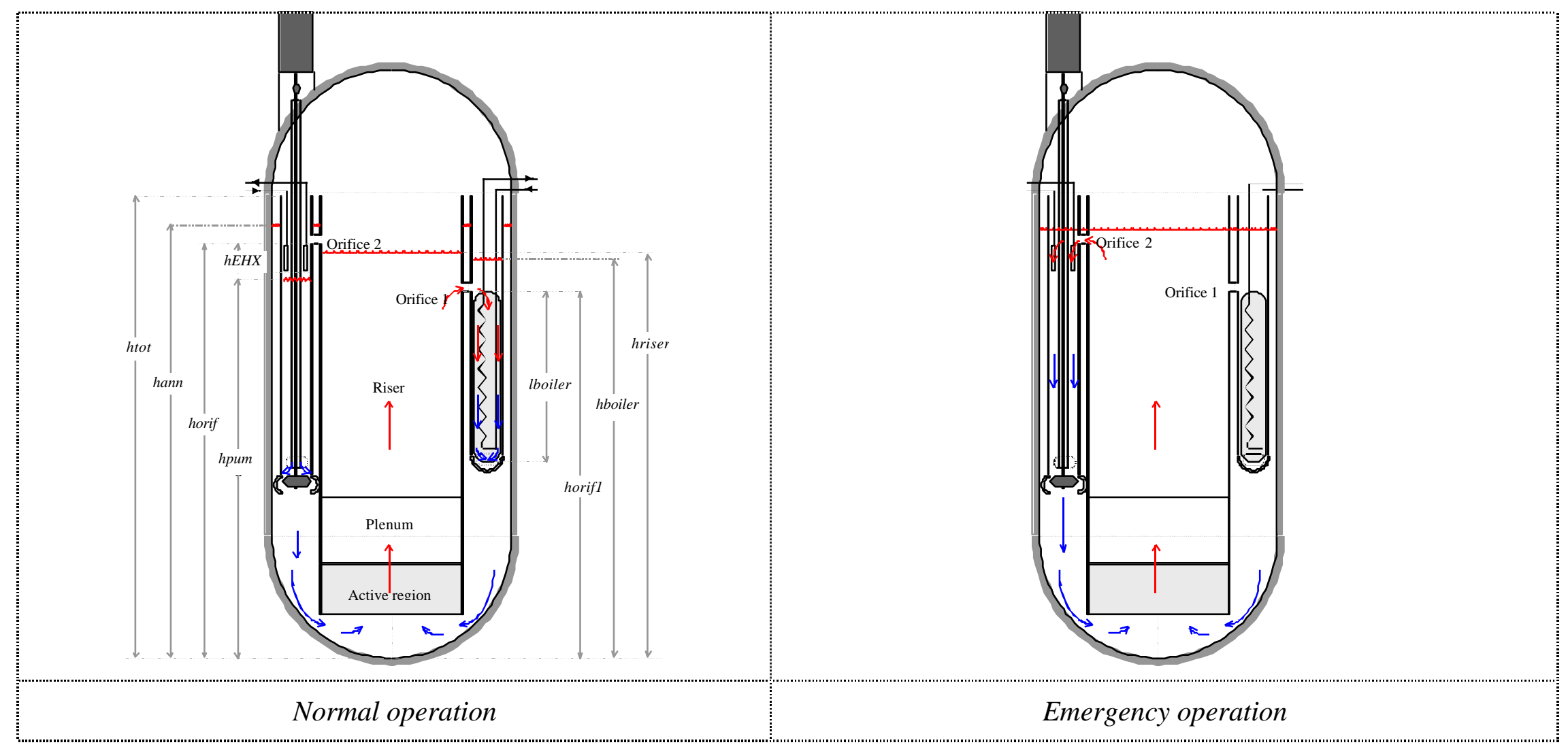

\begin{tabular}{|l|c|c|c|}
\hline Sodium level & $\begin{array}{c}\text { Normal } \\
\text { operation }\end{array}$ & $\begin{array}{c}\text { Emergency operation } \\
\text { (at the beginning) }\end{array}$ & $\begin{array}{c}\text { Emergency operation } \\
\text { (at the peak of temperature) }\end{array}$ \\
\hline Annulus region (hannu) & $7.45 \mathrm{~m}$ & $6.82 \mathrm{~m}$ & $7.08 \mathrm{~m}$ \\
\hline Riser region (hriser) & $6.41 \mathrm{~m}$ & $6.82 \mathrm{~m}$ & $7.08 \mathrm{~m}$ \\
\hline Boiler region (hboiler) & $6.36 \mathrm{~m}$ & $6.82 \mathrm{~m}$ & $7.08 \mathrm{~m}$ \\
\hline Pumps region (hpump) & $6.26 \mathrm{~m}$ & $6.82 \mathrm{~m}$ & $\mathrm{~m}$ \\
\hline
\end{tabular}

\begin{tabular}{|l|l|c|}
\hline Orifice 1 & horif 1 & $6.12 \mathrm{~m}$ \\
\hline Orifice 2 & horif2 & $6.52 \mathrm{~m}$ \\
\hline support & htot & $7.52 \mathrm{~m}$ \\
\hline height of EHX & lEHX & $40 \mathrm{~cm}$ \\
\hline
\end{tabular}

Figure 2-60. Sodium levels during normal reactor operation and during emergency decay heat removal. Sizes of the main components of the PHRS. 
The position (with respect to the bottom of the reactor vessel) of the components of the EHRS are shown in Figure 2-60. The level of the Orifice 1 (for normal operation), Orifice 2 (for decay heat removal) and the external shaft shell were dimensioned to optimize the sodium level variation during the transient, thus increasing the available space for the HXs. The sodium levels (reported in the table attached to Figure 2-60) were calculated accounting for the temperature and flow characteristics during normal and emergency operation, and the LMR-AMTEC geometric parameters. The temperature conditions at the beginning of the emergency operation and at the peak of temperature, $1050 \mathrm{~K}$ and $1223 \mathrm{~K}$ respectively, were determined from the transient model results (see Figure 2-64).

\subsubsection{Transient Response of the PHRS}

\subsection{The PHRS Model}

The performance of the PHRS was evaluated using the model schematized in Figure 2-61. This model estimates the temperatures of the fuel (centerline and for the hot channel), cladding, core, riser, HXs, down comer and in the air dumps HXs. Mass flow rates for the primary and the PHRS coolants are also determined. The main characteristics of the model are:

- Modeling of the natural convection transient of two loops coupled by a heat exchanger. Loop-1 represents the primary side components and loop-2 the PHRS loop.

- The reactor components including core, pumps, helical HX of the PHRS, air dumps HXs, pipes, etc. are modeled using lumped equations for the mass, momentum and energy balances.

- The primary coolant (sodium) flows through the shell side of the helical HX of the PHRS while the Na-K flows through the tube side. The wall and coolant temperatures (both in tube and shell side) are calculated. A heat transfer correlation for oblique flow of liquid metals through tube banks is used in the shell side. For friction losses, a correlation developed for oblique flow of water through tube banks is used (water friction correlation can be used for liquid metals).

- A pipe model is used in modeling the riser, the down-comer and the pipes of the PHRS. The pipe model consists of a pure delay $\tau$ on the fluid property (such as temperature or enthalpy) transmitted through the pipe:

$$
F_{\text {out }}(t)=F_{\text {in }}(t-\tau)
$$

where $F_{\text {out }}$ is any fluid property at the outlet of the pipe and $\tau$ is the transition time of the fluid calculated according to the pipe dimensions and the flow rate history. The buoyancy contribution of the pipe is calculated as function of the average fluid density and the effective pipe height. The friction losses are estimated using the standard correlations and the pipe length.

- The power released in the core corresponds to the nominal thermal power before initiation of the rod insertion. After shutdown (rods completely inserted) nuclear 
decay heat curves similar to the CRBR fuel elements after a full cycle is assumed. Between the beginning and the end of the rod insertion, a linear interpolation of the nominal power and the decay heat power is used.

- The fuel properties such as conductivity or heat capacity are calculated assuming a porosity of 0.1 and a $19.95 \%$ content of $\mathrm{Pu}$. Both parameters can strongly affect the centerline fuel temperature as shown in section 2.2.4.

- The flow coast-down after pump trip is modeled using the following equation:

$$
\frac{\dot{m}(t)}{\dot{m}_{o}} \cong \frac{1}{1+\beta \cdot t}
$$

where $\dot{m}_{o}$ is the initial primary mass flow rate. Core flow response depends on the flow distribution and pump characteristics. The value of $\beta$ for a loss of electric power is different from that for a pump seizure; somewhat quicker flow decay occurs for the later. Typical values for $\beta$ when the pumps are tripped are in the range of $0.2 \mathrm{~s}^{-1}$ to $0.5 \mathrm{~s}^{-1}$.

- After the sodium level reaches the 2 orifice of the riser (see Figure 2-60), the helical HX of the PHRS are activated and natural circulation flow can be established in the primary side and in the PHRS loop. The sodium level in the riser depends on the friction losses, i.e. on the flow rate. Therefore the model activates the heat transfer through the helical HX only below a critical core flow rate. The critical value of the core flow rate was estimated conservatively equal to $20 \%$ of the initial flow rate.

- Before the activation of the helical HX, the primary coolant flows through the AMBs. No heat transfer to the boilers is allowed during this period.

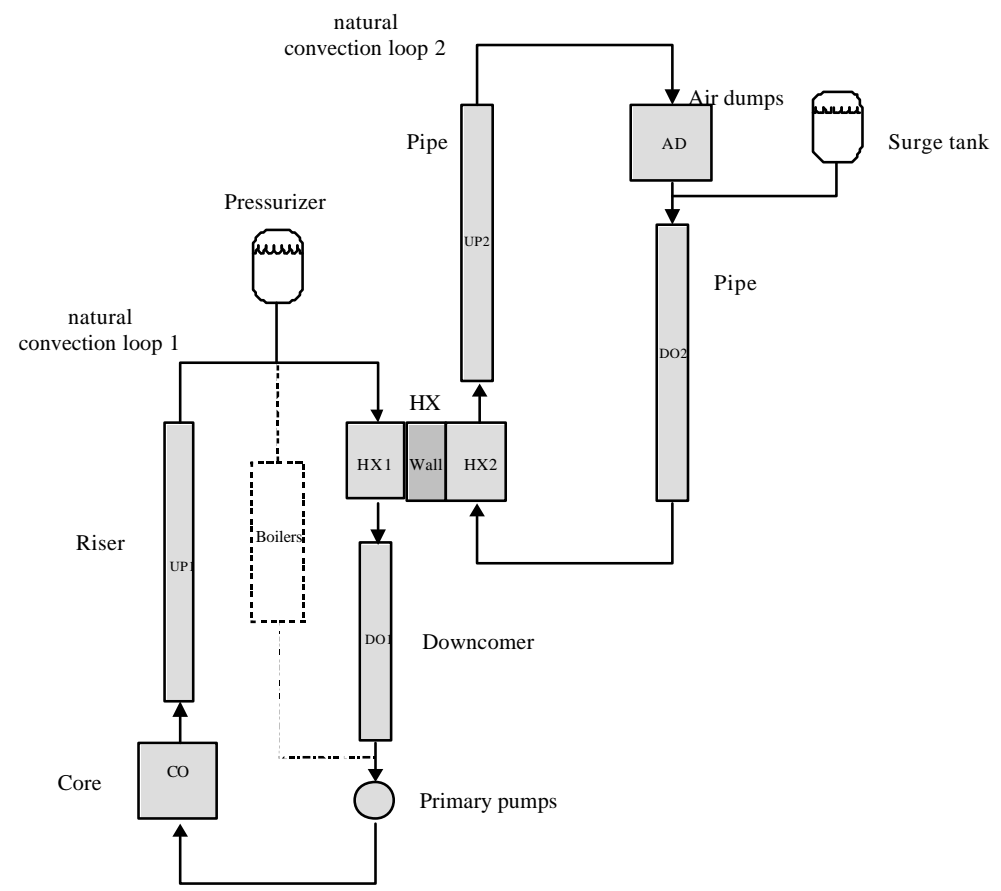

Figure 2-61. Model used for evaluating the PHRS response during postulated transient. 


\section{Loss of Flow Accident}

In order to study the performance of the PHRS, a Loss of Flow Accident (LOFA) due to a Loss of On-site and Off-site Electrical Power was studied. The main characteristics of the transient are:

- The primary pumps are tripped at $\mathrm{t}=0 \mathrm{~s}$. A pump flow coast-down is assumed until initiation of the natural circulation. The value of $\beta$ is set equal to $0.2 \mathrm{~s}^{-1}$ in the reference case.

- $\mathrm{AMBs}$ are supposed not available for heat transfer during the transient.

- The primary scram signal occurs $0.5 \mathrm{~s}$ after the LOFA. Conservatively, the reactor control rods are assumed to be completely inserted after $1.0 \mathrm{~s}$ of the primary scram signal (see [Tang78]). The power released in the core during the transient is shown in Figure 2-62.

- The temperature of the air dumps is conservatively fixed equal to $350 \mathrm{~K}$.

- The effective height of the PHRS loop (loop-2) is $5 \mathrm{~m}$.

Note that the secondary side of the PHRS (the Na-K coolant) could be operated in boiling regime, leading to a more compact PHRS secondary loop. This is because of the large buoyancy due to the density difference. In this design option, it is also predicted that the alkali metal vapor is condensed in double wall tubes placed inside a water tank, further reducing the size of the PHRS system as no air-dumps would be required.
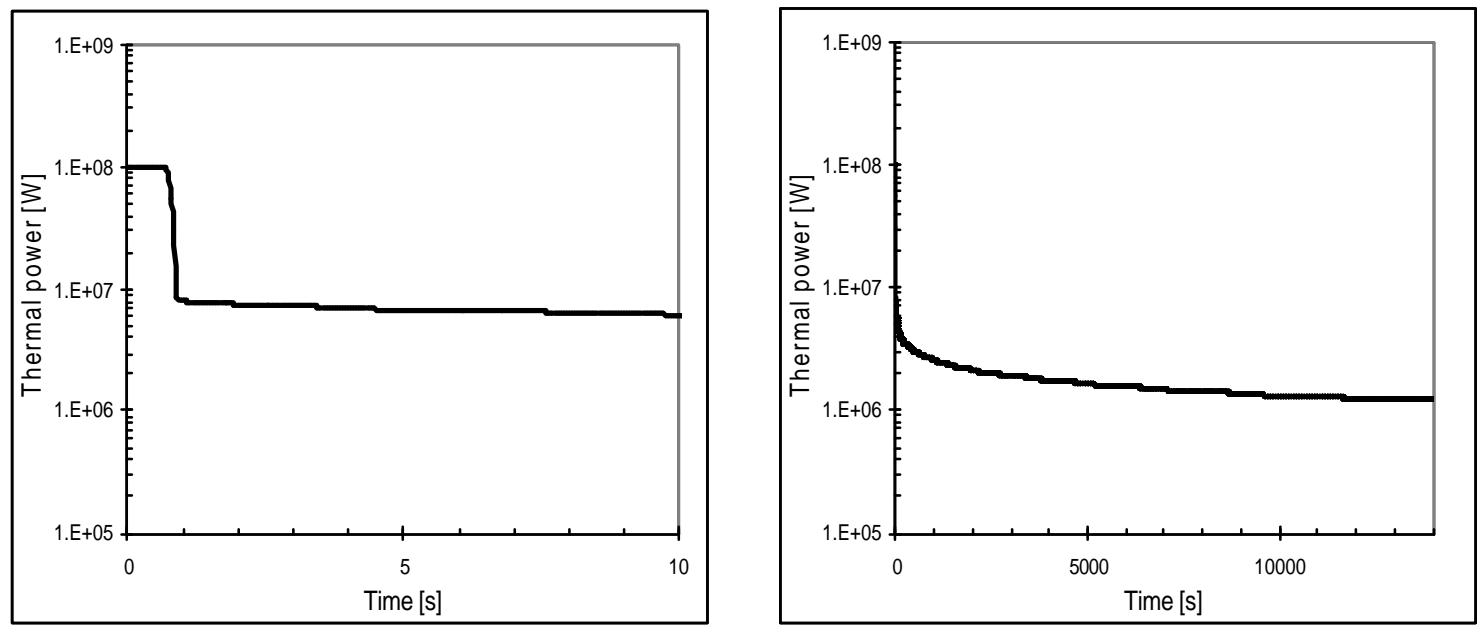

Figure 2-62. Core power a) 0-10 s, b) up to $14000 \mathrm{~s}$. After the reactor shutdown, decay heat power are used to estimate the core power.

Figure 2-63 and Figure 2-64 present the maximum fuel centerline and the cladding temperatures for the hot channel, the outlet core coolant temperature and the core mass flow rate during the first $50 \mathrm{~s}$ and $14000 \mathrm{~s}$ of the transient, respectively. As seen, the fuel and the cladding temperature tend to equalize after core shutdown. The core mass flow rate decreases according to the flow coast-down until the natural circulation 
starts establishing at about $20 \mathrm{~s}$. The maximum temperatures are reached at about $10000 \mathrm{~s}$ and are well below the coolant boiling point.

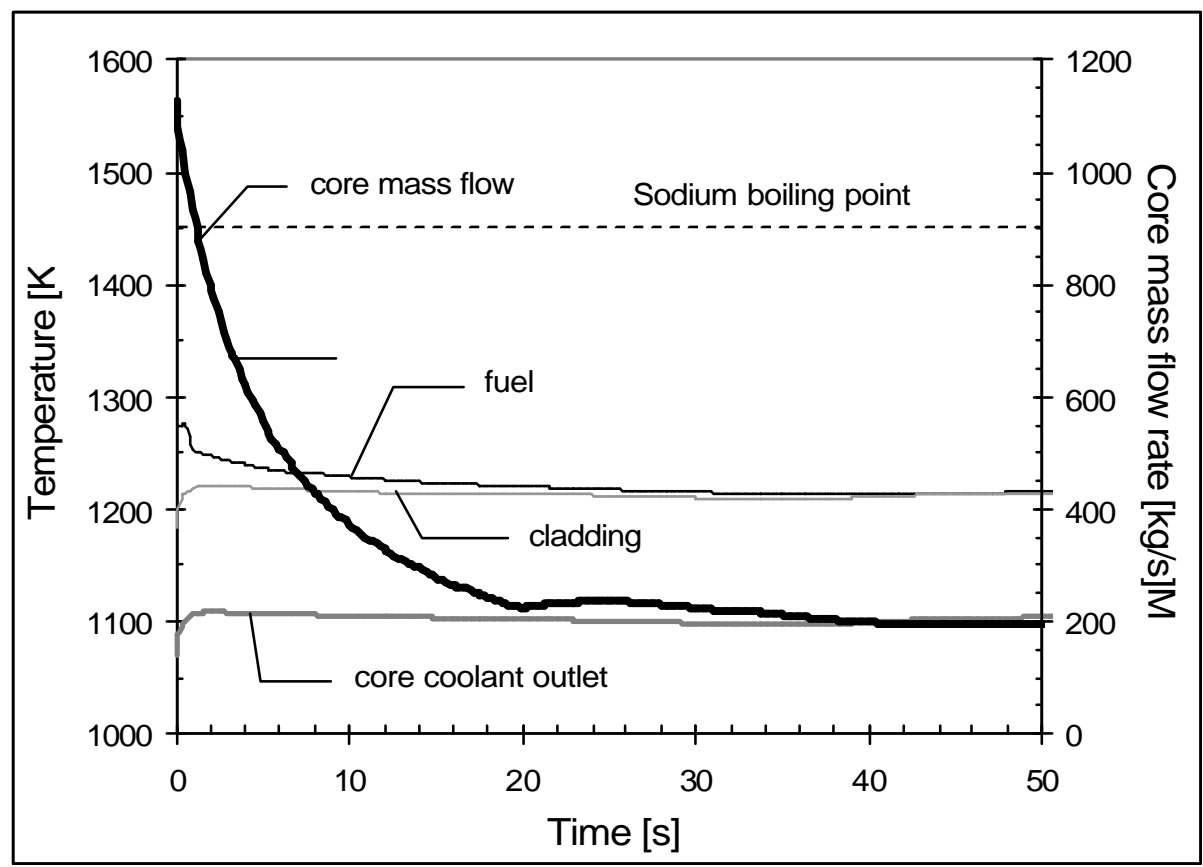

Figure 2-63. Temperature of the fuel, cladding, and the coolant at the core outlet. The fuel and cladding temperatures corresponds to the hot channel $(0 \mathrm{~s}<\mathrm{t}<50 \mathrm{~s})$.

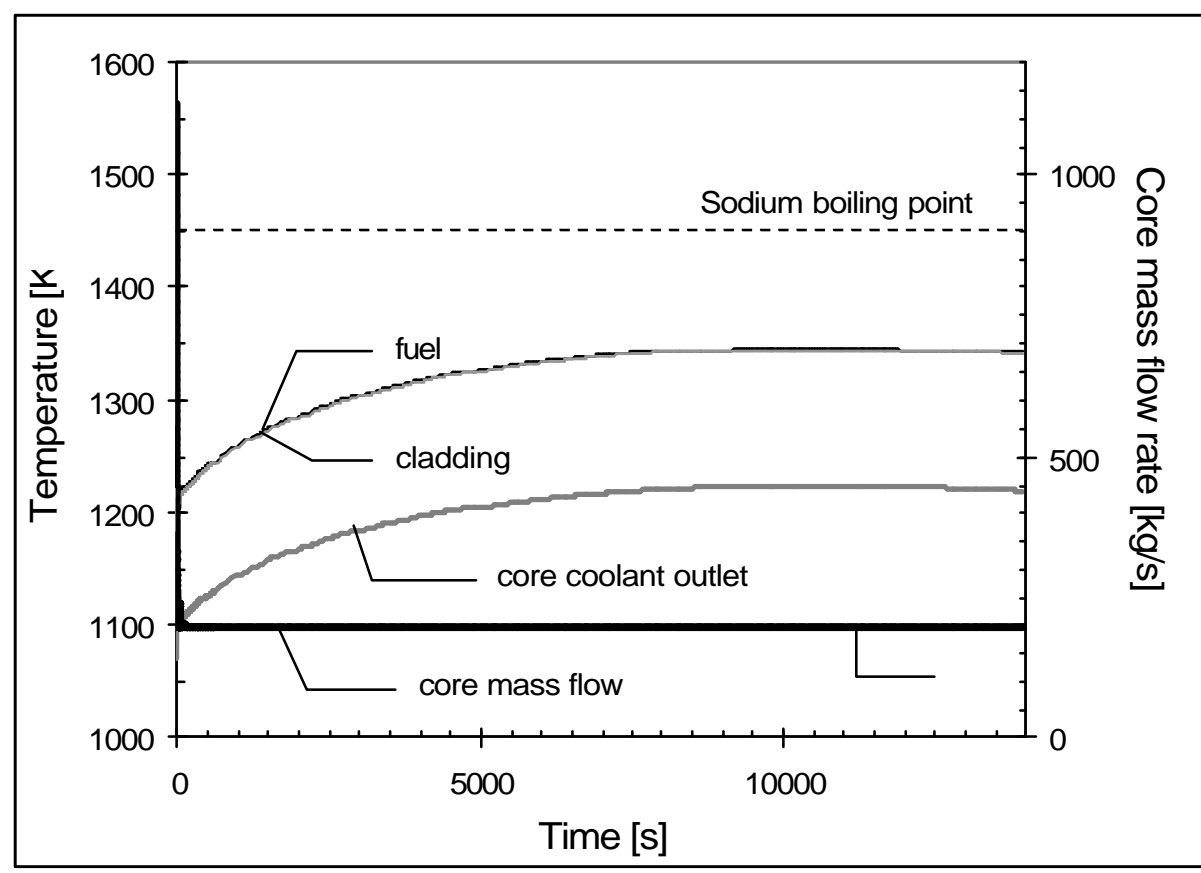

Figure 2-64. Coolant mass flow rate and temperature of fuel, cladding and coolant at the core outlet. The fuel and cladding temperatures corresponds to the hot channel $(0 \mathrm{~s}<\mathrm{t}<50 \mathrm{~s})$. 


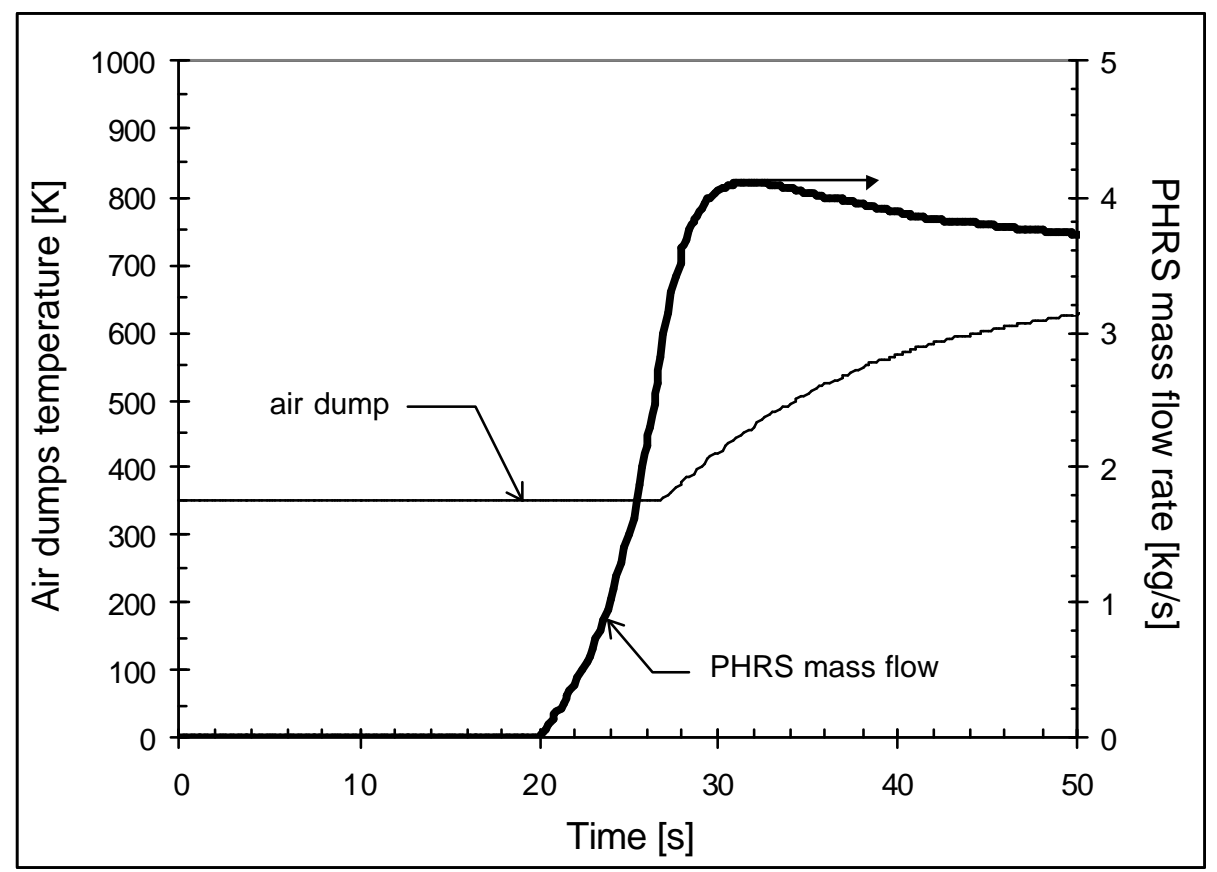

Figure 2-65. Coolant mass flow in the PHRS and average temperature of the air dumps $(0 \mathrm{~s}<\mathrm{t}<50 \mathrm{~s})$.

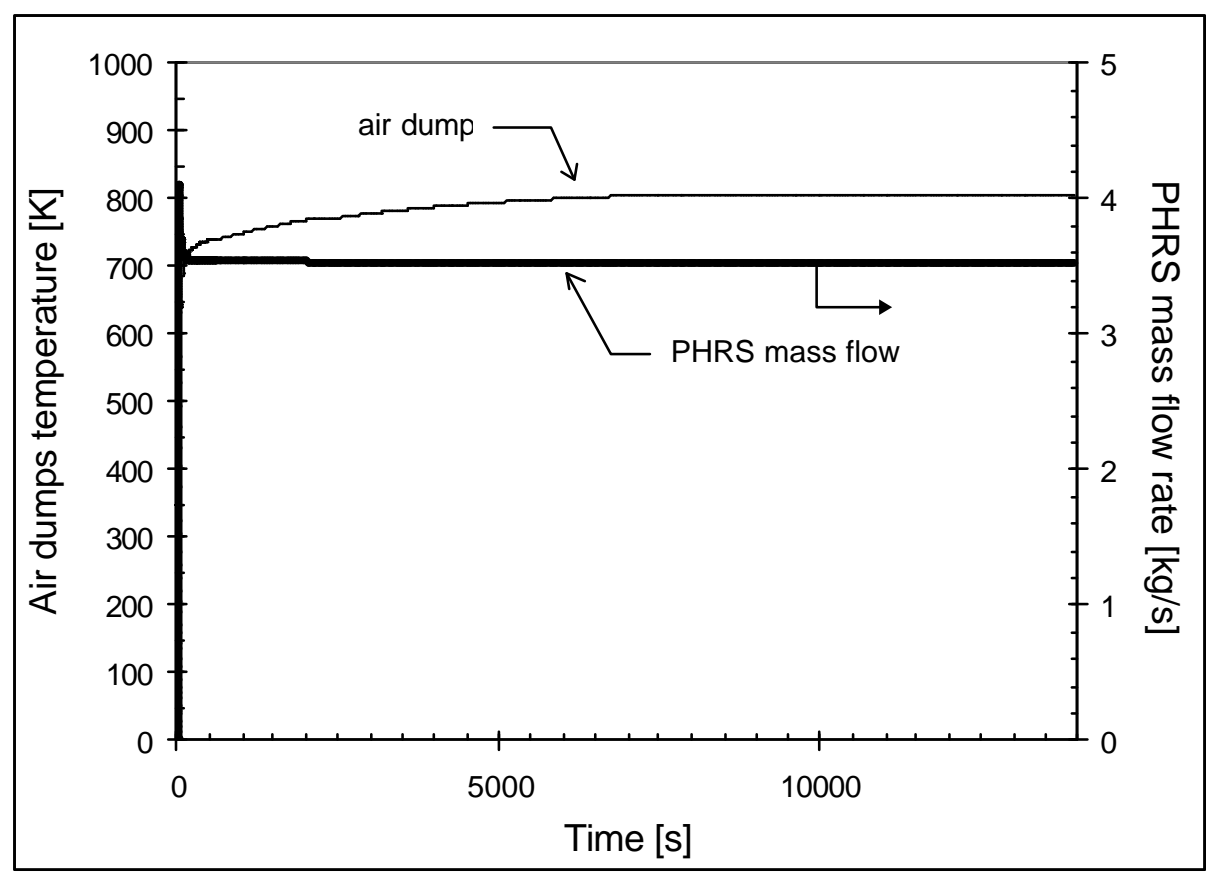

Figure 2-66. Coolant mass flow in the PHRS and average temperature of the air dumps $(0 \mathrm{~s}<\mathrm{t}<14000 \mathrm{~s})$.

Figure 2-65 and Figure 2-66 show the air dumps temperature and the PHRS mass flow rate during the transient. The natural circulation starts at about $20 \mathrm{~s}$ when the helical 
HXs are in contact with the sodium (i.e. the coolant level reach the Orifice 2). The air dumps temperature increases after a delay related to the transition time of the coolant in the upper pipe (see Figure 2-61).

The sensibility of the fuel and coolant temperatures with respect to the flow coastdown characteristics and the shutdown time (control rods completely inserted) was studied in the cases described in Table 2-34.

Table 2-34. Parameters used in the transient analyses .

\begin{tabular}{|c|c|c|c|}
\hline Name & $\begin{array}{c}\text { Time for } \\
\text { CRs } \\
\text { insertion }[\mathrm{s}]\end{array}$ & $\begin{array}{c}\text { Delay to } \\
\text { shutdown }[\mathrm{s}]\end{array}$ & $\beta\left[\mathrm{s}^{-1}\right]$ \\
\hline case 1 & 1.0 & 0.2 & 0.1 \\
\hline case 2 (reference) & $\mathbf{1 . 0}$ & $\mathbf{0 . 2}$ & $\mathbf{0 . 2}$ \\
\hline case 3 & 1.0 & 0.2 & 0.5 \\
\hline case 4 & 0.5 & 0.2 & 0.2 \\
\hline case 5 & 2.0 & 0.2 & 0.2 \\
\hline
\end{tabular}

Figure 2-67 and Figure 2-68 show the maximum centerline fuel temperature in the hot channel during the transient. Figure 2-69 and Figure 2-70 present the outlet coolant temperature during the transient. A significant difference exists only in the case where the sensitivity with respect to the time for CRs insertion was studied. According to available data on control assembly insertion time versus number of absorbent rod [Tang78], this value could not be larger than $0.9 \mathrm{~s}$ (corresponding to a CRs using $61 \mathrm{rod}$ ). Thus $1.0 \mathrm{~s}$ should be considered as a sufficient conservative value.

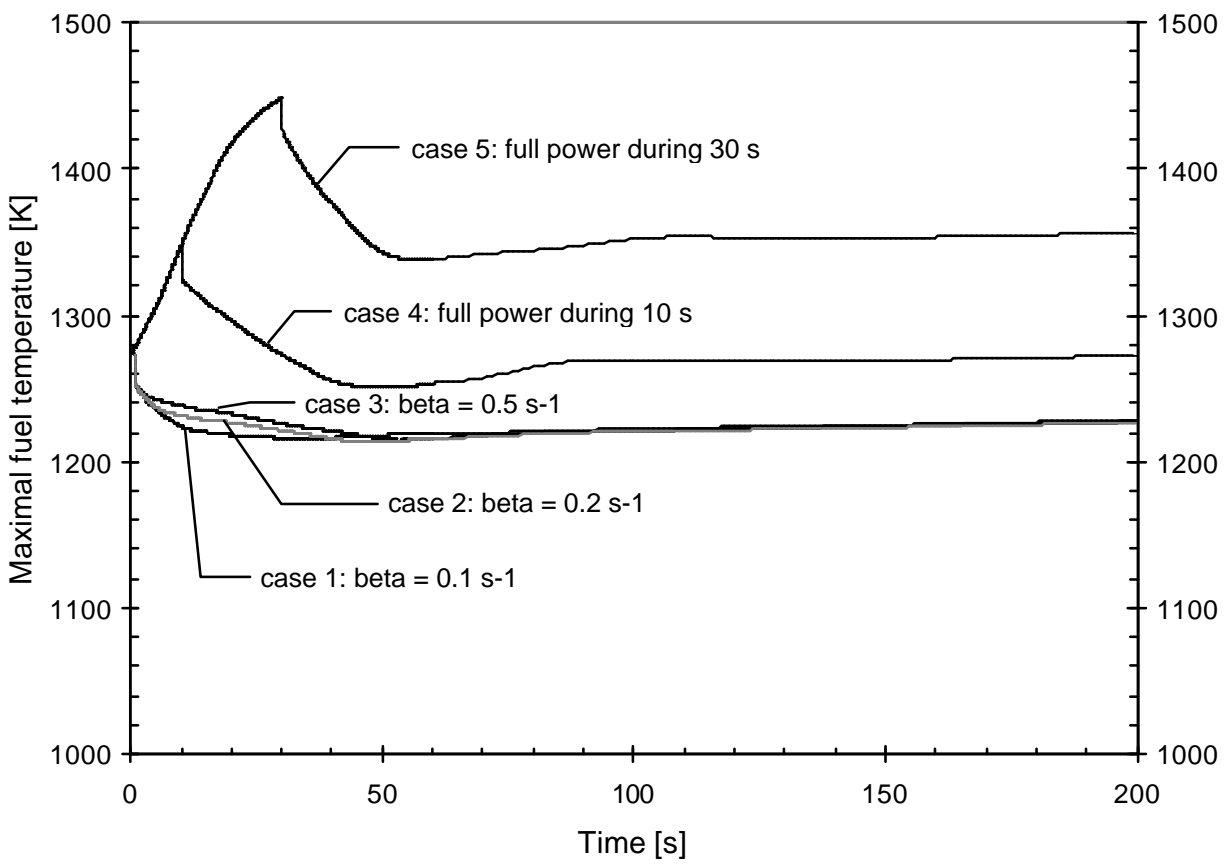

Figure 2-67. Maximum fuel temperature $(0 \mathrm{~s}<\mathrm{t}<200 \mathrm{~s})$. 


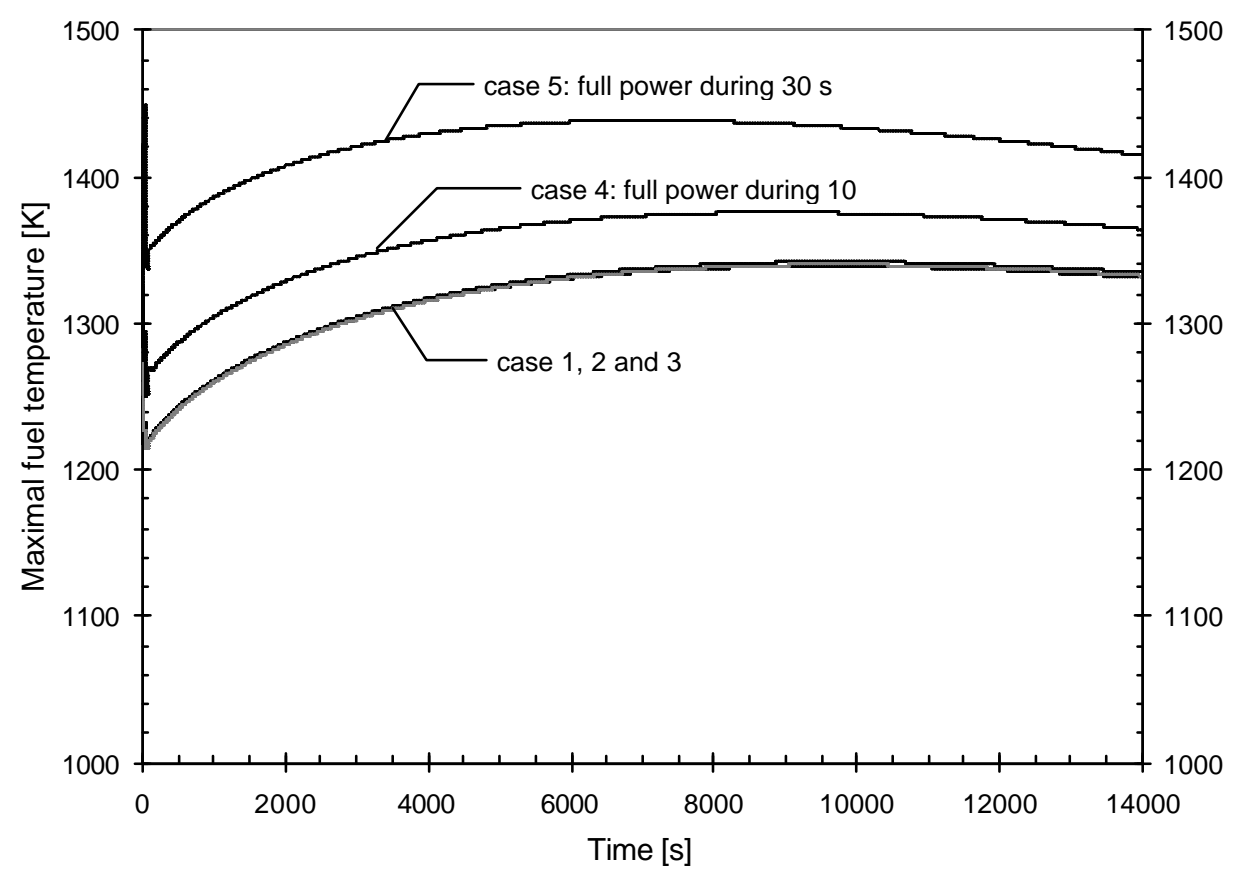

Figure 2-68. Maximum fuel temperature $(0 \mathrm{~s}<\mathrm{t}<14000 \mathrm{~s})$.

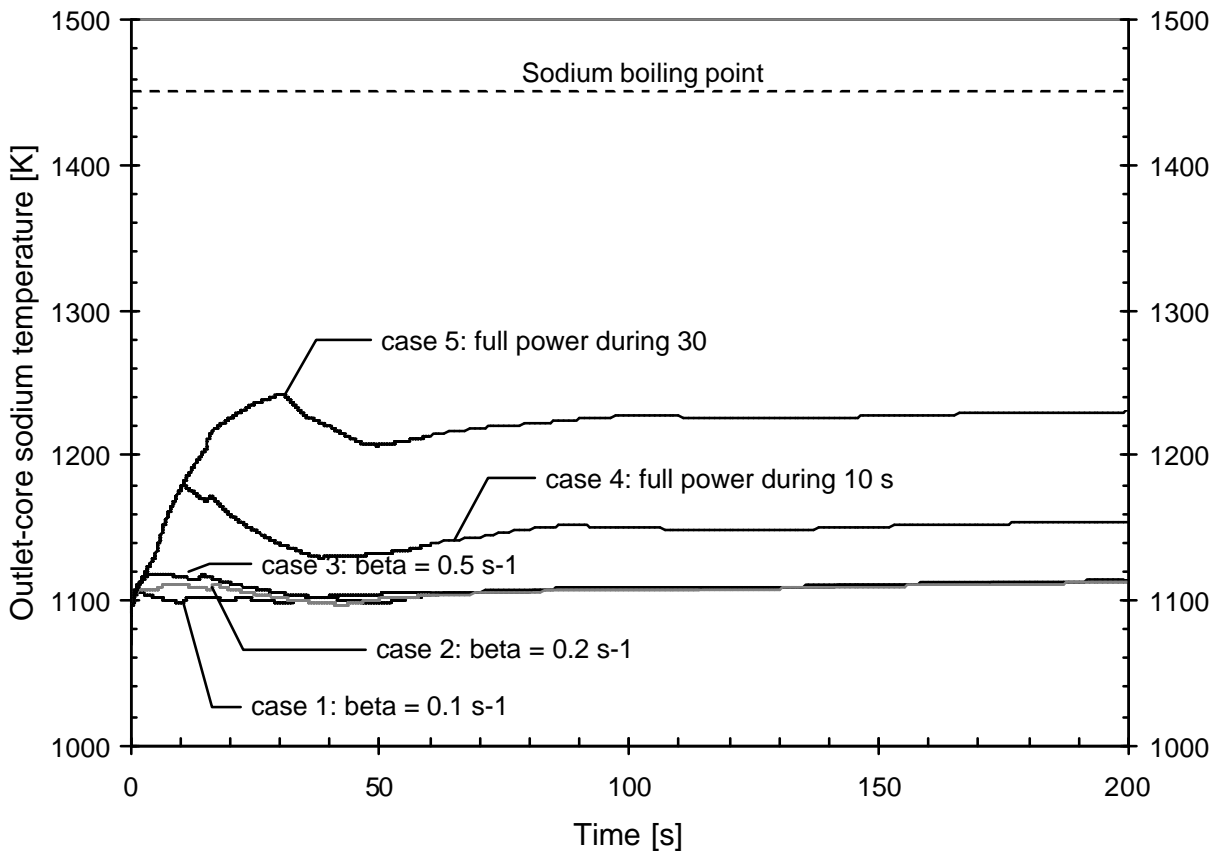

Figure 2-69. Average outlet core sodium temperature $(0 \mathrm{~s}<\mathrm{t}<200 \mathrm{~s})$. 


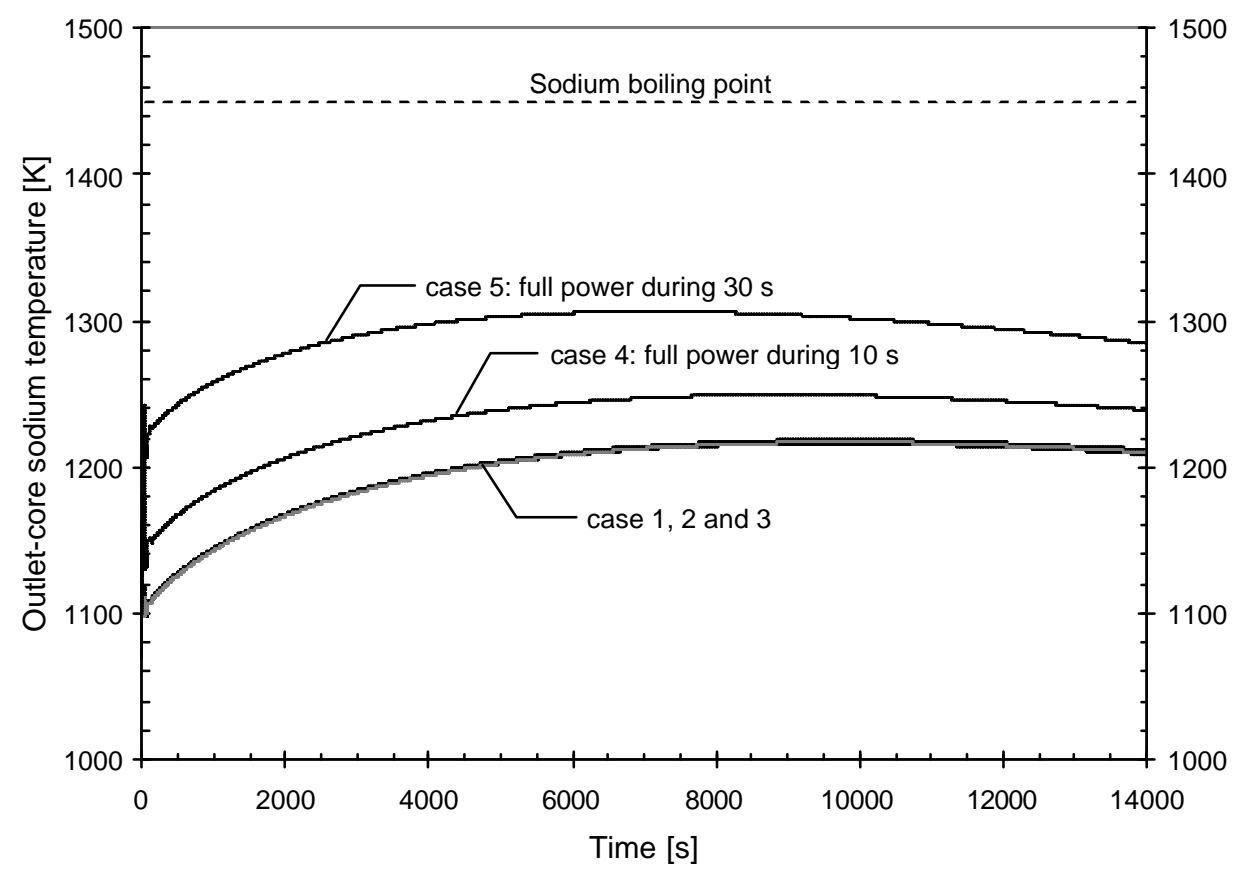

Figure 2-70. Average outlet core sodium temperature $(0 \mathrm{~s}<\mathrm{t}<14000 \mathrm{~s})$.

Key conclusions can be outlined from these results:

- The outlet average core coolant temperature during the transient in all cases was well below the boiling point. Furthermore, the maximum clad temperature was below the saturation temperature, even if one accounts for a $50 \mathrm{~K}$ margin (see section 2.2.3.3) accounting for uncertainties affecting the cladding temperature. Therefore, enough subcooling margin exists at the cladding surface temperature (boiling point of sodium at design condition is $\sim 1450 \mathrm{~K}$.) and cladding melting is precluded during the transient. Therefore the actual value of the primary pressure assures enough safety margin to prevent coolant boiling during these transients.

- In the reference case, based on the clad maximum temperature $(<1400 \mathrm{~K})$ and accounting for the internal pressure increase $(<670 \mathrm{psi})$, the total clad creep during the transient can be estimated to be less than $0.7 \%$. The circumferential stress was found to be three times lower than the ultimate tensile strength. These values are quite acceptable because no active system has been assumed to actuate in reducing the cooling down time and furthermore this event is considered to have a low frequency of occurrence.

- The results show that the PHRS behavior does not strongly depend on the flow coastdown characteristics.

- For the current design characteristics, the PHRS has enough capacity to passively remove decay heat working in natural circulation. The length of the transient could be reduced by using EM pumps. This option will allow the PHRS to work in either natural or forced convection. 


\subsubsection{Guard vessel}

Although the probability of a vessel leak is remote, the possible consequences of such an accident require the use of a Guard Vessel (GV) which surrounds the reactor vessel of the LMR-AMTEC. In the unlikely event that the reactor vessel was to leak, the GV would ensure that the sodium level does not fall below a minimum required to cool the reactor core. This minimum is determined by the position of the PHRS HX inside the vessel. In order to assure the mechanical integrity of the guard vessel during such an event, a Water Cooling Guard Vessel System (WCGVS) similar to that of the Phenix reactor is used (see Figure 2-71). This system employs integral cooling coils on the external surface of GV. The cooling water flows through the coils, eventually boils and then dumps its heat in a condenser tank (see Figure 2-59). The system would be designed to work in natural convection.

Both the GV and the WCGVS can be used in the LMR-AMTEC without penalizing the transportability and cost issues of the concept. In fact, the GV could be transported together with the reactor vessel thus reducing in-site work. As seen in Figure 2-59, two valves, normally closed, are used to avoid heat losses of the WCGVS during normal operation. To avoid excessive heating of the GV during normal operation, the heat transfer in the annulus between the reactor vessel and the GV must to be controlled. This can be done reducing the radiation heat transfer by using a reflective layer on the surfaces of the reactor vessel and the guard vessel. In addition, the heat conduction transfer can be controlled by the pressure of the inert gas (such as Helium) that fills the annulus. Furthermore, the guard vessel is surrounded by sodium- resistant concrete, which is water cooled, and which could eventually contain the consequences of a sodium leak from the GV. Finally, it must be noted that the GV and the WCGVS in the LMRAMTEC are used as a last resort for containing the reactor coolant and not as an RVACS.

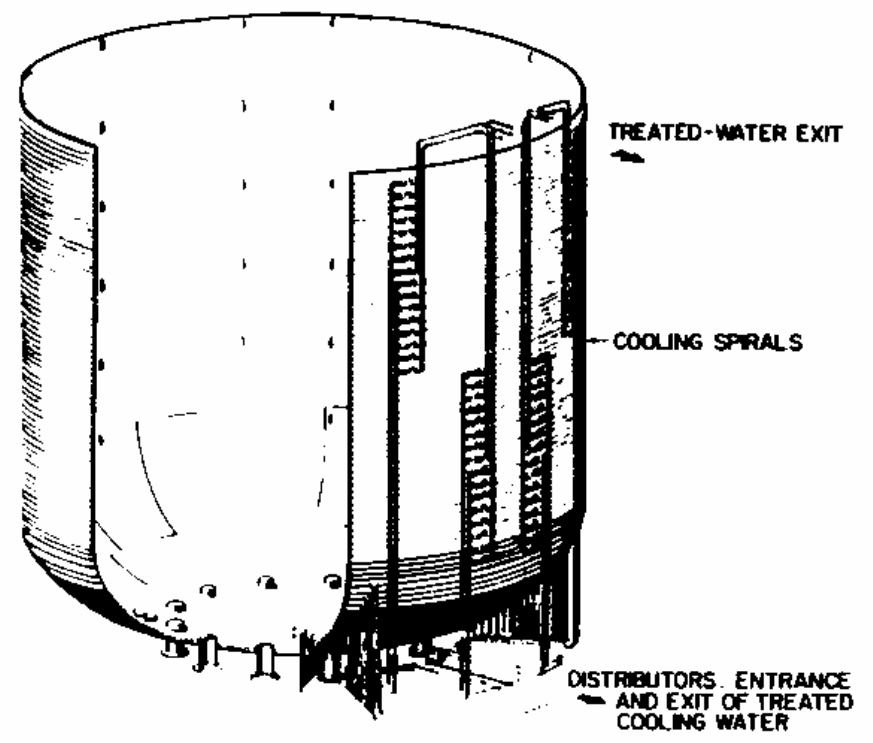

Figure 2-71. Water Cooling Guard Vessel System employed by the French PHENIX reactor. 


\title{
2.4 Transportation and Waste Disposal
}

\author{
Contributed by IERA, UNM
}

Liquid Metal Reactor-Alkali Metal Thermal-to-Electric Converter (LMR-AMTEC) is a new reactor concept under development, of which many aspects contribute to the final composition and configuration. Transportation of various reactor components is one of such aspects.

This section reviews the truck and/or rail transportation limitations of large components in the LMR-AMTEC reactors, from weight and size points of view, according to the laws and regulations of the Federal Administration and/or of individual States.

According to the Federal Highway Administration (FHWA) of the U.S. Department of Transportation (USDOT) there are currently about 3.9 million miles of public roads in the United States ${ }^{(1,2)}$. These major highways accommodate transportation between metropolitan areas, cities, and industrial centers. Nearly all of these Federal highways are paved and often have greater weight and traffic capacities than State and local roads ${ }^{(1)}$.

Highway infrastructure protection has been an important consideration in determining the parameters of truck size and weight (TS\&W) limits. Pavement wear increases with the axle weight, the number of axle loading; and the spacing within the axle group, such as tandem or tridem groups. Truck size also affects the design and fatigue of the bridges. As with pavements, the distribution of weight over the distance between the axles also affects the design and fatigue of the bridge. Therefore, the transportation of large LMR-AMTEC components will be restricted by the maximum permissible weight and size according to the USDOT rules and regulations (2).

The TS\&W analysis considers safety and efficiency of the total transportation system from both public and private sector point-of-views. Specifically, the TS\&W analysis covers the following:

- Safety of truck operation,

- Infrastructure impacts (pavements, bridges and geometric design) and how the costs of these impacts are recovered,

- Effects on productivity and efficiency for shippers and carriers,

- Federal and State roles in regulating traffic and equipment, as well as interstate and international commerce.

Freight transportation has become more complex since deregulation and the evolution toward a global marketplace. The complexity of TS\&W issues has also increased, especially with the advent of integrated, multi-modal transportation, increased international container movements, and the enactment of the North American Free Trade Agreement (NAFTA) and the General Agreement on Tariffs and Trade (GATT) (2). 


\subsubsection{Truck Transportation}

Various truck classes currently used in regular operations are illustrated in Figure 2-72; each of them has its own characteristics of permissible load, width, height and length as shown in Table 2-35. Not all States allow the various Longer Combination Vehicles (LVC) to be operated on their routes. Figure 2-73 illustrates the States allowing the various LVCs.

Table 2-35. Typical vehicle types and their weight and dimension specifications ${ }^{(2)}$

\begin{tabular}{|c|c|c|c|c|c|}
\hline Vehicle type & $\begin{array}{l}\text { Number } \\
\text { of axles }\end{array}$ & $\begin{array}{c}\text { Common Max. } \\
\text { Weight }\end{array}$ & $\begin{array}{l}\text { Max. } \\
\text { Length }\end{array}$ & Max. Width & $\begin{array}{l}\text { Max. } \\
\text { Height }\end{array}$ \\
\hline \multirow{2}{*}{$\begin{array}{l}\text { Single-Unit } \\
\text { Truck }\end{array}$} & 3 & 50,000 to 65,000 & \multirow[t]{2}{*}{45 feet } & \multirow{9}{*}{$\begin{array}{c}102 \text { inch } \\
\text { (except } \\
\text { Hawaii } \\
\text { which } \\
\text { applies } 108 \\
\text { inch as truck } \\
\text { width) }\end{array}$} & \multirow{9}{*}{14 feet } \\
\hline & 4 or more & 62,000 to 70,000 & & & \\
\hline \multirow[t]{2}{*}{ Semitrailer } & 5 & 80,000 to 100,000 & \multirow[t]{2}{*}{48 feet* $^{*}$} & & \\
\hline & 6 or more & 80,000 to 99,000 & & & \\
\hline $\begin{array}{l}\text { STAA } \\
\text { Double }\end{array}$ & 5,6 & 80,000 & $\begin{array}{l}28-28.5 \\
\text { feet/trailer }\end{array}$ & & \\
\hline $\begin{array}{l}\text { B-Train } \\
\text { Double }\end{array}$ & 8 & 105,500 to 137,800 & 84.3 feet & & \\
\hline $\begin{array}{l}\text { Rocky } \\
\text { Mountain } \\
\text { Double }\end{array}$ & 7 & 105,500 to 129,000 & 81 feet $^{* *}$ & & \\
\hline $\begin{array}{l}\text { Turnpike } \\
\text { Double }\end{array}$ & 9 & 105,500 to 147,000 & 95 feet $* *$ & & \\
\hline Triple & 7 & 105,500 to 131,000 & 95 feet $^{* *}$ & & \\
\hline
\end{tabular}

* Applied on the National Network (NN), grandfather lengths greater than 48 feet may apply on the NN

** For the trailer combination length 

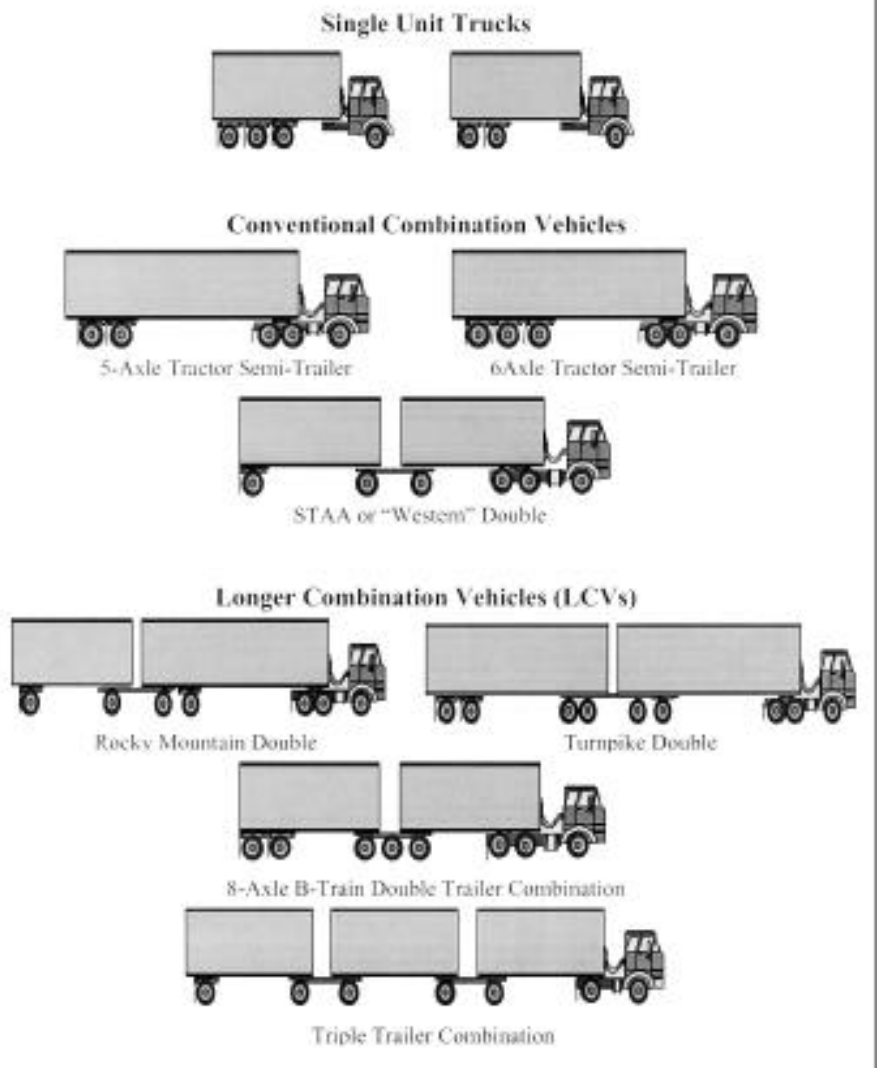

Figure 2-72. Illustrative Vehicle Configurations ${ }^{(2)}$
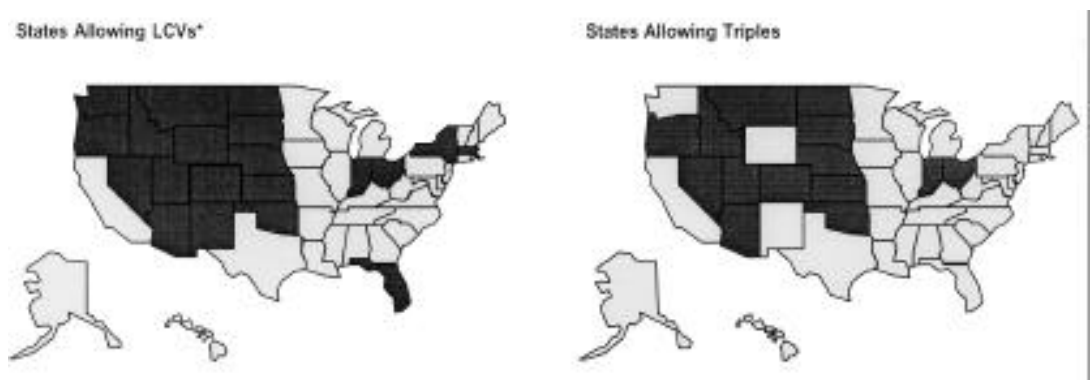

States Allowing Turnpike Doubles

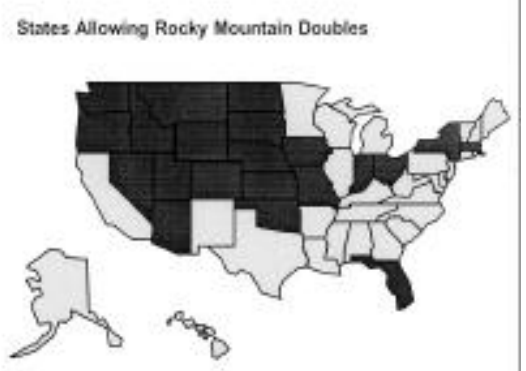

Figure 2-73: States Allowing Various Longer Combination Vehicles ${ }^{(2)}$

In addition to axle and maximum Gross Vehicle Weight (GVW) limits for Interstate highways, Federal law has adopted Bridge Formula B (BFB) that restricts the maximum 
weight allowed on any group of consecutive axles based on the number of axles in the group and the distance from the first to the last axle ${ }^{(2,3)}$.

\subsubsection{Federal Regulations}

Current Federal TS\&W laws now regulate TS\&W limits by specifying basic standards and exempting certain situations from those standards by grandfather right and provision for special permits. Federal laws governing truck weights apply to the Interstate System while Federal laws governing truck size apply to a legislated National Network (NN), which includes the Interstate system. The NN was designated under the authority of the same 1982 Act (STAA of 1982) that established the size limits. Current U.S. Federal TS\&W law, specifically, part 23 CFR 658 sections 658.13, 658.15 and 658.17 establish the following limits ${ }^{(2)}$ :

- 20,000 pounds for single axles on the Interstate.

- 34,000 pounds for tandem axes axles on the Interstate.

- Application of Bridge Formula B (BFB) for the axle group, up to the maximum of 80,000 pounds for GVW on the Interstate.

- 102 inches for vehicle width on the NN.

- 48 foot (maximum) for semitrailers in a semitrailer combination on the NN.

- 28 foot (maximum) for trailers in a twin-trailer combination on the NN.

\subsubsection{State Application}

Broadly speaking, many State provisions differ from Federal provisions; there are many regulatory differences among the States and these differences are increasing over time. These disparities exist because of differences in local and/or regional political choices that have been made balancing economic activities; freight movements; infrastructure design characteristics and status; traffic densities; mode options and engineering philosophies. Several States have higher weight limits off the Interstate System than Federal law allows on the Interstate System. While some States have both higher gross weights and higher axle limits, other States have the same gross weight limits, but different axle weight limits or vice versa. Overlying the gross vehicle weight and axle weight limits on Interstate and other highways are systems of overweight permits that are granted by each State. These permits are essential $\mathbf{b}$ allow non-divisible loads to be transported, and often come with strict conditions under which the moves can be made $(2,3)$.

Vehicle size and weight laws in each state are continually evolving due to several factors including, but not limited to, the natural resources, local industrial development, climate, the relative strength of special interest groups, and the national economy. 


\subsubsection{Rail Transportation}

The U.S. rail industry has a number of characteristics that sharply distinguish its production and supply of freight transportation services from other modes of freight transportation services. The most important difference is that, with some minor exceptions, the industry's infrastructure is privately owned and operated and access to the infrastructure is typically limited to the owner of the track. By contrast, trucking firms, airlines, and barge lines operate on infrastructure that is owned and operated by the government and can potentially serve any shipping point on the network. The combination of privately owned infrastructure and limited access are the primary characteristics that distinguish rail transportation from other transportation modes.

According to the Association of American Railroads (AAR), 553 railroads in the United States operated 173,896 miles of track in 1996. Ten Class I railroads operated nearly 73 percent, 32 regional railroads operated more than 11 percent, and 511 local line haul and switching and terminal railroads operated nearly 16 percent of the railway network (4). Railroads also benefit from economies in vehicle size, shipment size, and shipment distance. Economies from the size of the vehicle have resulted in a shift of hauling packages from boxcars to 70 ton covered hoppers to 100 ton covered hoppers, and finally to super-jumbo covered hoppers. Because no more labor is required to operate a train of 100 cars than one of 50 cars, and switching costs are the same regardless of the number of cars, railroads also have economies due to shipment size $(4,5)$. On average, a 286,000 pound covered hopper railcar can haul 10 percent more weight than 268,000pound railcars. As a comparison, standard size barges carrying grain on the U.S. inland waterway system loaded to a grain depth of 9 feet can carry 1,450 short-tons of grain, while a standard covered-hopper rail car will accommodate 95-100 tons of grain, and most grain trucks can be loaded with 22-25 tons of grain (6).

The maximum weight and size for rail shipment is determined by each individual railroad, based on the condition of their infrastructure. So one of the more newsworthy efficiency strategies has been the shift to larger rolling stock. This trend is expected to intensify and is straining the short line rail system, whose infrastructure is not equipped to safely handle the larger 286,000 pound and 315,000 pound gross weight railcars, and whose revenue levels make the necessary track and bridge upgrades prohibitive ${ }^{(7)}$.

\subsubsection{Transportation of the LMR-AMTEC Components}

We can summarize the transportable size and weight limits, by truck or rail, mandated by Federal or State (including Alaska) regulations as follows:

- The maximum weight limits for trucks in different states range from a Gross Vehicle Weight (GVW) of 80,000 pounds in 45 of the 50 states (slightly higher in the other 5 states) to a "Routine" Permit GVW (for any unit with sufficient axles before special review is required) ranging from 105,000 pounds in Oregon to 250,000 pounds in Arizona, with a median value of 135,500 pounds (as determined by analysis of the Routine" Permit GVW values for the different states). 
- The maximum truck length limits for all state regulations range from 58 feet in Michigan to 115.5 feet in Colorado for truck tractor and 3 trailer units.

- The maximum truck width limit is 102 inches, except for Hawaii where it is 108 inches according to Surface Transportation Assistance Act of 1982.

- There is no Federal height limit, so States may set their own. Most of them range from 13 feet 6 inches to 14 feet, with the exception of lower clearance on particular roads.

- The maximum weight and size for a rail shipment is determined by each individual railroad, based on the condition of their infrastructure. But the best railroad infrastructure can carry about 286,000 pounds (143 tons).

- Due to the overall structure of Alaska's transportation industry, it is necessary to divide the issue into two broad categories: inter-state and intra-state transportation. Incoming interstate transportation relies primarily on the marine system using steamship and barges traveling between Alaska and the contiguous United States. Intra-state transportation is conducted largely by light aircraft moving cargo from the rail-belt to remote communities and along the railroads. The vast majority of the goods brought into Alaska arrive via steamship, and there are only four steamships that serve the entire state. Cargo is also brought into Alaska via barge, aircraft and independent container trucks (Table 1). As these steamships travel principally between Anchorage and Seattle/Tacoma, the Anchorage International Airport acts as a hub for the major freight carriers operating in the state.

Based on this information and according to the available information about the weights of the large components of the LMR-AMTEC (including the reactor vessel and the AMTEC units), we can suggest the following:

1. The reactor vessel unit, with an estimated weight of 80.8 tons, cannot be transported as one unit via truck system. But the unit can be transported as separated parts, their number dependent on the regulations applied in both the sending and receiving places as well as the type of vehicle used. On the other hand, the reactor vessel unit can be transported as one unit by rail where the railcar can carry weights reach up to 130 tons provided it is not restricted by the condition and provision of the used railroad infrastructure.

2. AMTEC units can not be transported in a single batch either via trucks or rails, from or to any state within the United States.

3. The transportation provisions and suggestions provided above also apply to the state of Alaska wherever marine transportation considerations do not change or affect other transportation provisions and conditions. 


\subsubsection{Bridge Formula B (BFB) and The Alternative TTI Formula}

Most highway bridges in the United States were designed according to the design manual guidelines of the American Association of State Highway and Transportation Officials (AASHTO). The AASHTO bridge specifications provide traffic-related loading to be used in the development and testing of bridge designs. The AASHO developed the Bridge Formula B (BFB) which restricts the maximum weight allowed on any group of consecutive axles based on the number of axles in the group and the distance from the first to the last axles. The formula for the BFB is given as:

$$
\mathrm{W}=500[\mathrm{LN} /(\mathrm{N}-1)+12 \mathrm{~N}+36]
$$

where:

$\mathrm{W}=$ maximum weight in pounds on any group of two or more consecutive axles,

$\mathrm{L}=$ distance in feet between the extremes of the axle group, and

$\mathrm{N}=\quad$ is the number of axles in the axle group.

Federal law specifies exceptions to the results given by the above formula: 68,000 pounds may be carried on two sets of tandem axles spaced at least 36 feet apart, and a single set of tandem axles spread no more that 8 feet is limited to 34,000 pounds.

In 1974, Congress adopted BFB when it increased the GVW limit to 80,000 pounds and the limits on single and tandem axles to 20,000 and 34,000 pounds respectively. The BFB is also referred to as the Federal Bridge Formula (FBF) or FBF B. BFB is based on assumptions about the amount by which the design loading can be safely exceeded for different bridge designs. BFB reflects the fact that increasing the spacing between axles generally results in a less concentrated loading and lowers stresses in bridge members. Also, BFB allows more weight to be carried as the number of axles is increased.

\section{Potential Alternative to BFB}

In 1990, the Transportation Research Board (TRB) recommended adoption of the formula developed by the Texas Transportation Institute (TTI) that would allow a 5\% overstress fr the HS-20 bridges, in conjunction with existing Federal axle limits for vehicles with GVWs of 80,000 pounds or less. The TRB report further recommended that the BFB continue to be applied to vehicles weighting more than 80,000 pounds.

The TTI formula is in the form of two equations for straight lines that meet at a wheelbase length of 56 feet. For a wheelbase less than 56 feet, the equation takes the form:

$$
\mathrm{W}=1,000(\mathrm{~L}+34)
$$

For a wheelbase equal to or greater than 56 feet, the equation takes the form:

$$
\mathrm{W}=1,000(\mathrm{~L} / 2+62)
$$

where:

$\mathrm{W}=$ is the allowable weight, and

$\mathrm{L}=\quad$ is the wheelbase for truck configuration. 


\subsubsection{Waste Disposal}

Like all industries, the thermal generation of electricity produces wastes. Whatever fuel is used, these wastes must be managed in ways, which safeguard human health and minimize their impact on the environment.

Based on the selected design of the LMR-AMTEC components and the coolant types, different wastes will be generated from LMR. These wastes must be classified and characterized according to the Code of Federal Regulation (CFR). The objectives of this document are to identify:

- Waste generated from the secondary potassium circuit

- Waste generated from the primary sodium circuit

- Waste contained by the cold trap unit

- Waste generation from the cover gas purification unit

- Waste generation from special trap units

- Spent Nuclear Fuel (SNF)

- Characterization of the wastes generated

- Applicable regulations that manage these wastes.

The objective of this section is to define and characterize the waste generated from the LMR-AMTEC as well as to identify the applicable US regulations governing waste transportation, treatment, storage and final disposition. The waste generated from the LMR-AMTEC is characterized as: (1) mixed waste which is generated from liquid sodium contaminated by fission products and activated corrosion products, (2) hazardous waste which is generated from liquid potassium contaminated by corrosion products, (3) spent nuclear fuel, and (4) low level radioactive waste which is generated from the packing materials; e.g. activated carbon in cold trap, purification units. The regulation of these wastes and its management are summarized in this section.

\subsubsection{Background}

In the ideal nuclear reactor all fission products and actinides are contained in the fuel elements. There are four processes in a nuclear power plant through which radioactivity leaves the reactor vessel, in all cases the coolant usually is the carrier of activity through one or more of the following processes:

- Inducing radioactivity in the cooling medium

- Corrosion products containing induced radioactivity

- Leaked fission products and actinides from faulty fuel elements

- Fission products of actinides deposited on surfaces in the core 
The waste generated from the LMR could be classified according to the waste characterizations as hazardous waste, mixed waste and radioactive waste. Corrosion and fission products exist in the form of dissolved ions and precipitate in the crud, depending on the chemistry of corrosion and fission products and sodium conditions. Most of the corrosion products giving rise to induced radioactivity within the coolant. The major activated corrosion products are ${ }^{51} \mathrm{Cr},{ }^{54} \mathrm{Mn},{ }^{59} \mathrm{Fe},{ }^{58} \mathrm{Co},{ }^{60} \mathrm{Co},{ }^{65} \mathrm{Zn}$ and ${ }^{124} \mathrm{Sb}$ and the main fission products are ${ }^{3} \mathrm{H},{ }^{131} \mathrm{I},{ }^{134} \mathrm{Cs}$ and ${ }^{137} \mathrm{Cs}$. Other fission products and actinides are released in minor amounts depending on the kind and size of fuel elements leaked. These products are continually removed by cleaning the purification systems. Eventually the radioactive material accumulates in the purification systems; therefore filters are backwashed and adsorbing materials such as activated carbon and Charcoal delay bed in purification systems changed through a remotely controlled system. So solid radioactive wastes are collected from the purification circuits of the LMR station, as well as other parts of the reactor.

Most of the waste generated from the decommissioned nuclear reactors is nonradioactive. Also large volumes can be decontaminated and declassified which permits them to be treated as normal industrial waste. Of the remaining waste, most contain low levels of relatively short-lived nuclides; hence it can be treated and disposed of. Some internal parts of the reactor have relatively high activity. When spent fuel is removed from a reactor, it is temporarily stored at the reactor site until a permanent disposal place is found (1).

\subsubsection{Description of the Sodium and Potassium Circuits}

Sodium and potassium were selected as the most suitable working fluids in the LMRAMTEC. Sodium was selected to be the core working fluid (primary coolant) because of its better neutronic performance and the great experience associated with sodium-cooled reactors.

The configuration of the sodium circuit is such that sodium liquid will be contaminated with different radionuclides of the structural materials that may be chosen for the primary circuit and the sodium will also be contaminated by many of the corrosion products.

Sodium is also considered as a hazardous waste due to it is reactivity according to 40 CFR 261.23, corrosivity according to 40 CFR 261.22 and ignitability according to 40 CFR 261.21. It is regulated under RCRA by the EPA, 40 CFR 261. Under the 1984 Amendments to RCRA, Land Disposal Restriction (LDR) regulations prohibit disposal of hazardous waste until it meets specific treatment standards.

The heat exchange between sodium and potassium takes place inside the boiler where the sodium in the primary coolant circuit flows through the shell side while the potassium secondary coolant circuit flows through the tube side of the boiler.

The structural materials of the main components that are involved in the secondary circuit would be Nickel alloys, SS310 or SS316 alloys, Cobalt alloys, and/or Refractory ( $\mathrm{Nb}-1 \mathrm{Zr}$ ) alloys, with no significant radionuclide activity in this area. Potassium is considered as a hazardous waste due to its reactivity according to $40 \mathrm{CFR}$ 
261.23, corrosivity according to 40 CFR 261.22 and an ignitability according to 40 CFR 261.21, and it is regulated under RCRA by the EPA, 40 CFR 261. Under the 1984 Amendments to RCRA, Land Disposal Restriction (LDR) regulations prohibit disposal of hazardous waste until it meets specific treatment standards.

\subsection{Waste Generated in the Liquid Sodium Circuit}

Sodium has been universally chosen as the coolant for the modern LMFBR because of its small size; sodium does not appreciably slowdown neutrons by elastic scattering. Since sodium is an excellent heat transfer material, the LMFBR can be operated at high power density. This, in turn, means that the LMFBR core can be comparatively small. Furthermore, because sodium has a very high boiling point, reactor coolant loops can be operated at high temperature and at essentially atmospheric pressure without boiling, and no heavy pressure vessel is required. Sodium absorbs neutrons, even fast neutrons, leading to the formation of the beta-gamma emitter ${ }^{24} \mathrm{Na}$ (with a half-life of 15 hours) generated by neutron irradiation of natural sodium ${ }^{23} \mathrm{Na}(\mathrm{n}, \gamma){ }^{24} \mathrm{Na}$. Sodium passes through the reactor core, and therefore becomes radioactive. But even after shutdown and sufficient time for decay of this radionuclide (for instance 20 half lives $=12.5$ days) access to certain parts of the plants for inspection, maintenance and repair work may be restricted because of gamma radiation from long lived system contamination ${ }^{(2)}$.

\section{Sources of Contamination and Radioactivity}

The main sources of radioactivity in the primary loop coolant, i.e., sodium comes from:

- Activation of the coolant

- Activation of dissolved or entrained impurities in the coolant as they pass through the core. These impurities include existing impurities such as $\mathrm{Li}, \mathrm{U}$ and entrained impurities due to corrosion. Specifying the purity of sodium can control the activation of impurities.

- Activation of corrosion products, which subsequently enter the coolant. The components of importance are: Fuel cladding, core structural materials, control rods, and wear resistance.

- Release of active fission products and fuel components from failed pins.

- Release of Tritium from $6 \mathrm{Li}$ reactions in the coolant, ternary fission in fuel and activation of boron in control rod materials.

\section{Activation of the Coolant}

The major radioactive isotope in the coolant is ${ }^{24} \mathrm{Na}$, with a half-life of $15-\mathrm{h}$ it is produced from the reaction ${ }^{23} \mathrm{Na}(\mathrm{n}, \gamma){ }^{24} \mathrm{Na}$. Additional, but lower activities come from ${ }^{22} \mathrm{Na}$ (halflife 2.6 years) produced by $\mathrm{n}, 2 \mathrm{n}$ reactions and from ${ }^{23} \mathrm{Ne}$ (half-life $38 \mathrm{~s}$ ) produced from

${ }^{23} \mathrm{Na}(\mathrm{n}, \mathrm{p}){ }^{23} \mathrm{Ne}$ reactions. Because of its short half-life the presence of any ${ }^{24} \mathrm{Na}$ in the sodium wetted film, covering component removed from the reactor should only present short-term problems and over the long term the lower energy isotope ${ }^{22} \mathrm{Na}$ will be the dominant species ${ }^{(1,3)}$. 
Control of impurities in the coolant during normal plant operation, by continuous or periodic cold trapping of the coolant, should ensure that increased levels of impurities will only occur if contaminants enter the circuit either during core changes, or from failed components or replacement items of plant. The presence of $\mathrm{Li}$ and $\mathrm{U}$ impurities in the coolant, although capable of producing extra tritium and fissile materials, have negligible effects on activity levels compared to levels produced from ternary fission and failed pins respectively ${ }^{(1,3)}$.

- ${ }^{24} \mathrm{Na}$ radioisotope

The major source of radioactivity in the sodium of the LMFBR is due to ${ }^{24} \mathrm{Na}(\sim 0.5$ $\mathrm{TBq} / \mathrm{m}^{3}$, as shown in Table 1 , and all the calculations for the biological protection of the reactor are based on the exposure to this nuclide. However because of its short half-life of only $15 \mathrm{~h}$, it is of less importance during reactor shutdown and only nuclides of half-life of at least several days are of interest during this period.

- ${ }^{22} \mathrm{Na}$ radioisotope

Because of its long half-life of $2.6 \mathrm{a}^{22} \mathrm{Na}$ radioactivity increases in sodium with reactor thermal power history. Typical reactor values are around $20 \mathrm{MBq} / \mathrm{kg}$, (Table 1). After a long reactor shutdown, its radioactivity in the aerosols deposited in the cover gas, can make maintenance work difficult ${ }^{(4)}$.

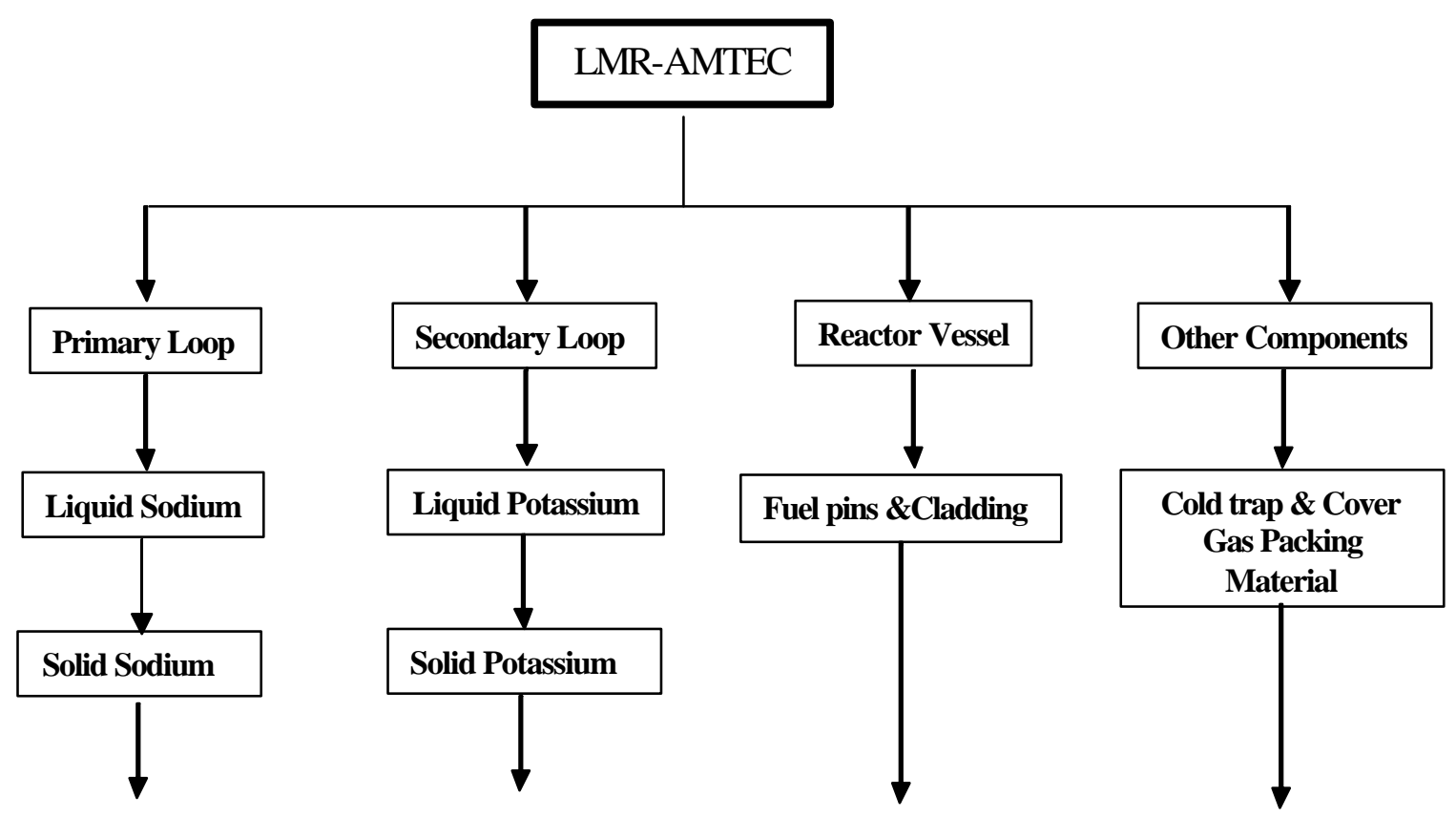

Figure 2-74. LMR-AMTEC Waste Management Flowcharts - Waste Generation 
Table 2-36. Maximum Activity Levels Measured in Sodium of LMFBRs ${ }^{(1)}$

\begin{tabular}{|c|c|c|c|c|c|c|c|c|}
\hline \multicolumn{2}{|l|}{ Isotope: } & ${ }^{24} \mathrm{Na}$ & ${ }^{22} \mathrm{Na}$ & ${ }^{65} \mathrm{Zn}$ & ${ }^{124} \mathrm{Sb}$ & ${ }^{131}$ I & ${ }^{137} \mathrm{Cs}$ & ${ }^{134} \mathrm{Cs}$ \\
\hline \multicolumn{2}{|l|}{ Half-life: } & $15 \mathrm{~h}$ & $2.6 \mathrm{a}$ & $244 \mathrm{~d}$ & $60.3 \mathrm{~d}$ & $8 \mathrm{~d}$ & $30 \mathrm{a}$ & $2.2 \mathrm{a}$ \\
\hline \multirow{2}{*}{$\begin{array}{l}\text { Reactor } \\
\text { Type }\end{array}$} & Unit: & $\mathrm{MBq} / \mathrm{kg}$ & $\mathrm{MBq} / \mathrm{kg}$ & $\mathrm{MBq} / \mathrm{kg}$ & $\mathrm{MBq} / \mathrm{kg}$ & $\mathrm{MBq} / \mathrm{kg}$ & $\mathrm{MBq} / \mathrm{kg}$ & $\mathrm{MBq} / \mathrm{kg}$ \\
\hline & Ref. & & & & & & & \\
\hline EBR-II & (5) & 100 & 5 & & 21 & 11 & 13 & 1.6 \\
\hline FFTF & (5) & 410 & 20 & & 0.026 & 1.8 & 8.1 & 5.5 \\
\hline KNK-II & $(6,7)$ & 2500 & 25 & 6.4 & 1.4 & 38 & 34 & \\
\hline PFR & $(8,9)$ & & 14 & & & & 11.7 & 2.2 \\
\hline RAPSODIE & 10 & & 40 & & & & 55 & \\
\hline PHENIX & $(10,11)$ & & 22 & & & & 1.7 & 0.33 \\
\hline BOR-60 & $(12,13)$ & & 22 & 0.74 & & 103 & 777 & 66 \\
\hline BN-350 & (14) & 370 & 28 & & & & & \\
\hline BN-600 & (13) & 750 & 26 & & 0.15 & 13 & 150 & 50 \\
\hline
\end{tabular}

\section{Activation of Corrosion Products}

Unless a reactor has operated for a long time with defective fuel elements, radioactive corrosion products are the major sources for system contamination. They can be formed in two ways:

- Transport of non-radioactive corrosion products mainly from heat exchanger into the core region and followed by activation.

- Corrosion of already activated material in the core region, like cladding and other core structure materials.

Although the first process is the most important for system contamination of water-cooled nuclear reactors, it is considered to be insignificant for LMFBRs. The second process is important in primary liquid sodium systems.

The major radionuclides produced by neutron-induced reactions in an LMFBR core are: ${ }^{51} \mathrm{Cr},{ }^{54} \mathrm{Mn},{ }^{59} \mathrm{Fe},{ }^{58} \mathrm{Co}$ and ${ }^{182} \mathrm{Ta}$. The relatively short half-lives of ${ }^{51} \mathrm{Cr}$ and ${ }^{59} \mathrm{Fe}$ (27.8 and $45 \mathrm{~d}$, respectively) and low level of $\mathrm{Ta}$ in steels $(k 0.1 \%$ ) means that the activity levels produced by these isotopes are only of interest during normal plant operation and once the plant is shutdown the longer-lived isotopes ${ }^{58} \mathrm{Co},{ }^{60} \mathrm{Co}$ and ${ }^{54} \mathrm{Mn}$ become the radionuclides of concern. 
- ${ }^{58}$ Co radioisotope

${ }^{58} \mathrm{Co}$ comes from the nickel content of the steel according to the reaction ${ }^{58} \mathrm{Ni}$ $(\mathrm{n}, \mathrm{p})^{58} \mathrm{Co}$. It has a modest half-life $(71 \mathrm{~d})$ and high gamma energy $(0.81 \mathrm{Mev})$, which makes it a major contributor to external dose rates once components have been removed from the reactor ${ }^{(1)}$.

- ${ }^{60}$ Co radioisotope

${ }^{60} \mathrm{Co}$ is derived solely from inactive $\mathrm{Co}$ and the ${ }^{60} \mathrm{Ni}$ content of steel alloy. The steel cobalt alloy, Stellite can also be a major source of ${ }^{60} \mathrm{Co}$. It has a long half-life (5.27 a) and therefore the build up of released activity to the coolant from this source is slow and never comes to equilibrium during typical fuel element lifetime ${ }^{(1)}$.

- ${ }^{54} \mathrm{Mn}$ radioisotope

${ }^{54} \mathrm{Mn}$ is the most prominent radioactive corrosion product (half-life $300 \mathrm{~d}$ ), with a gamma energy of $0.84 \mathrm{Mev}$ it approaches equilibrium at a faster rate and thus becomes a major contributor to activity levels in reactor circuits as in KNK-II as well as in other LMFBRs. ${ }^{54} \mathrm{Mn}$ deposits preferentially in the cooler sections of the primary system and it is responsible for the main part of the dose rate at the intermediate heat exchangers during shutdown periods ${ }^{(1,3)}$.

- ${ }^{65} \mathrm{Zn}$ radioisotope

${ }^{65} \mathrm{Zn}$ (half-life $244 \mathrm{~d}$ ) has been observed in various reactors, although its source is not always known. In KNK the main source was probably a zinc chromate protective coating used to cover the external surfaces of the ferritic components prior to assembly ${ }^{(15)}$. A further possible source was an oil leakage into the primary sodium from one of the primary pumps, the oil being stabilized with zinc dithiophosphate. The behavior of ${ }^{65} \mathrm{Zn}$ in sodium is similar to the behavior of ${ }^{137} \mathrm{Cs}$ in that its solubility in sodium is high $(100$ $\mu \mathrm{g} / \mathrm{g}$ at $\left.220{ }^{\circ} \mathrm{C}\right){ }^{(16)}$. It appears to plate out on steel surfaces by a chemisorption's process and its diffusion into stainless steel is also similar to that of ${ }^{137} \mathrm{Cs}$.

- ${ }^{124} \mathrm{Sb}$ radioisotope

Although the solubility of antimony in sodium is high, its deposition on nickel or steel surfaces is negligible. It is bund to co-precipitate with sodium oxide in cold trap and therefore its behavior is very dependent upon the oxide impurity level in the sodium. ${ }^{124} \mathrm{Sb}$ (half-life 60.3d) has been reported at PHENIX and it was found in Experimental Breeder Reactor, EBR-II, in 1984. In both instances its presence was due to a leaking SbBe neutron source used at start up of the reactor. At KNK, its presence was attributed to the use of "Molykote" (a lubricant containing antimony trisulfide and Molybdenum) for the bearing rings at the bottom of the sub-assemblies ${ }^{(6)}$.

\section{Active Fission Products from Failed Pins}

Fission products are generated during operation of a nuclear reactor inside the fuel. The nuclear fuel is usually enclosed in gastight fuel cladding tubes, e.g. $\mathrm{Zr}-\mathrm{Nb}$ alloy. During regular operation all fission products (except tritium) remain within the fuel pins. The inventories of fission products at any time in the life of a particular fuel pin can be calculated with a good certainty. For fast neutron fission the yields of the most abundant mass chains are known, within a few percent, for ${ }^{235} \mathrm{U},{ }^{238} \mathrm{U}$ and ${ }^{239} \mathrm{Pu}$. While operating several thousand fuel pins in large sodium cooled power reactor, one or several fuel pin claddings may fail. Fission products are then released into primary sodium. Open fuel failures expose fuel surfaces to the coolant, all kinds of fission products may be released 
into the sodium, and even fuel particles may be washed out from larger failures. Fission gas monitors in the coverage line may give larger signals, shorter-lived fission gases like ${ }^{138} \mathrm{Xe}$ and ${ }^{135 \mathrm{~m}} \mathrm{Xe}$ may be detected by gamma spectroscopy in the cover gas (Helium). The amount of activity released from a failed pin has been shown to depend upon the isotopic content of the fuel prior to failure, the quantity of elements present in the fuel clad gas gap, the rating of the pin and reactor history ${ }^{(17)}$. Irrespective of defect size the noble gases $(\mathrm{Xe}, \mathrm{Kr}$ ) are released fairly quickly and their limited solubility in sodium ensures rapid removal to the gas space. Fission products of high volatility behave in a similar manner and those elements of high yield, namely $\mathrm{Cs}$, I, Ba and Sr can also be released during the initial stages of fuel clad failures.

The level of activity circulating in the coolant depends upon the solubility behavior of the various isotopes. In principle, levels of activity produced by the partly soluble I, Te, Sb, Sn and Ag radionuclides can be reduced by operation of the cold trap. However, if fuel pin failures become excessive, there is a possibility that the cold trap may become a relatively ineffective sink and plate-out of the various products may occur elsewhere in the circuit.

Isotopes of major concern are ${ }^{134} \mathrm{Cs}$ and ${ }^{137} \mathrm{Cs}$ which have half-lives of 2 and $30 \mathrm{a}$, respectively. Both isotopes can produce radiation fields comparable to those produced by deposited corrosion products. The complete miscibility of Cs in sodium coupled with its high volatility points to complex behavior in sodium system. Other fission products such as the lanthanides, $\mathrm{Zr}, \mathrm{Nb}, \mathrm{Ru}$, Mo and fuel products have very low solubility in sodium.

\section{Tritium in LMR}

Although tritium is unlikely to present a major hazard during normal plant operation, the element is of biological importance and therefore knowledge of its transport behavior in an operating plant is required. In sodium-cooled reactors tritium originated principally in two locations, in the fuel and in the control rods. It is created in the fuel by ternary fission and in the control rods by neutron capture with the boron in $\mathrm{B}_{4} \mathrm{C}$. In addition, it will be produced by neutron activation of $\mathrm{Li}$ and boron impurities in the fuel and in the primary sodium $^{(3)}$.

At reactor operation temperatures tritium can permeate the fuel cladding and the walls of cooling systems. As a result, the possibility of escape of tritium must be considered. For complete surveillance tritium has to be measured in the sodium coolant, the helium in the reactor tank double wall, in the potassium coolant, secondary cover gas and in the AMTEC unit. Tritium is very well fixed in cold traps by coprecipitation with hydrogen $(\mathrm{NaH})$. In a reactor system tritium may escape from the sodium coolant:

- into the helium cover gas where the equilibrium is assumed to be instantaneous,

- into potassium coolant, by diffusion through the walls of heat exchangers,

- into the interspace gas between the vessels, by diffusion through the steel of the vessel and pipe work, and

- in the cold trap by crystallization of sodium tritide, or by isotopic exchange.

The generation of Tritium in LMFBR is much lower than in a PWR. In an LMFBR Tritium is produced by fission process and by neutron absorption in Boron (Boron Carbide is used in the control rod of LMR-AMTEC). The production depends on the fuel type; for example MOX type fuels can generate more Tritium due to Plutonium. The 
diffusion to the secondary side can be controlled by venting the Tritium in the cover gas (where it tends to concentrate).

\subsection{Waste Generated in the Liquid Potassium Circuit}

The potassium element is widely distributed in nature, being the seventh in abundance. It is found in the earth's crust at a level of $1.5 \%$ by weight. Most of potassium minerals are insoluble and the metal is extracted from the ore with great difficulty, mainly by electrolysis or by thermal methods. Potassium is never found in metallic form in nature due to its high reactivity. Potassium is solid under atmospheric temperature and pressure; its melting point is $63^{\circ} \mathrm{C}(336 \mathrm{~K})$ while it boils at $759^{\circ} \mathrm{C}(1032 \mathrm{~K})$. Potassium has good electrical and thermal conductivity.

Due to its electronic configuration and the presence of one electron in the outer most shell, potassium has high reactive chemical characteristics so it reacts very rapidly with water to form a colorless solution of $\mathrm{KOH}$ and $\mathrm{H}_{2}$

$$
2 \mathrm{~K}(\mathrm{~s})+2 \mathrm{H}_{2} \mathrm{O}-2 \mathrm{KOH}(\mathrm{aq})+\mathrm{H}_{2}(\mathrm{~g})
$$

This is an exothermic type of reaction; therefore, early in the reaction the potassium metal becomes so hot that it catches fire and burns with a characteristic lilac color. The heat released in this reaction is sufficient to ignite a hydrogen-oxygen reaction, which can be rather explosive. Potassium reacts with oxygen to form potassium superoxide $\left(\mathrm{KO}_{2}\right)$.

$$
\mathrm{K}(\mathrm{s})+\mathrm{O}_{2}(\mathrm{~g}) \longrightarrow \mathrm{KO}_{2}(\mathrm{~s})
$$

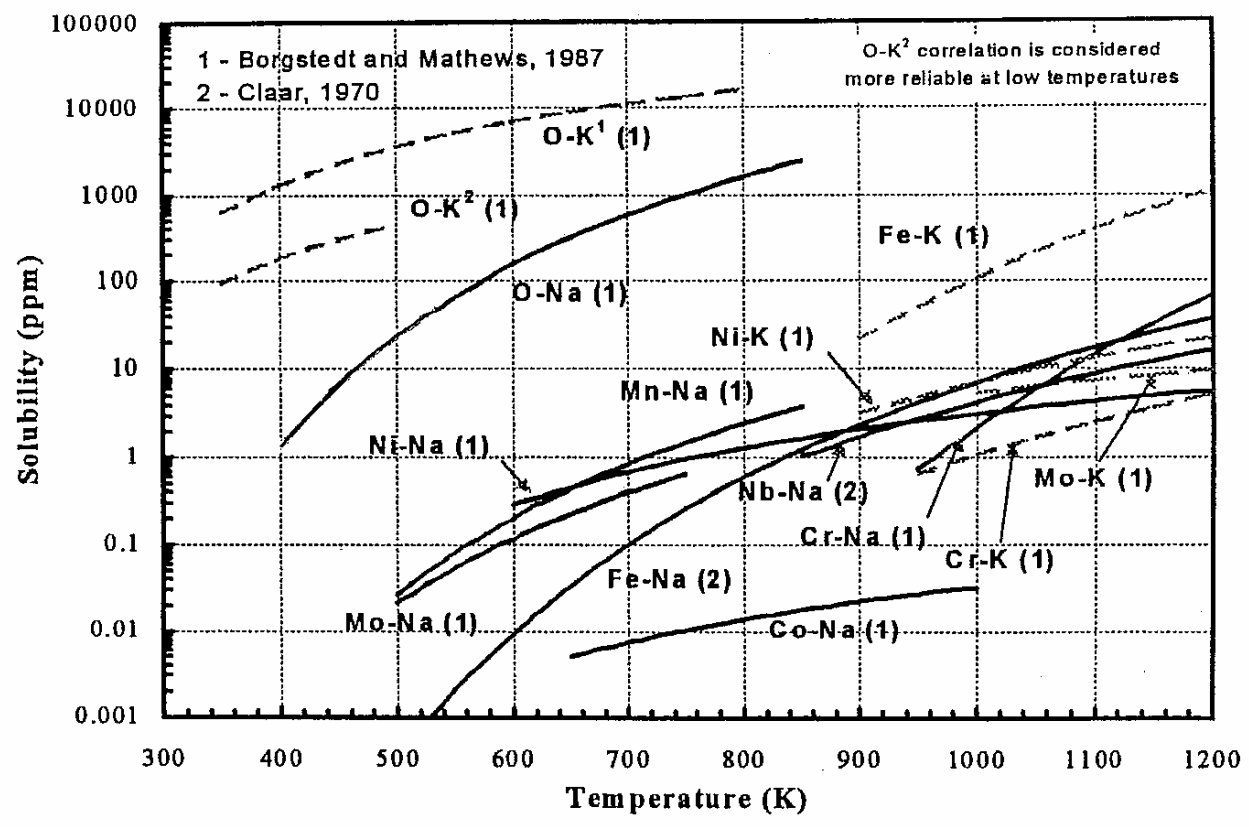

Figure 2-75. Solubility of Oxygen and Metallic Impurities in Liquid Sodium and Potassium. 


\section{Potassium Contamination by Corrosive Products}

The rate of corrosion by potassium is typically dependent on the type of structural material, oxygen content, flow rate and temperature where ${ }^{2}$

- Corrosion of stainless steel and super alloys includes preferential dissolution of nickel and chromium.

- Increased oxygen content and temperature increases corrosion rate.

- Increased nickel content typically increases corrosion rate but decreases the differentials of oxygen content on corrosion rate.

Experimental results and observations have shown clearly that corrosion by liquid metals is a strong function of oxygen content and is accelerated due to the presence of certain metallic elements, such as nickel. Cold trapping has been shown to be an effective method of controlling the oxygen and impurities levels, as in the Experimental Breeder Reactor (EBR-II), to an acceptable level ${ }^{(2)}$.

Figure 3 indicates that potassium liquid can corrode $\mathrm{Fe}, \mathrm{Ni}$, Mo and $\mathrm{Cr}$ elements especially at high oxygen content and high temperature. High temperatures increase oxygen dissolution, which results in failure of the cold trapping at $425 \mathrm{~K}$ to reduce the oxygen content of liquid potassium below $200 \mathrm{ppm}$. Hot trapping with oxygen gathering materials such as zirconium, hafnium or yttrium will be needed to maintain the oxygen concentration in liquid potassium at the desired levels ${ }^{(2)}$.

\section{Potassium Contamination by Radioactive Products}

Except for the probable contamination with tritium $\left({ }^{3} \mathrm{H}\right)$, there is generally no probability of contaminating liquid potassium by the fission products. Potassium liquid takes an independent route in the LMR-AMTEC configuration with no contact with sources of fission product contamination such as reactor fuel and liquid sodium.

Tritium is produced as a fission product from the fuel and at the reactor operating temperature, it can go through the fuel cladding and hence reach the sodium liquid. Due to its high penetration capability, tritium may also reach the liquid potassium during the heat exchange between sodium and potassium liquids inside the boiler by diffusion ${ }^{(3)}$.

\subsection{Waste Generation from Cold Trap}

A cold trap is a method used to purify the sodium in the first loop of LMFBRs as well as the potassium in the second loop. Although the principal role of the cold trap in the LMFBRs is to control the concentration of dissolved oxygen impurity and hence maintain acceptable levels of corrosion of steels and related radioactive corrosion products, a number of traps have been known to collect radioactive impurities. During cold trap operation, decreasing the sodium temperature in the cold trap causes crystallization of $\mathrm{Na}_{2} \mathrm{O}$ on the wires of the steel mesh. It is also established that cold traps are effective in controlling hydrogen impurities by precipitating sodium hydride, $\mathrm{NaH}$. A certain number of radioactive impurities have been observed to be cold trapped too, although cold traps are not designed with this intension. For the removal of certain radioactive constituents 
such as Cesium, Iodine, oxide forming fission products and radioactive corrosion products, a number of factors have to be considered in relation to this trapping behavior. The cold trap packing material, which is contaminated by radioactive fission products, corrosion products and $\mathrm{Na}_{2} \mathrm{O}$ and $\mathrm{NaH}$, is considered a low level waste after removing from the cold trap unit ${ }^{(3)}$.

\subsection{Waste Generation from Cover Gas Purification Units}

Although the activity resulting from corrosion products and other deposited species is contained within the primary vessel of pooltype LMFBRs, it is still necessary to control the build up of radioactivity in operating systems. Radioactive isotopes in cover gas, for example, are passed through gas purification units as part of normal reactor operations. The dominant radionuclides in the cover gas come from argon, isotopes of cesium and sodium and the noble gases such as, ${ }^{87} \mathrm{Kr},{ }^{88} \mathrm{Kr},{ }^{85 \mathrm{~m}} \mathrm{Kr},{ }^{135} \mathrm{Xe},{ }^{133 \mathrm{~m}} \mathrm{Xe}$ and ${ }^{133} \mathrm{Xe}$, as shown in Table 2. At RAPSOIDE (France) the use of helium for the cover gas allowed krypton to be trapped on charcoal in the cooled charcoal gas purification unit with an efficiency greater than $80 \%\left(100 \%\right.$ for Xe) ${ }^{(18)}$. At PHENIX and SUPER PHENIX, a gas purification unit is installed to trap radio-xenon on charcoal cooled by nitrogen while retention tanks allow for the decay of radio-krypton. The charcoal or activated carbon used in the purification unit, which is contaminated by isotopes of $\mathrm{Cs}$, $\mathrm{Na}$ and noble gases, is considered a low level waste. Table 3 illustrates the materials and techniques used in different LMFBRs around the world.

\subsection{Waste Generation from Special Traps}

Because radionuclides are deposited on the walls of the primary coolant system of LMFBRs, when liquid sodium is circulating at low temperatures, the gamma radiation fields may be high in the primary cell even when the sodium was drained into dump tank. Fission products, especially ${ }^{134} \mathrm{Cs},{ }^{137} \mathrm{Cs},{ }^{58} \mathrm{Co}$ and ${ }^{60} \mathrm{Co}$, are mainly responsible for the high gamma radiation in the primary sodium system. A cesium trap was designed for operation in the EBR-II primary sodium purification system. The trap contains $0.01 \mathrm{~m}^{3}$ of reticulated vitreous carbon, is about $26 \mathrm{~cm}$ in diameter and $34 \mathrm{~cm}$ long. It is thermally insulated and shielded with $11 \mathrm{~cm}$ of lead. The vitreous carbon used in the trap, which is contaminated with isotopes of Cs, is considered a low level waste ${ }^{(26)}$.

\subsection{Spent Nuclear Fuel}

The management of spent nuclear fuel from the LMR-AMTEC cores must comply with 10 CFR 72.108, 10 CFR 72.126, 10 CFR 72.128, 10 CFR 72.130, 10 CFR 72.166 and 10 CFR 60.135. A current concern is the safe disposal and isolation of spent fuel from reactors; under the Nuclear Waste Policy Act of 1982 the U.S. Department of Energy (DOE) has responsibility for the development of a national waste disposal system for spent nuclear fuel and high-level radioactive waste. Current plans call for the ultimate disposal of the wastes in solid form in deep, stable geological structures such as the proposed Yucca Mountain site in Nevada as specified in 10 CFR 63. Recently, Congress and the president of the US approved the site for high level waste disposal and it is going 
through the final phase of licensing application. It is expected that the site will be opened to accept high level waste in the year 2004.

Table 2-37. Maximum Activity Levels Measured in The Cover Gas of LMFBRS in GBq/m3

\begin{tabular}{|c|c|c|c|c|c|c|c|c|c|}
\hline \multirow{2}{*}{$\begin{array}{l}\text { Reactor } \\
\text { Name }\end{array}$} & Isotope: & ${ }^{23} \mathrm{Ne}$ & ${ }^{41} \mathrm{Ar}$ & ${ }^{133} \mathrm{Xe}$ & ${ }^{135} \mathrm{Xe}$ & ${ }^{85 m} \mathrm{Kr}$ & ${ }^{88} \mathbf{K r}$ & ${ }^{87} \mathbf{K r}$ & \multirow{2}{*}{ Ref. } \\
\hline & Half-life: & $38 \mathrm{~s}$ & $1.8 \mathrm{~h}$ & $5.2 \mathrm{~d}$ & $9 \mathrm{~h}$ & $4.5 \mathrm{~h}$ & $2.8 \mathrm{~h}$ & $1.3 \mathrm{~h}$ & \\
\hline FFTF & & 74000 & 15 & 1900 & 800 & 200 & 200 & 75 & (19) \\
\hline KNK-II & 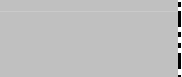 & & 7 & 1240 & 134 & 11 & 13 & 6 & $(20,21)$ \\
\hline PHENIX & & 7000 & 1.5 & 2500 & 1500 & 300 & 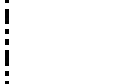 & 200 & $(13,18)$ \\
\hline BR-5 & & 26000 & 5.5 & 7400 & 4400 & & 3700 & 1800 & (22) \\
\hline BOR-60 & & & 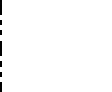 & 25000 & 2000 & 200 & 70 & & (23) \\
\hline BN-350 & & 18000 & 0.74 & 4000 & & & ! & & (24) \\
\hline BN-600 & & & 5.3 & 4800 & & & i & & (15) \\
\hline
\end{tabular}

Table 2-38. Different Techniques for Removal of Radioactivity in The Cover Gas (25)

\begin{tabular}{|l|l|l|}
\hline Reactor & Country & Purification Techniques \\
\hline RAPSODIE & France & Active carbon filter before discharge \\
\hline KNK-II & Germany & Active carbon filter \\
\hline MONJU & Japan & Charcoal delay bed \\
\hline EBR-II & USA & Cryo distillation \\
\hline FFTF & USA & Carbon delay bed and cold trap \\
\hline PHENIX & France & Active carbon bed and cryogenic effect \\
\hline ALMR & USA & Activated carbon bed \\
\hline SUPER-PHENIX1 & France & Active carbon bed and cryogenic effect \\
\hline SUPER-PHENIX2 & France & Sweeping and filtering \\
\hline BN-1600 & Russia & Aerosol filter \\
\hline BN-800 & Russia & Aerosol filter \\
\hline
\end{tabular}




\subsubsection{Waste Characterization for LMR-AMTEC}

The objective of this section was to define the waste generation and characterization from the LMR-AMTEC and to identify the applicable US regulations governing waste transportation, treatment, storage and final disposition. The waste generated from LMRAMTEC is characterized as: (1) mixed waste which is generated from liquid sodium contaminated by fission products and activated corrosion products, (2) hazardous waste which is generated from liquid potassium contaminated by corrosion products, (3) spent nuclear fuel, and (4) low level radioactive waste which is generated from the packing materials; e.g., activated carbon in cold trap and purification units. The regulations governing these wastes are summarized in this section.

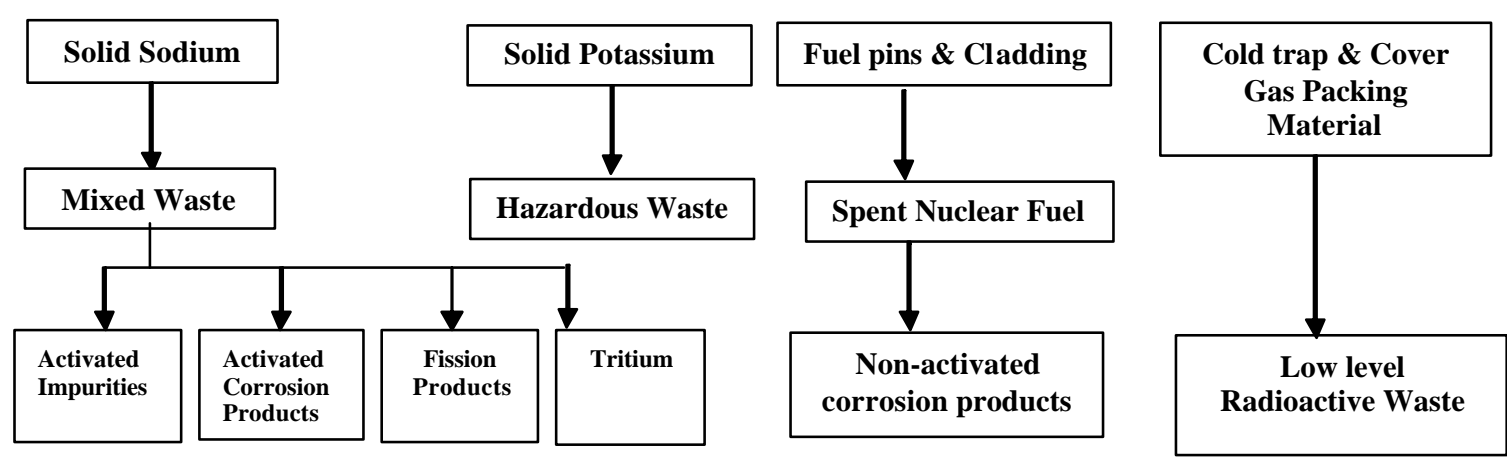

Figure 2-76. LMR-AMTEC Waste Management Flowchart - Waste Characterization

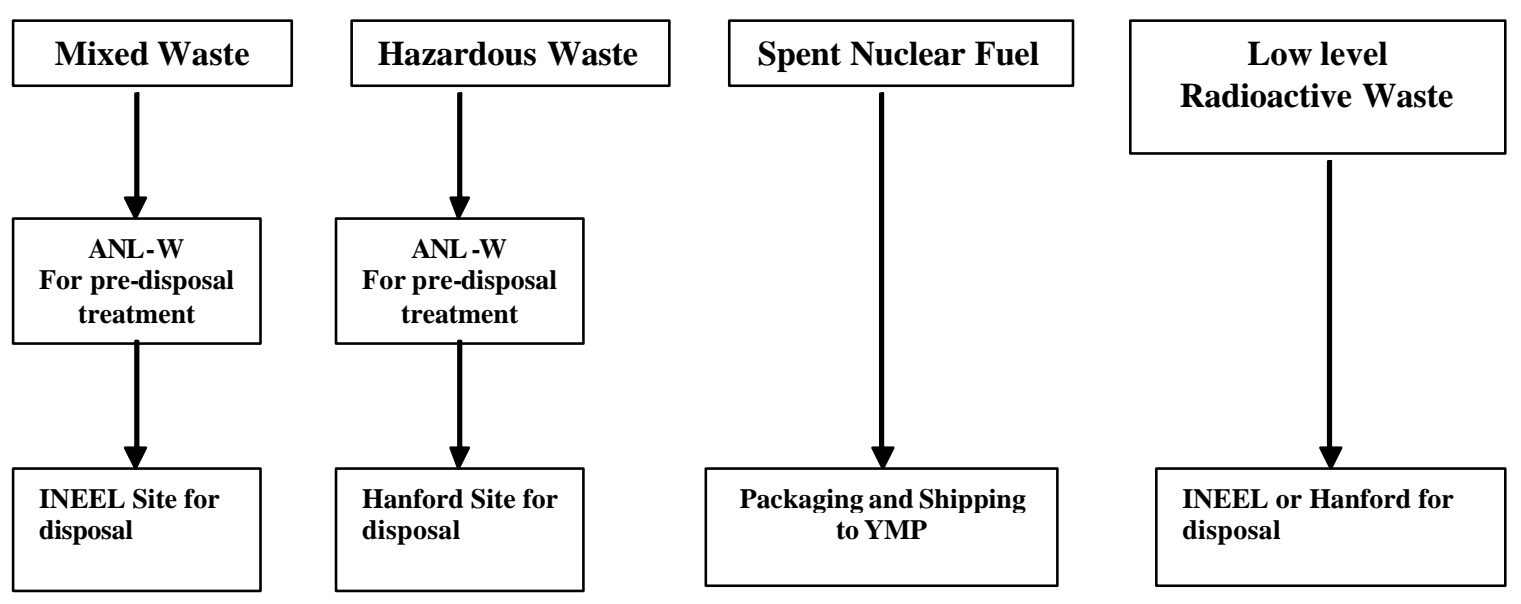

Figure 2-77. LMR-AMTEC Waste Management Flowchart - Waste Treatment 


\subsection{Mixed Waste}

Sodium liquid metal coolant, which is contaminated with fission products and activated corrosion products from the core meltdown of LMR, is classified as a mixed waste.

The principal regulatory agencies involved in the treatment and disposal of mixed waste are the EPA and NRC. DOE is subject to regulations promulgated by these agencies through the Federal Facility Compliance Act (FFCA) of 1992, which requires federal facilities to comply with the same regulations as non-federal facilities (FFCA, 1992). Thus, in dealing with its mixed waste, DOE's Office of Environmental Management (EM) must comply with EPA regulations for hazardous wastes and with NRC regulations for radioactive wastes. Further, the FFCA requires DOE to comply with applicable state regulations if they are more restrictive than federal regulations.

EPA has developed regulations for hazardous waste management and disposal principally under authority of the RCRA, as amended 1984. RCRA provides for the cradle to grave control of hazardous wastes by imposing management requirements on generators and transporters of hazardous waste and on owners and operators of treatment and storage facilities. The RCRA hazardous waste regulations are found in Title 40 of the Code of Federal Regulations. Parts 260 to 265 of Title 40 describe hazardous waste management, provide EPA's lists of hazardous wastes, and set standards that must be met by hazardous waste generators and managers. EPA's land disposal restrictions are given in 40 CFR 268 and its permit programs in 40 CFR 270. The NRC operates under the authority of the Atomic Energy Act (AEA) of 1954 and its subsequent amendments. NRC regulations that affect management of DOE mixed waste include: 10 CFR 61, Low-Level Waste Disposal Regulation, and 10 CFR 20, radiation Protection Standards. The NRC closely regulates stability and radioactive characteristics of low-level waste materials acceptable for near-surface land disposal through a combination of prescriptive and performance based requirements specified in 10 CFR 61.55-56.

Liquid sodium coolant containing high activity of corrosion and fission products is considered a mixed waste and regulated under RCRA and NRC. Waste with hazardous components prohibited from land disposal according to 40 CFR 268 Land Disposal Restrictions must be treated as specified according to 40 CFR 268, Subpart D, and Treatment Standards prior to disposal or interim storage. The Sodium Process Facility (SPF) at Argonne National Laboratory-West was designed and constructed to convert the elemental sodium from the EBR-II primary and secondary systems to sodium carbonate that is not RCRA regulated. This waste is acceptable for land disposal at the Radioactive Waste Management Complex at INEEL as low-level radioactive waste. Waste packages must meet all applicable provisions of DOT regulations in 49 CFR parts 171-178 and NRC regulations in $10 \mathrm{CFR} 71$. Transportation of mixed waste shall meet applicable DOT and USEPA regulatory requirements for hazardous components in addition to meeting regulatory requirements for radioactive materials. Mixed waste must be classified according to requirements for hazardous material as defined by Title $49 \mathrm{CFR}$ 173.2. The requirements of Title 40 CFR 264, subpart I Use and management of Containers shall be met for all mixed waste packages.

Sodium liquid metal coolant contaminated with fission products and activated corrosion products and classified as a mixed waste must be deactivated and converted to a contact- 
handled low level waste at the Argonne Sodium Component Maintenance shop and near surface land disposal at the Radioactive Waste Management Complex (RWMC). Treatment of the sodium involves converting the sodium to its respective hydroxide via reaction with air and water, followed by conversion to its respective carbonate via reaction with carbon dioxide. The resultant aqueous carbonate solution must be solidified in 55 gallon drums using Aquaset II-H (a product of Fluid Tech. Inc.) $(33,34)$. The waste that generated from first and second loop of LMR-AMTEC is similar to the waste generated from EBR-I and EBR-II. Treatment of the EBR-I NaK and EBR-II sodium was successful by accomplished by converting the mixed waste to a non-hazardous form and land disposal of the treated waste at the sodium facility in Argonne National Laboratory West (ANL-W) as LLW. The NaK of EBR-I containers consisting of two 55-gallon MIN Safety Appliance (MSA) drums, one 60 gallon and another 10-gallon closed pipe section.

The sodium carbonate, which is contaminated by fission products and activated corrosion products, is considered as a low level radioactive waste and could be transported to the INEEL RWMC for disposal using a type A container (55- gallon steel drum). Disposal criteria at the RWMC don't allow liquids to be land disposed.

INEEL site-specific requirements for disposal of LLW are specified in Ref. 35. These requirements identify the acceptance criteria for LLW and greater than Class C (GTCC) waste for disposal at the RWMC. The radiological performance assessment for RWMC was conditionally approved by DOE-HQ. The site-specific disposal limits, which are based on the conditionally approved performance assessment, have been developed in the INEEL RWAC. The requirements for classifying LLW at INEEL are the same as those defined in 10 CFR 61 and the clarification guidance provided in the various NRC's Branch technical position papers relating to LLW.

\subsection{Hazardous Waste}

Potassium liquid coolant is considered as a hazardous waste according to 40 CFR 261.2123. The potassium hazardous waste from the LMR-AMTEC must be treated before it can be sent to an RCRA-permitted land disposal facility (Land Disposal Restrictions, LDR, 40 CFR 268). Also like the mixed LLW, the Hanford Site currently does not have facilities to treat all types of hazardous waste. Therefore the untreated waste must be stored until it can be treated and become compliant with RCRA and state regulations. The potassium hazardous waste from LMR-AMTEC could be stored at the Hanford short term storage for up to 90 days, the maximum allowed for a non-permitted storage facility. Storage for more than 90 days requires a RCRA- permitted storage facility.

The treatment of potassium liquid coolant, which is considered as a hazardous waste, could be accomplished at the Argonne National Laboratory-West ANL-W. Treatment of the potassium involves converting the potassium to its respective hydroxide via reaction with air and water, followed by conversion to its respective carbonate via reaction with carbon dioxide. The resultant aqueous carbonate solution could be solidified in 55-gallon drums using Aquaset II-H (a product of Fluid Tech. Inc.) ${ }^{(33,34)}$.

The potassium liquid coolant can be transported in 55 gallon steel drums to the ANL-W for treatment. The container must be shaken to determine if potassium super-oxide $\left(\mathrm{KO}_{2}\right)$ is present which might present an explosion hazard during shipment and handling at 
ANL-W. The presence of potassium super-oxide would have been noted by an increase in container temperature after shaking it. The waste generator must adhere to the regulations specified in 40 CFR 264.176 and 264.281 concerning special requirements for ignitable, corrosive and reactive waste.

The management of hazardous waste will be chosen according to 40 CFR 264.170 to 175 . Packaging and transportation of hazardous wastes must meet the requirements in $49 \mathrm{CFR}$ 173 and 178. Each waste container must be clearly labeled with the words "Hazardous Waste", and meet other general requirements, which include: bar code, durability of labels and marks to remain intact and legible during handling of the waste before disposal. In addition, placement of labels, size of labels and labeling inner containers in lab packs, all must meet the acceptance criteria of Hanford Site Waste Acceptance Criteria or any site accepting this kind of waste.

The waste generator must also adhere to requirements; these include storage limitations under the regulation of 40 CFR 262.34 and perform record keeping and reporting, particularly the manifest under the regulation of 40 CFR 262.20 and 262.40.

\subsection{Spent Nuclear Fuel}

Spent nuclear fuel from the LMR-AMTEC must be transported in strong, thick-walled casks. The DOE requires extremely durable and massive transportation casks whose designs are certified by the NRC. The containers use multiple layers of lead and other materials to protect the spent fuel and confine radiation. Typically, for every ton of spent fuel there are approximately four tons of protective shielding materials.

The transport of spent nuclear fuel from LMR-AMTEC will be highly regulated by the DOT and the NRC. The DOT regulates all hazardous waste transportation, including radioactive materials, to ensure public health and safety. The NRC regulates all commercial nuclear activities, the transportation of spent nuclear fuel, the design, manufacture, and security of transportation casks, and any development and operation of Yucca Mountain ${ }^{(32)}$.

Spent nuclear fuel shipments from LMR-AMTEC must be tracked and escorted. DOE practice is to track and escort each shipment 24-hours a day. All spent nuclear fuel cask designs from LMR-AMTEC must be certified by the NRC, and must safely contain radioactive contents under hypothetical accident conditions that simulate the conditions of severe accidents. These conditions must be evaluated in sequence, on the same cask design:

- A 30-foot free fall onto an unyielding surface, landing on the cask's weakest point, which would be equivalent to a crash at 120 miles per hour into a concrete bridge abutment;

- A puncture test, during which the container must fall 40 inches onto a steel rod six inches in diameter;

- A 30-minute exposure to fire at 1,475 degrees Fahrenheit that engulfs the entire container; and

- Submergence of the same container under three feet of water. 
To achieve certification, a cask must prevent harmful release of radioactive material even when subjected to each of these tests. Figure 4 illustrates the transportation cask for spent nuclear fuel.

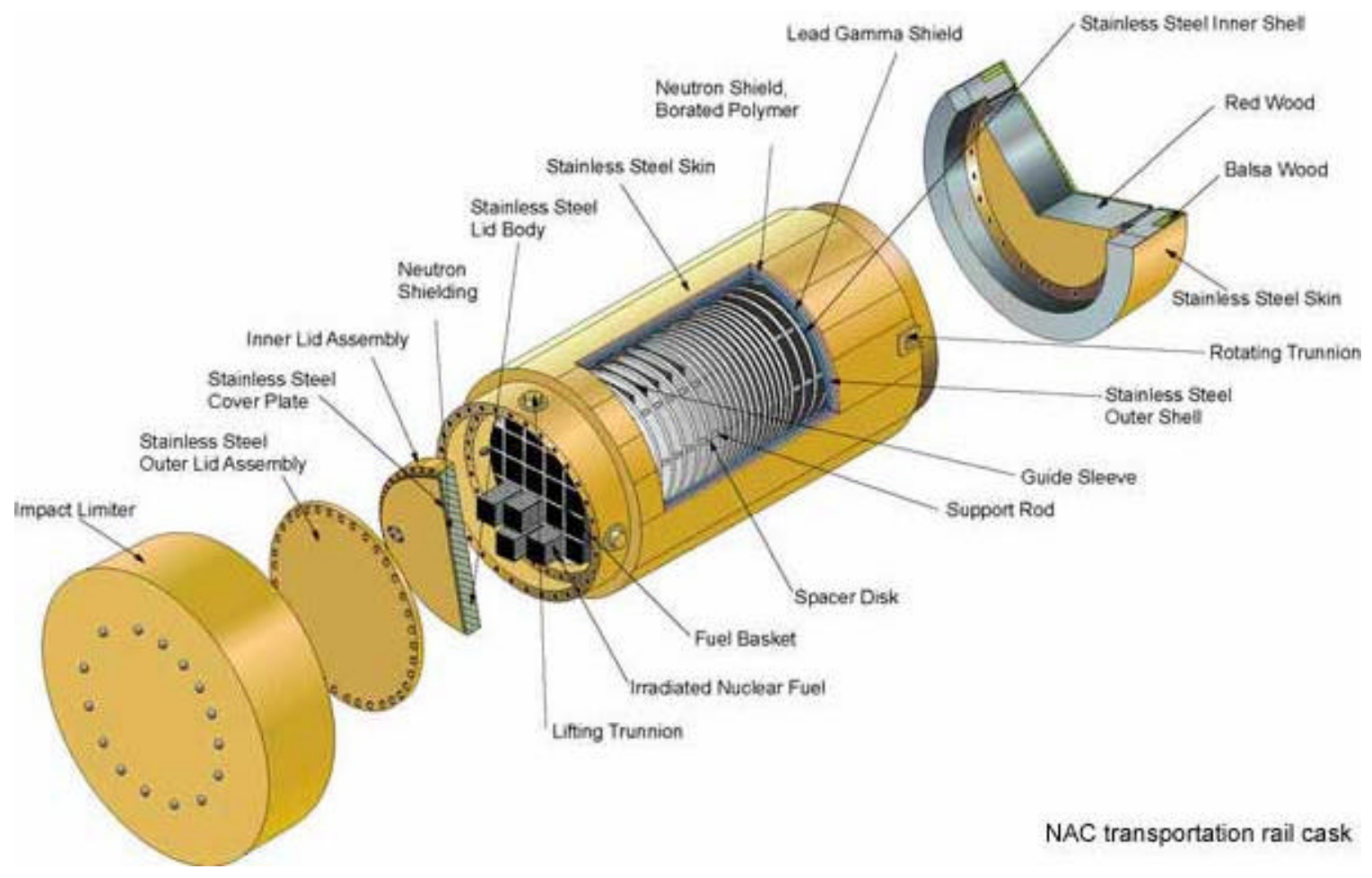

Figure 2-78. Spent Nuclear fuel Transportation Cask.

The spent nuclear fuel generated from LMFBRs, including LMR-AMTEC, contain elemental sodium which is a reactive material. Since reactive material is considered hazardous by EPA regulations it requires treatment before disposal in a geological repository. The EBR-II spent fuel, which is similar to spent fuel from LMR-AMTEC, was converted to three different products, low enrichment uranium, ceramic waste and metallic waste. The fuel treatment processes are operating in the Fuel Conditioning Facility (FCF) at ANL-W, a shielded hot cell facility at the Argonne site Idaho. The treatment process steps, which include fuel assembly dismantling element chopping, electrorefining, cathode processing and casting, are installed in FCF at $\mathrm{ANL}^{(29,30,31)}$.

Under 10 CFR 60, spent nuclear fuel is regulated as HLW. EPA has not yet determined whether SNF will be designated as mixed waste. When spent fuel is removed from the LMR-AMTEC, it should be placed in dry storage awaiting treatment in ANL-W and final disposal at the Yucca Mountain Site for high level waste. The waste generators must adhere to the waste acceptance criteria for the YMP site 10 CFR 63, such as storage of SNF according to 10 CFR 73.51, packaging, storage and transportation of SNF in certified containers and shipping regulations according to 10 CFR 961, 10 CFR 72.128, 10 CFR 72.108 and 10 CFR 60.135. 


\subsection{Summary}

Based on the results of the investigations presented in this chapter, the indirect interfacing arrangement through AMBs (located in the annulus between the riser and the reactor vessel wall) was selected for the LMR-AMTEC nuclear power plant for subsequent economical analysis. In the selected plant configuration, the nuclear reactor in a pool configuration is cooled by sodium working fluid, while the secondary working fluid is potassium. The hot potassium vapor from the AMBs is introduced directly into the high-pressure cavities of the AMTEC/TE converter units (open- unit design configuration). This configuration has the best performance with respect to net plant efficiency, vessel weight and corrosion behavior. The indirect interfacing arrangement (IC) with liquid-liquid IHXs and closed converter unit design was selected as an alternative design. In all interfacing arrangements, the design of the AMTEC/TE converter units is identical except for the feed in or circulation of the working fluid in the AMTEC units. Since the AMTEC units developed in this work are of the vapor-fed liquid anode type, they operate at the highest efficiency possible. Therefore, for the same BASE/evaporator temperature, the plant efficiency would be essentially the same, regardless of the interfacing arrangement, but economical and corrosion considerations would be different. The next Chapter reviews the work performed by UNM-ISNPS on the design of the sodium- and potassium-AMTEC/TE converter units, and presents detailed results of the optimization analysis of their overall performance.

\subsection{References}

Bailly H., Menessier D. and Prunier C., The Nuclear Fuel of Pressurized Water Reactors and Fast Reactors - Design and Behavior, Lavoisier Publishing, Paris, 1999.

Braquilanges B. and Bertrand R., "Decay Heat Removal and Natural Convection in Superphenix 1", Second Specialists' Meeting on Decay Heat Removal and Natural Convection in LMFBRs, New York, 1985.

Calamai G. J., "Final Report - Ultra Long Life Core and Plant Design Study," Report RA-81-80, Westinghouse Electric Corporation, 1981.

Carelli M. D., "Design Study of Advanced Small Or Medium Size FBR Concept", WAES-TR-91-0003, Westinghouse Electric Company, 1991.

Cardu M. and Dumitru M., "On a Simplified System for Steam production in Nuclear Power Plants with Pressurized Water Reactors", Energy Conversion \& Management, 40 (1999) 1931-1937.

Church A. H., Centrifugal Pumps and Blowers, John Wiley \& Sons, Inc., London 1944. 
Distefano J. R. and Devan J. H., "Refluxing Capsule Experiments with Refractory Metals and Boiling Alkali Metals", Nuclear Applications \& Technology, 8 (1970) 29-44.

Devans J. H., "Compatibility of structural Materials with boiling Potassium", Proceedings of the International Conference on liquid Metal Technology in Energy Production, May 1976.

Devans J. H., "Corrosion of iron and nickel-based alloys in high-temperature sodium and NaK", Proceedings of the Symposium on Alkali metal Coolants, IAEA, Vienna, 1967

Draley J. E. and Weeks J. R., Corrosion by Liquid Metal, Plenum Press, New York, 1970.

Dutt D., Road Map for SP-100 irradiation, WHC-SP-1083.

Emmet W. L. R., "Mercury Vapor for Central-Station Power", Mechanical Engineering, (1941) 351-356.

English R. E. and Weltmann R. N., "Experience in Investigation of Components of Alkali-Metal-Vapor Space Power Systems", Proceedings of the Symposium on Alkali Metal Coolant, IAEA, Vienna, 1967.

Fraas A. P., "Design Precepts for High-Temperature Heat Exchangers", Nuclear Science and Engineering, 8 (1960) 21-31.

Fraas A. P., "A Potassium-Steam Binary Vapor Cycle for a molten-Salt Reactor Power Plant”, Journal for Engineering for Power, (1966) 355-366.

Fraas A. P., "A Potassium-Steam Binary Vapor Cycle for Better Fuel Economy and Reduced Thermal Pollution”, Journal of Engineering for Power, (1973) 53-62.

Fraas A. P., "Comparison of Helium, Potassium, and Cesium Cycles”, IECEC75, 1975.

Fraas A. P., Engineering Evaluation of Energy Systems, McGraw-Hill, 1982.

Fraas A. P., Heat Exchanger Design, While-Interscience Publication, 1989.

Forster H. K. and Zuber N., "Dynamics of Vapor Bubbles and Boiling Heat transfer", A.I.Ch.E. Journal, 1 (1955) 531-535.

Foust O. J., Sodium-NaK Engineering Handbook Volume I: Sodium Chemistry and Physical Properties, Gordon and Breach, Science Publishers inc., 1978.

Foust O. J., Sodium-NaK Engineering Handbook Volume II: Sodium Flow, Heat Transfer, Intermediate Heat Exchangers, and Steam Generators, Gordon and Breach, Science Publishers inc., 1978.

Foust O. J., Sodium-NaK Engineering Handbook Volume IV: Sodium Pumps, Valves, piping, and Auxiliary Equipment, Gordon and Breach, Science Publishers inc., 1978. 
Foust O. J., Sodium-NaK Engineering Handbook Volume V: Sodium Purification, Material, Heaters, Coolers, and Radiators, Gordon and Breach, Science Publishers inc., 1978.

Ganic E. and Seider W. D., "Computer Simulation of Potassium-Steam Combined-Cycle, Electrical Power Plants", Computers and Chemical Engineering, 1 (1977) 161-169.

Ganguly C., Hegde P. V. and Sengupta A. K., "Status of (U,Pu)C and (U,Pu)N fuel development in BARC", Technical committee meeting on advance fuel for fast rectors: Fabrication and properties and their optimization, Vienna, November 1987.

Gluekler E. L., “ALMR Summary Plant Design Description”, GEFR-00941, GE Nuclear Energy, March 1995.

Golden G. H., "Elementary Neutronics Considerations in LMFBR Design," ANL-7532, Argonne National Laboratory, March 1969.

Holland F. A. and Chapman F. S., Pumping of Liquids, Reinhold Publishing Corporation, New York, 1966.

Kim J. and Lior N., "Some Critical Transitions in Pool Flash Evaporation", Int. J. Heat Mass Transfer, 40 (1997) 2363-2372.

Kutateladze S. S. and Borishanskii V. M., Liquid-Metal heat Transfer Media, Atomic Press, Moscow, 1958

Kuzina Y. A., Sorokin A. P. and Zhukov A. V., Numerical Simulation of the Thermohydraulics of Reactor Fuel Assemblies with blockages, Atomic Energy, 87 (1999) 808-822.

Lahey R. T. and Moody F. J., The thermal Hydraulics of a Boiling Water Nuclear Reactor, American Nuclear Society.

Lowell R. S., Tek M. R. and Balzhiser R. E., "Pressure Drops and Void Fractions in Horizontal Two-Phase flows of Potassium”, A.I.Ch.E. Journal, (1960) 50-58.

MacPherson R. E., Amos J. C. and Savage H. W., "Development Testing of liquid Metal and Molten Salt Heat Exchangers", Nuclear Science and Engineering, 8 (1960) 14-20.

MacPherson R. E., “Techniques for Stabilizing Liquid Metal Pool Boiling”, International Conference on the Safety of Fast Reactors, Aix en Provence, September 1967.

MCNP4B2: Monte Carlo N-Particle Transport Code System, CCC-660, Los Alamos National Laboratory, October 1997.

Ohse R. W., Handbook of Thermodynamic and Transport Properties of Alkali Metals, Blackwell Scientific Publications, Oxford, 1985. 
Paramonov D., Novel, Integrated Reactor/Power Conversion System (LMR-AMTEC): Year one Technical Progress Report, STD-ES-01-0028, July, 2001.

Peckner D. and Bernstein I. M., Handbook of Stainless steels, McGraw-Hill Book Company, 1959.

Peterson R. J., Grewalt S. S. and El-Wakil M. M., "Investigation of Liquid Flashing and Evaporation due to Sudden Depressurization", Int. J. Heat Mass Transfer, 27 (1984) 301$310,1984$.

Petrovic B. and Paramonov D., "Impact of Coolant Selection on Core Neutronics for Integrated LMR-AMTEC System", Trans. Am. Nucl. Soc., 84 (2001) 69-70.

Phillips W. M., "Some Alkali Metal Corrosion Effects in a Rankine Cycle Test Loop", Corrosion by Liquid Metals, Edited by Draley J. E. and Week J. R., Plenum Press, New york, 1970.

Saphier D. and Madell J. T., "The DSNP Simulation Language and its Application to Liquid-Metal Fast Breeder Reactor Transient Analyses”, Nuclear Technology, 56 (1982) 493-506.

Sorokin G. A. and Avdeev E. F., "Development of Thermo-hydraulic Codes for Modeling Liquid metal Boiling in LMR fuel Subassemblies", LMFR core thermohydraulics: Status and Prospects, IAEA, 2000.

SCALE: A Modular System for Performing Standardized Computer Analyses for Licensing Evaluations, NUREG/CR-0200, Rev. 5 (ORNL/NUREG/CSD-2/R5), Vols. F III (March 1997).

Smith L. S., Rasin T. M. and Balzhiser R. E., "Pressure Drops and Void Fractions in Horizontal Two-phase Flows of potassium”, A.I.Ch.E Journal, 12 (1960) 50-58.

Stewart W. A., "Natural Convection Cooling of AP-600 Containment", 87-8J0-PASIVRl, Engineering Division, Westinghouse Electric Company, April 1987.

Special Metals Corporation, www.specialmetals.com

Tang Y. S., Coffield R. D. and Markley R. A., Thermal Analysis of Liquid-Metal Fast Breeder Reactors, American Nuclear Society, 1978.

Tagawa H., "Phase Relations and Thermodynamic Properties of the Uranium-Nitrogen System", Journal of Nuclear Materials, 51 (1974) 78-89.

Vasiliev A., Alekseeve P., Mikityuk K., Fomichenko P. and Shestopalov A., "Theoretical Requirements to Tolerances to be Imposed on Fuel Rod Design Parameters for RBEC-M Lead-Bismuth Fast Reactor", ARWIF-2001, Chester, U.K., October 2001. 
Volney C. F., Pump Handbook, National Press, USA, 1948.

Wallenius J. and Pillon S., "N-15 Requirement for $2^{\text {nd }}$ Stratum ADS Nitride Fuels, Nuclear Applications in the New Millennium," AccAPP-ADTTA'01, Reno (Nevada), November 2001.

Wirtz K., Lectures on Fast Reactors, Kernforschungszentrum Karlsruhe, 1978.

Yarosh M. M., "Evaluation of the performance of liquid metal and molten salt heat exchangers", Nuclear Science and Engineering, 8 (1960) 32-43.

Yarosh M. M., "An Experiment Employing Ultrasonic Energy to Promote Boiling in Liquid Metals", Proceedings of the International Conference on the Safety of fast Reactors, Aix-en-Provence, France, 1967.

Yevick J. G. and A. Amorosi, Fast Reactor Technology: Plant Design, The M.I.T. Press, Cambridge, 1966.

\section{REFERENCES FOR TRANSPORTATION}

1. U.S. Department of Transportation, Federal Highway Administration: Federal size regulations for commercial Motor vehicles. http://www.ops.fhwa.dot.gov/freight/regulate/sw/federal1.pdf

2. U.S. Department of Transportation, publication number: FHWA-PL-00-029 HPTS/August 2000 "Comprehensive truck size and weight study" http://www.fhwa.dot.gov/reports/tswstudy/TSWfinal.htm

3. Western Association of State Highway and Transportation officials, 2000: Guide for uniform laws and regulations governing truck size and weight among WASHTO States, http://www.ops.fhwa.dot.gov/freight/regulate/sw/federal1.pdf

4. Association of American railroads: Analysis of Class I railroad, 1998, DC: AAR, 1999, http://www.aar.org.

5. Minnesota Department of Transportation: Minnesota rail service improvement program, rail line rehabilitation, http://www.dot.state.mn.us/ofrw/railroads.html

6. Marvin, P and Keith K., "Long-term trends in railroad service and capacity for U.S. agriculture" USDA, Agriculture Marketing Service, November, 2000

7. http://www.house.gov/transportation/rail/hearing/07-25-00/zarembski.html. 


\section{REFERENCES FOR WASTE MANAGEMENT}

1. IAEA-TECDOC-687 1993

2. LMR-AMTEC Year one Technical progress Report, research Grants Number DEFG03-99S21987, STD-CM-286, July 2001.

3. Kottowski H. M. "Safety Problem Related to Sodium Handling in Liquid Metal Fast Breeder Reactors and Large Test Facilities", Harwood Academic Publishers GmbH, Switzerland.

4. Stamm H. H., Stad K-Ch. "Corrosion Product behavior in the primary Circuit of the KNK nuclear reactor facility". Specialists meeting on fission and corrosion product behavior in the primary circuits of LMFBRs, Dimitrovgrad (USSR), 8-11 Sept 1975. IWGFR 7, IAEA Vienna, pp 36-63.

5. Brehm W. F., Colburn R. P., Maffei H. P., Stinson W. P., Bunch W. L. Bechtold R. A. "Corrosion and fission products in primary systems of liquid metal cooled reactors in the USA". Karlsruhe, Germany, 5-8 may 1987. IWGFR/64, IAEA, Vienna, KFK Report 4279, pp 75-92

6. Stamm H. H., Stad K-Ch. "Radiochemical surveillance of KNK primary sodium". Specialists meeting on fission and corrosion product behavior in the primary circuits of LMFBRs, Karlsruhe, Germany, 5-8 may 1987. IWGFR/64, IAEA, Vienna, KFK Report 4279, pp 385-418.

7. Stamm H. H., Hanke H. D., Clauss H., "First test of a radionuclide trap at KNKII.". 3rd Int Conf. On Liquid metal coolants, their transport within the circuits and removal from LMFBRs. Atomic Energy Review, 17, 3 (1979), 697-761.

8. Mason L., Morgan D. J., Marrison N. S., Steele K. B. Trevillion E. A. “ Impurity monitoring on the PFR primary circuit. 4th Int Conf. On Liquid Metal Eng.\& Tech., Avignon, France 17-21 Oct 1988. Ed SFEN F75724 Paris, pp 708/1-11.

9. Mason L., Trevillion E. A., Morrison N. S., Steele K. B., Green T. H., Woodrow T. "Measurements of fission products and activated corrosion products in the primary sodium circuit of the prototype Fast Reactor. Karlsruhe, Germany, 5-8 may 1987. IWGFR/64, IAEA, Vienna, KFK Report 4279, pp 297-320.

10. Clerc R., Guidez J., Maux P., Michaille P., Misraki J., Msika D. The French experience concerning the contamination of LMFBRs. Specialists meeting on fission and corrosion product behavior in the primary circuits of LMFBRs, Karlsruhe, Germany, 5-8 may 1987. IWGFR/64, IAEA, Vienna, KFK Report 4279, pp 93-141. 
11. Clerc R., Guidez J., Michaille P., Misraki J., Specialists meeting on fission and corrosion product behavior in the primary circuits of LMFBRs, Karlsruhe, Germany, 5-8 may 1987. IWGFR/64, IAEA, Vienna, KFK Report 4279, pp141185.

12. Krasnoyarov N. V., Polyakov V. I., Chetchetkin Yu, V., "Radioactive behavior during the operation of sodium cooled reactors of the USSR. Int. Conf. On Liquid Metal Technology in Energy Production, Champion (PA), USA, May 1976 Conf.760503, pp 30-34.

13. Bobkov Yu, Efimov I. A., Zagoroulko Yu I., Inutin E. I., Konovalov E. E., Lastin A. I., Zikunov A. G. "Radionuclides behavior in sodium coolant. Specialists meeting on fission and corrosion product behavior in the primary circuits of LMFBRs, Karlsruhe, Germany, 5-8 may 1987. IWGFR/64, IAEA, Vienna, KFK Report 4279, pp 41-60.

14. Vassilenko K. T., bolgarine V. I., Pomerantsev G. B., Samarkine A. A., Skorikov N. V., Chkolnik V. S., Khalov E. M., Antoufiev O. N. Proc. of the LMFBR Safety Tropical Meeting 19-23 July 1982, Lyon-Ecully (France) SFEN Ed, Vol. 1, pp 493505 .

15. Stamm H. H. "Sorption of radionuclides on sodium sampling systems". Int. Conf. On Liquid Metal Technology in Energy Production, Champion (PA), USA, May 1976 Conf.-760503, pp 271-277.

16. Allan C. G., Taylor T. A. H. "Activity deposition in DFR primary cold traps". Int. Conf. On Liquid Metal Technology in Energy Production, Champion (PA), USA, May 1976 Conf.-760503, p 35.

17. Powell H. J. "Fission product distribution in post reactor oxide fuels. Behavior and chemical state of irradiated ceramic fuels. IAEA, Vienna, 1974, p 379.

18. Michaille P., Clerc R. The French experience concerning the contamination by inactive and radioactive impurities and the purification of the cover gas of LMFBRs. Proc. of specialists Meeting on fast Reactor cover gas purification, Richland (WA) USA, sep 1986. IAEA, IWGFR/61, Vienna 1987, pp 21-63.

19. Bechtold R. A., Holt F. E., Meadows G. E., Schenter R. E., "Reactor cover gas monitoring at the Fast Flux test Facility". Proc. of specialists Meeting on fast Reactor cover gas purification, Richland (WA) USA, sep 1986. IAEA, IWGFR/61, Vienna 1987, pp 247-261.

20. Stade K-Ch, Richard H., Hoffmann G., Hacobi S., Schmitz G. " Radionuclides in the cover gas of KNK-II. Proc. 2nd Meeting on Liquid Techn. In Energy Production, Richland, WA, (USA) 1980 ERDA Conf. 800401, pp 14/38-45. 
21. Richard H., Stade K-Ch." Cover gas purification experience at KNK. Proc. of specialists Meeting on fast Reactor cover gas purification, Richland (WA) USA, Sept 1986. IAEA, IWGFR/61, Vienna 1987, pp 78-101.

22. Aristarkchov N. N., Efimov I. A., Krasnoyarov N. V., Schereshkov V. S., "An adsorption method for BR-5 primary cover gas decontamination from radioactive Xenon. Specialists meeting on fission and corrosion product behavior in the primary circuits of LMFBRs, Dimitrovgrad (USSR), 8-11 Sept 1975. IWGFR 7, IAEA Vienna, pp 157-162.

23. Chetchetkin Yu V., Konyashov V. V., Polyakov E. K. " Radioactivity of cover gases in fast reactors. Ibid, IWGFR/7, IAEA Vienna, pp 140-143.

24. Vassilenko K. T., Bolgarine V. I., Pomerantsev G. B., Samarkine A. A., Skorikov N. V., Chkolnik V. S., Khalov E. M., Antoufiev O. N. Proc. of the LMFBR Safety Topical Meeting 19-23 July 1982, Lyon-Ecully (France) SFEN Ed, Vol. 1, pp 493505.

25. IAEA-TECDOC-866, 1996

26. W.H Olson, W.E Ruther "Controlling Cesium in the coolant of EBR-II" Nuclear Technology, 46, 318-322 (1979)

27. http://www.nrc.gov/waste/low-level-waste.html

28. http://www.nrc.gov/waste/high- level-waste.html

29. Technical Report, ANL/TD/CP—86694, CONF-950919—8, 1995

30. Technical Report, ANL/RA/SUMM-94729, CONF-971125, 1997

31. Technical report, ANL/CMT/CP--85252; CONF-950919-11,1995.

32. http://www.nrc.gov/waste/spent-fuel-storage.html

33. Technical report, ANL/RA/CP--94687; CONF-980203, 1997

34. Technical report, ANL/ED/CP—95701; CONF-980249, 1998

35. DOE/ID-10381, "Recyclable Materials, and waste Acceptance Criteria (RRWAC)," INEEL Reusable Property, Rev. 5, October 1995" 


\title{
3 Static Energy Conversion System Design
}

\author{
by Institute for Space and Nuclear Power Studies, (UNM)
}

This chapter presents the work completed in Task 2c, "Energy Conversion Modules," and in Task 2e, "Thermoelectric Bottom Cycle." The high voltage output of $350-400$ V DC of the AMTEC converter unit design is based on the results of an experimental investigation of the breakdown voltage in alkali-metal vapor. The AMTEC/TE unit design is also developed to be cooled by natural convection of air, efficienctly minimizing maintenance requirements, while demonstrating an overall conversion efficiency in excess of $30 \%$.

Section 3.1 reviews the operation principle of AMTEC and the different unit design configurations, including vapor-anode and liquid-anode converters. Considerations pertaining to the selection of the AMTEC working fluid are discussed in Section 3.2. The design of the AMTEC converter unit and the layout of the thermoelectric bottom cycle are described in details in Section 3.3. Optimization analyses of the AMTEC/TE converter design for maximum overall efficiency are presented in Section 3.4. The numerical models developed at UNM-ISNPS for performing these optimizations are also described in this Section. The design and the performance of the reference sodium and potassium AMTEC/TE converter units selected, both using a total AMTEC electrodes' surface area of $37 \mathrm{~m}^{2}$, are compared in Section 3.5 at identical anode pressure and identical BASE temperature. Finally, Section 3.6 describes the electrical power modules and system of the LMR-AMTEC nuclear power plant. Materials Compatibility, lifetime issues and materials selection for the AMTEC converters are discussed in Section 3.7.

The optimized sodium AMTEC/TE converter unit is $7.1 \mathrm{~m}$ deep, $1.35 \mathrm{~m}$ wide and $0.79 \mathrm{~m}$ high, while the optimized potassium AMTEC/TE converter unit is $6.2 \mathrm{~m}$ deep, $1.57 \mathrm{~m}$ wide and $0.87 \mathrm{~m}$ high. These dimensions include the heat pipe cooling fins $(0.35$ m) of the P-TAGS-85/2N-PbTe TE bottom cycle by natural convection of ambient air. The Na-AMTEC converter is comprised of 672 elongated, dome-shaped, monolithic elements arranged in two rows, each with a $\mathrm{WRh}_{1.5}$ electrode surface area of $550 \mathrm{~cm}^{2}$, while the K-AMTEC converter has 744 elements of BASE elements arranged in three rows and connected electrically in series, each with a $\mathrm{WRh}_{1.5}$ electrode surface area of $498 \mathrm{~cm}^{2}$. Each BASE element consists of a thin BASE membrane (50-200 $\mu \mathrm{m}$ thick), deposited by plasma spraying or sputtering techniques onto a rigid porous anode substrate made of pressed and sintered molybdenum-rhenium powder. 
The sodium- and potassium- AMTEC/TE converters were optimized for operating at the same anode vapor pressure of $80.0 \mathrm{kPa}$. The corresponding BASE temperatures are $1127 \mathrm{~K}$ and $1006 \mathrm{~K}$ for the sodium and potassium AMTECs, respectively. At these operating conditions, the optimized sodium AMTEC/TE converter delivers a nominal electrical power of $69.5 \mathrm{~kW}_{\mathrm{e}}$, at an overall thermodynamic conversion efficiency of 33.7\%. Of this electrical power, the Na-AMTEC unit generates $61.0 \mathrm{~kW}_{\mathrm{e}}$ at a thermodynamic efficiency of $29.7 \%$ and $381 \mathrm{~V} \mathrm{DC}$, while the TE bottom cycle generates $8.5 \mathrm{kWe}$ at an efficiency of $6.4 \%$ and $322 \mathrm{~V} \mathrm{DC}$. At the same anode vapor pressure $\left(P_{a}=\right.$ $80.0 \mathrm{kPa})$, the optimized K-AMTEC/TE converter operates $121 \mathrm{~K}$ cooler than the NaAMTEC, at $T_{B}=1006 \mathrm{~K}$, and nominally generates $54.3 \mathrm{kWe}$, or $22 \%$ less power than the optimized Na-AMTEC/TE converter, but at nearly one percentage point higher thermodynamic efficiency of $34.4 \%$.

\subsection{Operation and Design of AMTEC}

This Section presents background information on the design and principle of operation of Alkali Metal, Thermal-to-Electric Converters (AMTECs). Using Beta-Alumina solid electrolytes in conjunction with liquid sodium $(\mathrm{Na})$ to convert heat to electricity had been demonstrated in a patent assigned to the Ford Motor Company (Kummer and Weber 1968). The operating principle of a liquid anode, Alkali-Metal Thermal-to-Electric Converter (AMTEC) cell, was first described by Weber (1974). Since the mid-1980s, a number of technology programs to develop the technology of liquid- and vapor-anode AMTECs have been initiated at several organizations: the Jet Propulsion Laboratory (Underwood et al. 1992, and Ryan et al. 1998a); the Electrotechnical Laboratory and Kyushu University in Japan (Kato et al. 1993); Advanced Modular Power Systems (AMPS) and the Environmental Research Institute of Michigan (Sievers et al. 1990 1999); the U. S. Air Force Research Laboratory (AFRL) (Schuller et al. 1996, and Merrill et al. 1997 and 1998); and the University of New Mexico's Institute for Space and Nuclear Power Studies (UNM-ISNPS) (Tournier and El-Genk 1999a - 1999f). Recently, the Bechtel-Bettis Atomic Laboratory is developing high-current AMTEC units for use in conjunction with a nuclear heat source. This effort is being conducted in collaboration with UNM-ISNPS. Furthermore, AMTEC technology is currently being developed as part of the recent NASA Space Nuclear Initiative (SNI) for both Advanced Radioisotope Power Systems (ARPSs) and Electric Propulsion Space Nuclear Reactor Power Systems (SNRPSs) for potential deployment in 2006 and beyond. The budget for the NASA SNI amounts to $\$ 950 \mathrm{M}$ for the next Fiscal Year starting in 2003. In addition, efforts to develop mixed-conducting (ionic and electronic) metal/oxide electrodes such as blends of $\mathrm{Mo} / \mathrm{Na}_{\mathrm{x}}-\mathrm{TiO}_{2}$ and $\mathrm{TiN} / \mathrm{Na}_{\mathrm{x}}-\mathrm{TiO}_{2}$ are underway at both the Jet Propulsion Laboratory (Ryan et al. 2001) and Advanced Modular Power systems (AMPS), Inc. (Fletcher and Schwank 2002 and 2003). These electrodes can be applied as a paint or slurry, are robust and inexpensive, and have demonstrated to date a performance equivalent to the best currently available refractory electrode $\left(\mathrm{WRh}_{\mathrm{x}}\right)$ (Ryan et al. 2001). Further studies on metal material selection, and optimization of the mixed-conducting electrodes' composition and thickness offer the promise for improved lifetime and performance of AMTEC devices. 


\subsubsection{Operation Principle}

An AMTEC is a high-temperature regenerative concentration device, which directly converts heat to electricity. The key component is the Beta"-Alumina Solid Electrolyte (BASE), a sodium-ion conductor whose ionic conductivity is high while its electronic conductivity is nil (Cole 1983). Sodium-BASE is a ceramic material with a nominal chemical formula of $\mathrm{Na}_{5 / 3} \mathrm{Li}_{1 / 3} \mathrm{Al}_{32 / 3} \mathrm{O}_{17}$ (Weber 1974). The K-Beta" and Na-Beta" Alumina Solid Electrolytes have a "spinel" crystal structure of extended layers of alumina $\left(\mathrm{Al}_{2} \mathrm{O}_{3}\right)$ separated by conduction planes containing only alkali metal and bridging oxygen ions (Figure 3.1). The alumina spinel layers (or blocks) consist of four close-packed oxygen layers each, in which the $\mathrm{Al}^{+3}$ and $\mathrm{O}^{-2}$ ions occupy both octahedral and tetrahedral positions. The spinel alumina layers are separated by planes containing loosely packed $\mathrm{Na}^{+}$(or $\mathrm{K}^{+}$) ions, with equal number of $\mathrm{O}^{-2}$ ions (Kummer, 1972). The spinel layers above and below the alkali metal ions conduction planes (Figure 3.1) produce low activation energy barriers for jumping $\mathrm{Na}^{+}$(or $\mathrm{K}^{+}$) ions from one site to the next, inducing rapid diffusion of the alkali metal ions within the conduction planes in the direction of

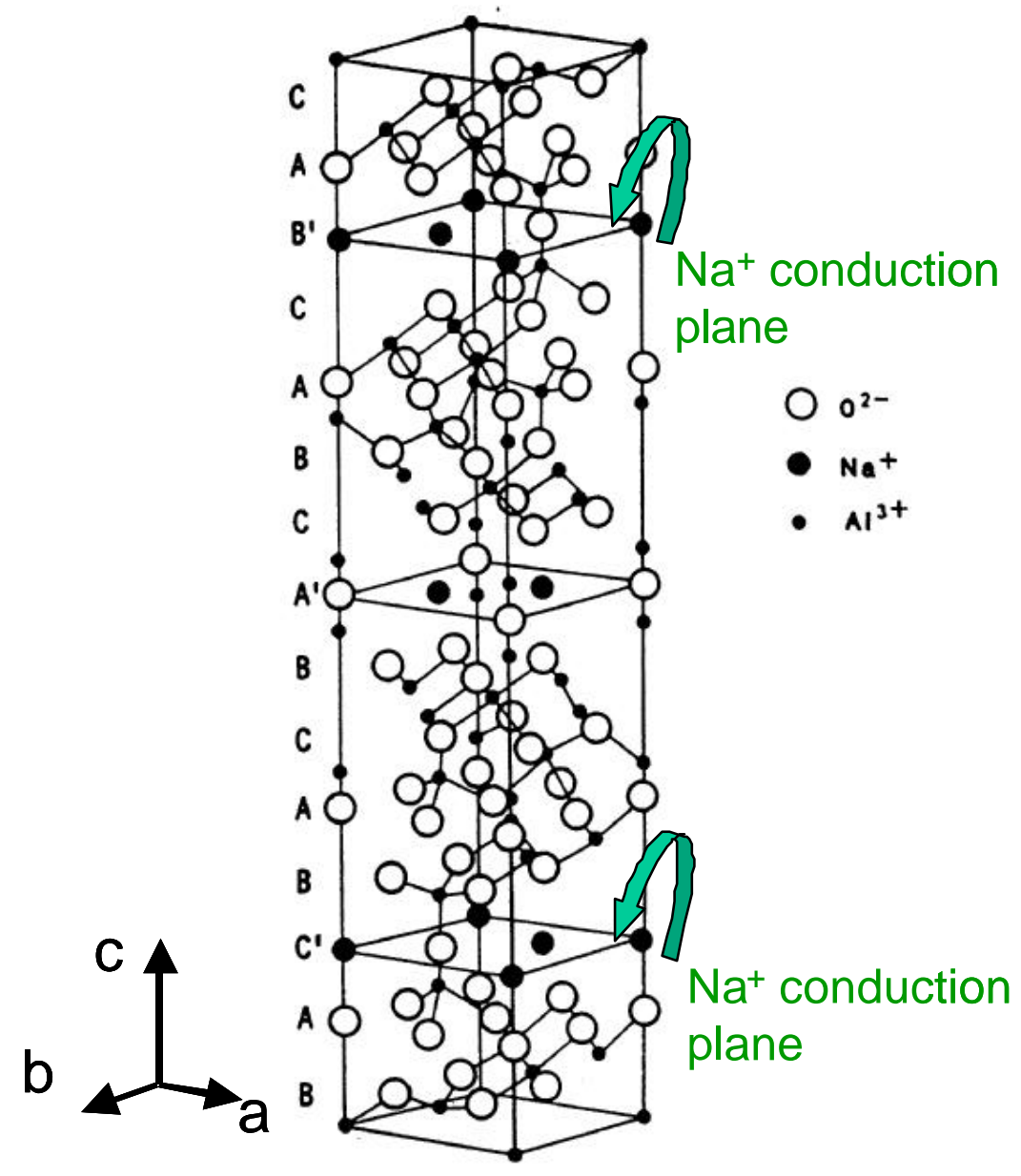

Figure 3-1. Lattice structure of sodium beta"-alumina solid electrolyte (Cole 1983). 
an applied pressure difference (Cole, 1983). The $\mathrm{Na}^{+}\left(\right.$or $\left.\mathrm{K}^{+}\right)$ions in the conduction planes diffuse by the vacancy mechanism, resulting in the BASE having high ionic conductivity. The ionic resistivities of the K-BASE and Na-BASE are $\sim 6.0 \Omega-\mathrm{cm}$ and $\sim$ $2.0 \Omega$-cm, respectively.

The BASE has a melting temperature of $\sim 2526 \mathrm{~K}$ and is inert to reaction with elemental alkali metal up to $1300 \mathrm{~K}$, which is one of the operation temperature limits in AMTEC. The K-BASE can easily be produced, by substituting $\mathrm{Na}^{+}$with $\mathrm{K}^{+}$, using $\mathrm{K}-\mathrm{Cl}$ vapor ion exchange techniques. For the same vapor pressure on the anode side of $80 \mathrm{kPa}$, operation temperatures are $1006 \mathrm{~K}$ and $1127 \mathrm{~K}$ for K- and Na-AMTEC, respectively.

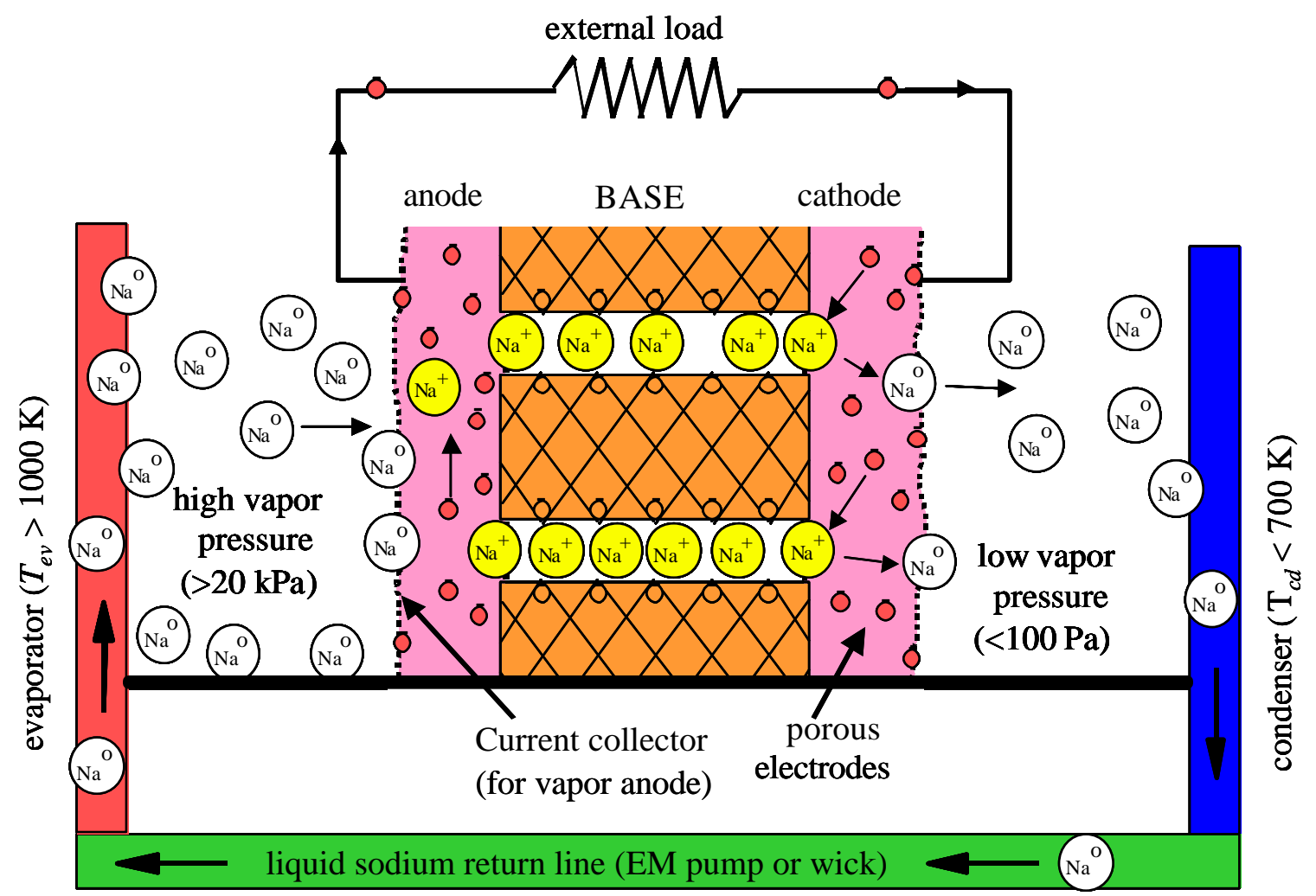

Figure 3-2. Schematic of a vapor anode AMTEC converter

In the AMTEC, the thin BASE membrane $(<1 \mathrm{~mm})$ divides the device into a hot (high-pressure) region, filled with sodium liquid or high-pressure vapor, and a cold (lowpressure) region containing sodium vapor (Figure 3.2). Electric power is produced by the isothermal expansion of sodium vapor across the BASE. 
A porous cathode electrode (Figure 3.3) covers the low-pressure (outer) side of the BASE, while a porous anode electrode covers the high-pressure side of the BASE in a vapor anode AMTEC. In a liquid anode AMTEC, however, since the hot liquid sodium comes directly in contact with the anode side of the BASE membrane, there is no need to use an anode electrode. The good electrical conductor liquid sodium transports the electrons stripped from the neutral atoms at the liquid sodium-BASE interface to the external load. The resulting sodium ons then diffuse through the BASE, where they undergo an almost isothermal expansion, developing an electric potential across it. In liquid anode AMTEC, care must be taken to electrically insulate the cathode electrode from the wall, since liquid sodium and all metals in contact with it will be at the same potential.

Electrical leads, which exit through the wall of the AMTEC unit, connect the cathode and anode electrodes to the external load. Electrons and sodium ions recombine at the interface between the BASE and the porous cathode electrode (Figure 3.2). The resulting low-pressure $(<90 \mathrm{~Pa})$ neutral sodium atoms transpire through the porous cathode electrode (1-5 $\mu \mathrm{m}$ thick) to the vapor space in the low-pressure cavity of the cell. This sodium vapor then flows to the low-temperature condenser surface, where it condenses, releasing its heat of vaporization. A wick structure or an electromagnetic pump returns the liquid $\mathrm{Na}$ from the condenser to the high pressure (>20 $\mathrm{kPa}$ ) side of the BASE, where it receives heat from the heat source, completing the cycle.

\subsubsection{Characteristics of AMTEC Electrodes}

Desirable electrodes properties include (Asakami et al. 1990, Fang and Knödler 1992, Fiebig et al. 1999, Ryan et al. 1992, 1999, 2000 and 2001, Williams et al. 1986 and 1990a):

(a) good electrical conductivity;

(b) good physical bonding with the BASE membrane;

(c) a thermal expansion coefficient that is close to that of BASE;

(d) high permeability to sodium vapor;

(e) high corrosion resistance to sodium vapor;

(f) low material loss rate by chemical reaction or sublimation; and

(g) slow grain growth and material migration. 
Refractory electrode materials such as $\mathrm{TiB}_{2}, \mathrm{TiC}$ and $\mathrm{NbN}$ offer many of these properties. Molybdenum electrodes have also some attractive characteristics for use in sodium- and potassium-AMTECs, particularly, the enhanced ionic and electronic conductivities of the molybdate compound.

Researchers at Corporate Research in Heidelberg, Germany (Fang and Knödler 1992), have proposed using refractory electrode materials, which exhibit high chemical stability at high temperature and high electronic conductivity (low sheet resistance). Based on its properties and thermal expansion coefficient that is almost identical to that of $\mathrm{BASE}, \mathrm{TiB}_{2}$ appears to be a promising electrode material. Corporate Research has performed some preliminary performance tests of $1 \mu \mathrm{m}$-thick sputtered $\mathrm{TiB}_{2}$ electrodes. Analyses of their data showed that these electrodes exhibit essentially no chargeexchange polarization losses (a charge-exchange current density of infinity) and a very low effective $G$ factor for vapor pressure losses of only $\sim 5$. Although no long-term experiments are performed to study the performance degradation of these electrodes, $\mathrm{TiB}_{2}$, like $\mathrm{TiN}$, is a refractory metal, and it is expected to exhibit good performance stability.

Table 3-1. Potential characteristics of AMTEC electrodes.

\begin{tabular}{|c|c|c|c|c|}
\hline $\begin{array}{c}\text { Electrode } \\
\text { Technology }\end{array}$ & $\begin{array}{c}\text { Charge-exchange } \\
\text { coefficient } B \\
\left(\text { A.K } \mathrm{K}^{1 / 2} / \mathrm{Pa} \cdot \mathrm{m}^{2}\right)\end{array}$ & $\begin{array}{c}\text { Electrode/collector } \\
\text { contact resistance } \\
R_{\text {cont }}\left(\Omega \cdot \mathrm{cm}^{2}\right)\end{array}$ & $\begin{array}{c}\text { Electrode } \\
\text { pressure loss } \\
\text { factor, } G\end{array}$ & $\begin{array}{c}\text { BASE thickness } \\
t_{B} \\
(\mu \mathrm{m})\end{array}$ \\
\hline $\begin{array}{c}\text { Current (TiN) } \\
\text { (Ryan et al. 1999a } \\
\text { and 2000) }\end{array}$ & 70 & 0.10 & 50 & 500 \\
\hline $\begin{array}{c}\text { Near-term (WRh) } \\
\text { (Ryan et al. 1999b } \\
\text { and 2000) }\end{array}$ & $\mathbf{9 0 - 1 1 0}$ & $\mathbf{0 . 0 6}$ & $\mathbf{1 0 - 2 5}$ & $\mathbf{2 0 0}$ \\
\hline $\begin{array}{c}\text { Advanced mixed } \\
\text { metal/oxide } \\
\text { electrodes (Ryan et } \\
\text { al. 2001, Fletcher and } \\
\text { Schwank 2003) }\end{array}$ & or higher ${ }^{(*)}$ & 0.01 & 10 & 50 \\
\hline
\end{tabular}

* does not require the use of glove boxes for assembly and application. 


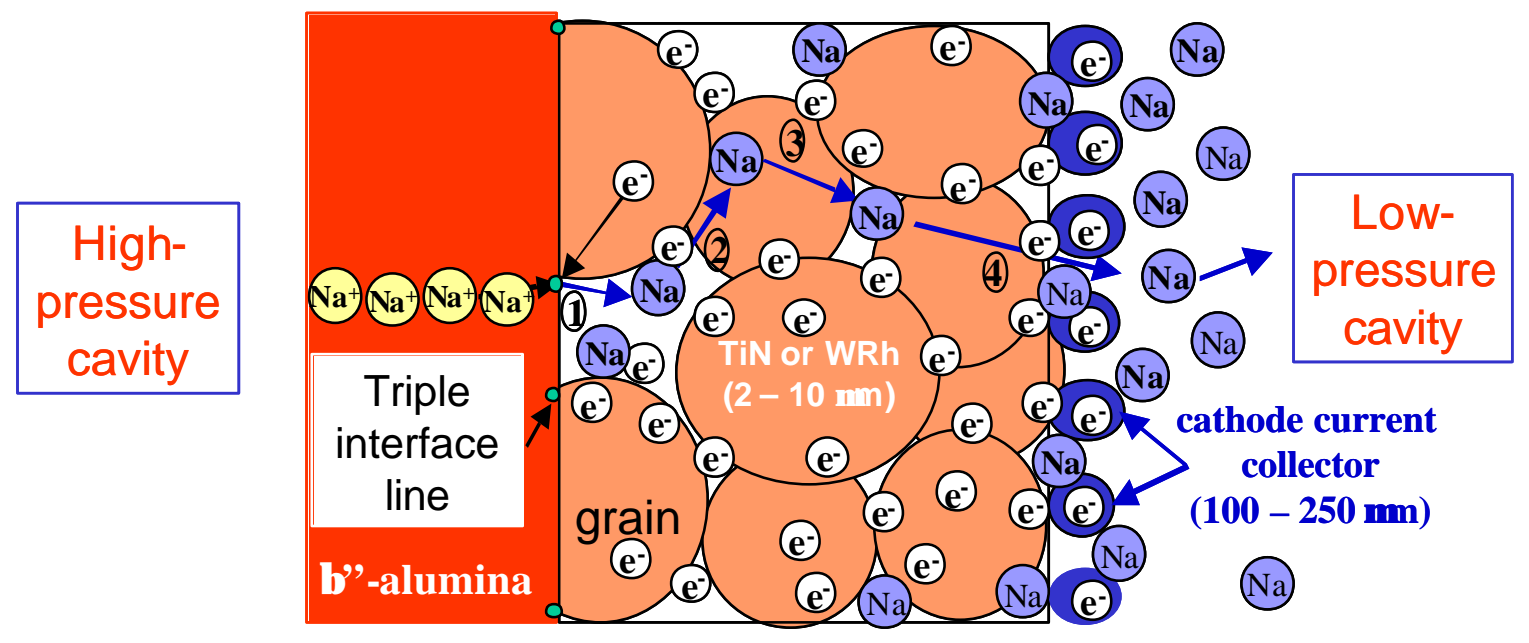

Figure 3-3. Transport processes in the cathode electrode. (1) Transfer of Na ions to the triple phase boundary (BASE-electrode-sodium vapor); (2) Surface diffusion of Na atoms on electrode grains; (3) Desorption of Na atoms from surface of electrode grains; and (4) Sodium vapor diffusion through the pores of the electrode.

Another promising electrode material is molybdenum. In sodium AMTEC converters, Mo electrodes have exhibited extremely high charge-exchange currents $(B$ $400 \mathrm{SI})$ in the presence of oxygen. This enhancement in performance has been attributed to the formation of sodium-molybdate, an extremely good conductor of sodium ions and electrons (Williams et al. 1986, Sievers et al. 1989). Unfortunately, this compound has a relatively high vapor pressure and evaporates rapidly, in a few hundred hours, at the operating temperatures of sodium-AMTEC devices.

It has been suggested that similar enhancement in performance could occur in potassium-AMTEC devices with Mo electrodes (Williams et al. 1994, Barkan et al. 1999). However, the potassium-molybdate compound has a much lower vapor pressure than sodium-molybdate, and is solid at the AMTEC operating temperatures of interest. Therefore, since potassium-AMTEC devices operate at 100-150 K lower BASE temperature than sodium-AMTEC converters, it is possible that Mo electrodes in the former could exhibit high performance, due to the presence of molybdate. Such an enhancement effect may occur over a long period time, possibly comparable to the lifetime of the power system.

Studies of refractory metal and alloy electrodes performed in Sodium Exposure Test Cells (SETC) and AMTEC devices at the Jet Propulsion Laboratory have determined that tungsten-rhodium co-sputtered films $(\sim 0.7-1.0 \mu \mathrm{m}$ thick $)$ are among the best performing electrodes to date (Ryan et al. 2000 and 2001), exhibiting $B$ values of $90-110$ (Figure 3.4), $R_{\text {cont }}=0.06 \Omega . \mathrm{cm}^{2}$, and $G \sim 10-25$. In these refractory electrodes, the requirement for ready transport of alkali metal atoms through the electrode must be balanced with that for efficient electrons conduction. The later may be enhanced by thickening the electrode, however this would in turn impede the alkali metal transport by vapor flow or surface or grain-boundary diffusion through the electrode. 


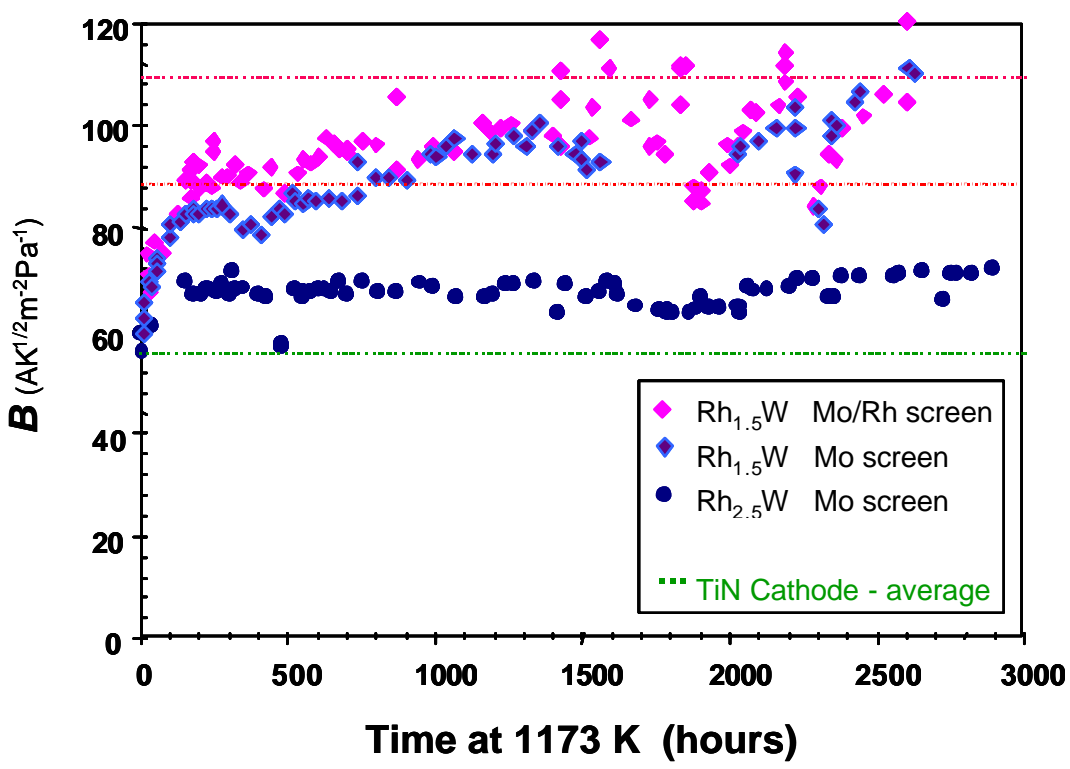

Figure 3-4. Measured values of charge-exchange current coefficient of $\mathrm{Rh}_{\mathrm{x}} \mathrm{W}$ porous electrodes at the Jet Propulsion Laboratory (Ryan et al. 2000).

The performance of an electrode can be improved if an ionic conductor material is incorporated in the electronically conducting electrode. The ionic conductor would allow sodium ions to travel through the electrode, away from the BASE surface, allowing the recombination of electrons and sodium ions to take place throughout the electrode (Ryan et al. 2001). These processes would in effect increase the magnitude of the chargeexchange current, $B$, by facilating ion-electron recombination and removal of sodium ions frm the BASE interface, and decrease the geometric loss factor, $G$, by providing an additional mechanism for alkali metal transport through the electrode. Efforts to develop such mixed-conducting (ionic and electronic) metal/oxide electrodes such as blends of $\mathrm{Mo} / \mathrm{Na}_{\mathrm{x}}-\mathrm{TiO}_{2}$ and $\mathrm{TiN} / \mathrm{Na}_{\mathrm{x}}-\mathrm{TiO}_{2}$ are underway at both the Jet Propulsion Laboratory (Ryan et al. 2001) and Advanced Modular Power systems (AMPS), Inc. (Fletcher and Schwank 2002 and 2003). These electrodes can be applied as a paint or slurry, are robust and inexpensive, and have demonstrated to date a performance equivalent to the best currently available refractory electrode $\left(\mathrm{WRh} \mathrm{h}_{\mathrm{x}}\right)(\mathrm{Ryan}$ et al. 2001). Further studies on metal material selection, and optimization of the mixed-conducting electrodes' composition and thickness offer the promise for improved lifetime and performance of AMTEC devices.

Based on the literature review performed in this work, potential characteristics of current state-of-the-art, near-term and advanced technology AMTEC electrodes are tabulated in Table 3.1. Current TiN electrodes exhibit a charge-exchange current coefficient, $B=70$, a current collector/electrode contact resistance, $R_{\text {cont }}=0.10 \Omega . \mathrm{cm}^{2}$, and a pressure loss factor of $G \sim 50$. Current BASE tubes are $\sim 0.5 \mathrm{~mm}$ thick. Electrodes of near-term technology are expected to exhibit $B$ values of $90-110, R_{\text {cont }}=0.06 \Omega . \mathrm{cm}^{2}$, and $G \sim 10-25$. A sputtered BASE thickness of $200 \mu \mathrm{m}$ can be readily achieved by CVD techniques (Table 3.1). Advanced mixed-conducting metal/oxide electrodes could offer $B$ values of 100 , or higher, lower contact resistance $\left(R_{\text {cont }}=0.01 \Omega . \mathrm{cm}^{2}\right)$, and $G \sim$ 10. Sputtered BASE layers as thin as $50 \mu \mathrm{m}$ would result in negligible ionic resistance 
and allow higher packing density of BASE electrodes in a given converter volume. In all subsequent analyses, it is assumed that the BASE electrodes are of the near-term technology (see Table 3.1). Such performance has recently been achieved with tungstenrhodium $\left(\mathrm{WRh}_{1.5}\right)$ electrodes developed and tested at the Jet Propulsion Laboratory (Ryan et al. 1999b and 2000). Some of the design options of the AMTEC converter developed and investigated are discussed in the following sub-sections.

\subsubsection{Liquid-Fed, Liquid Anode AMTEC Converters}

The simpler AMTEC unit design is that of a liquid-fed, liquid-anode. In this converter design, a liquid metal film is maintained onto the high-pressure surface of the BASE solid electrolyte. Since alkali metals are good electrical conductors, there is no need to provide an anode electrode. The alkali-metal liquid in direct contact with the BASE membrane may fill the pores of a wick structure bonded to the BASE membrane. For this converter design to be efficient, a large fraction of the coolant mass flow rate needs to be ionized and diffuse through the BASE membrane. The challenge in the design of a liquid-fed AMTEC is the difficulty of electrically insulating different BASE electrodes from the converter wall, while connecting them in series to increase the output voltage to a usable value.

\subsubsection{Vapor-Fed AMTEC Converters}

Vapor-Fed AMTEC converters offer the design flexibility of connecting many BASE electrodes in series to provide high output voltage, and hence reduce the internal electrical losses in the converter. Two design options of the vapor fed AMTEC converters are possible, namely: vapor anode and liquid anode. In the former, a secondary heat flow path is provided to the BASE membranes to keep them at a higher temperature than the liquid metal evaporator in the converter. The temperature margin (temperature difference between the cold end of the BASE and the converter evaporator surface) should be positive to avoid shorting the BASE electrodes, at the expense of lower performance of the cell.

In a vapor anode AMTEC, the liquid sodium returning from the condenser is converted into a high pressure vapor by the heat supplied by the heat source (Figures 3.2 and 3.5). This vapor then travels to the anode side of the BASE, which is maintained at a slightly higher temperature than the cell evaporator to prevent condensation of sodium in the anode cavity.

In a multi- tube AMTEC, the BASE electrodes are connected in series to increase the output voltage of the cell $(2.5-3.5 \mathrm{~V})$, and the BASE tubes are electrically insulated from each other and from the metal support plate (Figure 3.5). Using more than one BASE tube, however, is not possible in liquid anode cells, since liquid sodium is a good electrical conductor. Consequently, vapor anode, multi-tube cells have lower specific mass $\left(\mathrm{kg} / \mathrm{W}_{\mathrm{e}}\right)$ than single tube cells, and are more attractive for a number of space missions, because of their relatively high output voltage $(2-5-3.5 \mathrm{~V})$. Vapor anode, multi-tube AMTECs are being considered for providing electric power on board the NASA's Europa spacecraft, scheduled for launch early this century. 


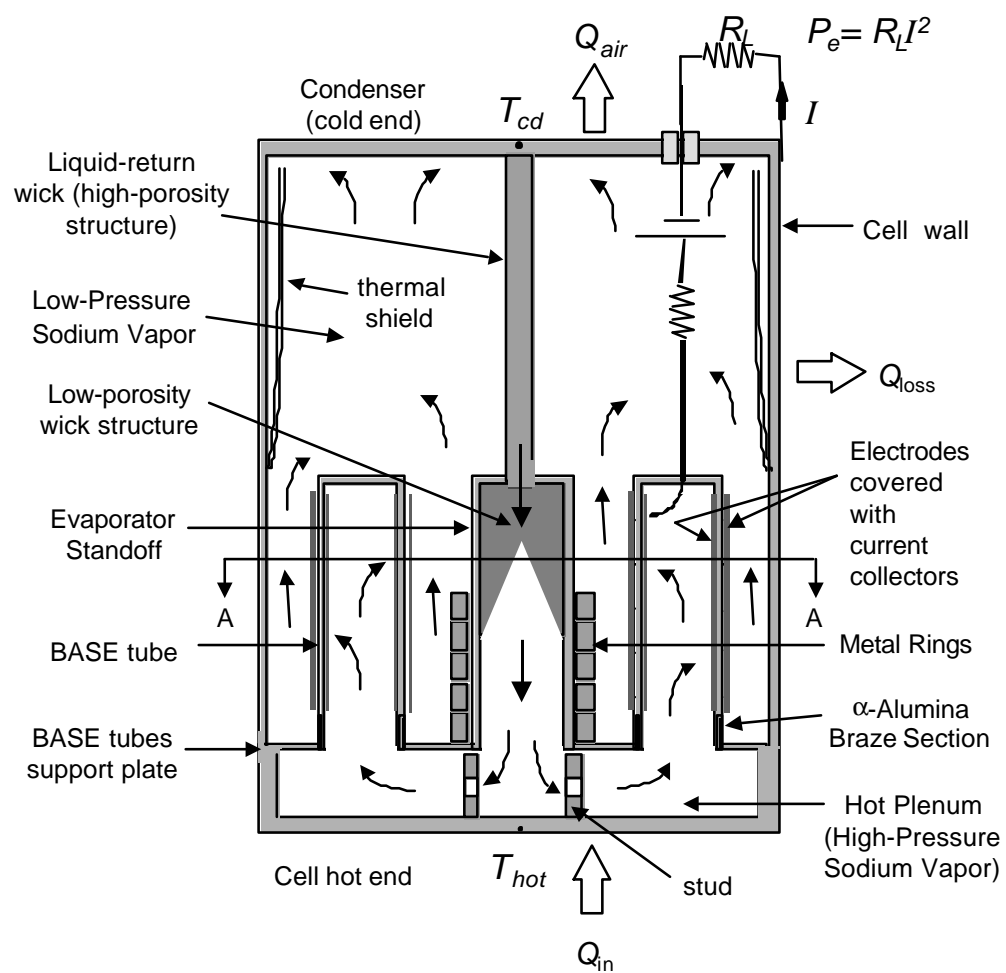

(a) Elevation

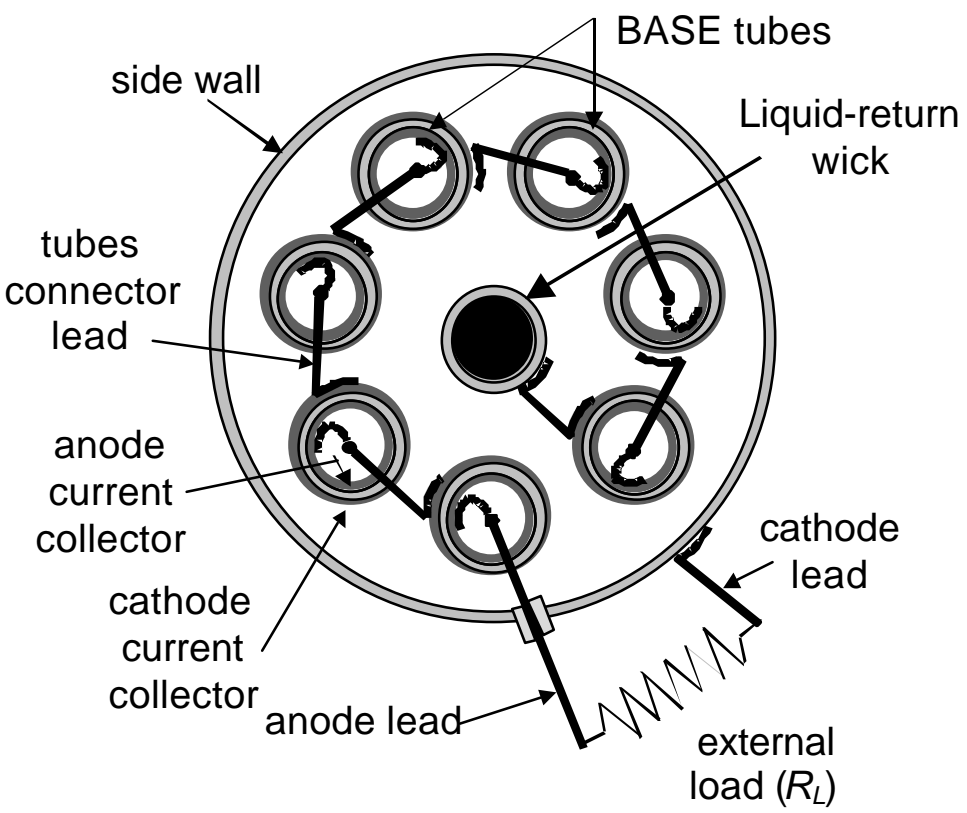

(b) Plan View (Section A-A)

Figure 3-5. Cross-section views of a vapor anode, multi-tube AMTEC (not to scale). 
In vapor anode, multi-tube PX-type AMTECs, the BASE tubes (5 to 7 tubes per cell) and the housing of the evaporator wick are brazed to a stainless steel (SS) support plate (Figure 3.5). The TiN porous electrodes on the anode and cathode sides of the BASE are covered with molybdenum mesh current collectors, to minimize internal electrical losses. Heat is transported from the hot end of the cell, by conduction and radiation to the support plate, then to the BASE tubes and evaporator structure. The radiation shield, laid against the cell wall above the tubes, reduces parasitic heat losses through the wall.

The liquid sodium working fluid is circulated in the AMTEC via a capillary structure. The liquid-return wick has a smaller pore size than the condenser wick structure. However, the pore size and the permeability of the former must be selected carefully, since they have competitive effects. For example, decreasing the pore size of the wick increases its capillary pumping power, but reduces its effective permeability, hence increasing the pressure losses in the wick. Such increase in pressure losses might exceed the capillary pressure head of the wick, causing the evaporator to dry out. Therefore, in order to keep the pressure losses in the liquid-return wick low, while providing high capillary pressure head, composite wick structures are being used (Figure 3.5a). The liquid transport section of the wick, that extends from the AMTEC condenser to near the evaporator surface, is made of a relatively large pore size, high permeability metal wick structure. The evaporator wick, however, is made of a very small pore size structure to provide high capillary pumping power and generate higher vapor pressure. The maximum capillary pressure rise across the liquid-vapor meniscus is directly proportional to the surface tension of liquid sodium at the evaporator temperature, and inversely proportional to the maximum radius of evaporator wick pores.

To enhance heat conduction from the hot plate to the evaporator and the BASE tubes, recent vapor anode, multi- tube AMTECs employ a solid conduction stud between the hot end and the tubes support plate. In addition, several solid metallic rings are placed around the evaporator standoff to further enhance the heat conduction from the support plate to the cell evaporator. The conical evaporator structure provides a larger evaporation surface area than flat evaporators (Figure 3.5a). Thus, the depth of the evaporator cone can be adjusted commensurate with the desired cell electric power output.

The BASE tubes in a vapor anode, multi-tube AMTEC are connected electrically in series (Figure 3.5b), to provide up to $3-4 \mathrm{~V}$ at an electric power of up to $9 \mathrm{~W}_{\mathrm{e}}$. The dimensions and the number of BASE tubes in the cell, and the length of the electrodes per tube can be selected to provide the desired electrical voltage and current for the load. Recent cells tested at AFRL/PRS used TiN electrodes and between 5 and 7 BASE tubes, $7.62 \mathrm{~mm}$ in diameter $\left(0.3^{\prime}\right.$ ') $0.508 \mathrm{~mm}$-thick $\left(0.02^{\prime}\right.$ '), $27 \mathrm{~mm}$ to $40 \mathrm{~mm}$ long. The electrodes are typically $\sim 12.7 \mathrm{~mm}(0.5$ ") to $25.4 \mathrm{~mm}(1$ ") in length, and have a specific power density of $0.15 \mathrm{~W}_{\mathrm{e}} / \mathrm{cm}^{2}$. The cell specific power density is $\sim 27 \mathrm{~W}_{\mathrm{e}} / \mathrm{kg}$. 


\subsubsection{Vapor-Fed, Liquid Anode AMTEC Converters}

In a vapor-fed, liquid anode AMTEC, the alkali metal vapor in the high pressure cavity is condensed into a porous structure covering the anode side of the BASE. This process has been successfully demonstrated in laboratory test at Advanced Modular Power systems, Inc. (AMPS) and is referred to as the "AMTEC internal self-heat pipe," (Sievers et al. 1999). It is an attractive process to ensure uniform temperature and the possible use of extremely thin BASE membranes. Providing heat to the anode side by condensation of the liquid metal vapor in the underlying porous structure is also more efficient than by conduction or radiation. In this design, careful thermal analysis is required to ensure that the condensing liquid sodium will not cause electrical shorting of the BASE electrodes.

A vapor-fed, liquid anode AMTEC design offers the following advantages:

(a) No anode electrode needed. Thus, there are no contact losses, current collector losses and charge-exchange losses on the anode side of the converter. The vapor feed feature allows connecting the BASE elements electrically in series. Thus, the AMTEC unit can deliver a high voltage up to $400 \mathrm{~V}$ DC (Momozaki and El-Genk 2002; see also Section 3.3.3) at a relatively low current, reducing the internal electrical losses in the AMTEC unit and increasing the conversion efficiency.

(b) The efficient heat transfer to the BASE anodes by condensation of the working fluid results in a nearly uniform temperature of the BASE elements. Such a uniform temperature increases the converter efficiency, for a given coolant exit temperature from the nuclear reactor, reducing the induced thermal stresses, and increasing converter life.

Based on these considerations, a vapor-fed, liquid anode AMTEC converter design is selected for use in the LMR-AMTEC power plant design developed in this work and documented in this report. Considerations pertaining to the selection of the AMTEC working fluid are discussed next. 


\subsection{Selection of AMTEC Working Fluid}

In this Section, the thermophysical and radiological properties of alkali metal (sodium, potassium and lithium) working fluids for use in the LMR-AMTEC were reviewed. Based on this review, lithium was eliminated from consideration, sodium was selected as the first choice and potassium was selected as the second choice. Sodium has been used extensively in liquid metal reactors and AMTEC. Potassium has higher vapor pressure (Figure 3.6) and lower latent heat of vaporization and specific heat than sodium (Figure 3.7), which result in a lower heat input, and higher AMTEC conversion efficiency than in sodium AMTEC when operated at the same hot side temperature. When operated at the same anode vapor pressure, both sodium and potassium AMTECs would perform similarly. However, the condenser temperature of the potassium AMTECs could be $\sim 90$ $\mathrm{K}$ lower than sodium AMTECs, resulting in a lower conversion efficiency of the TE bottom cycle. In addition, there is limited experience with potassium coolant and KBASE solid electrolyte (Barkan et al. 1999, Briant and Farrington 1980, Crosbie and Tennenhouse 1982, DiStefano 1989, Williams et al. 1992, 1994 and 1995) which has higher ionic resistivity than Na-BASE (Figure 3.8).

\subsubsection{Potential Alkali Metal Working Fluids}

When comparing potential alkali metal working fluids for the AMTECs the following conclusions were reached.

The lithium working fluid is eliminated from consideration for the following reasons:

(a) It has high melting temperature $(\sim 454 \mathrm{~K})$ and is compatible with refractory structural material $(\mathrm{Nb}-1 \% \mathrm{Zr})$. Besides being very expensive, this refractory alloy experiences embrittlement and failure when exposed to oxygen rich environment (> $10 \mathrm{ppm}$ ); a better refractory alloy is Mo-Re.

(b) The generation of helium and tritium gas in the nuclear reactor would require employing an active gas removal system from the primary coolant loop.

(c) The relatively very low vapor pressure of lithium makes it unsuitable for use in AMTEC converters. The evaporation temperature of lithium at a typical anode vapor pressure of $50 \mathrm{kPa}$ is very high $\sim 1490 \mathrm{~K}$ (Figure 3.6). Operating at such temperature in the reactor core would require using very thick cladding and result in high nuclear fuel swelling and fission gas release in the fuel rods. Under such operating conditions, it would be difficult to attain long operation life for the nuclear reactor core and it would be necessary to employ refractory alloys for the cladding and the reactor core structure.

(d) There is very little experience with the fabrication and use of Li-BASE material. 


\section{Both sodium and potassium working fluids are suitable coolants in Liquid Metal Reactors (LMRs) and AMTECs:}

(a) The current experience with sodium coolant in LMRs is extensive, compared with a limited database on the use of potassium. In fact, potassium has never been used as coolant for commercial LMRs in the past.

(b) Both sodium and potassium have high heat transport capabilities and relatively low vapor pressures at the temperatures of interest in the AMTEC converters, keeping the nuclear reactor at a slightly higher pressure than atmospheric pressure $(0.2-0.4 \mathrm{MPa})$.

(c) Sodium melts at $371 \mathrm{~K}$ and at $0.1 \mathrm{MPa}$ it boils at $1135 \mathrm{~K}$, while potassium melts at lower temperature $(336 \mathrm{~K})$ and at $0.1 \mathrm{MPa}$ it boils at $1018 \mathrm{~K}$.

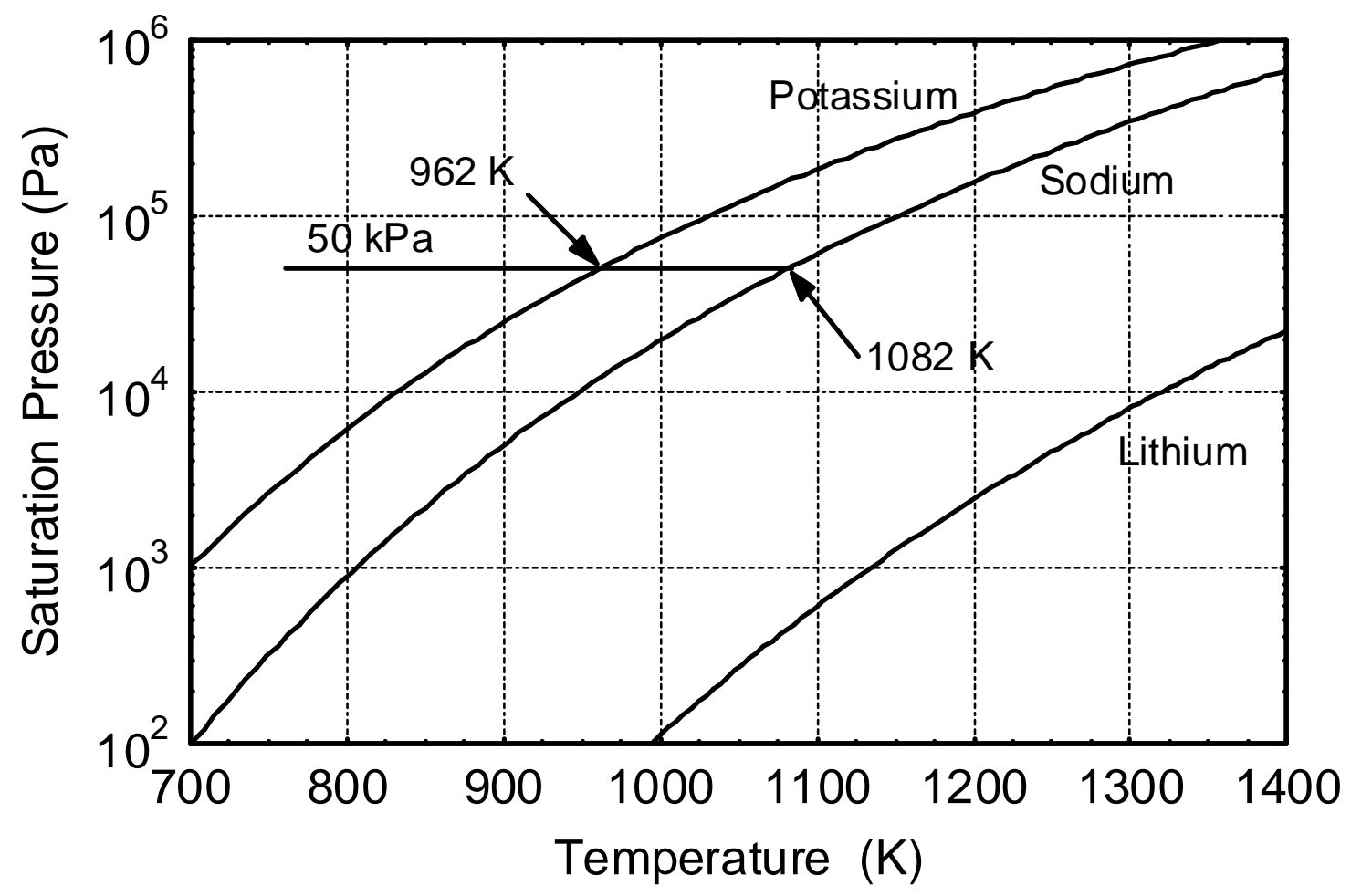

Figure 3-6. Vapor pressure of candidate alkali metal working fluids. 


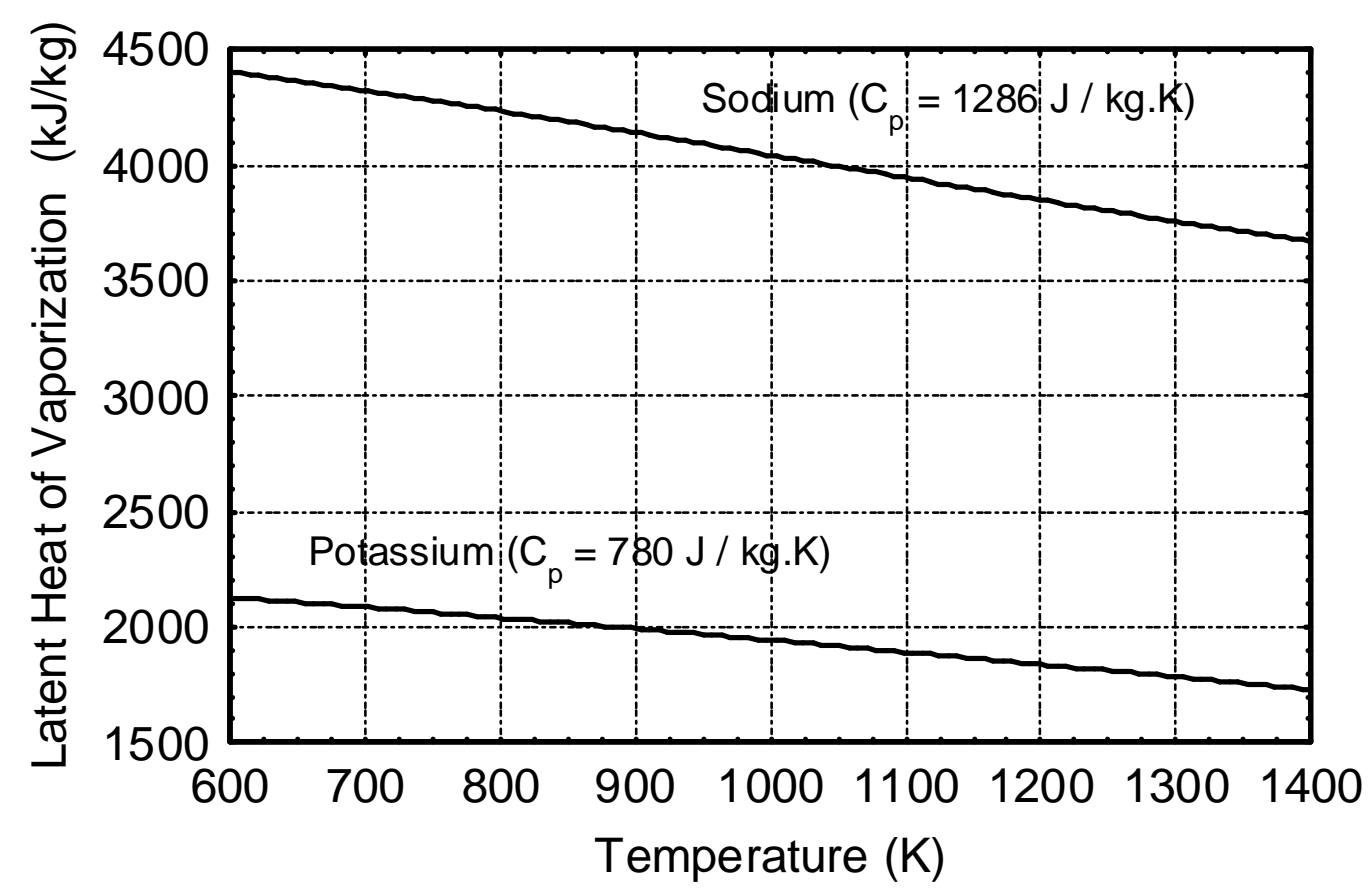

Figure 3-7. Latent heat of candidate alkali metal fluids.

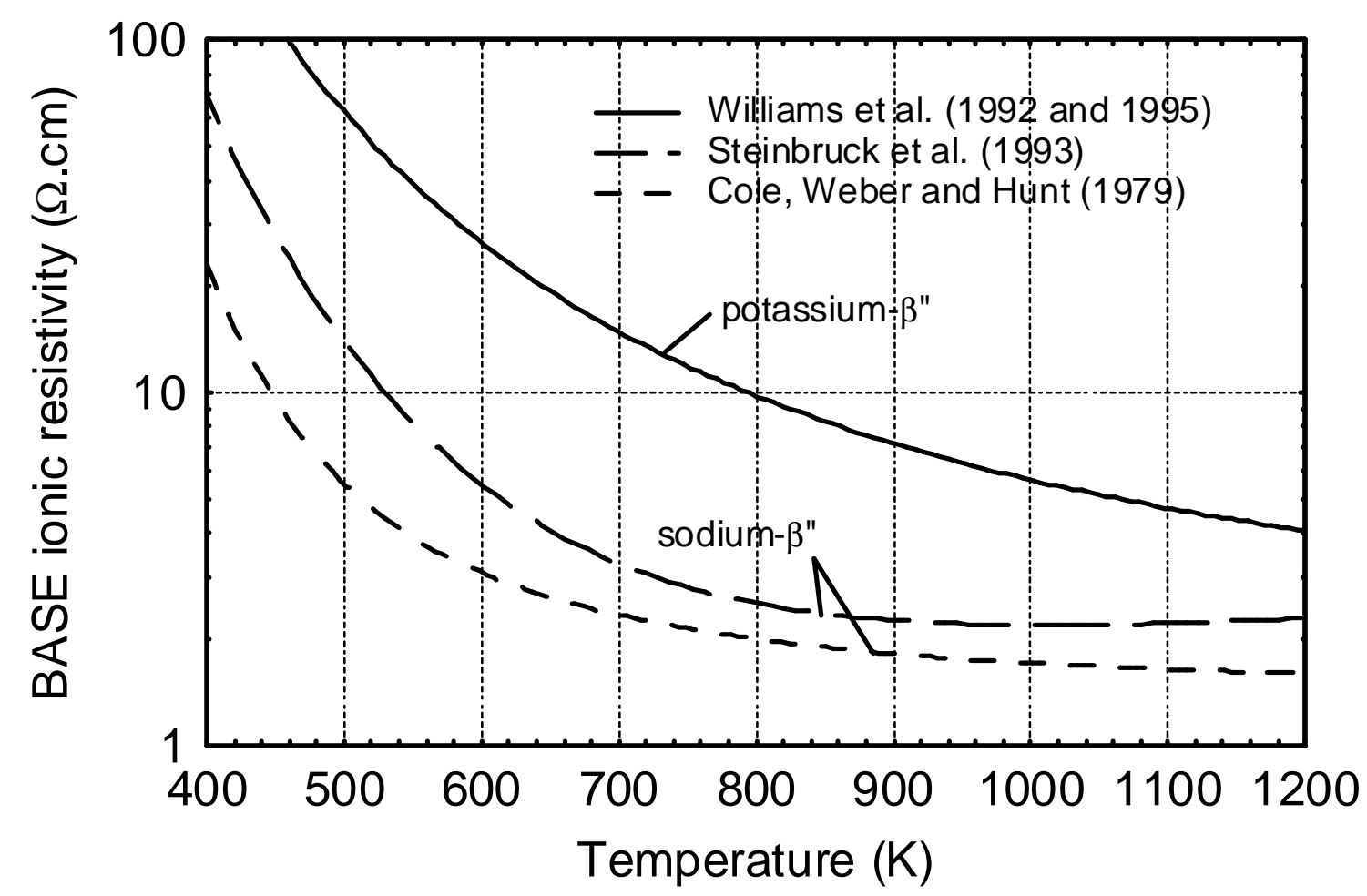

Figure 3-8. Ionic resistivity of sodium and potassium Beta"-alumina. 


\section{Direct feed of reactor coolant to AMTECs has disadvantages:}

(a) Both $\mathrm{Na}$ and $\mathrm{K}$ are prone to activation in the nuclear reactor core and are chemically active (Section 3.2.2). Natural sodium $\left({ }^{23} \mathrm{Na}\right)$ absorbs neutrons, and the product $\left({ }^{24} \mathrm{Na}\right)$ is a $\beta$ and $\gamma$ emitter $(2.76 \mathrm{MeV}$ and $1.38 \mathrm{MeV})$ with a $15 \mathrm{hrs}$ half-life. The same process occurs with ${ }^{41} \mathrm{~K}$, which has an abundance of $6.88 \%$ in natural potassium. The product resulting from neutron absorption $\left({ }^{42} \mathrm{~K}\right)$ is a $\beta$ and $\gamma(1.52 \mathrm{MeV})$ emitter with a 12.4 hrs half- life.

(b) The working fluid activity depends on flow rate and residence time in the reactor. Typically, the induced activity in potassium would be $\sim$ one fifth of that in sodium.

(c) Since induced radioactivity in the liquid metal coolant in the reactor core is a major safety concern, commercial, loop-type liquid-metal breeder reactors (LMFBRs) have used two separate coolant loops: a primary loop in which the reactor coolant is circulated and cooled in a heat exchanger by convection to a liquid metal working fluid in a secondary loop.

Another safety concern with the use of $\mathrm{Na}$ and $\mathrm{K}$ working fluids is that both are chemically active.

(a) Elements that are particularly soluble in hot $\mathrm{Na}, \mathrm{K}$ and $\mathrm{NaK}$ include cadmium, antimony, bismuth, copper, lead, silicon, tin and magnesium.

(b) The chief reason for attack/corrosion of structural materials is the ability of $\mathrm{Na}$ (and $\mathrm{Na}$ in $\mathrm{NaK}$ ) to dissolve oxygen. Materials are transported by dissolution in the hot regions and deposited in cooler regions of the primary coolant loop.

(c) Deposits and crystal growth in narrow, cool pipes in LMRs is a well-known problem.

(d) Because of the essential role of oxygen in corrosion, an inert-gas blanket (helium, argon) is kept over all free-surfaces in the reactor coolant loop.

(e) Based on these considerations, it is possible that corrosion problems in potassiumcooled reactors and potassium-AMTEC are similar to those in sodium-cooled systems.

Much experience has been gained in sodium-cooled LMRs and in sodium-AMTEC converters. This is not the case for potassium working fluid.

Potassium-AMTEC converters are promising and could be operated at a $\sim 120 \mathrm{~K}$ lower hot side temperature than sodium AMTECs. Conversely, the heat rejection temperature of the former will be $\sim 90 \mathrm{~K}$ lower than the later, resulting in a lower conversion efficiency of the TE bottom cycle. 
(a) Potassium has higher vapor pressure than sodium, therefore, in theory, a KAMTEC operating at a $~ 120 \mathrm{~K}$ lower hot-side temperature (see Figure 3.9) could deliver the same performance as a Na-AMTEC.

(b) Use of $\mathrm{K}$ in place of $\mathrm{Na}$ in AMTECs is therefore very attractive since the reactor core exit temperature affects fuel swelling and the reactor core lifetime.

(c) The fact that K-BASE has a higher (about 3 times) ionic resistivity than K-BASE (Figure 3.8) could be alleviated by designing new converters with thin BASE ( $30 \mu \mathrm{m}$-thick).

In summary sodium is ranked first choice for the working fluid in the AMTECs and as a reactor core coolant due to the following reasons:

- its moderate evaporation temperature at $50 \mathrm{kPa}(1082 \mathrm{~K})$,

- its extensive experience in liquid metal reactors and AMTECs for space,

- the experience with Na-BASE materials.

- Potassium came second due to the following reasons:

- its moderate/lower evaporation temperature at $50 \mathrm{kPa}(962 \mathrm{~K})$,

- its lower latent heat of vaporization ( $2000 \mathrm{~kJ} / \mathrm{kg}$ compared to $\sim 4000 \mathrm{~kJ} / \mathrm{kg}$ for sodium) which results, for a given AMTEC current, in a lower heat input, higher efficiency,

- its use in Rankine cycles in the space program is, however, limited (Angelo and Buden 1985, DiStefano 1989),

- There is limited experience with K-BASE, and

- K-BASE has higher ionic resistivity than Na-BASE.

The present effort focused initially on using sodium as a working fluid, to capitalize on the experience gained in Na-cooled LMRs and in Na-AMTEC. Of all candidate alkali metals, sodium has the highest boiling point after lithium. Since the boiling point of sodium (1135 K at $0.1 \mathrm{MPa}$ ) falls into the useful range of Na-BASE temperature, a direct coupling of the sodium cooled reactor core and the sodium AMTEC converters is possible. A small pressurization of the reactor coolant to $\sim 0.2 \mathrm{MPa}$ increase the sodium saturation temperature to $1230 \mathrm{~K}$ (Figure 3.6), increasing the conversion efficiency of the Na-AMTECs. Such increase in the conversion efficiency will come at the expense of increasing the cladding temperature and fuel swelling and fission gas release in the nuclear reactor core. Note that commercial LMFBRs are typically pressurized to $\sim 0.6$ $\mathrm{MPa}$ to offset the pressure losses in the primary coolant loop. In addition to the NaAMTEC unit design, a K-AMTEC unit design was developed and the performance of both units was investigated and compared. 


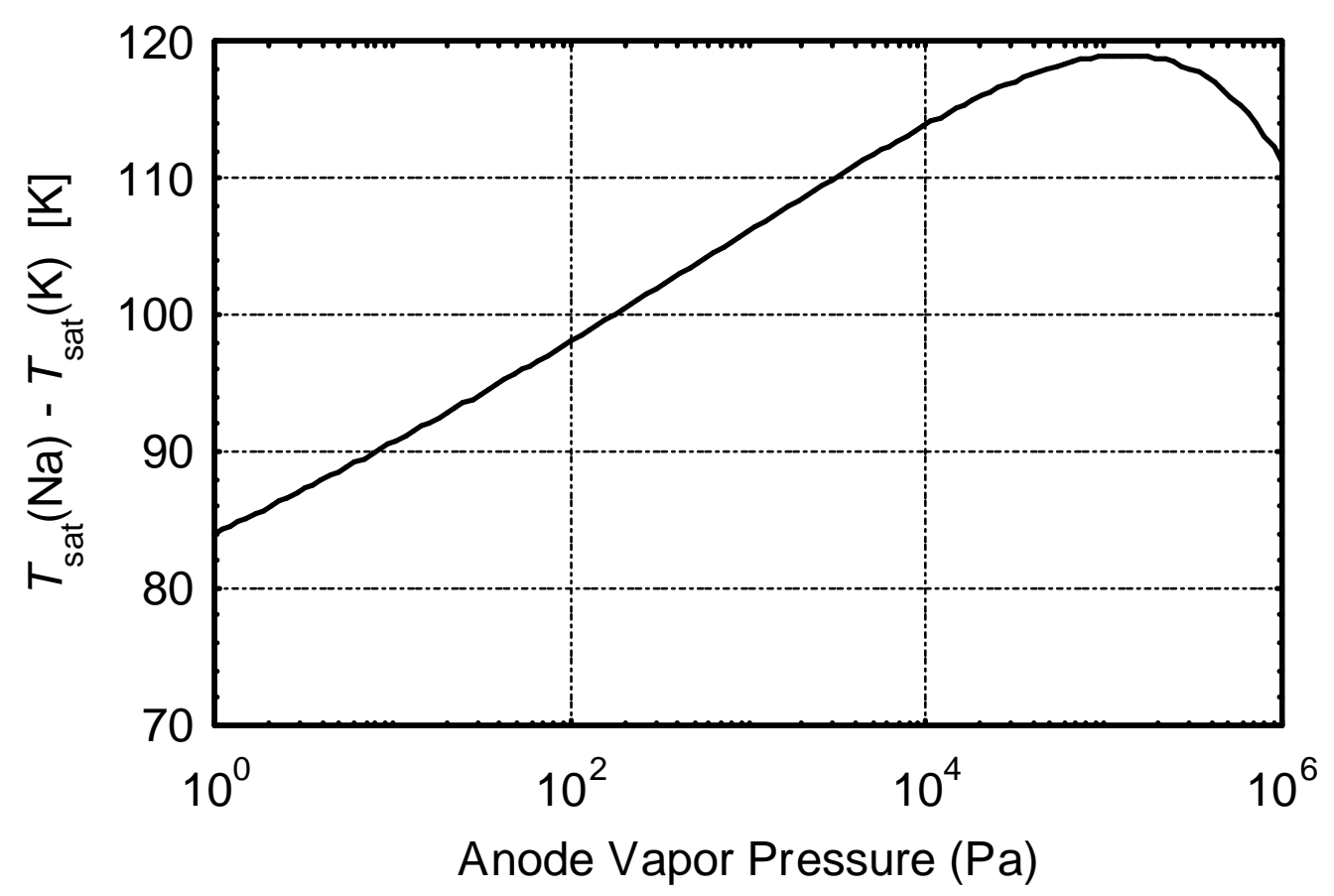

Figure 3-9. The difference in saturation temperature of sodium and potassium at same anode vapor pressure.

\subsubsection{Induced Radioactivity in Sodium and Potassium Coolants}

$\mathrm{Na}$ and $\mathrm{K}$ are both prone to induced radioactivity (El-Wakil 1978, Walker et al. 1988). All naturally occurring sodium is made up of the isotope ${ }^{23} \mathrm{Na}$. Natural sodium has a thermal neutron absorption cross-section of 0.534 barn and a fast-neutron cross section of about 1 millibarn at $0.25 \mathrm{MeV}$ (Table 3.2). In a nuclear reactor core, sodium absorbs neutrons according to the reaction:

$$
{ }^{23} \mathrm{Na}+{ }^{1} \mathrm{n} \rightarrow{ }^{24} \mathrm{Na}(14.96 \mathrm{hrs})+\gamma .
$$

The product $\left({ }^{24} \mathrm{Na}\right)$ is a $\beta$ and $\gamma$ emitter with a 14.96 hrs half-life (Table 3.2):

$$
{ }^{24} \mathrm{Na} \rightarrow{ }^{\mathrm{o}} \mathrm{e}^{-}(1.391 \mathrm{MeV})+{ }^{24} \mathrm{Mg}+\gamma(1.369 \mathrm{MeV} \text { and } 2.754 \mathrm{MeV})
$$

The decay product, magnesium- 24 , is stable and has a low activation cross section $(0.053$ barn). 
Table 3-2. Sodium $\left({ }_{11} \mathrm{Na}\right)$ isotopes (El-Wakil 1978, Walker et al. 1988).

\begin{tabular}{|c|c|c|c|c|c|c|c|}
\hline \multirow[t]{2}{*}{$\begin{array}{c}\text { Mass } \\
\text { number, } \\
\text { A }\end{array}$} & \multirow[t]{2}{*}{$\begin{array}{l}\text { Mass } \\
\text { (amu) }\end{array}$} & \multirow[t]{2}{*}{$\begin{array}{c}\text { Natural } \\
\text { abundance } \\
\text { (at.\%) }\end{array}$} & \multirow[t]{2}{*}{$\begin{array}{c}\text { Half- } \\
\text { life }\end{array}$} & \multicolumn{2}{|c|}{$\begin{array}{l}\text { Thermal neutrons } \\
\qquad(2,200 \mathrm{~m} / \mathrm{s}) \\
\text { cross-section (barn) }\end{array}$} & \multicolumn{2}{|c|}{$\begin{array}{c}\text { Fast neutrons } \\
(0.25 \mathrm{MeV}) \\
\text { cross-section (barn) }\end{array}$} \\
\hline & & & & Absorpt. & Scatt. & Absorpt. & Scatt. \\
\hline 20 & 20.00887 & - & $0.447 \mathrm{~s}$ & - & - & & \\
\hline 21 & 20.99760 & - & $22.48 \mathrm{~s}$ & - & - & & \\
\hline 22 & 21.99432 & - & $2.605 \mathrm{y}$ & 28,000 & - & & \\
\hline 23 & 22.98977 & 100 & - & 0.534 & 4.0 & 0.001 & \\
\hline 24 & 23.99102 & - & $14.96 \mathrm{~h}$ & - & - & & \\
\hline 25 & 24.98984 & - & $60.0 \mathrm{~s}$ & - & - & & \\
\hline 26 & - & - & $1.07 \mathrm{~s}$ & - & - & & \\
\hline
\end{tabular}

Table 3-3. Potassium ( $\left.{ }_{19} \mathrm{~K}\right)$ isotopes (El-Wakil 1978, Walker et al. 1988).

\begin{tabular}{|c|c|c|c|c|c|c|c|}
\hline \multirow[t]{2}{*}{$\begin{array}{c}\text { Mass } \\
\text { number, } \\
\text { A }\end{array}$} & \multirow[t]{2}{*}{$\begin{array}{l}\text { Mass } \\
\text { (amu) }\end{array}$} & \multirow[t]{2}{*}{$\begin{array}{c}\text { Natural } \\
\text { abundance } \\
\text { (at.\%) }\end{array}$} & \multirow[t]{2}{*}{ Half-life } & \multicolumn{2}{|c|}{$\begin{array}{l}\text { Thermal neutrons } \\
\qquad(2,200 \mathrm{~m} / \mathrm{s}) \\
\text { cross-section (barn) }\end{array}$} & \multicolumn{2}{|c|}{$\begin{array}{l}\text { Fast neutrons } \\
(0.25 \mathrm{MeV}) \\
\text { cross-section } \\
\text { (barn) }\end{array}$} \\
\hline & & & & Absorpt. & Scatt. & Absorpt. & Scatt. \\
\hline 37 & 36.97324 & - & $1.23 \mathrm{~s}$ & - & - & & \\
\hline 38 & 37.96905 & - & $7.63 \mathrm{~m}$ & - & - & & \\
\hline 39 & 38.96371 & 93.2581 & - & 1.94 & - & & \\
\hline 40 & 39.97400 & 0.0117 & $1.3 \times 10^{9} \mathrm{y}$ & 70.0 & - & & \\
\hline 41 & 40.96184 & 6.7302 & - & 1.24 & - & & \\
\hline 42 & 41.96352 & - & $12.36 \mathrm{~h}$ & - & - & & \\
\hline 43 & 42.96066 & - & $22.3 \mathrm{~h}$ & - & - & & \\
\hline 44 & 43.96192 & - & $22.1 \mathrm{~m}$ & - & - & & \\
\hline
\end{tabular}

Naturally occurring potassium is made of two stable isotopes, ${ }^{39} \mathrm{~K}(\sim 93$ at.\%) and ${ }^{41} \mathrm{~K}\left(\sim 6.73\right.$ at. \%). The remainder $(0.0117$ at. $\%)$ is radioactive ${ }^{40} \mathrm{~K}$, a $\beta$ and $\gamma$ emitter with a very long half-life (1.28 $\times 10^{9}$ years) and thus a very low level of activity (Table 3.3). 
Potassium-41 has a thermal-neutron absorption cross section of 1.24 barn, and converts according to the reaction (Table 3.3):

$$
{ }^{41} \mathrm{~K}+{ }^{1} \mathrm{n} \rightarrow{ }^{42} \mathrm{~K}(12.36 \mathrm{hrs})+\gamma
$$

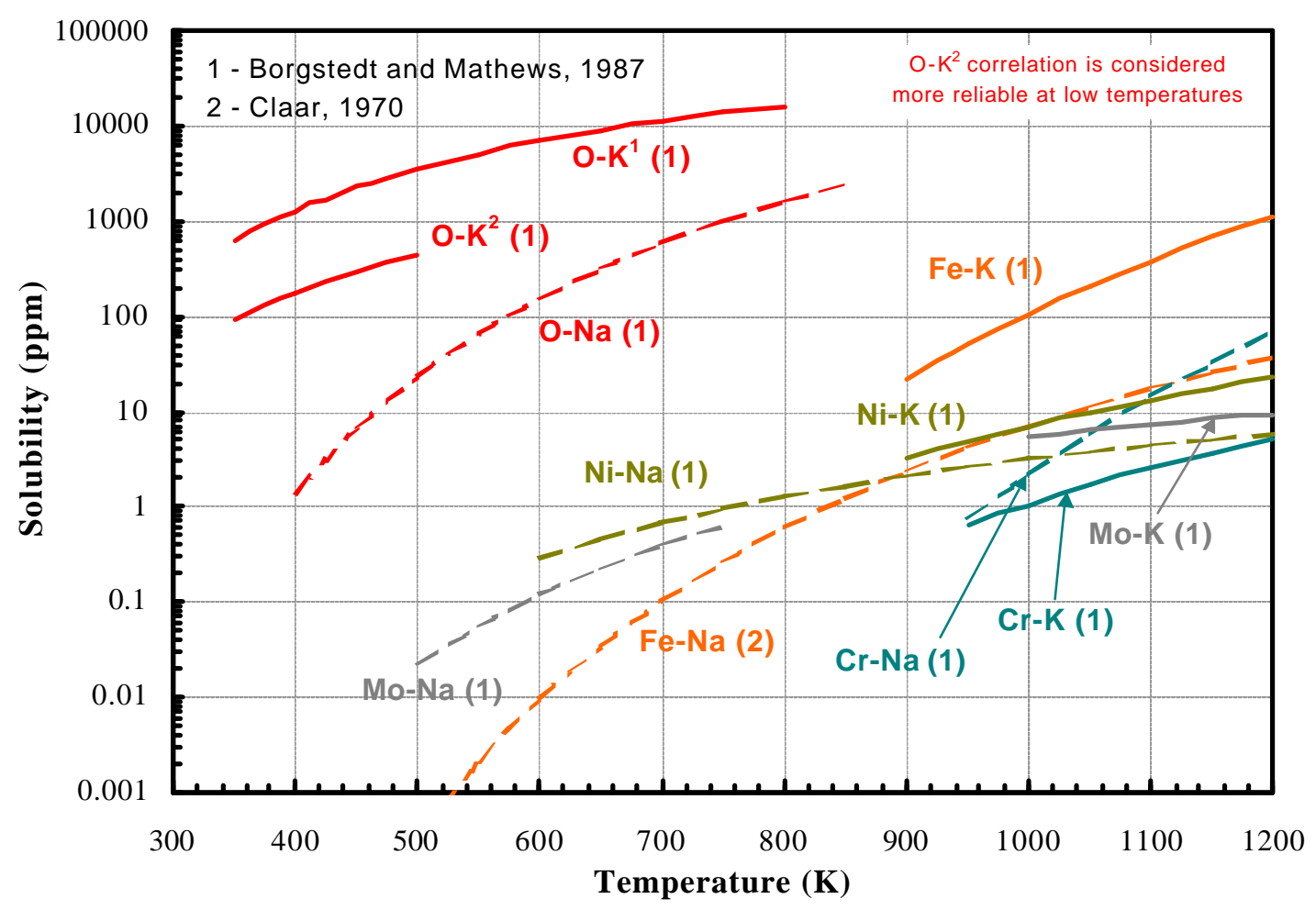

Figure 3-10. Solubility of oxygen and metallic impurities in liquid sodium and potassium.

The product $\left({ }^{42} \mathrm{~K}\right)$ is a $\beta$ and $\gamma$ emitter with a 12.36 hrs half-life:

$$
{ }^{42} \mathrm{~K} \rightarrow{ }^{\mathrm{o}} \mathrm{e}^{-}(3.52 \mathrm{MeV})+{ }^{42} \mathrm{Ca}+\gamma(1.525 \mathrm{MeV})
$$

The decay product, calcium-42, is stable and has a low activation cross section (0.7 barn). Note that, of the three naturally-occurring potassium isotopes, only ${ }^{41} \mathrm{~K}$ is converted to a radioactive isotope upon neutron irradiation. The most abundant isotope $\left({ }^{39} \mathrm{~K}\right)$ has a thermal-neutron absorption cross section of 1.94 barn, but converts to the long half-life (1.28 x $10^{9}$ years) ${ }^{40} \mathrm{~K}$ isotope (Table 3.3 ), thus resulting in a very low level of activity. Furthermore, while ${ }^{40} \mathrm{~K}$ has a large cross section (70 barns) for thermalneutron 
absorption, converting to ${ }^{41} \mathrm{~K}$ which reacts according to Reactions (3.3) and (3.4) above, its natural abundance is so low $(0.0117$ at.\%) that its contribution to activity is also unimportant (Table 3.3).

The working fluid activity depends on abundance, cross section and half-lives, as well as flow rate and residence time in the reactor. A rough estimate shows that the activity in potassium would be $\sim$ one fifth of that in sodium.

\subsubsection{Compatibility with Structural Materials}

A review of the materials compatibility and corrosion issues when using sodium and potassium working fluids suggested that $\mathrm{Na}$ and $\mathrm{K}$ exhibit similar corrosion mechanisms. Corrosion in alkali metals is mostly driven by the solubility of constituent elements and chemical reactions with oxygen, carbon and nitrogen impurities. The corrosion rate depends on temperature, the oxygen content and somewhat on the type of dissolved elements (particularly nickel). As an illustration, at $1000 \mathrm{~K}$, the rate of SS-316 loss in liquid sodium is $1 \mu \mathrm{m} /$ year at $1 \mathrm{ppm} \mathrm{O}_{2}$, and $\sim 10 \mu \mathrm{m} /$ year at $5 \mathrm{ppm} \mathrm{O}_{2}$. Cold trapping has been demonstrated in liquid sodium cooled reactors to be an effective means of maintaining the concentration of oxygen and metallic contaminants ( $\mathrm{Fe}, \mathrm{Cr}, \mathrm{Ni}$ and $\mathrm{Mn}$ ) to well below concern levels (see Figure 3.10). However, experience with cold trapping is limited in potassium systems. Since the solubility of each metallic element of concern in liquid potassium is comparable to that in liquid sodium (Figure 3.10), cold trapping (at $\sim 400 \mathrm{~K}$ ) would be an effective mean for removing metallic contaminants in liquid potassium. However, the solubility of oxygen in liquid potassium is nearly two orders of magnitude higher than in liquid sodium (Figure 3.10). Therefore, the amount of oxygen in liquid potassium cannot be controlled by cold trapping. Instead, active oxygen getters (such as zirconium, hafnium or yttrium) are required in liquid potassium systems to maintain oxygen levels below concern limits.

The AMTEC/TE converter unit design developed in this work is described in details in the next Sub-Section.

\subsection{AMTEC/TE Converter Design}

The topping cycle of the AMTEC/TE energy conversion units developed in this work is comprised of high-power (> $50 \mathrm{kWe})$, high-voltage ( $400 \mathrm{~V})$, Alkali Metal Thermal-toElectric Converter (AMTEC) units, which operate at an anode pressure of $\sim 80 \mathrm{kPa}$, corresponding to a hot (or beta"-alumina solid electrolyte, BASE) temperature of $1006 \mathrm{~K}$ and $1127 \mathrm{~K}$, for potassium and sodium working fluids, respectively. For these working fluids, the exit coolant temperature from the nuclear reactor core would be about $50-100$ $\mathrm{K}$ higher than the BASE temperature in the AMTEC converter units. Based on the recent voltage breakdown test results obtained at UNM-ISNPS (see Section 3.3.3), a terminal 
voltage of $400 \mathrm{~V}$, or even higher, could be used when the housing of the AMTEC converters is negatively biased relative to the BASE elements.

In the AMTEC unit design, the BASE elements are enclosed in a dome-shaped, thin metallic thermal radiation shield, with orifices for the low-pressure alkali-metal vapor to flow out to the condenser. This dome-shaped shield minimizes the internal radiation losses, hence, increasing the efficiency of the AMTEC to 25-30\%. The alkali metal vapor that flows out of the low-pressure cavity, through the orifices in the thermal radiation shield, condenses on the inside of the two, long vertical side walls. The condenser temperatures of $\sim 530 \mathrm{~K}$ and $\sim 600 \mathrm{~K}$, for potassium and sodium working fluids, respectively, are selected based on maximizing the conversion efficiency of the combined AMTEC/TE converter (see Section 3.4.5).

The heat imparted to the sidewalls of the AMTEC unit, by condensation of working fluid and by radiation and conduction is transmitted to a multitude of TE unicouples that are conductively coupled to the AMTEC sidewalls. The TE modules are well insulated on the sides and cooled at the colder end by natural convection of ambient air. The condensation of working fluid on the side surfaces of the wall of the AMTEC unit ensures a uniform hot side temperature of the thermoelectric (TE) modules. The electricity generated by the TE bottom cycle could contribute between $10 \%$ and $20 \%$ of the total electric power of the AMTEC/TE converter unit, depending on the cold side temperature and type of AMTEC working fluid (sodium or potassium). The reference sodium AMTEC/TE unit in this work produces more than $50 \mathrm{kWe}$ at a terminal voltage of $400 \mathrm{~V}$ DC. The TE modules are cooled using a common water heat pipe conductively coupled to the TE cold side. The heat pipe provides uniform cold side temperature of the TE modules, eliminates hot spots, and ensures that the heat is rejected to the ambient air at almost the same surface temperature. The outer surface of the heat pipe enclosure in made into isothermal, longitudinal fins to increase the surface area, enhance heat rejection and minimize the temperature differential with the ambient air. This arrangement increases the temperature differential across the TE unicouples, hence their efficiency for converting heat into electricity. The heat pipe fins are lightweight and are compartmentalized to ensure redundancy in the heat rejection and the operation of the energy conversion subsystem. When $35-\mathrm{cm}$ long fins that are $1-\mathrm{cm}$ wide and separated by a $2.54-\mathrm{cm}$ air gap are used, and assuming an ambient air temperature of $300 \mathrm{~K}$, the excess heat is removed from the cold side of the TE modules at 350-400 K. The TE modules are grouped in combinations of series and parallel electric connections to increase the terminal voltage and redundancy, and reduce Joule losses in the interconnecting leads. The AMTEC/TE converter unit design is described in more details next.

\subsubsection{Design of AMTEC Unit}

The developed AMTEC unit design is a vapor-fed, liquid anode type, which combines the advantages of both the liquid anode, and the vapor fed, vapor anode devices, and avoids their inherent disadvantages. In the liquid-fed, liquid-anode AMTEC, the alkali metal working fluid on the high-pressure side in direct contact with the BASE is liquid. 
Since alkali metals are good electrical conductors, there is no need to use an anode electrode, reducing the internal resistance of the converter, since the polarization/concentration voltage losses and the contact or current collector losses on the anode side of the BASE are eliminated. Furthermore, the temperatures of the BASE and of the high-pressure liquid can both be very close to that of the heat source, increasing the output voltage of the device. Each BASE element typically delivers $<0.7 \mathrm{~V}$ voltage, but the electrical current is proportional to the surface area of the BASE/cathode electrode. Unfortunately, liquid-fed, liquid-anode AMTECs are low-voltage, high-current devices, subjected to high internal Joule losses and high electrical losses on the cathode side. Because liquid alkali metals are good electronic conductors, it is extremely difficult to electrically connect the BASE electrodes in such a device in series, in order to increase the terminal voltage.

Conversely, in vapor fed, vapor anode AMTECs, the BASE anode electrode is connected to the cathode of the neighboring BASE, forming a series connection of a multitude of elements, resulting in a high voltage output. Unlike a liquid anode, a vaporanode AMTEC has porous anode electrodes and current collectors. In addition, the BASE temperature should be kept at least $20 \mathrm{~K}$ hotter than the evaporator wick temperature to avoid condensation of the working fluid on the BASE elements and shortcircuiting the device. Another disadvantage of the vapor fed, vapor anode AMTECs is the need to use an electrode and current grid collector on the anode side of the BASE, increasing the internal electrical losses.

In the present vapor-fed, liquid anode unit design (Figures 3.11 and 3.12), the alkali metal vapor generated in (closed-unit design) or introduced into (open- unit design) the high-pressure cavity of the AMTEC unit condenses into liquid and saturates a porous anode structure that is covered by a thin BASE membrane $(\sim 200 \mu \mathrm{m}$ thick) (Sievers 1993, U. S. Patent No. 5,228,922). This arrangement has been demonstrated successfully in laboratory tests at Advanced Modular Power Systems, Inc. (AMPS), and is referred to as the "AMTEC internal self-heat pipe" (Sievers et al. 1999). It ensures uniform temperature of the BASE and makes it possible to use extremely thin BASE membranes that could be sputtered or deposited onto the surface of the porous anode structure (molybdenum-rhenium alloy) using Chemical Vapor Deposition (CVD) or similar techniques. Careful design is required to ensure that any excess liquid alkali metal in the porous anode element (which does not diffuse through the BASE) will not drip down or pool, causing electrical shorting of the BASE electrodes, which are connected electrically in series.

The present AMTEC/TE converter unit design (Figures 3.11 and 3.12) is a high voltage, high efficiency static converter made of a vapor fed, liquid anode AMTEC unit that is thermally coupled to a multitude of thermoelectric (TE) conversion unicouples. The AMTEC unit employs alkali metal working fluid (e.g. sodium or potassium) and operates between 1006-1127 K on the hot side and 530-600 K on the condenser (or cooler) side. The condenser is thermally and conductively coupled to a multitude of solid state, TE conversion unicouples, operating between the temperature of the condenser and a heat rejection radiator temperature of $350-400 \mathrm{~K}$, and the waste heat is rejected by natural convection of air to the environment. 


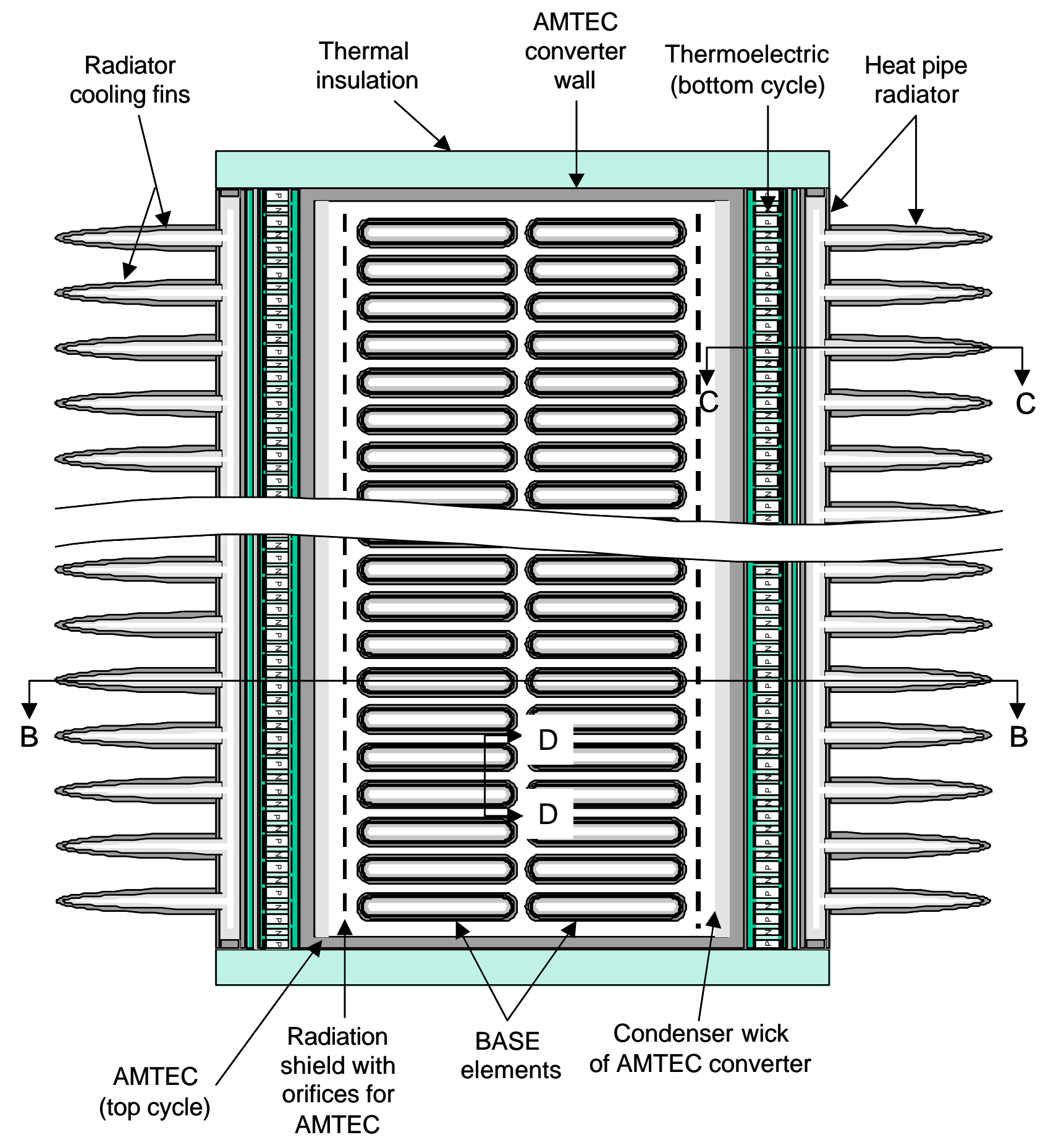

Figure 3-11.. A plane view of the reference AMTEC/TE converter unit design developed in this work (Section A - A in Figure 3.12). 


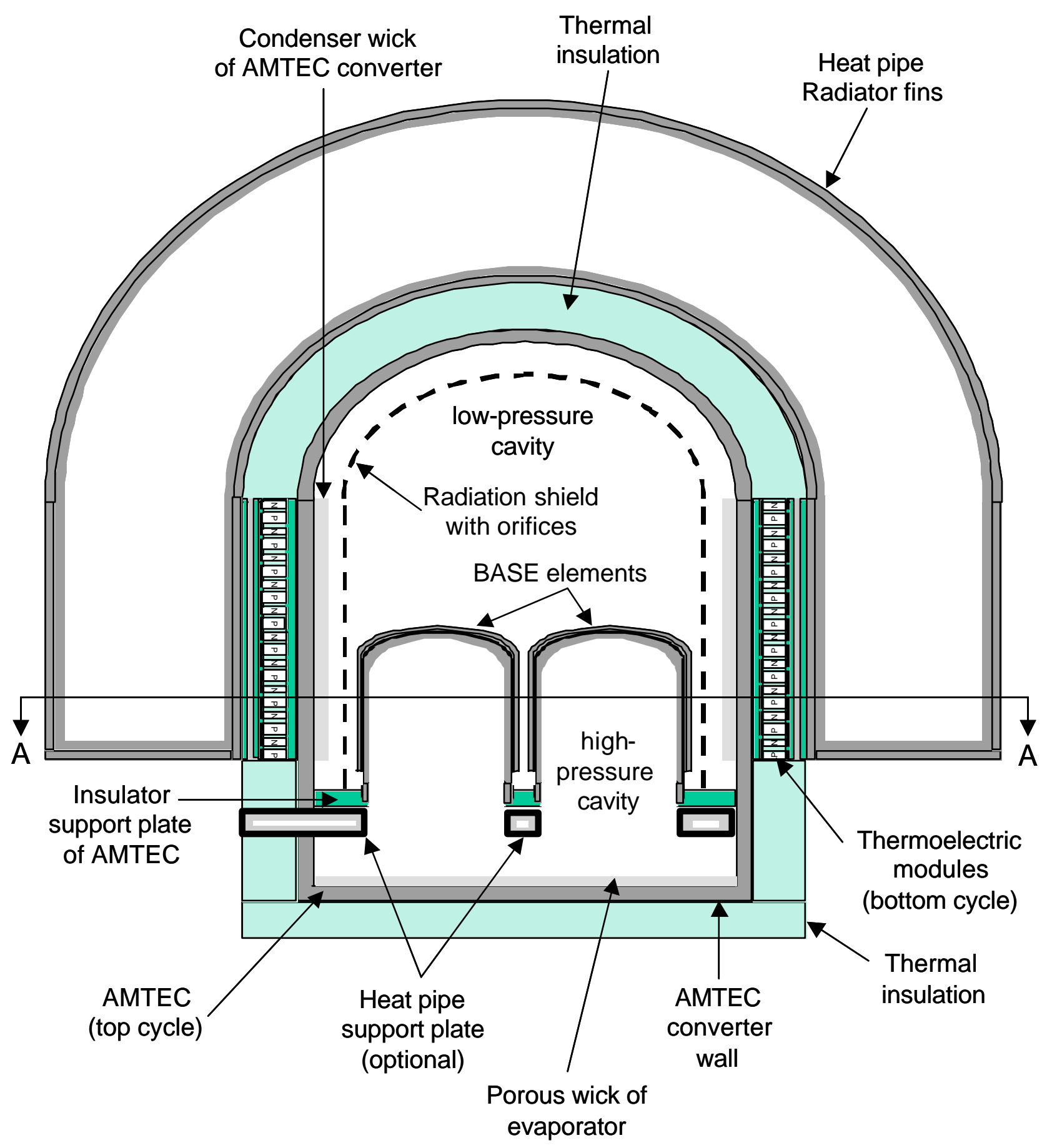

Figure 3-12. A vertical cross-section of the reference AMTEC/TE converter unit design developed in this work (Section B - B in Figure 3.11). 
The AMTEC unit (Figure 3.11) is comprised of a number of elongated, domeshaped, monolithic elements (Figure 3.13) arranged in two or more rows, each having an open cavity to the high vapor pressure zone of the unit. The proposed design of these elements significantly reduces the manufacturing steps needed and simplifies the series electrical connection of the elements. Each element is made up of a dome-shaped, liquidsaturated, rigid porous substrate which serves as a liquid anode, welded to a rubber-band like, non-porous (anode) metal structure (Figure 3.13). A thin film of Beta'-Alumina Solid Electrolyte (BASE) is deposited onto the outer surface of the porous anode by stateof-the-art plasma spraying or sputtering techniques, such as CVD (Figure 3.13). The BASE is a barrier that is impervious to the alkali metal, is an electron insulator, and a conductor of alkali metal ions.

There is no metal-eramic brazing required between the BASE solid electrolyte in the monolithic elements and the perforated insulator support plate (Figure 3.13). In addition, the dome shape of the monolithic elements provides mechanical strength and durability to the ceramic BASE membrane. The applied BASE layer should be preferably thin $(\sim 50-200 \mu \mathrm{m})$, but hermetically sealed to prevent non-electrolytic transport of alkali metal. A thin BASE layer would have negligible ionic resistance. In the event that some of the beta"-alumina is converted to beta'-alumina over time, the ionic resistivity would still be small, even though beta'-alumina has higher ionic resistivity than beta"-alumina. The composite electrolyte membrane herein also offers a number of advantages over current state-of-the-art hollow BASE tubes, including more rugged and stronger designs, and better seals, making them ideal for use in long-life, high-power, remote power systems.

The BASE is covered by a cathode made of a thin metal electrode of fine-grain $(0.05-0.2 \mu \mathrm{m})$ porous material (e.g. $\left.\mathrm{WRh}_{1.5}\right)$. The cathode electrode is applied preferably by a state-of-the-art sputtering technique, and is overlaid by a porous metallic current collector mesh or grid that is electrically connected to the anode of the next BASE element (Figure 3.13). A metal (anode) connector is connected to the anode support structure, and another metal (cathode) connector is electrically connected to the current collector grid. The anode connector of one monolithic BASE element is electrically connected to the cathode connector of the adjacent BASE element (Figure 3.13), electrically connecting them in series. The electrical connections between the monolithic BASE elements can be achieved by a number of metal leads in parallel to reduce the Joule losses, or by strip metal connectors along the straight, long edge of the dome-shaped elements. The porous anode substrate of the BASE element has a small gutter that extends beyond the insulator support plate to drain excess liquid alkali metal from the porous anode, thus preventing droplets of alkali metal from causing a short circuit between adjacent BASE elements.

As indicated earlier, the multitude of BASE elements in an AMTEC unit (Figure 3.11 ) are connected electrically in series to provide a high voltage output of $\sim 400 \mathrm{~V}$. The BASE elements in the AMTEC unit are enclosed in a pressure-tight vessel and are mounted onto a perforated, electrically insulating support plate (Figure 3.12), which divides the unit into high-pressure and low-pressure cavities. One possible way of mechanically connecting the BASE elements into the support plate is illustrated in Figure 3.13. The porous metal substrate is fused at the bottom to an anode metal strip shaped 
into a rubber band (or ribbon) that fits tightly into the open groove of the support plate. The pressure seal in this configuration may be obtained by using a braze, or by the differential thermal expansion between the metal ribbon and the support plate, in conjunction with an adequate compression of the support plate provided by the vessel wall. The support plate is made of an insulating ceramic material such as alumina or zirconia to electrically isolate the BASE elements.

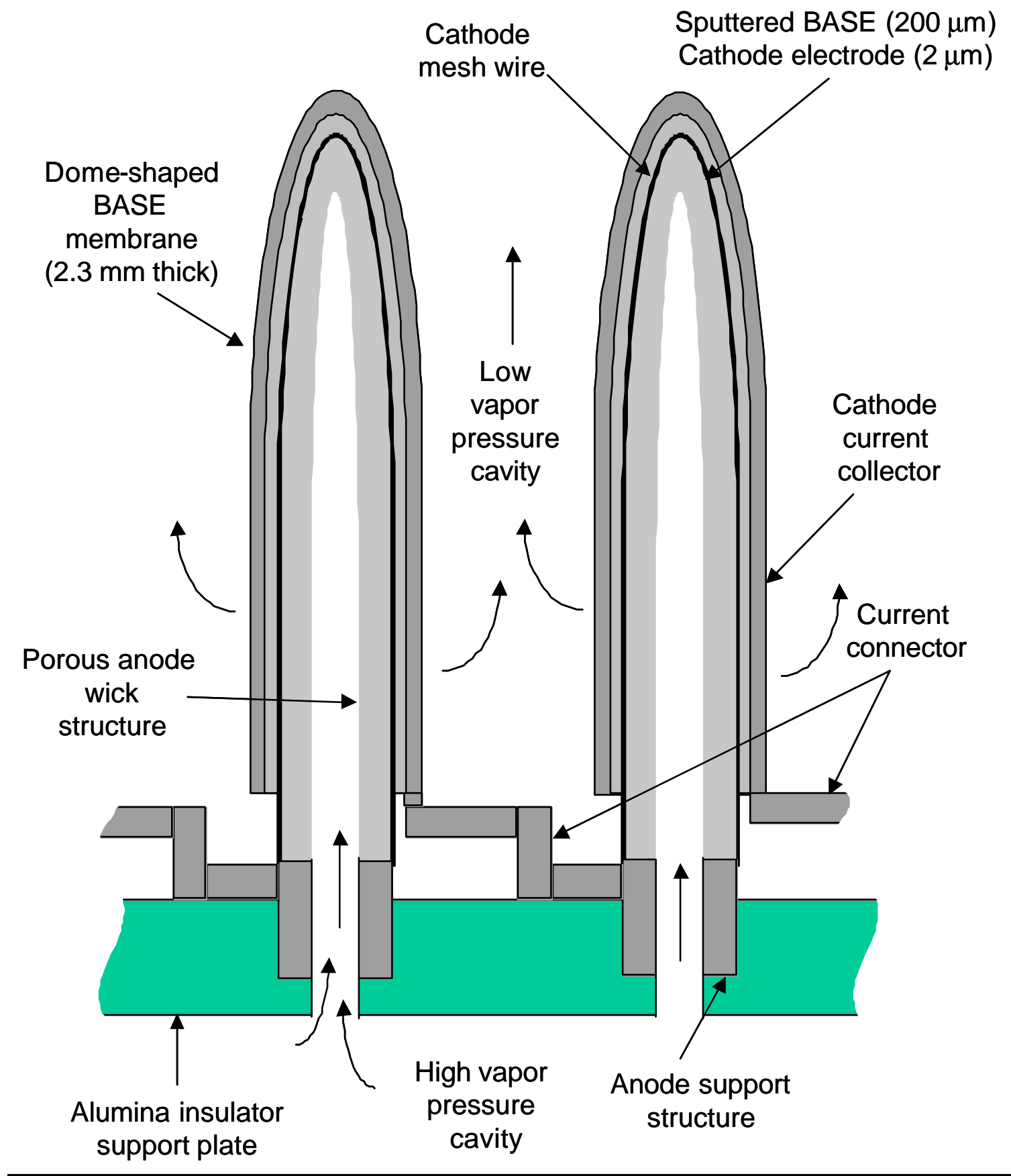

Figure 3-13. Cross-section view of two BASE elements connected in series (Section D - D in Figure 3.11). 
In the upper, low-pressure cavity of the AMTEC unit, the BASE elements are enclosed by a radiation and drip shield that is permeable to alkali metal vapor (Figure 3.12). The size and number of the orifices in the shield provide adequate flow area for the low-pressure vapor to circulate out to the condenser, but a small radiation view factor between the BASE elements and the condenser wall, thus minimizing the amount of radiative energy streaming through the orifices. The low-pressure vapor diffuses through the orifices in the shield and condenses on the two sidewalls of the AMTEC unit. Because the sidewalls of the AMTEC unit are vertical, the condensate flows down under the effect of gravity and pools at the bottom of the low-pressure cavity. Alternatively, the condenser wall may be covered with a high-porosity porous wick (Figures 3.11 and 3.12), or with specially-shaped grooves to maintain a continuous film of alkali-metal liquid on the inside surfaces of the walls. An alkali-metal liquid film is highly reflective $(>95 \%$ reflective), further reducing the radiation heat losses in the low-pressure cavity of the AMTEC unit.

The top sections of the radiation shield and the converter's roof are cylindrical surfaces of constant curvature, to help reflect thermal radiation back towards the BASE elements/support plate. The roof structure is made of titanium $(\sim 2 \mathrm{~mm}$ thick $)$, which has higher reflectivity than stainless steel. To prevent the alkali metal vapor flow through the shield orifices from reaching the sonic limit, the total orifices' flow area amounts to $33 \%$ of the total area of the vertical sides of the thermal shield. The radiation heat exchange model developed in this work accounts for the finite curvature of the top sections of the radiation shield and converter's roof, and for the geometric view factors through the shield orifices, between BASE elements, support plate and shield inner surface, under the shield, and the condenser walls, roof wall and support plate outside the shield enclosure. Since the temperatures of the radiation shield, roof structure, thermal insulation surfaces, and the radiator cooling fins are not known a priori, an iterative numerical solution was developed to solve the coupled radiation/conduction problem.

The porous artery lining of the inner surface of the converter sidewalls, between the condenser panels and the evaporator wick, returns the alkali metal liquid from the condenser to the evaporator wick in the high-pressure, hot cavity of the AMTEC unit In closed-circuit condition, when the alkali metal working fluid is circulating through the AMTEC converter, the condensate heats up to the evaporator temperature as it flows through the return artery. As a result, the conduction heat losses to the condenser through the arteries and the sidewalls are significantly reduced by the sensible heat of the circulating alkali metal. The conduction heat flow model of the AMTEC accounts for the liquid convection through the return arteries.

In the closed AMTEC unit design (Figures 2.3 and 2.5), the liquid working fluid in the evaporator wick is converted into high-pressure vapor by the heat supplied at the proper temperature from the plant's secondary loop coolant through an intermediate heat exchanger (indirect interfacing). The produced vapor condenses and saturates the porous anode, from which the alkali metal ions traverse the BASE membrane to the cathode, producing an electrical potential. At the cathode, the alkali-metal ions recombine with electrons circulating from the anode through the external load, to form neutral atoms. These neutral atoms evaporate (or desorb) from the cathode/BASE interface at a very low pressure and travel through the shield orifices to the condenser where they are converted into liquid (Figure 3.12). The resulting liquid in the condenser is circulated back to the 
high-pressure cavity by the capillary action developed at the liquid-vapor surface of the fine-pore evaporator wick.

In the open AMTEC unit design (Figure 2.4) used in conjunction with alkali metal boilers (AMB) concept (Figure 2.6), the high-pressure alkali metal vapor is introduced directly into the high-pressure cavity of the AMTEC unit, and condenses in the porous anodes of the BASE elements. The mass flow rate of working fluid introduced in the high-pressure cavity is greater than that diffusing through the BASE membranes, by the amount of electrical power generated and parasitic heat losses divided by the latent heat of vaporization of the working fluid. The excess working fluid forms a liquid pool at the bottom of the high-pressure cavity, and is recirculated back to the cold side of the AMB by an electromagnetic of mechanical pump. The fraction of the working fluid that has diffused through the BASE membranes is cooled and converted into liquid in the condenser wicks of the AMTEC unit, and is also recirculated back to the AMB by a pump after mixing with the hot liquid returning from the evaporator pool of the AMTEC unit. More details on the coupling schemes of the nuclear reactor and the AMTEC/TE converter units are given in Section 2.1.

\subsubsection{BASE Element and Composite BASE/Electrode}

Most AMTECs have used ceramic, hollow BASE tubes of sufficient thickness $(0.5-2.0$ $\mathrm{mm}$ ) to withstand the thermal and pressure loads. The BASE electrolyte, however, is a brittle material, and thus could fracture due to a thermal shock and/or induced strain. Also, the ionic resistance of the BASE is proportional to its thickness, contributing a nonnegligible fraction to the internal electrical losses in the AMTEC.

Recently, a composite electrolyte membrane (Figure 3.14), in which the electrolyte is reinforced by a porous metal structure as a means to improve its strength and durability, has been proposed (Sievers and Wright 1990, Sievers and Cooper 1990 and 1991). The porous metal structure serves as a compliant support for the BASE and provides high strength to accommodate the structural loads without failure. The metal substrate must have a good thermal expansion that matches that of the BASE and be sufficiently rigid to prevent cracking of the electrolyte due to substrate strains under applied loads. The metal substrate must be porous to allow the passage of sodium to the surface of the BASE. The metal substrate could be made of pressed and sintered powder of tantalum or molybdenum-rhenium. The BASE may be deposited onto the surface of the porous metal substrate by plasma spraying or sputtering techniques.

The applied BASE layer is physically very thin $(\sim 50-200 \mu \mathrm{m})$, but thick enough to be hermetically sealed to prevent non-electrolytic sodium transport from the high-

pressure to the low-pressure cavity (Figure 3.13). Such thin BASE has small or negligible ionic resistance. In the event that some of the beta"-alumina is converted to beta'-alumina, the ionic resistance would still be small, even though beta'-alumina has higher ionic resistivity than beta"-alumina. Composite electrolyte membranes also offer a number of advantages over current state-of-the-art hollow BASE tubes as in the PXtype AMTECs (Figure 3.5), including more rugged and stronger designs, and better seals, making them ideal for use in long-life, high-power, remote power systems. 
While pure $\mathrm{Nb}$ has a closely matching thermal expansion to that of the BASE, a niobium porous metal substrate is not recommended. Pure $\mathrm{Nb}$ exhibits accelerated erosion in the presence of oxygen (>10 ppm) and with liquid sodium (DiStefano 1989, Klueh 1968, Claar 1970). Instead, appropriate materials for the BASE membrane substrate are molybdenum alloys such as Mo-41\%Re (King and El-Genk 2001).

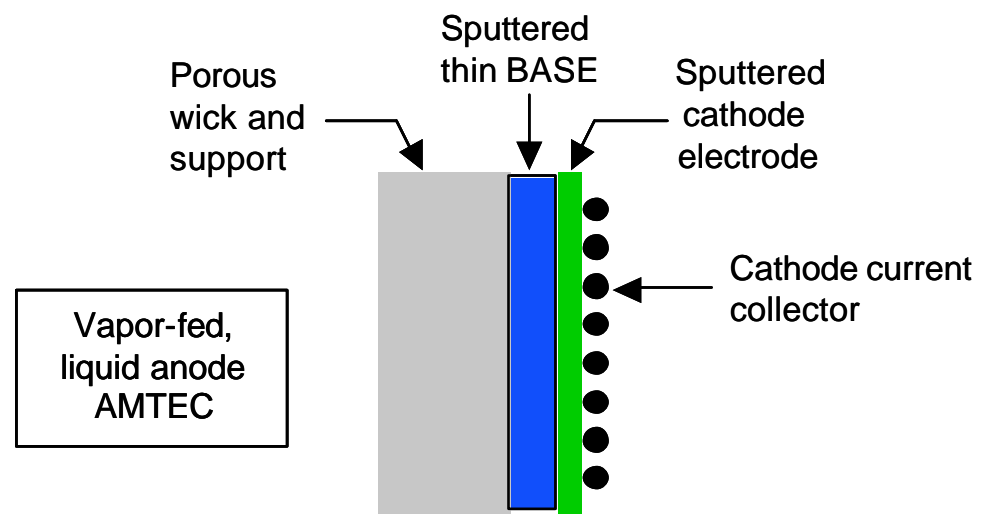

Figure 3-14. Close-up of a composite BASE/electrode.

Schematics of the narrow dome-shaped, composite BASE element developed for the reference vapor fed, liquid anode AMTEC unit design are shown in Figures 3.15 and 3.13. The composite BASE membrane is shaped as a dome that is much taller and longer than it is wide. The porous wick (metal) substrate is fused at the bottom to an anode metal strip shaped into a rubber band (or ribbon), that fits tightly into the open groove of the alumina support plate. The pressure seal in this configuration is obtained by a braze or the differential thermal expansion between the metal strip and the alumina plate, in conjunction with an adequate compression of the alumina plate provided by the AMTEC converter wall. The cathode wire mesh is held tightly against the cathode electrode by a strip-wire bus cage covering the dome (Figures 3.15 and 3.16). The strip wires (typically $1 \mathrm{~mm}$ thick) provide better electrical contact with the wire mesh collector and carry relatively high current ( 100 A for an electrode area of $400 \mathrm{~cm}^{2}$ per BASE element). Metal strip connectors are welded to the bus grid and the anode metal ribbon as shown in Figures 3.11 and 3.12 to electrically connect the BASE elements in the converter in series.

\subsubsection{Breakdown Voltage in Alkali Metal Vapor in Low- Pressure Cavity of AMTEC}

The electrical potential developing across a single BASE electrode is very small $(<0.7$ $\mathrm{V}$ ); however, a voltage output of a few tens to several hundred volts is desirable in order to minimize internal electrical losses and Joule heating, and, hence increase the efficiency of the converter. Such high terminal voltage is achieved in the present vapor anode, and in vapor-fed, liquid anode AMTEC unit design by connecting a multitude of BASE 
elements in series (Figure 3.11 to 3.13). However, the value of the terminal voltage of an AMTEC would depend on the electrical breakdown potential between the cathode

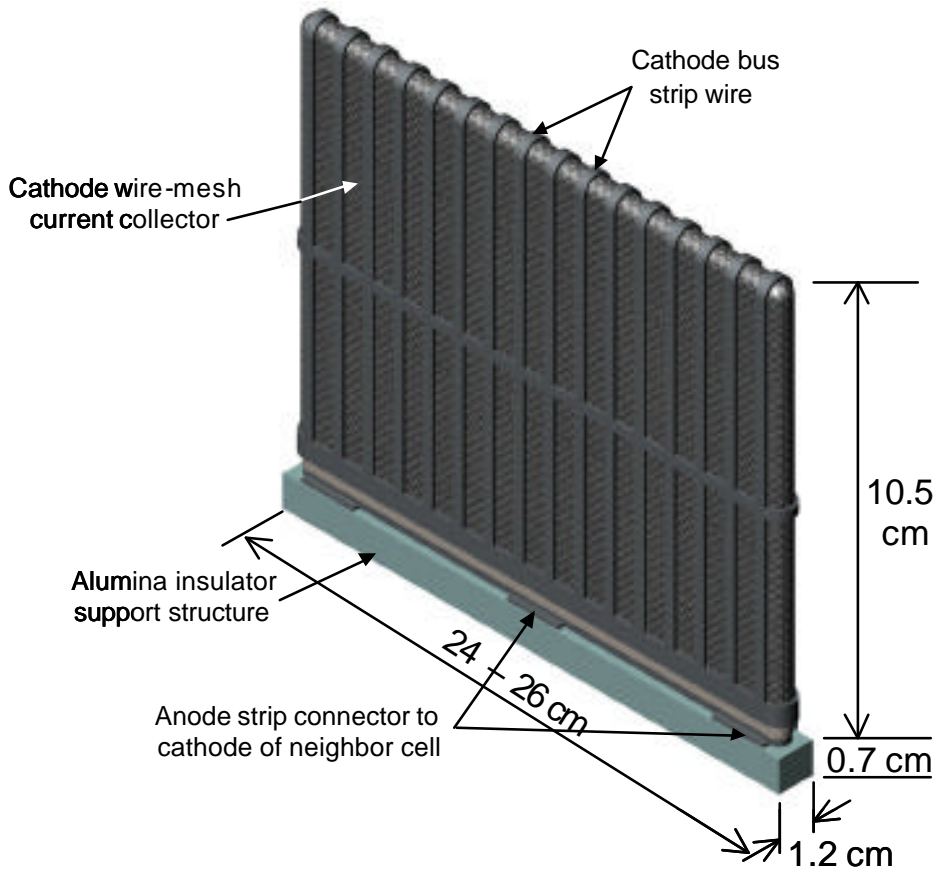

Figure 3-15. Isometric view of narrow dome-shaped, BASE element.

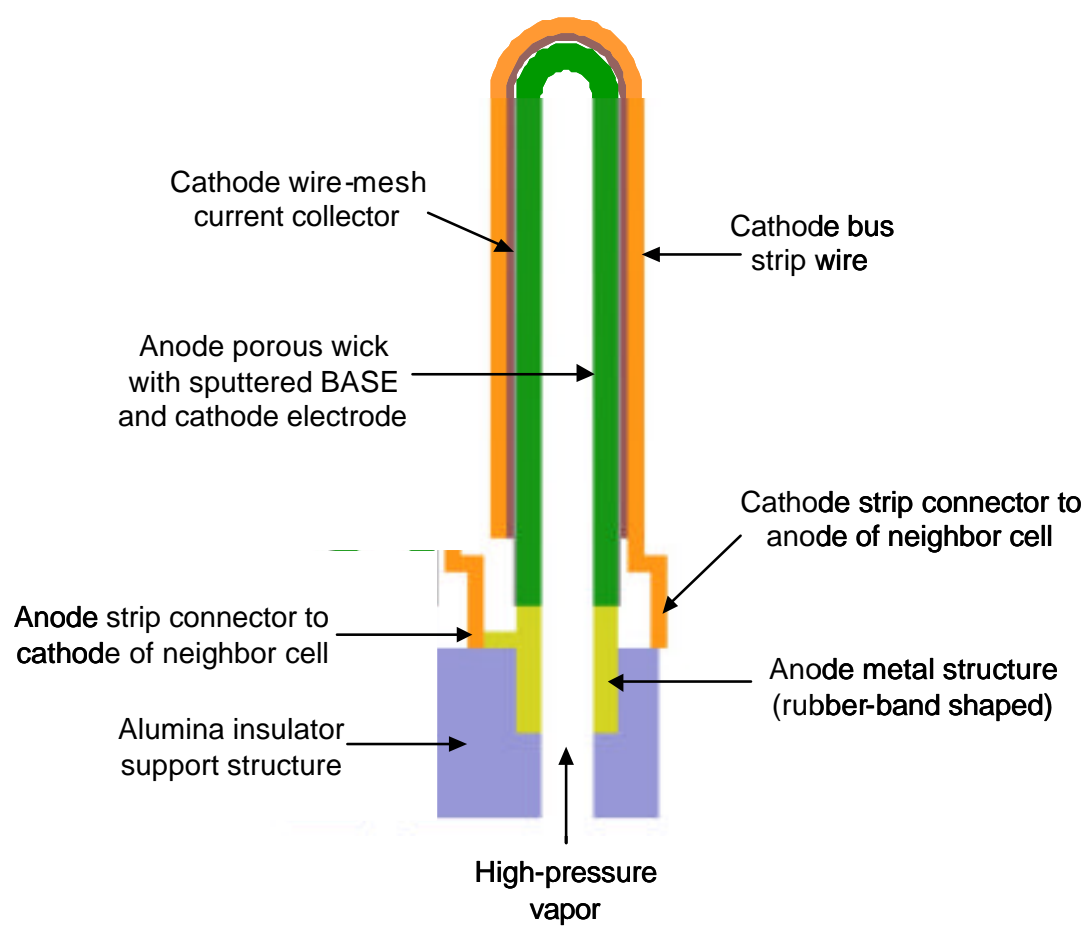

Figure 3-16. Vertical cross section of a narrow dome-shaped, composite BASE element. 
electrode with the highest voltage and the AMTEC wall, which is typically grounded. Therefore, the higher is the breakdown voltage in the low-pressure cavity of an AMTEC, the larger is the number of the BASE elements that could be connected in series, and, hence the terminal voltage. An electrical breakdown would increase the current leakage to the wall, hence decreasing the efficiency and the electrical power output of the converter, and could damage the BASE cathode electrodes.

An electrical breakdown is defined herein as an arc discharge resulting in a discharge current, typically $\geq 100 \mathrm{~mA} / \mathrm{cm}^{2}$. In addition to the temperatures of the cathode electrodes and the AMTEC wall, the breakdown voltage depends on the pressure of the alkali metal vapor in the low-pressure cavity and the polarity of the converter wall relative to the nearest cathode electrode. Previous work reported on DC electrical breakdown in alkali metal vapors is quite limited. A few investigations have been reported on the breakdown in cesium vapor (Lebedev and Gus'kov 1963, Gus'kov et al. $1965 \mathrm{a}$ and 1965b) and a few data have been found on DC electrical breakdown in sodium and potassium vapors in AMTECs, which are inconclusive (Williams et al. 1994, Barkan and Hunt 1998). Owing to the absence of useful data in the literature on the breakdown voltage in low-pressure (< $200 \mathrm{~Pa})$ alkali metal vapors, breakdown experiments were performed at the UNM-ISNPS energy conversion laboratory using existing capabilities. Results of these experiments were invaluable in determining a conservative value of the maximum design voltage in the AMTEC cell of $~ 400 \mathrm{~V}$ (Momozaki and El-Genk 2002). These experiments investigated the effect of the electrode temperatures, cesium vapor pressure, and the polarity of the electrodes on the breakdown voltage in cesium vapor. In addition, the applicability of the measurements to the design and operation of vapor anode, and vapor-fed, liquid anode AMTECs, which use either sodium or potassium working fluid, was studied. The present breakdown experiments used two planar molybdenum electrodes, $1.6 \mathrm{~cm}$ in diameter, separated by a $0.5 \mathrm{~mm}$ inter-electrode gap. During the measurements, one electrode was held at $1100 \mathrm{~K}$, while the other was kept at $625 \mathrm{~K}$, conditions typically encountered in the AMTEC. The cesium pressure was varied from 1.7 $\mathrm{Pa}$ to $235 \mathrm{~Pa}$. The discharge current and the breakdown voltage were measured, and their dependency on electrode temperatures, cesium pressure, and the polarity of the electrodes were obtained as well.

\subsubsection{Experimental Setup and Measurements}

The experiments measured the breakdown voltage in cesium vapor between a pair of planar, polycrystalline molybdenum electrodes, separated by a $0.5 \mathrm{~mm}$ gap. The electrodes are flat-end hollow cylinders. The top electrode was encased in a thin alumina guard tube, to limit the discharge to the planer surfaces of the electrodes, and electrically insulated from the rest of the test section using an alumina spacer (Figure 3.17). The planar surfaces of the electrodes are $2 \mathrm{~cm}^{2}$ in area and $16 \mathrm{~mm}$ in diameter. Both electrodes were fastened to the test section assembly, with the electrode gap and the surrounding cavity open to the cesium reservoir via a connecting tube. The cesium vapor pressure in the inter-electrode gap was adjusted by varying the temperature of the cesium reservoir and determined from the measured reservoir temperature.

The grounded electrode (bottom in Figure 3.17) is heated using a wound, helical tungsten 
filament, inserted in the bottom electrode cavity. A low voltage/high current AC power supply and a high voltage DC power supply were used to heat up the grounded electrode up to $1173 \mathrm{~K}$. During out-gassing the test chamber and the test section, prior to conducting the experiments, higher grounded electrode temperatures up to $1773 \mathrm{~K}$ were achieved using electron bombardment. In this heating mode, a large negative bias voltage was applied to the tungsten filament, which then functions as an electron gun. The top electrode is heated by radiation from the hot, grounded electrode. To control the temperature of the top electrode, a stainless steel, air-cooled cold finger, with an electric heater wound onto its outer surface, was inserted inside the cavity of the electrode. The temperatures of the two electrodes were measured using tungsten 5\%-rhenium $26 \%$ (type-C) thermocouples (TC), in contact with the inside of the electrodes' surfaces.

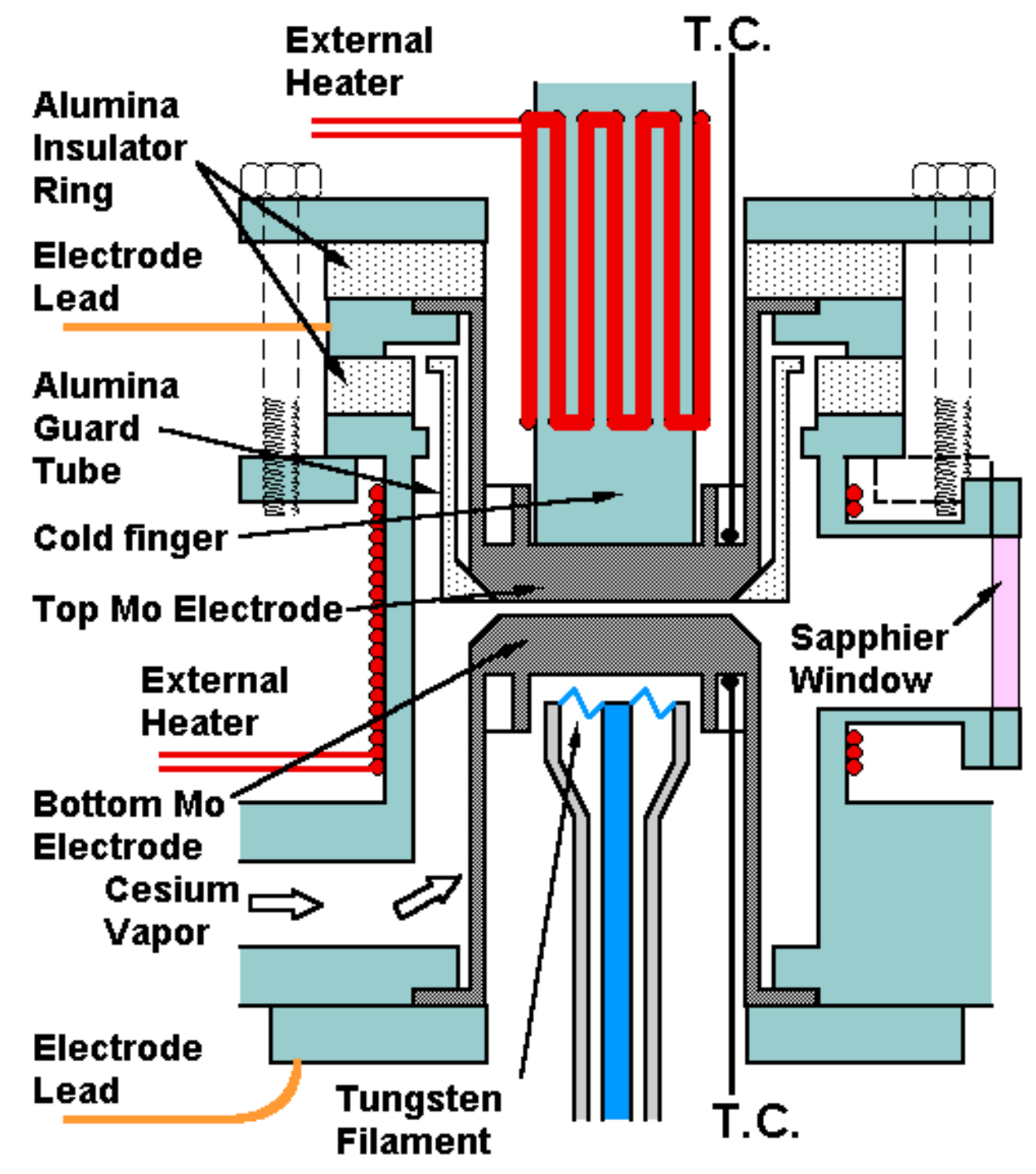

Figure 3-17. A cross-sectional view of the test section.

The test section was vacuum-sealed using four metal O-rings (Figure 3.17) and equipped with a sapphire viewport. The entire test section was placed inside a stainless 
steel vacuum chamber, and the viewport in the test section was aligned with that in the vacuum chamber to allow visual observation of the discharge from outside the chamber. The ion pump (Varian Star Cell VacIon pump, 230 liter/s) connected to the vacuum chamber creates a very high vacuum environment $\left(<10^{-6} \mathrm{~Pa}\right)$. Since it was not know priori how much voltage was needed to cause breakdown and how much current would be conducted through the inter-electrode gap when breakdown occurred, preliminary experiments were conducted to determine the applicable range of these parameters. In these experiments, a variable high voltage DC power supply capable of delivering up to $800 \mathrm{~V}$ was connected to the electrodes. In order to limit the current when arc discharge occurs, a $10.5 \mathrm{k} \Omega$ protective resistor was connected in series with the power supply (Figure 3.18). These experiments were conducted after all temperatures and cesium pressures reached steady state values. The applied voltage was increased manually, and the correspond ing values of the current were recorded. After determining the applicable range of the voltage and current for breakdown, a continuous measurement system for recording the voltage and the current in the experiments was assembled. The same variable high voltage DC power supply was used to apply high voltage, and a Tektronix TDS 420 digitizing oscilloscope was used for recording the values of the voltage and the current continuously with a voltage dividing circuit. This system of measurements was used in conjunction with the first set of experiments in which one electrode was held at $560 \mathrm{~K}$ and the other at $650 \mathrm{~K}$, while varying the cesium pressure from 0.71 to $29 \mathrm{~Pa}$.

In the second set of the experiments, performed at typical electrode and wall temperatures and vapor pressures in the low-pressure cavity of AMTECs, one electrode was held at $1100 \mathrm{~K}$ and the other at $625 \mathrm{~K}$, and the cesium pressure was varied from 1.7 to $235 \mathrm{~Pa}$. In these experiments, a variable, high voltage, $60 \mathrm{~Hz}$ AC power supply was used instead of the DC power supply used in the first set of experiments, in order to speed up the process of data collection (Figure 3.18). The time constants (or flight times through the inter-electrode gap) of the electrons and ions are much shorter than the rate of change in the applied voltage at $60 \mathrm{~Hz}$. Therefore, the voltage and current measurements are expected to emulate those taken in the first set of experiments at steady state conditions. A voltage sweep was carried out using one sinusoidal wave of the AC voltage, but in order to avoid the reverse bias voltage, a silicon diode was used to rectify the applied AC voltage (Figure 3.18).

The values of the applied voltage and corresponding electrical current were recorded using a Tektronix TDS 420 digitizing oscilloscope, synchronized with the AC power supply. Unfortunately, the AC power supply could only apply a maximum voltage of $396 \mathrm{~V}$ to the electrodes, compared to $800 \mathrm{~V}$ for the power supply used for the first set of experiments. The $396 \mathrm{~V}$ was assumed adequate for causing an electrical breakdown based on the measurements made in the first set of experiments. This assumption was found later not to be true.

\subsubsection{Experiments Conduct and Procedures}

Prior to the experiments, the entire test section, the vacuum chamber, and the cesium supply system were baked to facilitate out-gassing. The bottom electrode and the top electrode were baked at $\sim 1800 \mathrm{~K}$ and $1000 \mathrm{~K}$, respectively. After out-gassing was 
completed, both electrodes were kept at $\sim 600 \mathrm{~K}$, to stimulate Cs adsorption onto their surfaces, while keeping the Cs vapor pressure at $\sim 10 \mathrm{~Pa}$. After Cs adsorption onto the electrodes was completed, which typically takes several hours, the temperatures of the electrodes were raised to and held almost steady at the desired values in the experiment, while changing the temperature of the Cs reservoir to adjust the Cs vapor pressure in the inter-electrode gap. After the temperatures of the electrodes and the Cs reservoir reached steady values, the I-V curve was constructed based on the measured values of the applied voltage and current.

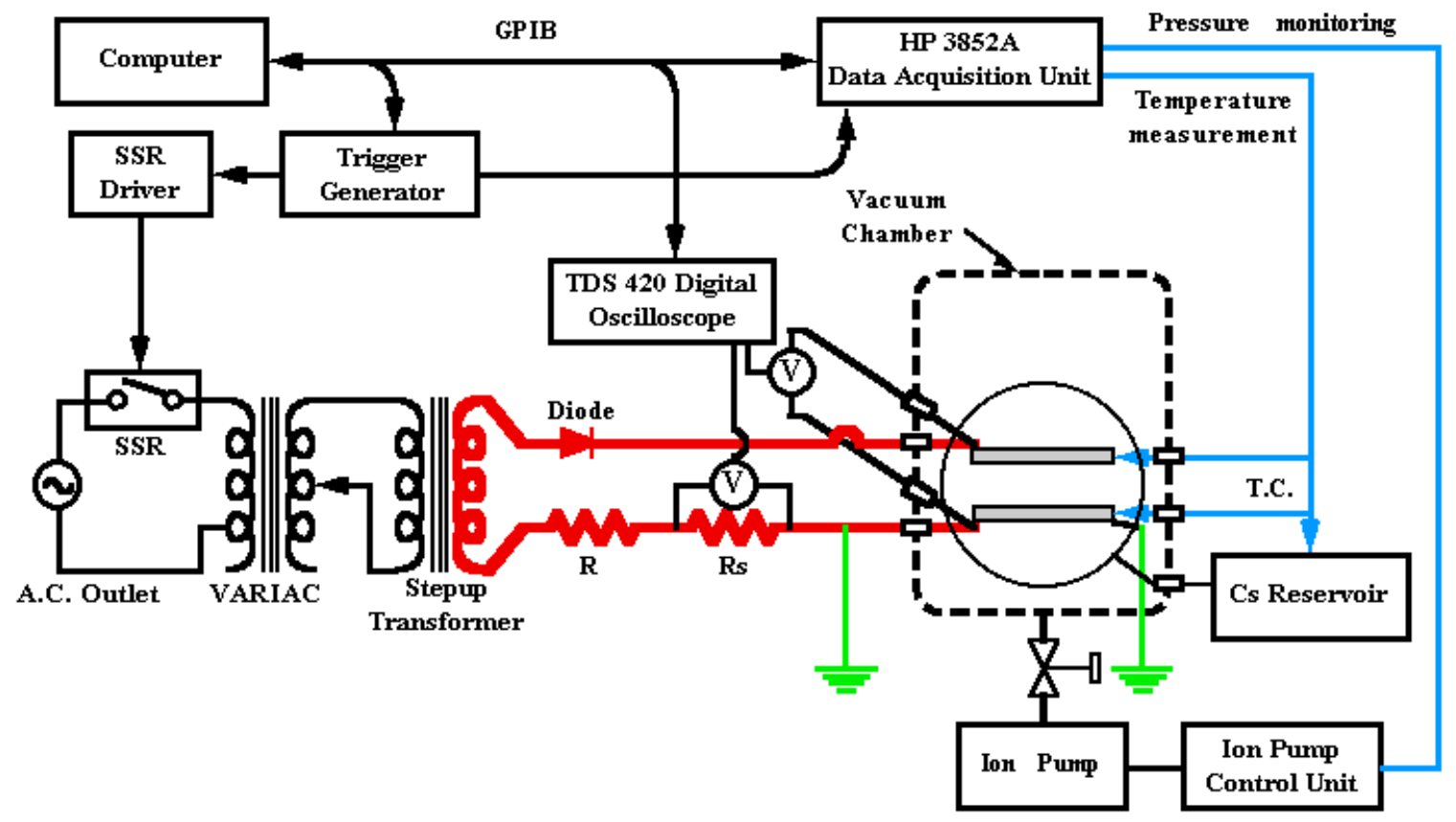

Figure 3-18. A schematic diagram of the control and measurement circuit.

When the measurements sequence began, a transistor-to-transistor evel (TTL) signal of $5 \mathrm{~V}$ was applied to a solid-state relay driver, which closed a solid-state relay (SSR) for $\sim 15-20 \mathrm{~ms}$, corresponding to a full sinusoidal wave at $60 \mathrm{~Hz}$. The TTL signal was simultaneously sent to the data acquisition system, a HP 3497A, to record the electrode and the cesium reservoir temperatures. While the SSR was closed, a high voltage sinusoidal wave was generated which was sent through the diode to be rectified. When rectified, only the positive (or the negative, depending on the direction of the diode) portion of the sinusoidal wave was applied to the electrodes. The applied voltage and the current flowing through the inter-electrode gap were recorded by a TDS 420 digitizing oscilloscope for a duration of $20 \mathrm{~ms}$ at a typical resolution of 25 data points per millisecond $(25 \mathrm{kHz})$. The measurement results are discussed next.

\subsubsection{Results and Discussion}

The measured current in the experiments as a function of the applied voltage is the sum of two component: (a) the discharge current through the cesium vapor in the inter- 
electrode gap, and (b) the leakage current through the cesium deposits at the inner surface of the alumina insulation ring (Figure 3.17). Thus, in order to determine the current voltage curves from the experimental measurement, each component of the measured current were calculated (El-Genk, Tournier and Momozaki 2001).
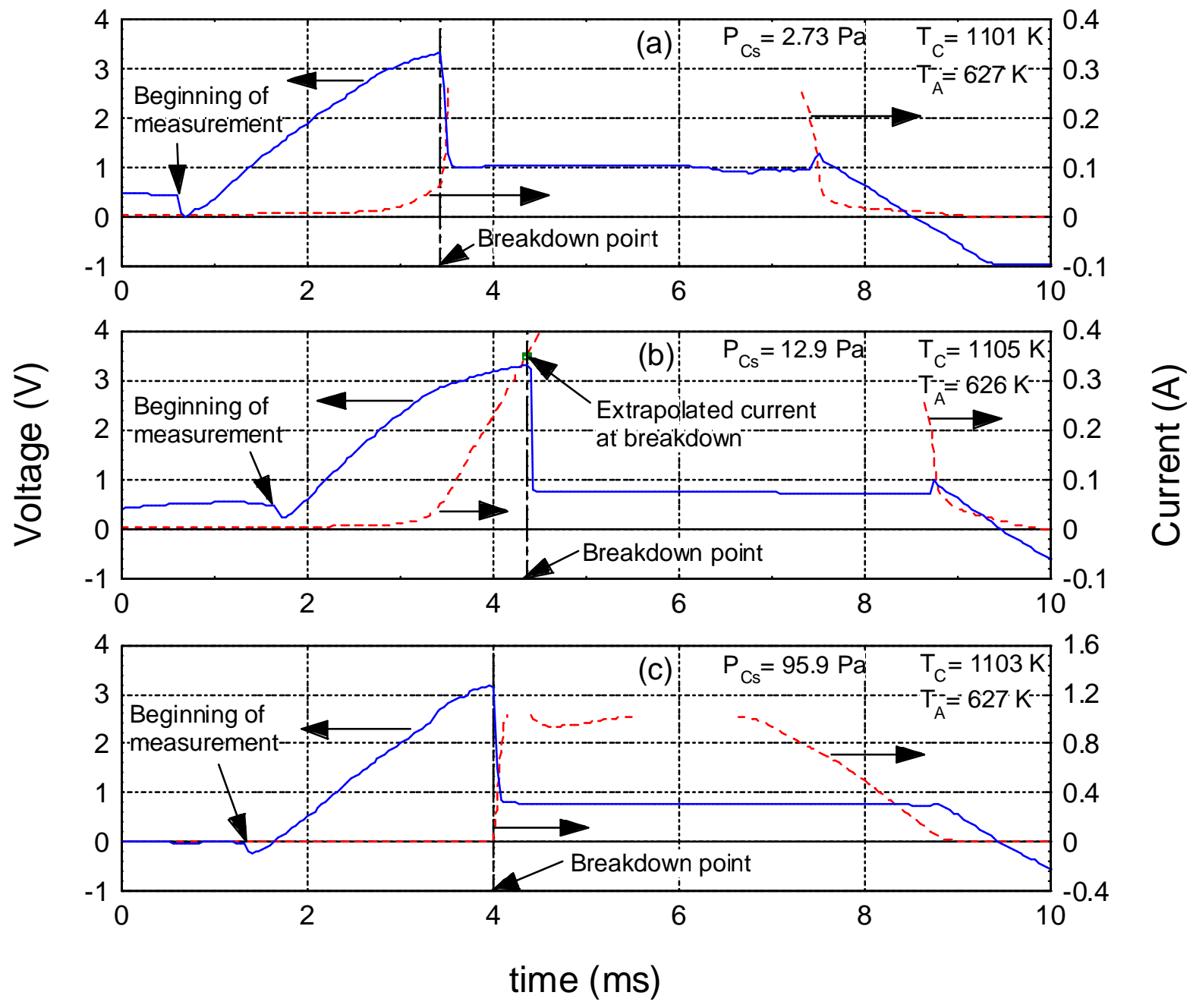

Figure 3-19. Examples of the histories of the current and voltage measurements.

The second set of experiments was performed at electrode and wall temperatures typically encountered in the low-pressure cavity of the AMTEC. In these experiments, one electrode was held at $1100 \mathrm{~K}$ and the other was kept at $620 \mathrm{~K}$, and the cesium pressure was varied from 1.7 to $235 \mathrm{~Pa}$. The AC power supply used in the higher cesium pressure experiments greatly reduced the electrical stress in the device as a result of shortening duration of the actual measurements. However, at $60 \mathrm{~Hz}$., the background noise could be a serious problem, especially in the discharge current measurements. The background noise in the voltage measurements of $\sim 10 \mathrm{mV}$ was very small compared to the values of the applied voltage, but that in the current measurements was comparable to the value of the current before breakdown. Therefore, prior to constructing the FV 
curves, the background noise in the current measurements and the leakage current were subtracted from the raw data to obtain the actual measured current.

Results indicated that when the cathode was the hotter electrode, electrical breakdown occurred at $\sim 2-5 \mathrm{~V}$, depending on the value of the cesium pressure in the inter-electrode gap. Conversely, when the cathode was the cooler electrode, electrical breakdown did not occur when the voltage limit of the power supply in the experiments was reached $(396 \mathrm{~V})$, suggesting that the breakdown voltage is $>396 \mathrm{~V}$. The electrical breakdown in the experiments was indicated by a sudden drop in the applied voltage and simultaneous increase in the measured current (Figures 3.19a-3.19c). These figures also show that increasing the cesium pressure slightly decreased the breakdown voltage (from $\sim 3.3$ at $2.73 \mathrm{~Pa}$ to $\sim 3.2$ at $95.9 \mathrm{~Pa}$ ). The relative changes in the measured current at breakdown, however, were significant; increasing from $64.4 \mathrm{~mA}$ at $2.73 \mathrm{~Pa}$ to as much as $348 \mathrm{~mA}$ at $12.9 \mathrm{~Pa}$ and decreasing to $7.09 \mathrm{~mA}$ at $95.9 \mathrm{~Pa}$.

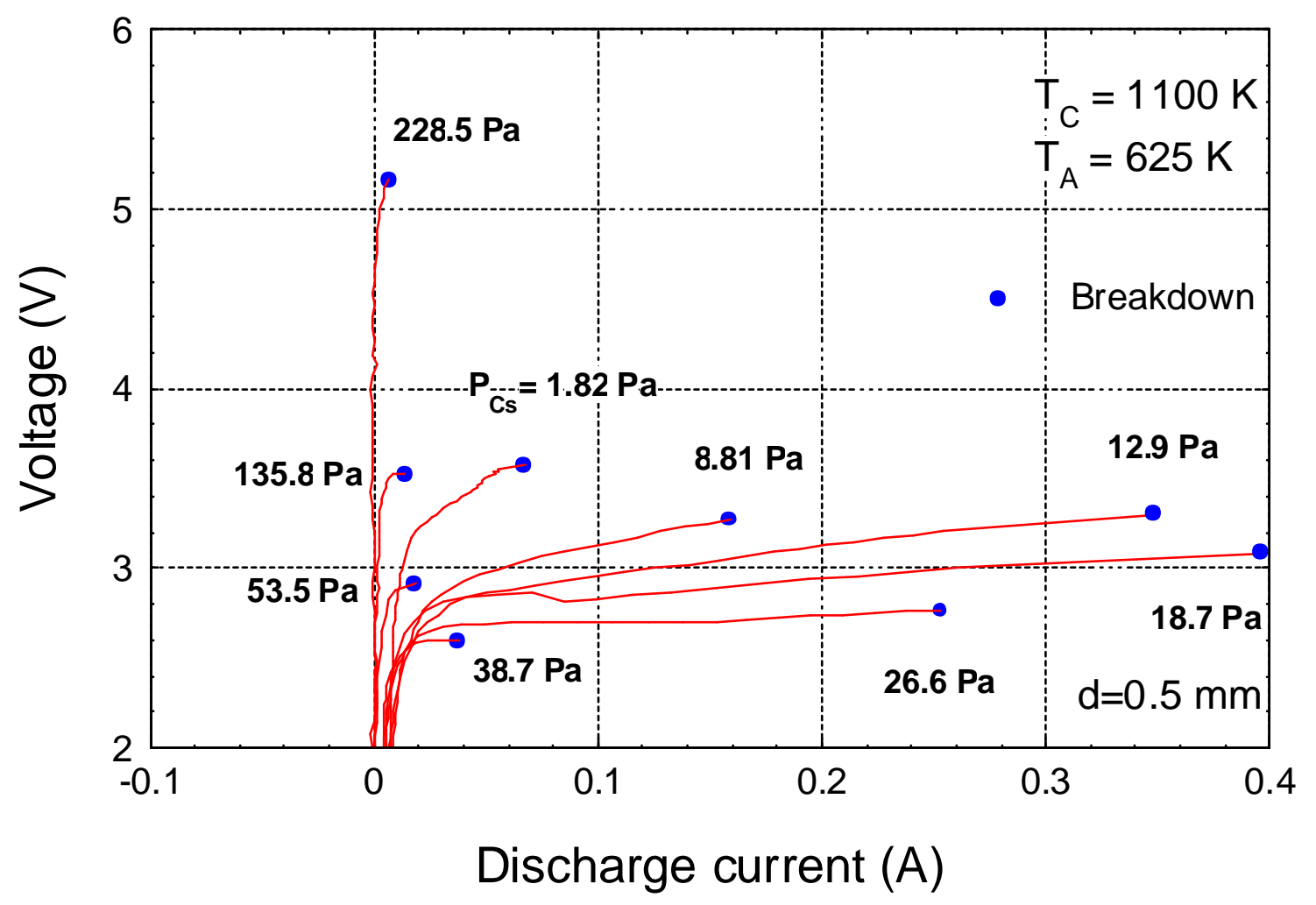

Figure 3-20. Effect of cesium pressure on I-V curves and breakdown voltage.

The obtained I-V curves, based on the measurements of the applied voltage and discharge current are delineated in Figure 3.17, for when the cathode was the hotter electrode. This figure indicates that when the cathode was the hotter electrode, increasing the cesium pressure shifted the $\mathrm{FV}$ curves to the right (higher discharge current and lower breakdown voltage) up to a cesium pressure of $18.7 \mathrm{~Pa}$. Beyond this value, further increases in the cesium pressure shifted the I-V curves to the left (lower discharge current and higher breakdown voltage). 
The breakdown voltage and the corresponding discharge current changed from $3.57 \mathrm{~V}$ and $67.2 \mathrm{~mA}$ at $P_{\mathrm{Cs}}=1.82 \mathrm{~Pa}$ to $3.09 \mathrm{~V}$ and $396 \mathrm{~mA}$ at $18.7 \mathrm{~Pa}$, then decreased gradually to $5.16 \mathrm{~V}$ and $6.73 \mathrm{~mA}$ at $228.5 \mathrm{~Pa}$. At a cesium pressure of $\sim 40 \mathrm{~Pa}$, the breakdown voltage reached a minimum of $\sim 2.6 \mathrm{~V}$, at which the discharge current was < $\sim 30 \mathrm{~mA}$. When the anode was the hotter electrode, the analysis of the data indicated that the measured current was almost entirely due to the leakage current, except when $P_{\mathrm{Cs}}<$ $20 \mathrm{~Pa}$. Therefore, it was not possible to quantify the discharge current in this case.

Figure 3.21 presents the estimated discharge current through the cesium vapor gap and the leakage current along the inner surface of the alumina insulation ring, separating the two electrodes (Figure 3.17), at breakdown as function of the cesium pressure.

As shown in Figures 3.20 and 3.21, at low cesium pressure $(<20 \mathrm{~Pa})$, the discharge current at electrical breakdown increased as the cesium vapor conductivity increased proportional to the square root of the cesium pressure, indicating that the measured current at breakdown was mostly the discharge current and the leakage current was insignificant. As delineated in Figure 3.19, doubling the cesium pressure from $\sim 10$ to $20 \mathrm{~Pa}$ increased the discharge current by more than $40 \%$.

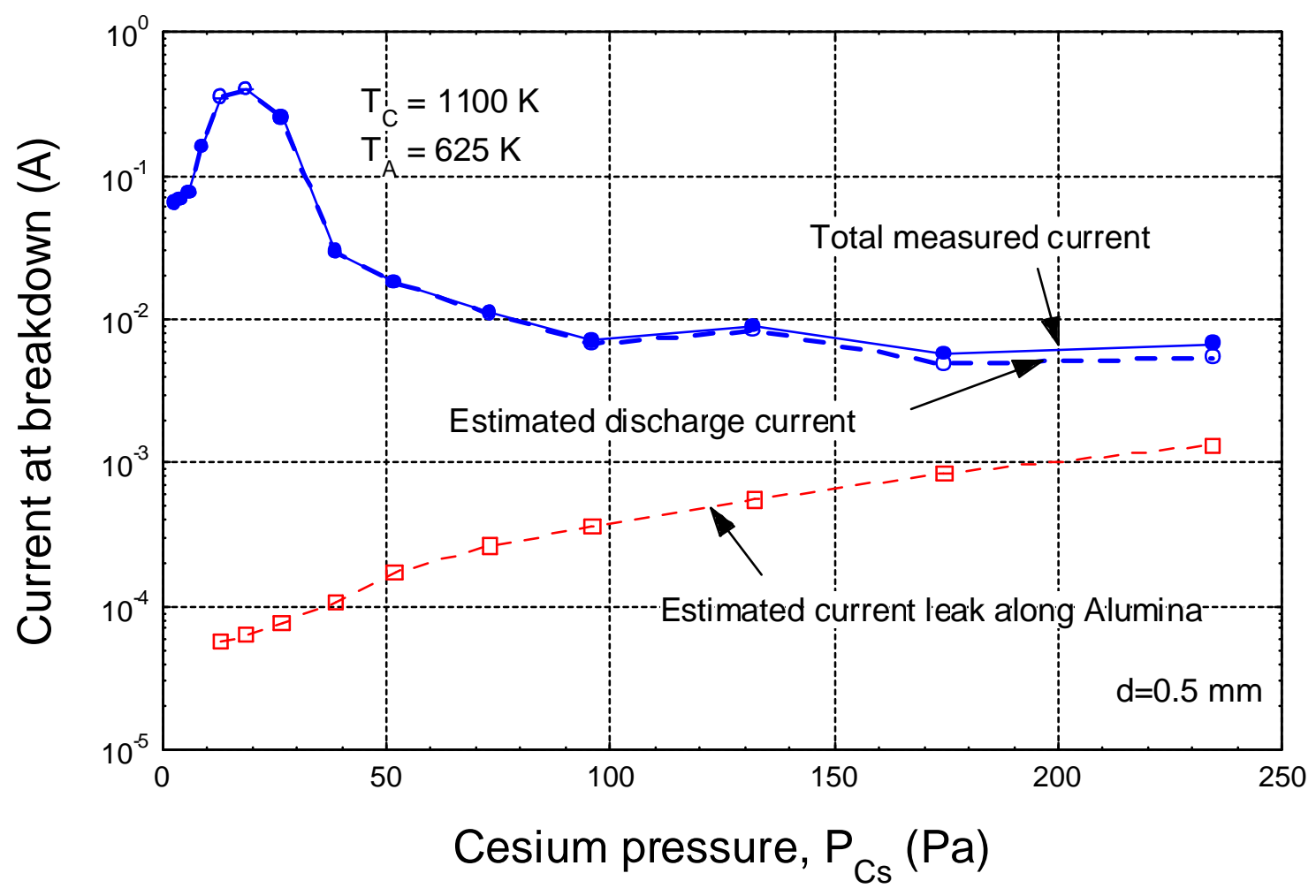

Figure 3-21. Estimated discharge and leakage currents

As the cesium pressure increased beyond $20 \mathrm{~Pa}$, the fraction coverage of the inner surface of the alumina insulation increased, increasing the leakage conductivity but decreasing the conductivity of the cesium vapor, thus causing the total and discharge 
currents at breakdown to decrease, but the leakage current to increase. As indicated in Figure 3.21, increasing the cesium pressure from 20 to $200 \mathrm{~Pa}$ decreased the discharge current at breakdown from $\sim 0.4 \mathrm{~A}$ to as little as $\sim 6.0 \mathrm{~mA}$. Conversely, for the same change in the cesium pressure, the leakage current increased by more than an order of magnitude, from $80 \mu \mathrm{A}$ at $20 \mathrm{~Pa}$ to $\sim 1.0 \mathrm{~mA}$ at $200 \mathrm{~Pa}$.

Figure 3.22 plots the measured values of the breakdown voltage and the estimated discharge current versus the cesium pressure in the experiments in which the cathode temperature was $1100 \mathrm{~K}$ and the anode temperature was $625 \mathrm{~K}$. The breakdown voltage data follow a Pachen-type curve, decreasing from slightly below the first ionization potential of cesium $(3.89 \mathrm{~V})$ at $1.1 \mathrm{~Pa}$ to a minimum of $\sim 2.6 \mathrm{~V}$ at $40 \mathrm{~Pa}$, then increasing almost linearly with the cesium pressure. The highest breakdown voltage measured was $5.2 \mathrm{~V}$ at a cesium pressure of $235 \mathrm{~Pa}$. Conversely, the discharge current at breakdown increased from $\sim 60 \mathrm{~mA}$ at $\mathrm{P}_{\mathrm{Cs}}<5.9 \mathrm{~Pa}$ to a maximum of $\sim 400 \mathrm{~mA}$ at $18.7 \mathrm{~Pa}$, then decreased rapidly with cesium pressure to $\sim 30 \mathrm{~mA}$ at $38.7 \mathrm{~Pa}$. Beyond this cesium pressure, the discharge current at breakdown continued to decrease, but at a much slower rate, as the cesium pressure decreased, reaching $<1.0 \mathrm{~mA}$ at $228.5 \mathrm{~Pa}$.

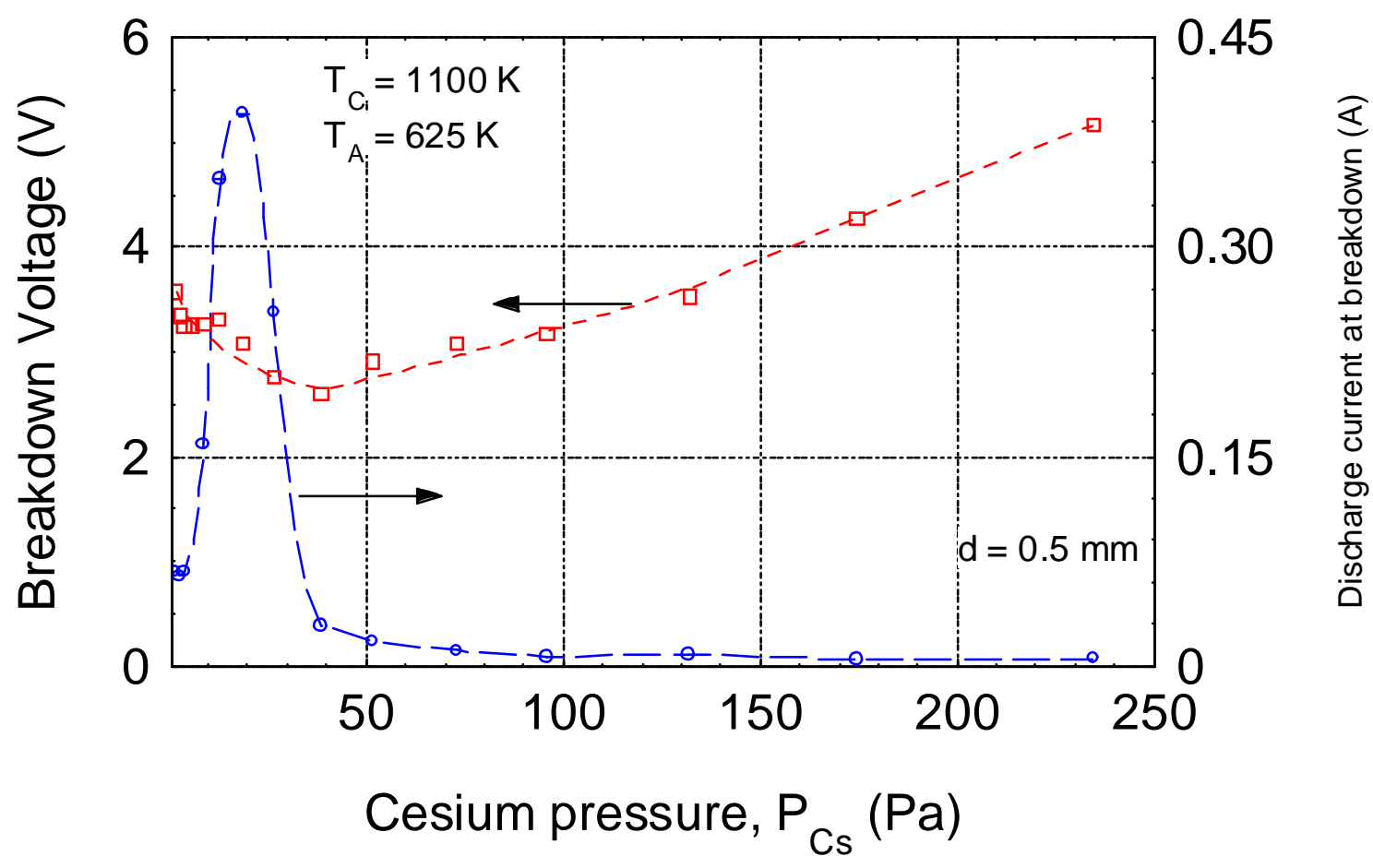

Figure 3-22. Measured breakdown voltage and discharge current as function of cesium pressure.

In summary, the experiments conducted at electrodes temperatures of $1100 \mathrm{~K}$ and $625 \mathrm{~K}$ have shown clearly that when the cooler electrode was regatively biased the breakdown voltage was beyond the limit of the power supply used (> $396 \mathrm{~V}$ ). In addition to the potentially very high breakdown voltage $(>400 \mathrm{~V})$, the corresponding discharge current was quite small $(<100 \mathrm{~mA})$. Conversely, when the cooler electrode 
was positively biased the breakdown voltage was in the single figure and followed a Pachen-type dependence on the cesium pressure. For the typical vapor pressures in the low-pressure cavity of an AMTEC of 20-60 Pa, the measured breakdown voltage in the cesium vapor could be between $2.8 \mathrm{~V}$ and $2.6 \mathrm{~V}$. These values are more than $30 \%$ lower than the first ionization potential of the cesium $(3.89 \mathrm{~V})$. The corresponding discharge currents at these values of the breakdown voltage were relatively high $\sim 200 \mathrm{~mA}$.

At the conditions in the experiments, measurements showed that when the hot electrode is negatively biased, the breakdown voltage is almost the same as the first ionization potential of cesium $(\sim 4 \mathrm{~V})$. However, when the cold electrode is negatively biased, no breakdown was observed up to $396 \mathrm{~V}$, the limit of the power supply used, suggesting that the breakdown voltage in this case would be $>400 \mathrm{~V}$.

\subsubsection{Application to Sodium and Potassium Vapors in AMTECs}

Since at the point of electrical breakdown the discharge is sustained by the volume ionization caused by the electron emission from the cathode, the breakdown voltage decreases when the electron emission and the volume ionization increase (Lieberman and Lichtenberg 1994). It is known that the effective work function of metals decreases greatly in an alkali metal vapor environment, due to the adsorption of alkali metal atoms onto the surface of the metal. Fehrs and Stickney (1971) have measured the reduction in the work function of tantalum in $\mathrm{Na}, \mathrm{K}$ and $\mathrm{Cs}$ vapors (Figure 3.23). The measurements shown in this figure clearly indicate that the effective work function of tantalum in the Cs vapor is the lowest, followed by that in potassium, then that in sodium vapor. The minimum in the work function occurred at a surface coverage of 0.6. Therefore, it may be argued that for the same electrode material and surface coverage, the electron emission from the electrode surface is the highest when in cesium vapor, followed by potassium vapor, then sodium vapor.

In addition, owing to the fact that the first ionization potential of cesium is the lowest among all three alkali metals, the volume ionization for $\mathrm{Cs}$ is the highest of all these alkali metal vapors at the same conditions. Zapesochnyi and Aleksakhin (1969) experimentally obtained the slopes of the ionization cross-section at the ionization threshold for $\mathrm{Na}, \mathrm{K}$ and $\mathrm{Cs}$, which were used to calculate the ionization rate constants from the ground state as a function of electron temperature (Figure 3.24). This figure clearly shows that cesium has the highest $(\mathrm{y}>$ one order of magnitude) ionization rate constant, indicating that Cs vapor would be the easiest to form the discharge, followed by potassium, then sodium. Based on the results presented in Figures 3.23 and 3.24, it is clear that the effective work function of the metal electrodes in the Cs vapor is lowest and the ionization rate constant for $\mathrm{Cs}$ is the highest, compared with those for potassium and sodium. Therefore, it may be argued that for the same electrodes temperatures and polarity and the same vapor pressures, the breakdown voltages in potassium and sodium vapors in the low pressure cavity of an AMTEC would be at least the same, but most likely higher than those reported in this work for cesium vapor. 


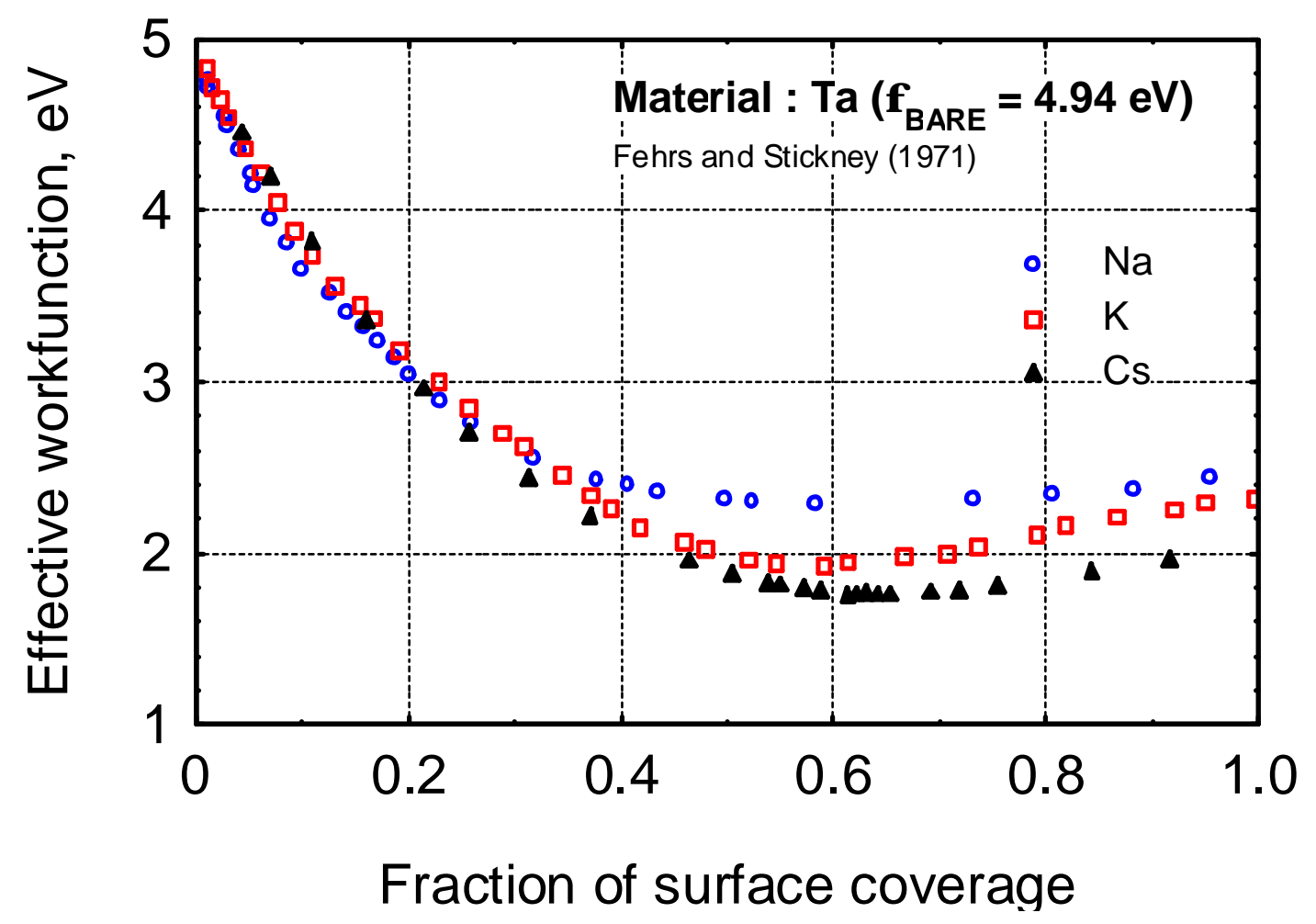

Figure 3-23. Effective work functions of tantalum in sodium, potassium and cesium vapors.

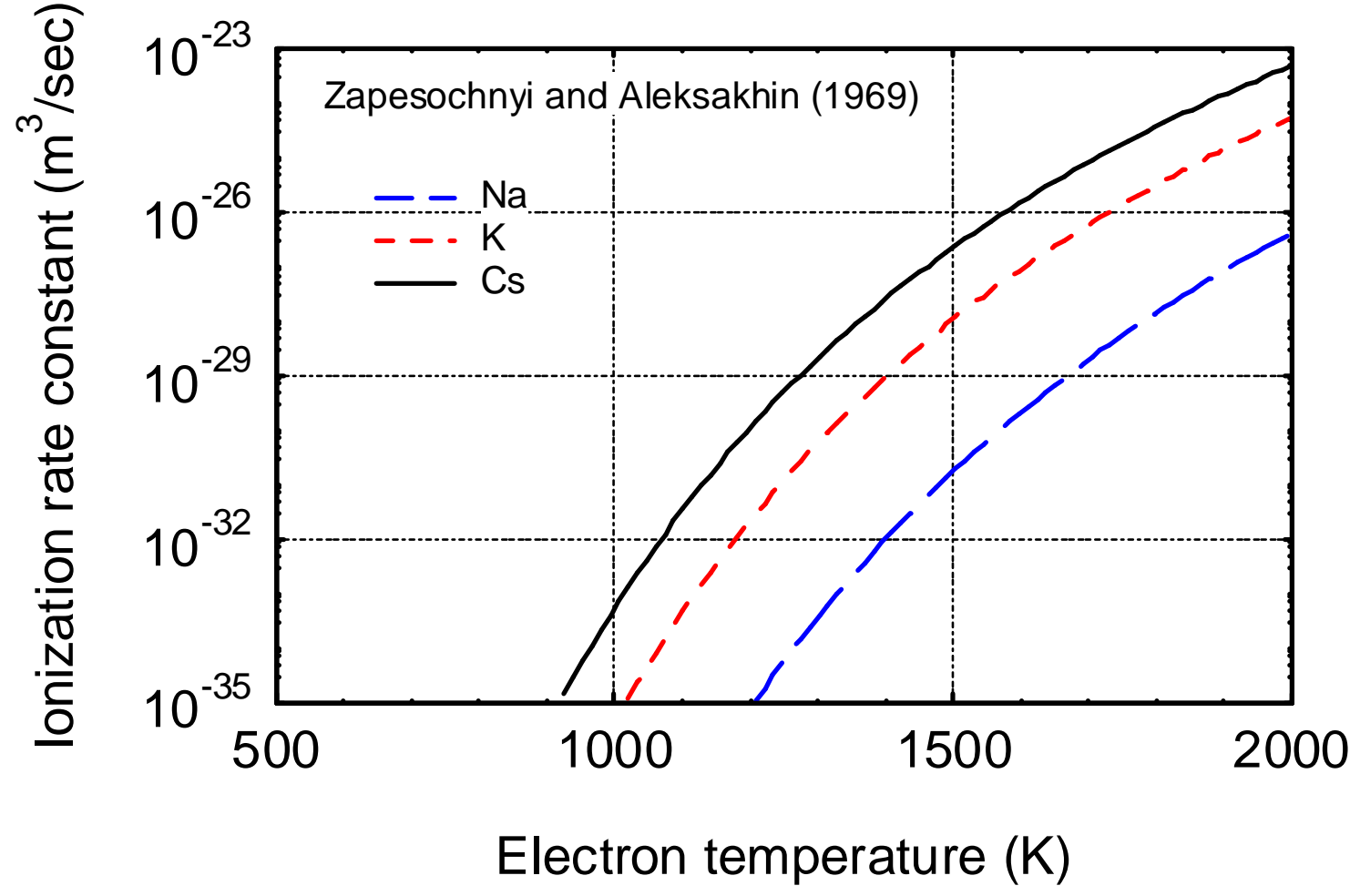

Figure 3-24. Ionization rate constants for sodium, potassium and cesium. 
Since cesium has the lowest first ionization potential of all alkali metals $(3.89 \mathrm{~V}$ versus $5.14 \mathrm{~V}$ for sodium and $4.34 \mathrm{~V}$ for potassium), the present experimental values of the breakdown voltage are conservative when applied to the breakdown in either sodium or potassium vapor, which are considered for use in the present AMTEC converters.

The present results suggest that when the AMTEC condenser wall, $~ 500 \mathrm{~K}$ cooler than the cathode electrodes $(\sim 1100 \mathrm{~K})$, is negatively biased, the breakdown voltage in the low-pressure cavity of the converter $(\sim 20-60 \mathrm{~Pa})$ could be in excess of $400 \mathrm{~V}$. Conversely, if the converter wall is positively biased relative to the nearest cathode electrode, the breakdown voltage in the low-pressure cavity could be $<5 \mathrm{~V}$ for both potassium and sodium working fluids. A DC electrical breakdown in the low-pressure cavity of an AMTEC would increase the leakage current to the wall, hence degrading the performance of the converter, in addition to potentially damaging the cathode electrodes and the converter wall. Based on the results of these breakdown experiments, The present AMTEC/TE converters were designed to deliver more than $50 \mathrm{kWe}$ each at a voltage output of $\sim 400 \mathrm{~V}$ and an overall conversion efficiency $>30 \%$.

\subsubsection{Design of Thermoelectric Bottom Cycle}

A thermoelectric (TE) bottom cycle is thermally coupled to the AMTEC top cycle to increase the electric power output and the conversion efficiency of the LMR-AMTEC electrical power plant. The TE modules are conductively coupled to the condenser wall of the AMTEC unit. The electric power output from the AMTEC units is fed to the grid or the end user separately from that generated by the TE modules, due to the difference in the values of the terminal voltage and current. Thermoelectric (TE) generators are static, semiconductor devices, which develop an electrical potential at the junction of two dissimilar semiconductor materials, typically the $\mathrm{N}$ - and P-doped semiconductors of the same base material, to counter the effect of the applied temperature differential. Therefore, the electrical potential developing across a TE unicouple is proportional to the applied temperature differential across. The proportionality coefficient, known as the material's Seebeck coefficient, is temperature dependent. The TE unicouples are electrically connected in series and in parallel to bring the voltage output to a usable value, while ensuring proper redundancy in the design. In the present AMTEC/TE converter design, a multitude of TE unicouples operate between the condenser temperature of the AMTEC unit and the temperature of the external radiator housing of the AMTEC/TE converter. A typical AMTEC unit that uses sodium working fluid may operate at a condenser temperature between 590 and $650 \mathrm{~K}$, while an AMTEC unit with potassium working fluid may operate at a condenser temperature between 510 and $570 \mathrm{~K}$. 
Both AMTEC units could provide a conversion efficiency of heat to electricity in excess of $25 \%$ when operating at an anode vapor pressure of $\sim 80 \mathrm{kPa}$.

The heat given up by condensation of the low-pressure vapor in the AMTEC is conducted at a uniform temperature to the multitude of TE unicouples in the bottom cycle. To ensure a uniform cold side temperature of the unicouples and for efficient operation and low converter weight, a metallic heat pipe spreader and cooling fins are used (Figure 3.25). They are charged with water working fluid, to reject the waste heat from the TE bottom cycle efficiently. The fins are cooled by natural convection of air for space or district heating. The heat rejected at the cold end of the TE legs would evaporate the working fluid in the housing wick against the two side (condenser) walls of the converter vessel (Figure 3.11). The resulting vapor would condense on the inside of the hollow fins, and be recirculated back through the porous wick to the bottom of the fins, with help from gravity, and then wicked back to the vertical wick surfaces facing the cold end of the TEs, by capillary action (Figure 3.12).

An illustration of the TE modules and the heat rejection housing of the converter is shown in Figure 3.25. The TE bottom cycle is comprised of alternating P- and N-legs that are electrically insulated from each other. The electrical insulator material is also a thermal insulator, to ensure that most of the heat supplied to the hot plate of the TE legs at the condenser wall is conducted through the legs. The TE legs are electrically connected in series and parallel using two electrical circuit boards made of checkerboard patterns of electrical conductor and insulator tabs, in direct contact with the hot side and cold sides of the TE legs (Figure 3.25). Each electrical circuit board is topped with a thin electrical insulator plate (Figure 3.25). The materials of the electrical insulator plates and of the insulator tabs of the circuit boards should be good thermal conductors, to reduce the temperature drops across these layers and maximize the temperature drop across the TE legs. To relieve the stresses caused by the differential thermal expansion between these different materials, a compliant pad is placed against one of the electrical insulator plates (Figure 3.25) and sandwiched by another electrical insulator plate if necessary (Truscello and Rutger 1992). Because of the different voltage and current provided by the AMTEC and the TE generators, the electrical outputs of these two cycles are preferably conditioned separately. With proper design, sizing, and optimization of the AMTEC/TE converter units, the temperature difference across the TE legs in the bottom cycle could be as much as $180 \pm 50 \mathrm{~K}$, depending on the ambient air temperature and the cooling method. At such temperature difference, lead telluride TE unicouples provide a net increase in the overall conversion efficiency of the AMTEC/TE converter unit by up to 5 percentage points. 


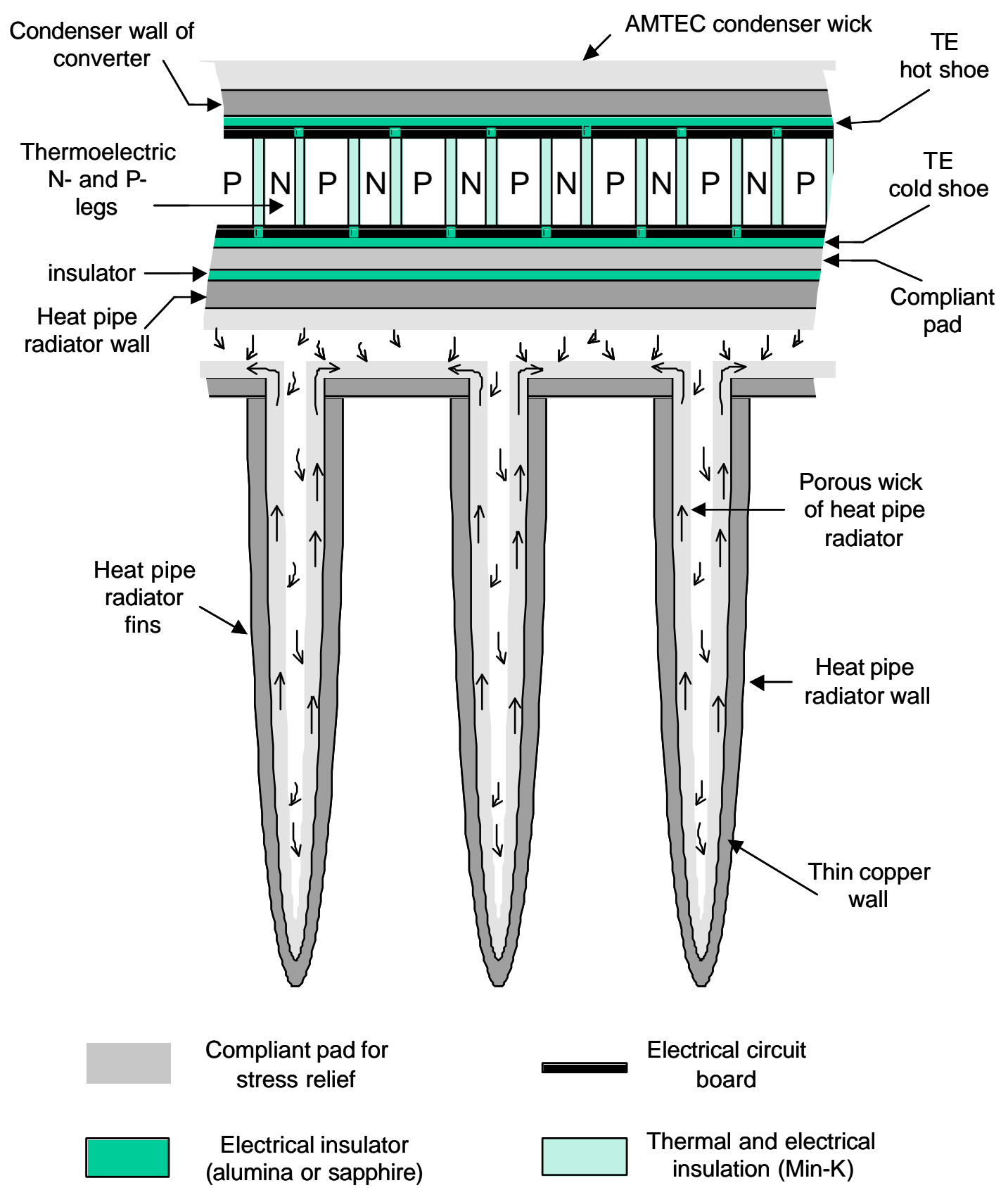

Figure 3-25. Cross-section of bottom cycle and heat pipe radiator (Section $\mathrm{C}-\mathrm{C}$ in Figure 3.11). 


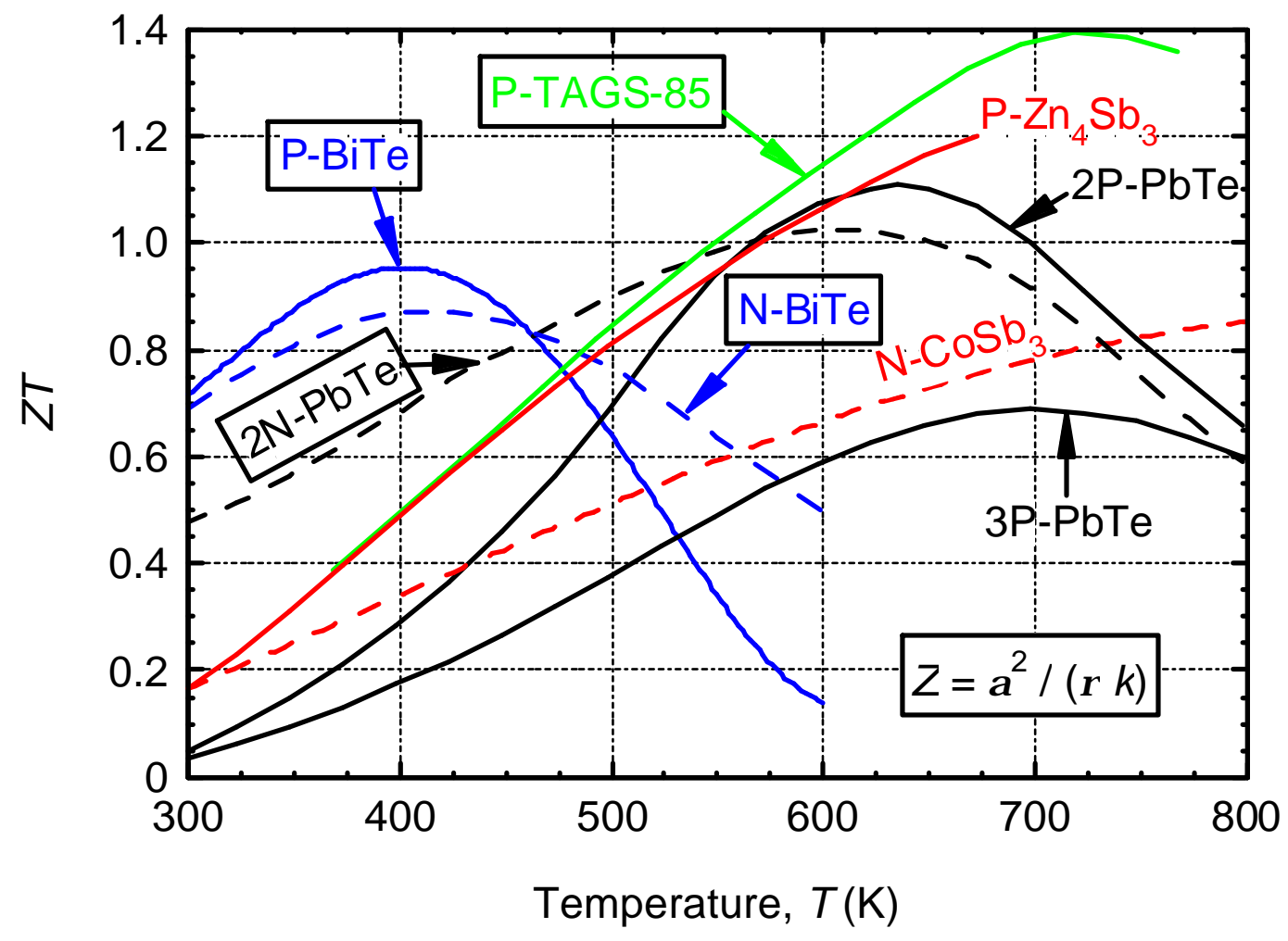

Figure 3-26. Figure-Of-Merit (FOM) of some thermoelectric materials of interest.

The present AMTEC/TE converter design provides high-voltage electricity at an efficiency of converting heat to electricity that is in the low thirty percentage points. These static AMTEC/TE converter units are an excellent option for terrestrial electric power generation using nuclear reactor heat sources, particularly in arid areas, with little or no water, on board a ship, or in auxiliary electric power sources. The present AMTEC/TE converters could also be used in dualpurpose (co-generation) plants for the production of electricity and the desalination of seawater.

\subsubsection{Selection of ThermoElectric Materials for Bottom Cycle}

The AMTEC condenser temperature (also the hot side temperature of the TE couples in the bottom cycle) for optimum overall efficiency of the AMTEC/TE converter unit is in the range $590-650 \mathrm{~K}$ for sodium, and in the range 510-570 K for potassium converters. The cold side temperature of the TE couples, also the AMTEC/TE heat rejection temperature to the ambient air, is 50 to $100 \mathrm{~K}$ above the ambient air temperature (that is in the range $350-400 \mathrm{~K}$ ) or even lower, depending on the location of the power plant and the seasonal ambient temperature. As a result, the temperature drop across the TE modules is in the range $140-240 \mathrm{~K}$. For these hot and cold side temperatures $\left(T_{\mathrm{h}} \leq 650\right.$ 
$\mathrm{K}$ and $T_{\mathrm{c}} \geq 350 \mathrm{~K}$ ), the following TE materials with the highest figure-of-merit were identified: P-BiTe, P-TAGS-85, 2P-PbTe and $\mathrm{P}_{2} \mathrm{Zn}_{4} \mathrm{Sb}_{3}$ for the P-leg, and $\mathrm{N}-\mathrm{BiTe}$ and $2 \mathrm{~N}-\mathrm{PbTe}$ for the N-leg (Figure 3.26). Lead telluride has one of the highest Figure-ofMerit of all TE materials available (Figure 3.26), and is the thermoelectric material of choice (Fritts 1959, Mayer and Ritchie 1961, Rowe 1995). Lead telluride TE couples have been used extensively in many industrial and medical applications (Rowe 1995) as well as in space power systems (Angelo and Buden 1985, Tournier et al. 1990). They have been used successfully in the SNAP-19 radioisotope generators which powered the Pioneer 10 and 11 space probes for over 13 years, and the Viking Mars Landers for over 7 years, and in the SNAP-27 radioisotope generators powering the ALSEP stations left on the surface of the Moon by the Apollo astronauts, which operated for over 5 years before they were disconnected (Angelo and Buden 1985). Another TE material that may be useful for the bottom cycle of the converter is bismuth telluride (Rowe 1995). This material has higher figure-of-merit than $\mathrm{PbTe}$ in the range 300-500 K (Figure 3.26), which may make up for the lower temperature drop across the TE legs in the potassium AMTEC/TE converter, compared to that in the sodium AMTEC/TE converter.

Both $2 \mathrm{P}-\mathrm{PbTe}$ and $\mathrm{P}-\mathrm{Zn}_{4} \mathrm{Sb}_{3}$ have a lower figure-of-merit than P-TAGS-85, and were therefore eliminated in favor of P-TAGS-85 (Figure 3.26). Both $2 \mathrm{~N}-\mathrm{PbTe}$ and PTAGS-85 exhibit a high figure-of-merit up to $\sim 750 \mathrm{~K}$, but their figure-of-merits fall off below that for BiTe below $\sim 470 \mathrm{~K}$. Based on these considerations, three different bottom cycle converter configurations were studied: a P-TAGS-85/2N-PbTe unicouple, a $\mathrm{P}-\mathrm{BiTe} / \mathrm{N}-\mathrm{BiTe}$ unicouple, and a segmented TE unicouple (STE) which uses P-TAGS85/P-BiTe in the P-leg, and 2N-PbTe/N-BiTe in the N-leg, with the BiTe materials in contact with the cold shoe (Figure 3.27).

In order to evaluate and compare the performance of these TE converter designs, a one-dimensional optimization model of segmented TE unicouple was developed, and benchmarked successfully against experimental data from the Jet Propulsion Laboratory (El-Genk and Saber 2002a). The model accounts for the change with temperature of the physical and thermoelectric properties of the TE materials used in the various segments of the TE unicouples, and assumes no side heat losses. The later condition is similar to that encountered in the actual fully-packed TE unicouples in the AMTEC/TE converter unit. For given hot side, cold side and interfacial temperatures, the model determines the optimum lengths of the various segments and the ratio of the legs' cross-sectional areas for maximum efficiency and for maximum electrical power operations.

Results delineated in Figure 3.28 show that up to a temperature drop of $150 \mathrm{~K}$ across the thermoelectric legs, corresponding to the nominal operating condition encountered in the potassium-AMTEC/TE converter unit, the P-TAGS-85/2N-PbTe unicouple delivers the highest efficiency of all three TE bottom cycles investigated. The efficiency of the P-BiTe/ N-BiTe unicouple is lowest, up to a temperature drop of $210 \mathrm{~K}$ across the legs. At the nominal operating condition encountered in the sodiumAMTEC/TE converter unit, corresponding to a temperature drop of $\sim 230 \mathrm{~K}$ across the legs, the efficiency of the P-BiTe / N-BiTe unicouple is 0.4 percentage point higher than that of the P-TAGS-85 / 2N-PbTe unicouple, and the efficiency of the segmented TE couple is about one percentage point higher $(7.4 \%)$ than that of the P-TAGS-85 / $2 \mathrm{~N}$ PbTe unicouple (6.4\%). The use of the segmented TE couple in the bottom cycle of the 
sodium-AMTEC/TE converter would only increase the unit's overall efficiency by $\sim 0.6$ percentage point, and this small gain does not justify the added complexity of the segmented design and associated issues of bonding the different TE materials. Thus, the P-TAGS-85 / 2N-PbTe unicouple was selected for both the sodium and the potassium converters. The single-segment TE unicouple can deliver peak efficiencies of $4.6 \%$ and $6.4 \%$ at temperature drops of $150 \mathrm{~K}$ and $230 \mathrm{~K}$ across the legs, respectively (Figure 3.28).

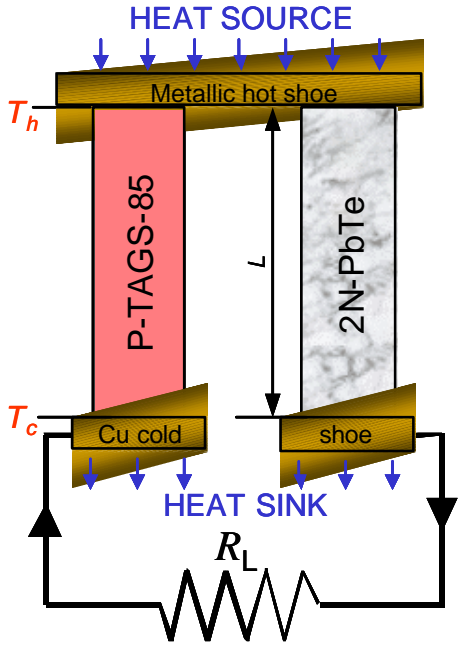

Lead telluride unicouple

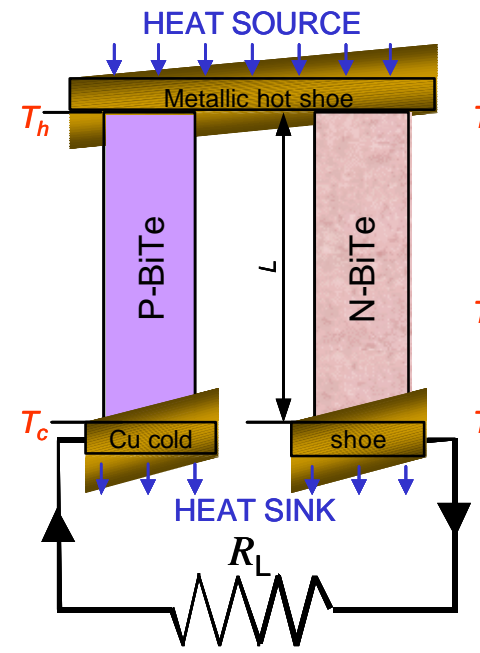

Bismuth telluride unicouple

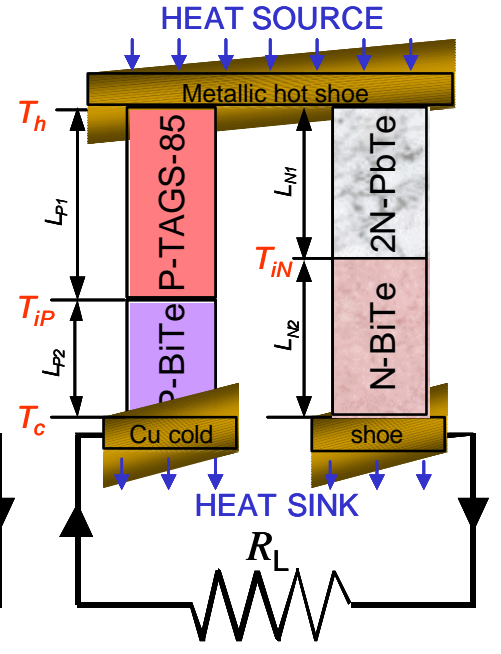

Segmented TE (STE)

Figure 3-27. Design of thermoelectrics for use in the bottom cycle of the AMTEC/TE converter unit.

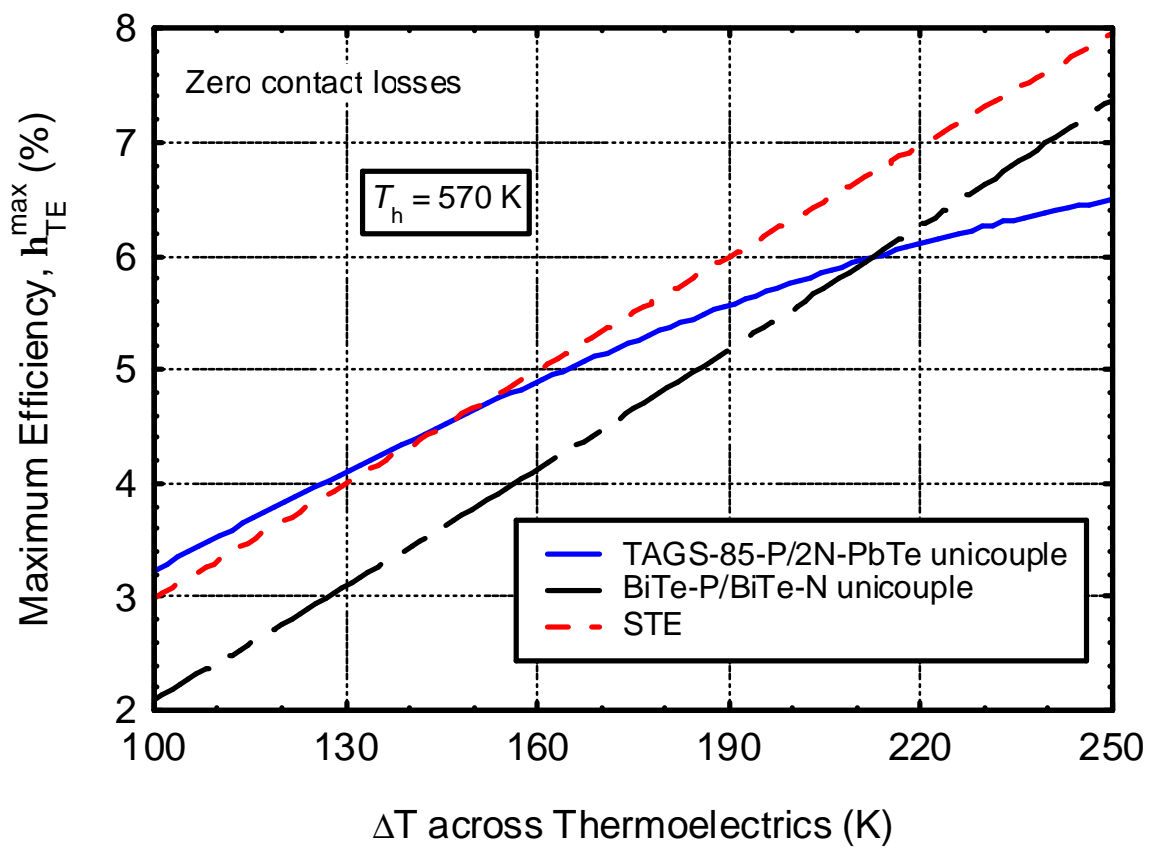

Figure 3-28. Maximum efficiency of thermoelectrics for use in the bottom cycle of the AMTEC/TE converter unit. 


\subsubsection{Cooling of AMTEC/TE Converter Unit by Natural Convection of Air}

The heat given up by condensation of the low-pressure vapor in the AMTEC condenser is conducted at a uniform temperature to the multitude of TE unicouples in the bottom cycle. To ensure a uniform cold side temperature of the unicouples, for efficient operation and low converter weight, a hollow metallic heat pipe shell with extruded fins is used. A cross-section of the converter housing is shown in Figure 3.25. The housing consists of a hollow, pressure-tight metallic shell. The metallic shell includes long, extended hollow fins spanning the two opposite sides and the rooftop of the converter (Figure 3.12), to provide the necessary surface area to reject the unconverted heat by natural convection of air. The inside wall of the metallic shell is lined with a porous wick structure. The later may be obtained using grooves or corrugations, or by a sand-blasting treatment for example. The role of the heat pipe shell is to ensure a nearly isothermal cold end of the TE legs and provide maximum utilization of the available surface area for heat rejection. The heat rejected at the cold end of the TE legs evaporates the water working fluid in the housing wick against the two side (condenser) walls of the converter vessel (Figure 3.11). The resulting vapor condenses on the inside of the hollow fins and is recirculated back through the porous wick to the bottom of the fins, with help of gravity, and then wicked back to the vertical wick surfaces facing the cold end of the TEs, by capillary action (Figure 3.12).

Vertical cross-sections of the outer wall of the heat pipe housing are shown in Figures 3.25 and 3.29. The extruded fins have a length $H_{\text {fin }}$, and a thickness $\delta_{\text {fin }}=1 \mathrm{~cm}$. The fins are equally distributed along the full length $(L)$ of the converter and separated by a distance $W_{\text {fin }}$, as a result the number of fins is equal to:

$$
N_{f i n s}=L / W_{f i n}
$$

and the air gap size between two adjacent fins is:

$$
\delta=W_{f i n}-\delta_{f i n}
$$

The top of the converter vessel is hemi-cylindrical for structural strength and to increase the heat rejection surface area. The diameter of the top section of the heat pipe housing is equal to:

$$
D_{\text {top }}=W+2 \times\left(t_{I N S}+t_{W}\right)
$$

The total surface area of the heat rejection heat pipe housing can then be calculated as (see Figure 3.28): 


$$
A_{\text {rad }}=L \times\left(2 H_{T E}+\frac{\pi}{2} D_{\text {top }}\right) \times\left[1+2 \frac{H_{f i n}}{W_{f i n}} \times\left(1+\frac{\pi}{2} \times \frac{H_{f i n}}{2 H_{T E}+\frac{\pi}{2} D_{t o p}}\right)\right]
$$

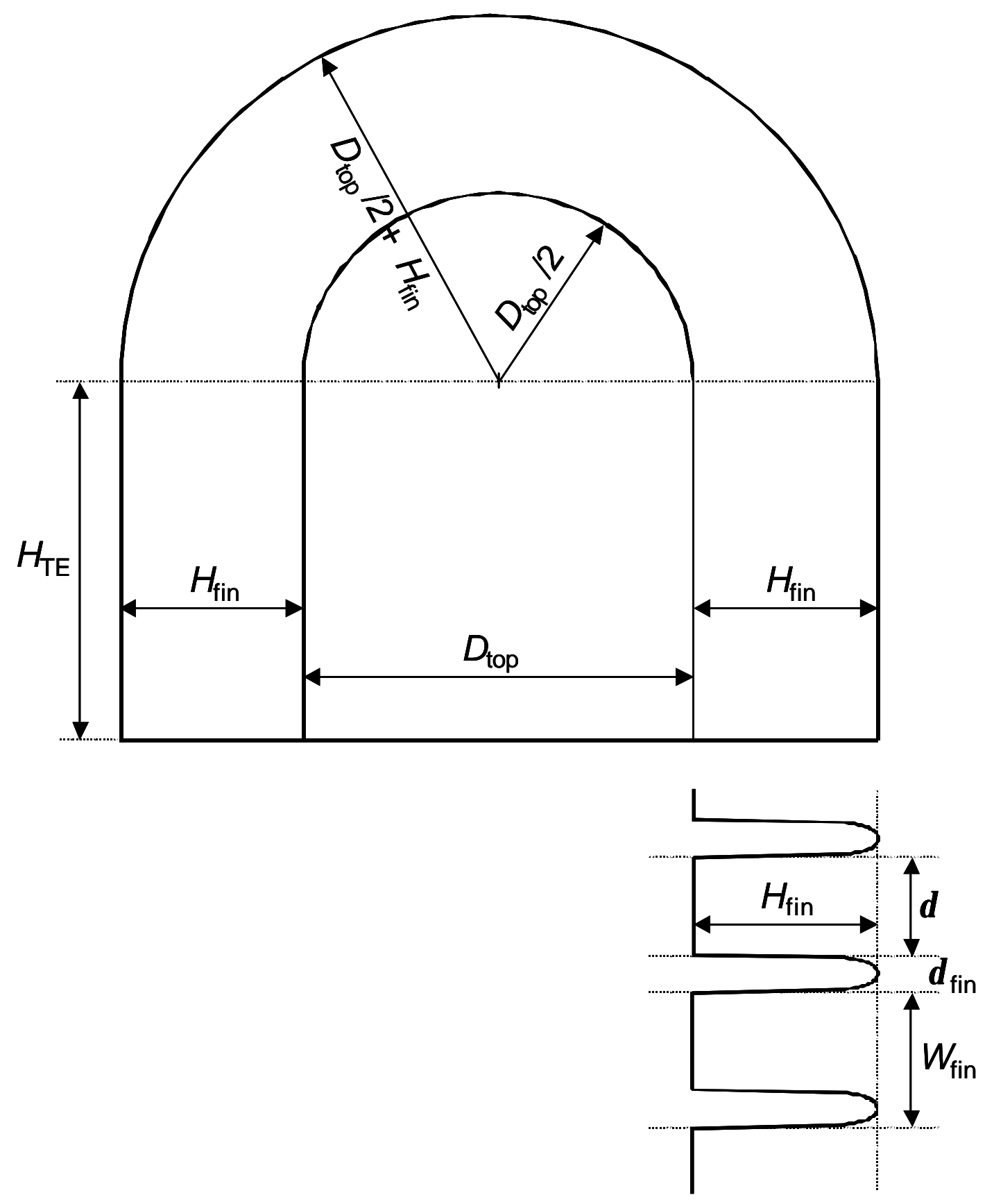

Figure 3-29. Cross-section views and dimensions of heat pipe radiator housing. 
The heat rejected by the radiator housing is then obtained from:

$$
Q_{\text {rad }}=A_{\text {rad }} \bar{h}^{C V}\left(T_{\text {rad }}-T_{\text {air }}\right)
$$

where $\bar{h}^{C V}$ is the average heat transfer coefficient for natural convection of air, which is determined in the next Section.

\subsubsection{Heat Transfer Coefficient for Natural Convection of Air}

Due to the large aspect ratio of the cooling fins $\left(H_{\text {fin }} / W_{\text {fin }}\right)$ of the AMTEC/TE converter unit (Figure 3.29), most of the heat rejection occurs from the vertical surfaces of the fins. A literature search was performed and the most appropriate heat transfer correlation for the present radiator design was found to be that for natural convection of air between two isothermal vertical and parallel plates with all four edges opened to air flow. The original analytical and experimental work for this classical problem was published in 1942 by W. Elenbaas (1942). It should be noted that the aforementioned analysis assumes plates of large width compared to their height, and does not account for air entrainment or flow at the two sides. The air properties are evaluated at the radiator surface (or wall) temperature. The Grashof number is calculated based on the gap width between plates (or fins), $\delta$ :

$$
G r=\frac{g \beta_{a i r}\left(T_{r a d}-T_{a i r}\right) \delta^{3}}{v_{\text {air }}^{2}},
$$

and the Rayleigh number has the expression:

$$
R a=G r \operatorname{Pr} \times \frac{\delta}{H_{T E}},
$$

where

$$
\operatorname{Pr}=\frac{\mu_{\text {air }} C_{p}^{\text {air }}}{k_{\text {air }}}
$$

is the Prandtl number of air. The ave rage Nusselt number is a function of the Rayleigh number: 


$$
\overline{N u}=\frac{R a}{24} \times\left[1-\exp \left(-\frac{25}{R a}\right)\right]^{3 / 4},
$$

and the average convective heat transfer coefficient is given by:

$$
\bar{h}^{C V}=\frac{k_{\text {air }} \overline{N u}}{\delta}
$$

The average Nusselt number is plotted as a function of the Rayleigh number in Figure 3.30. Numerical results obtained by Bodoia and Osterle (1962) using a finite difference technique and experimental data obtained by Levy et al. (1975) compared well with the correlation of Elenbaas (1942) and his own experimental data. For small values of the Rayleigh number $<20$ (small spacing $\delta$ between the plates), the flow becomes fully-developed before it reaches the top of the channel, and the Nusselt number is directly proportional to the Rayleigh number (see Figure 3.30). For larger values of $R a>$ 400 (larger distance between plates or fins), the boundary layers do not meet and the heat transfer correlation reduces to the well-known correlation for a single isothermal vertical plate in an infinite fluid (Figure 3.30).

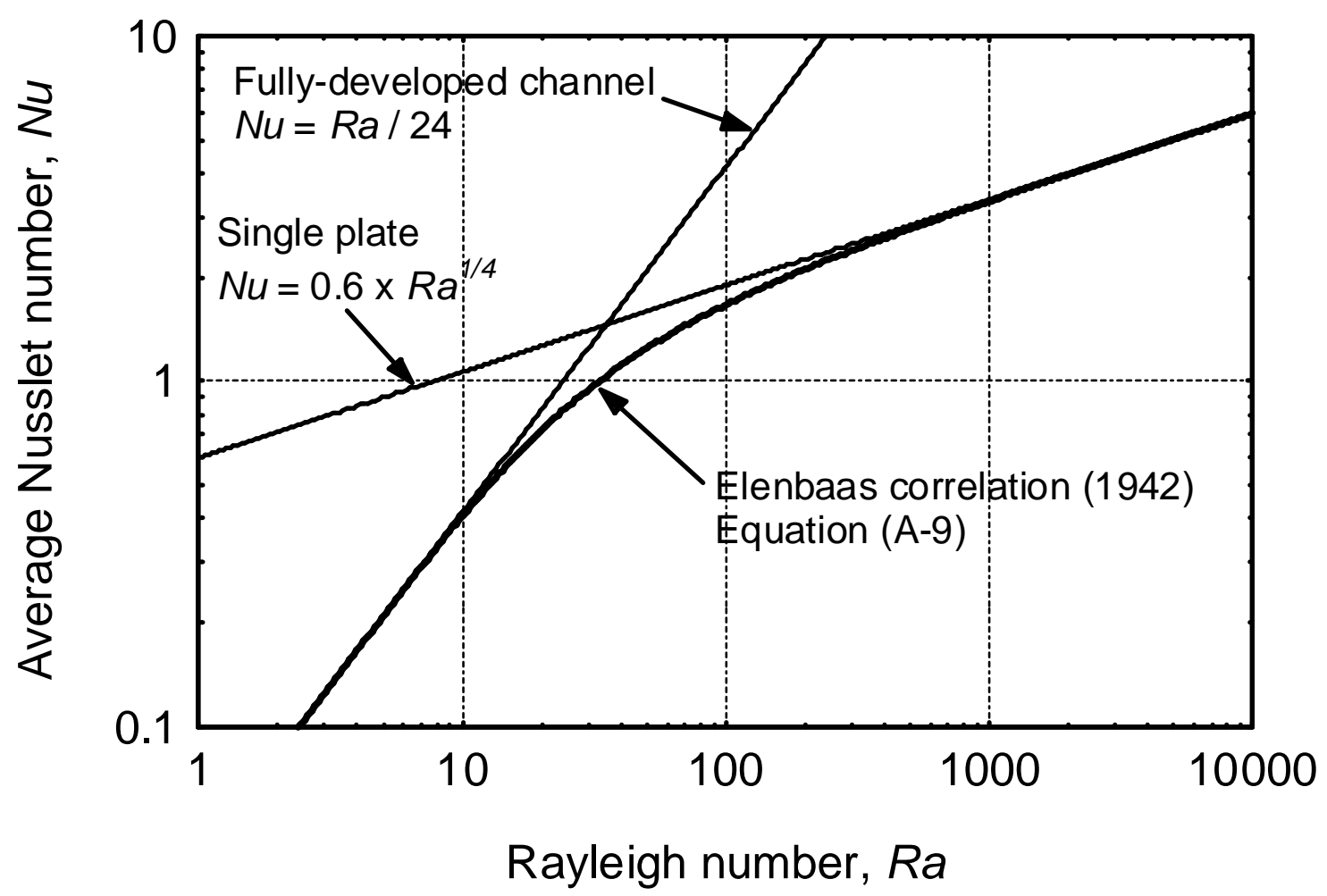

Figure 3-30. Heat transfer by natural convection of air between 2 isothermal, vertical parallel plates. 

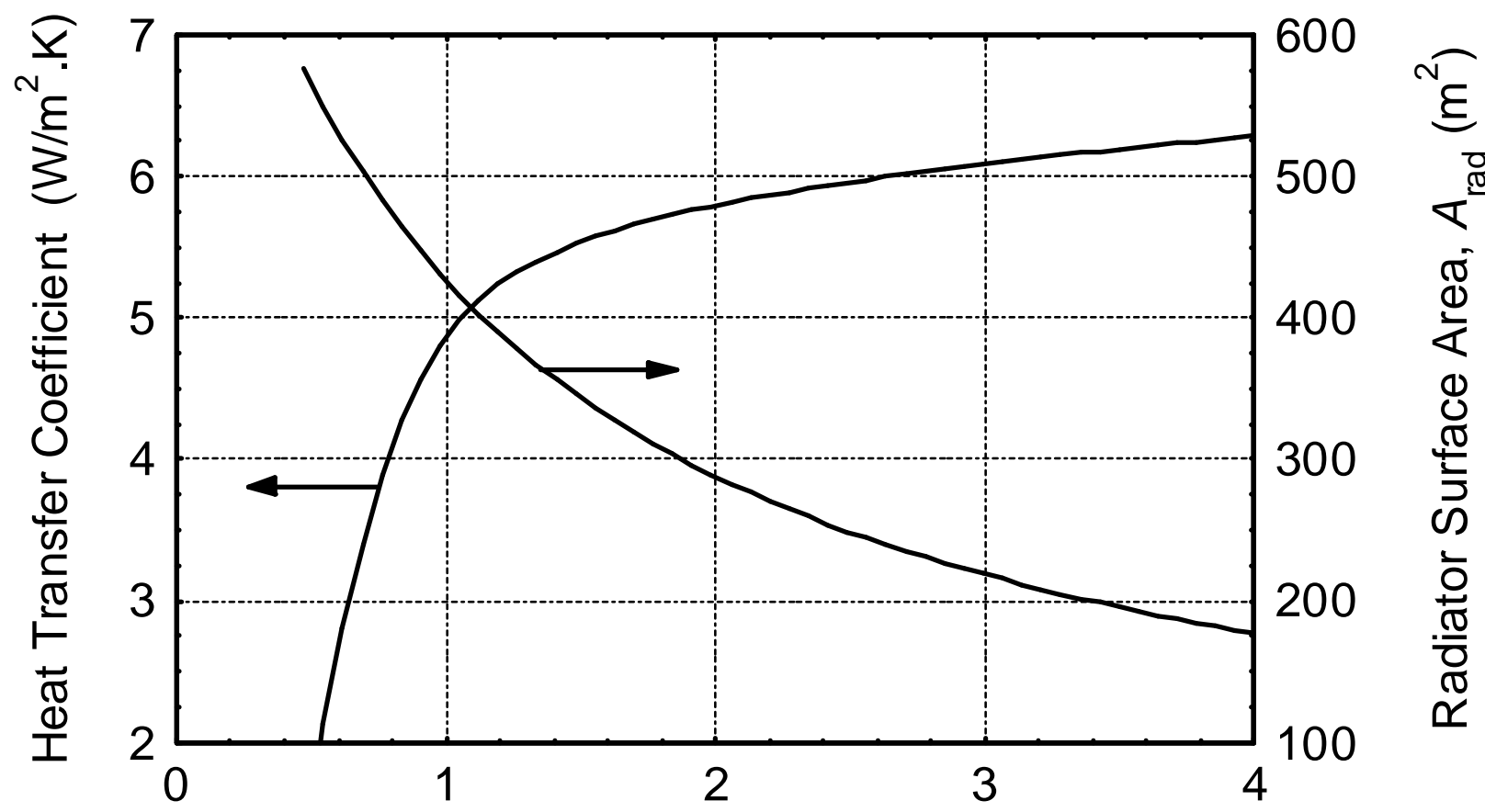

Air Gap between Fins, $\delta(\mathrm{cm})$

Figure 3-31. Heat transfer coefficient by natural convection of air and radiator surface area of 400-V reference sodium AMTEC/TE converter unit, as functions of separation distance between cooling fins $\left(Q_{\mathrm{rad}}\right.$ $\left.=109 \mathrm{~kW}, T_{\text {air }}=300 \mathrm{~K}\right)$.

\subsubsection{Optimum Gap Size between Vertical Cooling Fins}

The present radiator heat rejection model is used to find the optimum spacing between the cooling fins, $\delta$, to minimize the radiator housing temperature and maximize the temperature drop across the thermoelectric unicouples in the bottom cycle. The analysis is performed for the reference sodium AMTEC/TE converter unit, which consists of 2 long rows of $336 \mathrm{BASE}$ elements each, for a voltage output of $400 \mathrm{~V}$ DC. The BASE support plate has dimensions $L=6.7 \mathrm{~m}$ and $W=0.63 \mathrm{~m}$, the vessel wall is $2 \mathrm{~mm}$-thick $\left(t_{\mathrm{W}}=2 \mathrm{~mm}\right)$, the thermal insulation is 1 inch-thick $\left(t_{\mathrm{INS}}=2.54 \mathrm{~cm}\right)$, and the height of the condenser wicks and thermoelectric banks is $H_{\mathrm{TE}}=20 \mathrm{~cm}$. The converter uses sodium working fluid at an anode pressure of $76 \mathrm{kPa}\left(T_{\mathrm{B}}=1121 \mathrm{~K}\right)$, and cooling fins of length $H_{\text {fin }}=30 \mathrm{~cm}$. At these conditions, the waste heat rejected by the radiator housing is nearly constant, $Q_{\mathrm{rad}}=109 \mathrm{~kW}$. The ambient air temperature is taken as $T_{\text {air }}=300 \mathrm{~K}$. 
Figure 3.31 shows the effect of changing the separation distance between cooling fins on the heat transfer coefficient by natural convection of air and on the radiator surface area of the converter. Initially, the heat transfer coefficient increases rapidly with increasing the gap size, approaching an asymptotic value at larger gap sizes (Figure 3.31). Meanwhile, the radiator surface area decreases rapidly with increasing gap size, since the number of cooling fins along the length of the converter is inversely proportional to the distance between the fins (see Equations 3-5 and 3-8). As a result, the product $A_{r a d} \bar{h}^{C V}$ (or rate of heat rejection) peaks at some intermediate gap size, at which the temperature difference between the radiator housing and the ambient air is minimum (see Equation 39). Figure 3.32 and 3.33 shows that this situation occurs when the gap size, $\delta=1 \mathrm{~cm}(\Delta T$ $=52 \mathrm{~K}$ ), at a Rayleigh number $R a \sim 2500$. At such relatively high $R a$, the heat transfer is essentially that for a single isothermal vertical plate in an infinite medium, as shown in Figure 3.30.

Since the present correlation (Equation 3-9) pertains to the natural convection of air between two isothermal vertical and parallel plates with all edges opened to air flow, it does not directly apply to the geometry of the present radiator, as described in Figure 3.29. In an attempt to provide more conservative performance results of the AMTEC/TE converter units, a much larger gap size, $\delta=2.54 \mathrm{~cm}$ was selected for these calculations. For a fin length, $H_{\text {fin }}=30 \mathrm{~cm}$, and fins that are $1 \mathrm{~cm}$-thick $\left(\delta_{\text {fin }}=1 \mathrm{~cm}\right), W_{\text {fin }}=\delta+\delta_{\text {fin }}=$ $3.54 \mathrm{~cm}$, and the aspect ratio of the fins has a more realistic value, $H_{\text {fin }} / W_{\text {fin }}=8.5$. The calculated radiator housing temperature, $T_{\text {rad }}=375 \mathrm{~K}$ (Figure 3.32 ), is $23 \mathrm{~K}$ higher than its optimum value $(352 \mathrm{~K})$, which means that the temperature drop across the thermoelectric unicouples in the bottom cycle is $23 \mathrm{~K}$ lower than its optimum value.

\subsubsection{Estimates of Radiation Heat Exchange between Radiator and Environment}

In Equation (3-9), radiation heat exchange between the heat pipe radiator housing and the environment was not accounted for, which is a conservative approach during night-time, or when the converter housing is shaded from direct Sunlight during daytime. In these situations, some of the waste heat would also be removed from the radiator surface by radiation, reducing the radiator temperature and increasing the temperature drop across the thermoelectric couples in the bottom cycle and, hence, the electrical power output of the AMTEC/TE converter unit. In the daylight, however, when the radiator cooling fins are exposed to full Sun, net radiant energy may be absorbed by the radiator surface, increasing its temperature and reducing the electrical power output of the converter. A simple analysis is performed to estimate the magnitude of this radiative energy absorption. 


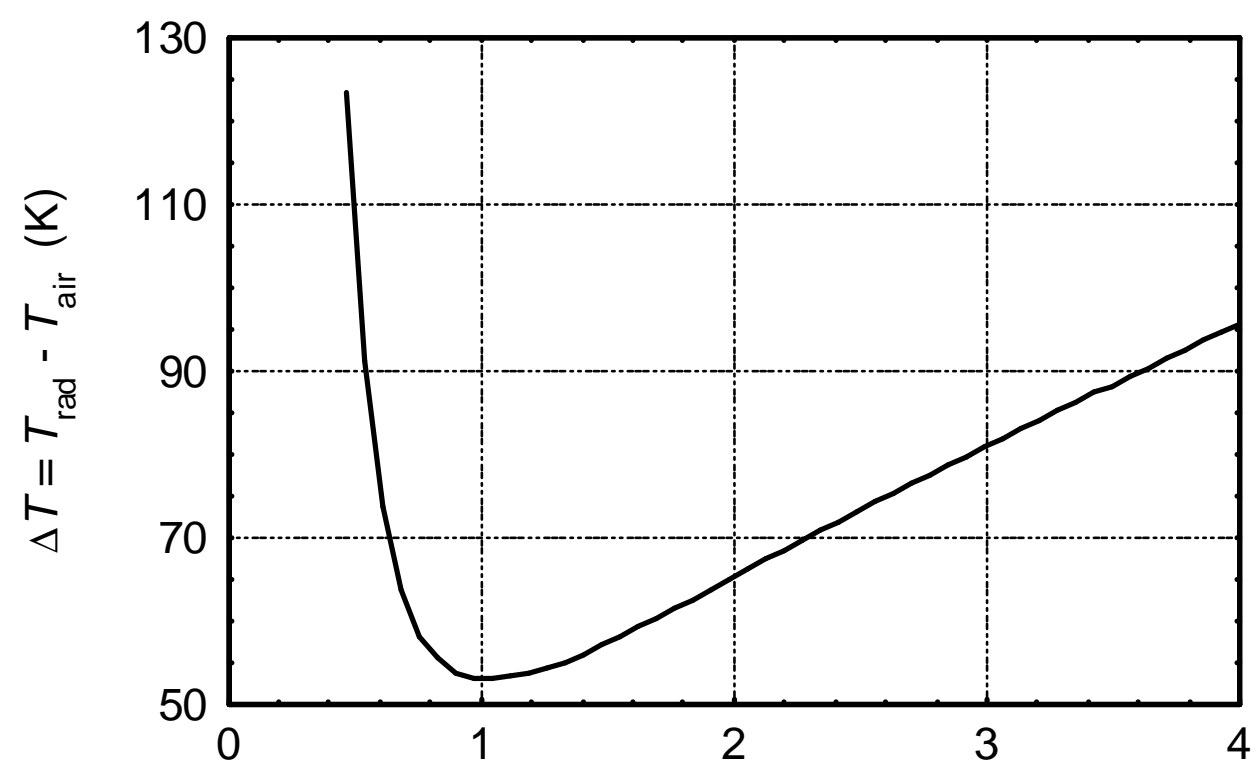

Air Gap between Fins, $\delta(\mathrm{cm})$

Figure 3-32. Radiator surface temperature of the reference sodium AMTEC/TE converter unit, as function of separation distance between cooling fins $\left(Q_{\mathrm{rad}}=109 \mathrm{~kW}, T_{\text {air }}=300 \mathrm{~K}\right)$.

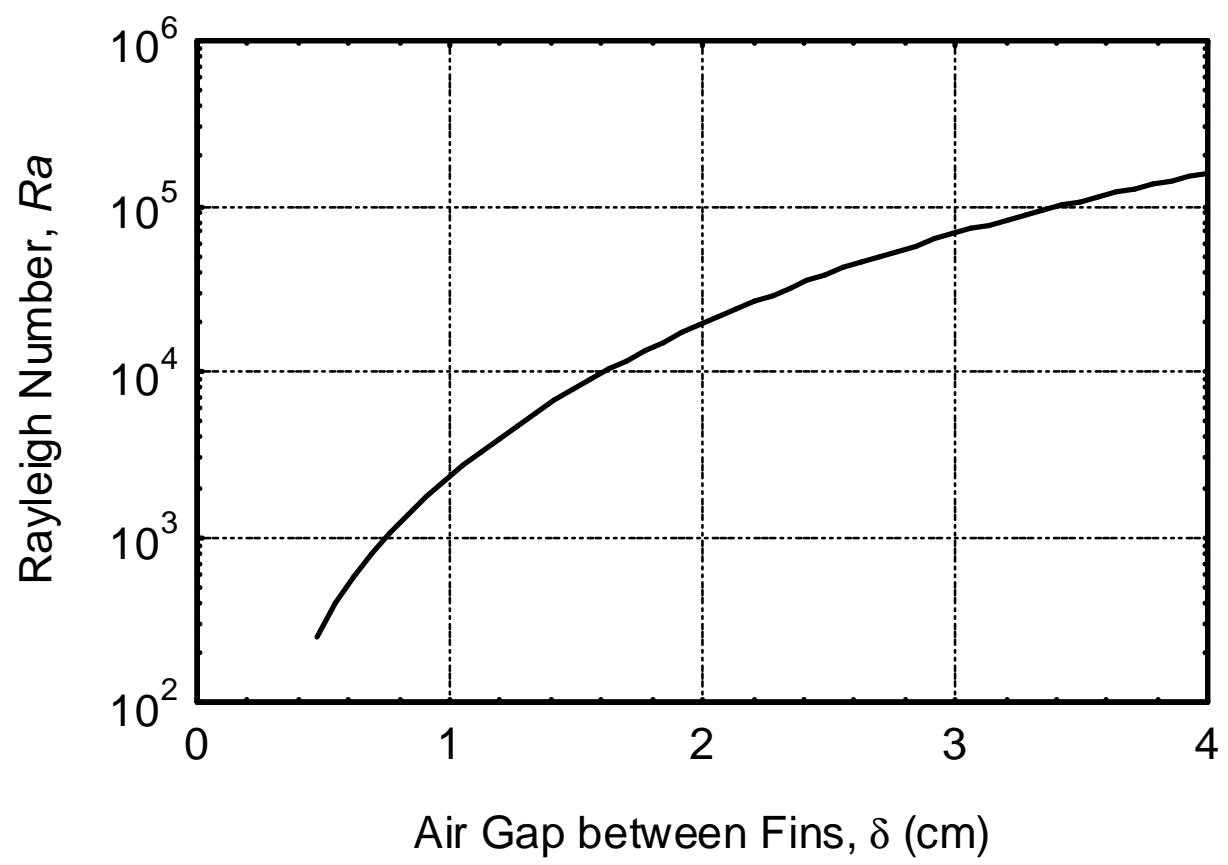

Figure 3-33. Air Rayleigh number at the radiator of the reference sodium AMTEC/TE converter unit, as function of separation distance between cooling fins $\left(Q_{\mathrm{rad}}=109 \mathrm{~kW}, T_{\text {air }}=300 \mathrm{~K}\right)$.

If we assume a typical insolation flux $q_{S u n}=1,400 . \mathrm{W} / \mathrm{m}^{2}$, incident onto the radiator surface, a fraction $\varepsilon_{\text {rad }} q_{S u n}$ would be absorbed by the radiator, while the 
remainder $\left(1-\varepsilon_{\text {rad }}\right) q_{S u n}$ would be reflected away. In addition, the radiator surface also emits a radiant energy flux $\varepsilon_{r a d} \sigma T_{r a d}^{4}$. As a result, the net radiant energy flux absorbed by the radiator surface would be:

$$
q_{\text {Net }}^{r a d}=\varepsilon_{\text {rad }}\left(q_{\text {Sun }}-\sigma T_{\text {rad }}^{4}\right)
$$

This net radiant energy flux is plotted in Figure 3.35 for a blackbody with an emissivity equal to 1 , and for a lightly-oxidized stainless steel surface (the structural material chosen for the housing of the water heat pipe radiator). The emissivity of stainless steel is shown in Figure 3.34. As shown in Figure 3.35, the net radiant energy flux absorbed by the radiator surface exposed to full Sun cancels to zero for a radiator surface temperature of $395 \mathrm{~K}$. A cooler radiator would absorb radiant energy, while a hotter surface would reject radiant energy to the environment.

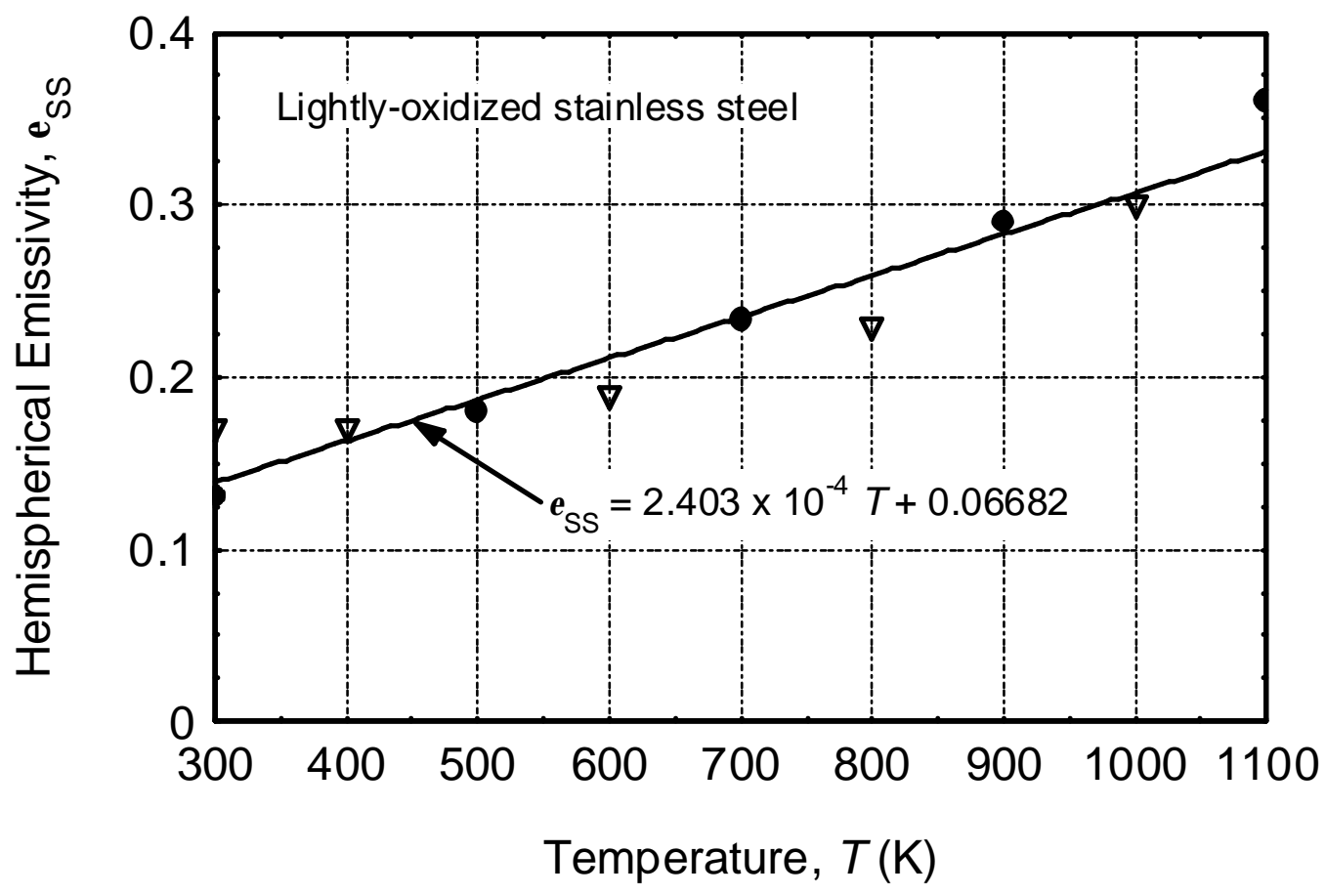

Figure 3-34. Hemispherical emissivity of lightly-oxidized stainless steel.

Note that this analysis is very conservative since the effect of the geometry was not taken into account. It is very unlikely that the full surface area of the radiator, including the sidewalls of all the cooling fins, would be exposed to direct solar radiation. In practice, 
the AMTEC/TE converter units designed in this work operate at a radiator surface temperature between $350 \mathrm{~K}$ and $400 \mathrm{~K}$ when the air ambient temperature is $T_{\text {air }}=300 \mathrm{~K}$, and the net radiant energy absorbed by the radiator is only a small fraction of the heat removed by the natural convection of air. For example, the reference 400-V sodium AMTEC/TE converter unit operates at a radiator temperature of $384 \mathrm{~K}$. The radiator surface area (including the cooling fins) is $A_{\text {rad }}=218.5 \mathrm{~m}^{2}$, and the heat removal by natural convection of air is $Q_{\mathrm{rad}}=107.3 \mathrm{~kW}$, corresponding to a convective heat flux $q_{C V}^{\prime \prime}=490 \mathrm{~W} / \mathrm{m}^{2}$. At these conditions, the maximum net radiant energy flux absorbed by the radiator surface would be $q_{N e t}^{r a d}=27 \mathrm{~W} / \mathrm{m}^{2}$, or only $5 \%$ of $q_{C V}^{\prime \prime}$.

In the following Section, performance and optimization analysis models of the AMTEC/TE converter are developed and used to predict the performance of and optimize the design of sodium and potassium AMTEC/TE units for the LMR-AMTEC power plant.

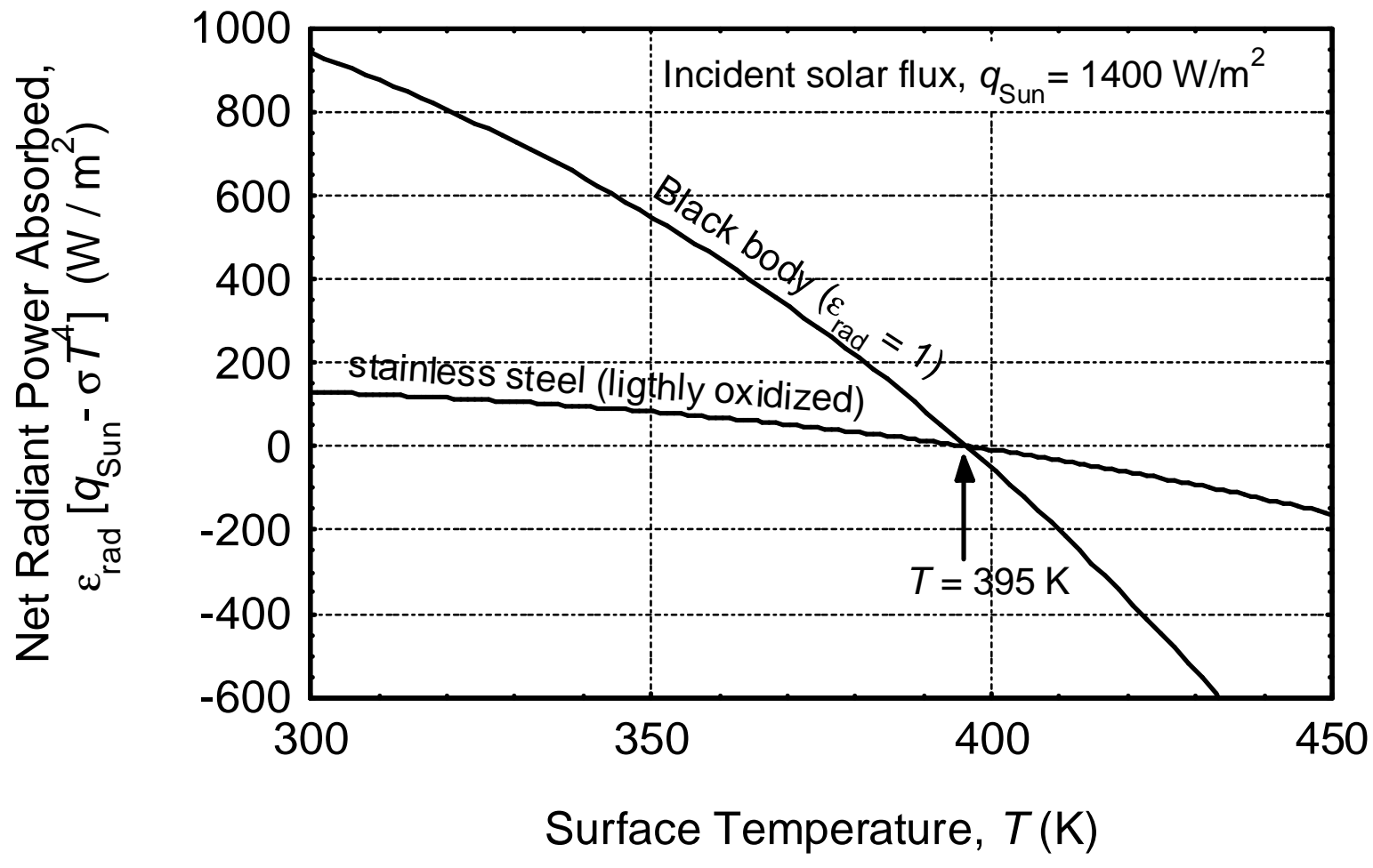

Figure 3-35. Estimate of net radiative power absorbed by heat pipe radiator housing. 


\subsection{Design and Optimization Analyses of AMTEC/TE Converters}

Optimization analysis models for the AMTEC/TE converter unit design were developed for a constant condenser temperature and maximum conversion efficiency of the TE bottom cycle. The length of the TE legs is calculated by matching the heat flux at the condenser / TE hot shoe interface. The models were used to optimize the design of the AMTEC/TE converter unit for maximum overall conversion efficiency. Design parameters optimized included the aspect ratio of the BASE support plate, $(W / L)$, controlled by the number of rows of BASE elements $\left(N_{\text {rows }}=1,2,3\right.$ and 4) (Figure 3.11), the aspect ratio of the dome-shaped BASE elements $\left(H_{\mathrm{B}} / L_{\mathrm{B}}\right)$, the separation distance between the BASE elements $(b)$, the height of the condenser side walls and of the TE modules $\left(H_{\text {TE }}\right)$, the size $\left(D_{\text {orif }}\right)$ and number $\left(N_{\text {orif }}\right)$ of orifices in the internal radiation shield, and the spacing $\left(W_{\text {fin }}\right)$ and length $\left(H_{\text {fin }}\right)$ of the cooling fins of the AMTEC/TE converter unit. The AMTEC units use $\mathrm{WRh}_{1.5}$ electrodes of current technology $(B=90$ A.K ${ }^{1 / 2} / \mathrm{Pa} . \mathrm{m}^{2}, G_{\mathrm{E}}=10$ and $R_{\mathrm{cont}}=0.06 \Omega . \mathrm{cm}^{2}$ ) with a total electrodes' surface area of 37 $\mathrm{m}^{2}$. The AMTEC units are coupled to "off-the-shelf," commercially available lead telluride thermoelectric modules for the bottom cycle. The developed models were used to optimize and compare the overall performance of the sodium and potassium AMTEC/TE converters.

\subsubsection{AMTEC COnverter Model (ACOM)}

The performance models developed in this work capitalize on the UNM-ISNPS previous experience in modeling multi-tube, vapor anode Pluto/Express (PX) cells (El-Genk et al. 2000). The developed high-power AMTEC model is based on the AMTEC Performance and Evaluation Analysis Model, APEAM which had been developed at UNM-ISNPS to predict and optimize the performance of PX-series cells (Tournier and El-Genk 1999a to 1999f). The predictions of APEAM have been successfully benchmarked with the experimental data obtained at the Air Force Research Laboratory, Kirtland AFB, Albuquerque NM (Tournier and El-Genk 1999e and 1999f). The AMTEC COnverter Model (ACOM) developed in this work consists of four major, interactively coupled submodels:

(a) Pressure loss model, which calculates the alkali-metal vapor pressure at the interface between the cathode electrode and the BASE solid membrane, as a function of vapor flow rate and temperatures (Tournier and ElGenk 1999b). This model takes into account the effect of non-uniform vapor injection along the cathode electrode;

(b) Electrochemical model, which calculates the voltage differential across the BASE as a function of AMTEC top cycle's electric current, BASE and condenser temperatures, and sodium pressure differential across the BASE (Tournier and El-Genk 1999c);

(c) Electrical model, which calculates the ionic resistance of the BASE, the electrical resistance of the current collectors, and the AMTEC unit's internal resistance and 
total electrical current, as function of the external load resistance (Tournier and ElGenk 1999c); and finally

(d) Thermal heat flow model, which estimates the heat losses by conduction and by thermal radiation exchange inside the AMTEC/TE converter unit. Knowledge of these heat losses is necessary to estimate the conversion efficiency of the converter. Because of the condensation of alkali-metal vapor in the porous substrate (anode) in the high-pressure side of the BASE membrane (heat pipe effect), the BASE membrane will be isothermal. The temperature of the BASE would be almost the same as that of the flash evaporator or liquid pool at the bottom of the AMTEC converter unit. The present analyses are therefore performed at fixed BASE/evaporator temperature and a fixed condenser temperature.

The sub-models of the different physical processes in the AMTEC/TE converter are coupled to those for calculating the temperature-dependent, material thermophysical and radiative properties, using an efficient iterative solution procedure (Tournier and El Genk 1999d). ACOM calculates the load electric current and terminal voltage as functions of the converter design, dimensions, electrode characteristics, and the BASE, condenser and air ambient temperatures. Highlights of the constitutive equations in ACOM are given next.

\subsubsection{ACOM Constitutive Equations}

The alkali metal vapor emerges from the BASE/cathode electrode interfaces at a mass flow rate of $\dot{m}_{I}=N_{B} \times M I_{A M T E C} / F$ and pressure $P_{c}^{c c}$. The alkali metal ions diffusing through the BASE develop an electro-chemical potential (or undergo isothermal expansion at $\left.T_{B}\right)$, which balances the pressure differential between the anode $\left(P_{a}\right)$ and

cathode $\left(P_{c}^{c c}\right)$. The ideal specific electrochemical work $[\mathrm{J} / \mathrm{kg}]$ produced is expressed as (Cole 1983):

$$
w=\frac{R_{g} T_{B}}{M} \ln \frac{P_{a}}{P_{c}^{c c}} .
$$

The corresponding electric power generated is then:

$$
P e=\dot{m}_{I} \times w=N_{B} \times M I_{A M T E C} \times w / F=N_{B} \times I_{A M T E C} \times V_{e m f}^{c c} .
$$

The closed-circuit electrochemical potential per BASE element, $V_{e m f}^{c c}$, is given by:

$$
V_{e m f}^{c c}=\frac{R_{g} T_{B}}{F} \ln \frac{P_{a}}{P_{c}^{c c}}
$$


The electric power generated in the BASE/electrodes assembly is the sum of: (a) the charge-exchange polarization losses in the cathode electrodes, $N_{B} \xi_{c}>0$; (b) the ionic Joule losses in the BASE elements; (c) the Joule losses in contact resistance between cathode electrode and current collector, in current collectors, and in the leads connecting the BASE elements; and (d) the electric power delivered to the external load. The polarization losses, $\xi_{c}$, are represented as an electrical potential loss in the closed-circuit electrochemical potential $V_{e m f}^{c c}$, thus:

$$
V_{o}^{c c}=V_{e m f}^{c c}-\xi_{c}
$$

The potential loss $\xi_{c}$ is caused by the accumulation of sodium ions at the BASE/cathode electrode interface, and is expressed as (Williams et al. 1990):

$$
\xi_{c}=\frac{R_{g} T_{B}}{F} \ln \left\{\frac{1}{2} \frac{J}{J^{e x}} \frac{P_{c}^{o c}}{P_{c}^{c c}}\left[\frac{J}{J^{e x}}+\sqrt{\left(\frac{J}{J^{e x}}\right)^{2}+4 \frac{P_{c}^{c c}}{P_{c}^{o c}}}\right]+1\right\} .
$$

The charge-exchange current density $\left(J^{e x}\right)$ is a measure of the effectiveness of ions recombination at the triple BASE/cathode electrode/ vapor interface line, and can be expressed as (Williams et al. 1990):

$$
J^{e x}=B \times\left[\frac{P_{c}^{o c} P_{s a t}\left(T_{B}\right)}{T_{B}}\right]^{1 / 2} .
$$

The coefficient $B$, characteristic of the type and material of the cathode electrode, is determined experimentally. For the $\mathrm{WRh}_{1.5}$ electrode, a stable value of $B=90 \mathrm{~A} \cdot \mathrm{K}^{1 / 2}$ / Pa. ${ }^{2}$ has been reported (Ryan et al. 2000). The so-called concentration losses are caused by the increase in the vapor pressure at the cathode side of the BASE, compared to open-circuit operation, such that:

$$
P_{c}^{c c}=P_{c}^{o c}+\Delta P_{c}^{\text {loss }}
$$

For a constant condenser temperature, $T_{c d}$, the condenser vapor pressure during both open- and closed-circuit operations is the same and equal to the vapor saturation pressure at $T_{c d}, P_{s a t}\left(T_{c d}\right)$. During open-circuit operation, the vapor pressure at the BASE/cathode electrode interface is higher because of the increase in the specific volume of vapor as it equilibrates with the $\operatorname{BASE}\left(T_{B}>T_{c d}\right)$, thus:

$$
P_{c}^{o c}=P_{s a t}\left(T_{c d}\right) \sqrt{T_{B} / T_{c d}}
$$


During closed-circuit operation, the pressures losses due to evaporation at the BASE/cathode surface, diffusion through the porous cathode, vapor flow in the lowpressure cavity, and condensation, increase the vapor pressure at the cathode side of the BASE to $P_{c}^{c c}$ :

$$
P_{c}^{c c}=P_{s a t}\left(T_{c d}\right) \sqrt{T_{B} / T_{c d}}+\frac{3 G_{\text {total }}}{8 \pi}\left(\frac{2 \pi R_{g} T_{B}}{M}\right)^{1 / 2} \times \frac{M}{F} J .
$$

In this equation, the dimensionless, geometric pressure loss coefficient $G_{\text {total }}$ is given as:

$$
G_{\text {total }}=G_{E}+G_{\text {cavity }}+\frac{8 \pi}{3}\left[\frac{1}{\varepsilon_{E}}+\frac{N_{B} A_{E}}{A_{c d}}\right] .
$$

The coefficient $G_{\text {cavity }}$ accounts for the vapor pressure losses in the low-pressure cavity and is calculated numerically using the Dusty-Gas-Model (Tournier and El-Genk 1999a). In the present analysis, $G_{\text {cavity }}$ is dominated by the pressure losses due to the flow area expansion above the BASE elements, and to the vapor flow between BASE elements. The $G_{E}$ coefficient accounts for pressure losses in the porous cathode electrode, and is measured experimentally. For the $\mathrm{WRh}_{1.5}$ electrode, $G_{E}=10$ (Ryan et al. 2000). The third term in Equation (3.25) accounts for the pressure losses due to vaporization at the BASE/cathode interface and condensation at the remote condenser. For the referenc KAMTEC unit operating at $T_{B}=1006 \mathrm{~K}$ and $I_{A M T E C}=126.8 \mathrm{~A}, G_{E}=10, \varepsilon_{\mathrm{E}}=0.85, N_{B}=$ $744, A_{E}=498 \mathrm{~cm}^{2}$, and $A_{c d}=2.48 \mathrm{~m}^{2}$, the total pressure loss coefficient is $G_{\text {total }}=197$.

The liquid anode pressure, $P_{a}$ in Equation (3.18) is given by:

$$
P_{a}=P_{e v}=P_{s a t}\left(T_{e v}\right)
$$

The load electrical power is given as:

$$
P e_{A M T E C}=V_{A M T E C} \times I_{A M T E C},
$$

where the load voltage is given by:

$$
V_{\text {AMTEC }}=N_{B} \times\left\lfloor V_{o}^{c c}-R_{\text {int }}^{\prime} \times J\right]
$$

and the specific internal resistance per BASE element (in $\Omega . \mathrm{m}^{2}$ ) is: 


$$
R_{\mathrm{int}}^{\prime}=\left[\sigma_{B} \times t_{B}+R_{\text {cont }}^{\prime}+R_{\text {coll }}^{\prime}\right]+R_{\text {leads }} \times A_{E} / N_{B} .
$$

In the optimized potassium- AMTEC/TE converter unit operating at $T_{\mathrm{B}}=1006 \mathrm{~K}$ and $I_{A M T E C}=126.8 \mathrm{~A}$, the internal resistance of the AMTEC top cycle is $0.406 \Omega$, and the ionic, contact, leads and collector electrical losses amount to $41.1 \%, 22.1 \%, 22.0 \%$ and $14.8 \%$ of the internal Joule losses in the AMTEC converter, respectively. The thermal heat flow model developed to estimate the radiation and conduction heat losses in the AMTEC/TE converter unit is described briefly next.

\subsubsection{Radiation and Heat Conduction Model}

The radiation and conduction heat losses model of the present AMTEC/TE converter design provides input to predicting the conversion efficiency and corresponding electrical power output, for both potassium and sodium AMTECs. This model accounts for parasitic heat losses by conduction through the sidewalls and the thermal insulation on the outside (1 inch-thick Kaowool blanket), for heat rejection by radiation and/or natural convection to the ambient air, for conduction heat losses to the condenser walls (ElGenk, Tournier and Momozaki 2001, Appendix B), by radiation between the support plate/BASE elements and the inner shield surface, and between the outer shield surface and both the condenser and inner surfaces of the wall. This model also allows for streaming radiation heat transfer through the orifices of the heat shield (Figures 3.11 and 3.12).

Since at the typical AMTEC operating temperatures ( 1000-1130 K evaporator temperature and $\sim 520-650 \mathrm{~K}$ condenser temperature) the radiation heat transfer in the unit could be significant, a sophisticated enclosure radiation model is developed for the low-pressure cavity of the converter. Since the BASE support plate has a relatively large aspect ratio $(L / W$ between 7 and 11), and the height of the AMTEC unit is much smaller than its length, thus radiation heat losses to the two end faces of the converter vessel could be neglected. The radiation model developed in this work calculates the radiation heat exchange between the BASE elements/support plate and the internal, dome-shaped thermal shield, and between the shield and the rooftop and condenser walls of the AMTEC converter. The radiative surfaces of interest in the low-pressure cavity are divided into elementary surface areas, and the view factors between these elementary surfaces are calculated analytically using tabulated view factor formulas available in the literature (Howell 1982), and closed-form algebraic relations and elementary flux algebra. The developed view factors ensure that all geometrical enclosure and reciprocity relationships in the low-pressure cavity are satisfied. More details on the radiation and conduction heat loss model can be found in El-Genk, Tournier and Momozaki 2001 (Appendix B). Since the temperatures of the dome-shaped radiation shield, thermal insulation surfaces, and the radiator cooling fins are not known a priori, an iterative numerical solution is developed to solve the coupled radiation/conduction problem.

The molybdenum internal radiation shield in the AMTEC converter unit is assumed to be thin enough $(\leq 250 \mu \mathrm{m})$ that conduction heat flow from the BASE support 
plate is negligible. The conservation of radiant energy in the low-pressure cavity dictates that all radiant energy lost by the BASE elements and their support plate $\left(Q_{B A S E}^{\text {rad }}\right)$ must be reabsorbed at the surfaces of the condenser walls supporting the banks of thermoelectrics ( $Q_{c d}^{r a d}$ ) and of the AMTEC containment rooftop ( $Q_{\text {roof }}^{\text {rad }}$ ).

The performance model of the present AMTEC/TE converter unit also calculates the heat losses by conduction through the four sidewalls of the converter's evaporator cavity, $Q_{e v}^{\text {loss }}$, and the heat losses by conduction through the two end faces of the AMTEC/TE unit, $Q_{\text {ends }}^{\text {loss }}$, by matching the conduction heat flux through the metallic converter vessel and through the Kaowool insulation blanket to the heat removal by natural convection of air and radiation to the ambient air. The model also calculates the conduction heat losses through the rooftop of the AMTEC/TE unit to the heat pipe radiator, $Q_{\text {roof }}^{\text {loss }}$. The conduction heat losses, $Q_{\text {roof }}^{\text {cond }}$ to the AMTEC condenser walls from the rooftop of the AMTEC containment are then obtained from the energy balance of the rooftop wall:

$$
Q_{\text {roof }}^{\text {cond }}=Q_{\text {roof }}^{\text {rad }}-Q_{\text {roof }}^{\text {loss }} .
$$

The heat flow model also calculates the conduction heat losses to the AMTEC condenser walls from the BASE support plate, $Q_{e v}^{c o n d}$. More details on the radiation and conduction heat flow model can be found in the FY2001 Progress Report (El-Genk, Tournier and Momozaki 2001, Appendices A and B).

\subsubsection{Overall Energy Balance and Performance of the AMTEC/TE Converter}

The heat rejected to (or absorbed by) the condenser walls in the AMTEC unit is calculated as the sum of the thermodynamic heat of the AMTEC top cycle and the radiation and conduction heat losses, e.g.:

$$
Q_{r e j}=Q_{T D}+Q_{c d}^{r a d}+\left(Q_{e v}^{\text {cond }}+Q_{\text {roof }}^{\text {cond }}\right) .
$$

The thermodynamic heat of the AMTEC top cycle, $Q_{T D}$, accounts for the latent heat imparted to the condenser and for the decrease in liquid enthalpy before returning back to the evaporator, and is given as:

$$
Q_{T D}=\dot{m}_{I} h_{f g}\left(T_{B}\right)+\dot{m}_{I} C_{p}\left(T_{B}-T_{c d}\right) .
$$

The heat losses, $Q_{\text {losses }}$, which are not absorbed into the condenser walls of the AMTEC/TE converter are the sum of those from the sidewalls of the evaporator cavity 
and from the two end faces of the converter, and conduction losses through the rooftop wall of the AMTEC containment to the heat pipe radiator:

$$
Q_{\text {losses }}=Q_{e v}^{\text {loss }}+Q_{\text {ends }}^{\text {loss }}+Q_{\text {roof }}^{\text {loss }} .
$$

The thermal heat input to the AMTEC/TE unit is then calculated as follows:

$$
Q_{\text {input }}=P e^{A M T E C}+Q_{\text {rej }}+Q_{\text {losses }} .
$$

This equation can also be written as:

$$
Q_{\text {input }}=P e^{A M T E C}+Q_{T D}+Q_{B A S E}^{\text {rad }}+Q_{e v}^{\text {cond }}+\left(Q_{e v}^{\text {loss }}+Q_{e n d s}^{\text {loss }}\right),
$$

since the conservation of radiant energy in the low-pressure cavity of the converter and Equation (3.29) allow to express $Q_{B A S E}^{r a d}$ as:

$$
Q_{B A S E}^{\text {rad }}=Q_{c d}^{\text {rad }}+Q_{\text {roof }}^{\text {rad }}=Q_{c d}^{\text {rad }}+\left(Q_{\text {roof }}^{\text {loss }}+Q_{\text {roof }}^{\text {cond }}\right)
$$

The electrical current and power produced by the AMTEC unit are calculated as functions of $T_{\mathrm{ev}}=T_{\mathrm{B}}, T_{c d}$, and the external load resistance, $R_{\mathrm{L}}$, using the developed electrochemical and electrical circuit models (Section 3.4.1.1). The conversion efficiency of the AMTEC unit is then obtained as:

$$
\eta_{\text {AMTEC }}=\frac{R_{L} I_{\text {AMTEC }}^{2}}{Q_{\text {input }}}=\frac{P e^{A M T E C}}{Q_{\text {input }}}
$$

\subsubsection{Performance of the Thermoelectric Bottom Cycle}

In order to estimate the contribution of the thermoelectric bottom cycle to the overall performance of the AMTEC/TE converter unit, the optimum efficiency of the P-TAGS85/ 2N-PbTe thermoelectric bottom cycle is calculated as a function of the hot and cold shoe temperatures (Fritts 1959, Soo 1968) using the developed TE model (El-Genk and Saber 2002a, El-Genk, Saber and Caillat 2002). This approach accounts for the electrical resistances of the $\mathrm{P}$ and $\mathrm{N}$ legs, and assumes a contact resistance of $100 \mu \Omega . \mathrm{cm}^{2}$ at each leg/shoe interface and that the $\mathrm{P}$ and $\mathrm{N}$ legs are perfectly insulated on the side. 
For a volume fraction of the TE couples insulation of $26 \%$, the conduction heat losses through the insulation are small, $<0.7 \%$. This is because the thermal conductivities of the P- and N-type lead telluride legs $(0.5$ to $2 \mathrm{~W} / \mathrm{m} . \mathrm{K})$ are large compared to that of the Min-K (0.03-0.04 W/m.K) (Huang and El-Genk 2001) or other suitable thermal insulators used. Thus, the electrical power produced by the TE bottom cycle is given by:

$$
P e^{T E}=\eta_{T E}^{o p t} \times Q_{r e j} .
$$

The optimum conversion efficiency of a P-TAGS- $85 / 2 \mathrm{~N}-\mathrm{PbTe}$ unicouple is shown in Figure 3.36, showing that it could operate at a maximum efficiency of $3 \%$, $5.5 \%$ and $7.5 \%$ when the temperature differential across the $\mathrm{P}$ and $\mathrm{N}$ legs is $100 \mathrm{~K}, 200 \mathrm{~K}$ and $300 \mathrm{~K}$, respectively (see Figure 3.36), and the hot shoe temperature is $650 \mathrm{~K}$ (for the sodium-AMTEC converter unit). At a lower hot shoe temperature of $560 \mathrm{~K}$ (near the optimum condenser temperature for the potassium-AMTEC), the conversion efficiency of the TE unicouple decreases slightly, to $2.8 \%, 5 \%$ and $6.2 \%$ for a temperature differential across the legs of $100 \mathrm{~K}, 200 \mathrm{~K}$ and $300 \mathrm{~K}$, respectively (see Figure 3.36). These conversion efficiencies are used in the present optimization analysis to estimate the electrical power output of the TE bottom cycle, according to Equation (3.37).

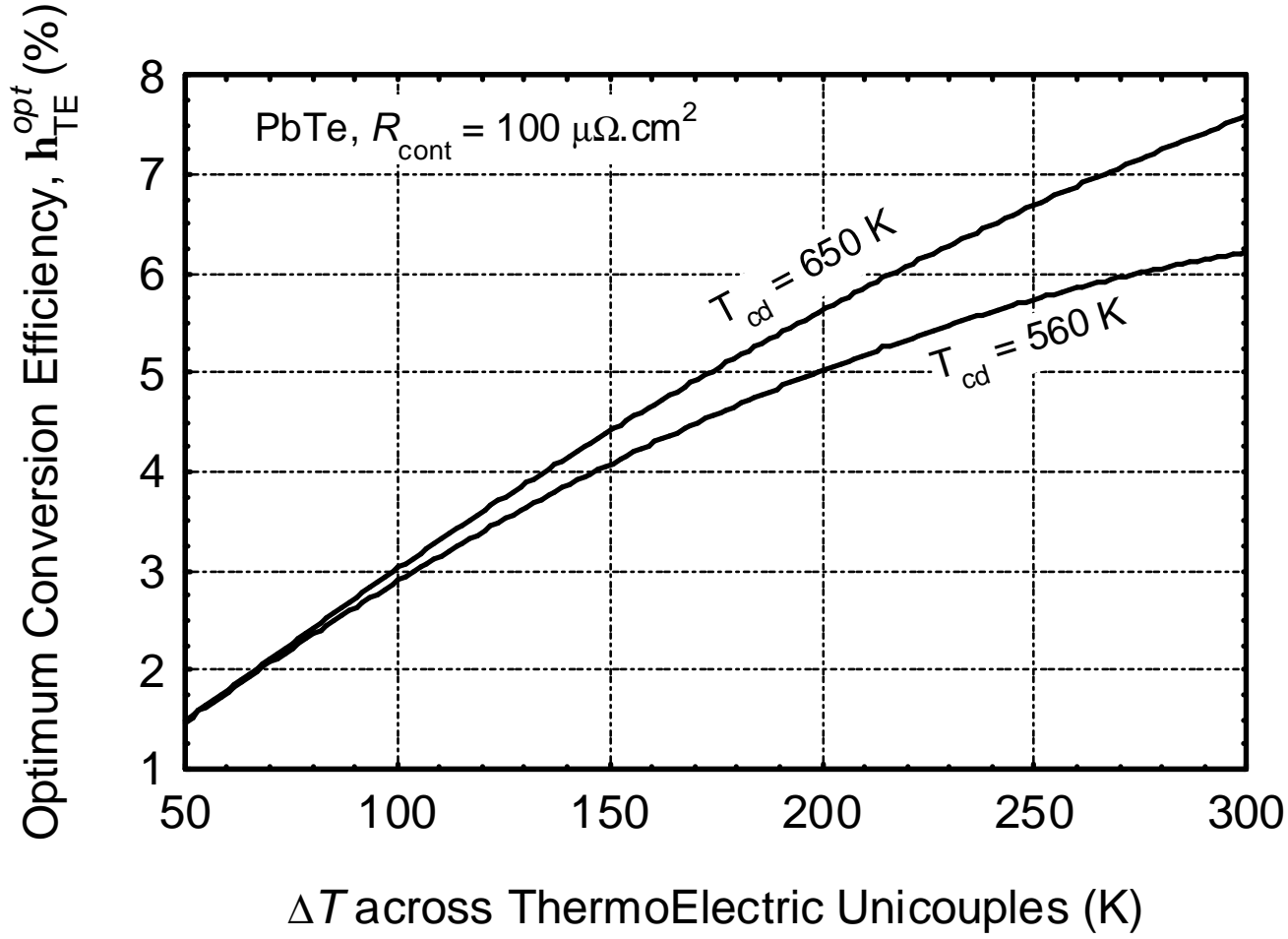

Figure 3-36. Optimum conversion efficiency of $\mathrm{PbTe}$ thermoelectric couples. 
The heat rejected by the outer housing of the heat pipe radiator from the cold shoe of the TE bottom cycle is then calculated as:

$$
Q_{\text {rad }}=Q_{\text {rej }}-P e^{T E}+Q_{\text {roof }}^{\text {loss }},
$$

which is used in conjunction with Equation (3-9) to calculate the heat pipe radiator temperature. Finally, the overall conversion efficiency of the AMTEC/TE converter unit is given by:

$$
\eta_{U N I T}=\frac{P e^{A M T E C}+P e^{T E}}{Q_{\text {input }}}=\frac{P e^{U N I T}}{Q_{\text {input }}} .
$$

As an illustration, the predicted heat flow rates and temperatures in the reference sodium and potassium AMTEC/TE converter units, at nominal operation, are shown in Figure 3.37 and Figure 3.38, respectively.

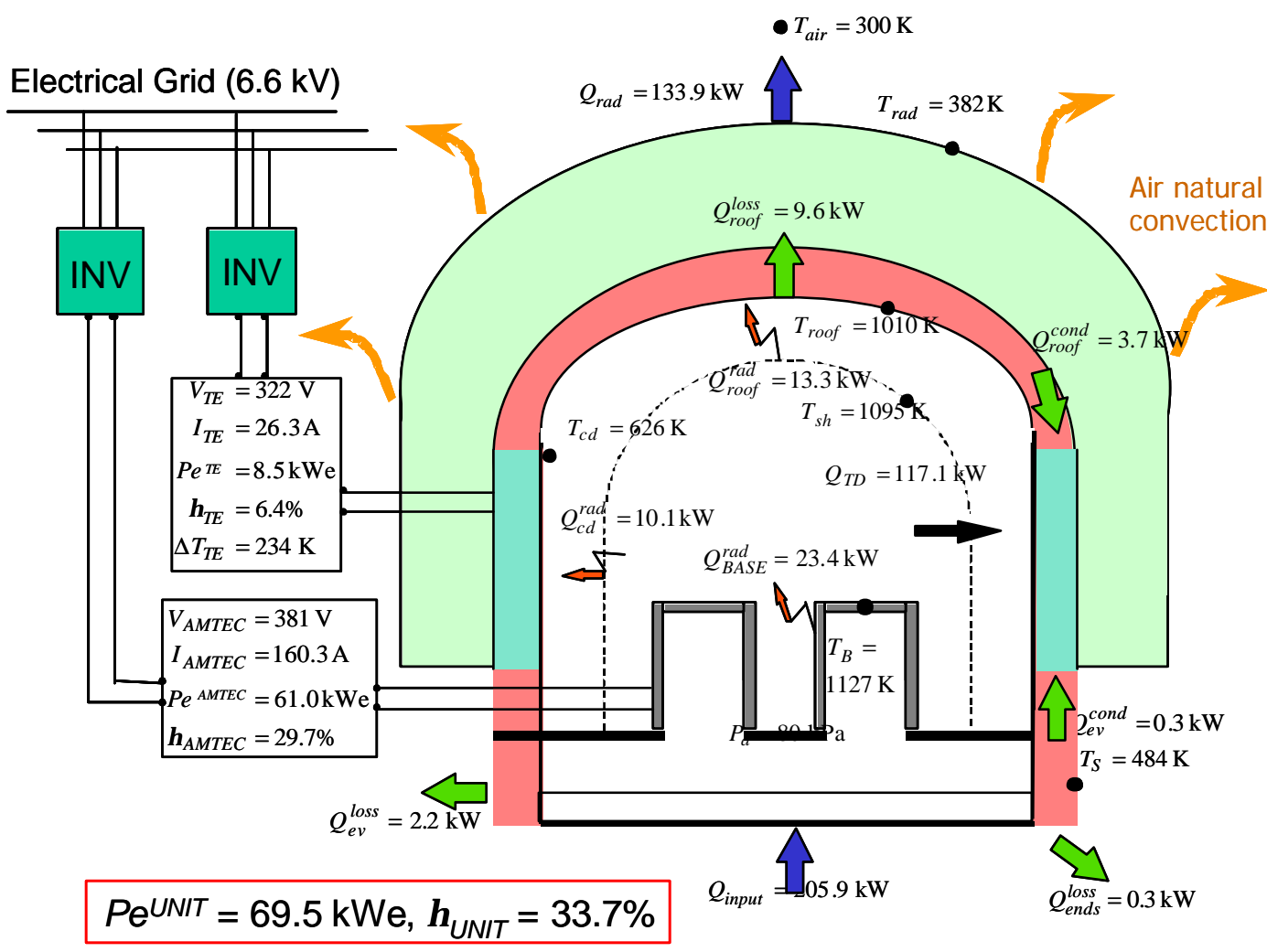

Figure 3-37. Predicted heat transfer rates and temperatures in the reference sodium AMTEC/TE converter $\left(N_{\text {rows }}=2, V_{\text {AMTEC }} \sim 400 \mathrm{~V}\right)$ at nominal operation and very near the peak efficiency $\left(T_{B}=1127 \mathrm{~K}, P_{a}=80\right.$ $\mathrm{kPa})$. 


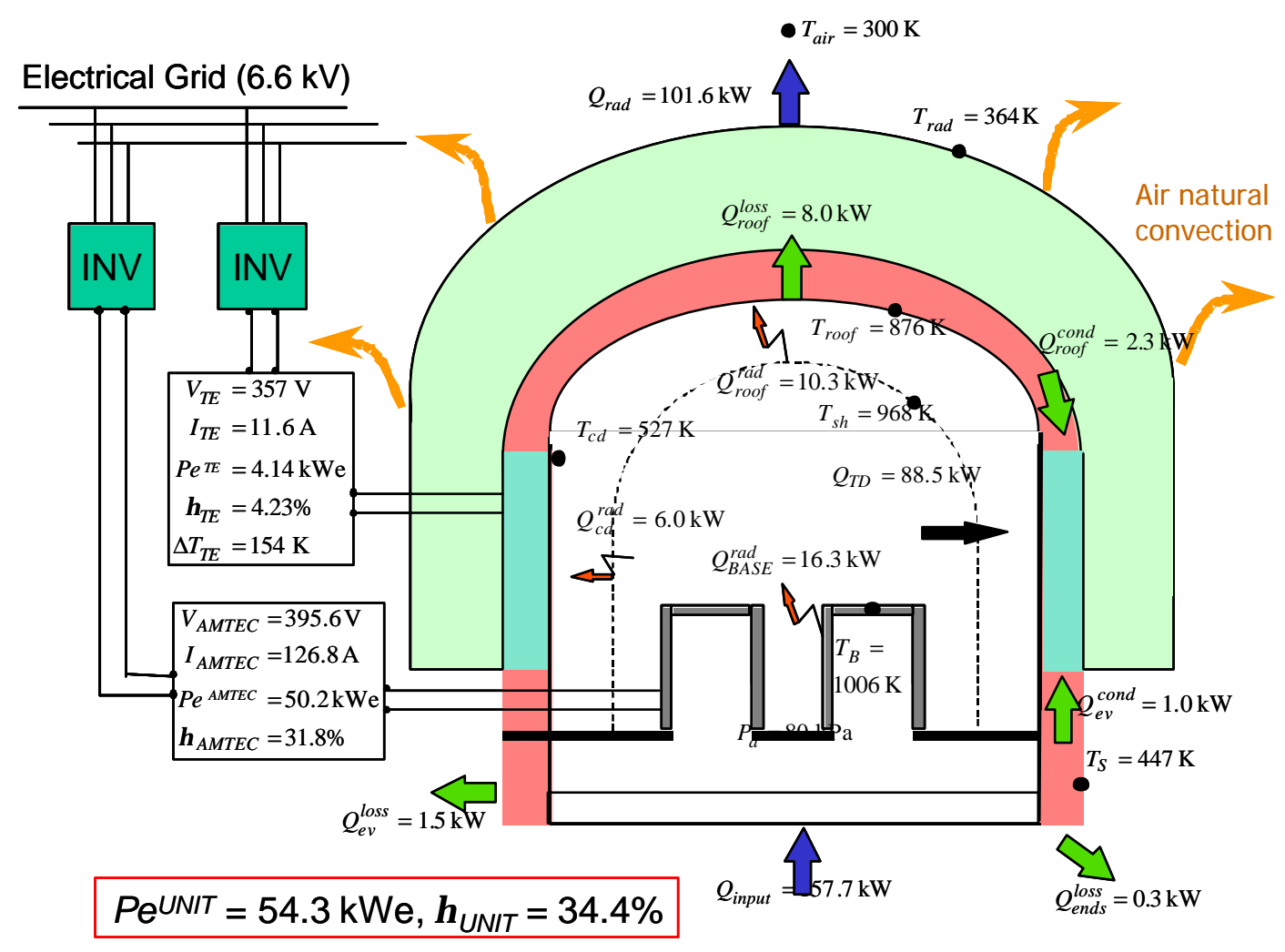

Figure 3-38. Predicted heat transfer and temperatures in the reference potassium AMTEC/TE converter $\left(N_{\text {rows }}=3, V_{\text {AMTEC }} \sim 400 \mathrm{~V}\right)$ at nominal operation and near the peak efficiency $\left(T_{B}=1006 \mathrm{~K}, P_{a}=80 \mathrm{kPa}\right)$.

\subsubsection{Optimization of AMTEC/TE Converter Design for Maximum Efficiency}

An optimization of the AMTEC/TE unit design (Figures 3.11 and 3.12) was performed for both the sodium and potassium AMTECs, for maximizing the conversion efficiency. The number of BASE elements electrically connected in series $(N)$ in the AMTEC unit is selected based on the desired voltage output and for an anode vapor pressure of $76 \mathrm{kPa}$ ( $T_{\mathrm{B}}=1121 \mathrm{~K}$ and $1000 \mathrm{~K}$ for the sodium and potassium AMTECs, respectively). The $\mathrm{WRh}_{1.5}$ electrode's surface area of each BASE element is calculated for a total electrodes' surface area of $37 \mathrm{~m}^{2}$ per unit. The height of the high pressure cavity of the AMTEC unit, including the thickness of the BASE support plate, is $H_{\text {pool }}=5 \mathrm{~cm}$, and the separation distance between the radiation heat shield and the condenser walls is $W_{\mathrm{CAN}}=$ $1.5 \mathrm{~cm}$ (Figures 3.11 and 3.12). The ambient air temperature is taken constant, at $T_{\text {air }}=$ $300 \mathrm{~K}$.

The sputtered BASE is $200 \mu \mathrm{m}$ thick, the anode porous substrate of the BASE elements is $2 \mathrm{~mm}$-thick, and the high-pressure inner spacing of the dome-shaped elements is $6.5 \mathrm{~mm}$ (see Figure 3.13). The current collector on the cathode side is made of 40-mesh molybdenum screen (254 $\mu \mathrm{m}$ wire diameter), and the molybdenum interconnecting leads between BASE elements have a specific voltage loss of $0.5 \mathrm{~V} / \mathrm{m}$. 
Such performance can be obtained with molybdenum leads with a total cross-section area of $50 \mathrm{~mm}^{2}$ (16 individual metal strips that are $3.12 \mathrm{~mm}^{2}$ in cross-section area). The analyses assume a condenser emissivity, $\varepsilon_{\mathrm{cd}}=0.20$, representative of a situation when the condenser surface is not perfectly wetted with a highly-reflective film of liquid alkali metal. The following design parameters are simultaneously optimized for maximum efficiency of the AMTEC/TE converter:

(a) aspect ratio of the BASE support plate $(W / L)$, controlled by the number of rows of BASE elements $\left(N_{\text {rows }}=1,2,3\right.$ and 4$)$;

(b) aspect ratio of the dome-shaped BASE elements $\left(H_{\mathrm{B}} / L_{\mathrm{B}}\right)$, with $H_{\mathrm{B}} \geq 10.5 \mathrm{~cm}$;

(c) separation distance between the BASE elements $(b)$;

(d) height of the condenser walls and of the thermoelectric modules $\left(H_{\mathrm{TE}}\right)$;

(e) size $\left(D_{\text {orif }}\right)$ and number $\left(N_{\text {orif }}\right)$ of orifices in the internal radiation shield;

(f) condenser temperature, $T_{\mathrm{cd}}$; and

(g) spacing $\left(W_{\text {fin }}\right)$ and the length $\left(H_{\text {fin }}\right)$ of the cooling fins.

To illustrate how these different parameters affect the performance of the AMTEC/TE unit, results of the parametric analyses performed are presented for the reference sodium AMTEC/TE unit, which consists of 2 rows of 336 BASE elements each, and operate at an output voltage of $400 \mathrm{~V} \mathrm{DC}$. The BASE support plate in the sodium-AMTEC (Figures 3.11 and 3.12) has dimensions $L=7.1 \mathrm{~m}$ and $W=0.64 \mathrm{~m}$, the vessel wall is $2 \mathrm{~mm}$-thick $\left(t_{\mathrm{W}}=2 \mathrm{~mm}\right)$, the Kaowool thermal insulation is $2.54 \mathrm{~cm}$ thick, and the height of the condenser wicks and thermoelectric modules is $H_{\mathrm{TE}}=20 \mathrm{~cm}$. The sodium anode pressure is $76 \mathrm{kPa}\left(T_{\mathrm{B}}=1121 \mathrm{~K}\right)$ and the condenser temperature, $T_{\mathrm{cd}}=650$ $\mathrm{K}$. The thickness of the cooling fins is $\delta_{\text {fin }}=1 \mathrm{~cm}$ and their length $H_{\text {fin }}=30 \mathrm{~cm}$; they are separated by a gap $\delta=3 \mathrm{~cm}\left(W_{\text {fin }}=4 \mathrm{~cm}\right)$. The current-voltage characteristic of the AMTEC converter is computed by varying the external load resistance, and the peak conversion efficiency of the AMTEC/TE combined cycle is calculated. Therefore, the parametric analysis curves shown and discussed next are a collection of the obtained performance parameters at the operating point corresponding to the overall peak conversion efficiency of the AMTEC/TE converter unit.

Note that the present optimization analyses, performed at constant hot side and condenser temperatures, are done to allow independent optimization of the performance of the AMTEC top and TE bottom cycles, and the selection of appropriate dimensions of the converter unit for maximizing the overall conversion efficiency. Once the optimum geometrical parameters are selected, an actual performance model is developed and used to predict the change in condenser temperature and output power of the converter unit in response to a change in external load. The results of such analysis are presented and discussed in Section 3.5.

\subsubsection{Effect of the Spacing between the BASE Elements}

The effects of varying the spacing, $b$, between the dome-shaped BASE elements in the AMTEC unit (Figures 3.11 and 3.12) on the thermal and electrical performance of the AMTEC/TE converter unit are shown in Figures $3.39-3.41$. Decreasing the spacing $b$ 
increases the resistance to the vapor flow between the BASE elements in the lowpressure cavity. As a result, the average pressure at the BASE/cathode interface increases sharply as $b$ decreases below $10 \mathrm{~mm}$ (Figure 3.39), and the electrical voltage and power outputs of the AMTEC top cycle decrease with decreasing this spacing (Figure 3.39).

On the other hand, the length of the converter, $L$, increases linearly and rapidly with increasing the spacing between the BASE elements, $b$ (see Figure 3.40), since there are 336 BASE elements (each is $11 \mathrm{~mm}$ wide) distributed along the length of the converter (see Figure 3.11). As a result, the radiation heat losses from he BASE elements and support plate increase linearly with increasing the spacing $b$, as well as the conduction heat losses through the side walls of the flash evaporator and to the condenser walls (Figure 3.40). Note that the electrical power output of the TE bottom cycle also increases with increasing $b$ (Figure 3.41), since the heat absorbed by the condenser (heat losses and heat of condensation due to increased AMTEC electrical current) increases. The net result is that the conversion efficiency of the AMTEC/TE converter unit peaks at some optimum value of $b=7 \mathrm{~mm}$ (Figure 3.41). Because the conversion efficiency curve is relatively shallow near the peak (Figure 3.41), a slightly higher value, $b=10 \mathrm{~mm}$ was selected in the present AMTEC unit design, to provide a higher electrical power output per converter and ensure enough spacing between the BASE elements to accommodate the current collectors and the electrical connectors (Figure 3.13).

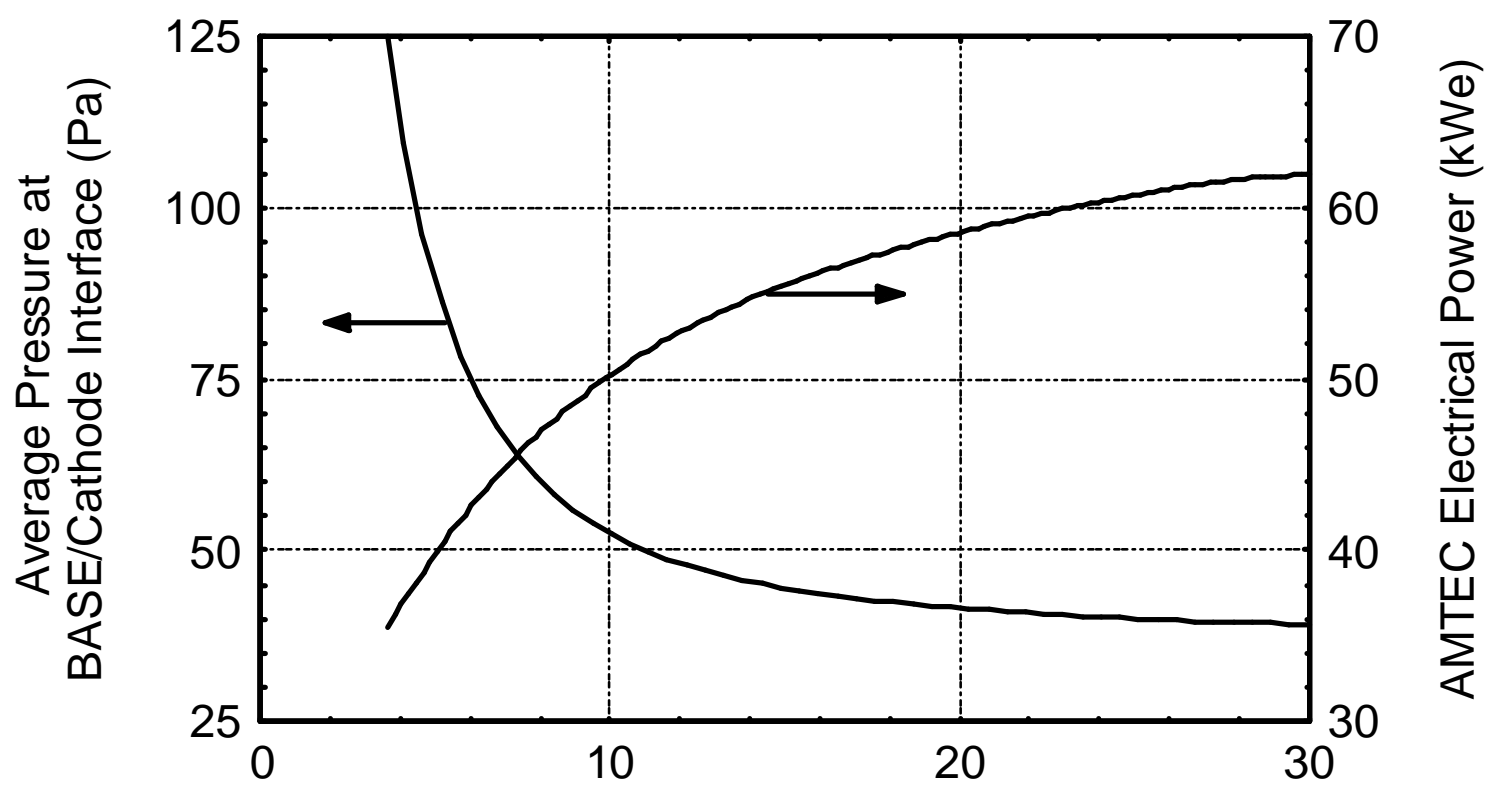

Spacing between BASE Elements, $b(\mathrm{~mm})$

Figure 3-39. Effect of the spacing between BASE elements, $b$ on the pressure at the BASE/cathode interface and on the power output of the AMTEC top cycle, at the peak efficiency. 


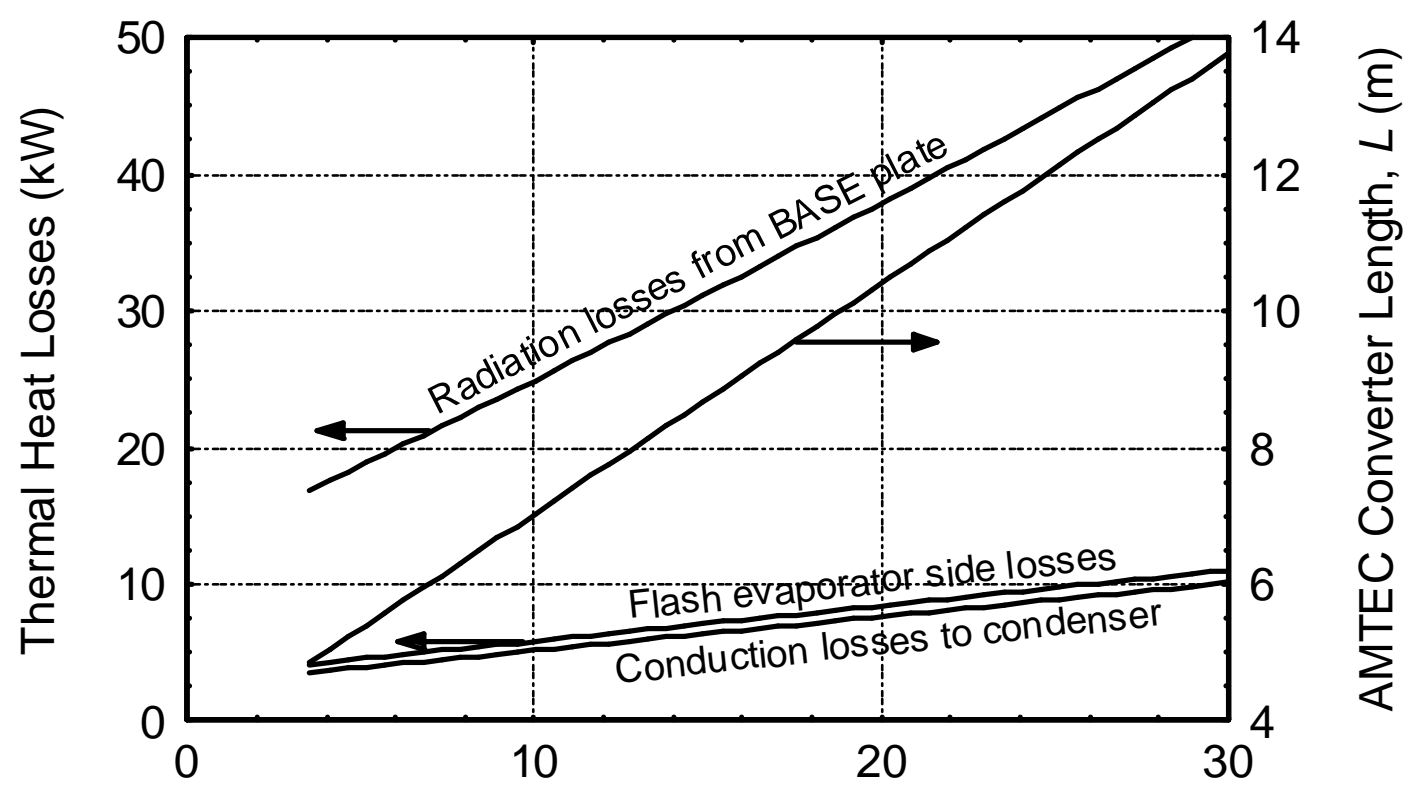

Spacing between BASE Elements, $b(\mathrm{~mm})$

Figure 3-40. Effect of the spacing between BASE elements, $b$ on the length of the converter and on the thermal heat losses, at the peak efficiency.

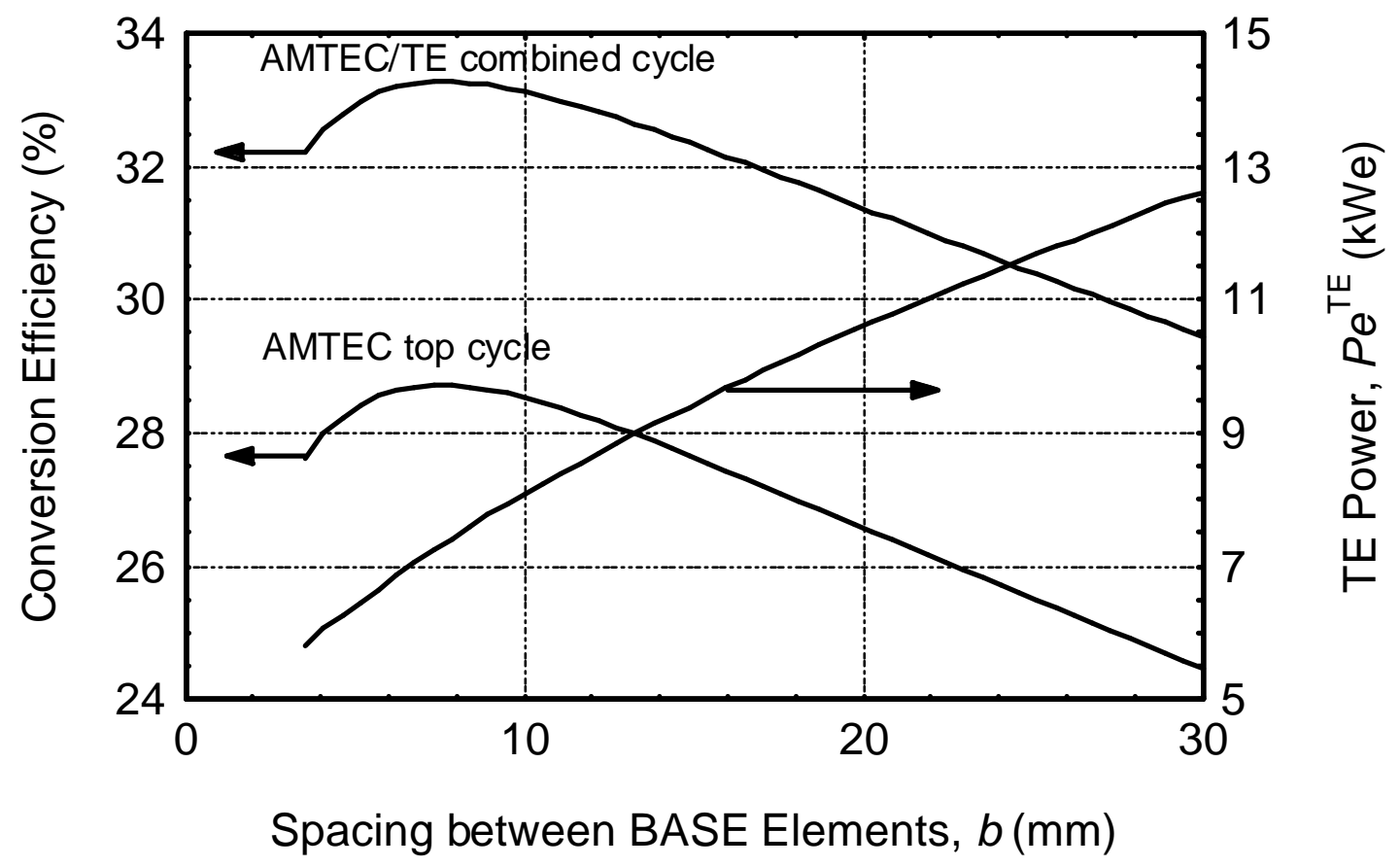

Figure 3-41. Effect of the spacing between BASE elements, $b$ on the power output of the TE bottom cycle and on the converter peak efficiency. 


\subsubsection{Effect of the Aspect Ratio of the Dome-Shaped BASE Elements}

The calculated effects of varying the aspect ratio $\left(H_{\mathrm{B}} / L_{\mathrm{B}}\right)$ of the dome-shaped BASE elements (Figure 3.16) on the thermal and electrical performance of the AMTEC/TE converter unit are shown in Figures 3.42 - 3.44. The lower the aspect ratio (the shorter and the wider are the BASE elements), the larger the width of the BASE support plate in the AMTEC unit, and the higher are the internal radiation heat losses (Figure 3.42).

On the other hand, increasing the aspect ratio of the BASE elements increases the path length $\left(H_{\mathrm{B}}\right)$ of the low-pressure vapor between the BASE elements, reducing the lateral flow area to the condenser, between the top of the BASE elements and the rooftop of the thermal radiation shield (Figure 3.12). As a result, the pressure at the BASE/cathode interface increases as the aspect ratio increases (Figure 3.43), and the output voltage and electrical power of the AMTEC top cycle decrease.

These two opposite effects cause the conversion efficiency of the AMTEC/TE converter to peak at an aspect ratio, $H_{\mathrm{B}} / L_{\mathrm{B}}=0.4$, however the peak is extremely shallow (Figure 3.44). Note that the electrical power output of the TE bottom cycle also decreases with increasing aspect ratio (Figure 3.44), since the heat to the condenser and the radiator surface area decrease. The peak in conversion efficiency is extremely shallow above $H_{\mathrm{B}} / L_{\mathrm{B}}=0.2$ and the electrical power output of the converter decreases with $H_{\mathrm{B}} / L_{\mathrm{B}}$, thus it is preferable to use a smaller aspect ratio, at the expense of decreasing the power density per unit area of BASE support plate. Therefore, a value $H_{\mathrm{B}}$ / $L_{\mathrm{B}}=0.4\left(H_{\mathrm{B}}=10.5 \mathrm{~cm}\right)$ is selected for our reference design, while keeping the height of the BASE elements $\geq 10.5 \mathrm{~cm}$.

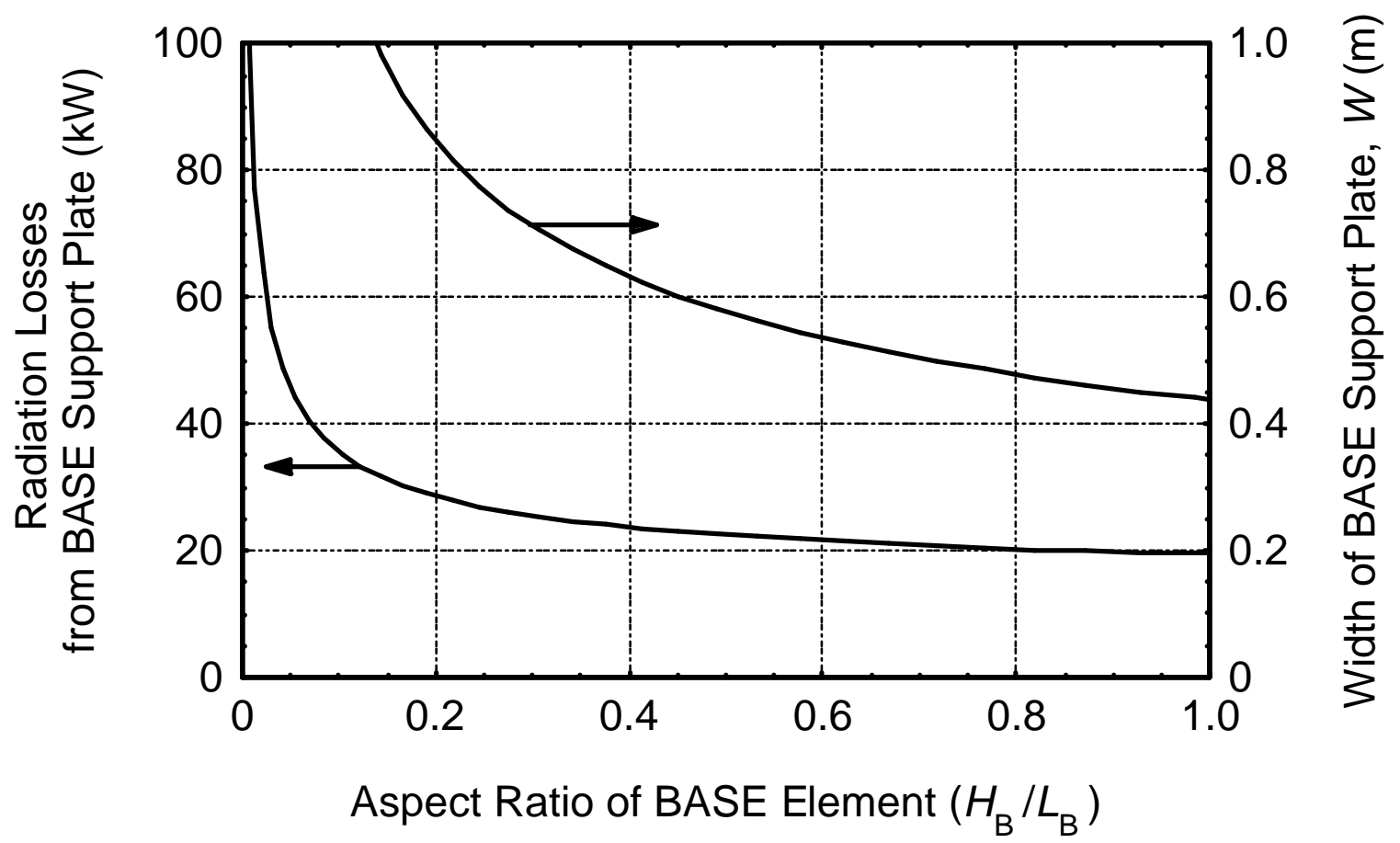

Figure 3-42. Effect of the aspect ratio of the BASE elements on the width of the converter BASE support plate and on the internal radiation losses, at the peak efficiency. 


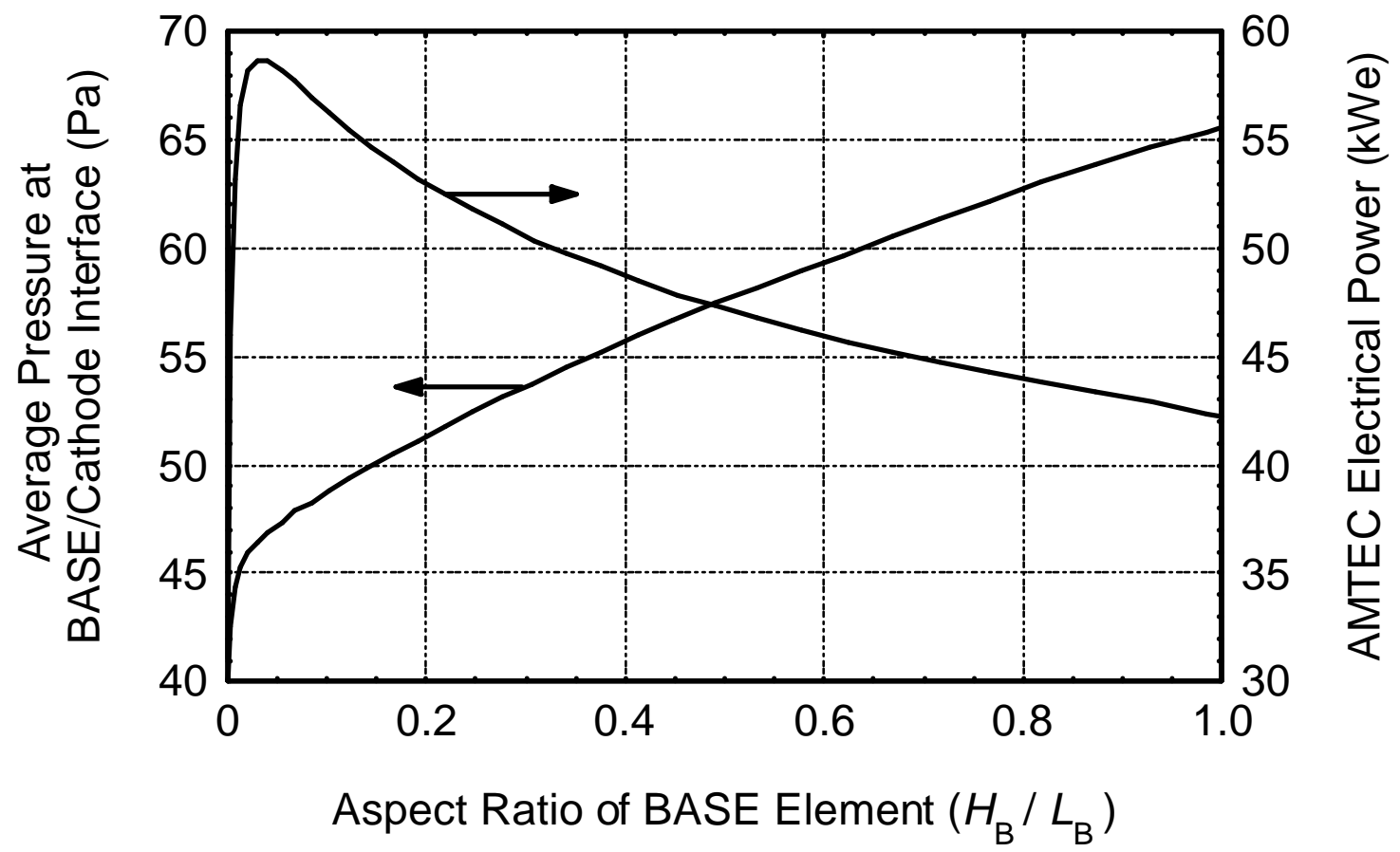

Figure 3-43. Effect of the aspect ratio of the BASE elements on the pressure at the BASE/cathode interface and on the power output of the AMTEC top cycle, at the peak efficiency.

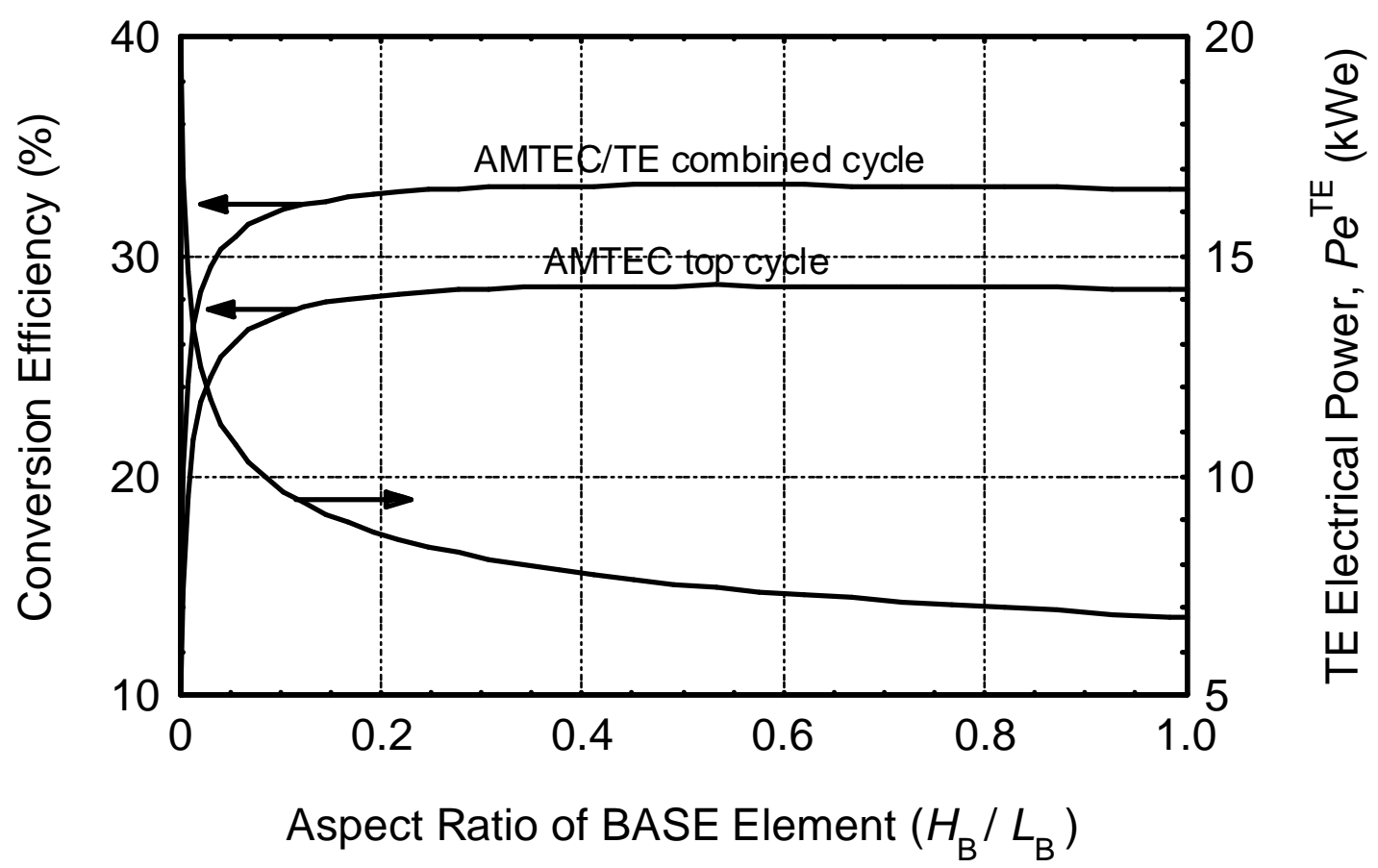

Figure 3-44. Effect of the aspect ratio of the BASE elements on the power output of the TE bottom cycle and on the converter peak efficiency. 


\subsubsection{Effect of the Height of the TE Modules/AMTEC Condenser Walls}

The predicted effects of varying the height of the AMTEC condenser walls, which is the same as the height of the thermoelectric modules, on the thermal and electrical performance of the AMTEC/TE converter unit, are shown in Figures 3.45 - 3.48. Decreasing the height of the condenser, $H_{\mathrm{TE}}$, reduces the lateral vapor flow area to the condenser, between the top of the BASE elements and the rooftop of the internal radiation shield (Figure 3.12), as well as reduces the area available for condensation of the alkali metal vapor. As a result, the pressure at the BASE/cathode interface decreases with increasing height $H_{\mathrm{TE}}$ (Figure 3.45), and the electrical voltage and power output of the AMTEC top cycle increase. The slight increase in the BASE/cathode interfacial pressure observed at $H_{\mathrm{TE}}>35 \mathrm{~cm}$ (Figure 3.45) is caused by the competing effects of decreasing flow resistance and increasing mass flow rate of the AMTEC working fluid, which is proportional to the electrical current of the AMTEC top cycle.

On the other hand, the internal radiation heat losses in the AMTEC unit increase linearly with $H_{\mathrm{TE}}$ (Figure 3.46), causing the heat input to the converter to increase. The conversion efficiency of both the AMTEC top cycle and the AMTEC/TE converter peaks at $H_{\mathrm{TE}}=11 \mathrm{~cm}$ (Figure 3.48).

The electrical power output of the TE bottom cycle also increases with increasing $H_{\mathrm{TE}}$ (Figure 3.46), since the heat absorbed by the condenser, which includes the radiation heat losses and latent heat of condensation, increases. Note that the electrical power output of the thermoelectric bottom cycle is not related to the number of thermoelectric couples, but only to the thermal power transferred to the TE modules from the AMTEC and the conversion efficiency of the TEs. Figure 3.47 shows that the temperature drop across the thermoelectric legs, and consequently the optimum conversion efficiency of the thermoelectrics, is essentially independent of $H_{\mathrm{TE}}$. This is because both the radiator area and the heat rejection rate increase commensurate with increasing $H_{\mathrm{TE}}$, and the radiator temperature is nearly constant. On the other hand, the average heat flux through the thermoelectric modules decreases with increasing $H_{\mathrm{TE}}$ (Figure 3.47), since the surface area of the TE modules increases faster than the heat flow to the TE modules. Therefore, for a given cross-section area of a lead telluride unicouple, a taller TE module requires using longer TE legs in order to operate at the optimum efficiency. This means that the volume of thermoelectric material required increases faster than the height of the TE modules, thus $H_{\mathrm{TE}}$ in the converter should be kept preferably small.

The optimum conversion efficiency and corresponding electrical power output of the AMTEC/TE converter are shown in Figure 3.48 as functions of the height of the TE modules. The peak efficiency of $33.5 \%$ occurs at $H_{\mathrm{TE}}=11 \mathrm{~cm}$, at which the electrical power output is $53 \mathrm{kWe}$. Since the electrical power output of the converter increases rapidly with $H_{\mathrm{TE}}$, a height of $H_{\mathrm{TE}}=20 \mathrm{~cm}$ is chosen for this design, which corresponds to a converter efficiency that is 0.3 percent point lower than the peak efficiency. At this design point, the converter electrical power output is $56.5 \mathrm{kWe}$, or $3.5 \mathrm{kWe}$ more than at the peak efficiency. This increase in electrical power output is well worth the small decrease in conversion efficiency (from $33.5 \%$ to $33.2 \%$ ). Economical analyses may investigate if the added cost of the increased volume of thermoelectric materials justifies 
the increase in the electrical power output of the AMTEC/TE converter, based on the estimated revenue per $\mathrm{kWhr}$.

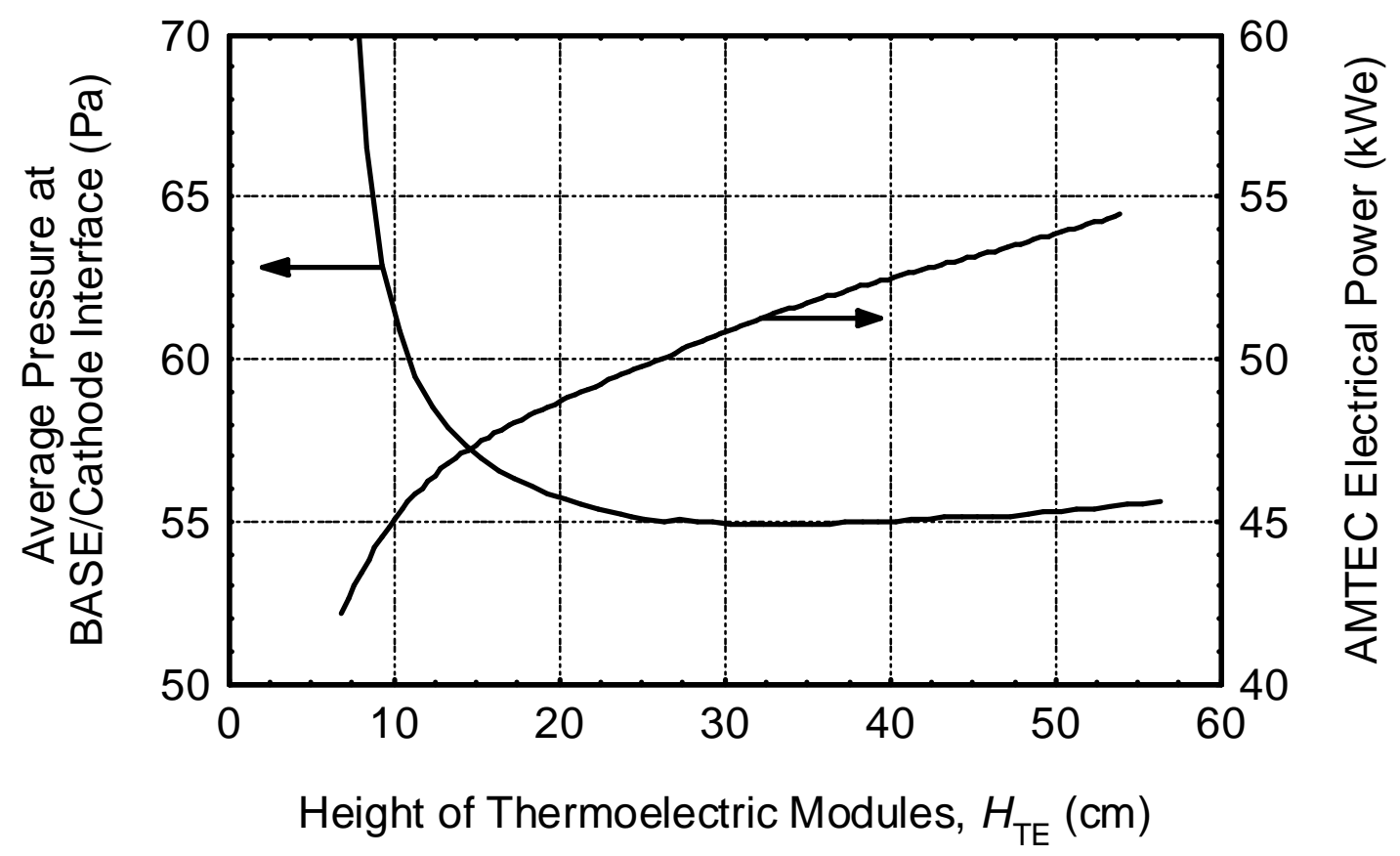

Figure 3-45. Effect of the height of thermoelectric modules on the pressure at the BASE/cathode interface and on the power output of the AMTEC top cycle, at the peak efficiency

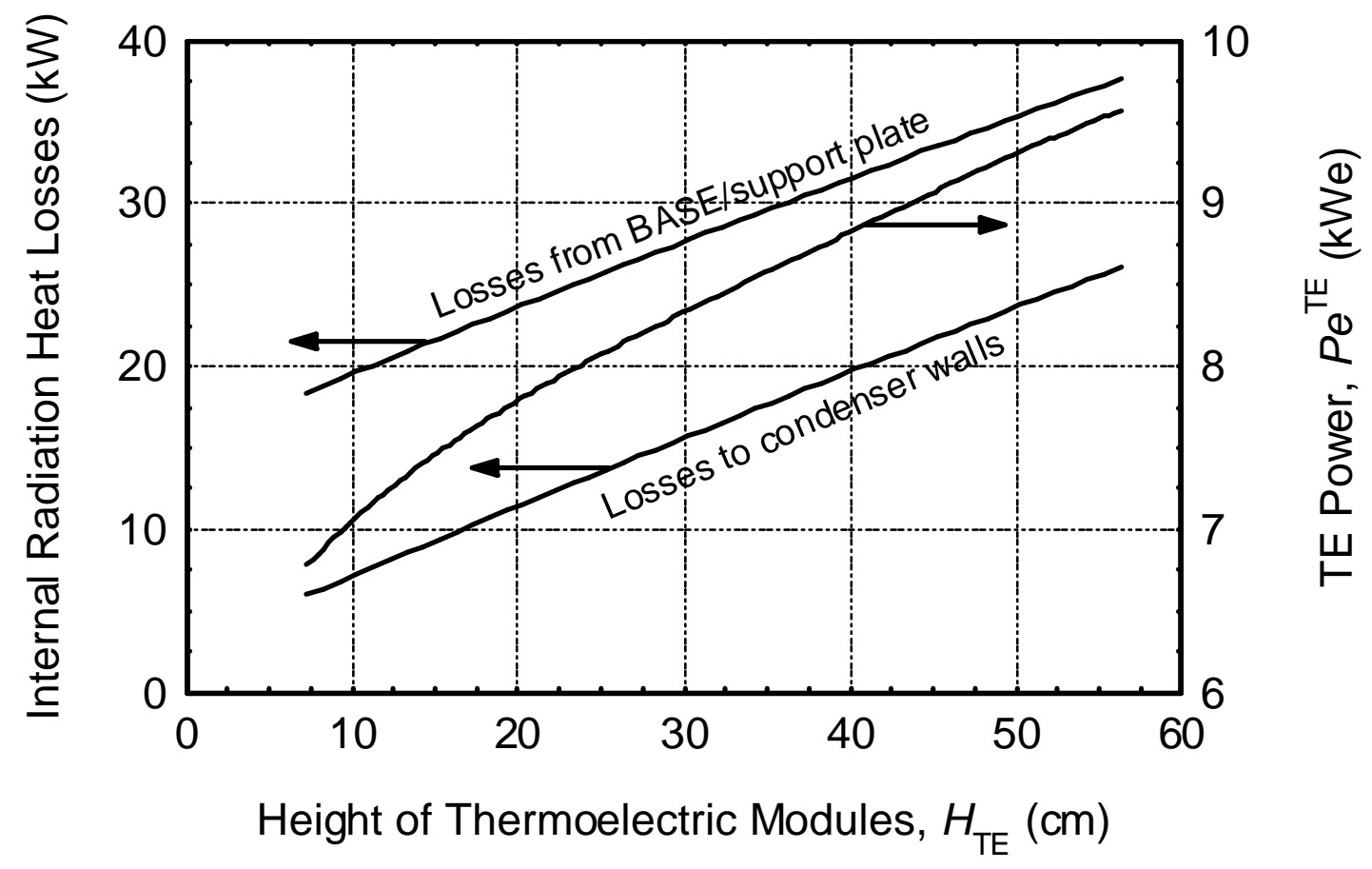

Figure 3-46. Effect of the height of thermoelectric modules on the internal radiation heat losses and on the power output of the TE bottom cycle, at the peak efficiency. 


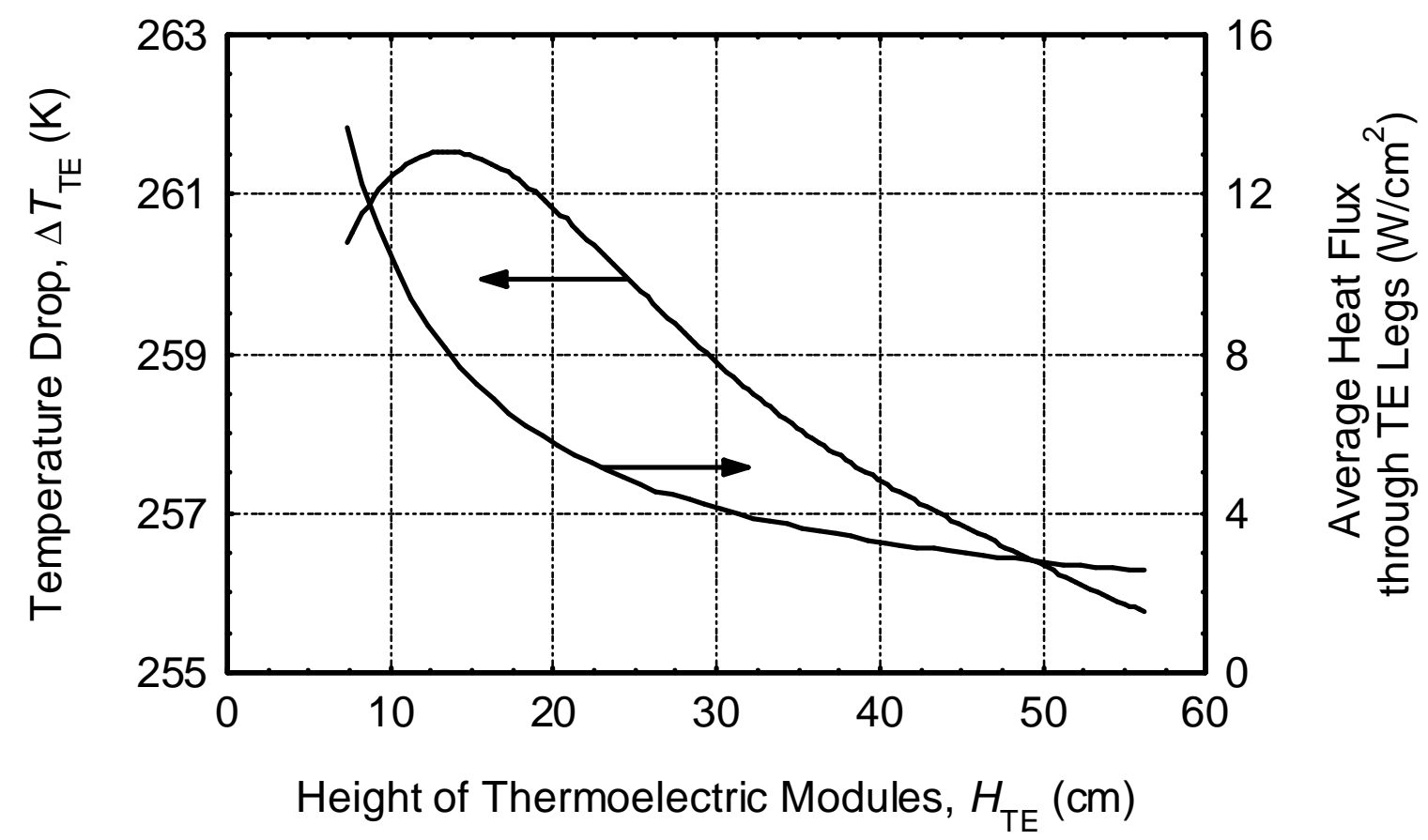

Figure 3-47. Effect of the height of thermoelectric modules on the temperature drop and average heat flux through the thermoelectric couples, at the peak efficiency.

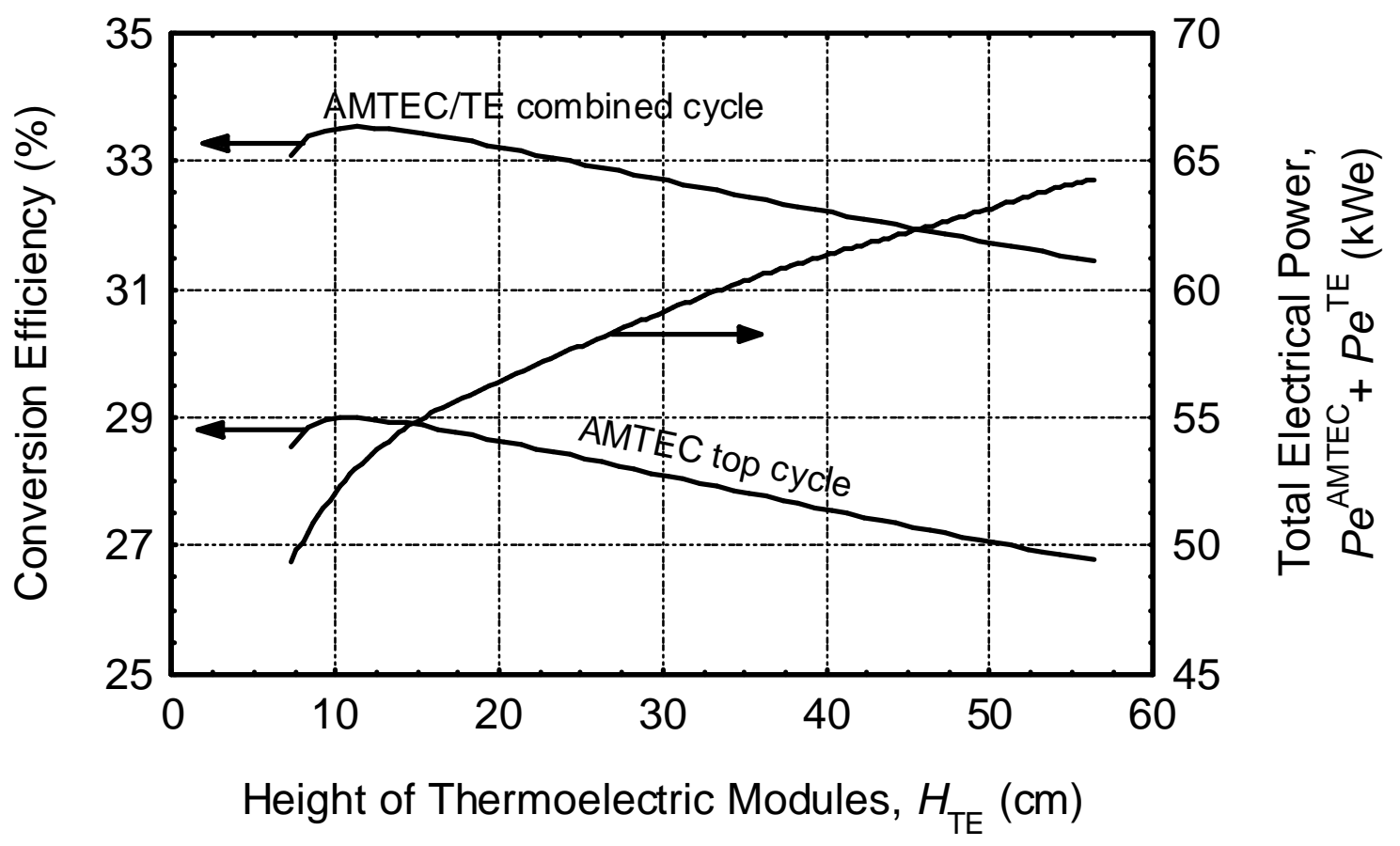

Figure 3-48. Effect of the height of thermoelectric modules on the power output of the converter and on the peak efficiency. 


\subsubsection{Effect of AMTEC Condenser Temperature}

The calculated effects of varying the AMTEC condenser temperature on the thermal and electrical performance of the AMTEC/TE converter are shown in Figures 3.49 - 3.52. As expected, radiation and conduction heat losses to the condenser walls of the AMTEC unit decrease as the condenser temperature increases (Figure 3.49). Below $T_{\mathrm{cd}}=600 \mathrm{~K}$, the condenser saturation pressure is essentially negligible compared to the pressure drop in the low-pressure cavity of the AMTEC, and both the average pressure at the BASE/cathode interface and the voltage output of the AMTEC unit are essentially constant (Figure 3.51). Above $600 \mathrm{~K}$, the condenser saturation pressure increases exponentially with $T_{\mathrm{cd}}$, and the pressure at the BASE/cathode interface increases rapidly with increasing $T_{\mathrm{cd}}$, causing the voltage (Figure 3.51) and the electrical power output (Figure 3.52) of the AMTEC unit to decrease. The decrease in heat losses and heat input to the converter as $T_{\mathrm{cd}}$ increases, in conjunction with the decrease in AMTEC electrical power output, causing the conversion efficiency of the AMTEC top cycle to peak at $T_{\mathrm{cd}}=$ $605 \mathrm{~K}$ (Figure 3.52).

On the other hand, the temperature drop across the thermoelectric bottom cycle increases almost linearly with increasing $T_{\mathrm{cd}}$ (which is also the hot shoe temperature of the thermoelectric unicouples), increasing the conversion efficiency of the TE bottom cycle (Figure 3.50). As a result, the total power output of the AMTEC/TE converter unit increases steadily with increasing condenser temperature up to $T_{\mathrm{cd}} \sim 700 \mathrm{~K}$ (Figure 3.52 ). The overall conversion efficiency of the AMTEC/TE converter unit peaks at a condenser temperature $T_{\mathrm{cd}}=640 \mathrm{~K}$ that is higher than that for the peak efficiency of the AMTEC top cycle $(605 \mathrm{~K})$. As shown in Figure 3.52, the efficiency curve near the peak is relatively shallow. A slightly higher condenser temperature, $T_{\mathrm{cd}}=650 \mathrm{~K}$ was selected for the sodium AMTEC/TE converter unit, resulting in a higher electrical power output of $56.5 \mathrm{kWe}$.

\subsubsection{Pressure Drop through the Orifices of AMTEC Radiation Heat Shield}

Another incentive for selecting a higher condenser temperature is to increase the vapor pressure in the low-pressure cavity of the converter, and keeping the alkali-metal vapor flow in the low-pressure cavity subsonic. The highest Mach number in the low-pressure cavity occurs in the orifices of the radiation shield (Figures 3.11 and 3.12). The total flow area of the orifices is kept relatively small, to limit the streaming of radiant energy between the BASE elements and the condenser walls, but large enough to ensure a subsonic vapor flow. The pressure drop caused by the low-pressure vapor flow through the circular orifices of the AMTEC internal radiation heat shield is calculated using the Dusty-Gas-Model (Tournier and El-Genk 1996):

$$
\Delta P_{\text {orif }}=\frac{t_{S H}}{\boldsymbol{D}}\left(\frac{R_{g} T_{1}}{M}\right) \times \dot{m}_{\text {orif }}^{\prime \prime}
$$

where $t_{S H}$ is the thickness of the metallic shield and the flow diffusion coefficient, $\boldsymbol{D}$, is given by: 
$\boldsymbol{D}=\boldsymbol{D}^{v i s}+\boldsymbol{D}^{K} \times \frac{1+c_{1}^{K} \bar{P}}{1+c_{2}^{K} \bar{P}}$

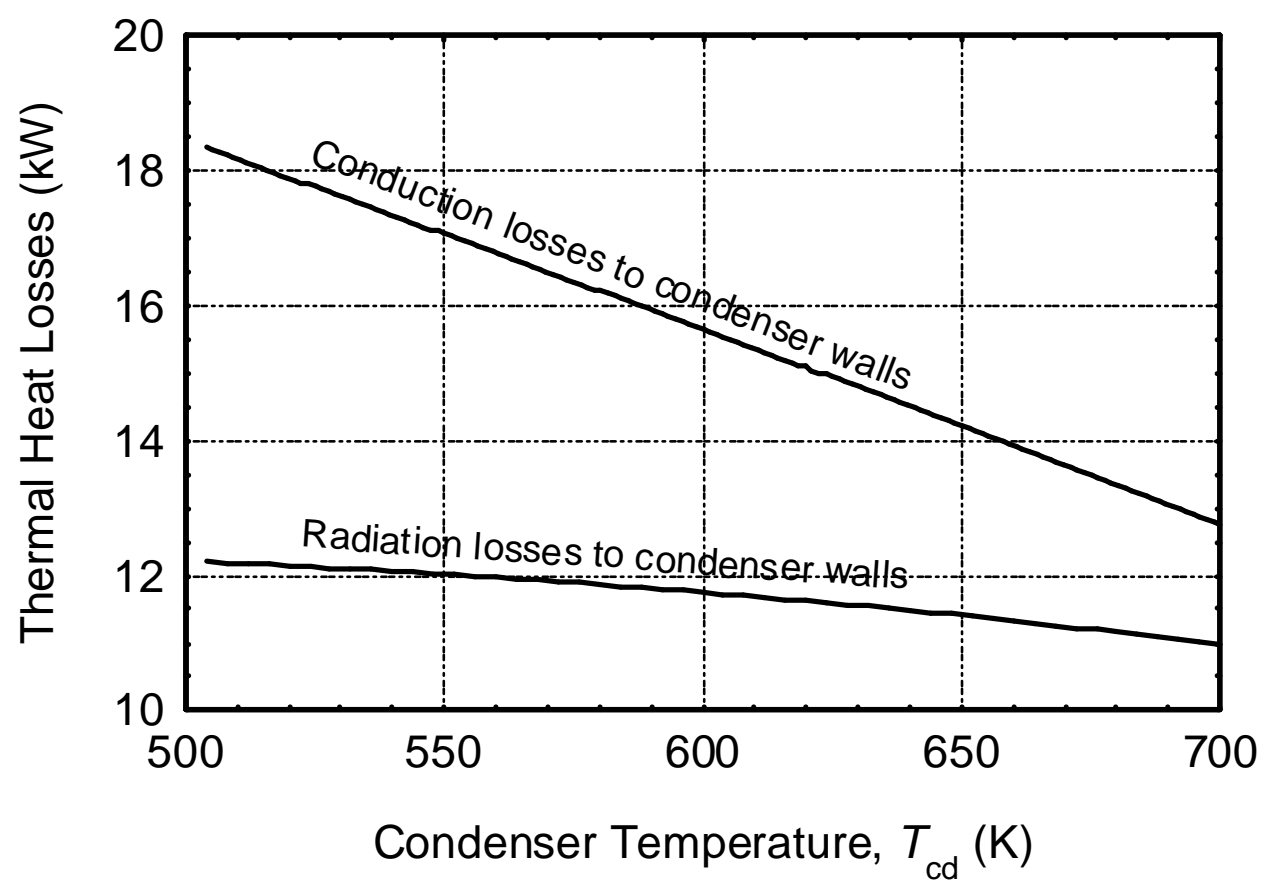

Figure 3-49. Effect of the condenser temperature of the converter on the conduction and radiation heat losses to the condenser walls, at the peak efficiency.

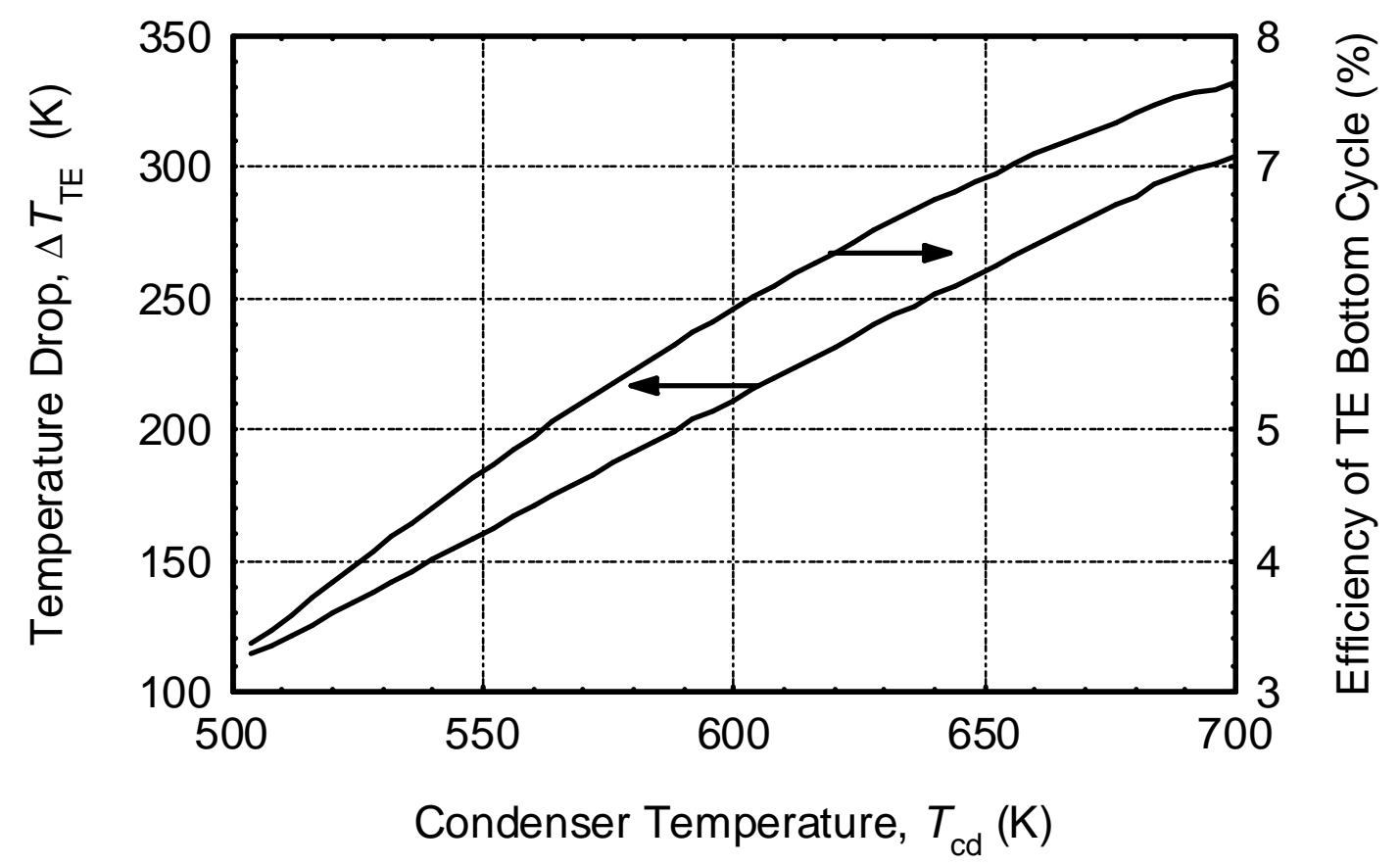

Figure 3-50. Effect of the condenser temperature of the converter on the temperature drop $\Delta T_{\mathrm{TE}}$ and on the efficiency of the thermoelectric bottom cycle. 


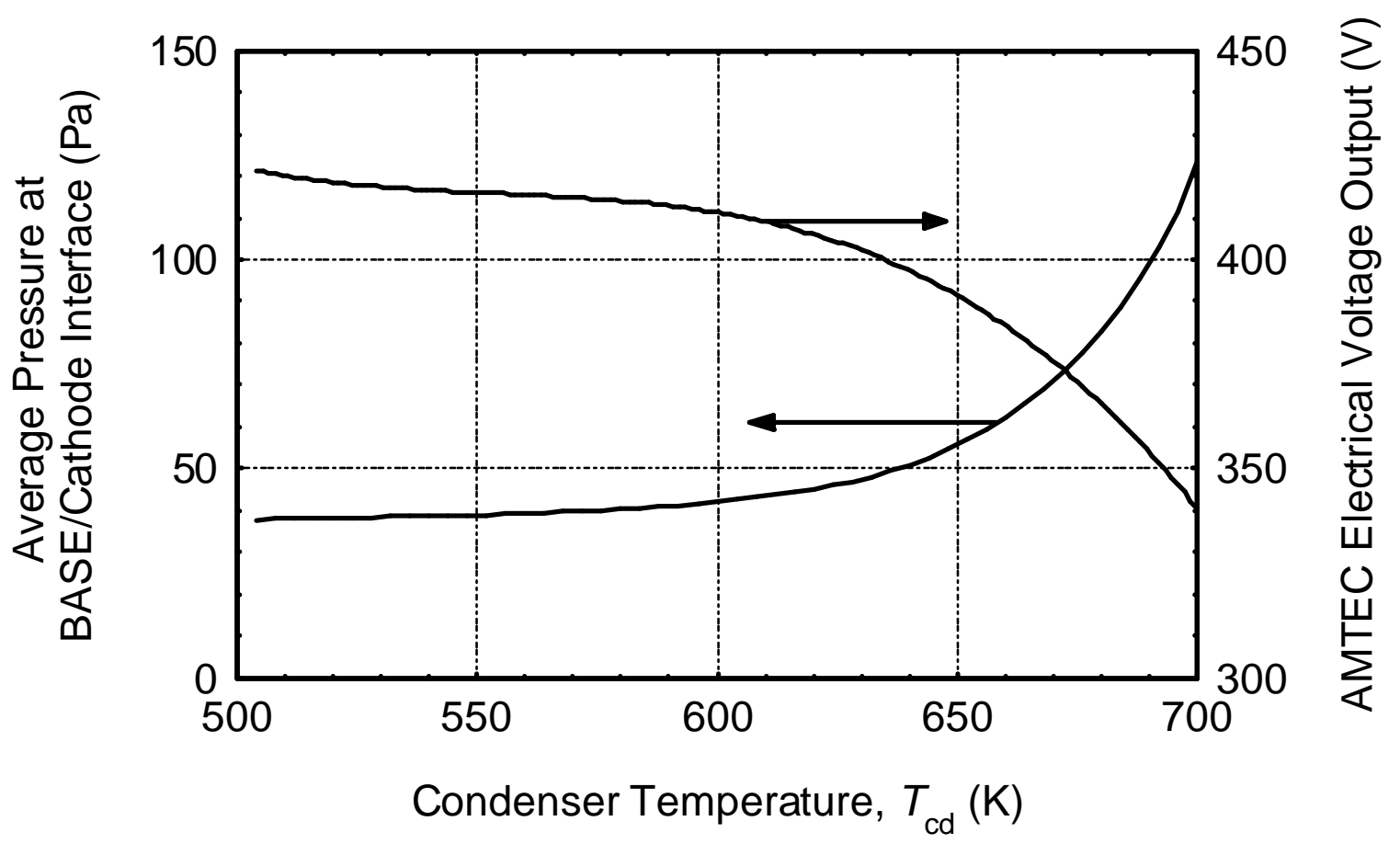

Figure 3-51. Effect of the condenser temperature of the converter on the pressure at the BASE/cathode interface and on the voltage output of the AMTEC top cycle, at the peak efficiency.

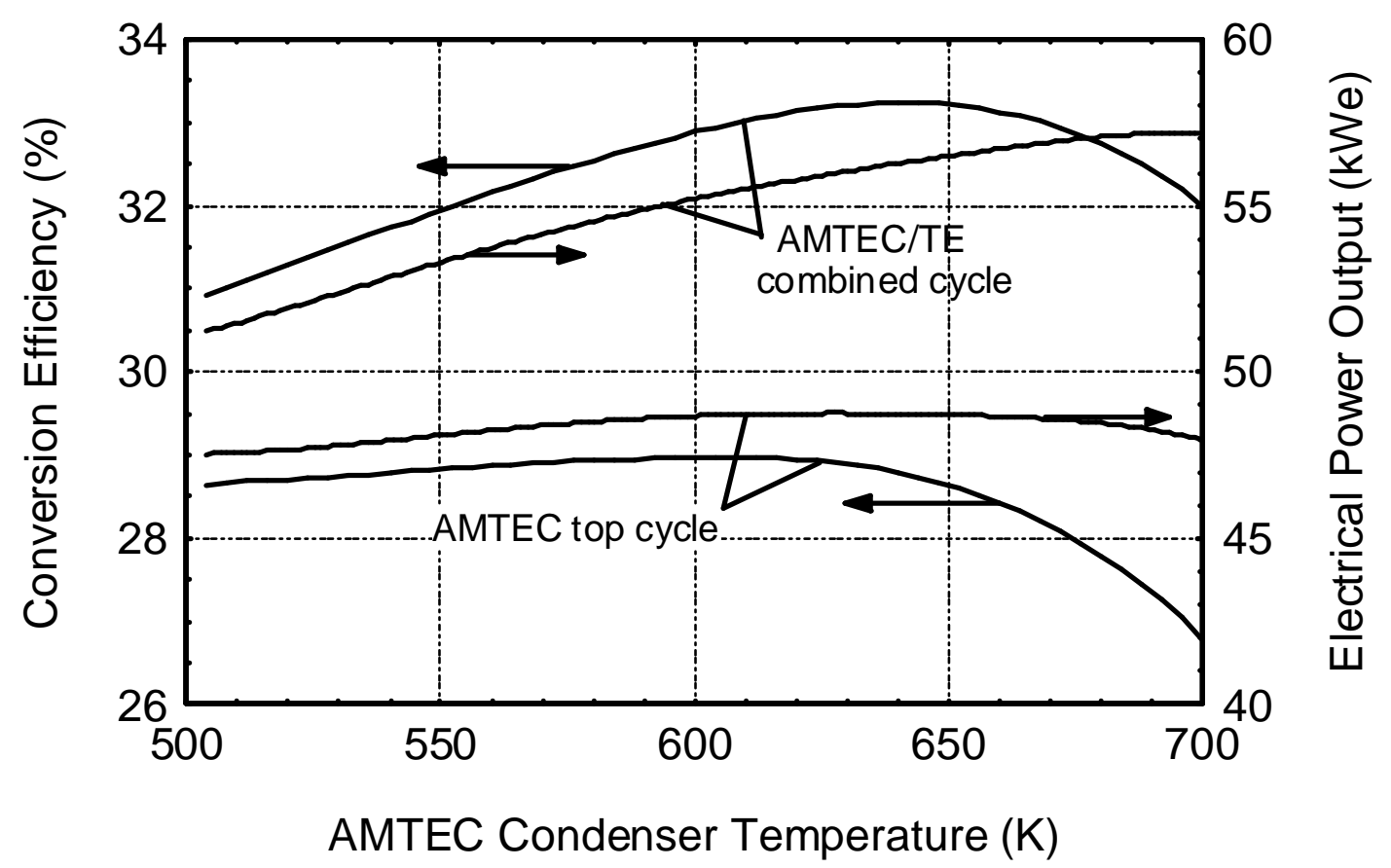

Figure 3-52. Effect of the condenser temperature of the converter on the electrical power output and peak conversion 
In Equation (3.41), $\bar{P}$ is the average vapor pressure in the orifices of the shield. The viscous and free-molecular flow diffusion coefficients are given by the Poiseuille and Dushman formulas, respectively (Tournier and El-Genk 1996, van Atta 1965, Tournier et al. 1999), as:

$$
\boldsymbol{D}^{v i s}=a^{K} \bar{P}=\frac{D_{\text {orif }}^{2}}{32 \mu_{V}} \bar{P}
$$

and

$$
\boldsymbol{D}^{K}=\frac{t_{S H}}{1+\frac{3 t_{S H}}{4 D_{\text {orif }}}} \times\left(\frac{R_{g} T_{1}}{2 \pi M}\right)^{1 / 2}
$$

Also,

$$
c_{2}^{K}=\frac{2 D_{\text {orif }}}{\mu_{V}} \times\left(\frac{M}{R_{g} T_{1}}\right)^{1 / 2}, \text { and } c_{1}^{K}=0.81 \times c_{2}^{K} .
$$

The Dusty Gas Model has been shown to accurately predict the pressure drop for gas flow in capillary tubes, in all 3 flow regimes, continuum, transition and free-molecule (Tournier and El-Genk 1996). These equations show that at very low vapor pressure, the flow diffusion coefficient, $\boldsymbol{D}$, reduces to the Knudsen diffusion coefficient for freemolecular regime, which is independent of pressure. At high pressure $(K n<0.02)$, however, the Knudsen diffusivity is small compared to the viscous flow diffusivity, which increases proportionally with the vapor pressure, and the flow becomes essentially continuum.

The mass flux of vapor molecules through the orifices is given by:

$$
\dot{m}_{\text {orif }}^{\prime \prime}=\dot{m}_{I} / A_{\text {orif }}
$$

where the total flow area through the orifices is:

$$
A_{\text {orif }}=N_{\text {orif }} \times \frac{\pi}{4} D_{\text {orif }}^{2}
$$

The area of the orifices must be small enough to minimize the direct view path between the BASE elements and the condenser walls, but large enough to ensure that 
$\Delta P_{\text {orif }}$ is a small fraction of the total pressure drop in the low pressure cavity, between the BASE/cathode interface and the condenser, and to ensure that the Mach number of vapor molecules through the orifices is $<1$. The vapor Mach number is given by:

$$
M a^{\text {orif }}=\frac{\dot{m}_{\text {orif }}^{\prime \prime}}{\bar{P}}\left(\frac{R_{g} T_{1}}{\gamma M}\right)^{1 / 2},
$$

where $\gamma$ is the specific heat ratio. For a monoatomic gas, $\gamma=5 / 3$.

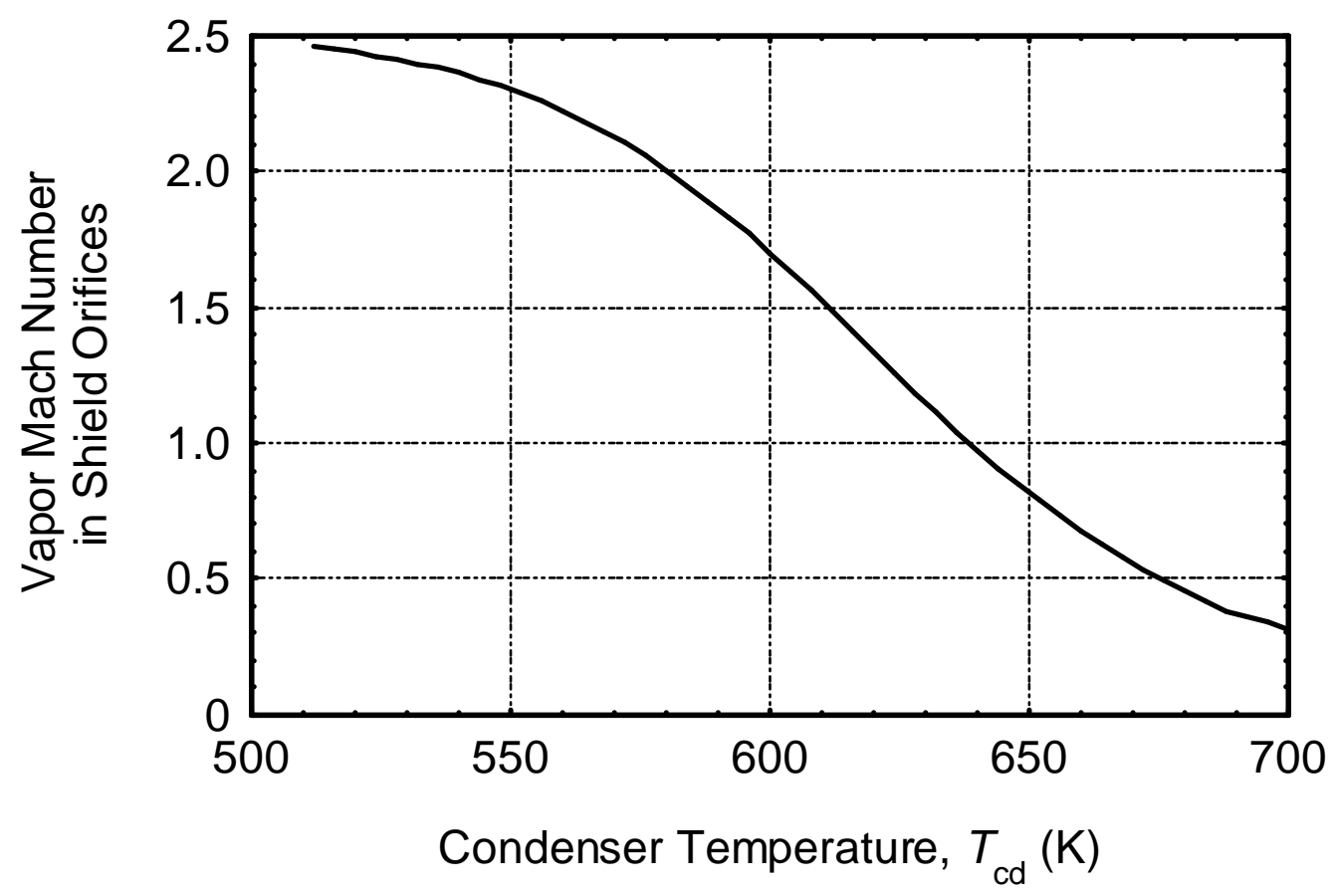

Figure 3-53. Effect of the condenser temperature of the converter on the vapor Mach number in the heat shield orifices.

The effect of condenser temperature on the Mach number of alkali-metal vapor flow in the orifices of the radiation shield in the AMTEC unit is shown in Figure 3.53. Such high Mach numbers arise because the vapor pressure in the low-pressure cavity of the converter is very low, $<100 \mathrm{~Pa}$ (Figure 3.51). Since the condenser saturation pressure increases exponentially with $T_{\mathrm{cd}}$, the Mach number decreases rapidly as the condenser temperature increases (Figure 3.53). The vapor flow in the low-pressure cavity is subsonic everywhere when $T_{\text {cd }} \geq 637 \mathrm{~K}$. 


\subsubsection{Effect of the Number of Orifices in the AMTEC Heat Shield}

The effect of the number of orifices in the heat shield, or the total vapor flow area through the orifices is shown in Figure 3.54. Increasing the number of the orifices decreases the vapor mass flux through the orifices and the vapor Mach number, according to Equations (3.47) - (3.49). Increasing the orifices area also increases the rate of radiant energy streaming through between the BASE elements and the condenser walls. To limit the rate of radiation heat losses from the BASE elements, the number of orifices in the radiation shield in the AMTEC unit is chosen $\left(N_{\text {orif }}=7000\right)$ such that the orifices' total area $\geq 10 \%$ the surface area of the two sides of the shield, while keeping the vapor flow subsonic (Figure 3.54).

\subsubsection{Effect of the Diameter of the Orifices in the AMTEC Heat Shield}

Equations (3.40) and (3.45) show that the pressure drop through the circular orifices of the radiation heat shield is inversely proportional to the product $\left(A_{\text {orif }} \times \boldsymbol{D}\right)$, which

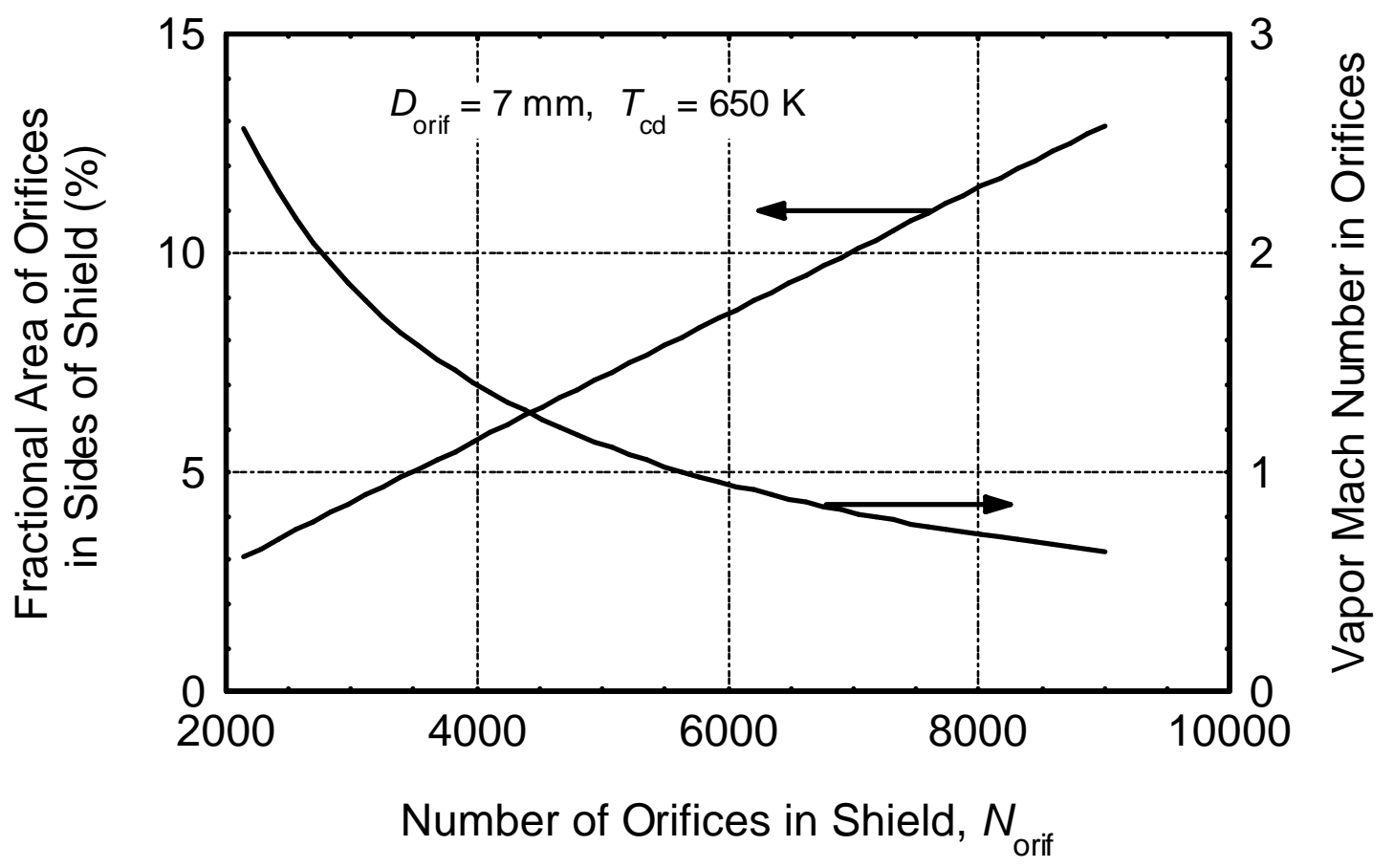

Figure 3-54. Effect of the number of orifices in the heat shield on the flow area and vapor Mach number in the orifices. 


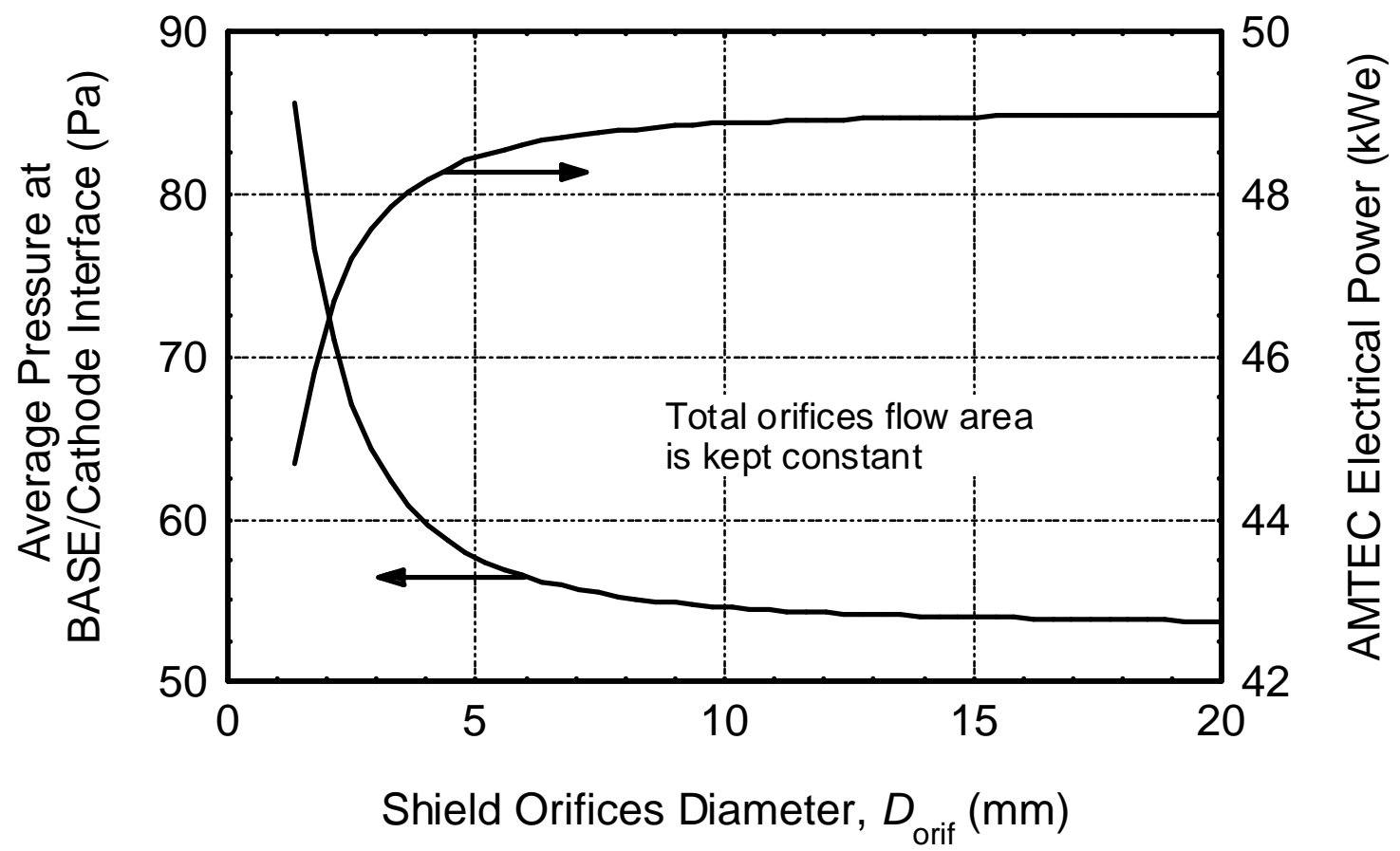

Figure 3-55. Effect of the orifices diameter in the heat shield on the pressure at the BASE/cathode interface and on the power output of the AMTEC top cycle, at the peak efficiency. The total orifices flow area is kept constant.

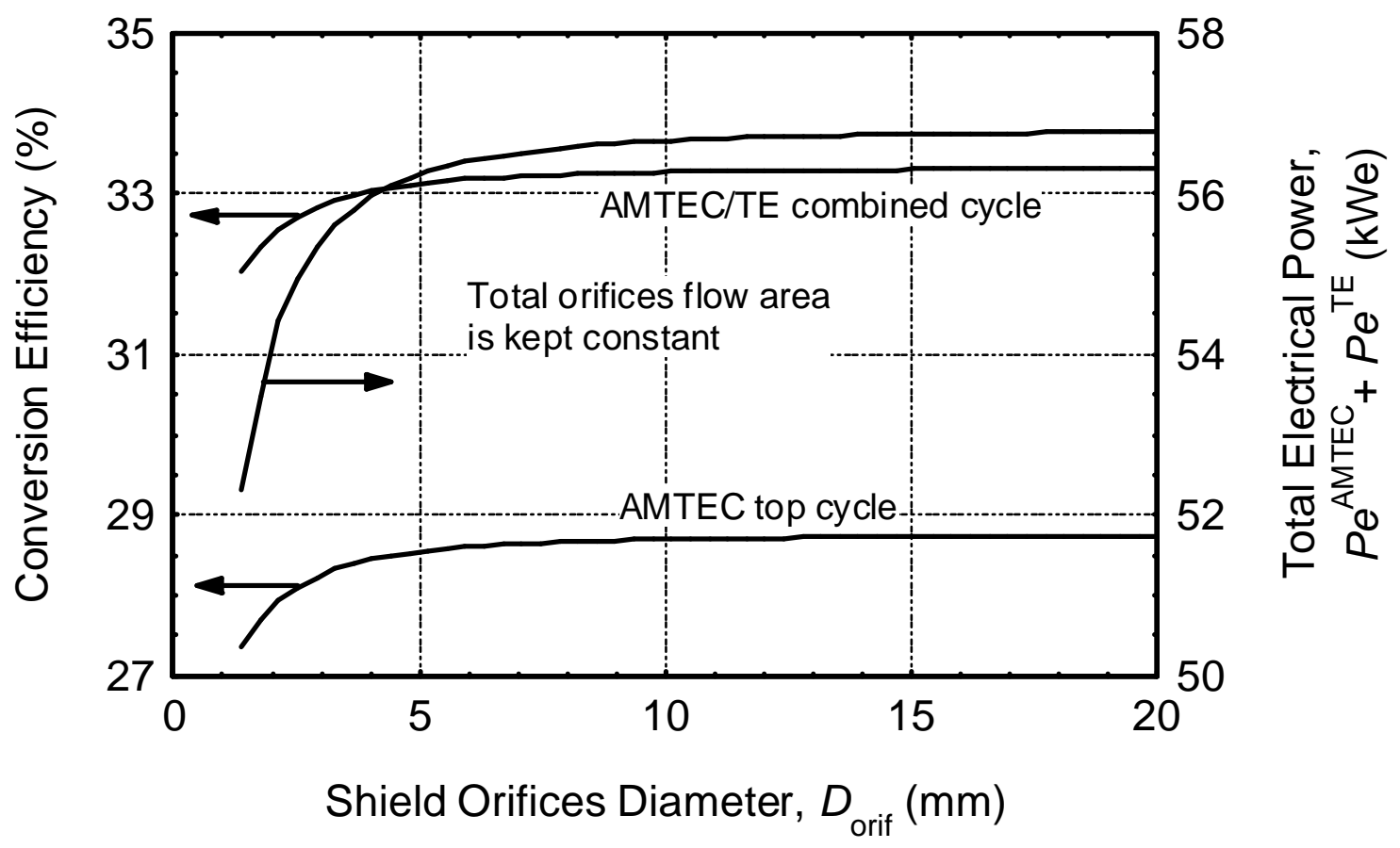

Figure 3-56. Effect of the orifices diameter in the heat shield on the peak efficiency and corresponding power output of the converter. The total orifices flow area is kept constant 
increases with the orifice diameter, $D_{\text {orif }}$. As a result, for a given flow area through the orifices, the pressure drop is lower when a fewer number of larger orifices is used. This effect is illustrated in Figure 3.55. For orifice diameters below $5 \mathrm{~mm}$, the average

pressure at the BASE/cathode interface increases rapidly as $D_{\text {orif }}$ decreases, causing a sharp decrease in the voltage and electrical power outputs of the AMTEC top cycle as well (Figure 3.55). For orifice diameters above $5 \mathrm{~mm}$, the peak conversion efficiency and the corresponding electrical power output of the AMTEC/TE converter increase very slowly, approaching asymptotic values (Figure 3.56). Based on these results, a value of $D_{\text {orif }}=10 \mathrm{~mm}$ was chosen for the reference AMTEC design.

\subsubsection{Effect of Radiator Cooling Fins' Geometry $\left(\mathrm{W}_{\text {fin }}\right.$ and $\left.\mathrm{H}_{\text {fin }}\right)$}

Since the performance optimization analyses discussed in Section 3.4.3 are performed at a fixed condenser temperature, $T_{\mathrm{cd}}$, the dimensions of the TE cooling fins (Figure 3.25) in the converter only affect the heat rejection radiator temperature and, therefore, the temperature drop across the thermoelectric unicouples, thus the performance of the TE bottom cycle. Obviously, increasing the length of the cooling fins, $H_{\text {fin }}$ increases the surface area of the cooling fins and reduces the surface temperature (or temperature of the cold shoe of the TE unicouples), increasing the conversion efficiency and electrical power output of the TE bottom cycle. A value of $H_{\text {fin }}=35 \mathrm{~cm}$ was selected and used in the optimized AMTEC/TE converter designs.

The heat rejection model from the TE bottom cycle was used to find the optimum spacing between the cooling fins to minimize the housing temperature and maximize the temperature drop across the thermoelectric unicouples. The results of this analysis, detailed in Sections 3.3.6 - 3.3.8, show that the optimum gap size between the "heat pipe" cooling fins is $\delta=1 \mathrm{~cm}\left(T_{\text {rad }}-T_{\text {air }}=52 \mathrm{~K}\right)$, at which the Rayleigh number, $R a \sim$ 2500 (see Figure 3.32), and the heat transfer is essentially that from a single isothermal vertical plate in an infinite media, as shown in Figure 3.30. Since the heat transfer correlation used in this work (Equation 3-13) pertains to natural convection of air between two isothermal, vertical parallel plates with all edges open to air flow, it does not directly apply to the present cooling fins as described in Figures 3.11 and 3.29. Therefore, a much larger gap size, $\delta=2.54 \mathrm{~cm}$ was selected. For a fin length, $H_{\text {fin }}=30$ $\mathrm{cm}$, and fins thickness $\delta_{\text {fin }}=1 \mathrm{~cm}, W_{\text {fin }}=\delta+\delta_{\text {fin }}=3.54 \mathrm{~cm}$, and the aspect ratio of the fins is $H_{\text {fin }} / W_{\text {fin }}=8.5$. The calculated housing temperature of the "heat pipe" coling fins, $T_{\mathrm{rad}}=375 \mathrm{~K}$ (Figure 3.32) for the reference sodium AMTEC/TE converter, is $23 \mathrm{~K}$ higher than its optimum value $(352 \mathrm{~K})$, which means that the temperature drop across the thermoelectric unicouples in the bottom cycle is $23 \mathrm{~K}$ lower than optimum.

The next Section reviews the design parameters and performance of the sodiumand potassium-AMTEC/TE converter designs for the LMR-AMTEC nuclear power plant. 


\subsection{Reference Sodium- and Potassium-AMTEC/TE Converters}

A fully-integrated performance model of the AMTEC/TE converter was developed, in which the condenser surface temperature is allowed to vary with the change in operating conditions. The condenser temperature was calculated by matching the heat flux at the condenser/TE hot shoe interface. The TE modules in the bottom cycle operated at their peak electrical power output, for which the external load resistance equals the modules' internal resistance. This performance model was then used to calculate the response of the AMTEC/TE converter to a change in the load demand of the AMTEC top cycle.

The nominal operation point was selected based on the following criteria:

(a) the hot shoe temperature of the P-TAGS- $85 / 2 \mathrm{~N}-\mathrm{PbTe}$ TE modules should not exceed $700 \mathrm{~K}$ when operating the AMTEC/TE converter units at $110 \%$ of nominal power. This condition applies in the Na-AMTEC/TE converter when the evaporator/BASE temperature $\left(T_{\mathrm{B}}\right)$ exceeded $1140 \mathrm{~K}$; and

(b) the overall efficiency of the AMTEC/TE converter units at $110 \%$ of nominal power equals the peak efficiency minus no more than one percentage point. This condition applies in the Na-AMTEC/TE converter when $T_{\mathrm{B}}<1140 \mathrm{~K}$, and always holds for the K-AMTEC/TE converter.

These selection criteria ensured that the AMTEC/TE converters operate within the load following portion of their characteristic (see Section 3.5.2.2), and can deliver an additional $10 \%$ of nominal power during peak demand, but at an efficiency that is no more than one percentage point below the peak efficiency.

\subsubsection{Design Parameters of Reference $\mathrm{Na}-$ and $\mathrm{K}$ - AMTEC/TE Converters}

The optimized sodium AMTEC/TE converter unit is $7.1 \mathrm{~m}$ deep, $1.35 \mathrm{~m}$ wide and $0.79 \mathrm{~m}$ high (Figure 3.57), while the optimized potassium AMTEC/TE converter unit is $6.2 \mathrm{~m}$ deep, $1.57 \mathrm{~m}$ wide and $0.87 \mathrm{~m}$ high (Figure 3.57). These dimensions include the heat pipe cooling fins $(0.35 \mathrm{~m})$ of the TE bottom cycle by natural convection of ambient air (Figure 3.59c). The Na-AMTEC converter is comprised of 672 elongated, dome-shaped, monolithic elements arranged in two rows (Figure $3.57 \mathrm{a}$ ), each with a $\mathrm{WRh}_{1.5}$ electrode surface area of $550 \mathrm{~cm}^{2}$, while the K-AMTEC converter has 744 elements of beta"alumina solid electrolyte (BASE) elements arranged in three rows, each with a $\mathrm{WRh}_{1.5}$ electrode surface area of $498 \mathrm{~cm}^{2}$. Other important design parameters are given in Table 3.4 .

The BASE elements in the potassium converter developed for the LMR-AMTEC nuclear power plant are $11 \mathrm{~cm}$ high and $1.2 \mathrm{~cm}$ wide in outside dimension, and separated by a 1.4 $\mathrm{cm}$ gap to allow the low-pressure, alkali-metal ( $\mathrm{Na}$ or $\mathrm{K}$ ) vapor to flow from the cathode electrode to the AMTEC's condenser (Figures 3.58a and 3.59a). The inside of the BASE elements is open to the high vapor pressure cavity of the AMTEC unit (Figure 3.58b). Each dome-shaped BASE element consists of a thin BASE membrane (50-200 $\mu \mathrm{m}$ 
thick), deposited by plasma spraying or sputtering techniques (Nicholson et al., 1995) onto a rigid porous anode substrate made of pressed and sintered molybdenum-rhenium powder.

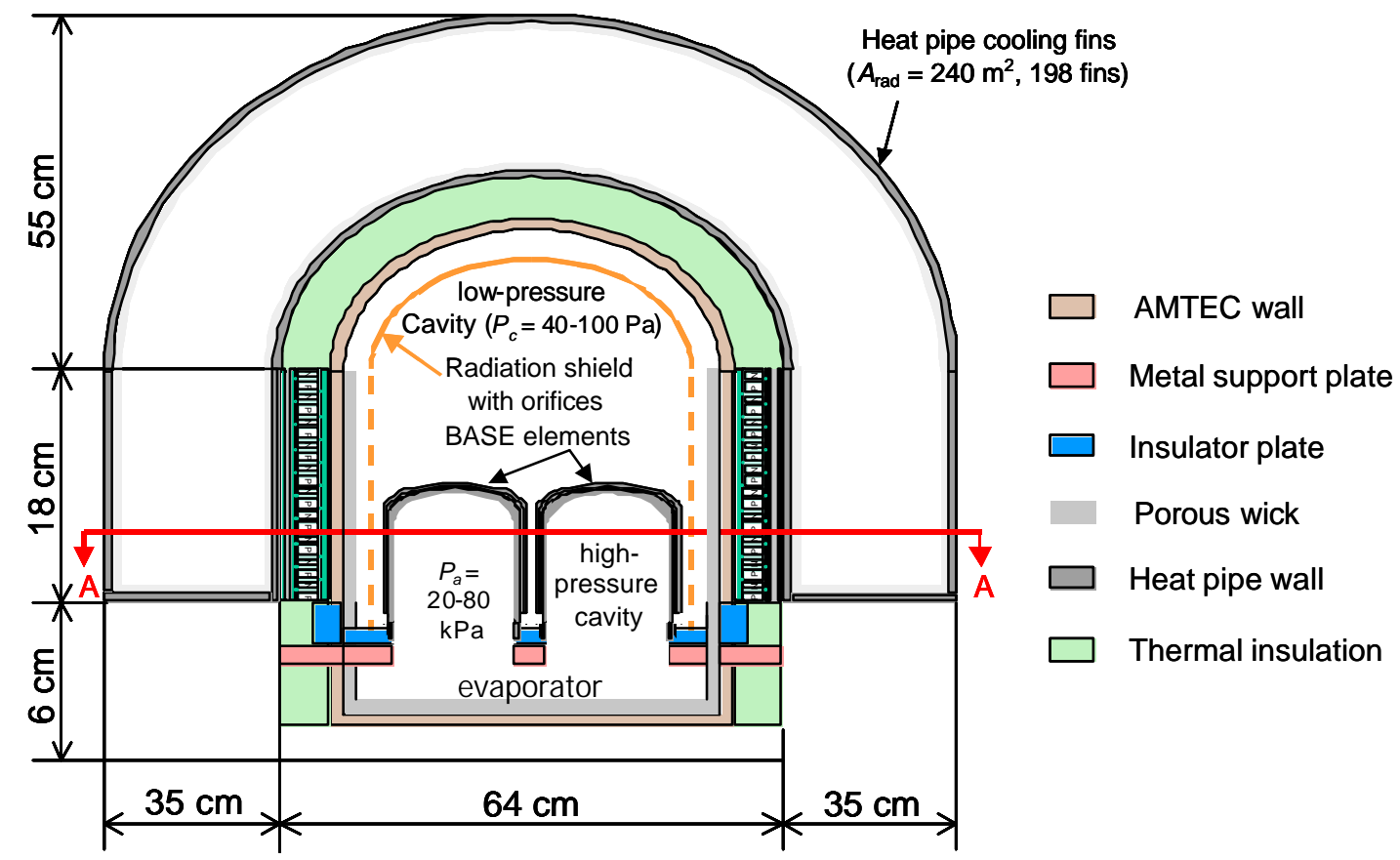

(b) Elevation view B - B

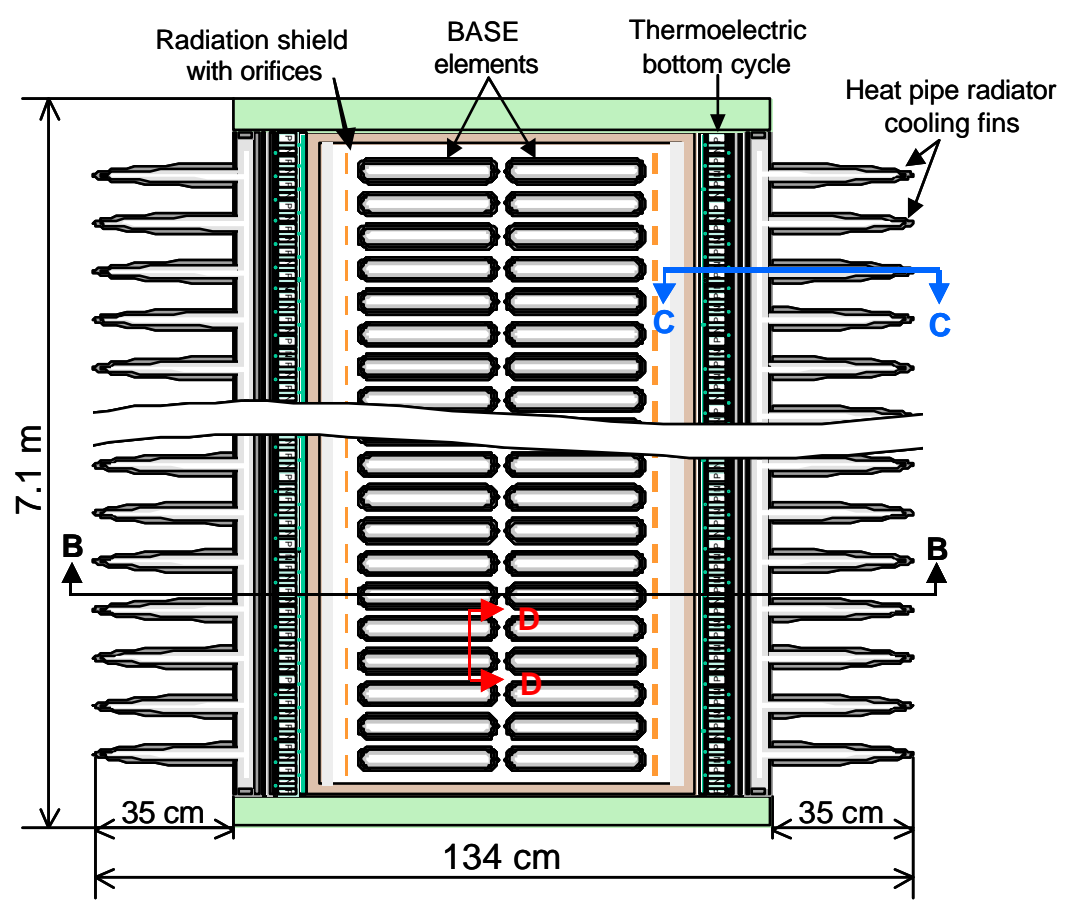

(a) Plane view $\mathrm{A}-\mathrm{A}$

Figure 3-57. Cross-sectional views of optimized sodium-AMTEC/TE converter. 


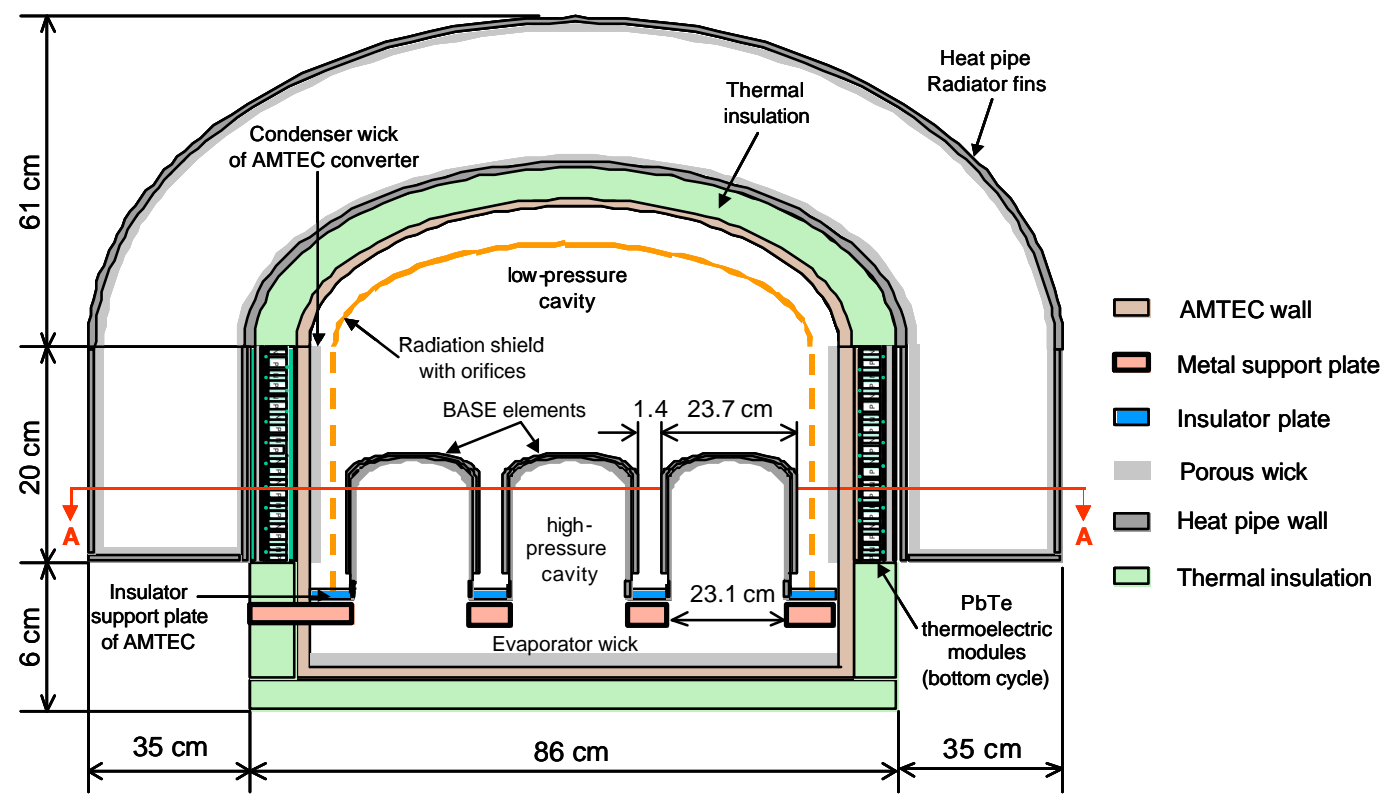

(b) Elevation view $\mathrm{B}-\mathrm{B}$

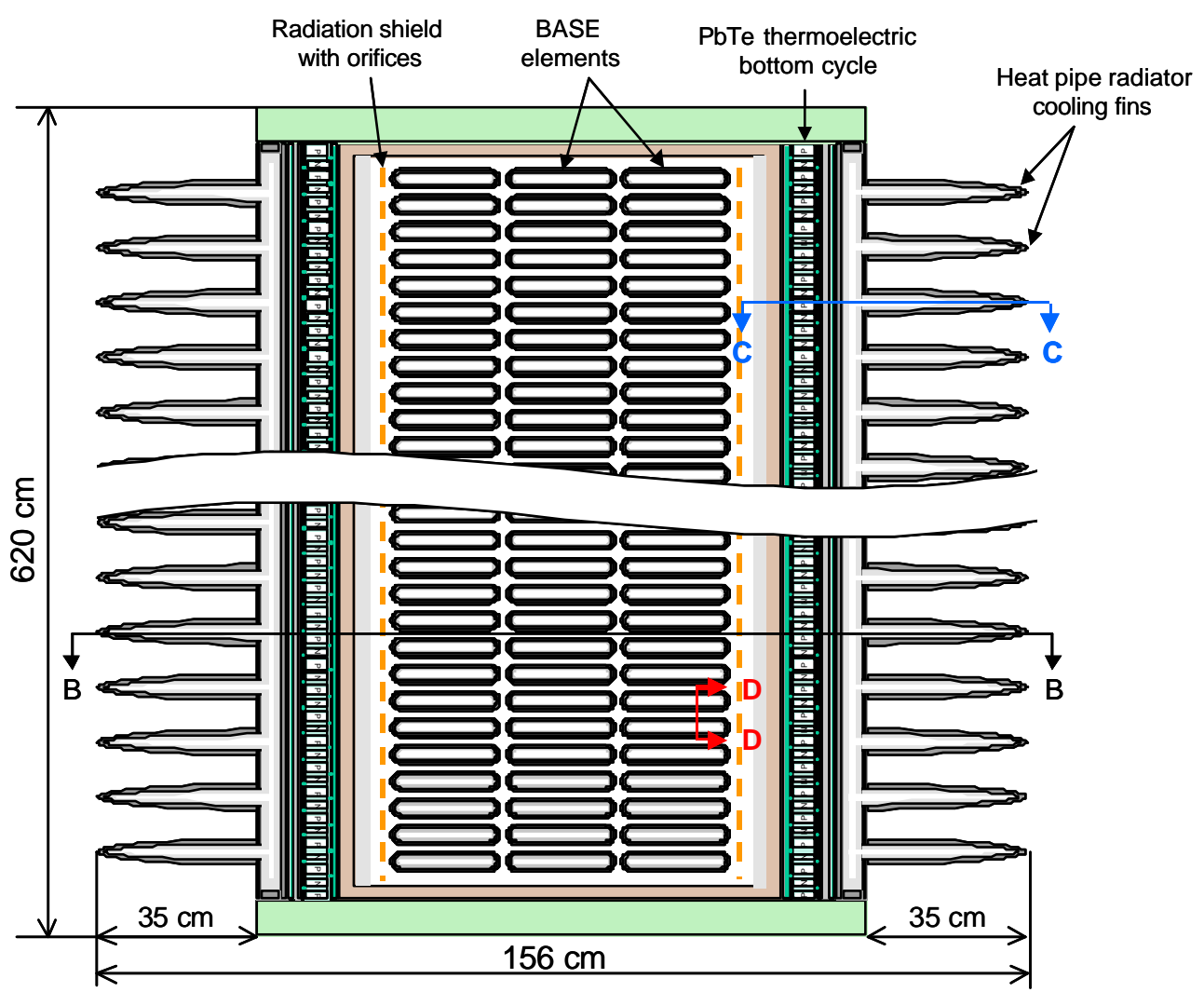

(a) Plane view $\mathrm{A}-\mathrm{A}$

Figure 3-58. Cross-sectional views of optimized potassium-AMTEC/TE converter. 


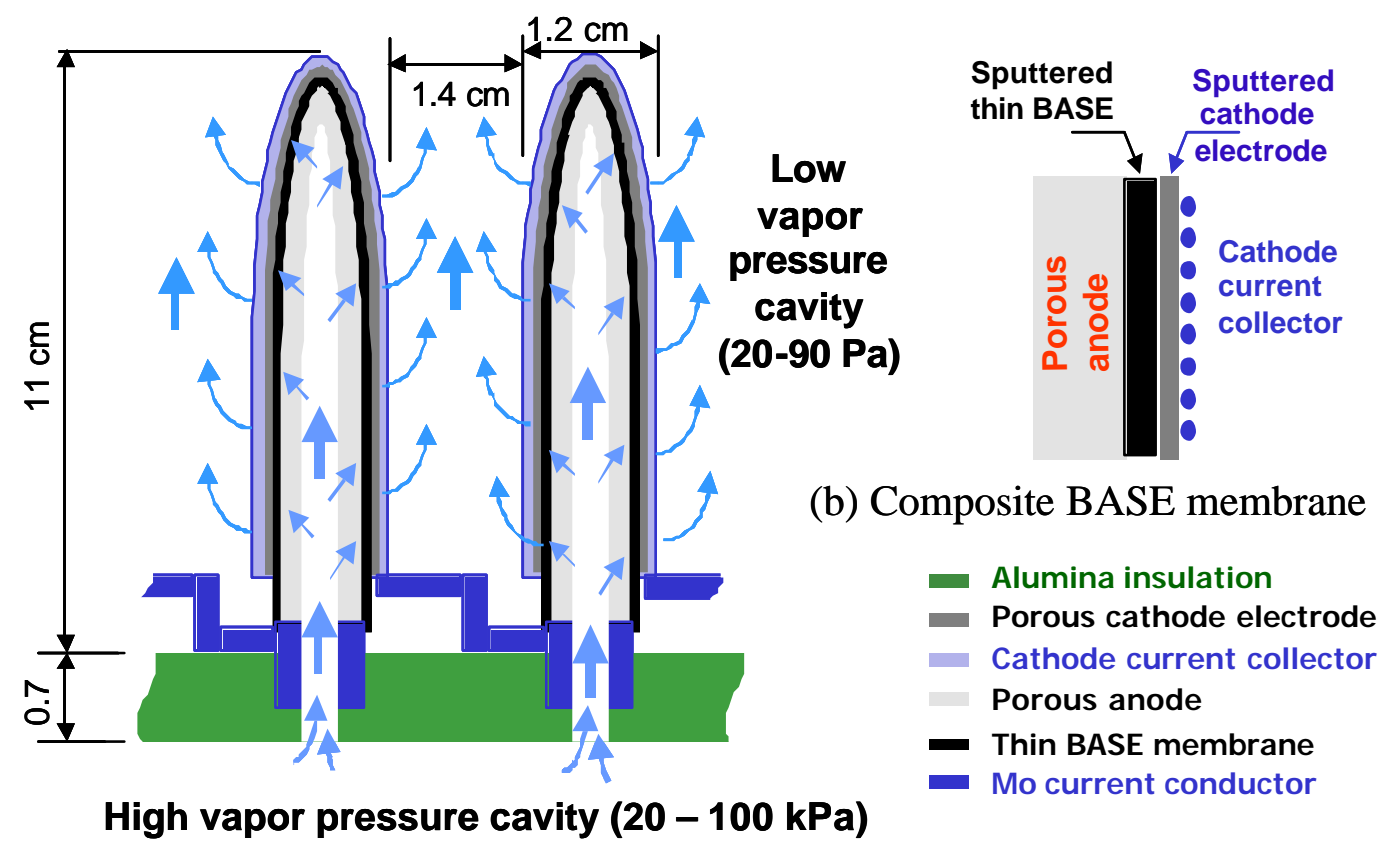

(a) Cross-sectional view D - D of two potassium-BASE elements connected in series

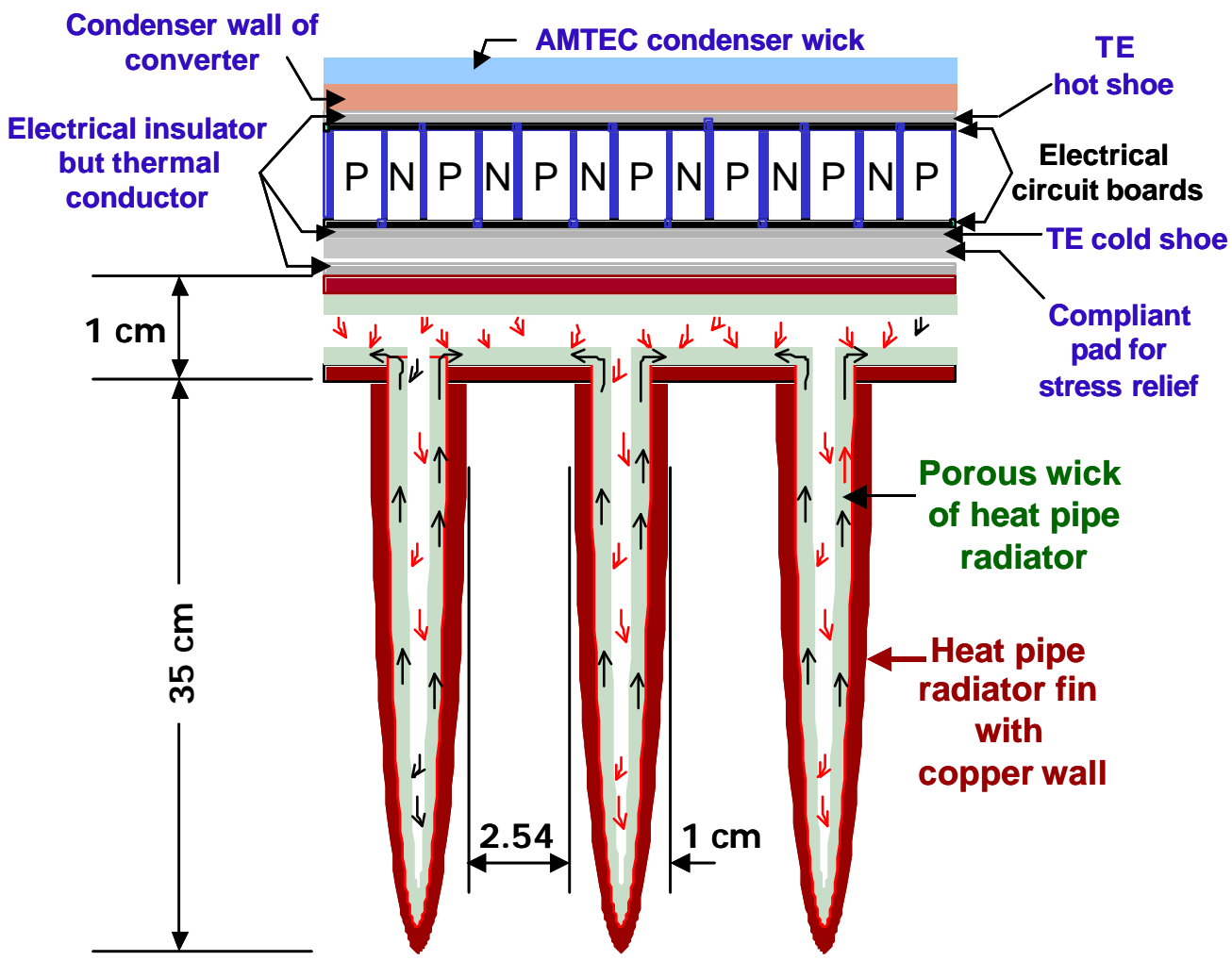

(c) Cross-sectional view $\mathrm{C}-\mathrm{C}$ of $\mathrm{TE}$ bottom cycle and heat pipe cooling fins

Figure 3-59. Cross-sectional views of BASE elements, TE bottom cycle and cooling fins. 
Table 3-4. Dimensions and nominal performance parameters of optimized AMTEC/TE converters operating at a BASE/evaporator temperature of $1006 \mathrm{~K}$.

\begin{tabular}{|c|c|c|}
\hline Design/Operation Parameter & Na-AMTEC/TE & K-AMTEC/TE \\
\hline Number of BASE rows & 2 & 3 \\
\hline Number of BASE elements & $2 \times 336=672$ & $3 \times 248=744$ \\
\hline Electrode's area per BASE $\left(\mathrm{cm}^{2}\right)$ & 550 & 498 \\
\hline BASE element dimensions $(\mathrm{cm})$ & $26.2 \times 1.2 \times 10.5$ & $23.7 \times 1.2 \times 10.5$ \\
\hline Spacing between BASE elements $(\mathrm{cm})$ & 1.0 & 1.4 \\
\hline Diameter of orifices in shield $(\mathrm{cm})$ & 1.0 & 1.0 \\
\hline Number of orifices in shield & 14,000 & 12,000 \\
\hline Converter's dimensions, excluding fins (m) & $7.09 \times 0.64 \times 0.44$ & $6.19 \times 0.86 \times 0.52$ \\
\hline BASE / condenser temperature $(\mathrm{K})$ & $1006 / 596$ & $1006 / 527$ \\
\hline Anode pressure $(\mathrm{kPa})$ & 21.3 & 80.0 \\
\hline Pressure at BASE/cathode interface $(\mathrm{Pa})$ & 40.8 & 35.2 \\
\hline Pressure loss factor on cathode side & 320 & 197 \\
\hline$I_{\mathrm{AMTEC}}(\mathrm{A}) / V_{\mathrm{AMTEC}}(\mathrm{V} \mathrm{DC})$ & $151 / 250$ & $126.8 / 395.6$ \\
\hline Electrode's power density $\left(\mathrm{We} / \mathrm{cm}^{2}\right)$ & 0.102 & 0.136 \\
\hline Number of cooling fins & 198 & 174 \\
\hline Cooling fin's length / pitch $(\mathrm{cm})$ & $35 / 3.54$ & $35 / 3.54$ \\
\hline Cooling fins' temperature $(\mathrm{K})$ & 375 & 364 \\
\hline Height of TE panels / condenser $(\mathrm{cm})$ & 18.0 & 20.0 \\
\hline$\overline{\text { Length of TE legs }(\mathrm{cm})}$ & $\overline{0.79}$ & $\overline{0.70}$ \\
\hline Cross section of P-TAGS-85 leg $\left(\mathrm{cm}^{2}\right)$ & 0.138 & 0.161 \\
\hline Cross section of $2 \mathrm{~N}-\mathrm{PbTe}$ leg $\left(\mathrm{cm}^{2}\right)$ & 0.20 & 0.20 \\
\hline$\Delta T$ across thermoelectrics $(\mathrm{K})$ & 211 & 154 \\
\hline$\overline{P e^{\text {AMTEC }}(\mathrm{kWe}) / \eta_{\text {AMTEC }}(\%)}$ & $37.7 / 22.6$ & $50.2 / 31.8$ \\
\hline$P e^{\mathrm{TE}}(\mathrm{kWe}) / \eta_{\mathrm{TE}}(\%)$ & $7.0 / 5.8$ & $4.14 / 4.23$ \\
\hline$P e^{\mathrm{AMTEC} / \mathrm{TE}}(\mathrm{kWe}) / \eta_{\mathrm{AMTEC} / \mathrm{TE}}(\%)$ & $44.7 / 26.8$ & $54.3 / 34.4$ \\
\hline
\end{tabular}


The porous anode provides additional structural support to the thin BASE membrane and serves as the liquid anode (Sievers et al.,1999). The BASE elements in the AMTEC unit are connected in series to operate at high output voltage, up to $400 \mathrm{~V}$ DC (Momozaki and El-Genk 2002), which also reduces internal electrical losses. This voltage is below that for discharge breakdown (> $400 \mathrm{~V}$ ) in the low-pressure (<100 Pa) alkali metal vapor (see Section 3.3.3).

The BASE membrane is covered on the outside by a thin porous cathode electrode (Figure 3.59b), made of $\mathrm{WRh}_{1.5}$ fine-grains (Ryan et al., 2000), that is applied using a state-of-the-art sputtering technique. The cathode electrode is overlaid by a current collector mesh or grid that is electrically connected to the anode of the adjacent BASE element. The BASE elements are mounted onto a perforated, electrically insulating plate, dividing the AMTEC unit into high vapor pressure $\left(P_{a}=20-80 \mathrm{kPa}\right)$ and low vapor pressure $\left(P_{c}=20-90 \mathrm{~Pa}\right)$ cavities (Figure 3.58a). A perforated metal plate of identical shape structurally supports the overlaying electrically insulating plate.

In the high-pressure cavity of the closed AMTEC/TE converter design in Figures 3.57 and 3.58, alkali metal liquid ( $\mathrm{Na}$ or $\mathrm{K}$ ) in the AMTEC's evaporator wick is converted into high-pressure vapor $(20-80 \mathrm{kPa})$, by the thermal power supplied by the circulating sodium in the secondary loop of the nuclear power plant at $\sim 1100 \mathrm{~K}$. In the open converter design, the high-pressure Alkali Metal vapor produced in a Boiler (AMB) is introduced directly into the high-pressure cavity of the AMTEC units. In both designs, the high-pressure, alkali metal vapor condenses and saturates the porous anode of the BASE elements. The alkali metal ions from the anode traverse the BASE to the cathode side (20-90 Pa), producing an electrical potential of $\sim 0.6 \mathrm{~V}$ per BASE element. This electrical potential is proportional to the BASE temperature and the natural log of the ratio of the anode and cathode vapor pressures (Cole 1983). At the cathode, alkali metal ions emerging from the BASE recombine with electrons to form neutral atoms. The resulting low-pressure alkali metal vapor traverses the low-pressure cavity, flows through a multitude of circular orifices in a molybdenum radiation heat shield (Figures 3.57 and 3.58 ), to the condenser, where it gives up the latent heat and converts into liquid at 500$650 \mathrm{~K}$. In the closed converter design, this liquid is circulated back to the high-pressure cavity through a porous wick, by the capillary action developed in the surface pores of the evaporator wick having an average pore size of $\sim 1.5 \mu \mathrm{m}$ (El-Genk and Tournier $2002 \mathrm{~b}$ ). In the case of the open converter design, the condensate is circulated back to the AMBs using an electromagnetic or mechanical pump.

\subsubsection{Thermo-Electric Modules of the Bottom Cycle}

The heat given up by the alkali metal vapor at the condenser of the AMTEC is conducted to the multitude of P-TAGS-85/2N-PbTe TE converters in the bottom cycle (Figures $3.59 \mathrm{c}$ and 3.60 ). The TE modules are cooled at $\sim 370 \mathrm{~K}$ by natural convection of ambient

air. These TE materials have a high Figure-of-Merit in the temperature range of 400 to $800 \mathrm{~K}$, and have been extensively used in many industrial and medical applications as 
well as in space power systems. To ensure a uniform heat rejection temperature for the TE bottom cycle, a heat pipe spreader and cooling fins are used (Figures 3.57 and 3.59c). The heat removed from these fins by natural convection of air is used for residential or industrial space heating. As indicated earlier, the heat pipe spreader and cooling fins are lined on the inside with a thin porous wick saturated with the water working fluid. The wick provides the capillary pressure head for passively circulating the water and water vapor in the heat pipe (Chi 1976, Peterson 1994, Faghri 1995).

A cross sectional view of the TE unicouple used in the bottom cycle of the NaAMTEC/TE converter is shown in Figure 3.61a. The optimized $\mathrm{n}$ - and p-legs are $7.9 \mathrm{~mm}$ long and operate nominally between $621 \mathrm{~K}$ and $387 \mathrm{~K}$, for a total temperature drop of $234 \mathrm{~K}$, when $T_{B}=1127 \mathrm{~K}$. The p-leg is made of a P-TAGS-85 alloy and the $\mathrm{nt}$ leg is made of a $2 \mathrm{~N}-\mathrm{PbTe}$ alloy. To avoid sublimation of PbTe in the latter, the hot side temperature of the TE bottom cycle in the optimized sodium- and potassium- AMTEC/TE converters is kept below $700 \mathrm{~K}$. The dimensions and performance parameters of both the Na- and K-AMTEC/TE converters are listed in Table 3.4.

The TE unicouple used in the bottom cycle of the K-AMTEC/TE converter uses $\mathrm{n}$ - and p-legs that are $7.0 \mathrm{~mm}$ long and operates nominally between $522 \mathrm{~K}$ and $368 \mathrm{~K}$, for a total temperature drop of $154 \mathrm{~K}$. At a BASE temperature of $1006 \mathrm{~K}$ and the selected nominal operation point, each TE unicouple in the condenser panels of the reference $\mathrm{K}$ AMTEC/TE unit operates at $26.6 \mathrm{mV}$ and $2.9 \mathrm{~A}$ (Figure 3.61b). To reduce the Joule losses and operate at high terminal voltage, all TE unicouples in the TE Module (TEM) are connected in series (Figures 3.60a and 3.60b). The TEM shown in these figures is comprised of $8 \times 70=560$ unicouples, and is $12 \mathrm{~mm}$ high, $50.8 \mathrm{~mm}$ wide, and $508 \mathrm{~mm}$ long. The thermal and electrical insulation on the sides of the n-and p-legs in the TEM is $0.49 \mathrm{~mm}$ thick. To accommodate the differential thermal expansions of the materials in the $\mathrm{n}$ - and p-legs and reduce thermal stresses, a compliant pad is placed at the cold end of the TEM (Truscello and Rutger 1992). Electrical insulator, but thermal conductor layers separate the hot shoe and the hot electrical connectors, the cold connectors and the compliant pad, and the compliant pad and the cold shoe. A single TEM nominally delivers $43.13 \mathrm{We}$ at $14.87 \mathrm{~V} \mathrm{DC}$, when operating at $\mathrm{Th}=522 \mathrm{~K}$ and $\mathrm{Tc}=368 \mathrm{~K}$. Each of the two TEM panels in the K-AMTEC/TE converter unit is comprised of 4 parallel strings of 12 TEMs each to deliver $2.07 \mathrm{kWe}$ at $178.5 \mathrm{~V}$ DC. The two TEM panels, however, are connected in series and deliver a total of $4.14 \mathrm{kWe}$ at $357 \mathrm{~V} \mathrm{DC}$ (Figure 3.63 and Table 3.4). 


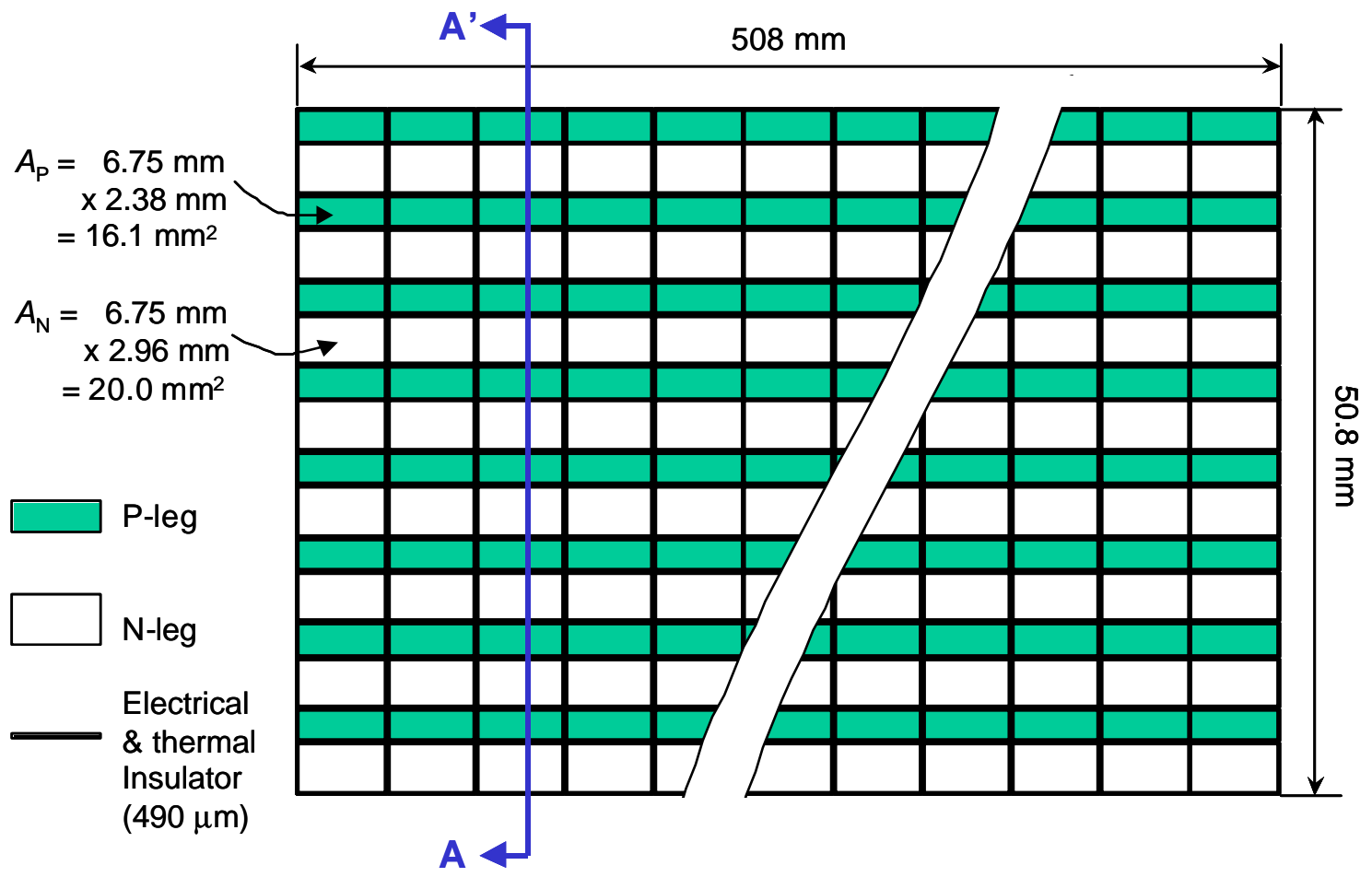

(a) Top view of TE Module (TEM)

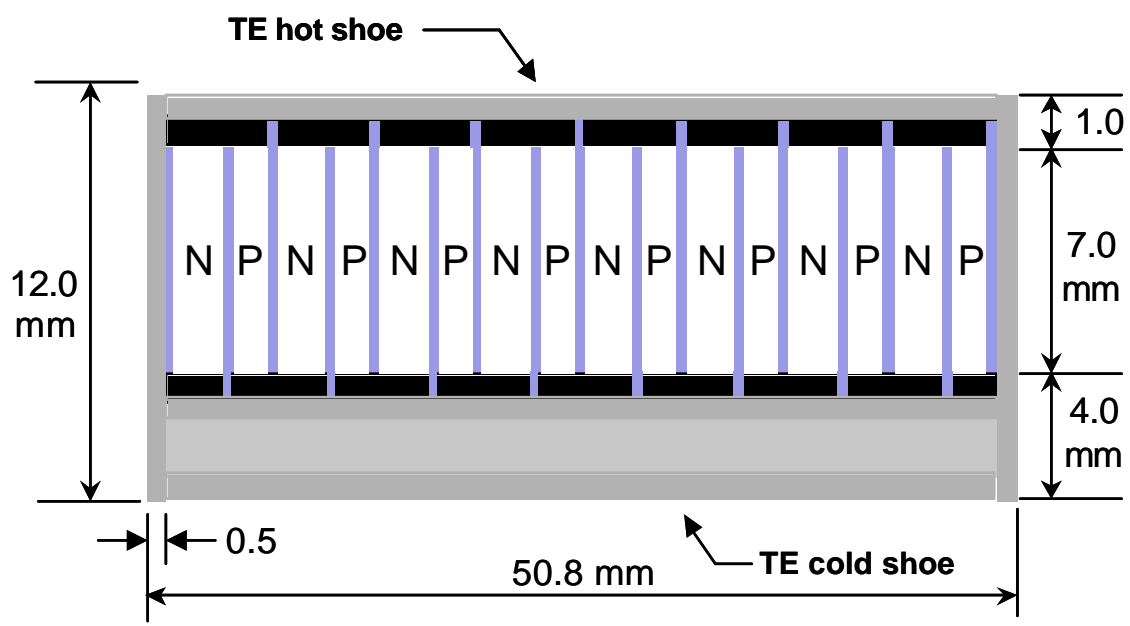

Electrical and thermal insulator

Electrical connectors

Compliant pad for stress relief

Electrical insulator but thermal conductor

(b) Cross-sectional view A - A

Figure 3-60. P-TAGS- $85 / 2 \mathrm{~N}-\mathrm{PbTe}$ thermoelectric module (TEM) in reference K-AMTEC/TE unit (560 unicouples connected in series, and $P e_{\text {STEM }}=43.13 \mathrm{We}$ at $\left.14.87 \mathrm{~V} \mathrm{DC}\right)$. 


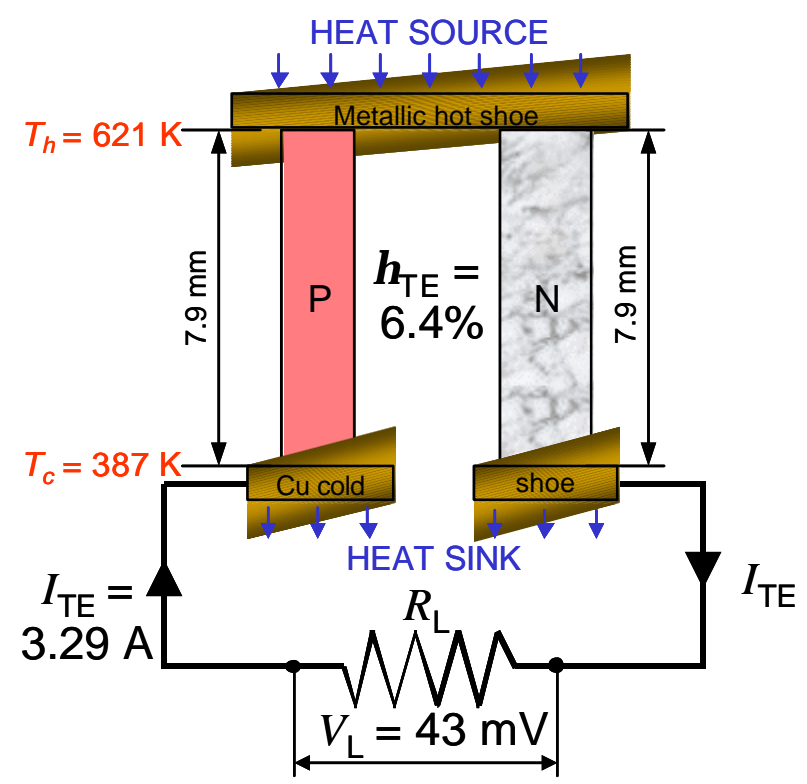

n-leg: $2 \mathrm{~N}-\mathrm{PbTe}, A_{N}=20.0 \mathrm{~mm}^{2}$

p-leg: P-TAGS-85, $A_{P}=13.8 \mathrm{~mm}^{2}$

(a) TE unicouple for Na-AMTEC/TE converter bottom cycle

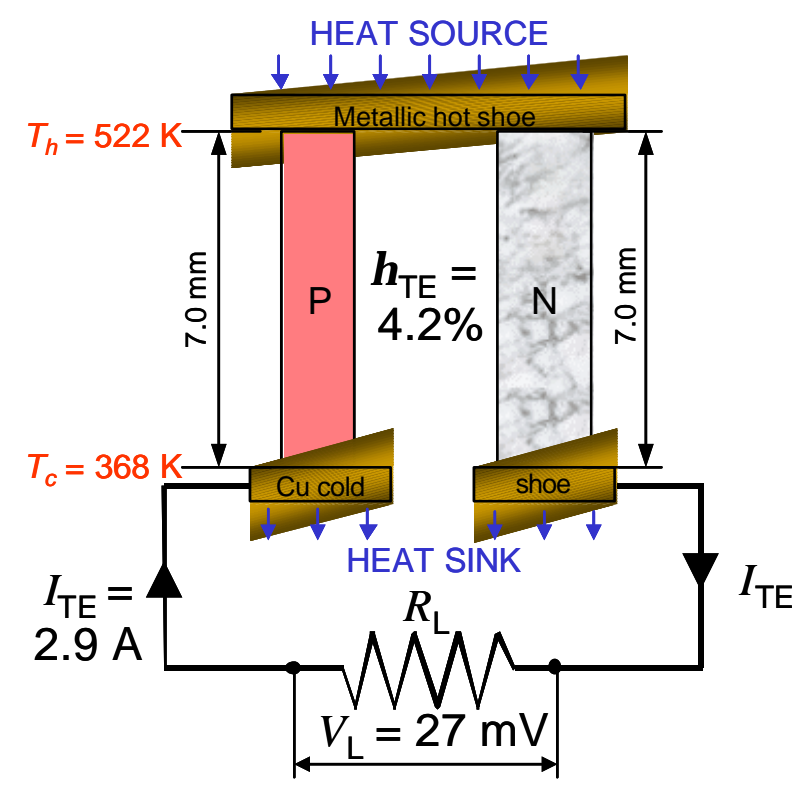

n-leg: $2 \mathrm{~N}-\mathrm{PbTe}, A_{N}=20.0 \mathrm{~mm}^{2}$

p-leg: P-TAGS-85, $A_{P}=16.1 \mathrm{~mm}^{2}$

(b) TE unicouple for K-AMTEC/TE converter bottom cycle

Figure 3-61. Unicouples for the bottom cycle of the optimized AMTEC/TE converters (nominal operation at $P_{a}=$ $80 \mathrm{kPa})$. 


\subsubsection{Heat Transfer Rates and Temperatures}

Owing to the high vapor pressure of potassium compared to sodium, at the same BASE/evaporator temperature of $1006 \mathrm{~K}$ (Figure 3.6), the anode vapor pressure in the KAMTEC $(80 \mathrm{kPa})$ is four times that in the Na-AMTEC $(21.3 \mathrm{kPa})$. Consequently, the KAMTEC unit is not only $30 \%$ more efficient, but also generates more electrical power than the Na-AMTEC unit (see Table 3.4). The optimized potassium- and sodiumAMTEC/TE converters operating at $T_{B}=1006 \mathrm{~K}$ are suitable for coupling to the LMRAMTEC power plants in which the core exit temperature could be kept at or below 1100 $\mathrm{K}$. The sodium- and potassium- AMTEC/TE converters were optimized for operating at the same anode vapor pressure of $80.0 \mathrm{kPa}$. The corresponding BASE temperatures are $1127 \mathrm{~K}$ and $1006 \mathrm{~K}$ for the sodium and potassium AMTECs, respectively. At these operating conditions, the optimized sodium AMTEC/TE converter delivers a nominal electrical power of $69.5 \mathrm{~kW}_{\mathrm{e}}$, at an overall thermodynamic conversion efficiency of $33.7 \%$. Of this electrical power, the Na-AMTEC unit generates $61.0 \mathrm{~kW}_{\mathrm{e}}$ at a thermodynamic efficiency of $29.7 \%$ and $381 \mathrm{~V} \mathrm{DC}$, while the TE bottom cycle generates $8.5 \mathrm{kWe}$ at an efficiency of $6.4 \%$ and $322 \mathrm{~V} \mathrm{DC}$ (Figure 3.62). The condenser in the NaAMTEC unit was at $626 \mathrm{~K}$ and the surface temperature of the heat pipe fins of the TE generators was $382 \mathrm{~K}$, for a temperature drop of $234 \mathrm{~K}$ across the TE generators. At the same anode vapor pressure $\left(P_{a}=80.0 \mathrm{kPa}\right)$, the optimized K-AMTEC/TE converter operates $121 \mathrm{~K}$ cooler than the Na-AMTEC, at $T_{B}=1006 \mathrm{~K}$, and nominally generates $54.3 \mathrm{kWe}$, or $22 \%$ less power than the optimized Na-AMTEC/TE converter, but at nearly one percentage point higher thermodynamic efficiency of $34.4 \%$.

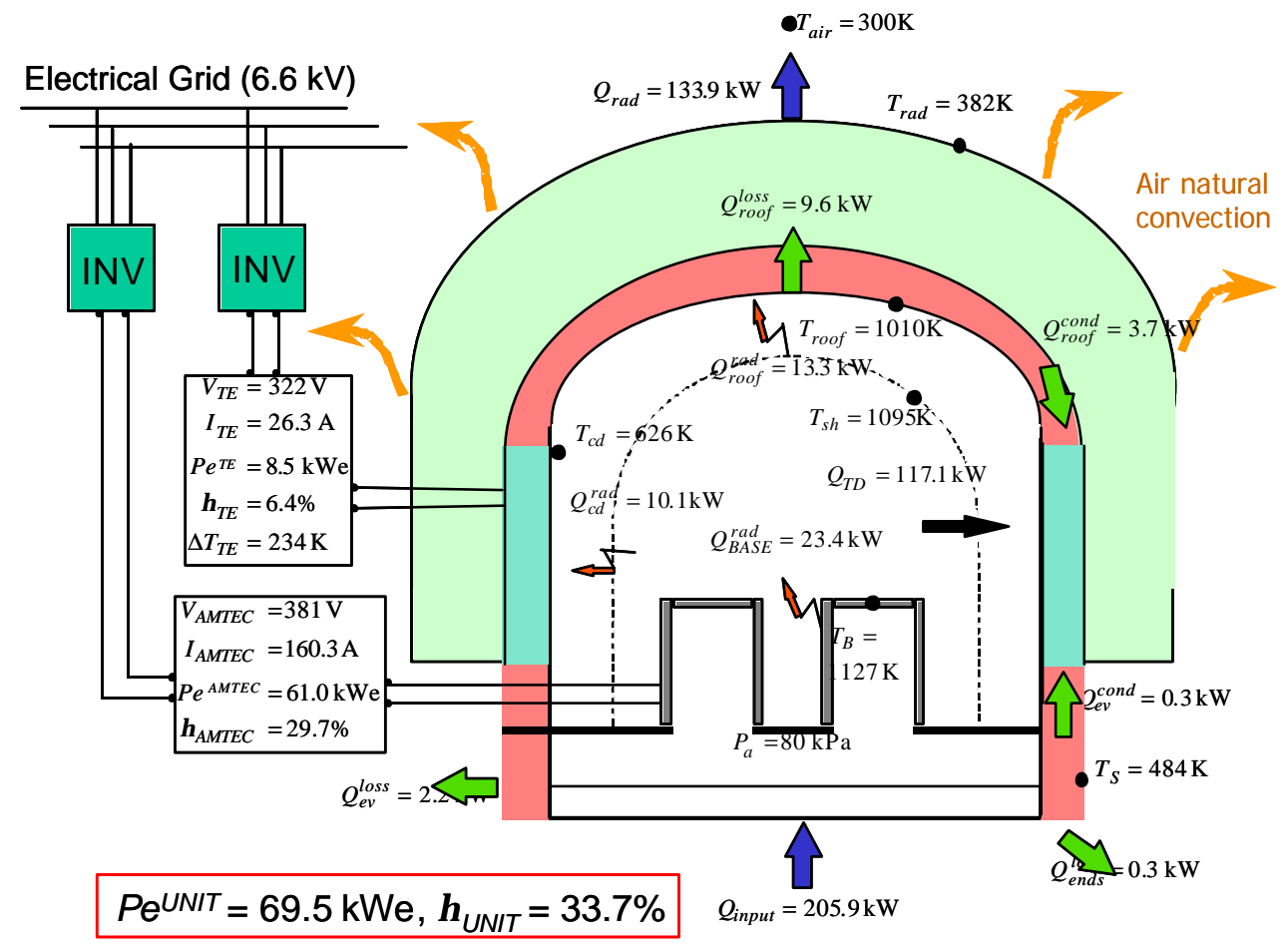

Figure 3-62. Predicted heat transfer rates and temperatures in the reference sodium AMTEC/TE converter $\left(N_{\text {rows }}=2, V_{\text {AMTEC }} \sim 400 \mathrm{~V}\right)$ at nominal operation, very near the peak efficiency $\left(T_{B}=1127 \mathrm{~K}, P_{a}=80 \mathrm{kPa}\right)$. 


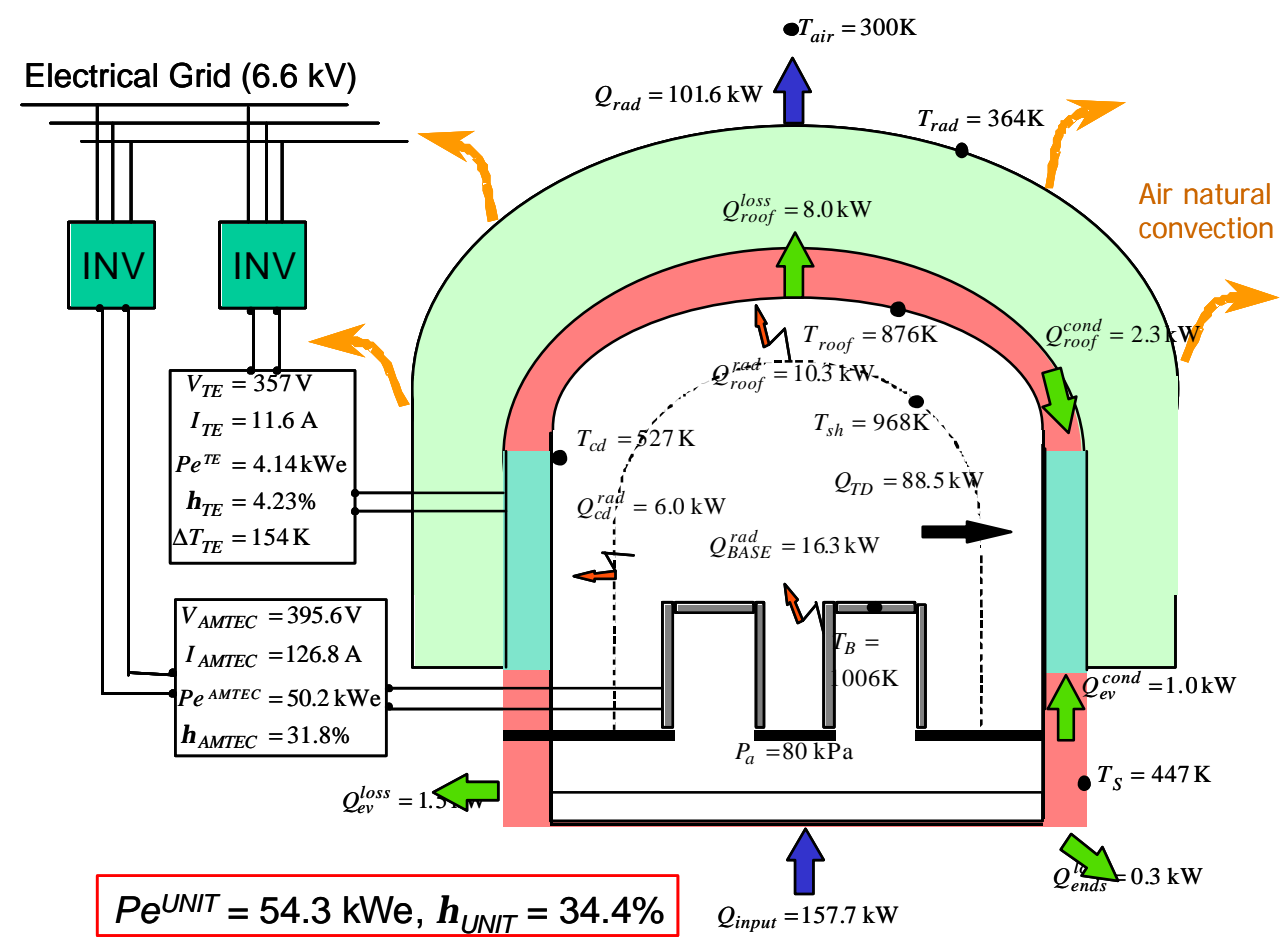

Figure 3-63. Predicted heat transfer rates and temperatures in the reference potassium AMTEC/TE converter $\left(N_{\text {rows }}=3, V_{\mathrm{AMTEC}} \sim 400 \mathrm{~V}\right)$ at nominal operation $\left(T_{B}=1006 \mathrm{~K}, P_{a}=80 \mathrm{kPa}\right)$.

The performance results of the optimized sodium and potassium AMTEC/TE converters when operating at the same BASE/evaporator temperature of $1006 \mathrm{~K}$ are listed in Table 3.4 and discussed next.

\subsubsection{Load-Following Characteristic of Converter}

Figure 3.64a plots the conversion efficiency of the optimized K-AMTEC/TE converter and the DC electrical power output, versus the electrical current of the K-AMTEC top cycle. The solid portions of the curves indicate the region in which the converter is load following. This means that an increase in the load electrical power demand will result in an increase in the electrical power output of the converter up to the peak value indicated by the solid triangle, without an active interference by the operator to change the thermal power of the nuclear reactor.

In addition, owing to the negative temperature reactivity feedback of the nuclear reactor, it is inherently load following, making the entire nuclear power plant with AMTEC/TE converters load following, so long as they operate in the region indicated by the solid portions of the curves in Figure 3.64a. For example, an increase in the load demand results in an increase in the electrical current supplied by the AMTEC/TE converter, causing the BASE temperature to decrease and the vapor production in the high-pressure cavity to increase. Such an increase in the vapor generation rate decreases 
the temperature of the sodium coolant in the underlying $\mathrm{HX}$, before returning to the secondary side of the intermediate heat exchanger of the plant. As a result, the inlet temperature to the nuclear reactor decreases, increasing the reactivity in the reactor core due to the negative temperature reactivity and, hence, the reactor thermal power, commensurate with the increase in the electrical load demand. Conversely, a decrease in the electrical load demand decreases the thermal power intput to the AMTEC/TE converter units, resulting in an increase in the inlet coolant temperature to the nuclear reactor. Such higher temperature introduces negative reactivity feedback in the reactor core, which decreases the reactor thermal power commensurate with the decrease in the electrical power demand.

While in the load following region of the performance curves in Figure 3.64, an increase in the load demand (or current) increases the electrical power output of the converter up to the peak power. Beyond the peak power, a further increase in the load demand or electric current decreases the electric power generated by the converter. The non-load following region is indicated in Figure 3.64 by the dashed portions of the performance curves.

\subsubsection{Selection of Nominal Operation Point}

The nominal operation point of the AMTEC/TE converter (indicated by the open triangles in Figures 3.64a and 3.64b) is selected to the left of the peak electrical power of the converter. This selection is based on ensuring that:

(a) when the AMTEC/TE converter is operating at $110 \%$ of the nominal electric power, the hot side temperature of the TE bottom cycle remains $\leq 700 \mathrm{~K}$ to avoid materials sublimation; and

(b) when operating at $110 \%$ of the nominal electric power, the decrease in conversion efficiency of the AMTEC/TE converter is less than one percentage point below its peak value (Figure 3.64a). The first condition is limiting only when the BASE temperature in the Na-AMTEC/TE converter is $\geq 1140 \mathrm{~K}$, while the second condition is limiting at lower BASE temperatures in the Na-AMTEC/TE converter, and always holds for the K-AMTEC unit.

This selection criteria ensures that the converters remain load following and operate nominally as close to the peak electrical power as possible, without risking a large drop in the conversion efficiency or overheating of the TE generators, as the load demand increases to $110 \%$ of nominal.

When operating at $T_{B}=1006 \mathrm{~K}$, the optimized $\mathrm{K}$-AMTEC/TE converter nominally delivers $54.2 \mathrm{~kW}_{\mathrm{e}} \mathrm{DC}$ at a thermodynamic conversion efficiency of $34.4 \%$, AMTEC condenser temperature of $527 \mathrm{~K}$, and a surface temperature of the cooling fins of the TE generators of $364 \mathrm{~K}$ (Table 3.4). 


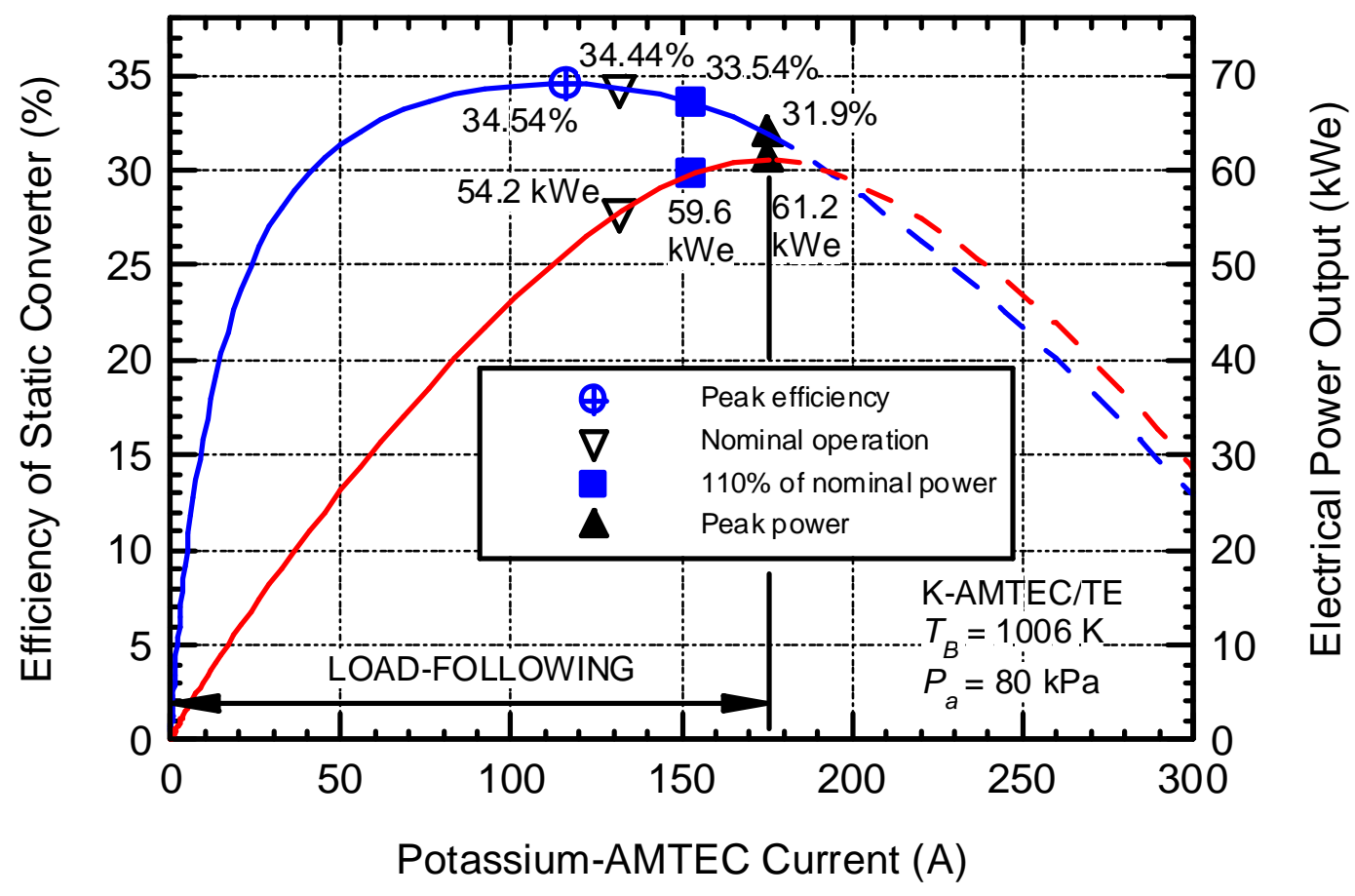

(a) Load-following characteristic of optimized K-AMTEC/TE converter

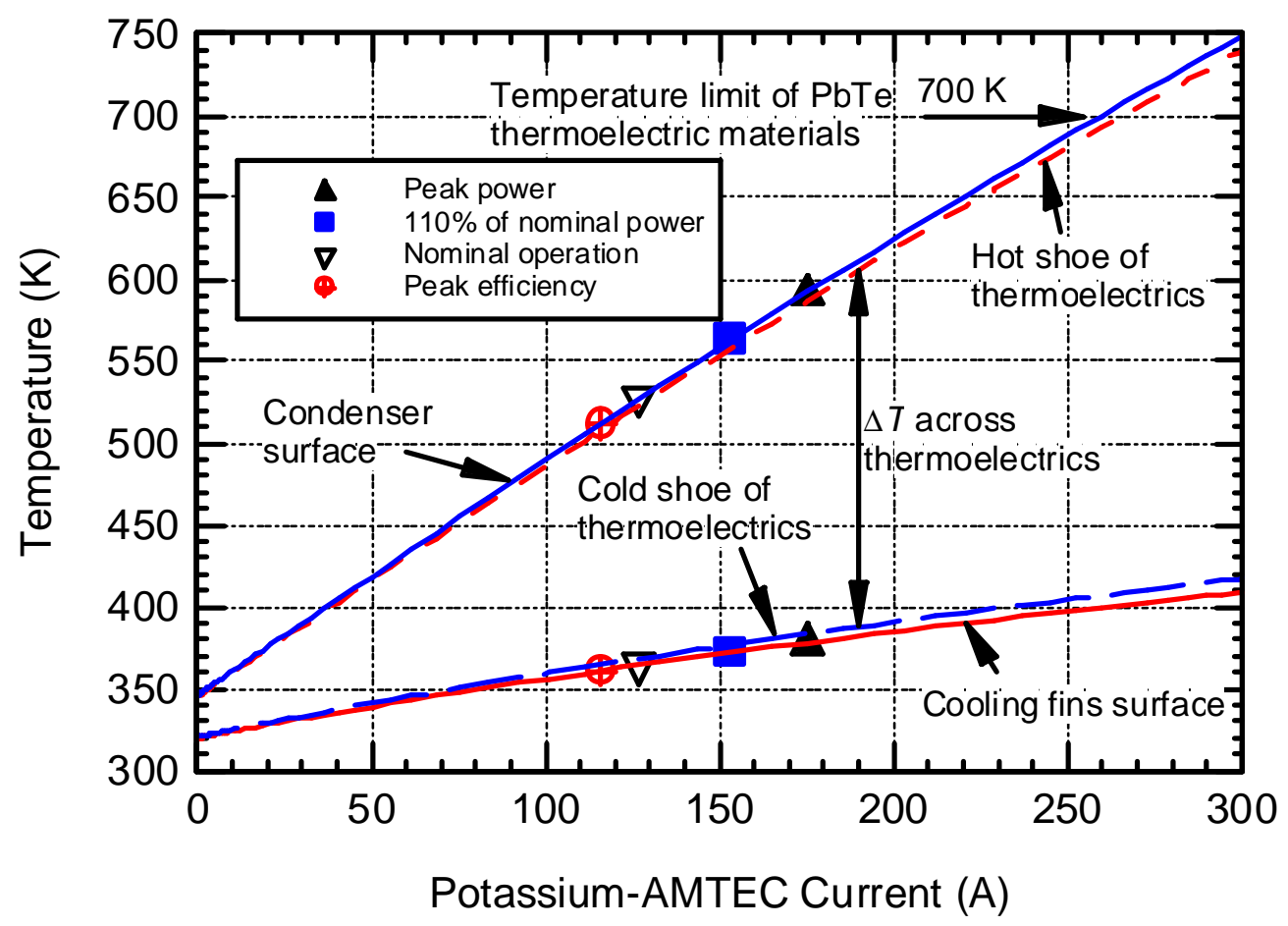

(b) Condenser and radiator temperature

Figure 3-64. Performance results of the optimized potassium-AMTEC/TE converter when operating at $T_{B}$ $=1000 \mathrm{~K}$. 
At $110 \%$ of the nominal electrical power $\left(59.6 \mathrm{~kW}_{\mathrm{e}}\right)$, the net efficiency of the $\mathrm{K}$ AMTEC/TE converter is only 0.9 percentage point lower, at $33.5 \%$, and the temperatures of the AMTEC condenser and cooling fins of the TE generators are $562 \mathrm{~K}$ and $372 \mathrm{~K}$, respectively (Figure 3.64b). The resulting $35 \mathrm{~K}$ increase in the condenser temperature and the increase in temperature drop across the TE modules from $154 \mathrm{~K}$ to $181 \mathrm{~K}$ (Figure 3.64b) increase the electrical power generated by the TE bottom cycle, partially offsetting the decrease in the electrical power generated by the AMTEC unit (Figure 3.64a).

\subsubsection{Comparison of the Performance of the Na- and K- AMTEC/TE Converters at Identical BASE Temperature}

Figure 3.65 plots the electrical power generated by the optimized Na-AMTEC/TE and KAMTEC/TE converters versus the conversion efficiency, in the load-following portion of their characteristics, when operating at $T_{B}=1006 \mathrm{~K}$. The solid triangles indicate the peak electrical powers and the crossed open circles indicate the peak conversion efficiencies.

Figure 3.65 indicates that at a BASE temperature of $1006 \mathrm{~K}$, the peak DC electrical power of the Na-AMTEC/TE converter unit $\left(50.2 \mathrm{~kW}_{\mathrm{e}}\right)$ is only $82 \%$ of that for the K-AMTEC/TE converter $\left(61.2 \mathrm{~kW}_{\mathrm{e}}\right)$. In addition, the peak conversion efficiency of the latter $(34.54 \%)$ is much higher than that of the former $(27.0 \%)$. As indicated earlier,

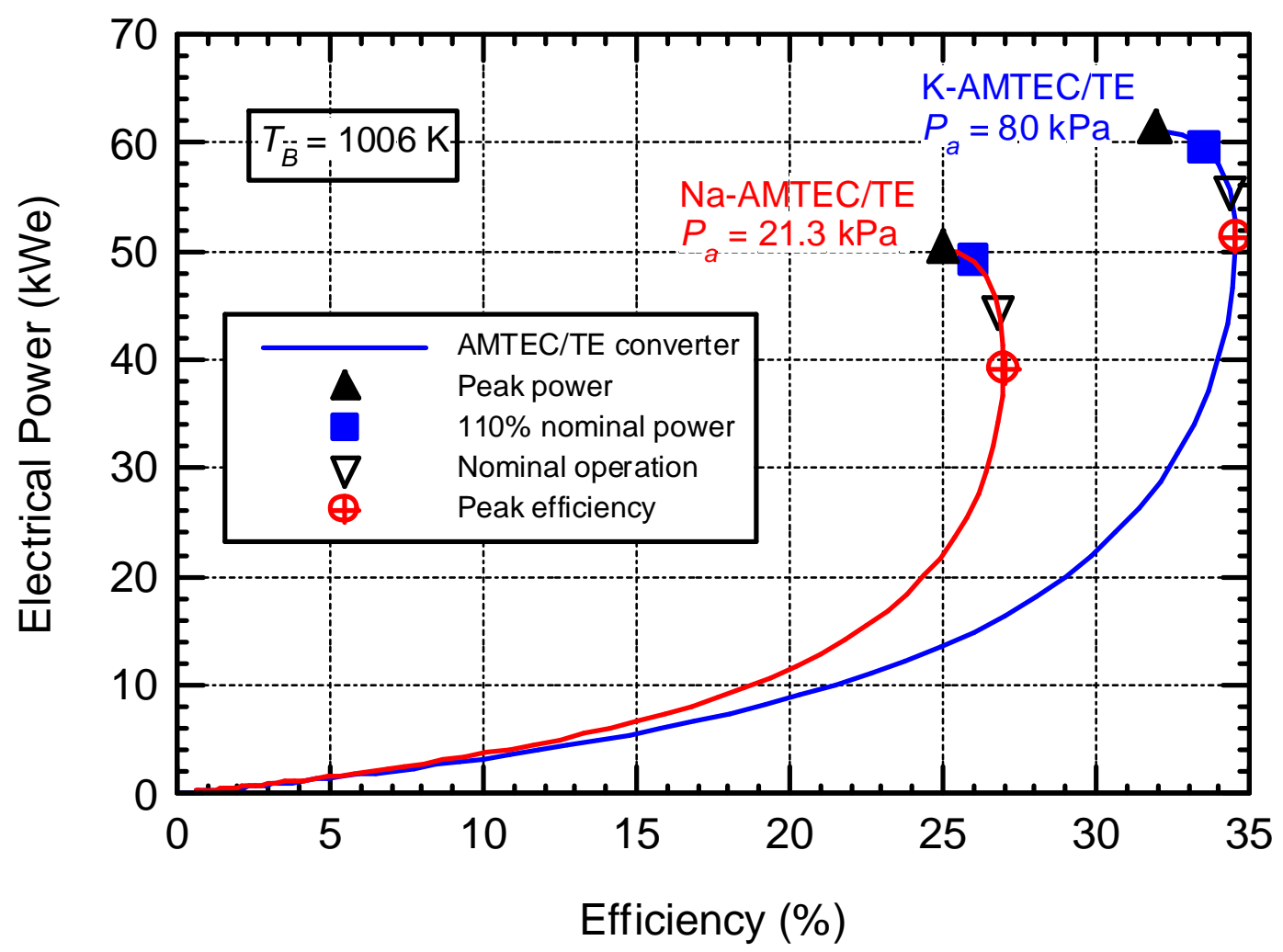

Figure 3-65. Performance comparison of the optimized sodium and potassium-AMTEC/TE converters when operating at $T_{B}=1000 \mathrm{~K}$. 
this is because of the higher anode vapor pressure of potassium (80 kPa versus $21.3 \mathrm{kPa}$ for sodium at $T_{B}=1006 \mathrm{~K}$ ), which generates higher potential across the BASE element and, hence, higher electrical power output.

Based on these results, the potassium-AMTEC/TE converter unit was selected for the LMR-AMTEC nuclear power plant. The optimized potassium-AMTEC/TE converter unit outperforms the optimized sodium converter in terms of conversion efficiency, while operating at up to a $120 \mathrm{~K}$ cooler evaporator and BASE temperature. The higher vapor pressure of potassium compared to sodium allows the nuclear reactor of the LMRAMTEC power plant with optimized potassium AMTEC/TE converter units to operate at a core exit temperature that is more than $100 \mathrm{~K}$ lower than that of a LMR-AMTEC plant with sodium converters, significantly reducing fuel swelling and increasing the reactor operation lifetime.

Note that the use of a highly-efficient potassium AMTEC top cycle does not preclude the use of a sodium-cooled nuclear reactor, when indirect interfacing between core and converters is selected. The use of intermediate alkali metal boilers (AMB) or liquid/liquid heat exchangers, which is recommended for obvious safety considerations, allows the LMR-AMTEC to capitalize on the best of two worlds: a sodium-cooled nuclear reactor and a liquid sodium primary loop or pool, which capitalize on the extensive experience and LMFBR technology gained over the past decades, and highlyefficient potassium AMTEC/TE converter units. Potassium converters operating at an even lower BASE temperature $\left(T_{\mathrm{B}}<1000 \mathrm{~K}\right)$ may still deliver a conversion efficiency near $30 \%$, further reducing the core exit temperature, and consequently reducing fuel swelling and increasing the reactor operation lifetime. The lower the operating BASE temperature, however, the lower is the electrical power output of each AMTEC/TE converter, requiring the use of a large number of static converters in the LMR-AMTEC plant, increasing its capital cost. The effect of the operating BASE temperature on the performance of the optimized potassium-AMTEC/TE converter unit designed for the LMR-AMTEC power plant is shown in Figures 3.66 - 3.69, at the nominal operation point in the load-following portion of the converter's operation. As shown in Figure 3.66 , the static converter delivers an efficiency of $34.4 \%$ and electrical power output of $54.3 \mathrm{kWe}$ DC when operating at $T_{B}=1006 \mathrm{~K}$. At these conditions, a 25-MWe LMRAMTEC would require the use of approximately 500 converter units. At a lower BASE temperature of $950 \mathrm{~K}$, the converter can still deliver a thermodynamic efficiency $>30 \%$ $(31.5 \%)$, but at a lower power output of $43.7 \mathrm{kWe}$, requiring the use of $24 \%$ more converter units (about 620 of them). A lower operating temperature increases the reactor and plant operation lifetime and may allow the use of less costly and or exotic structural materials. On the other hand, the capital cost of the power plant also increases due to the larger number of converter units needed. Therefore an optimization analysis of the overall performance and cost of the LMR-AMTEC power plant was performed by Westinghouse Electrical Company, using the nominal performance curves developed by UNM-ISNPS for the reference potassium- AMTEC/TE converter unit (Figures 3.66 3.69). The results of this optimization analysis are reported in Chapter 4. 


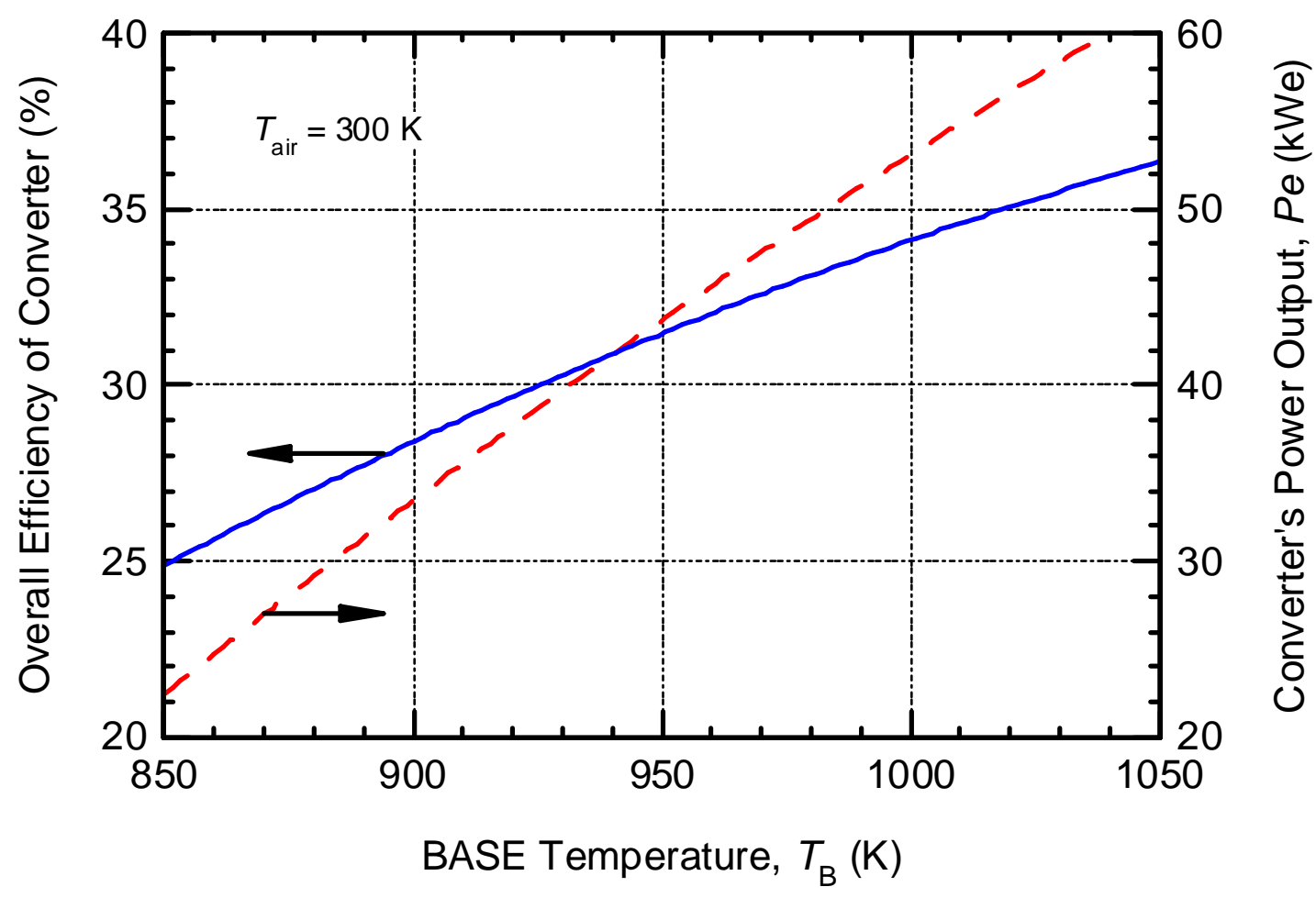

Figure 3-66. Performance of reference K-AMTEC/TE converter at nominal operation.

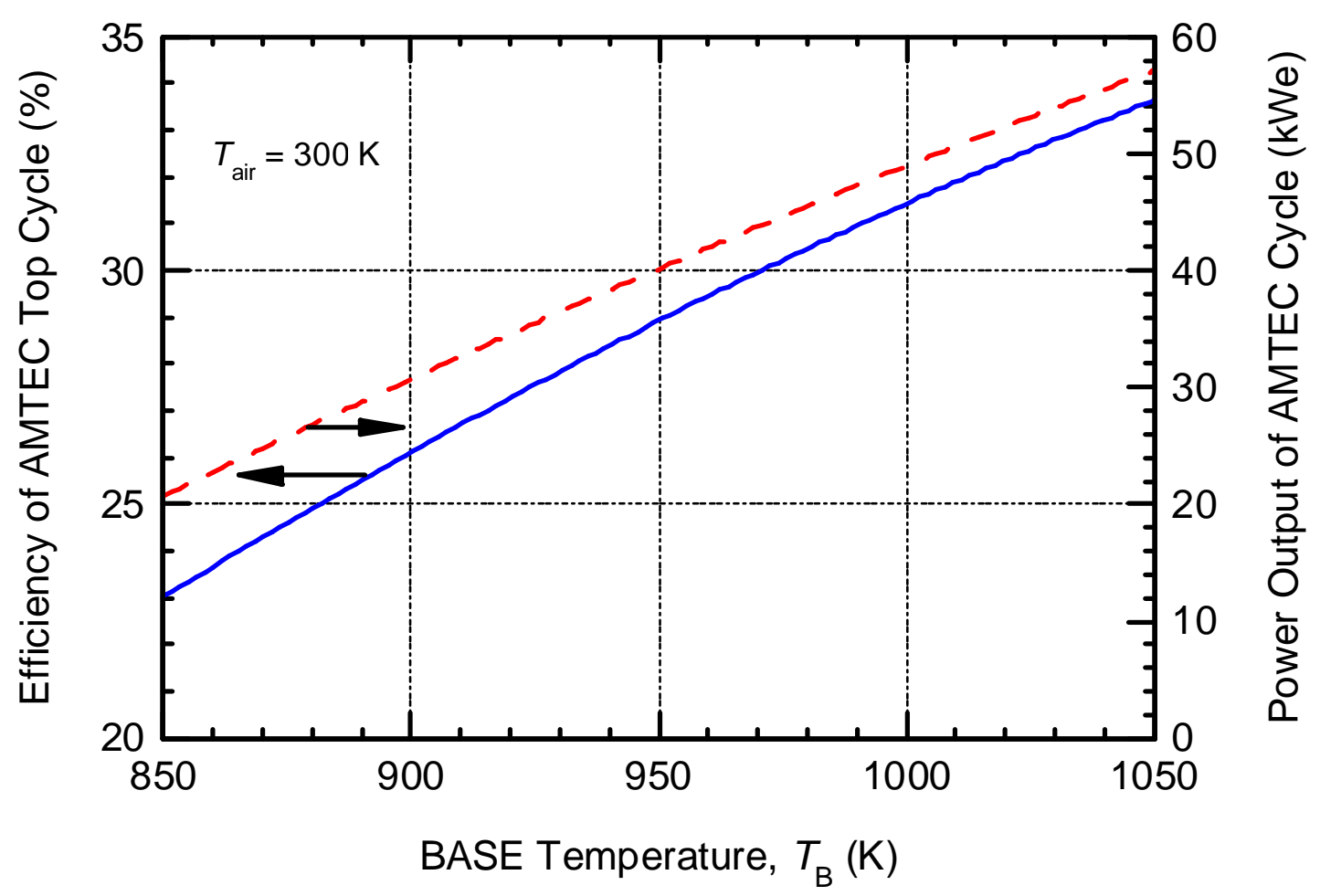

Figure 3-67. Performance of top cycle of reference K-AMTEC/TE converter at nominal operation. 


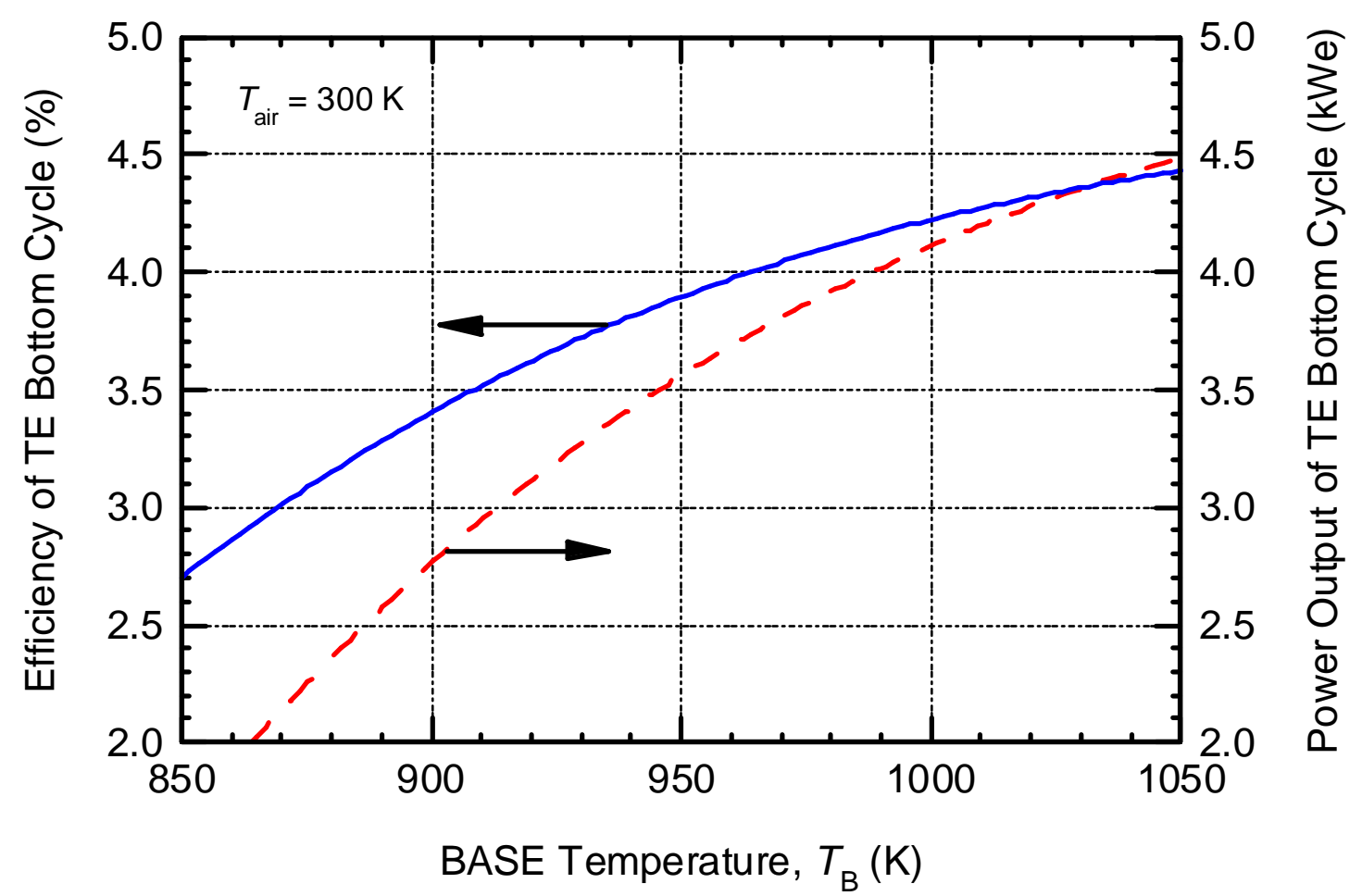

Figure 3-68. Performance of bottom cycle of reference K-AMTEC/TE converter at nominal operation.

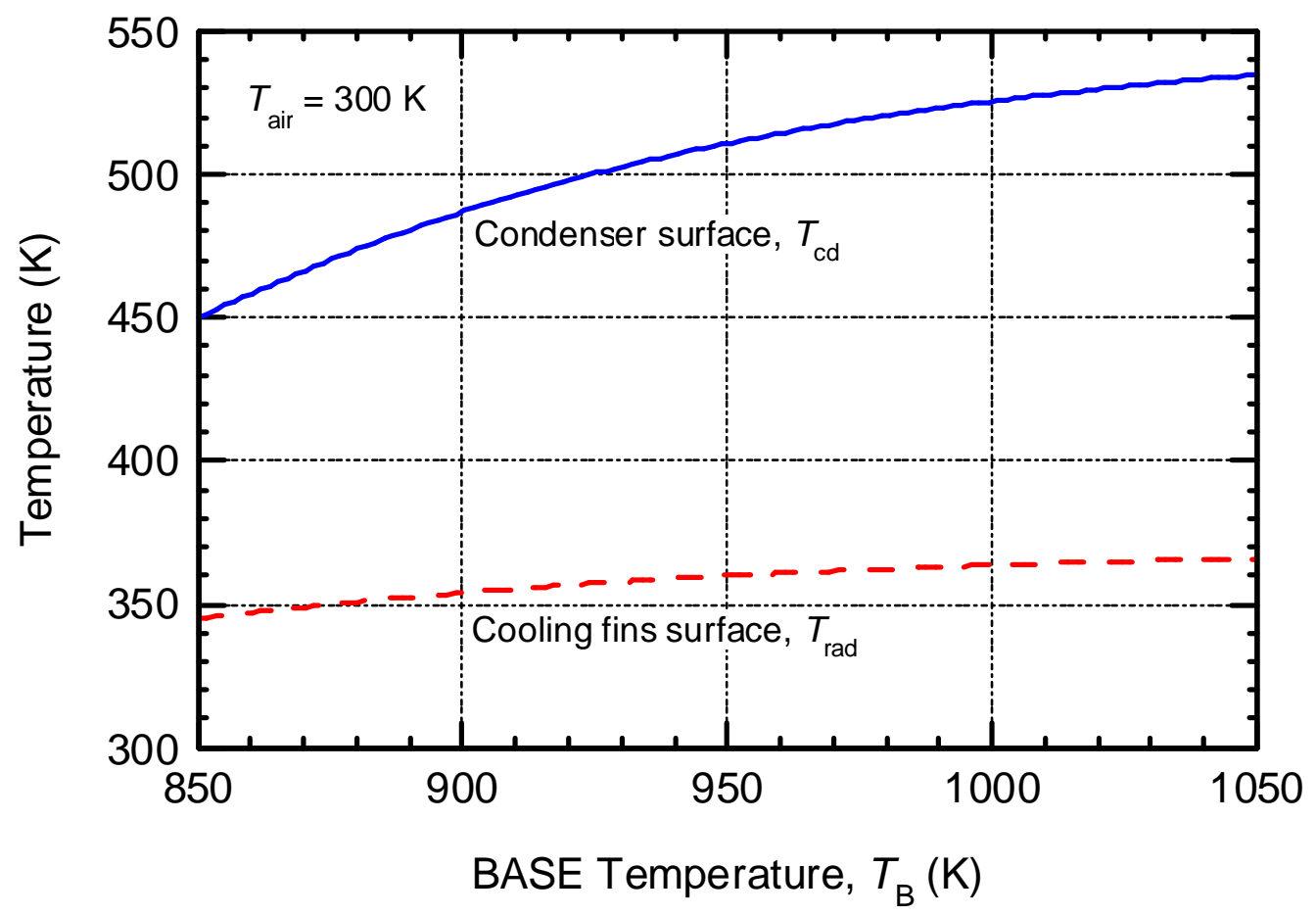

Figure 3-69. Temperatures in reference K-AMTEC/TE converter at nominal operation. 


\subsection{Integration of AMTEC/TE Converters into Power Modules}

The AMTEC/TE converter units were integrated into electrical power modules for the LMR-AMTEC nuclear power plant. The electrical power system consists of a number of electrical modules, each generating 1.04 MWe AC (Figure 3.70). The number of these modules was determined based on the desired electrical power output of the plant. For a 25 MWe power plant, 25 modules will be required. All modules are electrically connected in parallel to maximize redundancy in the electrical power system.

For the reference potassium-AMTEC/TE converter units described in the previous Section, which operate at a BASE temperature of $1006 \mathrm{~K}$, each electrical power module consists of $20 \mathrm{AMTEC} / \mathrm{TE}$ converter units and a DC-AC inverter/transformer (Figure 3.71). The output voltage of the module is $6.6 \mathrm{kV} \mathrm{AC}$ at $158.0 \mathrm{~A}$, assuming an efficiency of the DC/AC inverter/transformer of $96 \%$. Each potassium-AMTEC/TE converter unit generates $54.3 \mathrm{kWe}$ DC and all 20 units in a module are electrically connected in parallel (Figure 3.71).

The electrical power modules are all connected in parallel, and, if necessary, a stepup transformer may be used to raise the output voltage of the power plant to match the Grid voltage (typically $100-150 \mathrm{kV}$ AC.

Figure 3.72 illustrates the layout of the electrical power system for a $25 \mathrm{MWe}$ LMR-AMTEC power plant with potassium-AMTEC/TE converter units operating at $T_{\mathrm{B}}=$ $1006 \mathrm{~K}$. The AMTEC top cycle of these converters operates at $126.8 \mathrm{~A}$ and an output voltage of $395.6 \mathrm{~V} \mathrm{DC}$, while the TE bottom cycle operates at a lower current of $11.6 \mathrm{~A}$

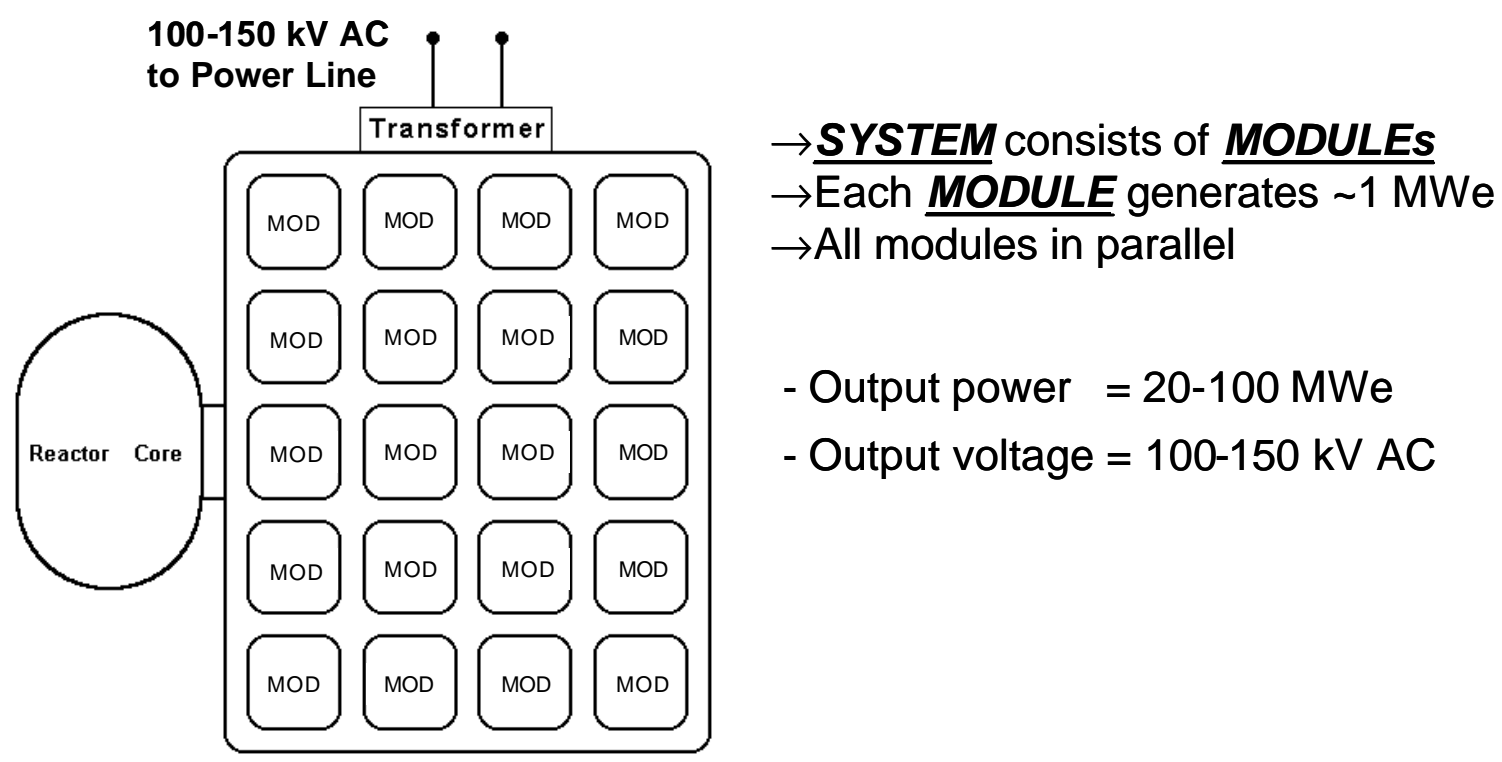

Figure 3-70. Configuration of the electrical power system for the LMR-AMTEC power plant. 


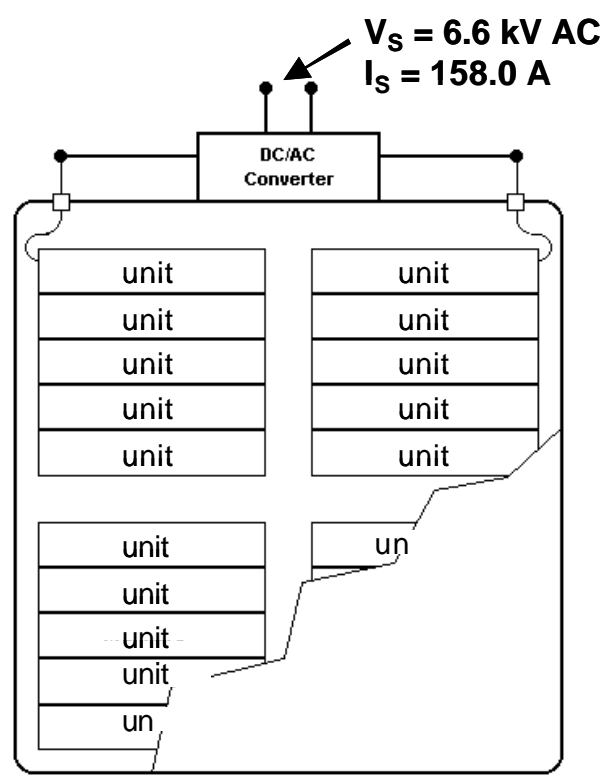

$\rightarrow$ MODULE consists of UNITS

$\rightarrow$ Each UNIT generates $54.3 \mathrm{kWe}$

$\rightarrow$ Each Module has its own DC/AC converter (efficiency $96 \%$

$\rightarrow$ All units are connected in parallel

- Output power per module $=1.043 \mathrm{MWe}$

- Output voltage $=6.6 \mathrm{kV} \mathrm{AC}$

- Output current $=158.0 \mathrm{~A} \mathrm{AC}$

- Number of units per module $=20$

Figure 3-71 . A schematic of a scalable, AMTEC/TE electric power module.

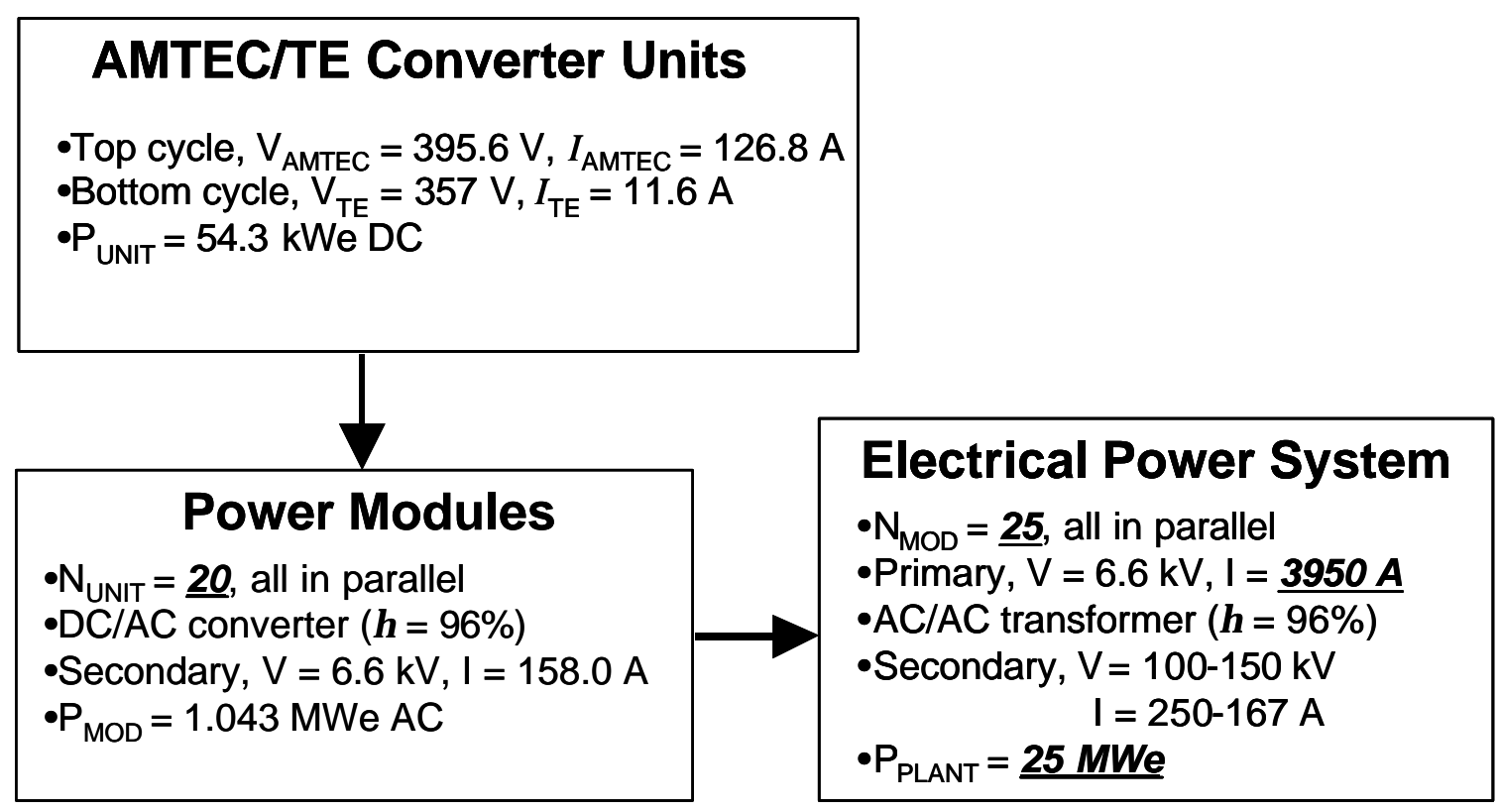

Figure 3-72. Integration of AMTEC/TE converter units in the electrical power system for a 25 MWe power plant. 
and $357 \mathrm{~V} \mathrm{DC}$, for a total converter's electrical power output of $54.3 \mathrm{kWe}$ DC. Each electrical power module is comprised of 20 AMTEC/TE converter units connected in parallel, and a DC/AC inverter and step-up transformer. Assuming a transformer's efficiency of $96 \%$, the secondary current and voltage of the module are $158.0 \mathrm{~A}$ and 6.6 $\mathrm{kV}$, producing 1.043 MWe AC. Twenty-five modules are connected in parallel in the power plant, generating a total of $26.1 \mathrm{MWe} \mathrm{AC}$ at $3950 \mathrm{~A}$ and $6.6 \mathrm{kV}$. A final stepup AC/AC transformer may be used, if needed, to increase the output voltage of the plant to match that of the Grid (typically 100-150 kV AC). This LMR-AMTEC nuclear power plant delivers $25 \mathrm{MWe}$ at an output voltage of 100-150 kV AC and current of 167-250 A (Figure 3.72).

\subsection{Materials Selection and Research for AMTEC Converters}

Structural and corrosion properties of stainless steels and superalloys structural materials, and potential degradation mechanisms of the AMTEC electrodes and BASE were reviewed. Also, properties of refractory metal and refractory alloy candidate materials for the porous structure of the BASE anode were reviewed and investigated. Properties of interest included: compatibility with alkali-metal vapor and liquid environment, thermal expansion coefficient match with the Beta"-Alumina Solid Electrolyte (BASE), strength and high creep resistance at operating temperature, low vapor pressure, volatiles and contaminants, cost and availability.

Estimates of the conversion efficiency of the static AMTEC/TE converter for the LMR-AMTEC power plant showed that values in excess of $30 \%$ could be achieved, which are based on conservative assumptions regarding the technology of the AMTECs and on using off-the-shelf lead-telluride (PbTe) TE modules (see Section 3.5). As more advances are made in the development of the porous metallic anode that is compatible with and has a thermal expansion coefficient that is similar to that of the BASE; the development of high performance cathode electrodes; the fabrication of thin BASE membrane that is less than $200 \mu \mathrm{m}$; and the fabrication of reliable metal-graded ceramic brazes of the anode metallic support structure with the ceramic insulating support plate, higher conversion efficiencies in excess of $35 \%$ for the combined AMTEC/TE converters with a long operation lifetime of 5-10 years, with little degradation, would be possible. Some of these technology advances have been reported very recently, and are being investigated, by industry, universities, and government research laboratories.

For example, it is currently possible to deposit thin layers $(<200 \mu \mathrm{m})$ of Beta"Alumina Solid Electrolyte (BASE) on a porous metallic anode at a rate one order of magnitude faster than in the technology currently being used in the space AMTECs, for both sodium- and potassium-BASE (Virkar et al. 2000). Furthermore, a number of small companies are currently developing ceramic-to-metal graded brazes, which would not be as susceptible to thermal stresses or mechanical strains as conventional brazes. Finally, recent work done at the Jet Propulsion Laboratory in Pasadena, California, has shown that unlike the TiN electrodes used in the AMTEC cells developed during the space 
program, the new tungsten/rhodium $\left(\mathrm{WRh}_{1.5}\right)$ electrodes have demonstrated excellent performance properties and long-term stability (minimal degradation) (Ryan et al. 2000). Furthermore, efforts to develop mixed-conducting (ionic and electronic) metal/oxide electrodes such as blends of $\mathrm{Mo} / \mathrm{Na}_{\mathrm{x}}-\mathrm{TiO}_{2}$ and $\mathrm{TiN} / \mathrm{Na}_{\mathrm{x}}-\mathrm{TiO}_{2}$ are underway at both the Jet Propulsion Laboratory (Ryan et al. 2001) and Advanced Modular Power systems (AMPS), Inc. (Fletcher and Schwank 2002 and 2003). These electrodes can be applied as a paint or slurry, are robust and inexpensive, and have demonstrated to date a performance equivalent to the best currently available refractory electrode $\left(W R h_{x}\right)(R y a n$ et al. 2001). Further studies on metal material selection, and optimization of the mixedconducting electrodes' composition and thickness offer the promise for improved lifetime and performance of AMTEC devices. All recent progresses in these technologies apply directly to the present LMR-AMTEC design. More details on the work performed and on the recent progresses in technologies are given in the following sub-sections.

\subsubsection{Materials Selection for the Cathode Electrode and Lifetime Degradation Predictions}

The development of the sodium AMTEC has been ongoing for more than a decade (Merrill et al. 1998, Sievers et al. 1998, El-Genk and Tournier 1998, Carlson et al. 1999, Hendricks et al. 1999, Schock et al. 1999, El-Genk and King 2001, Giglio et al. 2001). The first-generation sodium converters, known as the PX-series cells, used a stainless steel structure and TiN electrodes. These electrodes initially exhibited a charge-exchange current coefficient $B=120 \mathrm{~A} . \mathrm{K}^{1 / 2} / \mathrm{Pa} . \mathrm{m}^{2}$, which decreased to a stable value of $B \sim 70$ after operating for a few hundreds hours (Ryan et al. 1999). The TiN electrode has a pressure loss coefficient $G_{\mathrm{E}} \sim 50$, which characterizes the pressure drop due the diffusion of the neutral sodium atoms forming at the BASE-cathode interface through the porous electrode. However, in order to decrease the internal charge-exchange polarization and concentration losses, and hence, increase the net electric potential developed in the beta"alumina solid electrolyte (BASE) tubes, electrodes characterized by $B \geq 90 \mathrm{~A} . \mathrm{K}^{1 / 2} / \mathrm{Pa} \cdot \mathrm{m}^{2}$ and $G_{\mathrm{E}} \leq 20$ are desirable.

In the vacuum tests of the PX-type sodium AMTEC converters, gradual degradation in performance has been measured, with some converters failing prematurely (Merrill et al. 1998, ElGenk and Tournier 1998, Shields et al. 2001). For example in a test performed at $T_{\text {hot }}=1023 \mathrm{~K}$, the electrical power output of the PX-3A sodium converter decreased steadily $30 \%$ over a period of two-years, which is suspected to have been caused by degradation of the TiN cathode electrodes and of the BASE. One degradation mechanism of the TiN porous electrode has been attributed to grain growth with time at temperature. Such grain growth reduces the number of active sites available for the recombination of the sodium ins diffusing out of the BASE with the electrons circulating through the external load to the cathode side.

Ryan et al. (2000) predicted that the grain growth alone could degrade the performance of the TiN electrodes by $\sim 10 \%$ after 2 years and by $\sim 30 \%$ after 16 years, when operated at typical operating temperatures in sodium AMTEC converters. To resolve the lifetime and performance degradation issues of the TiN electrodes, high 
performance electrodes of different materials are being developed at the Jet Propulsion Laboratory (Ryan et al. 2000 and 2001). Studies of refractory metal and alloy electrodes performed in Sodium Exposure Test Cells (SETC) and AMTEC devices at the Jet Propulsion Laboratory have determined that tungsten-rhodium co-sputtered films $(\sim 0.7-$ $1.0 \mu \mathrm{m}$ thick) are among the best performing electrodes to date (Ryan et al. 2000 and 2001). These electrodes exhibit good stability at high temperature and demonstrated the highest performance of all refractory electrodes tested to date, with a stable chargeexchange current $B=90-110$ (Figure 3.73), and $G_{\mathrm{E}} \sim 10$ (Ryan et al. 2000). Based on these findings, the present analyses used $\mathrm{WRh}_{1.5}$ electrodes characterized by a conservative value, $B=90 \mathrm{~A} \cdot \mathrm{K}^{1 / 2} / \mathrm{Pa} \cdot \mathrm{m}^{2}$, and $G_{\mathrm{E}}=10$ (Ryan et al. 2000 ).

In these refractory electrodes, the requirement for ready transport of alkali metal atoms through the electrode must be balanced with that for efficient electrons conduction. The later may be enhanced by thickening the electrode, however this would in turn impede the alkali metal transport by vapor flow or surface or grain-boundary diffusion through the electrode. The performance of an electrode can be improved if an ionic conductor material is incorporated in the electronically conducting electrode. The ionic

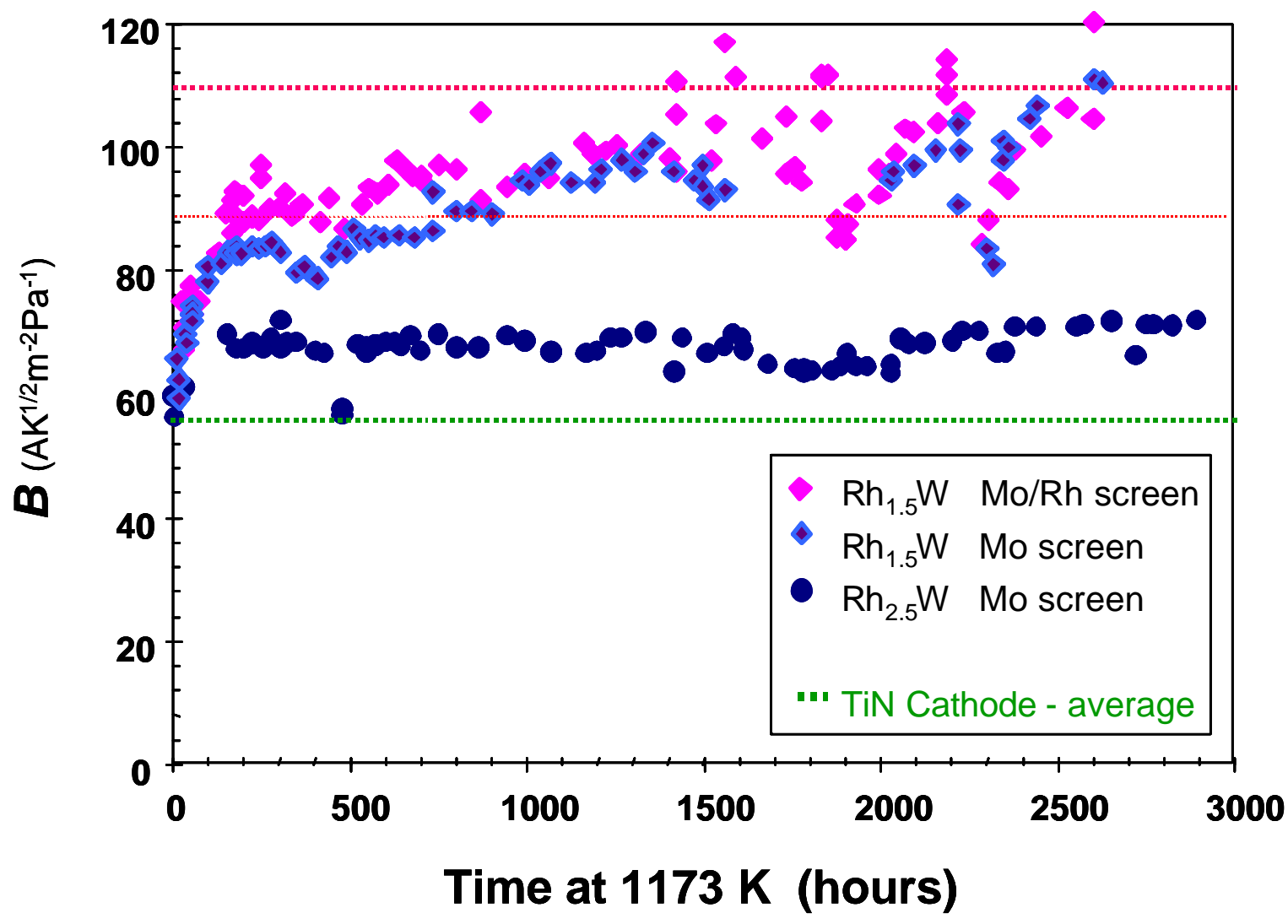

Figure 3-73. Measured values of charge-exchange current coefficient of $\mathrm{Rh}_{\mathrm{x}} \mathrm{W}$ porous electrodes at the Jet Propulsion Laboratory (Ryan et al. 2000). 
conductor would allow sodium ions to travel through the electrode, away from the BASE surface, allowing the recombination of electrons and sodium ions to take place throughout the electrode (Ryan et al. 2001). These processes would in effect increase the magnitude of the charge-exchange current, $B$, by facilating ion-electron recombination and removal of sodium ions frm the BASE interface, and decrease the geometric loss factor, $G$, by providing an additional mechanism for alkali metal transport through the electrode. Efforts to develop such mixed-conducting (ionic and electronic) metal/oxide electrodes such as blends of $\mathrm{Mo} / \mathrm{Na}_{\mathrm{x}}-\mathrm{TiO}_{2}$ and $\mathrm{TiN} / \mathrm{Na}_{\mathrm{x}}-\mathrm{TiO}_{2}$ are underway at both the Jet Propulsion Laboratory (Ryan et al. 2001) and Advanced Modular Power systems (AMPS), Inc. (Fletcher and Schwank 2002 and 2003). These electrodes can be applied as a paint or slurry, are robust and inexpensive, and have demonstrated to date a performance equivalent to the best currently available refractory electrode $\left(\mathrm{WRh}_{\mathrm{x}}\right)(\mathrm{Ryan}$ et al. 2001). Further studies on metal material selection, and optimization of the mixedconducting electrodes' composition and thickness offer the promise for improved lifetime and performance of AMTEC devices.

\subsubsection{Materials Selection for the Porous Anode}

The volatile alloying elements of manganese and chromium in the stainless steel structure of the PX-type, sodium converters could also have contributed to the observed degradation of the TiN electrodes and the BASE (Ryan et al. 1999). Several chemical reactions of these volatile elements with the BASE have been reported (Williams et al. 1999), which convert beta"- to alpha-alumina $\left(\mathrm{Ab}_{2} \mathrm{O}_{3}\right)$ and produce metal oxides. For example, chromium reacts with BASE to form a thin surface film of $\mathrm{Cr}_{2} \mathrm{O}_{3}$ and $\alpha-\mathrm{Al}_{2} \mathrm{O}_{3}$, impairing the passage of the sodium ions through and, hence, the converter performance over time. Manganese has also been shown to react with the BASE to form $\mathrm{MnAl}_{2} \mathrm{O}_{3}$ and possibly $\alpha-\mathrm{Al}_{2} \mathrm{O}_{3}$ (Williams et al. 1999). These reaction products are likely to increase the ionic resistance of the BASE, further degrading the converter performance. Owing to these concerns, stainless steel-316 and Haynes-25 super steel alloy (17-20\% Cr, $10-14 \% \mathrm{Ni}$ and $1.5-2 \% \mathrm{Mn}$ ) have been excluded in the second generation PX-type, sodium converters.

Instead, the niobium alloys $\mathrm{Nb}-1 \mathrm{Zr}$ and $\mathrm{C}-103$, and lately molybdenum-rhenium (Mo-Re) alloys were considered (Hendricks et al. 1999, Kramer et al. 2000, El-Genk and King 2001, Giglio et al. 2001, DiStefano and Chitwood 2001, Moore et al. 2002). The niobium alloys were later excluded on the ground that they become brittle during assembly in glove boxes at oxygen concentration as low as one ppm (Kramer et al. 2000). Also, the gettering effect of zirconium in the $\mathrm{Nb}-1 \mathrm{Zr}(1 \% \mathrm{Zr})$ and $\mathrm{C}-103(0.5 \% \mathrm{Zr})$ niobium alloys may deplete the oxygen in the BASE over a ong period of operation (Schock et al. 1999). In addition to being more resistive to embrittlement by the oxygen, Mo-Re alloys are stronger than the niobium alloys (Mo-41\%Re has twice the strength of Nb-1\%Zr), but heavier (Kramer et al. 2000, El-Genk and King 2001, DiStefano and Chitwood 2001). The oxidation resistance of Mo-41\%Re is much higher than $\mathrm{Nb}-1 \% \mathrm{Zr}$ because the oxidation rate of the former is slower, and the oxidation of Mo- $41 \% \operatorname{Re}$ is 
limited to a surface effect and does not reduce the strength nor the ductility of the alloy, as is observed with $\mathrm{Nb}-1 \% \mathrm{Zr}$ (Moore et al. 2002).

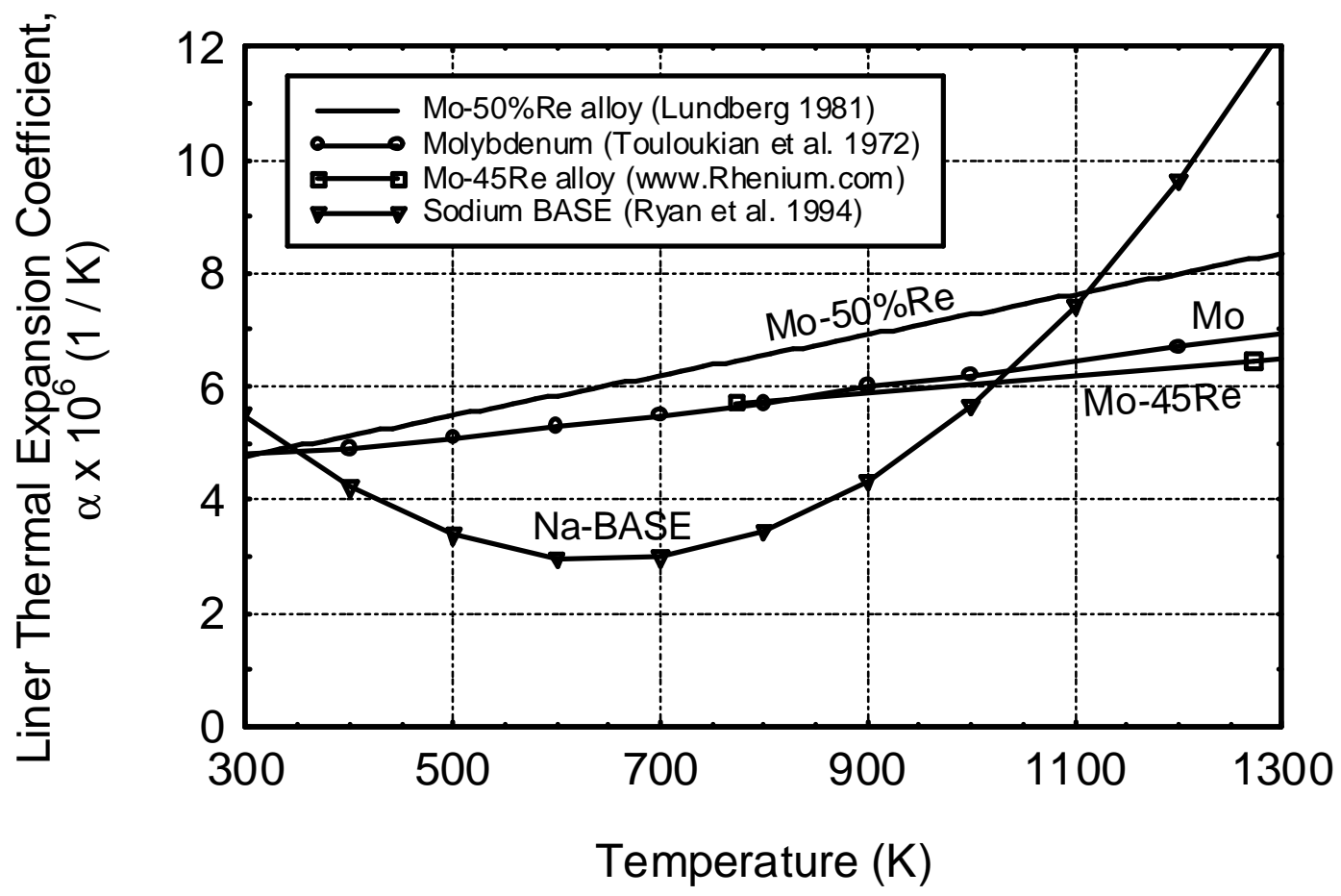

Figure 3-74. Thermal expansion coefficient of Na-BASE and molybdenum refractory alloys.

In addition to being compatible with the alkali metal working fluid and BASE, the refractory alloy candidate materials for the porous structure of the BASE anode must have a thermal expansion coefficient that matches closely that of BASE at the operating temperature of interest.

Figure 3.74 shows that, indeed, the molybdenum-rhenium refractory alloys have a thermal expansion coefficient that matches that of BASE in the temperature range 1000 $1127 \mathrm{~K}$ of interest. Recently, arc melted Mo-41\%Re has been produced at the Oak Ridge National Laboratory using a manufacturing process similar to that used for the production of iridium alloy for the encapsulation of plutonium dioxide in the General Purpose Heat Source (Moore et al. 2002). The Mo-41\%Re alloy was rolled into plates and sheets that were used for characterization and R\&D relevant to AMTEC converters. Initial tests indicated that Mo- $41 \%$ Re can be electron-beam welded without the porosity normally observed in welded powder metallurgy products. The welds exhibited good ductility (Moore et al. 2002). 


\subsubsection{Recent Advances in the Fabrication of Na-BASE and K-BASE}

An avenue to improve the conversion efficiency of the AMTEC converter, not seriously considered until now, is using potassium working fluid. Potassium has higher vapor pressure, and bwer latent heat of vaporization (2000 versus $\sim 4000 \mathrm{~kJ} / \mathrm{kg}$ ) than sodium. Therefore, a potassium converter may provide the same performance as a sodium converter, but at a lower hot side temperature. At a typical 80 to $120 \mathrm{~K}$ lower hot side temperature for the potassium converter, the materials compatibility and the BASE and electrodes performance degradation issues could be minimized considerably; also, the parasitic heat losses would be lower, increasing the conversion efficiency. The higher vapor pressure of potassium, however, would also lower the optimum condenser temperature by $\sim 90 \mathrm{~K}$ compared to that for the sodium converters, decreasing the conversion efficiency of the thermoelectric bottom cycle.

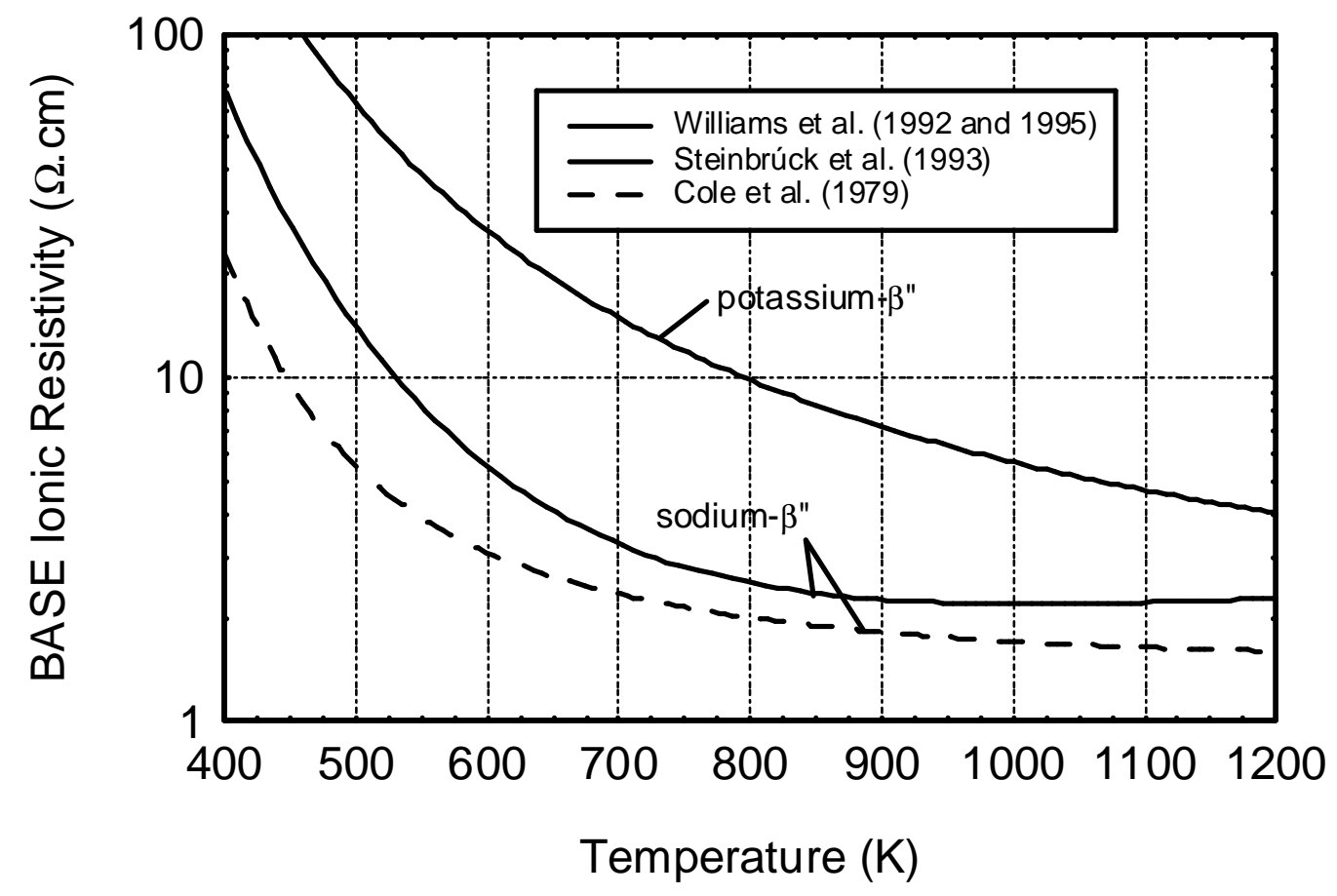

Figure 3-75. Ionic resistivity of sodium and potassium- $\beta$ "-alumina solid electrolytes (Cole et al. 1979, Steinbrück et al. 1993, Williams et al. 1992 and 1995).

One of the reasons that sodium converters have been investigated more intensively than potassium converters is that the development and fabrication techniques of the potassium $\beta$ "-alumina solid electrolyte (K-BASE) has been slow coming. However, the commercial availability of K-BASE in recent years makes it possible to design and fabricate potassium AMTEC converters. Historically, direct fabrication of the K-BASE using the sintering process used for Na-BASE has been extremely difficult due 
to the high vapor pressure of $\mathrm{K}_{2} \mathrm{O}$ at the sintering temperatures (Barkan et al. 1999). Although single crystals $\mathrm{Na} \beta$ - and $\beta$ "-alumina can be ion-exchanged to form K-BASE by immersion into molten nitrates, polycrystalline membranes of Na-BASE fracture on contact with molten $\mathrm{KNO}_{3}$. The current manufacturing technique syntheses leak-tight $\mathrm{K}$ BASE by exchanging polycrystalline membranes of $\mathrm{Na}$-BASE using a $\mathrm{KCl}$ vapor ion exchange process (Crosbie and Tennenhouse 1982, Williams et al. 1995). The reported ionic resistivity of the K-BASE at high temperature is nearly 3 times that of Na-BASE (see Figure 3.75). This drawback of K-BASE can be easily alleviated by applying a BASE layer that is preferably thin $(\sim 50-200 \mu \mathrm{m})$, but thick enough to be hermetically sealed to prevent non-electrolytic transport of alkali metal. A thin BASE layer would have small or negligible ionic resistance. Furthermore, in the event that some of the beta"-alumina is converted to beta'-alumina over time, the ionic resistance would still be small, even though beta'-alumina has higher ionic resistivity than beta"-alumina.

Recently, a new manufacturing technique for alkali-metal $\beta$ - and $\beta$ "-alumina was developed by Materials and Systems Research, Inc., in Salt Lake City, Utah (Virkar et al. 2000). In this technique, a ceramic composite of $\alpha$-alumina and an oxygen-ion conductor (such as zirconia, ceria, thoria, or any of their forms) is formed by a conventional green forming method. Both components are present in amounts suitable to form continuous matrices of each, providing two continuous, penetrating networks through the composite. The ceramic composite is then exposed to the appropriate alkali metal oxide vapor $\left(\mathrm{Na}_{2} \mathrm{O}\right.$ or $\mathrm{K}_{2} \mathrm{O}$ ) at a high temperature, between 1400 and $1773 \mathrm{~K}$. The vapor may contain a $\beta$ "'alumina stabilizer such as $\mathrm{Li}_{2} \mathrm{O}, \mathrm{MgO}$, and/or $\mathrm{ZnO}$. Alternatively, the stabilizer may be included in the ceramic composite. During the fabrication process, the oxygen ions are transported through the oxygen-ion conductor to the reaction interface, while the alkalimetal ions are conducted through the already formed $\beta$ "-alumina phase. In this manner, rapid paths are provided for both species, and the reaction kinetics are not controlled by their rates of diffusion, but primarily by the formation rate of $\beta$ "-alumina at the reaction front. Using this process, an $\alpha$-alumina sheet that is $75 \mu \mathrm{m}$ thick was converted into NaBASE in 16 hours at $1723 \mathrm{~K}$, compared to several weeks if using direct sintering processes. The final product is a ceramic composite of the $\beta$ "-alumina and the oxygenion conductor.

\subsubsection{Materials Compatibility and Lifetime Issues in AMTEC Components}

One of the key considerations in the selection of structural materials for the open-loop alkali metal thermal-to-electric converter (AMTEC) is the compatibility of the material with the operating conditions present inside the converter. These conditions include contact with liquid and vapor alkali metals at temperatures up to $1150 \mathrm{~K}$ and pressures up to $100 \mathrm{kPa}$ on the evaporator (anode) side of the converter. The condenser and liquid return structures of the converter will be in contact with liquid alkali metal at temperatures between 500-650 K. Other components on the cathode side of the converter will be exposed to liquid alkali metal at low pressure $(\sim 30 \mathrm{~Pa})$ and at a range of 
temperatures (from 500-650 K near the condenser to $\sim 1125 \mathrm{~K}$ near the boundary between the high and low pressure cavities).

Under these conditions, the converter's evaporator, support structures, condenser, liquid return structures and anode wicks will be subject to corrosion by liquid alkali metal. The beta" alumina solid electrolyte (BASE) and alumina insulators may be degraded by deposition of volatilized support structure constituents as well as chemical reaction with liquid alkali metal. The BASE and insulators may also release sodium oxide over time, which may influence the corrosion rate of other parts of the converter by liquid alkali metal. The converter electrodes can chemically react with deposited structural constituents and are life limited by high-temperature grain growth, which decreases the performance of the electrode. The contaminant-driven degradation mechanisms of BASE, $\alpha$-alumina insulator and AMTEC electrodes are reviewed next.

\subsubsection{Contaminant-Driven Degradation of AMTEC Components}

Several adverse contaminant/BASE reactions have been documented (Williams et al. 1998, Williams et al. 1999). Generally speaking, the known contaminants of concern react with BASE (composition $\left.\mathrm{Na}_{2} \mathrm{O}\left(\mathrm{Li}_{2} \mathrm{O}\right)_{0.176}\left(\mathrm{Al}_{2} \mathrm{O}_{3}\right)_{6.21}\right)$ to form a metal oxide and alumina (Al2O3). Chromium in contact with BASE reacts to form a thin surface film of $\mathrm{Cr} 2 \mathrm{O} 3$ and $\mathrm{ALO}_{2}$ (Williams et al. 1998). This film is likely to impair the passage of sodium ions into the electrolyte, reducing converter performance. Manganese and BASE have been shown to react to form MnA2O3 and probably Al2O3 (Williams et al. 1999). These reaction products are also likely to increase the ionic resistance of the BASE, impairing converter performance. The uptake of iron by BASE is slow and results in no detectable reaction product (Williams et al. 1999). The interaction of nickel and BASE has not been extensively studied. The important reactions between the $\alpha$-alumina insulator and potential contaminants are not known.

A number of adverse reactions between electrodes and contaminant materials are also known. The nature of these reactions is generally more diverse than the BASE/contaminant reactions. Mangane se and chromium react with and degrade titanium nitride (TiN) electrodes (Ryan et al. 1998). Nickel in contact with the advanced tungsten/rhodium (W/Rh) electrodes has been shown to cause rhodium diffusion towards the nickel along with increased sintering and void formation, resulting in reduced electrode performance (Ryan et al. 1992). Manganese in contact with W/Rh electrodes accelerates grain growth, which decreases electrode performance and lifetime (Ryan 1999).

During the lifetime of the AMTEC converter, contaminants (such as nickel, chromium and manganese released from a steel or superalloy converter structure) will have the opportunity to come into contact with the BASE, the electrical insulators and the electrodes. These materials may be dissolved and transported in the liquid alkali metal 
working fluid or they may evaporate from the converter structure and be transported by diffusion to the BASE/insulator/electrode assemblies. It is important that these contaminants be as immobile and as non-detrimental as possible. The evaporation and transport of structural material constituents and the resulting degradation of the BASE and electrodes has received considerable attention in the development of multitube, vapor-anode AMTEC cells for space applications and has been a driving factor in materials selection (King and El-Genk 2000). Fortunately, the design of the proposed terrestrial converter is likely to be mitigating. The use of a flash evaporator and heat-pipe style heat transfer is likely to trap many of the contaminants in the evaporator pool. Likewise, the use of an open-loop style converter in conjunction with alkali metal boilers will allow for cold-trapping or filtering to prevent the build up of contaminants in the working fluid. Unlike space applications, the terrestrial converters may be replaced, although shortened converter life will increase the overall cost of the system.

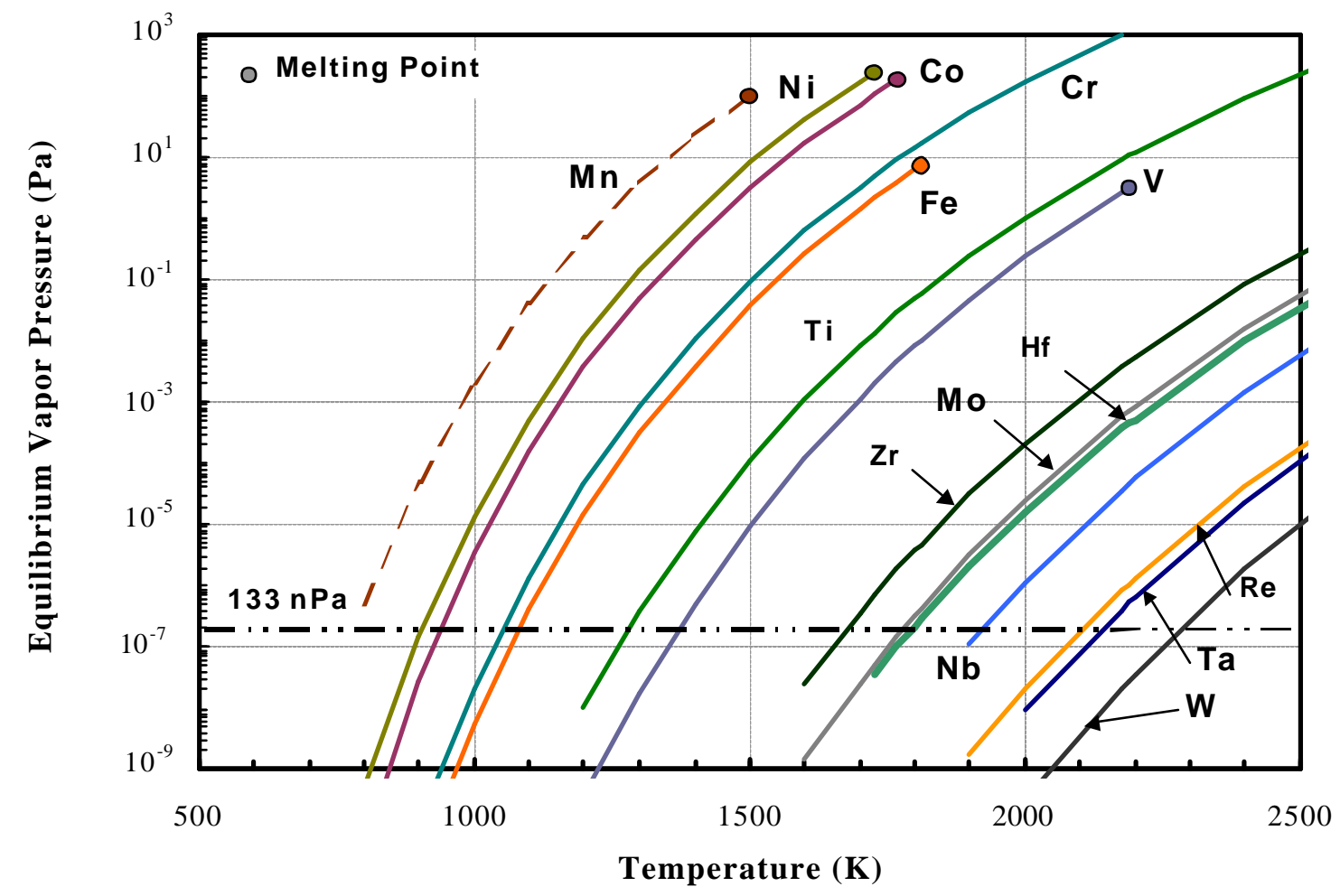

Figure 3-76. Vapor pressure of structural metallic elements .

The amounts of metallic contaminants present in the AMTEC converter can be estimated based on their vapor pressure. The vapor pressures of several base and alloying metals are shown in Figure 3.76. From this figure, it is apparent that the refractory metals (niobium, tantalum and molybdenum) are several orders of magnitude less volatile than the typical components of stainless steel and superalloys (iron, nickel, cobalt and chromium). A pressure of $10^{-9}$ torr $(133 \mathrm{nPa})$ has been recommended by researchers at 
the Jet Propulsion Laboratory as an upper limit on vapor pressure in order to prevent material transport and deposition in vapor-anode AMTEC cells for space applications (Ryan 1999). This limit eliminates the stainless steels and superalloys from further consideration as AMTEC structural materials for space applications (King and El-Genk 2000). This limit is probably overly conservative when considered for the proposed terrestrial converters. Degradation mechanisms of the beta"-alumina solid electrolyte (BASE), and their contribution to oxygen levels in the AMTEC converter are discussed next.

\subsubsection{Thermal Degradation of BASE}

The Jet Propulsion Laboratory has extensively studied the thermal degradation of the beta"-alumina solid electrolyte. The main mechanism for degradation of the electrolyte is the slow loss of sodium oxide at high temperatures with or without a corresponding change in phase (Williams et al. 2000). An evenly distributed phase change in a small amount of electrolyte would result in a slight reduction in the overall ionic conductance of the electrolyte. A massive phase change or the formation of different phase surface layers would significant impair the performance of the electrolyte. The loss of sodium oxide, whether or not a phase change is involved, will add oxygen to the liquid metal system and may result in an increase in the rate of structural material corrosion.

At the expected operating temperatures of the converter $(\sim 1000 \mathrm{~K})$, the degradation of the beta'-alumina solid electrolyte has been shown to be slow and nearly negligible except when contaminants such as chromium or manganese are in contact with the electrolyte (Williams et al. 2000). One of the major degradation mechanisms of AMTEC porous electrodes, slow grain growth at operating temperature, is discussed next.

\subsubsection{Grain Growth in AMTEC Electrodes}

Grain growth in the electrodes has been predicted to be one of the significantly life limiting processes in an AMTEC converter (Ryan et al. 1999). As grain growth in an AMTEC electrode proceeds, the temperature-independent charge-exchange coefficient (B) will decrease as shown in Figure 3.77 for Mo, W/Rh and W/Pt electrodes. It is expected that the $B$ coefficient for TiN electrodes will also decrease with increasing grain radius (Ryan et al. 2000). These changes will increase the charge-exchange overpotential at the BASE/cathode electrode interface, and decrease the performance of the converter.

Based solely on electrode grain growth models and assuming a lifetime performance loss of 10\%, the Jet Propulsion Laboratory predicts a TiN electrode lifetime of approximately one year at an operating temperature of $1125 \mathrm{~K}$ (Ryan et al. 2000) with an initial $B$ value of 60 . Under the same conditions, an advanced $\mathrm{W} / \mathrm{Rh}$ electrode is 
predicted to have a lifetime exceeding 100 years with an initial $B$ value of 100 . If a $30 \%$ lifetime performance decrease is acceptable, the expected lifetimes are extended to $>10$ and >1000 years, respectively (Ryan et al. 2000). These results could be highly speculative since they are not supported with long-duration experimental data. However, the above discussion indicates that the relative operational lifetime of W/Rh (for the assumed percent degradation) is likely to be much longer than that of TiN.

Operation and lifetime issues in AMTEC converters pertaining to the surface tension and wetting behavior of liquid alkali metal are discussed next.

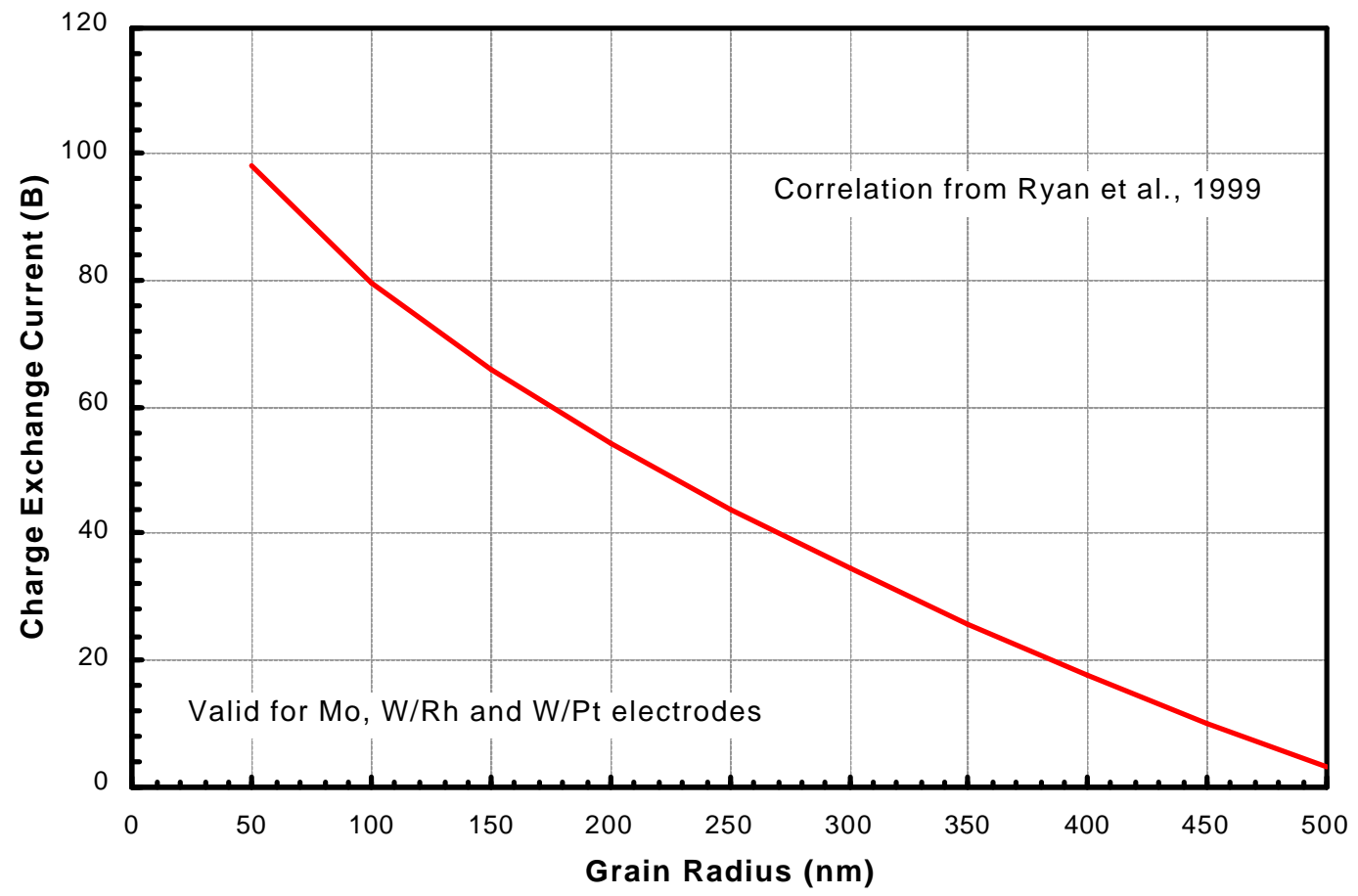

Figure 3-77. Effect of electrode grain growth on charge-exchange coefficient, $B$.

\subsubsection{Effect of Surface Tension and Wetting}

The surface tension and wetting behavior of alkali metal liquids is strongly affected by impurities such as oxygen and the formation of oxide deposits on the surface (Viswanathan and Virkar 1982, Mailhe et al. 1987). As a rule of thumb, liquid metals easily wet metals but poorly wet oxides. In sulfur/sodium batteries operated at 425-625 $\mathrm{K}$ (which also use a sodium beta"-alumina solid electrolyte), reduced battery performance has been attributed to poor wetting of the BASE by liquid sodium (Mailhe et al. 1987). Poor wetting has also been implicated in reduced battery electrolyte lifetime resulting from the propagation of pressurized sodium filled cracks (Viswanathan and Virkar 1982). While it is reasonable that similar effects may occur in a liquid anode AMTEC, better quantitative data on the effect of impurities is needed to make a proper assessment. 
Important findings and conclusions on the materials compatibility and lifetime issues pertaining to the components of the AMTEC converter are summarized in the next chart.

The important material issues and recommendations made under Task $2 \mathrm{~d}$, pertaining to the lifetime of the AMTEC converters and the selection of suitable structural materials, are summarized next.

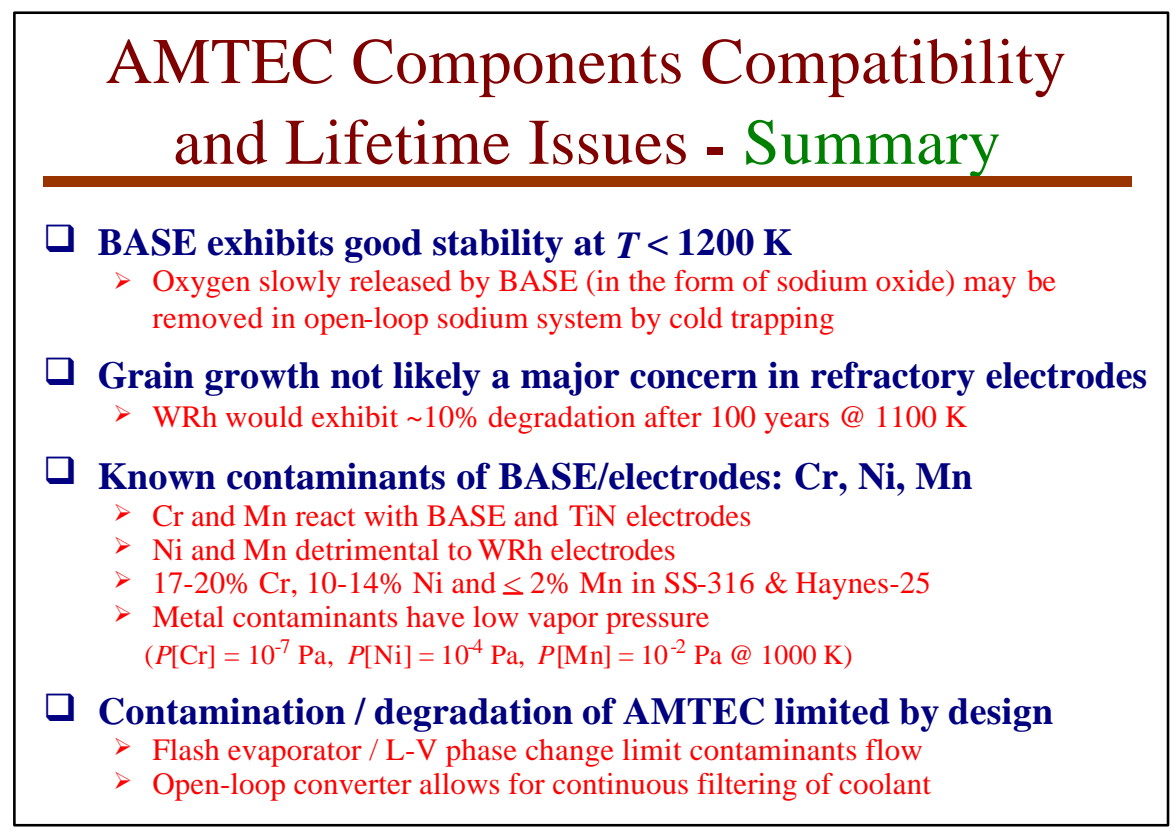

\subsubsection{Degradation Mechanisms in AMTEC Cells}

An important part of the present effort to develop a conceptual design of the highperformance AMTEC cells is assessing and identifying potential degradation mechanisms of the various components of the proposed static converter. Important material degradation issues identified in this work are as follows:

(a) corrosion of structural materials (refractory metals and alloys) exposed to ambient air;

(b) corrosion of structural materials by liquid alkali metal, whose rate strongly depends on the oxygen content in the system. As indicated earlier, the oxygen content in sodium working fluid could be kept well below 1 ppm using cold traps. By contrast, the oxygen content in potassium working fluid can only be controlled to such levels by using active oxygen getters;

(c) strength and creep of structural materials at typical operating temperature of 1000 - $1150 \mathrm{~K}$; chemical reactions between AMTEC electrodes/BASE materials and deposited, volatilized structural constituents. All these concerns could be dealt with through proper selection of materials, understanding of degradation mechanisms, and proper preventive maintenance of the electric conversion units; 
(d) grain growth in the electrodes' material at high temperature; and

(e) loss of oxygen and sodium oxide from BASE and electrical insulators, which affects the properties of these components and also contributes to oxygen present in the cell (which may affect corrosion in other parts of the cell). Corrosion by the liquid alkali metal may occur by dissolution, chemical reaction with impurities (oxygen, but also carbon and nitrogen), and mass transport driven by differences in chemical potential and temperature gradients.

From an operation point of view, it is preferable to keep the core exit temperature as low as possible (fuel swelling increases with $\sim T^{3}$ ). The literature on structural materials for use in sodium-LMR systems (EBR2, Phenix, Super-Phenix,...) was reviewed, and a database on their thermophysical and compatibility properties was compiled. In particular, the levels of contaminants in the liquid metal incoming from the core in operating sodium systems were compiled. During this compilation process, data pertaining to potassium working fluid were also collected for future use and documentation. Most of the information pertaining to potassium corrosion and materials compatibility originated from the research and development programs of PotassiumRankine cycles in the sixties and seventies (Angelo and Buden 1985, DiStefano 1989).

A review of the literature has shown that Na-BASE material is expected to exhibit good stability up to $1200 \mathrm{~K}$ (Williams et al. 1998b and 1999). However, there are no conclusive data available at the present time. The oxygen slowly released by BASE over time may be removed by cold trapping in an open-loop sodium system. One degradation mechanism of the AMTEC electrodes is the growth of the grains of the electrode materials, reducing the available area for recombination and evaporation of sodium atoms on the cathode side of the BASE and increasing the pressure drop by the diffusion of these atoms through the porous electrode. Ryan et al. (2000) predicted that such grain growth alone could degrade the performance of TiN electrodes by $\sim 10 \%$ after 2 years and by $\sim 30 \%$ after 16 years. Higher performance electrodes such as tungsten/rhodium $\left(\mathrm{WRh}_{\mathrm{x}}\right.$, where $x$ varies between 1 and 2$)$ are currently being tested at the Jet Propulsion Laboratory (Ryan et al. 1999b, 2000 and 2001). Extrapolated results of accelerated tests suggest that these electrodes would degrade because of grain growth by only $10 \%$ after 100 years (Ryan et al. 2000). Therefore, grain growth is not likely to be a major concern in AMTEC with WRh refractory electrodes.

Several adverse contaminant/BASE reactions have been reported (Williams et al. $1998 \mathrm{~b}$ and 1999), which involve volatile alloying elements in stainless steel and form metal oxides and alumina $\left(\mathrm{Al}_{2} \mathrm{O}_{3}\right)$. For example, chromium reacts with $\mathrm{BASE}$ to form a thin surface film of $\mathrm{Cr}_{2} \mathrm{O}_{3}$ and $\mathrm{Al}_{2} \mathrm{O}_{3}$, impairing the passage of sodium ions into the BASE solid electrolyte and, hence, reducing the converter performance over time. Manganese has also been shown to react with the BASE material to form $\mathrm{MnAl}_{2} \mathrm{O}_{3}$ and probably $\mathrm{Al}_{2} \mathrm{O}_{3}$ (Williams et al. 1999). In addition, the reaction products are likely to increase the ionic resistance of the BASE, further impairing the converter performance. Chromium $(\mathrm{Cr})$, nickel $(\mathrm{Ni})$ and manganese $(\mathrm{Mn})$ are also known contaminants of AMTEC electrodes (Ryan et al. 1992, 1994, 1999 and 2000). Cr and Mn react with TiN electrodes, and $\mathrm{Ni}$ and $\mathrm{Mn}$ are detrimental to WRh electrodes. These contaminants are present as alloying elements in SS-316 and Haynes-25 (17-20\% Cr, 10-14\% Ni and $\leq 2 \%$ 
$\mathrm{Mn})$, however their vapor pressures are relatively low $\left(P[\mathrm{Cr}]=10^{-7} \mathrm{~Pa}, P[\mathrm{Ni}]=10^{-4} \mathrm{~Pa}\right.$, and $P[\mathrm{Mn}]=10^{-2} \mathrm{~Pa}$ at $\left.1000 \mathrm{~K}\right)$.

The contamination and degradation of AMTEC cells can be limited by design, proper selection of materials of various components, and preventive maintenance of the electric conversion units. In the proposed design of the indirect coupling of the vapor-fed AMTEC cells with the reactor core, liquid-vapor phase change of the alkali metal in the boilers (AMBs) would limit the flow of contaminants to the BASE and AMTEC electrodes. Most contaminants will reside in the liquid pool at the bottom of the AMBs. These contaminants could be removed using cold-trapping and/or active getters. In such system, continuous filtering of the coolant can be conducted easily.

\subsection{References}

Alger, D. L. (1997) "Some Corrosion Failure Mechanisms of AMTEC Cells," in Proceedings of the 32nd Intersociety Energy Conversion Engineering Conference, 1997, American Chemical Society, Paper No. 97014, 2:1224-1229.

Anderson, W. G., Bland, J. J., Ivanenok, J. F., III, and Sievers, R. K. (1993) "Design of a Wick-Pumped, Vapor-Fed AMTEC System," Proc. $28^{\text {th }}$ Intersociety Energy Conversion Engineering Conference, American Chemical Society, Paper No. 93038, 1: 835-841.

Angelo, J. A., and D. Buden (1985) Space Nuclear Power, Orbit Book Company, Inc., Malabar, Florida, pp. 88-93, 133-157, 197-202, and 256-257.

Asakami, O., K. Tsuchida, and A. Kato (1990) "Materials for Electrode of Alkali Metal Thermoelectric Converter (AMTEC) (II)," Journal of Materials Science Letters, 9 (8), 892-894.

ASME (1974), ASME Boiler and Pressure Vessel Code Section VIII, American Society of Mechanical Engineers, New York, 1974.

Atkin, S.D., Puigh, R.J., and Grossbeck, M.L. (1989) "SP-100 Phase I Materials Irradiation Experiment," Space Nuclear Power Systems 1988, edited by M.S. El-Genk and M.D. Hoover, Orbit Book Company, Malabar, FL, 1989, pp. 257-279.

Ault, G.M. (1966) A Decade of Progress in Refractory Metals, American Society for Testing and Materials, Philadelphia, PA, 1966, pp. 5-6.

Baker, H. (1978), senior editor, Metals Handbook, Ninth Edition, Vol. 1, American Society for Metals, Metals Park, OH, 1978, pp. 655.

Bankston, C. P., T. Cole, R. Jones, and R. Ewell (1983) "Experimental and Systems Studies of the Alkali Metal Thermoelectric Converter for Aerospace Power," Journal of Energy, 7 (5): 442-450.

Bankston, C. P., T. Cole, S. K. Khanna, and A. P. Thakoor (1985) "Alkali Metal Thermoelectric Conversion (AMTEC) for Space Nuclear Power Systems," in Space Nuclear Power Systems 1984, M. S. El-Genk and M. D. Hoover, Eds., Orbit Book Company, Malabar, FL, Vol. II, pp. 393-402. 
Barkan, A., and T. K. Hunt (1998) "Investigations of DC Electrical Discharges in LowPressure Sodium Vapor With Implications for AMTEC Converters," in Proceedings of the $33^{\text {rd }}$ Intersociety Energy Conversion Engineering Conference, American Nuclear Society, LaGrange Park, IL, 1998.

Barkan, A., T. K. Hunt, and B. Thomas (1999) "Potassium AMTEC Cell Performance," Proceedings of the $34^{\text {th }}$ Intersociety Energy Conversion Engineering Conference, Society of Automotive Engineers, Inc., Paper No. 1999-01-2702.

Bates, J. B., Wang, J.-C., and Dudney, N. J. (1982) "Solid Electrolytes - the Beta Aluminas," Physics Today, July 1982, pp. 46-53.

Bates, J. B., G. M. Brown, T. Kaneda, W. E. Brundage, and J. C. Wang (1979) "Properties of Single Crystal Beta"-Alumina," in Fast Ion Transport in Solids: Electrodes and Electrolytes, P. Vashishta, J. N. Mundy, and G. K. Shenoy, Eds., Elsevier North-Holland, Inc., pp. 261-266.

Bechtold, R.A., Meadows, G.E., and McCown, J.J. (1984) "Experience With Chemistry Monitoring at FFTF," in Liquid Metal Engineering and Technology - Proceedings of the Third International Conference held in Oxford on 9-13 April 1984, British Nuclear Energy Society, London, 1984, vol. 1, pp. 39-43.

Benjamin, D. (1980), senior editor, Metals Handbook, Ninth Edition, Vol. 3, American Society for Metals, Metals Park, OH, 1980, pp. 213-223.

Bird, R. B., W. E. Stewart, and E. N. Lightfoot (1960) Transport Phenomena, John Wiley @ Sons, New York, NY, Chapter 7, pp. $208-240$.

Bodoia, J. R., and J. F. Osterle (1962) "The Development of Free Convection between Heated Vertical Plates," ASME Journal of Heat Transfer, 84, 40-44.

Borgstedt, H.U., and Mathews, C.K. (1987) Applied Chemistry of the Alkali Metals, New York, Plenum Press, 1987, pp. 75-224.

Briant, J. L., and G. C. Farrington (1980) "Ionic Conductivity in $\mathrm{Na}^{+}, \mathrm{K}^{+}$, and $\mathrm{Ag}^{+}$Beta"Alumina," Journal of Solid State Chemistry, 33, 385-390.

Brown, S. C. (1966) Introduction to Electrical Discharges in Gases, John Wiley \& Sons, Inc., NY.

Brown, Sanborn C. (1966) Introduction to Electrical Discharges in Gases, John Wiley \& Sons, Inc., New York, NY.

Bouchiat, M. A., Guena, J., Jacquier, Ph., Lintz, M. and Papoyan, A. V. (1999) "Electrical Conductivity of Glass and Sapphire Cells Exposed to Dry Cesium Vapor," Applied Physics B, 68, 1109-1116.

Caillat, T., Borshchevsky, A., Snyder, J., and Fleurial, J.-P. (2001) "High Efficiency Segmented Thermoelectric Couples," in Proceedings of Space Technology and Applications International Forum, M. El-Genk, Ed., AIP-CP-552, American Institute of Physics, New York, NY, 2001, pp. 1107-1112.

Carlson, M. E., J. C. Giglio, and R. K. Sievers (1998) "Design and Fabrication of MultiCell, AMTEC Power Systems for Space Applications," in Proceedings of the 3rd Space 
Technology and Applications International Forum (STAIF-98), CONF-980103, M. S. ElGenk, Ed., American Institute of Physics, New York, NY, AIP Conference Proceedings No. 420, 3: 1486-1490.

Carlson, E.C., Hendricks, T.J., Sievers, R.K., and Svedberg, R.C. (1999) "Design of the EPX-1 AMTEC Converter for the Advanced Radioisotope Power System," Proc. $34^{\text {th }}$ Intersociety Energy Conversion Engineering Conference, Society of Automotive Engineers, Inc., Paper No. 1999-01-2654.

Chi, S. W. (1976) Heat Pipe Theory and Practice: A Sourcebook, Hemisphere Publishing Corporation, Washington, DC, Chapter I, pp. 1-28.

Chi, J. W. H., K. Kesavan, R. E. Morgan, and J. E. Schmidt (1987) "Comparison of Multimegawatt Space Nuclear Power Systems," in Space Nuclear Power Systems 1986, M. S. El-Genk and M. D. Hoover, Eds., Orbit Book Company, Malabar, FL, Vol. V, pp. $417-425$.

Christopoulos, C. (1979) "The Electrical Conductivity of Caesium Plasma," Physics Letters, vol. 71A, No. 2-3, pp. 227-230.

Christopoulos, C. and Endean, M. A. (1980) "Prebreakdown Electrical Conductivity of Nearly Saturated Caesium Vapour," IEE Proceedings, vol. 127, No. 2, Part A, pp. 95101.

Claar, T.D. (1970) "Solubilities of Metallic Elements in Liquid Sodium," Reactor Technology, 13 (2), 124-146.

Cobine, J. D. (1958) Gaseous Conductors: Theory and Engineering Application, Dover Publications, Inc., New York, NY.

Cole, T. (1983) "Thermoelectric Energy Conversion with Solid Electrolytes," Science, 221: 915-920.

Condliff, A.F., and Marsh, R.J. (1987) "Selection of Structural Materials for Space Nuclear Power Generators," Space Nuclear Power Systems 1986, edited by M.S. El-Genk and M.D. Hoover, Orbit Book Company, Malabar, FL, 1987, pp. 275-281.

Crosbie, G. M., and G. J. Tennenhouse (1982) "Potassium Beta"-Alumina Membranes," Journal of the American Ceramic Society, 65 (4), 187-191.

Crowley, C. J., and M. G. Izenson (1993) "Condensation of Sodium on a Micromachined Surface for AMTEC," in Proceedings of the 10th Symposium on Space Nuclear Power and Propulsion, held in Albuquerque, NM, January 10-14, 1993, Conference No. 930103, El-Genk, M. S., and M. D. Hoover, Eds., American Institute of Physics, New York, NY, 2: 897-904.

DeVan, J.H., DiStefano, J.R., and Hoffman, E.E. (1984) "Compatibility of Refractory Alloys with Space Reactor Coolants and Working Fluids," in Proceedings of the Symposium on Refractory Alloy Technology for Space Nuclear Power Applications, edited by R.H. Cooper and E.E. Hoffman, Report CONF-8308130, U.S. Department of Energy, 1984, pp. 34-85. 
DiStefano, J. R. (1989) "Review of Alkali Metal and Refractory Alloy Compatibility for Rankine Cycle Applications," in Space Nuclear Power Systems 1988, edited by M.S. El Genk and M. D. Hoover, Orbit Book Company, Malabar, FL, 1989, pp. 299-310.

DiStefano, J. R., and Chitwood, L. D. (2001) "Oxidation of Mo-Re at Reduced Oxygen Pressures," Proc. Space Technology and Applications International Forum (STAIF2001), M. S. ElGenk, Ed., American Institute of Physics, New York, NY, AIP Conference Proceedings No. 552, 1: 1076-1081.

Elenbaas, W. (1942) "Heat Dissipation of Parallel Plates by Free Convection," Physica, Holland, 9(1): 1-28 (January 1942).

El-Genk, M. S., and King, J. C. (2001) "Performance Analyses of a Nb-1\%Zr/C-103, Vapor Anode, Multi-Tube Alkali-Metal Thermal-to-Electric Conversion Cell," Journal of Energy Conversion and Management, 42: 721-739.

El-Genk, M. S., and Saber, H. (2002a) "Performance Optimization of Segmented Thermoelectric Unicouples," in Proc. of Space Technology and Applications International Forum, M. El-Genk, Ed., AIP-CP-608, American Institute of Physics, Melville, NY, 2002, pp. 980-988.

El-Genk, M. S., and Saber, H. (2002b) Personal Communications, Institute for Space and Nuclear Power Studies, The University of New Mexico, Albuquerque, NM, USA, April 24.

El-Genk, M. S., Saber, H., and Caillat, T. (2002) "A Performance Comparison of SiGe and Skutterudite Based Segmented Thermoelectric Devices," in Proc. of Space Technology and Applications International Forum, M. El-Genk, Ed., AIP-CP-608, American Institute of Physics, Melville, NY, 2002, pp. 1007-1015.

El-Genk, M. S. and J.-M. Tournier (1998a) "Parametric Analysis of Vapor Anode, Multitube AMTEC Cells for Pluto/Express Mission," in Proceedings of the 3rd Space Technology and Applications International Forum (STAIF-98), CONF-980103, M. S. EGenk, Ed., American Institute of Physics, New York, NY, AIP Conference Proceedings No. 420, 3: 1461-1470.

El-Genk, M. S., and J.-M. Tournier (1998b) "Recent Advances in Vapor Anode, MultiTube, Alkali-Metal Thermal-to-Electric Conversion Cells for Space Power," in Proceedings of the 5th European space Power Conference (ESPS-98), held 21-25 September 1998, in Tarragona, Spain, Paper No. 98-1046, SP-416, European Space Agency Publications Division, pp. 257-264.

El-Genk, M. S., and Tournier, J.-M. (2000) "Design Optimization and Integration of Nickel/Haynes-25 AMTEC Cells into Radioisotope Power Systems," Journal of Energy Conversion and Management, 41: 1703-1728.

El-Genk, M. S., J.-M. Tournier, J. King and Y. Momozaki (2000) Novel, Integrated Reactor/Power Conversion System (NIR/PCS): Alkali Metal Thermal-to-Electric Energy Conversion - Progress Report, Performance Period: September 1999 - August 2000, Report Number UNM-ISNPS-3-2000, U. S. Department of Energy Award No. 99-0198, Institute for space and Nuclear Power Studies, the University of New Mexico, Albuquerque, NM 87131. 
El-Genk, M. S., Tournier, J.-M., and Momozaki, Y. (2001) Novel, Integrated Reactor/Power Conversion System (NIR-PCS): Design and Analyses of AMTEC Units and Interfacing with Nuclear Reactor, Progress Report II No. UNM-ISNPS-2-2001, Institute for Space and Nuclear Power Studies, University of New Mexico, Albuquerque, NM, October 2001.

El-Genk, M. S. and J.-M. Tournier (2001) "Analysis of a Vapor Anode, Multi-tube, Potassium Refractory AMTEC Converter for Space Applications," in Proceedings of the 2001 Space Technology and Applications International Forum (STAIF-01), M. S. ElGenk, Ed., American Institute of Physics, New York, NY, AIP Conference Proceedings No. 552, 1: 1066 - 1075.

El-Genk, M. S. and Tournier, J.-M. (2002a) "Static Converter for High Energy Utilization, Modular, Small Nuclear power Plants," in Proc. of $10^{\text {th }}$ International Conference on Nuclear Engineering (ICONE-10), Arlington, VA, American Society of Mechanical Engineers, NY, 14-18 April, Paper No. ICONE10-22040.

El-Genk, M. S. and Tournier, J.-M. (2002b) "High Energy Utilization, Modular, CoGeneration Nuclear Power Plants with Static Energy Conversion," in Proc. International Congress on Advanced Nuclear Power Plants (ICAPP), 2002 ANS Annual Meeting, held in Hollywood, FL, USA, June 9-13, 2002, American Nuclear Society, La Grange Park, IL, USA.

El-Genk, M. S., and Tournier, J.-M. (2002c) "Performance Comparison of Potassium and Sodium vapor Anode Multi-tube AMTEC Converters," J. Energy Conversion and Management, 43, 1931-1951.

El-Wakil, M. M. (1978) Nuclear Heat Transport, The American Nuclear Society, La Grange Park, Illinois, USA, pp. 233-234, Chapter 10, pp. 259-290, Chapter 12, pp. 363365, Appendix B, pp. 432-433.

Evans, R.W., and Wilshire, B. (1993) Introduction to Creep, The Institute of Materials, London, 1993, pp. 1-6.

Faghri, A. (1995) Heat Pipe Science and Technology, Taylor and Francis, Washington, DC.

Faibish, R.S., Konishi, T., and Gasparini, M. (2002) "Application of Nuclear Energy for Seawater Desalination - Design Concepts of Nuclear Desalination Plants," in Proc. of $10^{\text {th }}$ International Conference on Nuclear Energy (ICONE-10), Arlington, VA, American Society of Mechanical Engineers, NY, 14-18 April, Paper No. ICONE10-22071.

Fang, Q.I., and R. Knödler (1992) "Porous $\mathrm{TiB}_{2}$ Electrodes for the Alkali Metal Thermoelectric Converter,” Journal of Material Sciences, 27 (24), 6725-6729.

Fehrs, D. L. and Stickney, R. E. (1971) "Contact Potential Measurements of The Adsorption of Alkali Metals on Ta(110) and W(100) Crystals," Surface Science, 24, 309331.

Fiebig, B., M. Schuller, M. A. Ryan, R. Williams and P. Hudson (1999) "Advanced Electrodes for AMTEC," in Proceedings of the Space Technology and Applications International Forum (STAIF-1999), M. S. ElGenk, Ed., American Institute of Physics, New York, AIP Conference Proceedings No. 458, pp. 1312-1318. 
Fletcher, R.W., and Schwank, J.W., "Mixed Conductor Electrode Development for the Alkali Metal Thermal Electric Converter (AMTEC)," Topical Conference Proceedings on "Fuel Cell Technology: Opportunities and Challenges", AIChE Spring, 2002 National Meeting, March 10-14, 2002, pp. 328-338.

Fletcher, R. W., and Schwank, J. W. (2003) "Recent Developments in Mixed Ionic and Electronic Conducting Electrodes for the Alkali Metal Thermal Electric Converter (AMTEC)," in Proc. of the Space Technology and Applications International Forum (STAIF-2003), M. S. El-Genk, Ed., American Institute of Physics, Melville, NY, 2003, in print.

Foust, O. J., Ed. (1972) Sodium-NaK Engineering Handbook, Vol. 1 Sodium Chemistry and Physical Properties, Gordon And Breach Science Publishers, INC., N.Y.

Fritts, R. W. (1959) "Design Parameters for Optimizing the Efficiency of Thermoelectric Generators Utilizing P-Type and N-Type Lead Telluride," Transactions of the American Institute of Electrical Engineers, 78: 817, Part I.

Giglio, J. C., Sievers, R. K., and Mussi, E. F. (2001) "Update of the Design of the AMTEC Converter for Use in AMTEC Radioisotope Power Systems," Proc. Space Technology and Applications International Forum (STAIF-2001), M. S. El-Genk, Ed., American Institute of Physics, New York, NY, 2001, AIP Conference Proceedings No. 552, 1: 1047-1054.

Greenberg, S., Ruther, W.E., and Levin, H.A. (1967) "Corrosion of Vanadium Base Alloys in Sodium at 550E to 750EC," in Alkali Metal Coolants - Proceedings of the Symposium on Alkali Metal Coolants, International Atomic Energy Agency, Vienna, Austria, 1967, pp. 63-84.

Gus'kov, Yu. K., Lebedev, M. A. and Stakhanov, I. P. (1965a) "Low-Voltage Arc in Cesium Vapors," Soviet Physics-Technical Physics, 9 (8), 1122-1129.

Gus'kov, Yu. K., Lebedev, S. Ya. and Rodionova, V. G. (1965b) "Electrical Breakdown Through a Gap in Glass in an Atmosphere of Cesium Vapor," Soviet Physics-Technical Physics, 10(1), 123-124.

Hage 1, W.C., Shields, J.A., and Tuominen, S.A. (1984) "Processing and Production of Molybdenum and Tungsten Alloys," Proceedings of the Symposium on Refractory Alloy Technology for Space Nuclear Power Applications, edited by R.H. Cooper and E.E. Hoffman, Report CONF-8308130, U.S. Department of Energy, 1984, pp. 98-113.

Hatsopoulos, G. N. and Gyftopoulos, E. P. (1973) Thermionic Energy Conversion, Vol. 1: Processes and Devices, Cambridge, MIT Press.

Haynes International, Inc. (2000), alloys property data on company website, http://www.haynesintl.com.

Hendricks, T., Huang, C., and Huang, L. (1999) "AMTEC Converter Optimization for Advanced Radioisotope Power System (ARPS) Design," Proc. 34th Intersociety Energy Conversion Engineering Conference, Society of Automotive Engineers, Inc., Paper No. 1999-1-2655. 
Hoffman, E.E. (1985) "Technology Status of Tantalum Alloys for Space Nuclear Power Applications," Space Nuclear Power Systems 1984, edited by M.S. El-Genk and M. D. Hoover, Orbit Book Company, Malabar, FL, 1985, pp. 349-357.

Holmes, J.T., Smith, C.R.F., Osterhout, M.M., and Olson, W.H. (1977) "Sodium Purification By Cold Trapping at the Experimental Breeder Reactor II," Nuclear Technology, 32, 304-314.

Howell, J. R. (1982) A Catalog of Radiation Configuration Factors, McGraw-Hill, New York, NY, Factors C1 to C3, pp. 90 - 91.

Huang, L., and M. S. El-Genk (2001) "Thermal conductivity Measurements of Alumina Powders and Molded Min-K in Vacuum," Energy Conversion and Management, 42, 599-612.

Hunt, T. K., N. Weber, and E. N. Sickafus (1982) Research and Development Program of a Sodium Heat Engine, Final Report No. DOE/ER/10347-T1, Ford Motor Company, Dearborn, MI 48121, October 1982.

Hunt, T. K., R. F. Novack, J. R. McBride, D. J. Schmatz, W. B. Copple, J. T. Brockway, N. Arnon and G. A. Grab (1990) "Test Results of a Kilowatt-Scale Sodium Heat Engine," in Proceedings of the $25^{\text {th }}$ Intersociety Energy Conversion Engineering Conference, American Institute of Chemical Engineers, Vol. 2, pp. 420-425.

Idelchik, I. E. (1986) Handbook of Hydraulic Resistance, $2^{\text {nd }}$ Edition, Revised and Augmented, Hemisphere Publishing Corporation, New York, NY, p. 132, pp. 154 - 156, pp. $323-408$, pp. 433 - 436, p. 614.

Inco Alloys International (2000), alloys property data on company website, http://www.incoalloys.com.

Ivanenok, J. F., III, and T. K. Hunt (1994) "High Voltage Terrestrial AMTEC," in Proceedings of the $29^{\text {th }}$ Intersociety Energy Conversion Engineering Conference, held August 7-11, 1994, in Washington, D.C., American Institute of Aeronautics and Astronautics, Paper No. AIAA-94-3903-CP, pp. 904-908.

Johnson, G. A. (1992) "The Alkali Metal Thermoelectric Converter (AMTEC) Radioisotope Thermo electric Generator (RTG)," in Proceedings of the 27th Intersociety Energy Conversion Engineering Conference, held in San Diego, CA, August 3-7, 1992, Paper No. 929063, Society of Automotive Engineers, Inc., 3: 203-207.

Kalandarishvill, A. G. (1996) "Working Medium Circuit for Alkali Metal Thermal-toElectric Converters (AMTEC)," in Proceedings of the $31^{\text {st }}$ Intersociety Energy Conversion Engineering Conference, held August 11-16, 1996, in Washington, D.C., Institute of Electrical and Electronics Engineers, Paper No. 96130, pp. 885-889.

Kamins, T. I. (1968) "Electrical Properties of Cesium Adsorbed on Insulating Substrates," Journal of Applied Physics, 39 (10), 4536-4546.

Kato, A., H. Nakata, and K. Tsuchida (1993) "Characteristics of Ceramic Electrode for AMTEC. II: Structure of Electrode and Power Density,"in Proceedings of the 28th Intersociety Energy Conversion Engineering Conference, American Chemical Society, Paper No. 93027, 1, 809-814. 
Kennard, E. H. (1938) Kinetic Theory of Gases, McGraw-Hill Book Company, Inc., New York, pp. 60-67.

King, J.C., and El-Genk, M.S. (2000) "A Review of Refractory Materials for VaporAnode AMTEC Cells," in Proceedings of the Space Technology and Applications International Forum (STAIF-2000), edited by M.S. El-Genk, AIP Conference Proceedings No. 504, American Institute of Physics, New York, 2000, pp. 1391-1401.

King, J. C., and El-Genk, M. S. (2001) "Review of Refractory Materials for Alkali Metals Thermal- to-Electric Conversion Cells," J. Propulsion and Power, 17(3), 547-556.

Klopp, W.D. (1985) “Technology Status of Molybdenum and Tungsten Alloys," in Space Nuclear Power Systems 1984, edited by M.S. ElGenk and M. D. Hoover, Orbit Book Company, Malabar, FL, 1985, pp. 359-370.

Klueh, R.L. (1968) "The Effect of Oxygen in Sodium on the Compatibility of Sodium with Niobium," Proceedings of the International Conference on Sodium Technology and Large Fast Reactor Design, ANL-7520 Part I, Argonne National Laboratory, Argonne, IL, 1968, pp. 171-176.

Kramer, D. P., McDougal, J. R, Booher, B. A., Ruhkamp, J. D., Howell, E. I., and Kwiatkowski, J. J. (2000) "Electron Beam and Nd-YAG Laser Welding of Niobium1\%Zirconium and Molybdenum-44.5\%Rhenium Thin Sheet Metal," Proc. 35 Intersociety Energy Conversion Engineering Conference, American Institute of Aeronautics and Astronautics, Paper No. AIAA-2000-2971, pp. 956-961.

Kramer, D.P., Ruhkamp, J.D., McNeil, D.C., Howell, E.I., Williams, M.K., McDougal, J.R., and Booher, R.A. (2000b) "Investigation of Molybdenum-44.5\% Rhenium as Cell Wall Material in an AMTEC Based Space Power System," in Proceedings of Space Technology and Application International Forum (STAIF-2000), edited by M.S. El-Genk, American Institute of Physics, Melville, NY, 2000, AIP Conference Proceedings No. 504, Part 2, pp. 1402-1407.

Kummer, J. T., and N. Weber (1968), U. S. Patent No. 3,458,356 assigned to the Ford Motor Company.

Kummer, J. T. (1972) "Beta-Alumina Electrolytes," in Progress in Solid State Chemistry, Volume 7, H. Reiss and J. O. McCaldin, Eds., Pergamon Press, New York, NY, Chapter 5, pp. 141-175.

Künstler, K., Lang, H.-J., Heyne, H., Ananiev, V.D., Kirpikov, E.B., and Kulpin, J.V. (1993) "Monitoring and Control of Impurities in the Primary Circuit of the IBR-2 Reactor," Journal of Nuclear Materials, 200, 169-176.

La Bar, M. P. and, Simon, W. A. (1997) “The Modular Helium Reactor for the $21^{\text {st }}$ Century," in Proc. Uranium Institute's $22^{\text {nd }}$ Annual International Symposium, held in London, 3-5 September 1997, World Nuclear Association's Symposium Proceedings, London, United Kingdom.

Lebedev, M. A. and Gus'kov, Yu. K. (1963) "Electrical Breakdown in Cesium Vapor," Soviet Physics-Technical Physics, 8 (12), 1086-1087. 
Levy, E. K. (1971) "Optimum Plate Spacings for Laminar Natural Convection Heat Transfer from Parallel Vertical Isothermal Flat Plates," Journal of Heat Transfer, Trans. ASME, Series C, 93(4): 463-465 (November 1971).

Levy, E. K., P. A. Eichen, W. R. Cintani, and R. R. Shaw (1975) "Optimum Plate Spacings for Laminar Natural Convection Heat Transfer from Parallel Vertical Isothermal Flat Plates: Experimental Verification," Journal of Heat Transfer, 97(3): 474475 (August 1975).

Lieberman, M. A. and Lichtenberg, A. J. (1994) Principles of Plasma Discharges and Materials Processing, John Wiley \& Sons, Inc..

Lundberg, L.B. (1981) A Critical Evaluation of Molybdenum and Its Alloys for Use in Space Reactor Core Heat Pipes, Los Alamos Scientific Laboratory Report LA-8685-MS, Los Alamos, NM, January 1981, pp. 5-12.

Mailhe, C., Visco, S., and De Jonghe, L. (1987) "Oxygen Activity and Asymmetric Polarization at the Sodium/Beta"-Alumina Interface," Journal of the Electrochemical Society, 134, 1121-1127.

Mayer, S. E., and I. M. Ritchie (1961) "The Use of High-Temperature TE-Materials (Silicides) for Power Generation in Space," Energy Conversion for Space Power, Progress in Astronautics and Rocketry, 3: 63-72, edited by Nathan W. Snyder, Academic Press Inc., London.

Merrill, J., M. J. Schuller, R. K. Sievers, C. A. Borkowski, L. Huang, and M. S. ElGenk (1997) "Vacuum Testing of High-Efficiency Multi-BASE Tube AMTEC Cells," in Proceedings of the 32nd Intersociety Energy Conversion Engineering Conference, 1997, American Chemical Society, Paper No. 97379, 2:1184-1189.

Merrill, J., M. J. Schuller, and L. Huang (1998) "Vacuum Testing of High-Efficiency Multi-tube AMTEC Cells: February 1997 - October 1997," in Proceedings of the 3rd Space Technology and Applications International Forum (STAIF-98), CONF-980103, M. S. El-Genk, Ed., American Institute of Physics, New York, NY, AIP Conference Proceedings No. 420, 3: 1613-1620.

Metals Handbook (1979) 9th Ed., Vol. 2 - Properties and Selection: Nonferrous Alloys and Pure Metals, American Society for Metals, Metals Park, OH.

(These data are also reproduced in the following: An analysis of startup from the frozen state and transient performance of heat pipes, a thesis presented by Jong Hoon Jang, Georgian Institute of Technology, February 1988.)

Miyatake, O. (1994) "Comparative Study of Flash Evaporation Rates," Desalination, 96: 163-171.

Momozaki, Y., and El-Genk, M. S. (2002) "An Investigation of Breakdown Voltage in AMTECs," in Proc. of the Space Technology and Applications International Forum (STAIF-2002), M. S. El-Genk, Ed., AIP-CP-608, American Institute of Physics, Melville, NY, 2002, pp. 972-979.

Moore, J. P., J. F. King, J. R. DiStefano, and E. K. Ohriner (2002) "Production and Properties of Mo-41\%Re for AMTEC Cell Construction," in Proceedings of the 2002 
Space Technology and Applications International Forum (STAIF-02), to be held February 2002 in Albuquerque, NM, M. S. El-Genk, Ed., American Institute of Physics, New York, NY, in prints.

Nesmeyanov, A.N. (1963) Vapor Pressure of the Chemical Elements, edited by R. Gary, New York, Elsevier Publishing Company, 1963, pp. 150-456.

Nicholls, D. R. (2001) "The Pebble Bed Modular Reactor," Nuclear News Magazine, 44(10), 35-40, September 2001.

Nicholls, D. R. (2002) "Status of the Pebble Bed Modular Reactor," J. Nuclear Energy, 39(4), 231-236.

Nicholson, P. S., A. Tan, and C. K. Kuo (1995) "Method of Forming a Single Crystal Film of Sodium-Beta'-Alumina," U. S. Patent No. 5,415,127; assigned to Ontario Centre for Materials Research, Kingston, Canada, May 16, 1995.

Ohse, Roland W., Ed. (1985) Handbook of Thermodynamic and Transport Properties of Alkali Metals, International Union of Pure and Applied Chemistry, Blackwell Scientific Publications, Oxford.

Osterhout, M.M. (1978) "Control of Oxygen, Hydrogen and Tritium in EBR-II Sodium Systems," Transactions of the American Nuclear Society, 30, 159-160.

Osterhout, M.M. (1980) "Control of Oxygen, Hydrogen and Tritium in Sodium Systems at Experimental Breeder Reactor II,” Nuclear Technology, 49, 47-50.

Oto, A., N. Naohara, M. Ishida, T. Kuroki, K. Katsuki and R. Kumazawa (1995) "Sodium-Immersed Self-Cooled Electromagnetic Pump Design and Development of a Large-Scale Coil for High Temperature," Nuclear Technology, 110, 159-167.

Papoular, R. (1965) Electrical Phenomena in Gases, American Elsevier Pub. Co., NY.

Paramonov, D. (2000) Novel, Integrated Reactor/Power Conversion System, Grant DEFC03-99SF21987 - DOE Program Review, presented June 23, 2000, at the U.S. Department of Energy Headquarters, Germantown, Washington, DC.

Paramonov, D., et al. (2001) Novel, Integrated Reactor/Power Conversion System (LMRAMTEC), Year One Technical Progress Report, Grant DE-FC03-99SF21987, Report No. STD-ES-01-0028, Westinghouse Electric Company, LLC, Pittsburgh, PA, July 2001.

Peckner, D., and Bernstein, I.M. (1977) Handbook of Stainless Steels, McGraw-Hill Book Company, New York, 1977, pp. 21.5-21.9.

Peterson, G. P. (1994) An Introduction to Heat Pipes: Modeling, Testing, and Applications, Wiley Series in Thermal Management of Microelectronic and Electronic Systems, New York, NY.

Rhenium Alloys Inc. (2001), Webb Site: www.Rhenium.com.

Rowe, D. M., Editor (1995) CRC Handbook of Thermoelectrics, CRC Press, Inc., New York, NY, Chapter 19, pp. 211 - 238, Chapter 21, pp. 257 - 266, and Chapter 36, pp. 459 470. 
Ryan, M. A., Jeffries-Nakamura, B., Williams, R. M., Underwood, M. L., O’Connor, D. and Kikkert, S. (1992) "Advances in Materials and Current Collecting Networks for AMTEC Electrodes," Proc. 27th Intersociety Energy Conversion Engineering Conference, Society of Automotive Engineers, Inc., Paper No. 929007, 3, 7-12.

Ryan, M. A., Williams, R. M., C. Saipetch, A. Kisor, D. O'Connor, M. L. Underwood and B. Jeffries-Nakamura (1994) "Development in AMTEC Devices, Components and Performance," in Proceedings of the $11^{\text {th }}$ Symposium on Space Nuclear Power Systems, CONF-940101, M. S. El-Genk and M. D. Hoover, Eds., American Institute of Physics, New York, AIP Conference Proceedings No. 301, Part III, pp. 1495-1500.

Ryan, M. A., R. M. Williams, M. L. Homer, W. M. Phillips, L. Lara, and J. Miller (1998a) "Lifetime Modeling of TiN Electrodes for AMTEC Cells," in Proceedings of the 3rd Space Technology and Applications International Forum (STAIF-98), CONF980103, M. S. ElGenk, Ed., American Institute of Physics, New York, NY, AIP Conference Proceedings No. 420, 3: 1607-1612.

Ryan, M.A., Williams, R.M., Lara, L., Cortez, R.H., Homer, M.L., Shields, V.B., Miller, J., and Manatt, M.S. (1998b) "The Sodium Exposure Test Cell to Determine Operating Parameters for AMTEC Electrochemical Cells," in Proceedings of the $33^{\text {rd }}$ Intersociety Energy Conversion Engineering Conference, American Nuclear Society, LaGrange Park, IL, 1998, Paper No. 98-335.

Ryan, M.A. (1999), Personal Communication, Pasadena, CA, Jet Propulsion Laboratory, March 18, 1999.

Ryan, M.A., Williams, R.M., Homer, M.L., Shields, V.B., Wright, S., Lara, L., and Miller, J. (1999a) "Performance Parameters of TiN Electrodes for AMTEC Converters," Proc. Space Technology and Applications International Forum (STAIF-99), M.S. ElGenk, Ed., American Institute of Physics, New York, AIP Conference Proceedings No. 458, pp. 1301-1305.

Ryan, M.A., Shields, V.B., Cortez, R.H., Lara, L., Homer, M.L., and Williams, R.M. (1999b) "Lifetimes of AMTEC Electrodes: Rhodium-Tungsten and Titanium Nitride," in Proceedings of the $34^{\text {th }}$ Intersociety Energy Conversion Engineering Conference, Society of Automotive Engineers, Inc., Paper No. 1999-01-2704.

Ryan, M. A., Shields, V. B., Cortez, R. H., Lara, L., Homer, M. L., and Williams, R. M. (2000) "Lifetime of AMTEC Electrodes: Molybdenum, Rhodium-Tungsten, and Titanium Nitride," Proc. Space Technology and Applications International Forum (STAIF-2000), M.S. El-Genk, Ed., American Institute of Physics, Woodbury, AIP Conference Proceedings No. 504, pp. 1377-1382.

Ryan, M. A., Williams, R. M., Lara, L., Fiebig, B. G., Cortez, R. H., Kisor, A. K., Shields, V. B., and Homer, M. L. (2001) "Advances in Electrode Materials for AMTEC," Proc. Space Technology and Applications International Forum (STAIF-2001), M. S. ElGenk, Ed., American Institute of Physics, New York, NY, AIP Conf. Proc. No. 552, 1: 1088 - 1093.

Sakai, T. (1977) "Surface Electrical Resistivity of Alumina Oxide Ceramics in Cesium Vapor," Japan. J. Appl. Phys., 16 (5), 859-860. 
Sasakawa, E., M. Kanzaka, A. Yamada, H. Tsukuda and M. Hiramatsu (1992) "Performance of the Terrestrial Power Generation Plant using the Alkali Metal ThermoElectric Conversion (AMTEC)," Proceedings of the 27 th Intersociety Energy Conversion Engineering Conference, Society of Automotive Engineers, Inc., Paper No. 929062, P259, Vol. 3, pp. 143-149.

Schmidt, J. E., J. W. H. Chi, R. E. Morgan, J. P. Hanson, and T. K. Hunt (1987) "A Liquid Metal Cooled Reactor-Alkali Metal Thermoelectric Space Power System Concept for Multimegawatt Applications," in Space Nuclear Power Systems 1986, M. S. El-Genk and M. D. Hoover, Eds., Orbit Book Company, Malabar, FL, Vol. V, pp. 409-415.

Schock, A., Noravian, H., Or, C., and Kumar, V. (1999) "Recommended OSC Design and Analysis of AMTEC Power System for Outer-Planet Missions," Proc. Space Technology and Applications International Forum (STAIF-99), M.S. El-Genk, Ed., American Institute of Physics, New York, AIP Conference Proceedings No. 458, pp. 1534-1554.

Sessions, C.E., Reynolds, S.D., Hebbar, M.A., Lewis, J.F., and Kiefer, J.H. (1981) "Materials Development for a Fast Breeder Reactor Steam Generator Concept," Nuclear Technology, 55, 270-279.

Sheafer, E.F., and Stearns, J.D. (1972) "Piping Materials for Fast Breeder Reactor Liquid Metal Coolant," Heating/Piping/ Air Conditioning, 44, 67-73.

Sherman, A.J., Tuffias, R.H., and Kaplan, R.B. (1999) "CVD Processing, Properties, and Applications of Rhenium" [online document], URL: http://www.ultramet.com/rhenium2.htm.

Shields, V. B., A. K. Kisor, B. Fiebig, R. M. Williams, M. A. Ryan, and M. L. Homer (2001) "Metallurgical Examination of an AMTEC Unit," Proc. Space Technology and Applications International Forum (STAIF-2001), M. S. El-Genk, Ed., American Institute of Physics, New York, NY, 2001, AIP Conference Proceedings No. 552, 1: 1094-1099.

Schuller, M., et al. (1996) "Vacuum Testing of High Efficiency AMTEC Cells," in Proceedings of the $31^{\text {st }}$ Intersociety Energy Conversion Engineering Conference, Institute of Electrical and Electronics Engineers, Inc., Paper No. 96184, 2: 877-884.

Siegel, R., and J. R. Howell (1981) Thermal Radiation Heat Transfer, $2^{\text {nd }}$ Edition, Hemisphere, New York, NY, Chapters 7 and 8, pp. 172 - 275.

Sievers, R. K., R. A. Markley, J. E. Schmidt, N. Weber, J. R. Rasmussen, S. L. Olsen, and T. K. Hunt (1989) "Alkali Metal Thermoelectric Converter Design for Space Power Systems Applications," in Space Nuclear Power Systems 1988, M. S. El-Genk and M. D. Hoover, Eds., Orbit Book Company, Malabar, FL, Vol. IX, pp. 531-540.

Sievers, R. K., and M. H. Cooper (1990) "Advanced AMTEC Design Options with Composite Electrolyte Membranes," in Proceedings of the th Symposium on Space Nuclear Power Systems, CONF-900109, M. S. El-Genk and M. D. Hoover, Eds., American Institute of Physics, Part I, pp. 239-244.

Sievers, R. K., and R. F. Wright (1990) "High Power Density Alkali Metal Thermal-toElectric Converter," in Proceedings of the 25 $5^{\text {th }}$ Intersociety Energy Conversion Engineering Conference, American Institute of Chemical Engineers, Vol. 2, pp. 426-430. 
Sievers, R. K., and M. H. Cooper (1991) “Advanced AMTEC Design Options with Composite Electrolyte Membranes," in Space Nuclear Power Systems 1990, M. S. ElGenk and M. D. Hoover, Eds., Orbit Book Company, Malabar, FL, Vol. XI, pp. 239-244.

Sievers, R. K., T. K. Hunt, R. M. Williams, and M. L. Underwood (1992) "Assessment of the U.S. Industrial Program for AMTEC Development," in Proceedings of the 27th Intersociety Energy Conversion Engineering Conference, held in San Diego, CA, August 3-7, 1992, Paper No. 929065, Society of Automotive Engineers, Inc., 3: 215-220.

Sievers, R. K., Rasmussen, J. R., Borkowski, C. A., Hendricks, T. J., and Pantolin, J. E. (1998) "PX-5 AMTEC Cell Development," Proc. Space Technology and Applications International Forum (STAIF-98), M. S. El-Genk, Ed., American Institute of Physics, New York, NY, 1998, AIP Conference Proceedings No. 420, 3: 1479-1485.

Sievers, R. K., T. K. Hunt, T. J. Hendricks, A. Barkan and S. Siddiqui (1999) "AMTEC Internal Self-Heat Pipe," in Proceedings of the $34^{\text {th }}$ Intersociety Energy Conversion Engineering Conference, Society of Automotive Engineers, Inc., Paper No. 1999-012660.

Soo, S. L. (1968) Direct Energy Conversion, Prentice-Hall, Inc., Englewood Cliffs, N. J., Chapter 5, pp., 114-127.

Stade, K. C., E. Zimmermann, and J. Schindewolf (1984) "Cleaning and Requalification of KNK Primary Cold Traps," in Liquid Metal Engineering and Technology, Proceedings of the $3^{\text {rd }}$ International Conference, held 9-13 April 1984 in Oxford, the British Nuclear Energy Society, London, England, Paper No. 189, Vol. 2, pp. 407 - 412.

Steinbrück, M., V. Heinzel, F. Huber, W. Peppler, H. Hill and M. Voss (1993) "Investigations of Beta-Alumina Solid Electrolytes for Application in AMTEC Cells," Proceedings of the $28^{\text {th }}$ Intersociety Energy Conversion Engineering Conference, American Chemical Society, Paper No. 93108, Vol. 1, pp. 799 - 80.

Svedberg, R. C., D. A. Schaupner, and A. J. Appleby (1997) "Accelerated Testing for Extended-Service AMTEC Cells," in Proceedings of the 32nd Intersociety Energy Conversion Engineering Conference, Paper No. 97458, American Chemical Society, 2: 1230-1235.

Svedberg, R.C., and Sievers, R.K. (1998) "Refractory Metals for ARPS AMTEC Cells," Proceedings of the $33^{\text {rd }}$ Intersociety Energy Conversion Engineering Conference, American Nuclear Society, LaGrange Park, IL, 1998, Paper No. 98-397.

Thorley, A.W. and Tyzack, C. (1973) "Corrosion and Mass Transport of Steel and Nickel Alloys in Sodium Systems," in Liquid Alkali Metals; Proceedings of the International Conference, British Nuclear Energy Society, London, 1973, pp. 257-273.

Tietz, T.E., and Wilson, J.W. (1965) Behavior and Properties of Refractory Metals, Stanford University Press, Stanford, CA, 1965, pp. 28-39, 377-409.

Titran, R.H, Stephens, J.R., and Petrasek, D.W. (1988) Refractory Metal Alloys and Composites for Space Nuclear Power Systems, NASA Technical Memorandum 101364, NASE Lewis Research Center, Cleveland, OH, 1988, pp. 5-6. 
Touloukian, Y. S., Ed. (1970a) Thermophysical Properties of Matter, the TPRC data, Vol. 1: Thermal Conductivity-Metallic Elements and Alloys; Vol. 4: Specific HeatMetallic Elements and Alloys, Plenum Publ., Corp.

Touloukian, Y.S., Powell, R.W., Ho, C.Y., Klemens, P.G. (1970) Thermophysical Properties of Matter, the TPRC Data Series, Vol. 1, 7 \& 12, IFI/Plenum, New York, 1970.

Tournier, J.-M., M. S. El-Genk and F. Carré (1990) "An Assessment of Thermoelectric Conversion for the ERATO-20 kWe Space Nuclear Power System," 25th Intersociety Energy Conversion Engineering Conference, Paper No. 900019, Reno, Nevada, August 12-17, 1990, AIChE Proceedings, Part 1, pp. 141-146.

Tournier, J.-M. (1996), HPTAM, a Two-Dimensional Heat Pipe Transient Analysis Model, Including the Startup from a Frozen State, a Dissertation submitted in partial fulfillment of the requirements for the Degree of Doctor of Philosophy in Engineering, the University of New Mexico, Albuquerque, NM, May 1996.

Tournier, J.-M. and M. S. El-Genk (1996) “A Vapor Flow Model for Analysis of LiquidMetal Heat pipe Startup from the Frozen state," International Journal of Heat and Mass Transfer, 39 (18), 3767-3780.

Tournier, J.-M., El-Genk, M.S., and Huang, L. (1999) Experimental Investigations, Modeling, and Analyses of High-Temperature Devices for Space Applications, Final Report No. UNM-ISNPS-1-1999, University of New Mexico, Albuquerque, NM, pp. 260-317. Also Final Report No. AFRL-VS-PS-TR-1998-1108, Air Force Research Laboratory, Space Vehicles Technologies Directorate, Kirtland AFB, Albuquerque, NM (Federal Contract No. F29601-96-K-0123).

Tournier, J.-M. and M. S. El-Genk (1999b) "Sodium Vapor Pressure Losses in a MultiTube, Alkali Metal Thermal-to-Electric Converter," Journal of Thermophysics and Heat Transfer, 13 (1), 117-125.

Tournier, J.-M. and M. S. El-Genk (1999c) "An Electric Model of a Vapor-Anode, Multi-Tube Alkali-Metal Thermal-to-Electric Converter," Journal of Applied Electrochemistry, 29 (11), 1263-1275.

Tournier, J.-M. and M. S. El-Genk (1999d) "Radiation Heat Transfer in Multi-Tube, Alkali Metal Thermal-to-Electric Converter," Transactions of the ASME - Journal of Heat Transfer, 121 (1), 239-245.

Tournier, J.-M. and M. S. El-Genk (1999e) "Performance Analysis of Pluto/Express, Multi-Tube AMTEC Cells," Journal of Energy Conversion and Management, 40, 139173.

Tournier, J.-M. and M. S. El-Genk (1999f) "Analysis of Test Results of a Ground Demonstration of a Pluto/Express Power Generator," Journal of Energy Conversion and Management, 40, 1113-1128.

Tournier, J-M., and El-Genk, M. S. (2002) 'Capillary Limit of Evaporator Wick in Alkali Metal Thermal-to-Electric Converters," J. Thermophysics and Heat Transfer, 16(1), $141-153$. 
Truscello, V. C., and L. L. Rutger (1992) “The SP-100 Power system," in Proceedings of the 9th Symposium on Space Nuclear Power Systems, held in Albuquerque, NM, January 12-16, 1992, M. S. El-Genk and M. D. Hoover, Eds., American Institute of Physics, New York, NY, AIP Conference Proceedings No. 246, Part 1, 1992, pp. 1-23.

Tsukuda, H., M. Kanzaka, A. Yamada, H. Kikuchi and E. Sasakawa (1992) "Fundamental Study of the Terrestrial Power Generation Plant using the Alkali Metal Thermo-Electric Conversion (AMTEC)," Proceedings of the $27^{\text {th }}$ Intersociety Energy Conversion Engineering Conference, Society of Automotive Engineers, Inc., Paper No. 929061, P-259, Vol. 3, pp. 135-142.

Underwood, M. L., R. M. Williams, B. Jeffries-Nakamura, Bankston, C. P., and Cole, T. (1988) "Progress in AMTEC Electrode Experiments and Modeling," in Proceedings of the $23^{\text {rd }}$ Intersociety Energy Conversion Engineering Conference, D. Y. Goswami, Ed., American Society of Mechanical Engineers, New York, 1: 227-233.

Underwood, M. L., R. M. Williams, B. Jeffries-Nakamura, M. A. Ryan, and D. O'Connor (1991) "Performance Projections of Alternative AMTEC Systems and Devices," in Proceedings of the $8^{\text {th }}$ Symposium on Space Nuclear Power Systems, CONF-910116, M. S. El-Genk and M. D. Hoover, Eds., American Institute of Physics, New York, AIP Conference Proceedings No. 217, Part I, pp. 472-481.

Underwood, M. L., R. M. Williams, M. A. Ryan, B. Jeffries-Nakamura and D. O'Connor (1992) "An AMTEC Vapor-Vapor, Series Connected Cell," in Proceedings of the $9^{\text {th }}$ Symposium on Space Nuclear Power Systems, CONF-920104, M. S. El-Genk and M. D. Hoover, Eds., American Institute of Physics, New York, AIP Conference Proceedings No. 246, Part III, pp. 1331-1336.

van Atta, C. M. (1965) Vacuum Science and Engineering, McGraw-Hill Book Company, New York, NY, Chapter 2, pp. 23-61.

Vargaftik, N. B. (1975) Tables on the Thermophysical Properties of Liquids and Gases In Normal and Dissociated States, 2nd Edition, Hemisphere Publishing Corp., Washington, DC, pp. 600-631.

Virkar, A. V., J-F Jue, and K-Z Fung (2000) "Alkali-Metal- $\beta$ - and $\beta$ "-Alumina and Gallate Polycrystalline Ceramics and Fabrication by a Vapor Phase Method," United States Patent No. 6,117,807; September 12, 2000, assigned to Materials and Systems Research, Inc., Salt Lake City, Utah.

Viswanathan, L. and Virkar, A.V. (1982) "Wetting Characteristics of Sodium on $\beta$ "Alumina and Nasicon,” Journal of Materials Science, 17, 753-759.

Walker, F. W., J. R. Parrington, and F. Feiner (1988) Chart of the Nuclides, Knolls Atomic Power Laboratory, operated by the General Electric Company, San Jose, CA, $14^{\text {th }}$ Edition, Revised to April 1988.

Waltar, A.E., and Reynolds, A.B. (1981) Fast Breeder Reactors, Pergamon Press, New York, 1981, pp. 350-395, pp. 454-460, pp. 463-502, and pp.768-782.

Walters, L.C. (1999) "Thirty Years of Fuels and Material Information From EBR-II," Journal of Nuclear Materials, 270, 39-48. 
Weber, N. (1974) "A Thermoelectric Device Based on Beta-Alumina Solid Electrolyte," Energy Conversion, 14 (1), 1-7.

Wilkinson, W.D. (1969) Properties of Refractory Metals, Gordon and Breach Science Publishers, New York, 1969, pp. 29-138.

Williams, R. M., G. Nagasubramanian, S. K. Khanna, C. P. Bankston, A. P. Thakoor and T. Cole (1986) "The Role of Oxygen in Porous Molybdenum Electrodes for the Alkali Metal Thermoelectric Converter," J. Electrochemical Society: Electrochemical Science and Technology, 133 (8), 1587-1595.

Williams, R. M., Loveland, M. E., Jeffries-Nakamura, B., Underwood, M. L., Bankston, C. P., Leduc, H., and Kummer, J. T. (1990a) "Kinetics and Transport at AMTEC Electrodes I. The Interfacial Impedance Model," Journal of the Electrochemical Society 137(6), 17091716.

Williams, R. M., Jeffries-Nakamura, B., Underwood, M. L., Bankston, C. P., Leduc, H., and Kummer, J. T. (1990b) "Kinetics and Transport at AMTEC Electrodes - II. Temperature Dependence of the Interfacial Impedance of $\mathrm{Na}(\mathrm{g}) / \mathrm{Porous} \mathrm{Mo/Na-Beta"-Alumina,"} \mathrm{Journal}$ of the Electrochemical Society 137(6), 1716-1723.

Williams, R. M., Jeffries-Nakamura, B., Underwood, M. L., Ryan, M. A., O’Connor, D., and Kikkert, S. (1992) "High Temperature Conductivity of Potassium-Beta"-Alumina," Solid State Ionics 53-56, 806-810.

Williams, R. M., Jeffries-Nakamura, B., Ryan, M. A., Underwood, M. L., Kisor, A., O'Connor, D., and Kikkert, S. (1993) "Advances in Studies of Electrode Kinetics and Mass Transport in AMTEC Cells," Proc. 28 th Intersociety Energy Conversion Engineering Conference, American Chemical Washington DC, 1: 819-822.

Williams, R. M., Kisor, A., Ryan, M. A., Jeffries-Nakamura, B., Kikkert, S. and O'Connor, D. (1994) "Potassium Beta"-Alumina/Potassium/Molybdenum Electrochemical Cell," in Proceedings of the $29^{\text {th }}$ Intersociety Energy Conversion Engineering Conference, American Institute of Aeronautics and Astronautics, Paper No. AIAA-94-3833-CP, pp. 888-893.

Williams, R. M., Kisor, A., and Ryan, M. A. (1995) "Time Dependence of the High Temperature Conductivity of Sodium and Potassium Beta"-Alumina Ceramics in Alkali Metal Vapor," J. Electrochemical Society, 142 (12), 4246-4251.

Williams, R. M., Homer, M. L., Lara, L., Cortez, R. H., Miller, J., Jeffries-Nakamura, B., Underwood, M. L., Kisor, A., O'Connor, D., Shields, V. B., Manatt, K. S., and Ryan, M. A., (1998a) "Sodium Transport Modes in AMTEC Electrodes," Proc. 33 ${ }^{\text {rl }}$ Intersociety Energy Conversion Engineering Conference, American Nuclear Society, LaGrange Park, IL, Paper No. 98-332.

Williams, R. M., Ryan, M. A., Homer, M. L., Lara, L., Manatt, K., Shields, V., Cortez, R. H., and Kulleck, J. (1998b) "The Thermal Decomposition of Sodium Beta"-Alumina Solid Electrolyte Ceramic," Proc. 33 ${ }^{\text {rd }}$ Intersociety Energy Conversion Engineering Conference, American Nuclear Society, LaGrange Park, IL, Paper No. 98-333.

Williams, R. M., Ryan, M. A., Homer, M. L., Lara, L., Manatt, K., Shields, V., Cortez, R. H., and Kulleck, J. (1999) "The Thermal Stability of Sodium Beta"-Alumina Solid 
Electrolyte Ceramic in AMTEC Converters," Proc. Space Technology and Applications International Forum (STAIF-99), M.S. El-Genk, Ed., American Institute of Physics, New York, AIP Conference Proceedings No. 458, pp. 1306-1311.

Williams, R.M., Homer, M.L., Kulleck, J., Lara, L., Kisor, A.K., Cortez, R.H., Shields, V.B., and Ryan, M.A. (2000) "Thermal Stability of Beta"-Alumina Solid Electrolyte Under AMTEC Operating Conditions," in Proceedings of the Space Technology and Applications International Forum (STAIF-2000), edited by M.S. El-Genk, AIP Conference Proceedings No. 504, American Institute of Physics, New York, 2000, pp. 1408-1411.

Woloshun, K. A., M. A. Merrigan, and E. D. Best (1989) "HTPIPE - A Steady-State Heat Pipe Analysis Program," in Space Nuclear Power Systems 1988, M. S. El-Genk and M. D. Hoover, Eds., Orbit Book Company, Malabar, FL., Vol. IX, pp. 395-403.

Zapesochnyi, I. P. and Aleksakhin, I. S. (1969) "Ionization of Alkali Metal Atoms by Slow Electrons," Soviet Physics JETP, 28 (1), 41-45. 
[ This page intentionally blank ] 


\title{
4 Performance Evaluation of the LMR-AMTEC Concept
}

\author{
By \\ Westinghouse Electric Company \\ and \\ Institute for Space Nuclear Power Studies (UNM)
}

\subsection{Preliminary Economic Evaluation of the LMR- AMTEC (WEC)}

The results of the LMR-AMTEC cost estimation are presented is this section. The objective of this economic evaluation is to define a figure that can be use to optimize the reactor parameters. At this point in the design phase, any cost estimation must be on a very general scale, and therefore is preliminary. The LMR-AMTEC design need to be further developed so the detail design of the components is better known and then a more accurate cost estimate can be made. Other important difficulties in determining the cost estimation were the large uncertainties of the price of components like the AMTEC or the TE converter which are still in development stage. In determining the modular plant costs, some basic ground rules were followed:

- The DOE EEDB program Code of Accounts was used as guideline for the structure used for cost estimation (see Table 4-1). This allows for a more consistent method of cost estimation and also to compare with other power plant designs. As the design of the LMR-AMTEC becomes more detailed, these guidelines will be more closely adhered to.

- The cost estimations, procedures and assumptions of the Power Reactor Innovative Small Module (PRISM) and the Sodium Advanced Fast Reactor (SAFR) were used as 
reference for the components of the LMR-AMTEC which are similar to those of a convectional LMR.

- The cost estimates were established in constant January 2003 US dollars.

The next section briefly discusses the DOE approach to plant cost estimation which was used to estimate the base construction cost. Then some consideration on the benefices of the factory fabrication and modular construction of the LMR-AMTEC are described and the main approximations used in the analysis discussed. Finally some results from the economic optimization of the reactor parameters are presented and discussed.

\subsubsection{EEDB Cost Account Definitions}

The EEDB Cost Account is presented in Table 4-1.The base construction cost Cost $_{\text {base }}$ is divided into the Direct Costs $\left(\right.$ Cost $\left._{\text {direct }}\right)$ and the Indirect Cost $\left(\operatorname{Cost}_{\text {indirect }}\right)$ :

$$
\operatorname{Cost}_{\text {base }}=\text { Cost }_{\text {direct }}+\text { Cost }_{\text {indirect }}
$$

From the base construction costs, the overnight cost is in generally estimated as:

$$
\text { Cost }_{\text {overnight }}=\text { Cost }_{\text {base }}+\text { Cost }_{\text {contingency }}
$$

where Cost $_{\text {contigency }}$ is a construction contingency allowance that is applied on both the direct and indirect costs. In the present analysis the contingency cost are not considered.

\subsubsection{Direct Cost Accounts}

Direct cost accounts include those construction and installation costs directly associated with the operating plant structures, systems, and components. Equipment costs include the costs for all design, analysis, fabrication, documentation preparation, predelivery testing and follow-up engineering performed by equipment vendors; materials for all plant equipment ; equipment; transportation an insurance expenses; provision of shipping fixtures and skids; warranties; preparation of maintenance and operations manuals and handling instructions; delivery of startup and acceptance test equipment; onsite unloading and receiving inspection expenses; and overhead expenses.

All plant equipments items, whether directly associated with the power generation systems or the facility systems, such as heating and ventilation, are included in this 
category. For the equipment fabricated and/or assembled at an on-site fabrication facility, all the associated costs are included as equipment costs, including the fabrication and/or assembly costs and the costs to move the equipment within the facility its on-site receiving or storage point. The on-site labor related to installation of shop fabricated modules should be included in the field labor and not as factory equipment. Field labor rates should be used for any on-site fabrication facility.

The site labor portion of the construction and equipment installation costs includes all on-site activities related to permanent plant structures, systems, and equipment required for all aspects of power plant operation.

\subsubsection{Indirect Cost Accounts}

The indirect costs account include those construction support activities required to design and build the structures and systems described in the direct cost accounts. At the two-digit account level of the detail, the indirect costs accounts collect the cost for construction services, home office engineering and services, and field office engineering and services.

Indirect costs covers all cost items no identified with any direct construction activity for a permanent plant facility, such as temporary facilities, construction services, design, engineering and startup. Construction services costs and field office and services costs, are both a function of the plant size, direct site labor, and construction duration.

The home office services indirect costs for the first commercial plant were also linearly levelized over the first power blocks to arrive at the lead plant cost. In addition there are some site specific indirect costs, engineering activities required to adapt standard plant design to factors associated with the particular site such as: cooling water sources, location and quality; utility grid interfaces; soil and ground water characteristics; environmental conditions; and licensing and permitting. The home office services cost are function of total direct cost, equipment cost, site labor direct cost, construction services cost, and field office costs. The owner's cost is 10 percent of the sum of the total direct cost, other indirect costs and the cost of sodium. The cost for reactor manufacture's engineering is assumed to include: services for systems engineering, technical integration, design, analysis, plant level engineering, and supporting functions. 


\section{DIRECT COSTS (EEDB 20 - 26)}

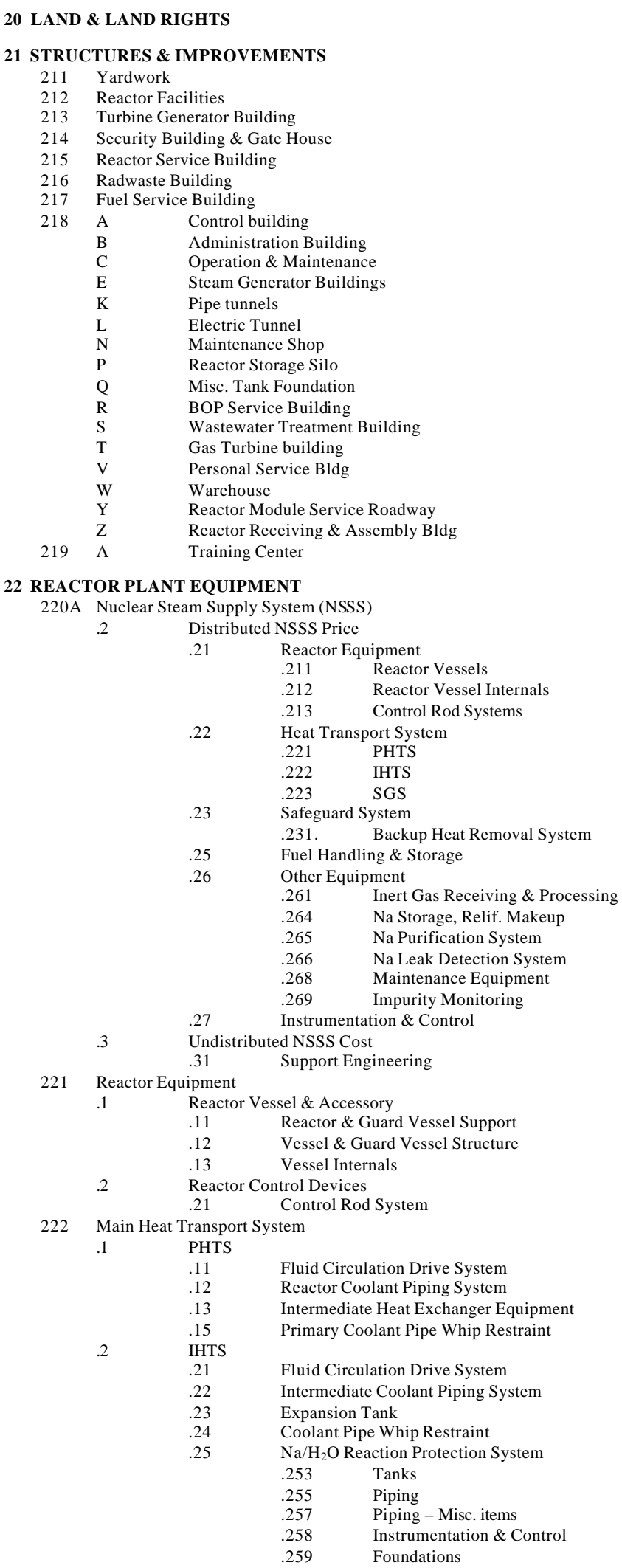

Table 4-1. U.S. DOE EEDB program Code of Accounts.

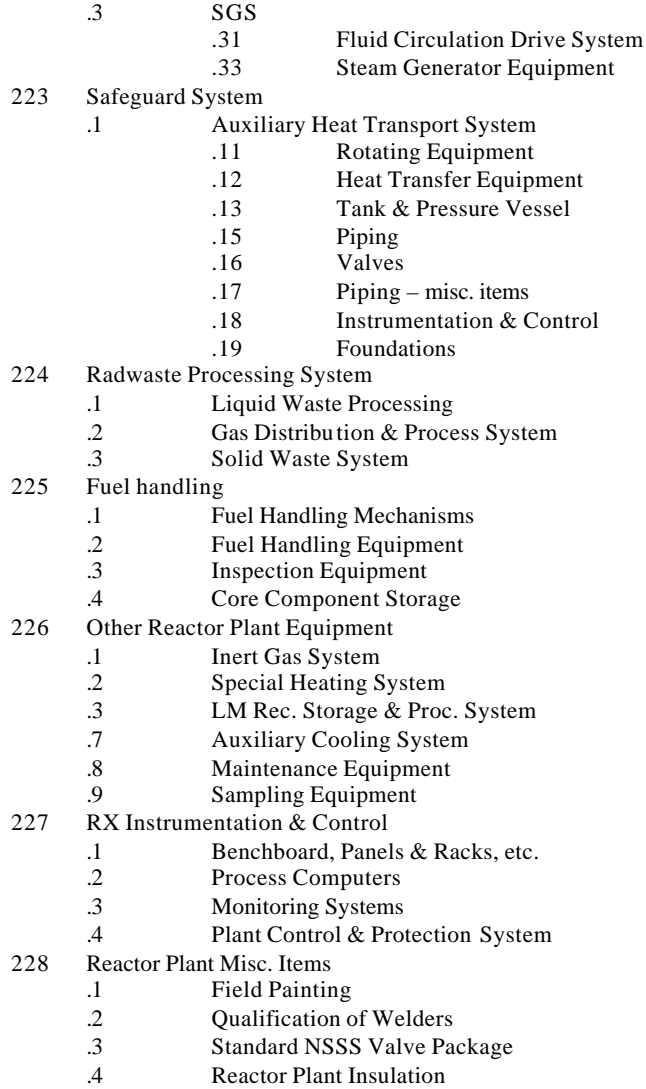

26 MAIN CONDENSER HEAT REJECT SYSTEM

261 Structures

262 Mechanical Equipment

INDIRECT COST (EEDB 91 - 95)

91 Construction Services

92 AE Home Office Engr. \& Service

93 Field Office Supr. \& Service

94 Owner' Expenses

95 RM Home Office Engineering \& Services 


\subsubsection{Factory Fabrication and Modular Construction}

The LMR-AMTEC design characteristics (like size, pool configuration, etc.) allow the many advantages of factory fabrication and modular construction to be taken. Relative to field construction, a factory has a stable work force that is familiar with the procedures and equipment; work which is not affected by weather conditions; and work which is more easily monitored. The end result is more predictable costs and schedules for factory work, and a higher quality product. In addition, factory work can be performed in parallel with construction work leading to reduced construction schedules. Factory fabrication also offers many opportunities for reduced costs. In general a manufacturer with high volume and continuous production will have lower overhead and higher productivity. In addition to this, the fabrication of a reactor like the LMR-AMTEC can be modularized. This approach provides the advantage of lower capital cost, a shorter construction schedule, and improved field work productivity as the learning benefits (i.e. the increased labor productivity gained from performing repetitive work) would be fully exploited by the manufactures, further reducing the costs. The standardized modular construction approach combined with extensive factory fabrication can result in a plant design with has lower costs.

In order to realize the benefits of modularization, the lead plant module equipment cost can be averaged over the first several modules manufactured in a dedicated facility. These cost include factory capital equipment cost amortization, factory special tooling, startup costs, and initial reactor manufacturing engineering. In essence the plants containing these reactor modules are all lead plants. This approach was adopted in reactors like PRISM or AP600 and consists in levelizing first-of-a-kind costs over nplants.

\subsubsection{Determination of the Overnight Cost}

In determining the overnight cost of the plant the DOE EEDB program Code of Accounts was used as guideline for the structure used for cost estimation. The cost estimates were established in constant January 2003. A summary of the accounts used in the preliminary cost estimation is showed in Table 4-2.

For the ordinary LMR-AMTEC components (i.e., those common to others LMRs) the cost estimations, procedures and assumptions of the Power Reactor Innovative Small Module (PRISM) and the Sodium Advanced Fast Reactor (SAFR) were used as reference (scaled according to the reactor power). In particular, the SAFR has several common features with the LMR-AMTEC as both are pool reactors. The SAFR plant concept employs a 350-MWe reactor unit as the basic module and each unit consists of a poof type reactor assembly. The cost estimation procedure employed for the SAFR accounted for the reduction fuel labor, shortening the construction schedule and reducing the indirect costs. 
For those components unique to the LMR-AMTEC the following assumption were made:

- Fuel cost: The fuel cost first core cost was included in the direct cost as the reactor is a one cycle type. In the present analysis it was assumed that the fuel is fabricated from recycled fuel from PWR. The fuel cost included the cost of the source material ( $\$ 3300$ per $\mathrm{Kg} \mathrm{Pu}$ ) and the cost of fabrication. The cost of fabrication was obtained by adding to the estimated MOX fabrication cost ( $\$ 1100$ per $\mathrm{kg} \mathrm{HM})$, the cost due to the use of $\mathrm{Nb}-1 \mathrm{Zr}$ for the cladding and the cost of the enrichment of $\mathrm{N}^{15}$. This last was estimated equal to $10 \%$ of the fabrication cost. The use of UN fuel is not expect to introduce important savings on the fuel cost as the presence of $\mathrm{Nb}-1 \mathrm{Zr}$ deteriorates the neutron economy and then increases the required UN enrichment and the cost of this fuel. On the contrary, the cost of the $(\mathrm{U}, \mathrm{Pu}) \mathrm{N}$ is independent of the enrichment.

- Reactor vessel cost: The reactor vessel cost was estimated from the RV weight and assuming that Inconel alloy is used as structural material and $\mathrm{Nb}-1 \mathrm{Zr}$ as lining material to protect against corrosion.

- Alkali metal Boilers: the cost was estimated from their total weight and assuming that the structural material is $\mathrm{Nb}-1 \mathrm{Zr}$.

- AMTEC/TE converter units: The AMTEC cost was estimated from the total BASE surface. At present there is not a good estimation of the cost of an AMTEC unit having the characteristics of the converter used in the LMR-AMTEC. It has been suggested [Subramanian 82] that the cost of some designs of the NA-AMTEC can be conservative estimated to $1000 \$ \mathrm{Kwe}$. In our analysis this cost was estimated to be in the range between $1000 \$ / \mathrm{kWe}$ to $2000 \$ / \mathrm{kWe}$. These values were used as reference to determine the cost per unit surface of the BASE. On the other hand, the cost of the TE converters was lumped with the AMTEC unit.

- Electric power conversion system: No a good estimation of the cost of this component exist. Therefore this cost was also lumped with the cost AMTEC/TE unit by increasing the last.

\begin{tabular}{|l|c|c|c|}
\hline Account description & $\begin{array}{c}\text { Scaling } \\
\text { Parameters }\end{array}$ & $\begin{array}{c}\text { Scaling } \\
\text { factor }\end{array}$ & Reference \\
\hline DIREC COSTS & & & \\
\hline Fuel Cost & $\begin{array}{c}\text { heavy metal } \\
\text { weight }\end{array}$ & 1 & [EPRI96] [Chow95] [Calamia81] \\
\hline Reactor Vessel & weight & 1 & [Fraas89] [Brunings87] \\
\hline Alkali Metal Boilers & weight & 1 & [Fraas89] \\
\hline AMTEC/TE converters & total BASE & 1 & [Subramanian82] \\
\hline Other Direct Costs & thermal power & $0.5-0.8$ & [Asamoto87] [Brunings87] \\
[Carelli91] [Yevick66]
\end{tabular}

Table 4-2. Accounts used in the preliminary cost estimation of the LMR-AMTEC. 


\subsubsection{Optimization of the Reactor Design Parameters}

The economic evaluation analysis was added to the plant evaluation model (section 2.2.8) in order to perform the optimization procedure shown in Figure 4-1 (similar to the one described in section 2.1.3). In this procedure, for a given RPV mass, a vessel diameter and height are chosen and the AMB tube length is calculated. The overnight cost of the plant is then determined for different values of PDR, tube Outer Diameter (OD) and secondary saturation temperature. Once the minimum overnight cost is found, the optimization is repeated for new values of vessel diameter and height.

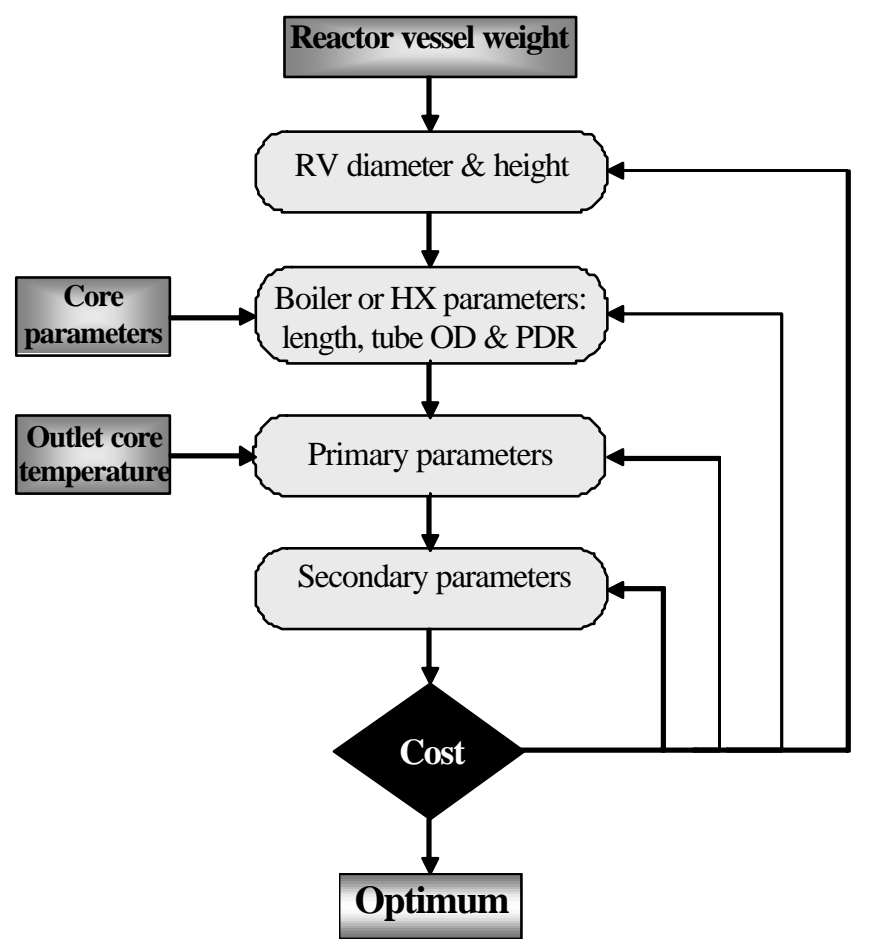

Figure 4-1. Optimization procedure used in determining the LMR-AMTEC operating parameters.

Using this procedure the reactor parameters were determined. A list of these parameters is given in section 2.2.8.2. In the next section to examples of calculations are presented.

\subsubsection{Comparison Between Once-through and Recirculation AMB Operation}

The economic effect of operating the alkali metal boilers in once-through or in recirculation mode was investigated. As part of the length of the once-through AMBs is used to superheat the vapor (see 2.2.5.3), then for the same vessel weight the effective exchange surface is lower than the recirculation AMB. Therefore the once-through design achieves a lower net efficiency (Figure 4-2) and has higher cost (Figure 4-3). 


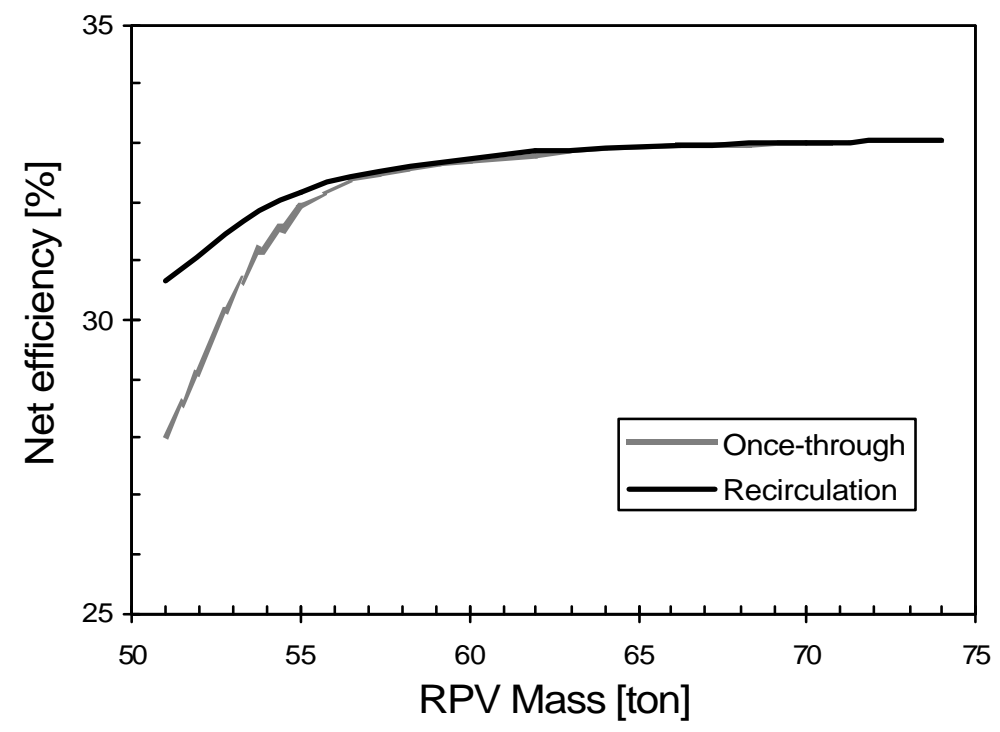

Figure 4-2. Net efficiency versus the reactor vessel mass for AMBs operating in recirculation or in oncethrough mode.

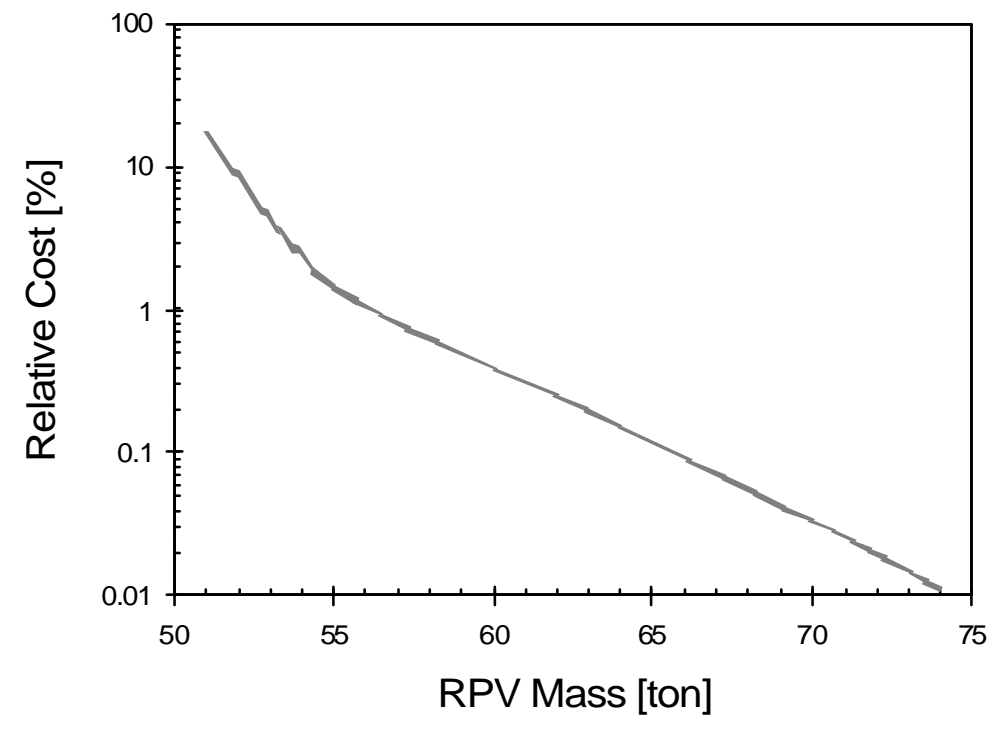

Figure 4-3. Cost increment of the LMR-AMTEC design with AMBs operating once-through versus the reactor vessel. The LMR-AMTEC with boilers operating in recirculation is used as reference.

\subsubsection{Effect of the Tube Diameter of the Alkali Metal Boilers}

An important parameter of the alkali metal boilers is the tube external diameter. As can be seen in Figure 4-4, reducing the diameter of the tubes allows reducing the cost as the exchange surface is increased and therefore the reactor efficiency. Due to the limit imposed by fabrication and by the pressure drop in the secondary side, the value was set to $2.5 \mathrm{~cm}$. 


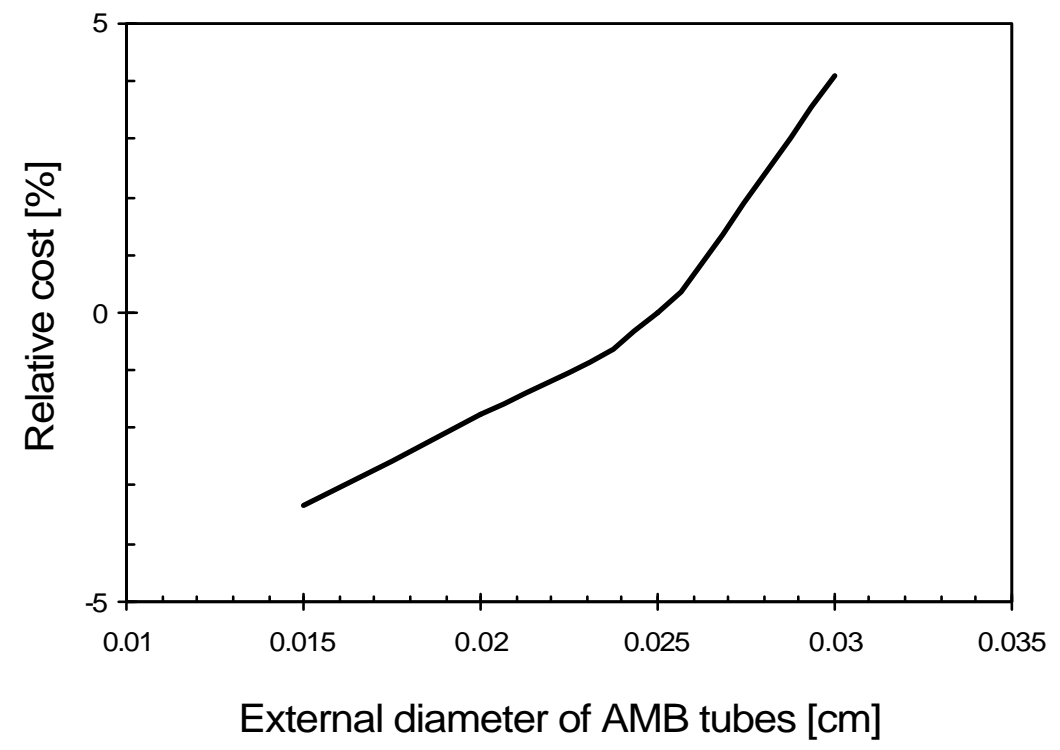

Figure 4-4. Relative overnight cost versus the external diameter of the Alkali Metal Boilers. The reference LMR-AMTEC design is used as reference $(2.5 \mathrm{~cm})$.

\subsubsection{Summary of the Preliminary Economic Evaluation}

The economic analysis described in the precedent section was added to the LMRAMTEC plant model to determine the optimal design parameters of the plant. The plant evaluation showed that a moderate electric efficiency could be achieved by design if the core working temperature is maintained about $1000 \mathrm{~K}$. Even so, when the economic aspects of the concept are considered together with the net electric production, the plant shows a poor overall performance. This preliminary study showed that the capital cost of the reactor is situated between $150 \%$ and $250 \%$ (depending on the cost range of the AMTEC/TE) of the cost of a classic LMR having similar thermal power. Two reasons explain this poor performance: first, the high capital costs of both the LMR and the AMTEC/TE converter units and, second, the relatively low conversion efficiency of the AMTEC/TE converters when compared with other conversion technologies at equivalent working temperatures such a Rankine cycle. The AMTEC/TE capital cost represents between 20 to $35 \%$ of the total cost. In the case of the LMR, the high capital cost is driven mainly by the conversion efficiency and to a lesser extent by the long life core requirement. In particular, operational requirements of the AMTEC push the LMR design toward a very high working temperature which requires the use of very expensive refractory alloys as structural material. Furthermore, as explained in this report, the use of $\mathrm{Nb}-1 \mathrm{Zr}$ deteriorates the neutron economy, hence increasing the fuel cost when $\mathrm{UN}$ is used. In summary, the preliminary economic studies showed that the expected cost savings arising from the simplification on the LMR design (no intermediate loop) and the lower operation and maintenance costs are well overwritten by costs derived from the high working temperature and the AMTEC/TE capital cost. 


\subsection{Study on the High-Energy Utilization of LMR-AMTEC for Developing Countries and Remote Areas (ISNPS- UNM)}

Small nuclear power plants with static energy conversion could meet the energy mix in underdeveloped countries, including electricity, residential and industrial space heating, seawater desalination, and/or high temperature process heat or steam for industrial uses. In this Section, analyses are performed for three Liquid Metal Cooled Reactors (LMR) with an alkali-metal/alkali-metal intermediate heat exchanger (IHX) to examine cogeneration options and calculate and compare the total energy utilization of the plants. Conversion of the reactor thermal power to electricity is accomplished using the present closed-design, static AMTEC/TE converters developed for the LMR-AMTEC, and segmented thermoelectric converters for the desalination preheating units. The total energy utilization of these plants of 95\% includes low-voltage $(\sim 400 \mathrm{~V})$ DC electrical power at a net plant efficiency of up to $32.7 \%$, and co-generation options such as residential and industrial space heating at $<400 \mathrm{~K}$, seawater desalination at $365 \mathrm{~K}$, and/or high temperature process heat or steam at $>500 \mathrm{~K}$.

In the LMR plants, which use the long-life sodium-cooled nuclear reactor developed in this work, the sodium coolant exits the reactor at $1130 \mathrm{~K}\left(85{ }^{\circ} \mathrm{C}\right)$. The sodium in the secondary loop exits the IHX at $1113 \mathrm{~K}\left(840{ }^{\circ} \mathrm{C}\right)$, enters the heat exchangers of the AMTEC/TE modules at $1109 \mathrm{~K}$, and exits at $1014 \mathrm{~K}$. The corresponding evaporator temperature of the AMTEC converters is $1006 \mathrm{~K}$. At this temperature, the K-AMTEC/TE converters provide higher power output and efficiency than the sodium converters.

\subsubsection{Production of Electricity and Co-Generation Thermal Power}

High-energy utilization, small nuclear power plants for electricity production and cogeneration heat for low and high temperature applications offer an attractive and economical option for underdeveloped countries. In addition to their obvious environmental advantages, these nuclear power plants with a high total utilization of the nuclear reactor power would be competitive with fossil plants. The total energy utilization is defined herein as the sum of the percentages of the reactor thermal power converted into DC electrical power and those used in co-generation options such as residential and industrial space heating $(<400 \mathrm{~K})$, seawater preheating prior to desalination $(\sim 365 \mathrm{~K})$, and/or high temperature process heat or steam at $>500 \mathrm{~K}$. 
Underdeveloped countries and small communities in remote parts of the world, representing more than $60 \%$ of the world population, have small or nonexistent electrical grids, inadequate socio-economical infrastructure, and limited or diminishing water resources. Current and future energy needs for these populations of more than 3.5 billions include both electricity and co-generation applications. These needs could effectively be met using small nuclear reactor power plants generating a few to tens of megawatt of electricity and co-generation thermal power for a multitude of uses. Cogeneration options may include residential and/or industrial space heating, low temperature process heat for seawater desalination in arid regions (Faibish et al. 2002), and high temperature process heat or steam for a variety of industries. Examples include food processing, agriculture and fertilizer industries, oil refineries and petrochemical industry, chemical, textile and paper industries, aluminum smelters and metal foundries, and medical, pharmaceuticals, and manufacturing applications. Small nuclear power plants of simple designs are easy to operate and maintain, and could be fabricated and partially or totally assembled in the factory and shipped to the site by rail or on barge. The nuclear reactors in these plants could make extensive use of passive cooling means for the removal of decay heat, and be designed proliferation proof and to operate continuously without refueling for 5-10 years, or even longer.

While high voltage AC electricity is suitable for reducing transmission losses in distributing grids, which extend over vast distances, it is not suitable for direct use in aluminum and metals foundries, and in future all-digital societies. For these uses, nuclear power plants could generate low-voltage DC electricity using static conversion and supply it directly to the end user. Static conversion is modular, has no moving parts, and could be cooled by natural convection of ambient air. Static conversion of the reactor thermal power into electricity further enhances these plants' reliability and could markedly decrease maintenance frequency and cost. In addition to its inherent modularity, redundancy, and low or no maintenance, static conversion could provide DC electricity at a net efficiency in the high twenties to low thirties percent range, depending on its type, the reactor's exit temperature, and the co-generation options provided for in the plant. The higher the temperature rise across the reactor core, the larger is the fraction of the reactor power that could be used for co-generation applications, but the smaller is the fraction converted into electricity, and vice versa. High efficiency, static converters in small nuclear reactor plants are also of interest to undersea, marine, and naval uses requiring quiet operation and both electricity and co-generation applications.

Small nuclear power plants may not compete in electricity cost with large commercial nuclear power plants in metropolitan areas, but they could provide a specific mix of energy needs in remote locations and underdeveloped countries, with no or limited electrical Grid. In these regions, where large power plants are neither economical nor practical, the emphases should be placed not only on design simplicity, low maintenance, inherent safety, passive cooling, non-proliferation, and long life, but also on providing both electricity and process heat for a multitude of uses in order to create jobs and stimulate economical growth. 


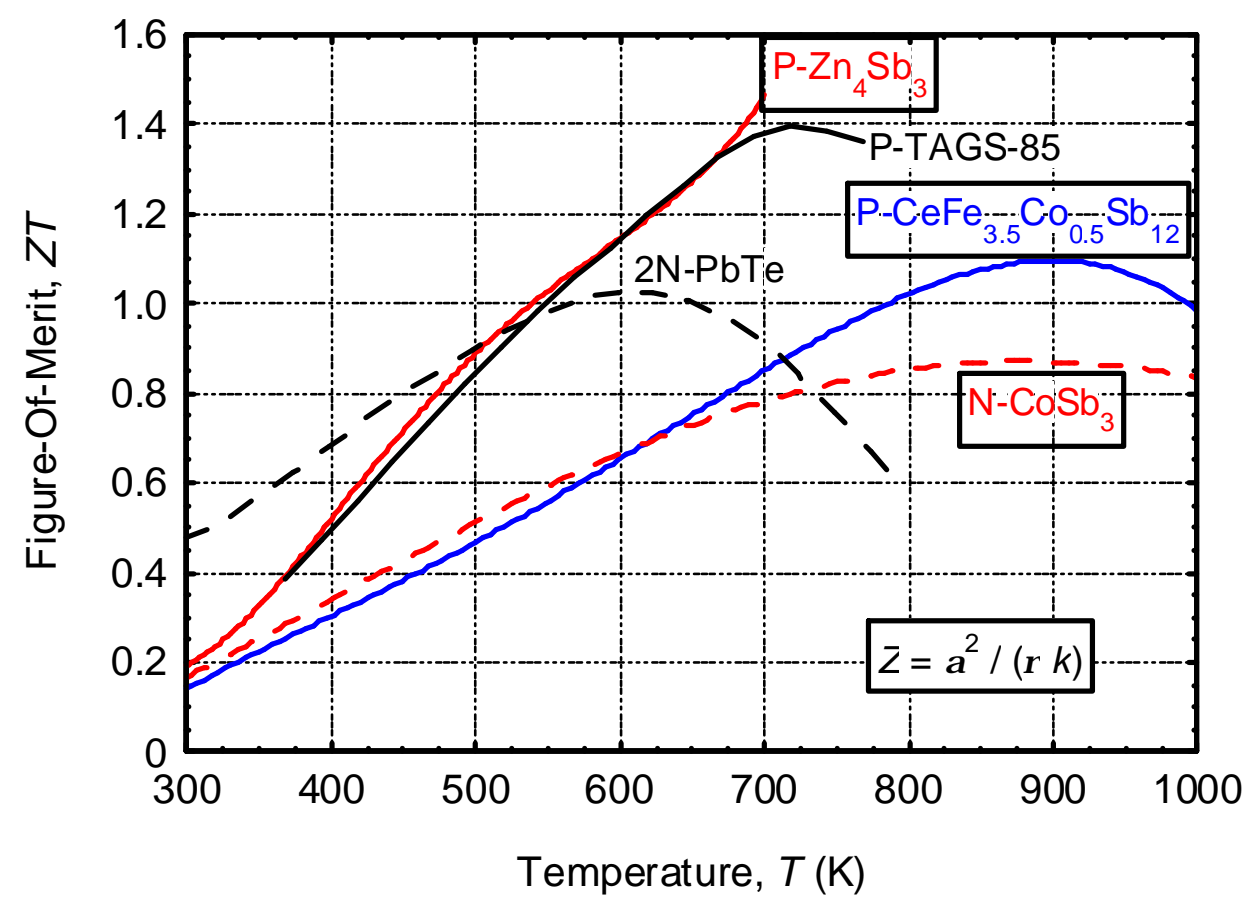

(a) Figure-of-merit of selected thermoelectric materials

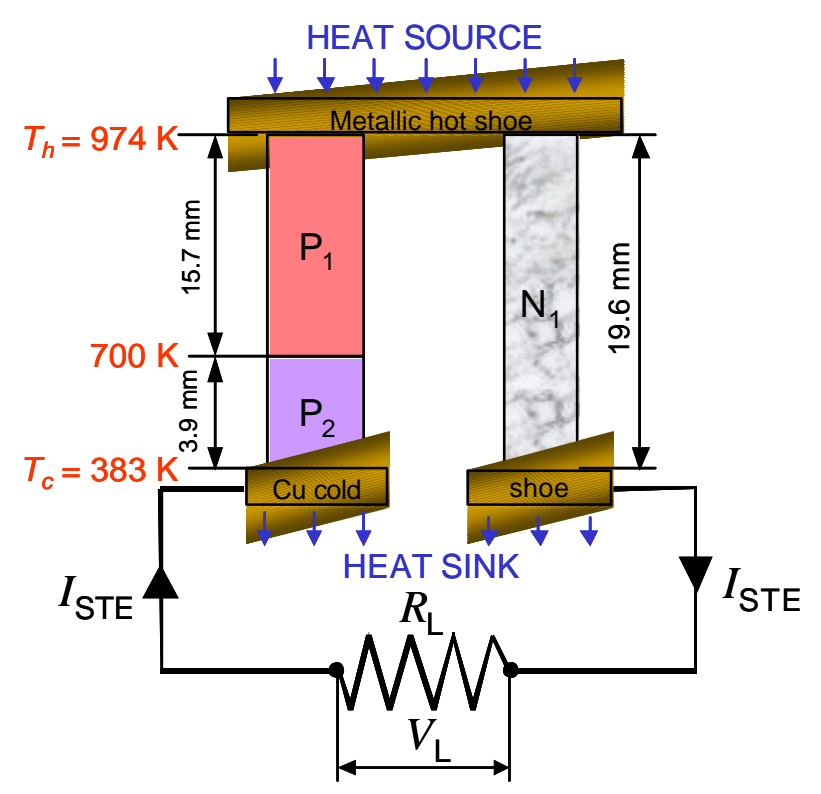

n-leg: $\mathrm{CoSb}_{3}, A_{N}=10.0 \mathrm{~mm}^{2}$

p-legs: $\mathrm{P}_{1}: \mathrm{CeFe}_{3.5} \mathrm{Co}_{0.5} \mathrm{Sb}_{12}, \mathrm{P}_{2}: \mathrm{Zn}_{4} \mathrm{Sb}_{3}$, $A_{P}=15.44 \mathrm{~mm}^{2}$

(b) Segmented thermoelectric unicouple used with seawater desalination

Figure 4-5. Characteristics of segmented TEs used in seawater desalination units. 
The small nuclear power plants considered herein use the optimized sodium- and potassium-AMTEC/TE converters described in Chapter 3. The heat rejected from the TE bottom cycle is removed by natural convection of ambient air and used for industrial or residential space heating. The AMTEC top and TE bottom cycles operate at terminal voltages of $\sim 350-400 \mathrm{~V} \mathrm{DC}$, which may be delivered directly to the end user. The reference potassium- AMTEC/TE units, each generating more than $50 \mathrm{kWe}$, are grouped into modules, each comprised of 20 units connected electrically in parallel. The total DC electricity generated by the module is $1.087 \mathrm{MWe}$, including those generated by the AMTEC top cycle and the TE bottom cycle. The AMTEC top cycle operates at $2.54 \mathrm{kA}$ and $396 \mathrm{~V}$ DC and produces a total of $1.004 \mathrm{MWe}$ DC, and the TE bottom cycle operates at $232 \mathrm{~A}$ and $357 \mathrm{~V} \mathrm{DC}$ and produces a total of $83 \mathrm{kWe} \mathrm{DC}$.

Additional static converters employed in these plants include segmented thermoelectrics (STE) of high figure-of-merit materials (El-Genk and Saber 2002a, ElGenk et al. 2002, Caillat et al. 2001) (Figures 4.5a and 4.5b), used in the units for

preheating seawater prior to desalination by multi-stage flash evaporation. In addition to calculating the total energy utilization of the plants, the comparisons of the LMR plants include estimates of the fraction of the reactor power converted into DC electricity and those used for various co-generation options such as space heating $(<400 \mathrm{~K})$, seawater desalination $(\sim 365 \mathrm{~K})$, and/or high temperature process heat or steam $(>500 \mathrm{~K})$.

\subsubsection{High Figure-Of-Merit Segmented Thermoelectric Modules for Seawater Desalination}

In the plant shown in Figure 4.11, co-generation heat is also used for preheating seawater to $365 \mathrm{~K}$ prior to desalination using multi-stage flash evaporation. The thermal energy supplied by the liquid sodium in the secondary loop of the nuclear power plant is partially converted into electricity using segmented thermoelectric modules (STEMs) (Figure 4.8), and the waste heat is used for seawater preheating (Figure 4.11). The STE modules are sandwiched between the secondary $\mathrm{Na}$ loop heat exchanger and that for the incoming seawater (Figure 4.7). Seawater is preheated to $365 \mathrm{~K}$ by the heat rejected by the STEMs, before entering the first stage of the desalination unit. The relatively large temperature drop $(\geq 500 \mathrm{~K})$ across the STEMs produces an electrical potential by the Seebeck phenomena (Rowe 1995), which is proportional to the temperature drop across the STEMs. In addition to this temperature drop, the performance of the STEMs depends on the Figure-of-Merit, $Z T$, of the thermoelectric materials used in the n- and p-legs (Figure 4.5a). For given dimensions and temperature difference, the higher ZT materials give higher conversion efficiency. However, since there is no single thermoelectric material that has highest $Z T$ over a wide temperature range, STEMs are employed in conjunction with the seawater desalination units in the plant delineated in Figure 4.11. As indicated in Figure 4.5a, each thermoelectric material typically possesses highest $Z T$ within a certain temperature range. Therefore, in order to obtain high conversion efficiency, STEs in the temperature range of interest (383 K-974 K) are used, in which the $\mathrm{n}$ - and $\mathrm{p}$-legs are comprised of a single segment of $\mathrm{CoSb}_{3}$ and two segments of $\mathrm{CeFe}_{3.5} \mathrm{Co}_{0.5} \mathrm{Sb}_{12}$ and $\mathrm{Zn}_{4} \mathrm{Sb}_{3}$, respectively (Figure 4.5b) (Caillat et al. 2001, El-Genk and Saber 2002a and 2002b). 


\subsubsection{Performance of STE Unicouple}

In the STEMs developed herein (Figures $4.5 \mathrm{~b}$ and 4.8 ), the dimensions of the $\mathrm{n}$ - and $\mathrm{p}$ legs as well as the lengths of the segments in the p-leg are determined for maximizing the conversion efficiency when operating at $T_{h}=974 \mathrm{~K}$ and $T_{c}=383 \mathrm{~K}$, for a total temperature drop of $591 \mathrm{~K}$. The $\mathrm{n}$ - leg and p-leg of the STE are $19.6 \mathrm{~mm}$ long and the hot segment in the $\mathrm{p}$-leg, made of $\mathrm{CeFe}_{3.5} \mathrm{Co}_{0.5} \mathrm{Sb}_{12}$, operates between $T_{h}=974 \mathrm{~K}$ and $\sim 700$ $\mathrm{K}$ while the bottom segment, made of $\mathrm{Zn}_{4} \mathrm{Sb}_{3}$, operates between $\sim 700 \mathrm{~K}$ and $T_{c}=383 \mathrm{~K}$. The interface temperature of $700 \mathrm{~K}$ is determined using a global optimization methodology of STEs developed at the University of New Mexico's Institute for Space and Nuclear Power Studies (El-Genk and Saber 2002b). The n- and p-legs are perfectly insulated on the sides and have constant, but different cross-sectional areas (see Table 4.3 and Figure 4.8).

Hot Shoe Temperature, $T_{h}(\mathrm{~K})$

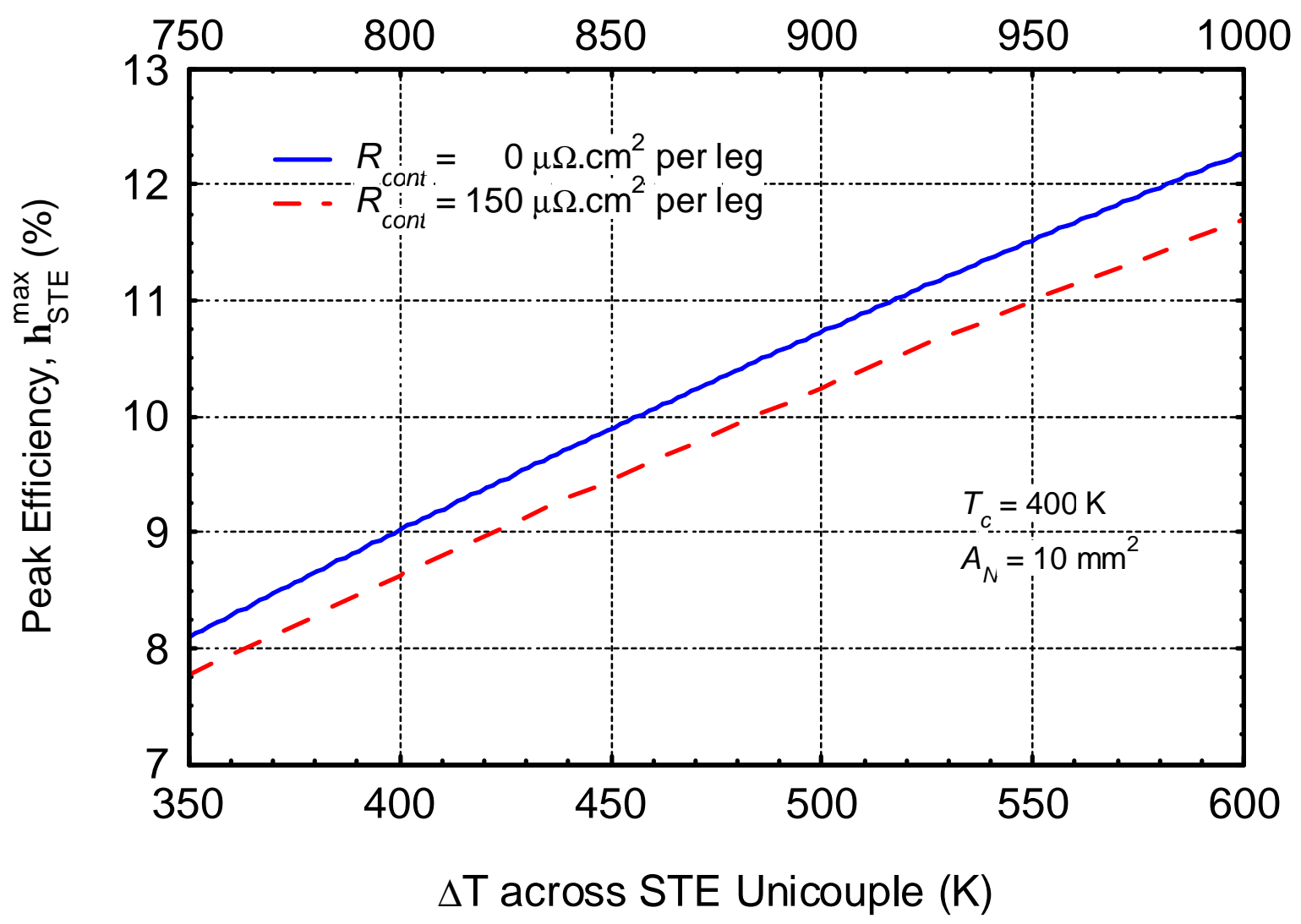

Figure 4-6. Peak conversion efficiency of STE and SiGe unicouples.

The predicted performance of the optimized STEs (Figure 4.5b) is shown in Figures 4.6, 4.9a and 4.9b, for a total interfacial resistance of zero and $150 \mu \Omega . \mathrm{cm}^{2}$ per leg, assuming zero side heat losses, and constant hot and cold side temperatures of $974 \mathrm{~K}$ 
and $383 \mathrm{~K}$, respectively. In these figures, the crossed circles indicate the peak conversion efficiency, while the solid squares indicate the peak electrical power. The load following portions of these curves are to the left of the peak electrical powers (Figure 4.9a). The selected operation point, in order for the STEM to provide up to $110 \%$ of nominal power in response to an increase in the load demand, is indicated by the solid squares in Figures 4.9a and 4.9b. The nominal operation points, indicated by the open triangles, are slightly to the left of the peak efficiency (at a current of $\sim 2 \mathrm{~A}$ ). When the interfacial resistance per leg is $150 \mu \Omega . \mathrm{cm}^{2}$, a STE unicouple (Figure 4.5b) delivers nominally $0.28 \mathrm{We}$, at a conversion efficiency of $11.5 \%$. This conservative value of the contact resistance accounts for the electrical losses at the interfaces between the metallic hot and cold shoes and the $\mathrm{n}$ - and $\mathrm{p}$-legs, and in the connectors between adjacent unicouples in the STEM.

Figure $4.9 \mathrm{~b}$ plots the predicted electrical power output of the optimized STE unicouple in Figure $4.5 \mathrm{~b}$, as a function of the calculated conversion efficiency. This figure indicates that for zero interfacial resistance, the STE unicouple could operate at a peak thermodynamic conversion efficiency of $12.2 \%$, or a peak electrical power of 0.32 $\mathrm{W}_{\mathrm{e}}$, at which the efficiency is slightly lower at $12.0 \%$. Increasing the total interfacial resistance to $150 \mu \Omega . \mathrm{cm}^{2}$ per leg, however, decreases the peak conversion efficiency by a half percentage point to $11.7 \%$. The optimized STE unicouples (Figure 4.5b) are assembled into STEMs, which are described next.

\subsubsection{STEM Design}

As shown in Figure 4.9a, at the selected nominal operation point, each STE unicouple operates at $0.141 \mathrm{~V}$ and $2.0 \mathrm{~A}$. To reduce the Joule losses and operate at high terminal voltage, all STE unicouples in the STEM are connected in series (Figures 4.8a and 4.8b). The STEM shown in these figures is comprised of $8 \times 160=1280$ unicouples, and is 25.4 $\mathrm{mm}$ high, $43.7 \mathrm{~mm}$ wide, and $874 \mathrm{~mm}$ long. The thermal and electrical insulation on the sides of the $\mathrm{n}$-and $\mathrm{p}$-legs in the STEM is $0.275 \mathrm{~mm}$ thick. To accommodate the differential thermal expansions of the materials in the $\mathrm{n}$ - and $\mathrm{p}$-legs and reduce the induced thermal stresses, a compliant pad is placed at the cold end of the STEM (Truscello and Rutger 1992). Electrical insulator, but thermal conductor layers separate the hot shoe and the hot electrical connectors, the cold connectors and the compliant pad, and the compliant pad and the cold shoe.

A single STEM nominally delivers $360 \mathrm{We}$ at $180 \mathrm{~V} \mathrm{DC}$, when operating at $T_{h}=$ $974 \mathrm{~K}$ and $T_{c}=383 \mathrm{~K}$. Each of the two STEM panels in the seawater desalination unit is comprised of 18 STEM converters in parallel to deliver a total of $6.5 \mathrm{kWe}$ at $180 \mathrm{~V} \mathrm{DC}$. The two STEM panels, however, are connected in series and deliver a total of $13 \mathrm{kWe}$ at 360 V DC (Figure 4.7 and Table 4.3). 


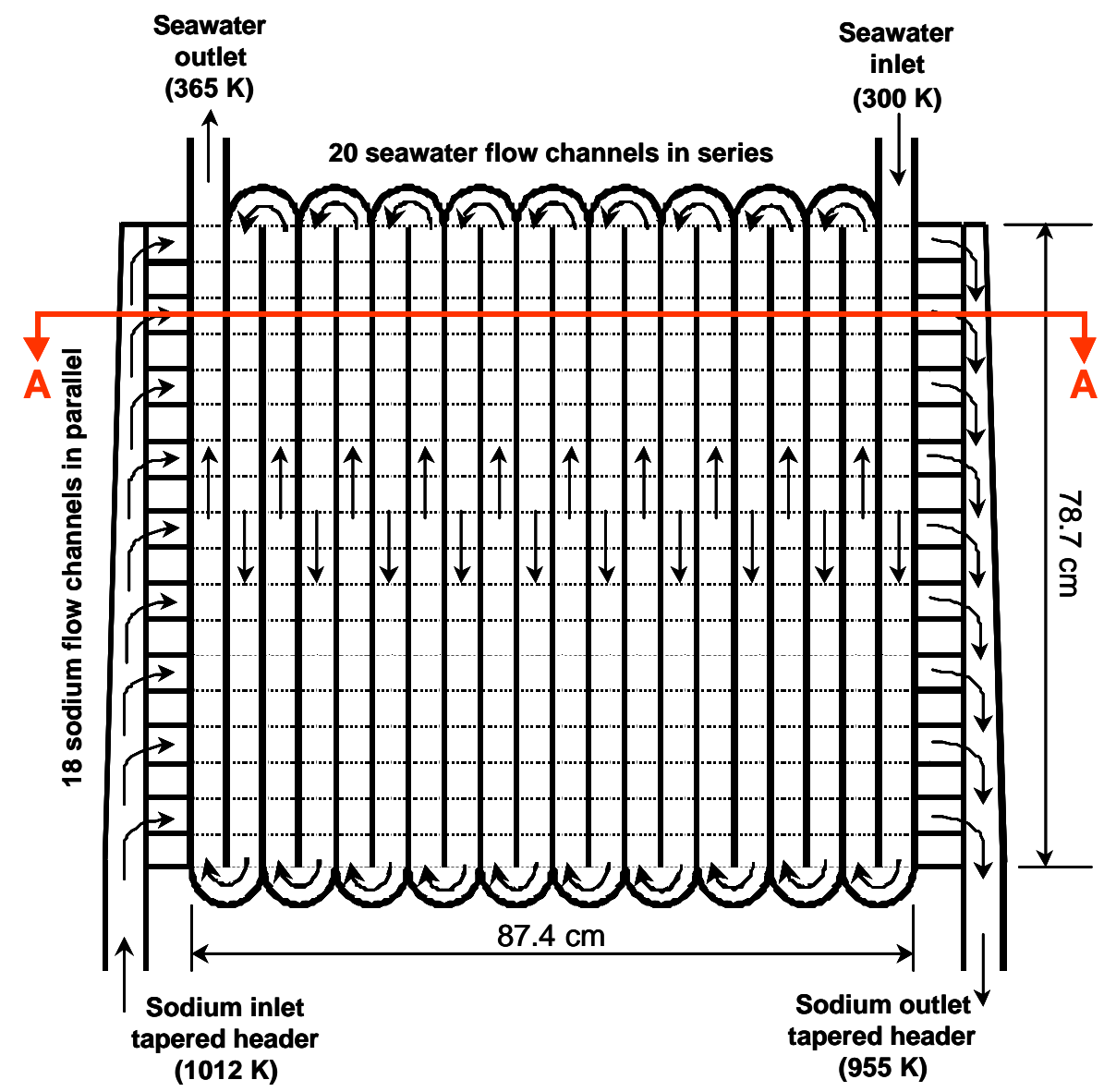

(a) Plan- view $B$ - B showing seawater and sodium flows arrangement

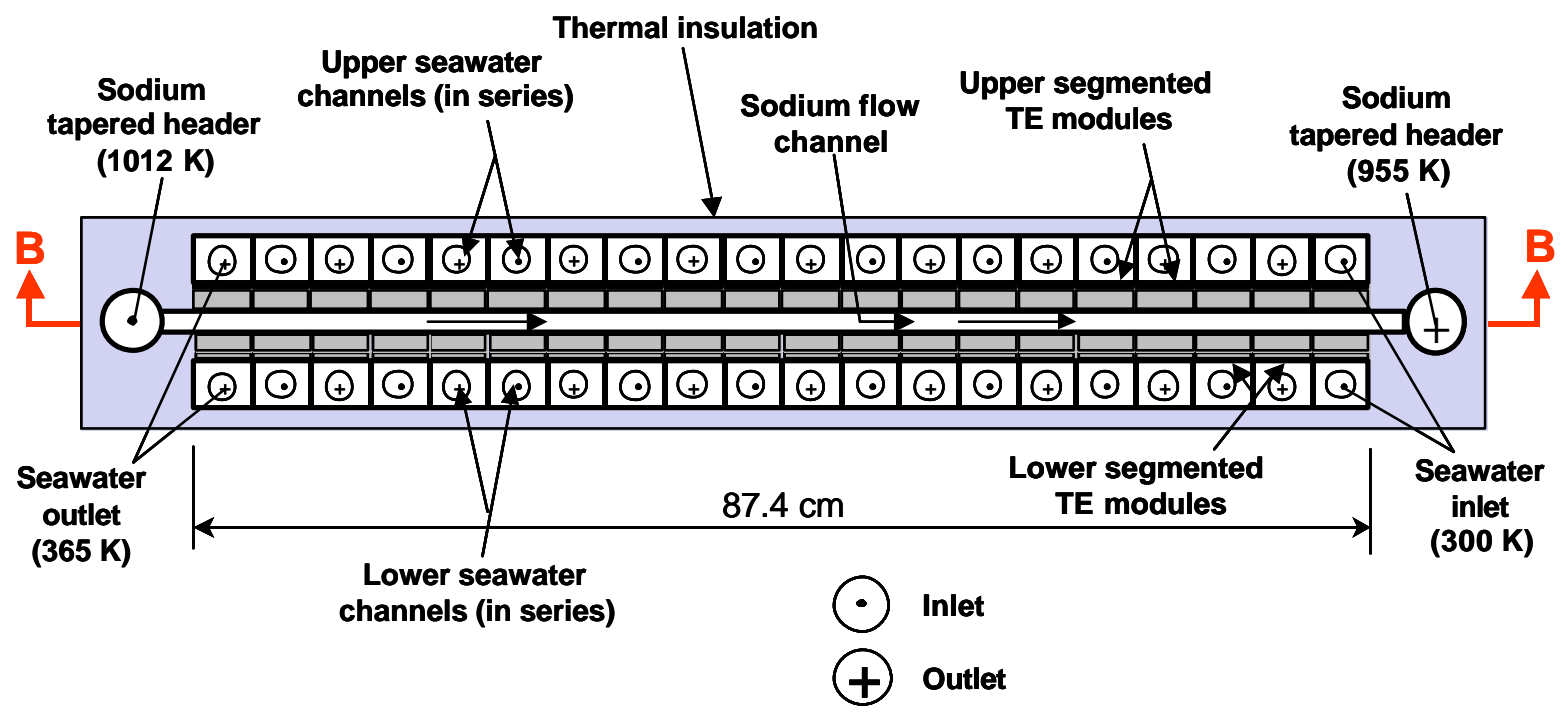

(b) Cross-sectional view $\mathrm{A}-\mathrm{A}$

Figure 4-7. Cross-sectional views of STEM/desalination preheating unit, comprised of 2 STEM panels of 18 STEMs each $\left(P e=13 \mathrm{kWe}\right.$ at $\left.360 \mathrm{~V} \mathrm{DC}, Q_{\text {water }}=100 \mathrm{~kW}\right)$. 


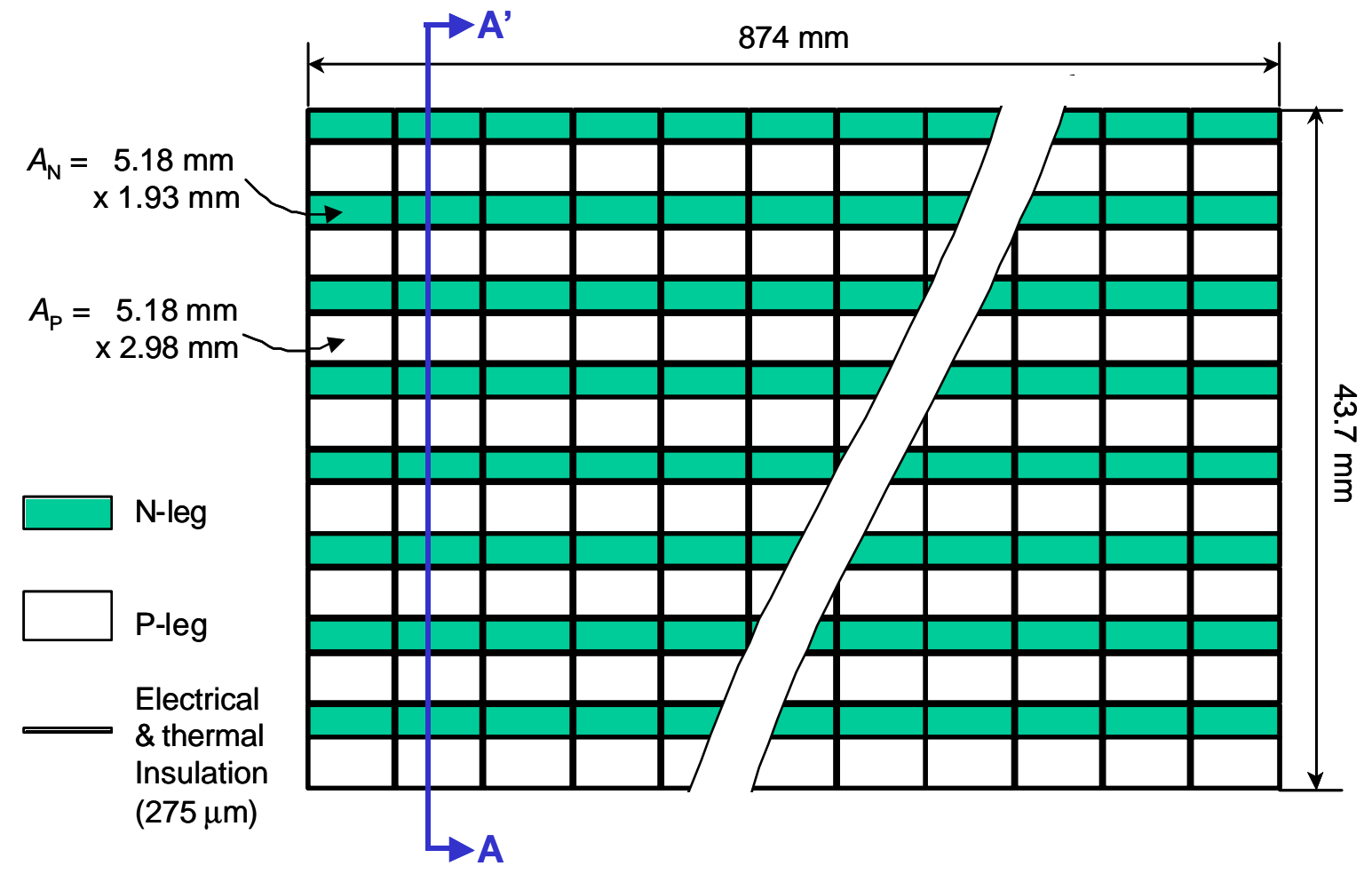

(a) Top view of STE Module (STEM)

TE hot shoe

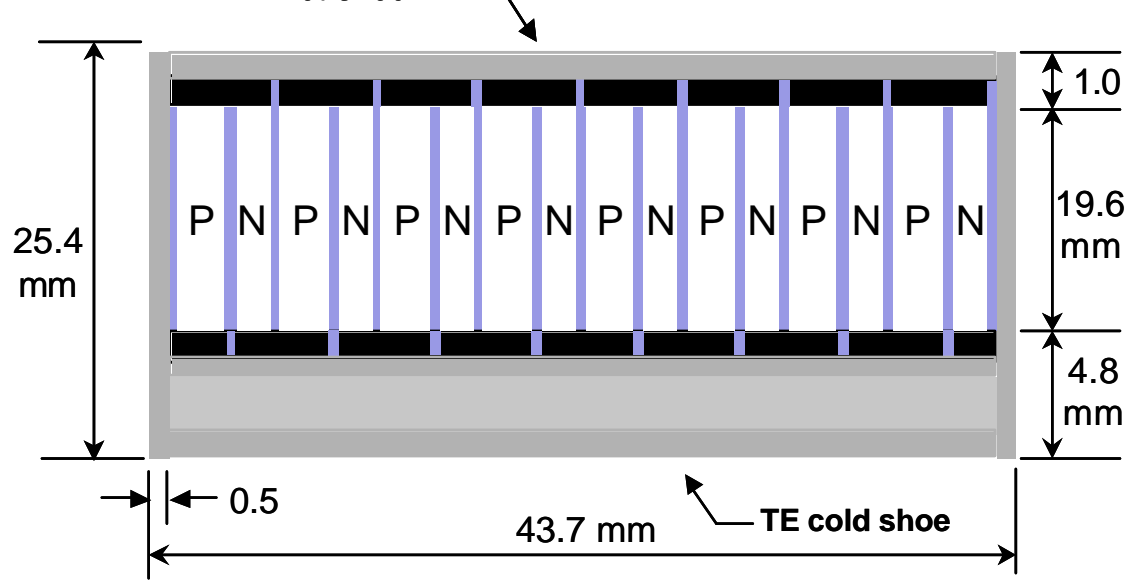

Electrical and thermal insulator

Electrical connectors

Compliant pad for stress relief

Electrical insulator but thermal conductor

(b) Cross-sectional view A - A

Figure 4-8. Segmented ThermoElectric Module (STEM) (1280 unicouples connected in series, and $P e_{\text {STEM }}$ $=360 \mathrm{We}$ at $180 \mathrm{~V} \mathrm{DC})$. 
Table 4-3. Dimensions and performance parameters of STEM/desalination preheating unit at nominal operation.

\begin{tabular}{|c|c|c|}
\hline Parameter & Liquid sodium & Seawater \\
\hline \multicolumn{3}{|l|}{ (a) Dimensions and Operation } \\
\hline Number of channels & 18 & 40 in 2 panels \\
\hline Channel width $\mathrm{x}$ height $(\mathrm{mm})$ & $41.7 \times 5.0$ & $41.7 \times 10.0$ \\
\hline Total mass flow rate $(\mathrm{kg} / \mathrm{s})$ & 1.54 & 0.367 \\
\hline Inlet / Outlet temperature (K) & $1012 / 955$ & $300 / 365$ \\
\hline Thermal power lost / gained by fluid $(\mathrm{kW})$ & 113.0 & 100.0 \\
\hline Average flow velocity $(\mathrm{m} / \mathrm{s})$ & 0.525 & 0.401 \\
\hline Average Reynolds number & 17,900 & 13,600 \\
\hline Average Nusselt number & 12.4 & 69.9 \\
\hline Convective heat transfer coeff. $\left(\mathrm{kW} / \mathrm{m}^{2} . \mathrm{K}\right)$ & 78.0 & 2.80 \\
\hline \multicolumn{3}{|l|}{ (b) Temperature Drops } \\
\hline$\Delta T$ between sodium and STE hot shoe $(\mathrm{K})$ & \multicolumn{2}{|c|}{9.6} \\
\hline$\Delta T$ across STE legs $(\mathrm{K})$ & \multicolumn{2}{|c|}{$591 \pm 17$} \\
\hline$\Delta T$ between STE cold shoe and seawater $(\mathrm{K})$ & \multicolumn{2}{|c|}{51} \\
\hline \multicolumn{3}{|l|}{ (c) Performance } \\
\hline STEM's output voltage (V) / current (A) & \multicolumn{2}{|c|}{$180 / 2.0$} \\
\hline STEM panels output voltage (V) / current (A) & \multicolumn{2}{|c|}{$360 / 36$} \\
\hline$P e(\mathrm{kWe}) / \eta(\%)$ for 2 STEM panels & \multicolumn{2}{|c|}{$13.0 / 11.5$} \\
\hline
\end{tabular}

\subsubsection{STEM Assembly}

The liquid Na heat exchanger for the STEMs assembly consists of two thermallyinsulated, tapered inlet and outlet headers connected to 18 rectangular cross channels, each $41.7 \mathrm{~mm} \times 5.0 \mathrm{~mm}$ in cross-section and $87.4 \mathrm{~cm}$ long (Figure $4.7 \mathrm{a}$ ). The crosssectional flow areas of the headers are gradually reduced to ensure identical mass flow rates and average liquid sodium temperatures in the cross flow channels. One STEM is laid out on the top and bottom sides of each flow channel. The 18 STEMs on each side are connected electrically in parallel to form a STEM panel, which measures $87.4 \mathrm{~cm}$ by $78.7 \mathrm{~cm}$. 


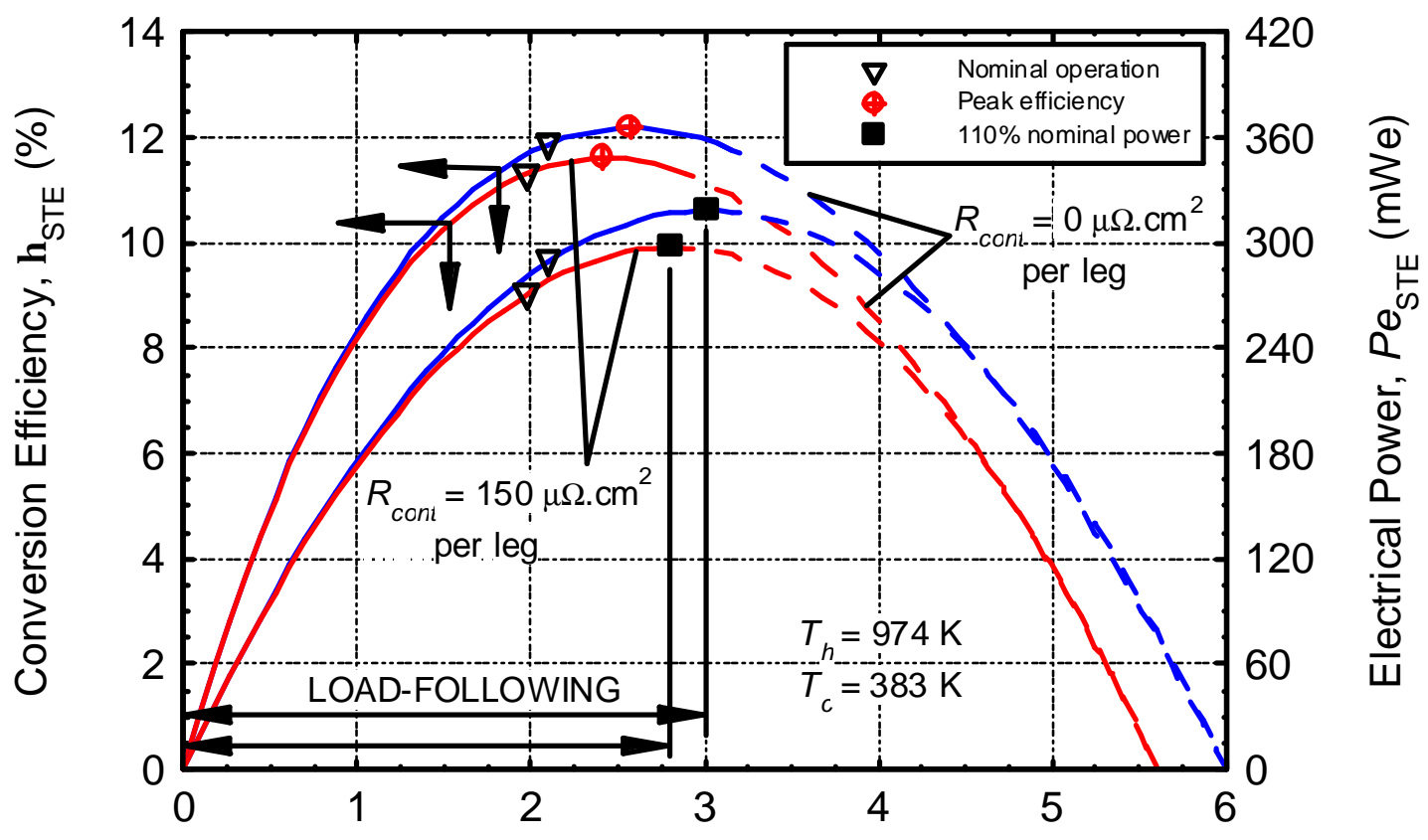

Electrical Current per unicouple, $I_{\text {STE }}(A)$

(a) Load-following characteristic of optimized STEs

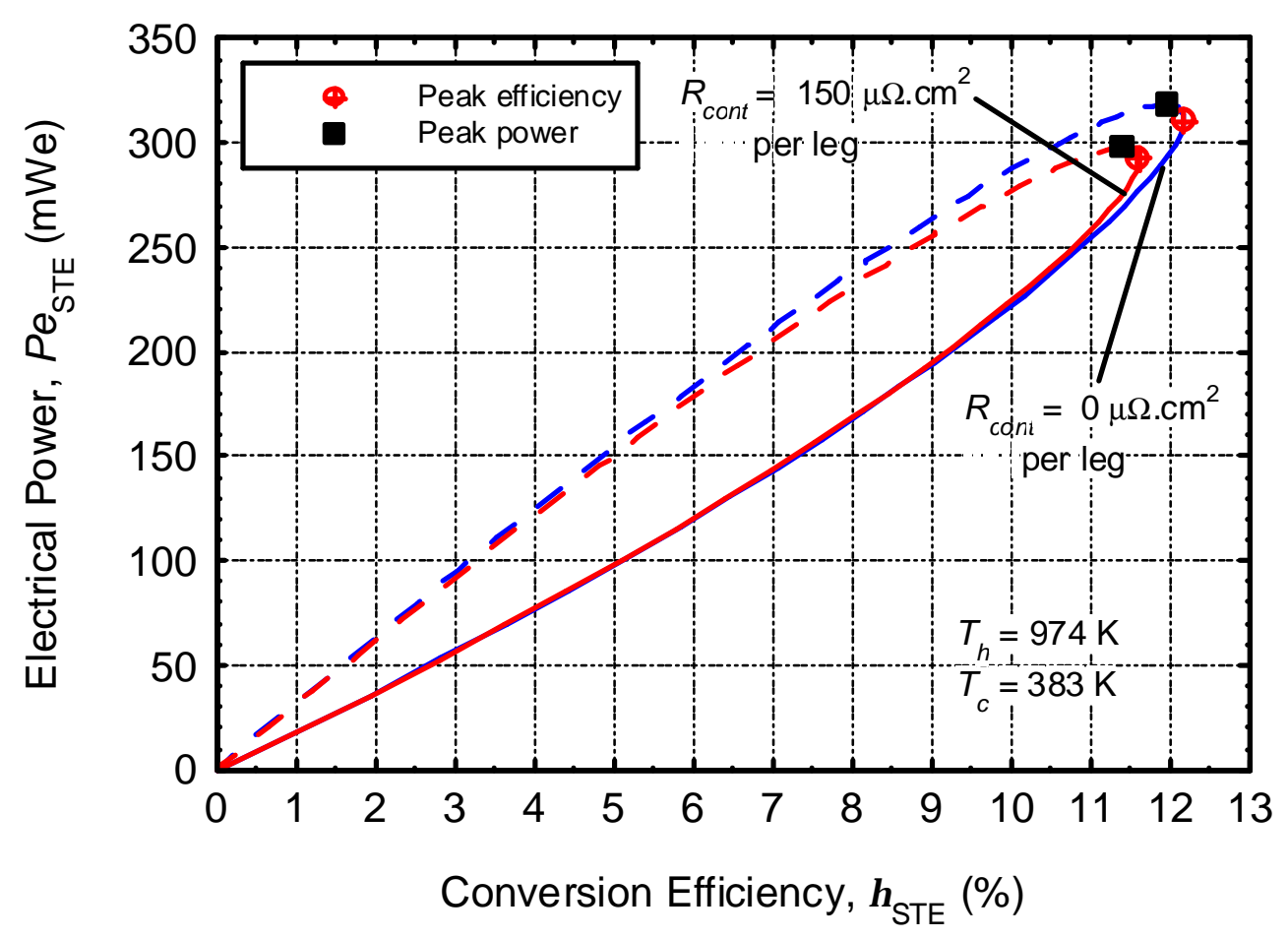

(b) Effect of contact resistance on performance of optimized STE

Figure 4-9. Performance of optimized STEs used in conjunction with seawater desalination. 
The cold side of each STEM panel is mounted to the seawater heat exchanger, having 20 rectangular flow channels (Figures $4.7 \mathrm{a}$ and $4.7 \mathrm{~b}$ ), each $78.7 \mathrm{~cm}$ long. The flow of the incoming seawater in the heat exchanger channels is turbulent at a Reynolds number of 13,600 , , resulting in a convective heat transfer coefficient equal to 2.80 $\mathrm{kW} / \mathrm{m}^{2} . \mathrm{K}$ (Table 4.3). At these conditions, the variation in the cold side temperature across the 36 STEMs is only $\pm 4 \mathrm{~K}$. As a result, the STEMs in the panels generate a near identical electrical power output. The unicouples in the STEM panels experience a temperature drop of $591 \pm 17 \mathrm{~K}$, and the hot side temperature of the unicouples in a STEM varies between $945 \mathrm{~K}$ and $1000 \mathrm{~K}$, while the cold shoe temperature varies from $351 \mathrm{~K}$ to $416 \mathrm{~K}$. Other performance parameters of interest are listed in Table 4.3.

As indicated earlier, the top and bottom STEM panels are connected electrically in series, delivering $13.0 \mathrm{kWe}$ at $360 \mathrm{~V} \mathrm{DC}$ and an efficiency of $11.5 \%$. The thermal power delivered to the incoming seawater flow through the STEM assembly is $\sim 100 \mathrm{~kW}$. Additional performance figures and dimensions are listed in Table 4.3. In a nuclear power plant with co-generation for seawater desalination (Figure 4.11), a multitude of the 13-kWe STEM assemblies could be used, commensurate with the thermal power of the nuclear reactor. Such modularity in both electricity generation and co-generation for seawater desalination is an added advantage of the small nuclear power plants with static energy conversion. The reactor thermal power is the sum of the DC electrical power generated, co-generation thermal powers, and thermal losses. Several options of these plants are presented and their performance figures are discussed next.

\subsubsection{Potential Performance of High-Energy-Utilization LMR-AMTEC Plant}

In this section, the performance results of three LMR plants with static conversion are presented and discussed. The layout and a summary of the energy utilization of each of these plants with potassium AMTEC/TE converters are shown in Figures 4.10 - 4.12, and the results are compared in Table 4.4 with those employing sodium AMTEC/TE converters.

In the LMR plant delineated in Figure 4.10, the coolant temperature rise in the nuclear reactor core and, hence, across the IHX, is $100 \mathrm{~K}$. The primary sodium coolant exits the reactor at $1130 \mathrm{~K}\left(857^{\circ} \mathrm{C}\right)$ and enters at $1030 \mathrm{~K}\left(757^{\circ} \mathrm{C}\right)$. The sodium in the secondary loop exits the intermediate heat exchanger (IHX) at $1113 \mathrm{~K}\left(840{ }^{\circ} \mathrm{C}\right)$ and enters at $1013 \mathrm{~K}\left(740{ }^{\circ} \mathrm{C}\right)$. The heat losses in the connecting lines decrease the temperature of the secondary sodium entering the heat exchangers of the AMTEC/TE converters by $4 \mathrm{~K}$ to $1109 \mathrm{~K}$. The heat losses in the secondary loop are assumed conservatively at $5 \%$ in all the plants presented herein.

Performance results for the LMR plant delineated in Figure 4.10 are summarized in the figure and listed in Table 4.4. These results indicate that $32.7 \%$ of the reactor thermal power is converted into DC electrical power, while $62.3 \%$ of the reactor thermal power is used for space heating, for a plant total energy utilization of 95.0\%. For the same temperatures shown in Figure 4.10, when sodium AMTEC/TE converters replace 
the K-AMTEC/TE converters, the fraction of the reactor thermal power converted into DC electricity (or the plant net efficiency) decreases to $24.7 \%$, but the fraction used for space heating increases to $70.3 \%$, for the same plant total energy utilization of $95.0 \%$ (Table 4.4).

Figure 4.11 presents the layout and performance parameters of a LMR plant with both potassium AMTEC/TE converters and STEM assemblies, and co-generation power for space heating and for preheating seawater to $365 \mathrm{~K}$ prior to desalination by multistage flash evaporation. This figure shows that the temperature rise across the IHX (160 $\mathrm{K}$ ) in this plant is higher than that in the LMR plant shown in Figure 4.10, but the coolant temperature drop across the AMTEC/TE HX $(95 \mathrm{~K})$ and the reactor core exit temperature $(1130 \mathrm{~K})$ are the same as in Figures 4.10 and 4.12. Figure 4.11 and Table 4.4 show that adding the STEM assemblies and the desalination co-generation option reduces the fraction of the reactor's thermal power converted into electricity (the net plant efficiency) to only $24.5 \%$. However, the sum of the fractions for co-generation, including space heating and seawater desalination, increases to $70.5 \%$. After deducting the thermal losses (5\%), the total energy utilization of the LMR plant shown in Figure 4.11 is again $95.0 \%$. In this plant, when substituting sodium-AMTEC/TE converters for the potassiumAMTEC/TE converters, the plant's net efficiency decreases to $19.5 \%$, while the cogeneration power for space heating and seawater desalination increases to $75.5 \%$.

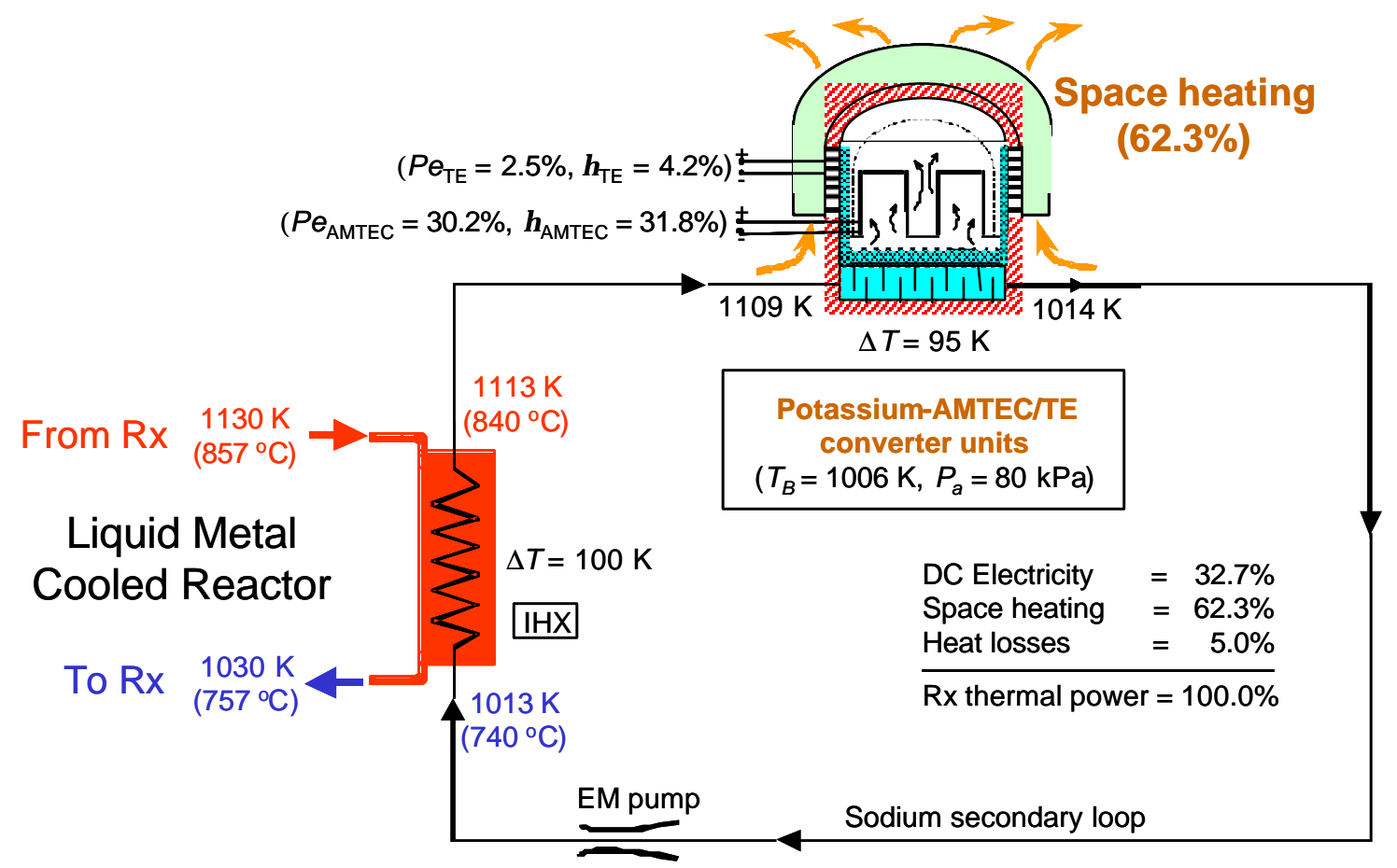

Figure 4-10. LMR plant with potassium AMTEC/TE converters. 


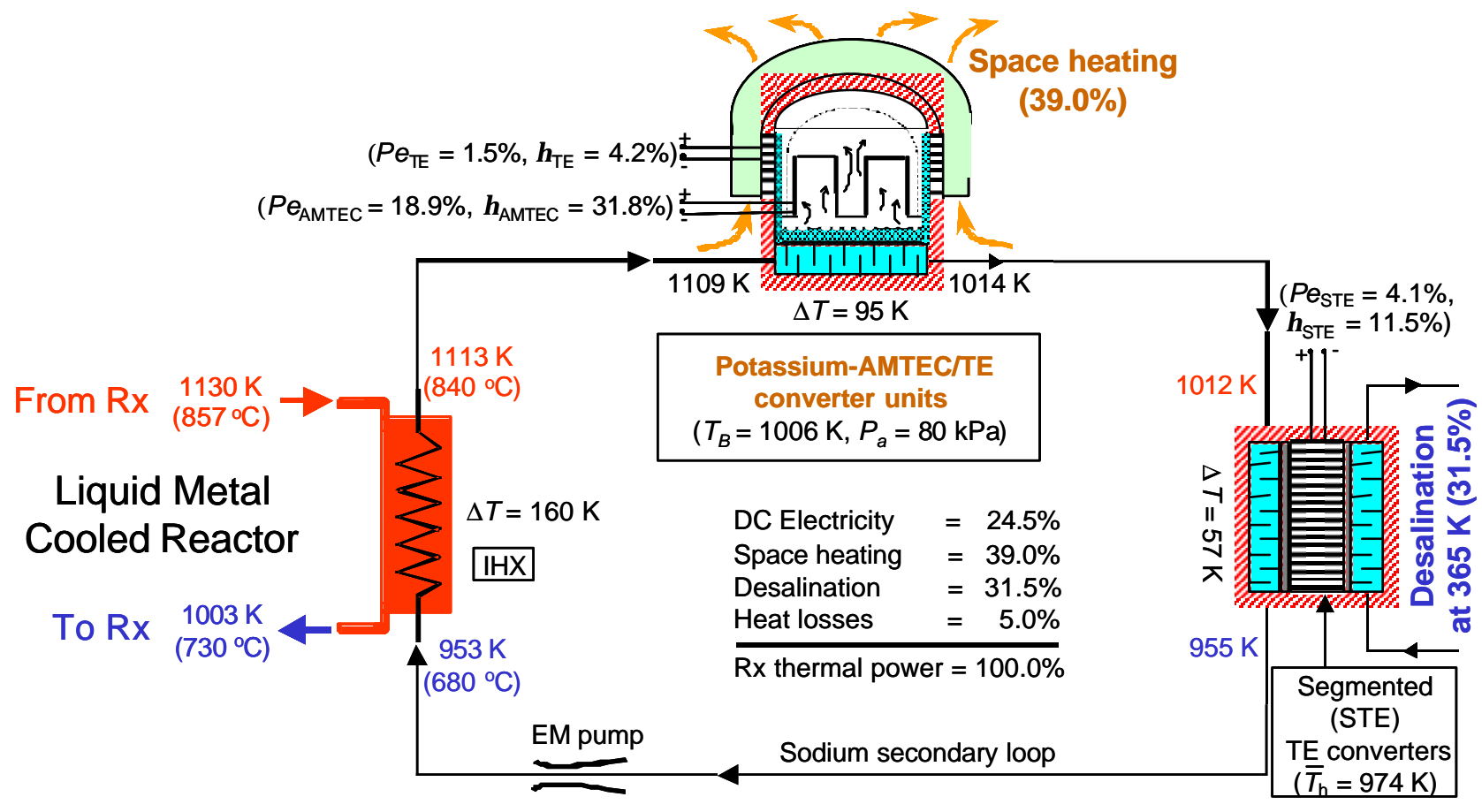

Figure 4-11. LMR plant with potassium AMTEC/TE converters and STEM/seawater desalination.

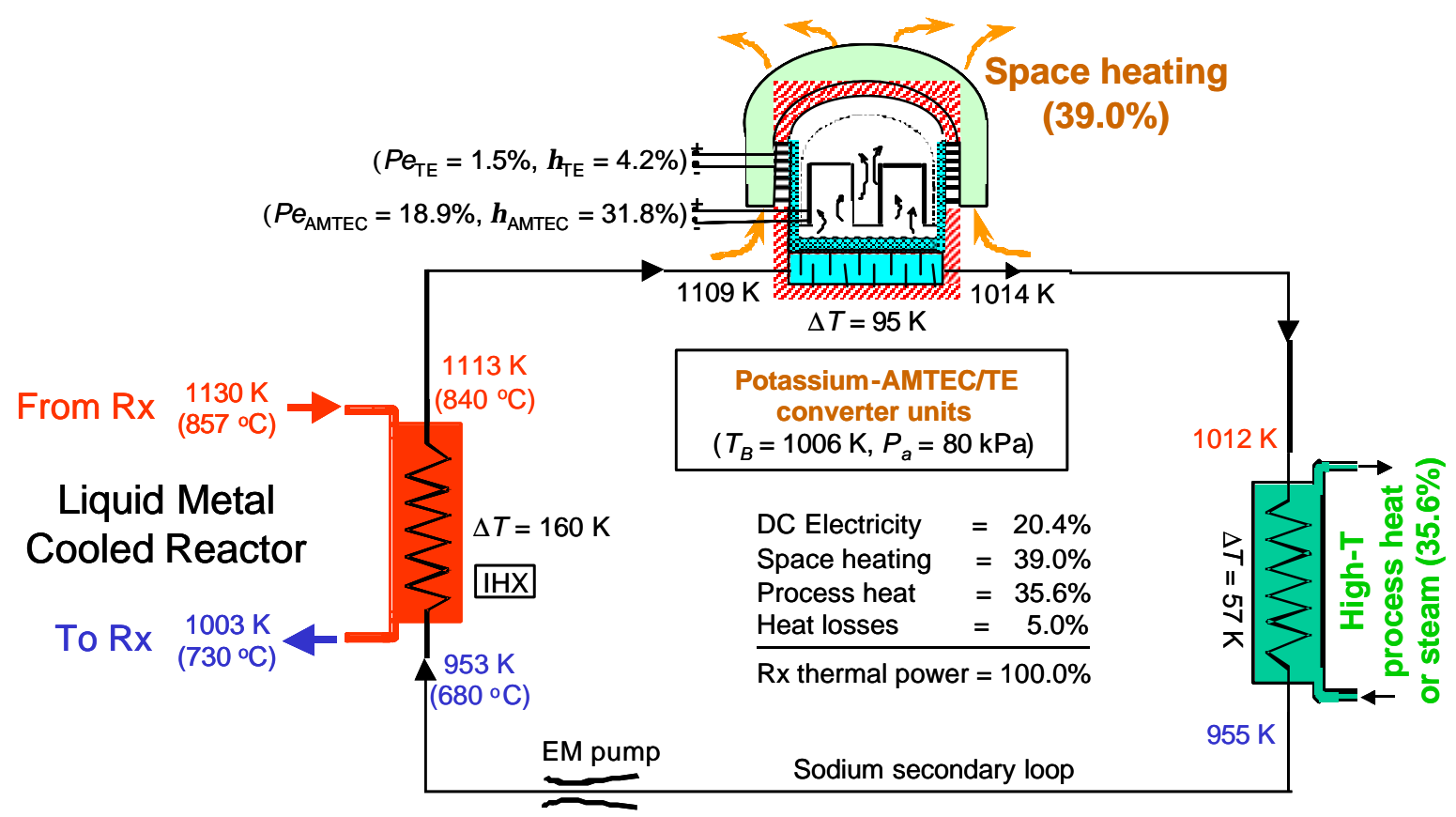

Figure 4-12. LMR plant with K-AMTEC/TE converters and high-temperature process heat/steam. 
Table 4-4. Performance comparison of high-energy-utilization plants $\left(\eta_{\mathrm{th}}=95 \%\right)$ with sodium- and potassium-AMTEC/TE converter units.

\begin{tabular}{|c|c|c|c|c|c|c|}
\hline \multirow[t]{2}{*}{ Uses of reactor thermal power } & \multicolumn{2}{|c|}{$\begin{array}{l}\text { LMR plant } \\
\text { (Fig. 4.6) }\end{array}$} & \multicolumn{2}{|c|}{$\begin{array}{l}\text { LMR plant } \\
\text { (Fig. 4.7) }\end{array}$} & \multicolumn{2}{|c|}{$\begin{array}{l}\text { LMR plant } \\
\text { (Fig. 4.8) }\end{array}$} \\
\hline & $\mathrm{Na}$ & $\mathrm{K}$ & $\mathrm{Na}$ & $\mathrm{K}$ & $\mathrm{Na}$ & $\mathrm{K}$ \\
\hline I. DC Electricity output & 24.7 & 32.7 & 19.5 & 24.5 & 15.4 & 20.4 \\
\hline II. Co-generation heat & & & & & & \\
\hline (a) Space Heating (\%) & 70.3 & 62.3 & 44.0 & 39.0 & 44.0 & 39.0 \\
\hline (b) Desalination (\%) & $?$ & $?$ & 31.5 & 31.5 & $?$ & $?$ \\
\hline $\begin{array}{l}\text { (c) High Temperature } \\
\text { Process Heat/Steam }(\%)\end{array}$ & ? & ? & ? & ? & 35.6 & 35.6 \\
\hline III. Plant Thermal Losses & 5.0 & 5.0 & 5.0 & 5.0 & 5.0 & 5.0 \\
\hline IV. Energy Balance of Plant & & & & & & \\
\hline (a) DC Electricity (\%) & 24.7 & 32.7 & 19.5 & 24.5 & 15.4 & 20.4 \\
\hline (b) Co-Generation Heat (\%) & 70.3 & 62.3 & 75.5 & 70.5 & 79.6 & 74.6 \\
\hline & 5.0 & 5.0 & 5.0 & 5.0 & 5.0 & 5.0 \\
\hline Total Sum $(\%)$ & 100 & 100 & 100 & 100 & 100 & 100 \\
\hline V. Total Energy Utilization (\%) & 95.0 & 95.0 & 95.0 & 95.0 & 95.0 & 95.0 \\
\hline
\end{tabular}

*: Table values are percentages of the nuclear reactor's thermal power.

Figure 4.12 presents a layout of a LMR power plant employing potassium AMTEC/TE converters and providing co-generation power for space heating and the production of high temperature process heat or steam at $>500 \mathrm{~K}$, for use in industrial applications. In this plant, the reactor coolant exit temperature, the temperature rise across the IHX, and the coolant temperature drop across the AMTEC/TE HX are the same as in the LMR plant shown in Figure 4.11. Figure 4.12 and Table 4.4 show that the percentage of the reactor power converted into DC electricity (or plant net efficiency) is only $20.4 \%$, while the sum of co-generation power for space heating and the production of high temperature process heat/steam is $74.6 \%$ (Figure 4.12 and Table 4.4). 
When the potassium-AMTEC/TE converters are replaced with sodium AMTEC/TE converters in the LMR plant in Figure 4.12, the percentage of the reactor power converted into electricity (or plant net efficiency) decreases to $15.4 \%$. On the other hand, the sum of co-generation powers for space heating (44.0\%) and the production of high temperature process heat or steam (35.6\%) increases to $79.6 \%$. Owing to the relatively small temperature rise across the IHX, the LMR plants in Figures 4.11 and 4.12 can only provide for either seawater desalination or high-temperature process heat/steam, but not for both in line. In the LMR plants shown in these figures, providing co-generation power for both seawater desalination and the production of process heat/steam simultaneously may be achieved by splitting the sodium secondary line downstream of the AMTEC/TE modules into two parallel lines, one supporting the STEM/ desalination units and the other for the steam generators. Such a plant with KAMTEC/TE units would provide a net efficiency between $20.4 \%$ and $24.5 \%$, depending on the sodium mass flow rates in the two parallel lines.

\subsubsection{Summary}

Performance analyses of three LMR small nuclear power plants with static energy conversion were conducted. The plants use static conversion units comprised of an AMTEC topping cycle and a thermoelectric bottom cycle, as well as segmented thermoelectric (STE) conversion modules. The reference potassium-AMTEC/TE converter units, each generating more than $50 \mathrm{kWe}$ DC, are grouped into modules, each comprised of 20 units connected electrically in parallel. The total DC electrical power generated by one module is $1.087 \mathrm{MWe}$, including those generated by the AMTEC top cycle and the TE bottom cycle. The AMTEC top cycle operates at $2.54 \mathrm{kA}$ and $396 \mathrm{~V}$ DC and produces a total of $1.004 \mathrm{MWe}$ DC, and the TE bottom cycle operates at $232 \mathrm{~A}$ and $357 \mathrm{~V} \mathrm{DC}$ and produces a total of $83 \mathrm{kWe}$ DC. The number of modules in the plant will depend on its installed electrical capacity and the thermal power of the nuclear reactor. For example, a $100 \mathrm{MWe}$ plant will use one hundred 1.1 MW AMTEC/TE modules. These modules are mounted on top of an exchanger heated by the liquid sodium secondary loop. Thus, a failure or a replacement of one or more AMTEC/TE converter unit(s) could be accomplished without having to shutdown the nuclear reactor or disturbing the liquid sodium in the secondary loop of the plant. These static conversion modules are cooled by natural conversion of ambient air, and could deliver a net conversion efficiency of $\sim 34 \%$ when operating at an evaporator temperature of $1000 \mathrm{~K}$. The heated air is used for space or district heating.

The analyses of the plants were performed at the same exit temperature of the secondary sodium coolant in the IHX $(1113 \mathrm{~K})$, and the same coolant temperature drops across the HX for the AMTEC/TE converters $(95 \mathrm{~K})$ and in the STEM/seawater desalination units $(57 \mathrm{~K})$. The temperature drop across the co-generation unit for the production of high-temperature process heat or steam in the LMR plants is $57 \mathrm{~K}$. In the LMR plants used only for electricity production and co-generation power for space heating, the coolant temperature rise across the reactor $\left(\Delta T_{\text {core }}\right)$ and the IHX is $100 \mathrm{~K}$, versus $160 \mathrm{~K}$ when co-generation power is provided also for either preheating seawater 
prior to desalination or high-temperature process heat/steam, reducing the inlet temperature to the IHX from $1013 \mathrm{~K}$ to $953 \mathrm{~K}$.

Results indicate that the LMR plants operating at a lower coolant temperature rise through the core (100-160 K) offer an advantage when the emphasis is on converting a large fraction of the reactor's thermal power into electricity (or higher net plant efficiency). In these LMR plants, providing co-generation power for both seawater desalination and the production of process heat/steam simultaneously may be achieved by splitting the sodium secondary line downstream of the AMTEC/TE units into two parallel lines, one supporting the STEM/desalination units, the other having a steam generator or $\mathrm{HX}$ for generating steam or process heat, respectively.

The results of the present analyses showed that small nuclear power plants with static energy conversion could achieve a total utilization of the nuclear reactor's thermal power in excess of $90 \%$. In addition to the DC electricity generated, it includes cogeneration power for space heating $(<400 \mathrm{~K})$, preheating seawater to $365 \mathrm{~K}$ prior to desalination by multi-stage flash evaporation, and/or the production of high-temperature process heat or steam at $>500 \mathrm{~K}$.

Based on the results of the present analyses, it might be argued that small nuclear power plants with static conversion could indeed be a valuable choice for providing the appropriate mix of electricity and co-generation power needed in underdeveloped countries and in remote communities, with no or limited electrical Grid, and little or no access to reasonably priced fossil fuels. Although such small, high-energy-utilization nuclear power plants may not compete with large commercial nuclear power plants in electricity cost in metropolitan areas, they could meet the electrical and thermal energy needs for a variety of applications, where large power plants are neither economical nor practical. These small nuclear power plants could be designed and built with emphases on simplicity, low maintenance, inherent safety, passive cooling, non-proliferation, and long life, and could provide both electricity and process heat for a variety of uses, creating jobs and stimulating economical growth.

\subsection{References}

Asamoto R., Hutchins B.A., Law D.D., Pavlenco G.F. and Snyder C.R., "Economics of the PRISM Modular Nuclear power Plant", Experience Gained and Path to Economical Power Generation, Septembre 13-17, 1987.

Brunings J.E., Foster .K.W. and Larson E.M., "Sodium Advanced Fast Reactor Economics", Experience Gained and Path to Economical Power Generation, Septembre 13-17, 1987.

Calamai G.J. et als, "Ultra Long Life Core and Plant design Study", DGRA-60, Westinghouse Electric Corporation, 1981.

Caillat, T., Borshchevsky, A., Snyder, J., and Fleurial, J.-P. (2001) "High Efficiency

Segmented Thermoelectric Couples," in Proceedings of Space Technology and 
Applications International Forum, M. El-Genk, Ed., AIP-CP-552, American Institute of Physics, New York, NY, 2001, pp. 1107-1112.

Carelli M. and Soto J., "Design Study of Advanced small or Medium Size FBR Concepts", WAES-TR-91-0003, 1991.

Chow B.G., "Plutonium Economics and the Civilian Nuclear Future", Global95 Proceedings, Paris, 1995.

El-Genk, M. S., and Saber, H. (2002a) "Performance Optimization of Segmented Thermoelectric Unicouples," in Proc. of Space Technology and Applications International Forum, M. El-Genk, Ed., AIP-CP-608, American Institute of Physics, Melville, NY, 2002, pp. 980-988.

El-Genk, M. S., and Saber, H. (2002b) Personal Communications, Institute for Space and Nuclear Power Studies, The University of New Mexico, Albuquerque, NM, USA, April 24.

El-Genk, M. S., Saber, H., and Caillat, T. (2002) "A Performance Comparison of SiGe and Skutterudite Based Segmented Thermoelectric Devices," in Proc. of Space Technology and Applications International Forum, M. El-Genk, Ed., AIP-CP-608, American Institute of Physics, Melville, NY, 2002, pp. 1007-1015.

EPRI, "A review of the Economic Potential of Plutoniumin Spent Nuclear Fuel", EPRI report, p 4.11, B-4, C-4, Chapter C 5.2 Palo Alto, 1996.

Faibish, R.S., Konishi, T., and Gasparini, M. (2002) "Application of Nuclear Energy for Seawater Desalination - Design Concepts of Nuclear Desalination Plants," in Proc. of $10^{\text {th }}$ International Conference on Nuclear Energy (ICONE-10), Arlington, VA, American Society of Mechanical Engineers, NY, 14-18 April, Paper No. ICONE10-22071.

Fraas A.P., Heat Exchanger Design, Whiley-Interscience Publication, 1989, New York.

Hren R.R., "SAFR Plant Construction", Experience Gained and Path to Economical Power Generation, Septembre 13-17, 1987.

Inoue M., Ono K. Fujioka T. Sato K. and Asaga T., "Feasibility Study on Nitrogen-15 Enrichment and Recycling System for Innovative FR Cycle System with nitride Fuel", ICONE-10, Arlington, 2002.

Subramanian K. and Hunt T.K., "Solar Residential Total Energy System Using the sodium Heat Engine - A Conceptual Study," Proceedings of the $17^{\text {th }}$ Intersociety Energy Conversion Engineering Conference (IECEC-82), Los Angeles, August 8-12, 1982.

Truscello, V. C., and L. L. Rutger (1992) "The SP-100 Power system," in Proceedings of the 9th Symposium on Space Nuclear Power Systems, held in Albuquerque, NM, January 12-16, 1992, M. S. El-Genk and M. D. Hoover, Eds., American Institute of Physics, New York, NY, AIP Conference Proceedings No. 246, Part 1, 1992, pp. 1-23.

Westlen D., Gudowski W., Wallenius J. and Tucek K., "A cost Benefit Analysis of an Accelerator driven Transmutation System", Royal Institute of technology, Stockholm centre for Physics, Astronomy and Biotechnology.

Yevick J., Fast Reactor Technology: Plant Design, The MIT Press, 1966. 


\section{Conclusions and Recommendations}

This Final Scientific/Technical Report presented the work performed for the LMR-AMTEC project. The overall objectives of the project were to assess the feasibility, develop a conceptual plant layout and engineering solutions, and determine a range of potential applications for a Novel Integrated Reactor/Energy Conversion System. The main goals of the project were the development of a long life (up to 10 years) LMR coupled with a static energy conversion subsystem comprising an Alkali Metal Thermat to-Electric (AMTEC) topping cycle and a Thermoelectric (TE) Bottom cycle.

The research effort of Westinghouse Electric Company LLC (WEC) on the LMR conceptual design included the evaluation of different reactor design options. These options included direct and indirect couplings of the LMR and the AMTEC/TE converters, the reactor configuration (pool or loop), the coolant types, the alkali metal vapor generation (expanders or alkali metal boilers), the cladding and fuel materials and the structural materials. The performance of these design options were compared and consequently an Indirect Coupling (IC) plant with Alkali Metal Boilers (AMB) was chosen as the reference design because of its superior performance and safety characteristics. The adopted design uses a pool configuration for the reactor, placing the AMBs and the primary pumps in the annulus between the riser and the reactor vessel. The primary coolant is sodium while the secondary coolant is potassium. The refractory alloy $\mathrm{Nb}-1 \mathrm{Zr}$ was selected as the cladding material and a nitride fuel $(\mathrm{U}, \mathrm{Pu}) \mathrm{N}$ was adopted as fuel pellet. The following plant parameters and components were studied and determined: working temperatures, flow rates and pressures, core design (fuel and control rods), alkali metal boiler design and operation, primary pump characteristics, flowinduced vibrations in fuel elements and AMB tubes, corrosion allowance, reactor vessel design, and in-vessel layout. A preliminary economical analysis of the plant was also performed. In addition to the performance studies, safety aspects of the design were analyzed. As part of these activities the reactivity control systems were assessed and the reactivity behavior upon sodium removal was studied. This study showed an acceptable core reactivity response. Finally, a passive residual heat removal system (PHRS) suitable for the high operating temperatures of the LMR-AMTEC was designed. This novel system is based on the coolant level variations inside the reactor vessel. The transient studies showed that the PHRS has enough capacity to passively remove the decay heat by natural circulation.

The work performed by the Institute for Space and Nuclear Power Studies (University of New Mexico) included the design of the AMTEC/TE energy conversion and of the electrical converter modules for different coupling options with the LMR. The energy conversion subsystem consists of an AMTEC top cycle and a TE bottom cycle conductively coupled to the AMTEC condenser. The top cycle uses a vapor-fed, liquid anode AMTEC design, in which the hot alkali metal vapor in the high-pressure cavity is condensed into a porous structure covering the anode side of dome-shaped, composite 
BASE elements. This design essentially eliminates the electrical losses on the anode side, and allows connecting a large number of BASE elements in series, to deliver a voltage output of $\sim 400 \mathrm{~V} \mathrm{DC}$ and electrical power $>40 \mathrm{kWe}$. Furthermore, the efficient heat transfer to the BASE anodes by condensation of the working fluid results in a nearly uniform temperature of the BASE. This in effect increases the converters' efficiency for a given coolant core exit temperature, reduces the induced thermal stresses in the BASE membrane, and increases converter life. Furthermore, the AMTEC/TE converter was designed specifically for cooling by natural convection of air, to extent its usefulness for developing countries or remote areas with limited water resources. An open-unit design configuration was developed for the LMR-AMTEC power plant with Alkali Metal Boilers (AMBs), in which the hot vapor generated by the AMBs condenses inside the porous anodes of the BASE elements, and excess working fluid forms a liquid pool at the bottom of the unit's high-pressure cavity. The fraction of the mass flow rate introduced into the AMTEC which does not diffuse through the BASE membranes, and the condensate are both recirculated back to the AMBs using mechanical pumps.

Laboratory tests were performed at UNM-ISNPS to investigate the breakdown voltage in low-pressure cesium vapor and relate the results to sodium and potassium vapor in the AMTEC converters. A DC electrical breakdown in the low-pressure cavity of the AMTEC would increase the leakage current to the wall, hence degrading the performance of the converter, in addition to potentially damaging the cathode electrodes and the converter wall. The experiments conducted at electrodes temperatures of $1100 \mathrm{~K}$ and $625 \mathrm{~K}$ showed clearly that when the cooler electrode was negatively biased the breakdown voltage was beyond the limit of the power supply used (> $396 \mathrm{~V}$ ). In addition to the potentially very high breakdown voltage $(>400 \mathrm{~V})$, the corresponding discharge current was quite small. Conversely, when the cooler electrode was positively biased the breakdown voltage was in the single figure and followed a Pachen-type dependence on the cesium pressure. For the typical vapor pressures in the low-pressure cavity of an AMTEC of 20-60 Pa, the measured breakdown voltage in the cesium vapor was almost the same as the first ionization potential of cesium $(3.89 \mathrm{~V})$. The corresponding discharge currents at these values of the breakdown voltage were relatively high. Since cesium has the lowest first ionization potential of all alkali metals $(3.89 \mathrm{~V}$ versus $5.14 \mathrm{~V}$ for sodium and $4.34 \mathrm{~V}$ for potassium), and since the effective work function of tantalum in cesium vapor is the lowest, followed by that in potassium, then that in sodium vapor, it is expected that for the same electrodes temperatures and polarity and the sme vapor pressure, the breakdown voltages in potassium and sodium vapors in the low-pressure cavity of an AMTEC would be at least the same, but most likely higher than those reported in this work for cesium vapor. Based on the results of these breakdown experiments, The present AMTEC/TE converter units were designed to deliver more than $50 \mathrm{kWe}$ each at a voltage output of $\sim 400 \mathrm{~V}$ DC and a conversion efficiency $>30 \%$.

The heat rejected by the condenser of the AMTEC flows to the TE bottom cycle, through a conductive coupling arrangement. The electricity generated by the TE bottom cycle, which is cooled by natural convection of ambient air, contributes between $7 \%$ and $14 \%$ of the total electric power generated by the AMTEC/TE converters. The electric power output from the AMTEC units is fed to the grid or the end user separately from that generated by the TE modules. The TE couples are electrically connected in series and in parallel to bring their voltage output to a usable value ( $\sim 350 \mathrm{~V}$ DC), while 
ensuring proper redundancy in the design. To ensure a uniform cold side temperature of the TE couples and for efficient operation and low converter weight, a metallic heat pipe spreader and cooling fins are used. They are charged with water working fluid, to reject the waste heat from the TE bottom cycle efficiently. The fins are cooled by natural convection of air for space or district heating. Three different bottom cycle converter configurations were studied: a P-TAGS-85/2N-PbTe unicouple, a P-BiTe/N-BiTe unicouple, and a segmented TE unicouple (STE) that uses P-TAGS-85/P-BiTe in the Pleg, and $2 \mathrm{~N}-\mathrm{PbTe} / \mathrm{N}-\mathrm{BiTe}$ in the $\mathrm{N}$-leg. A one-dimensional optimization model of segmented TE unicouple was developed, and benchmarked successfully against experimental data from the Jet Propulsion Laboratory. The model determines the optimum lengths of the various segments and the ratio of the legs' cross-sectional areas for maximum efficiency and for maximum electrical power operations. Results showed that the use of the STE in the bottom cycle of the sodium-AMTEC/TE converter would only increase the unit's overall efficiency by $\sim 0.6$ percentage point, and this small gain does not justify the added complexity of the segmented design and associated issues of bonding the different TE materials. Thus, the P-TAGS-85/2N-PbTe unicouple was selected for both the sodium and the potassium converters. The single-segment TE unicouple can deliver peak efficiencies of $4.6 \%$ and $6.4 \%$ at temperature drops of $150 \mathrm{~K}$ and $230 \mathrm{~K}$ across the legs, respectively.

Numerical analysis models of the AMTEC/TE converter unit were developed and used to optimize the design for maximum overall thermodynamic efficiency, compare the performances of the Na- and K-AMTEC/TE converter units, and determine the operation regime in which the static AMTEC/TE converters were load-following. Estimates of the conversion efficiency of the static converters for the LMR-AMTEC power plant showed that values in excess of $30 \%$ could be achieved at operating temperatures of the beta"alumina solid electrolyte (BASE) of $\sim 1000 \mathrm{~K}$ and $\sim 1123 \mathrm{~K}$, for the potassium and sodium working fluids, respectively, based on conservative assumptions regarding the technology of the AMTECs and on using off-the-shelf lead-telluride TE modules. As more advances are made in the development of thin composite BASE membranes, high performance AMTEC electrodes, and the fabrication of reliable metal graded ceramic brazes, higher conversion efficiencies in excess of $35 \%$ for the combined AMTEC/TE converters with a long operation lifetime of 5-10 years, with little degradation, would be possible. Some of these technology advances have been reported very recently, and are being investigated, by industry, universities, and government research laboratories. Recent work done at the Jet Propulsion Laboratory in Pasadena, California, has shown that unlike the TiN electrodes used in the AMTEC cells developed during the space program, the new tungsten/rhodium $\left(\mathrm{WRh}_{1.5}\right)$ electrodes have demonstrated excellent performance properties and long-term stability. Furthermore, efforts to develop mixed-conducting (ionic and electronic) metal/oxide electrodes such as blends of $\mathrm{Mo} / \mathrm{Na}_{\mathrm{x}}-\mathrm{TiO}_{2}$ and $\mathrm{TiN} / \mathrm{Na}_{\mathrm{x}}-\mathrm{TiO}_{2}$ are underway at both the Jet Propulsion Laboratory and Advanced Modular Power systems (AMPS), Inc.. These electrodes can be applied as a paint or slurry, are robust and inexpensive, and have demonstrated to date a performance equivalent to the best currently available refractory electrode $\left(\mathrm{WRh}_{1.5}\right)$.

The interfacing arrangement of the LMR with the converters subsystem developed and investigated by the UNM-ISNPS used an intermediate liquid/liquid heat exchanger (IHX) between the LMR and the AMTEC/TE converter units. The overall 
thermal and electrical performances of the plant were evaluated using a thermal-hydraulic model of the primary and secondary loops of the LMR-AMTEC. In these designs, the secondary sodium or potassium liquid exiting the IHX is partially flash evaporated as it is introduced through an orifice in the high-pressure cavity of each AMTEC/TE converter unit. These studies showed that a $\mathrm{Na} / \mathrm{Na}$ plant (sodium in the primary loop and potassium in the secondary loop) operating at a core exit temperature of $1208 \mathrm{~K}$ could deliver a net power output of $25 \mathrm{MWe}$ at an overall conversion efficiency of $27.7 \%$, while for a $\mathrm{Na} / \mathrm{K}$ plant, which operates at a core exit temperature of $1087 \mathrm{~K}$, the net electrical power output is $25.4 \mathrm{MWe}$ at an overall plant efficiency of $28.6 \%$. In addition, these analyses showed that the K-AMTEC/PbTe converter units deliver higher efficiency (34.9\%) than the NaAMTEC/PbTe converter units (33.6\%). Even though this particular interfacing arrangement between the nuclear reactor and the AMTEC/TE converter units was not selected for the LMR-AMTEC power plant, the following important conclusions could be drawn from these analyses. The higher vapor pressure of potassium compared to sodium allows the nuclear reactor of the LMR-AMTEC plant with K-AMTEC/TE converter units to operate at a core exit temperature that is $\sim 120 \mathrm{~K}$ lower than that in the LMR-AMTEC plant with Na-AMTEC/TE converters, significantly reducing the fuel swelling and the materials compatibility issues, and increasing the reactor and plant operation lifetime. However, a K-AMTEC/PbTe converter unit delivers an electrical power output of 54.3 $\mathrm{kWe}$, which is lower than that delivered by the $\mathrm{Na}-\mathrm{AMTEC} / \mathrm{PbTe}$ converter $(69.5 \mathrm{kWe})$, thus requiring the use of $30 \%$ more converter units in the $\mathrm{Na} / \mathrm{K}$ plant.

The UNM-ISNPS also investigated potential applications of the LMR-AMTEC nuclear power plant for both electrical power and co-generation heat for a variety of uses. Analyses of three LMR plants with an alkali-metal/alkali-metal intermediate heat exchanger (IHX) were performed and examined various co-generation options. The very high total energy utilization of these plants of 95\% includes low-voltage $(\sim 400 \mathrm{~V}) \mathrm{DC}$ electrical power at a net plant efficiency of up to $32.7 \%$, and co-generation options such as residential and industrial space heating at $<400 \mathrm{~K}$, seawater desalination at $365 \mathrm{~K}$, and/or high temperature process heat or steam at $>500 \mathrm{~K}$.

The performance evaluation of the interfacing arrangement with AMBs selected for the LMR-AMTEC power plant was accomplished by Westinghouse Electric Company LLC (WEC) using a plant model, which integrates the LMR and the AMTEC/TE conversion units and calculates different working parameters and main component characteristics. This model was used to determine the optimal design parameters of the plant. The results showed that a moderate electric efficiency could be achieved by design if the core working temperature is maintained over $1000 \mathrm{~K}$. Even so, when the economic aspects of the concept are considered together with the net electric production, the plant shows a poor overall performance. Two reasons explain this poor performance: first, the high capital costs of both the LMR and the AMTEC/TE converter units and, second, the relatively low conversion efficiency of the AMTEC/TE converters when compared with other conversion technologies at equivalent working temperatures such a Rankine cycle. In the case of the LMR, the high capital cost is driven mainly by the conversion efficiency and to a lesser extent by the long life core requirement. In particular, operational requirements of the AMTEC push the LMR design toward a very high working temperature which requires the use of very expensive refractory alloys as structural material. Furthermore, as explained in this report, the use of $\mathrm{Nb}-1 \mathrm{Zr}$ 
deteriorates the neutron economy, hence increasing the fuel cost especially if UN is used $((\mathrm{U}, \mathrm{Pu}) \mathrm{N}$ costs are least sensible to enrichment). In summary the studies showed that the expected cost savings arising from the simplification on the LMR design (no intermediate loop) and the lower operation and maintenance costs are overwritten by costs derived from the high working temperature and the AMTEC/TE capital cost.

From these results, the following recommendations can be made:

- The WEC's plant performance studies showed that, at the present time and in the near term, this concept appears not sufficiently attractive from an economic point of view. Thus the LMR-AMTEC should only be considered as an option for terrestrial electric generation if the capital cost and conversion efficiency are substantially improved.

- In that case the research effort should be oriented toward:

- The evaluation of the use of a less expensive alloy for the fuel cladding. This will allow improving the neutron economy and reducing the reactor capital costs.

- Detailed studies of the fuel rod mechanical and corrosion behavior.

- Further theoretical and experimental studies on mechanics and corrosion behavior of the Alkali Metal Boilers to assess and improve the reliability of the selected design.

- An evaluation on the use of lead-bismuth as primary coolant.

- An evaluation of shorter cycle lengths allowing refueling. This will further reduce the solicitation on the fuel design and the control systems. Also the possibility of reprocessing the fuel should be evaluated.

- The design and testing of a compact AMTEC electric power conversion subsystem, to minimize the capital cost of the converters.

- The design of an electric power conversion system which avoids large electric power losses.

- Fabrication and performance evaluation of the current KAMTEC/TE converter design to confirm the predictions made in this report.

- Simplifying the fabrication and reducing the cost of the converters through the use of mixed metaloxide electrodes, instead of the refractory WRh1.5 electrodes which require clean, well-controlled Glove Boxes, thus representing a measurable fraction of the fabrication cost of the AMTEC units. Mixed-conducting (ionic and electronic) metal/oxide electrodes, such as $\mathrm{Mo} / \mathrm{Nax}-\mathrm{TiO} 2$ or TiN/Nax-TiO2 are currently being investigated with very promising results at both the Jet Propulsion Laboratory and Advanced Modular Power systems (AMPS), Inc.. These electrodes could be simply brushed onto the cathode side of the BASE at room temperature without a need to use Glove Boxes. 
- Developing production procedures and protocol for volume production, which could significantly reduce the fabrication cost by more than 50\%; a good target is $<100-200 \$ / \mathrm{kWe}$ for the AMTEC/TE converter. 


\section{Appendix I: ISNPS's Power Plant Thermal-Hydraulic Model for IC with HXs}

To estimate the pumping power requirements and the overall thermal and electrical performance of the IC plant arrangement with IHXs, thermal-hydraulic loop models of the LMR-AMTEC indirect interfacing arrangement were developed at UNMISNPS. The components of the plant simulated in the model include the nuclear reactor core, IHX, EM-pumps that are 40\% efficient, cold traps, feed headers and collectors, pipes, bends and valves. Also, a model of the coolant flash evaporation in the AMTEC units was developed and coupled to the thermal-hydraulic loop model of the plant. The sub-models of the different components of the power plant were tested and verified individually, and are described in details in the FY2001 Progress Report, Appendix D. An efficient iterative solution procedure was developed to obtain the steady-state coolant pressure and temperature at all locations in the primary and secondary loops of the plant for given operating parameters in each loop.

Analyses were performed to evaluate and compare the overall conversion efficiency and core exit temperature of a $25-\mathrm{MWe}, \mathrm{Na} / \mathrm{Na}$ plant which uses sodium in both the primary and secondary loops, and a $25-\mathrm{MWe}, \mathrm{Na} / \mathrm{K}$ plant which uses sodium in the primary loop and potassium in the secondary loop. Both plants use AMTEC/PbTe converters in the open-unit design configuration. Results, which are summarized in Table I.1, show that the main contributors to reducing the plants' electrical and thermal performance are the electrical power supplied to the electromagnetic pumps and the heat losses by the coolant, as it passes through the cold traps or crystallizers. In order to eliminate metallic and non-metallic impurities, a fraction of the working fluid, typically $0.5 \%$, is circulated through the cold traps. The traps must be cooled to a temperature as low as $\sim 350 \mathrm{~K}$ in order to be effective. Some of that heat, but not all, is recovered by the coolant through an economizer, before exiting the traps. The heat lost from the coolant in the traps is transferred to the auxiliary coolant of the trap. The results showed that the $\mathrm{Na} / \mathrm{Na} 25-\mathrm{MWe}$ plant could deliver an overall conversion efficiency of $27.7 \%$, while operating at a core exit temperature and pressure of $1208 \mathrm{~K}$ and $200 \mathrm{kPa}$, respectively, and core thermal power of $90 \mathrm{MW}$ (Table 2.2). The sodium AMTEC/PbTe converter units operate at a flash evaporator temperature of $1127 \mathrm{~K}$ and a conversion efficiency of $33.6 \%$. The thermal efficiency of the plant is $95.8 \%$, due to the coolant heat losses in the primary (2.4 MW) and secondary (2.1 MW) cold traps, and the electrical efficiency of the plant is only $86.0 \%$, due to the electrical losses in the DC-DC (? DC-DC $=0.97)$, DC-AC $(\eta \mathrm{DC}-\mathrm{AC}=0.96)$ and $\mathrm{AC}-\mathrm{AC}$ transformers $(\eta \mathrm{AC}-\mathrm{AC}=0.95)$, and the electrical power supplied to the primary (158 kWe) and secondary (516 kWe) EM-pumps.

Results also show that the temperatures of the primary and secondary coolants in the $\mathrm{Na} / \mathrm{K}$ plant are generally $120 \mathrm{~K}$ cooler than in the $\mathrm{Na} / \mathrm{Na}$ plant, alleviating material issues and fuel swelling in the core, and increasing lifetime (Table 2.2). In particular, the lower core exit temperature in the $\mathrm{Na} / \mathrm{K}$ plant $(1087 \mathrm{~K})$ allows the primary sodium loop to operate at a pressure below atmospheric $(71.3 \mathrm{kPa}$ at core inlet), a safety advantage in case of a leak in the primary radioactive loop.

The potassium AMTEC units operate at a flash evaporator temperature and pressure of $1006 \mathrm{~K}$ and $80 \mathrm{kPa}$, respectively, and deliver a higher efficiency than the 
sodium AMTEC units operating at $T_{e v}=1127 \mathrm{~K}$ and the same anode pressure, $P_{e v}=80$ $\mathrm{kPa}$, due to lower radiation and conduction heat losses in the converters, as well as lower operating electrical current. Even though the PbTe bottom cycle of the potassium converters is less efficient due to the lower optimum condenser temperature $(527 \mathrm{~K}$ versus $626 \mathrm{~K}$ in the sodium converters), the potassium converters deliver an efficiency of $34.9 \%$ that is 1.3 points higher than that of the sodium converters $(33.6 \%)$. On the other hand, the sodium AMTEC/PbTe converters deliver an electrical power output of 69.5 $\mathrm{kWe}$ each, that is $28 \%$ higher than that delivered by the potassium AMTEC/PbTe converters $(54.3 \mathrm{kWe}$ each), requiring the use of $\sim 30 \%$ more converter units in the $\mathrm{Na} / \mathrm{K}$ plant (a total of 550, versus 425 in the $\mathrm{Na} / \mathrm{Na}$ plant, see Table I.1). As a result, the cost per kilowatt-hour of the $\mathrm{Na} / \mathrm{K}$ plant will be higher than that of the $\mathrm{Na} / \mathrm{Na}$ plant.

Results also show that the IHX can be designed and operated at appropriate primary and secondary flow conditions, such that the temperature difference between the primary coolant at the inlet and the secondary coolant at the outlet is minimal, $<20 \mathrm{~K}$. In the present analyses, this temperature difference is only $12 \mathrm{~K}$ for the $\mathrm{Na} / \mathrm{Na}$ plant, and 13 $\mathrm{K}$ for the $\mathrm{Na} / \mathrm{K}$ plant. Therefore, the use of an IHX causes only a small increase in the core exit temperature. The thermal efficiency of the $\mathrm{Na} / \mathrm{K}$ plant $(97.2 \%)$ is higher than that of the $\mathrm{Na} / \mathrm{Na}$ plant $(95.8 \%)$, since the heat losses in the cold traps of the former are $20 \%$ lower than in the latter, even though the potassium secondary mass flow rate is $62 \%$ higher than the sodium secondary flow rate. The cold traps cool the working fluid to 350 $\mathrm{K}$, and the coolant in the $\mathrm{Na} / \mathrm{K}$ plant enters the traps at a temperature that is $\sim 120 \mathrm{~K}$ cooler than in the $\mathrm{Na} / \mathrm{Na}$ plant. The pressure losses in the potassium secondary loop, however, are higher than in the sodium secondary loop, and the potassium EM-pumps require about twice as much electrical power than the sodium pumps (1.14 MWe versus $0.52 \mathrm{MWe})$, causing the electrical efficiency of the $\mathrm{Na} / \mathrm{K}$ power plant $(84.3 \%)$ to be lower than that of the $\mathrm{Na} / \mathrm{Na}$ plant $(86.0 \%)$.

In summary, the $\mathrm{Na} / \mathrm{K}$ plant with liquid-liquid IHXs, which uses a total of $550 \mathrm{~K}$ AMTEC/PbTe converter units, may operate at a core exit temperature and pressure of $1087 \mathrm{~K}$ and $65 \mathrm{kPa}$, respectively, and delivers a net electrical power output of $25.4 \mathrm{MWe}$ at an overall plant efficiency of $28.6 \%$. By contrast, the $\mathrm{Na} / \mathrm{Na}$ plant uses a total of 425 $\mathrm{Na}$-AMTEC/PbTe converter units, operates at $T_{\text {core }}^{e x}=1208 \mathrm{~K}$ and $P_{\text {core }}^{e x}=200 \mathrm{kPa}$, and delivers 25.0 MWe at an overall efficiency of 27.7\%; this efficiency is about 1 point lower than that of the $\mathrm{Na} / \mathrm{K}$ plant. Therefore, the $\mathrm{Na} / \mathrm{K}$ plant is expected to have a longer operation life, but a higher cost per kilowatt-hour than the $\mathrm{Na} / \mathrm{Na}$ plant. These results clearly show the advantage of using potassium as the working fluid for the AMTEC top cycle, compared to using sodium. Also, the LMR-AMTEC plants with liquid-liquid IHXs exhibit a relatively high temperature difference between core exit and converters' BASE elements of $\sim 80 \mathrm{~K}$ (Table 2.2). This temperature difference may be reduced somewhat by substituting AMBs in place of the IHXs in the LMR-AMTEC plant. 
Table I.1- Performance Comparison of 25-MWe, $\mathrm{Na} / \mathrm{Na}$ and $\mathrm{Na} / \mathrm{K}$ power plants.

\begin{tabular}{|c|c|c|c|}
\hline \multicolumn{2}{|c|}{ Performance parameter } & \multirow{3}{*}{$\begin{array}{c}\mathrm{Na} / \mathrm{Na} \text { plant } \\
25 \times 17=425 \\
69.5 \mathrm{kWe}\end{array}$} & \multirow{3}{*}{$\begin{array}{c}\mathrm{Na} / \mathrm{K} \text { plant } \\
25 \times 22=550 \\
54.3 \mathrm{kWe}\end{array}$} \\
\hline Number of converter units & $N=N_{M O D} \times N_{U N I T}$ & & \\
\hline Brut power output per unit & $P e_{U N I T}$ & & \\
\hline \multicolumn{2}{|l|}{ Primary/secondary coolant } & $\mathrm{Na} / \mathrm{Na}$ & $\mathrm{Na} / \mathrm{K}$ \\
\hline Core thermal power & $Q_{\text {core }}$ & 90.3 MW & 88.8 MW \\
\hline Core mass flow rate & $\dot{m}_{P}$ & $983.9 \mathrm{~kg} / \mathrm{s}$ & $966.2 \mathrm{~kg} / \mathrm{s}$ \\
\hline Core temperature rise & $\Delta T_{\text {core }}$ & $72 \mathrm{~K}$ & $72 \mathrm{~K}$ \\
\hline Core exit temperature & $T_{\text {core }}^{e x}$ & $1208 \mathrm{~K}$ & $1087 \mathrm{~K}$ \\
\hline Core exit pressure & $P_{\text {core }}^{e x}$ & $200 \mathrm{kPa}$ & $65 \mathrm{kPa}$ \\
\hline Loss through primary traps & $Q_{P}^{\text {traps }}$ & $2.40 \mathrm{MW}$ & $1.96 \mathrm{MW}$ \\
\hline IHX thermal power & $Q_{I H X}$ & $88.1 \mathrm{MW}$ & 87.0 MW \\
\hline Secondary-loop flow rate & $\dot{m}_{S}$ & $918.9 \mathrm{~kg} / \mathrm{s}$ & $1488 \mathrm{~kg} / \mathrm{s}$ \\
\hline IHX temperature rise & $\Delta T_{I H X}^{S}$ & $75 \mathrm{~K}$ & $75 \mathrm{~K}$ \\
\hline IHX exit temperature & $T_{I H X}^{e x, S}$ & $1196 \mathrm{~K}$ & $1074 \mathrm{~K}$ \\
\hline IHX exit pressure & $P_{I H X}^{e x, S}$ & 179 kPa & $179 \mathrm{kPa}$ \\
\hline AMTEC flash evaporator & $T_{e v} / P_{e v}$ & $1127 \mathrm{~K} / 80 \mathrm{kPa}$ & $1006 \mathrm{~K} / 80 \mathrm{kPa}$ \\
\hline AMTEC condenser & $T_{c d}$ & $626 \mathrm{~K}$ & $527 \mathrm{~K}$ \\
\hline \multicolumn{2}{|c|}{ Fraction of flow diffusing through BASE } & $1.1 \%$ & $1.4 \%$ \\
\hline Loss through secondary traps & $Q_{S}^{\text {traps }}$ & $2.10 \mathrm{MW}$ & $1.80 \mathrm{MW}$ \\
\hline Power to primary pumps & $P e_{P}^{p u m p s}$ & $0.16 \mathrm{MW}$ & $0.13 \mathrm{MW}$ \\
\hline Power to secondary pumps & $P e_{S}^{\text {pumps }}$ & $0.52 \mathrm{MW}$ & $1.14 \mathrm{MW}$ \\
\hline Net electrical power output & $P e_{N E T}^{\text {plant }}$ & $25.0 \mathrm{MW}_{\mathrm{e}}$ & $25.4 \mathrm{MW}_{\mathrm{e}}$ \\
\hline Overall plant efficiency & $\eta_{\text {plant }}$ & $27.7 \%$ & $28.6 \%$ \\
\hline Converters' efficiency & $\eta_{U N I T}$ & $33.6 \%$ & $34.9 \%$ \\
\hline Thermal efficiency & $\eta_{t h}$ & $95.8 \%$ & $97.2 \%$ \\
\hline Electrical efficiency & $\eta_{\text {elec }}$ & $86.0 \%$ & $84.3 \%$ \\
\hline
\end{tabular}


[ This page intentionally blank ] 